\title{
PHYSICAL, CHEMICAL, AND BIOLOGICAL DATA FOR DETAILED STUDY OF IRRIGATION DRAINAGE IN THE KLAMATH BASIN, CALIFORNIA AND OREGON, 1990-92
}

By Dorene E. MacCoy

U.S. GEOLOGICAL SURVEY

Open-File Report 93-497

U.S. GEOLOGICAL SURVEY

U.S. FISH AND WILDLIFE SERVICE

U.S. BUREAU OF RECLAMATION

U.S. BUREAU OF INDIAN AFFAIRS

8
$\delta$
$\delta$
$\delta$ 


\title{
U.S. DEPARTMENT OF THE INTERIOR BRUCE BABBITT, Secretary
}

\author{
U.S. GEOLOGICAL SURVEY \\ ROBERT M. HIRSCH, Acting Director
}

Any use of trade, product, or firm names in this publication is for descriptive purposes only and does not imply endorsement by the U.S. Government.

U.S. Geological Survey

Earth Science Information Center

Open-File Reports Section

Box 25286, MS 517

Denver Federal Center

Denver, CO 80225

For additional information write to:

District Chief

U.S. Geological Survey

Federal Building, Room W-2233

2800 Cottage Way

Sacramento, CA 95825 


\section{CONTENTS}

$\begin{array}{cc}\text { Abstract } \mathbf{1} & \\ \text { Introduction } \mathbf{1} & \\ \text { Background } \mathbf{1} & \\ \text { Purpose and scope } & \mathbf{3} \\ \text { Acknowledgments } & \mathbf{3}\end{array}$

Study area $\mathbf{3}$

Sampling sites $\mathbf{3}$

Sample collection, preservation, and analysis $\mathbf{8}$

Sediment 8

Water 8

Biota 9

Compilations of physical, chemical, and biological data 11

References cited 34

\section{FIGURES}

1-5. Maps showing:

1. Location of Klamath Basin study area 2

2. Tule Lake National Wildlife Refuge, agricultural area, and frog call survey sites 4

3. Lower Klamath National Wildlife Refuge, agricultural area, and frog call survey sites $\mathbf{5}$

4. Tule Lake upper sump leaseland waterway sampling sites 6

5. Tule Lake lower (south) sump leaseland waterway sampling sites 7

6. Diagram of in situ test chamber 11

7. Graphs showing diel $\mathrm{pH}$, temperature, and dissolved-oxygen data from selected sites in Klamath Basin, 199112

8. Graphs showing diel specific conductance, $\mathrm{pH}$, temperature, and dissolved-oxygen data from selected sites in Klamath Basin, 199217

\section{TABLES}

1. Surface-water sampling sites and types of samples collected at each site, 1990-92 38

2. Principal biological tissue sampling locations, 1990-92 41

3. Concentrations of carbon and organochlorine compounds in bottom sediment, 199044

4. Particle-size distribution in bottom sediment, 199046

5. Moisture content, arsenic, mercury, and chlorophenoxy acid, organochlorine, organophosphate, and carbamate pesticides in bottom sediment, $1992 \mathbf{4 6}$

6. Field measurements of $\mathrm{pH}$ and dissolved oxygen in surface water from sediment-collection sites, 199047

7. Field and laboratory measurements of selected properties and constituents in surface water from primary and additional sampling sites, 1991-92 $\mathbf{4 8}$

8. Water-quality data from frog call survey sites, 1991-92 $\mathbf{7 6}$

9. Concentrations of arsenic and mercury in surface water from selected sites in Lower Klamath National Wildlife Refuge, February 28, 199178

10. Concentrations of total and dissolved nitrogen, phosphorus, and organic carbon in surface water, 1991-92 79

11. Concentrations of major dissolved constituents in surface water, 199287

12. Concentrations of pesticides and related compounds detected in surface water, 1991-92 
13. Concentrations of acrolein $\left(\right.$ Magnicide- $\mathrm{H}^{\circledR}$ ) in sites downstream from an acrolein application in the $\mathrm{J}-7$ Canal, July 199293

14. Concentrations of methamidophos recovered from deposition samplers placed in leaseland waterways at Tule Lake National Wildlife Refuge, 199293

15. Concentrations of trace elements in blue-green algae (Aphanezomenon flos-aquae) samples, September 199193

16. Moisture content and concentrations of arsenic, mercury, and selenium in biological tissue samples, 1990-92 94

17. Moisture content, lipid content, and concentrations of organochlorine compounds in biological tissue samples, 1990-92 97

18. Cholinesterase (ChE) activity of waterfowl brain tissue collected, 1991-92 103

19. Eggshell thickness of avian eggs collected, 1990-92 104

20. Benthic invertebrate surveys, 1990-91 105

21. Water-column invertebrate surveys, $1992 \mathbf{1 2 4}$

22. Frog call surveys, 1991-92 $\mathbf{1 3 0}$

23. Fish surveys, 1991-92 132

24. Fish health surveys of native fathead minnows, 1991-92 135

25. Microtox $^{\circledR}$ sediment (solid phase) bioassay, 1991138

26. Microtox $^{\circledR}$ sediment porewater bioassay, $1991 \quad 138$

27. Microtox $^{\circledR}$ sediment porewater bioassay, 1992139

28. Aquatic plant, invertebrate, and vertebrate laboratory and in situ bioassays, 1991-92 140

29. Sodium chloride positive-control bioassays, 1991-92 148

30. Frog embryo (Xenopus laevis) positive-control bioassays, 1991-92 149

31. Chironomus tentans sediment bioassay, $1991 \mathbf{1 5 0}$

32. Chironomus tentans sediment bioassay, $1992 \mathbf{1 5 3}$

33. Mallard duckling bioassay, $1991 \mathbf{1 5 5}$

34. In situ bioassay in sites downstream from an acrolein (Magnicide- $\mathrm{H}^{\circledR}$ ) application in the J-7 Canal, July 13-14, 1992165

35. In situ bioassay in leaseland waterways at Tule Lake National Wildlife Refuge, $1992 \mathbf{1 6 6}$

36. Mallard duckling bioassay in leaseland waterways at Tule Lake National Wildlife Refuge, 1992168

\section{Conversion Factors and Abbreviations}

\begin{tabular}{rll}
\hline Multiply & By & To obtain \\
\hline centimeter $(\mathrm{cm})$ & 0.3937 & inch \\
gram $(\mathrm{g})$ & 0.03527 & ounce avoirdupois \\
kilometer $(\mathrm{km})$ & 0.6214 & mile \\
millimeter $(\mathrm{m})$ & 0.03937 & inch \\
\hline
\end{tabular}

Temperature is given in degrees Celsius $\left({ }^{\circ} \mathrm{C}\right)$, which can be converted to degrees Fahrenheit $\left({ }^{\circ} \mathrm{F}\right)$ by the following equation:

$$
{ }^{\circ} \mathrm{F}=1.8\left({ }^{\circ} \mathrm{C}\right)+32
$$

\footnotetext{
Abbreviations

$\mu \mathrm{m}$ - micrometer

mg - milligram

$\mathrm{mL}$ - milliliter
} 


\title{
PHYSICAL, CHEMICAL, AND BIOLOGICAL DATA FOR DETAILED STUDY OF IRRIGATION DRAINAGE IN THE KLAMATH BASIN, CALIFORNIA AND OREGON, 1990-92
}

\author{
By Dorene E. MacCoy
}

\begin{abstract}
Physical, chemical, and biological data were collected between 1990 and 1992 as part of a detailed study by the U.S. Department of the Interior of the effects of irrigation drainage on aquatic resources in the Klamath Basin of California and Oregon. Most of the sites for data collection were in and around the upper and lower sump of Tule Lake, in Tule Lake National Wildlife Refuge, and along major drains in Lower Klamath National Wildlife Refuge.
\end{abstract}

The physical and chemical data consist of particle-size determinations and concentrations of carbon, arsenic, mercury, and chlorophenoxy acid, organochlorine, organophosphate, and carbamate pesticides in bottom sediment; and concentrations of organophosphate, carbamate, and pyrethroid pesticides, major and trace inorganic constituents, nitrogen, phosphorus, and organic carbon in water. Continuous dissolved oxygen, $\mathrm{pH}$, specific conductance, and temperature data from selected sites in 1991 and 1992 are presented in graphical form to summarize the diel water-quality conditions. The biological data consist of concentrations of inorganic constituents and organochlorine pesticides in tissue, invertebrate and fish surveys, fish health surveys, frog call surveys, eggshell thickness of avian eggs, and in situ and laboratory toxicity bioassay data collected in 1991 and 1992 using aquatic bacteria, plants, invertebrates, fish, frog, and bird species as test organisms.

\section{INTRODUCTION}

\section{BACKGROUND}

In response to increasing concern about the effects of contaminated irrigation drainwater on human health, fish, and wildlife, the U.S. Department of the Interior established the Irrigation Water Quality Program to investigate irrigation-related water-quality problems at National Wildlife Refuges (NWR) and migratory bird use areas potentially affected by irrigation projects constructed or managed by the Department of the Interior. The Klamath Basin (fig. 1) was one of the areas chosen for investigation. A reconnaissance-level study completed in 1988 identified elevated trace-element concentrations in local areas and potential problems related to pesticide contamination and water-quality degradation in highly eutrophic waters (Sorenson and Schwarzbach, 1991).

A more detailed follow-up study was begun in 1990. The objective of the detailed study was to determine the extent, severity, and magnitude of irrigation-induced water-quality problems caused by pesticide contamination, eutrophic conditions, and trace-element contamination in the irrigation system. The study was also designed to determine sources and environmental fate of identified contaminants.

Three basic approaches were used to evaluate the existence and effect of water-quality problems: (1) Measurements were made to determine water-quality characteristics and the presence of chemical contaminants in the environment and biota that could cause adverse effects to living organisms; (2) toxicity of 


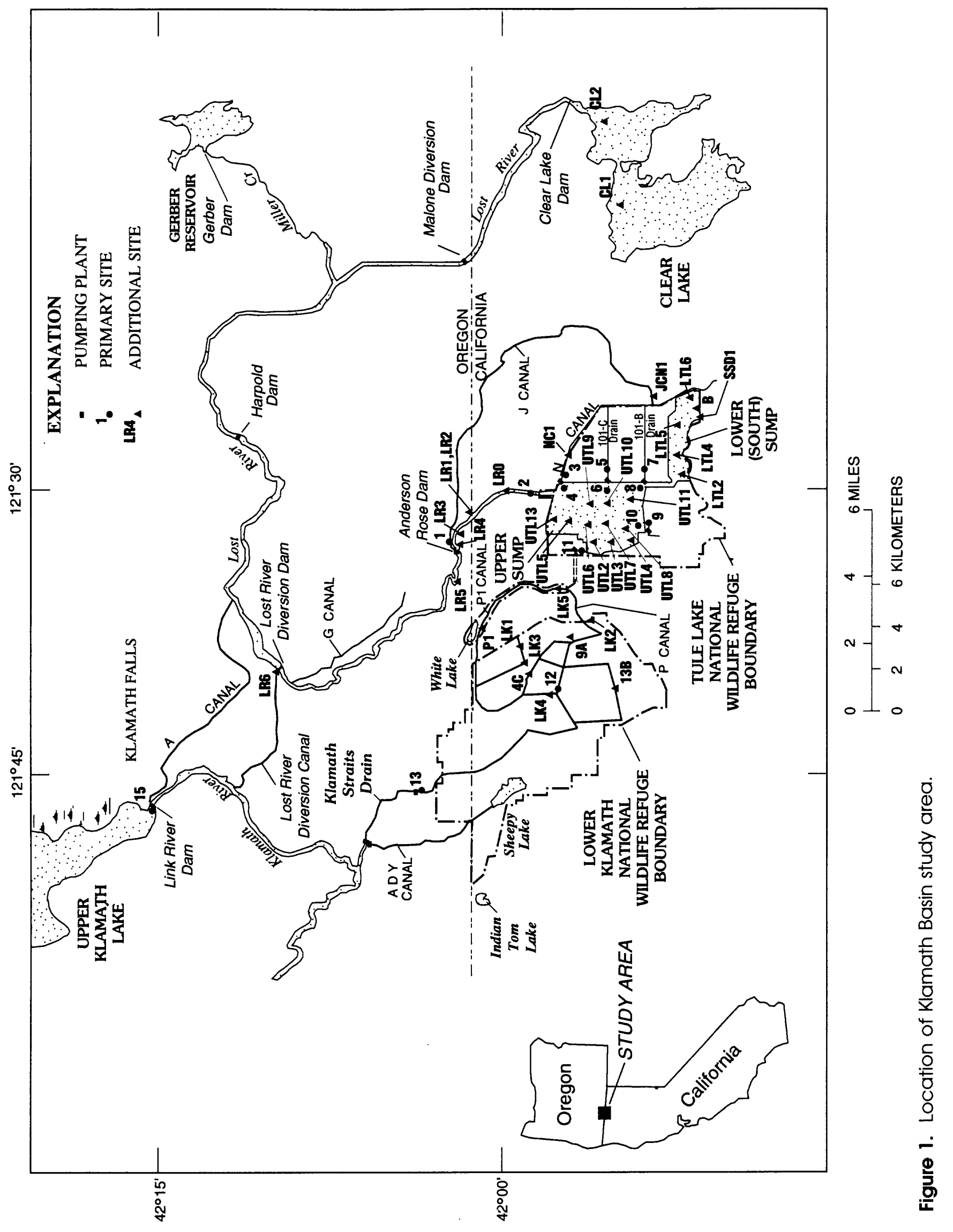


water and sediment were measured using bioassay techniques on a variety of test organisms; and (3) assessments of resident biological communities were made to evaluate the health of the ecosystem exposed to drainwater. Data from each of these approaches complement one another and contribute to a comprehensive evaluation of water-quality problems in the Klamath Basin.

The study was done by research teams from the U.S. Geological Survey (USGS) and the U.S. Fish and Wildlife Service (USFWS) with support from the U.S. Bureau of Reclamation and the U.S. Bureau of Indian Affairs.

\section{PURPOSE AND SCOPE}

The purpose of this report is to present, in tabular and graphical form, the data that were collected during the 1990-92 study of irrigation drainwater in the Klamath Basin. This report contains physical, chemical, and biological data from water-quality and biological monitoring sites in the Tule Lake and Lower Klamath National Wildlife Refuges (fig. 1), as well as the major tributaries supplying water to the area.

The method detection limits for chemical analysis are given in the tables using the "less than" $(<)$ sign. These limits vary according to the date, type of analysis, and sensitivity of the instrumentation. The determination of the method detection limit by the USGS National Water Quality Laboratory (NWQL) can be found in the Code of Federal Regulations (1990).

The method validation for the analysis of pesticides and related compounds in surface water has been completed and is in the process of being approved by the NWQL. All other analytical methods used by the NWQL have been validated and approved (Fishman and Friedman, 1985; Wershaw and others, 1987).

\section{ACKNOWLEDGMENTS}

-

The author would like to acknowledge the following individuals for their contributions to the collection and preparation of data during the 3 years of study in the Klamath Basin: Staci Kawaguchi, Darian LaBrie, Deblyn Mead, and Stephen Sorenson, of the USGS; John Henderson, Elizabeth Materna, Thomas Maurer, Steven Schwarzbach, and Judy Sefchick of the
USFWS; Jewel Bennett, Robin Boyer, Therese Littleton, and Meri Moore of the Washington Cooperative Fish and Wildlife Research Unit; and volunteers Chetna Acharya, Stephanie Ciccarello, Robin McWilliams, Karin Podlesch, and Nina Woodgate from the Student Conservation Association.

\section{STUDY AREA}

The Klamath Basin study area (fig. 1) is located on the border between California and Oregon and includes part of the Klamath River Basin and all of the Lost River Basin. This study was done in two key wildlife areas-the Tule Lake and Lower Klamath National Wildlife Refuges (figs. 2 and 3). Also included in the study area is the outlet for Upper Klamath Lake at the A Canal and sites in Clear Lake and the Lost River (fig. 1). Upper Klamath Lake and Clear Lake provide the majority of irrigation water to the Klamath Basin. These lakes and waterways are important for migratory waterfowl that use the Pacific flyway and provide habitat for two endangered fish, the shortnose sucker and Lost River sucker.

Within the Klamath Basin is the Klamath Basin Irrigation Project administered by the U.S. Bureau of Reclamation. This project was started in 1905 to drain and reclaim the lake bottoms of Tule and Lower Klamath Lakes for agricultural use. This was accomplished through the construction of a series of canals and drains that provided flood control and irrigation water to the newly reclaimed land. In 1964, homesteading of reclaimed land was discontinued and the remaining wetland areas of Tule and Lower Klamath Lakes were preserved as USFWS National Wildlife Refuges. Part of the land now included in the refuges continues to be farmed under a lease agreement with the Bureau of Reclamation, a situation unique in the national wildlife refuge system.

\section{SAMPLING SITES}

The principal data-collection sites for the detailed study are given in table 1. Site locations are shown in figures 1, 4, and 5. Sampling locations and sample numbers for biological tissue collected in 1990-92 are given in table 2; these locations have no corresponding site numbers but they can be located in figures 1,2 , and 3 . Sites where frog call surveys were done are shown in figures 2 and 3 .

In 1990-91, bottom-sediment and benthic invertebrate samples were collected in and around Tule Lake 


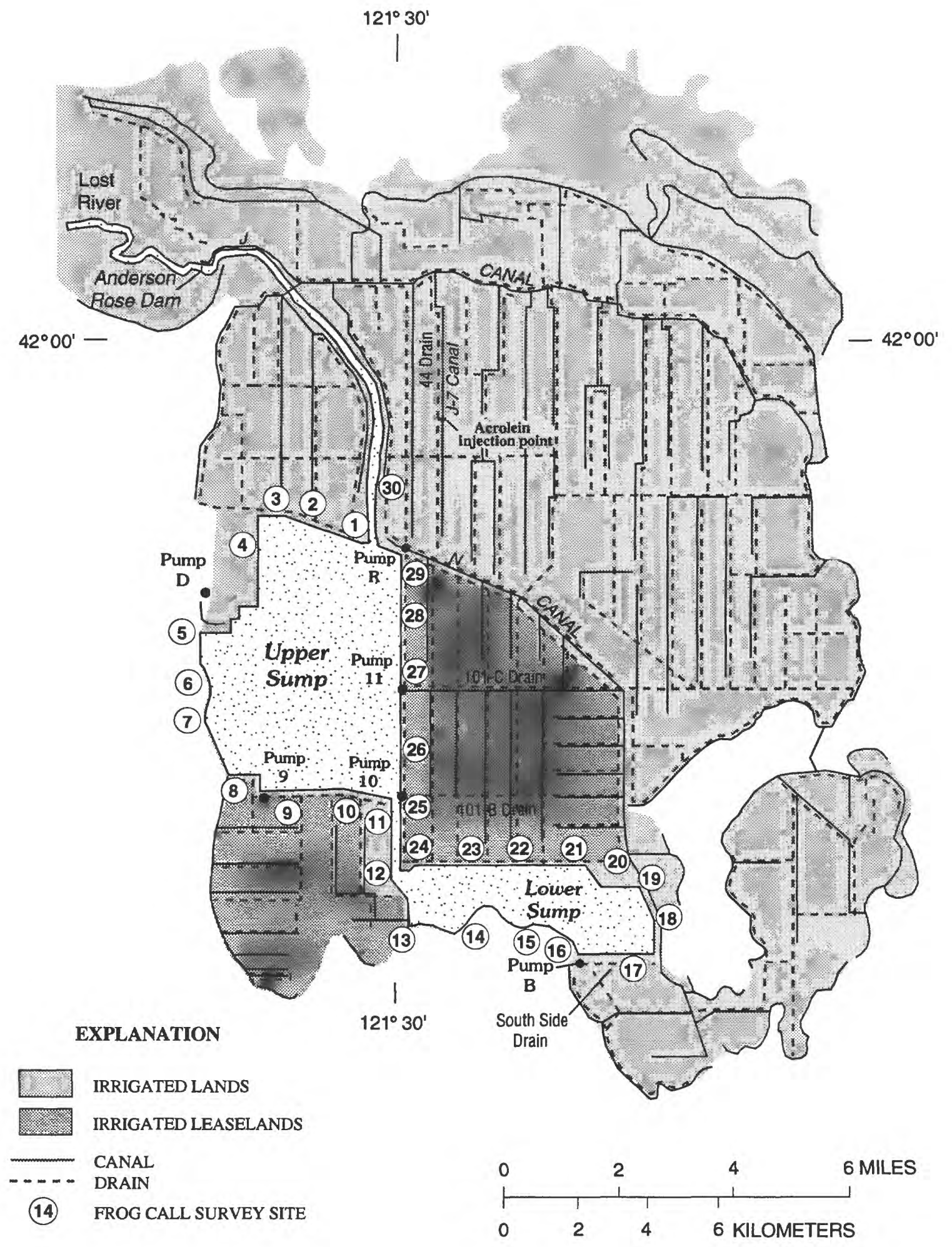

Figure 2. Tule Lake National Wildlife Refuge, agricultural area, and frog call survey sites. 


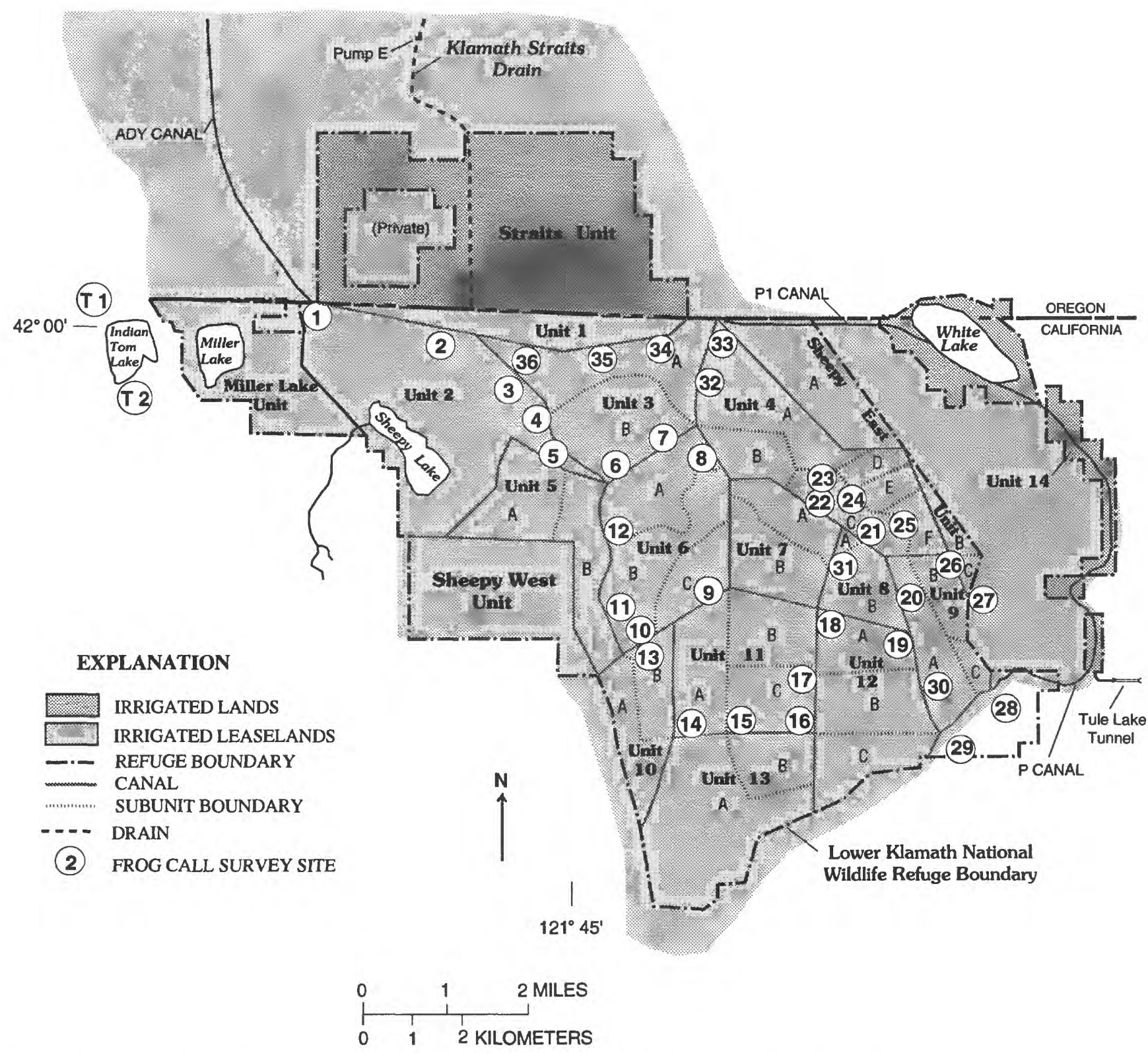

Figure 3. Lower Klamath National Wildlife Refuge, agricultural area, and frog call survey sites.

and Lower Klamath Refuges; a more intense monitoring of surface water and bioassays began in June 1991. The water was sampled weekly during June, July, and August (13 weeks) in 1991 and 1992. These months represent the peak agricultural irrigation season and the period of greatest eutrophic water conditions.

Water and sediment were sampled at strategic locations along the irrigation system to identify potential gradients in water quality and toxicity. There were three categories of sampling sites: (1) Above most agricultural inputs (sites 1, 2, and, in 1992, 15); (2) where irrigation return flow from agricultural fields enters Tule Lake upper sump (sites 3, 4, 5, 6, 7, 8, 9, and 10); and (3) at outflows from Tule Lake and Lower Klamath Refuges (sites 11, 12, and 13). Of these sites, nine were sampled in 1991 and ten in 1992 on a weekly basis (sites $1,2,3,5,7,9,11,12$, 13 , and, in 1992, site 15). Also in 1992, water-quality data were collected in the irrigated leaselands of Tule Lake NWR in waterways adjacent to fields; the general area of these collection sites is shown in figure 2 and the individual sites are shown in figures 4 and 5 . 




Figure 4. Tule Lake upper sump leaseland waterway sampling sites. 


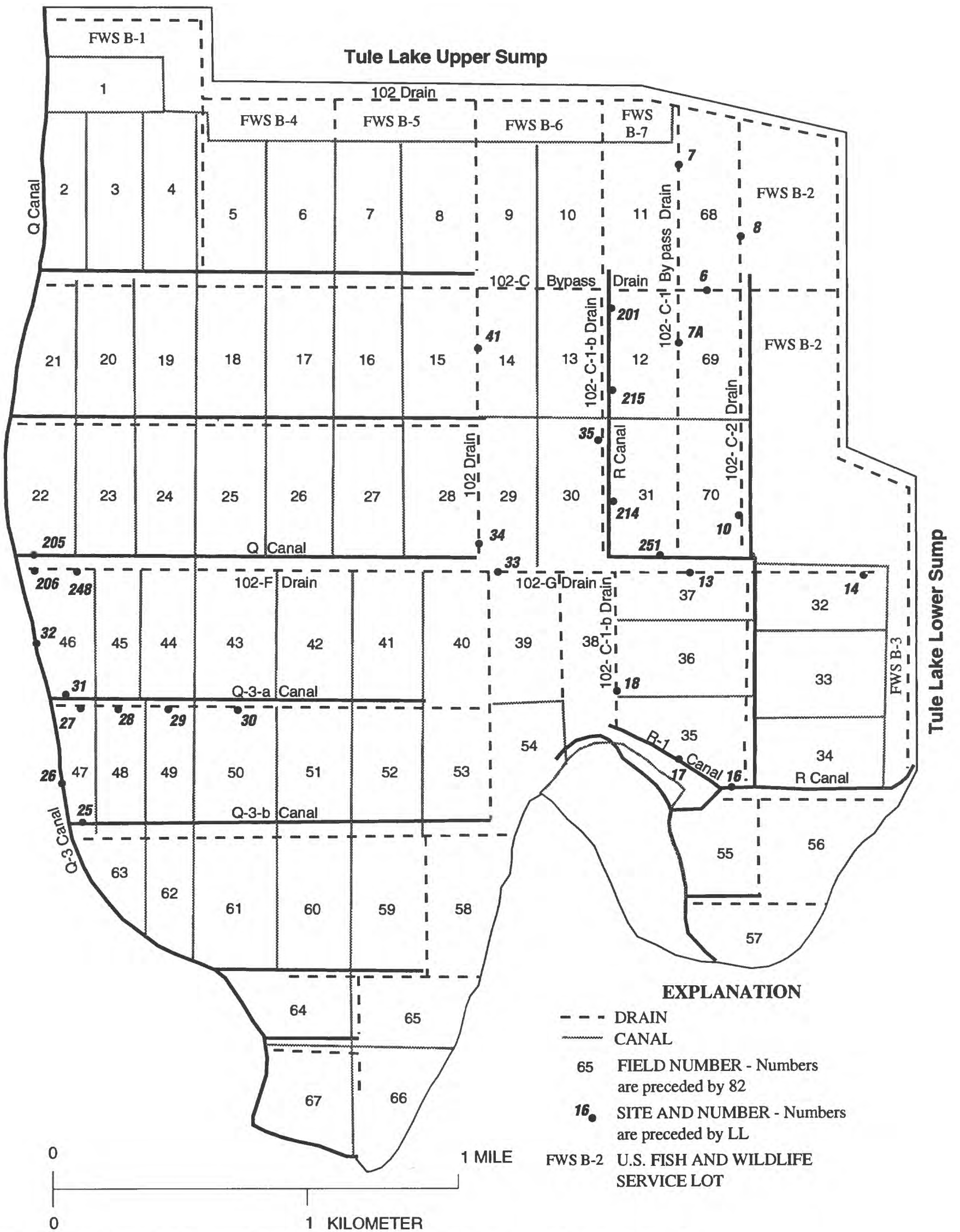

Figure 5. Tule Lake lower (south) sump leaseland waterway sampling sites. 
SAMPLE COLLECTION, PRESERVATION, AND ANALYSIS

The field and laboratory procedures used for measurement of water quality (specific conductance, $\mathrm{pH}$, water temperature, and dissolved oxygen) and collection, preservation, and analysis of samples of surface water, bottom sediment, and biota were similar to those used during the 1988-89 reconnaissance investigation (Sorenson and Schwarzbach, 1991). USGS quality-assurance practices were followed for all surface-water and sediment samples sent to the USGS NWQL (Friedman and Erdman, 1982). Additions to those procedures and sampling frequency are indicated below.

\section{SEDIMENT}

Bottom sediment was collected using a stainless steel Ekman grab sampler and bucket. Three grabs at each site were composited. Four subsamples for Microtox ${ }^{\circledR}$ bioassay (Microbics Corporation, 1988a), chironomid bioassay, organic analyses, and inorganic analyses were placed in 125-mL jars or whirlpak bags (inorganics). Porewater was removed from sediment for the Microtox ${ }^{\circledR}$ bioassay by centrifuge (in 1991 and 1992) or vacuum filtration through an air stone (in 1991).

Bottom-sediment samples were analyzed for organic and inorganic compounds using analytical procedures based on U.S. Environmental Protection Agency (1986 and 1991), U.S. Fish and Wildlife Service (1989a), Hazelton Laboratories America, Inc. (1991a,b), and other internal laboratory procedures approved and monitored by U.S. Fish and Wildlife Service Patuxent Analytical Control Facility.

\section{WATER}

Water-quality measurements (specific conductance, $\mathrm{pH}$, water temperature, and dissolved oxygen) were made with handheld instruments up to three times a week, and more frequently during special studies, at each sampling site. Diel fluctuations in specific conductance, $\mathrm{pH}$, water temperature, and dissolved oxygen at the primary sampling sites were monitored continuously during select in situ bioassays done in 1991 and 1992. Four monitors were rotated systematically to different sampling sites each week. Recorded data were recovered and the monitoring instruments calibrated prior to redeployment. The procedures used for set-up, calibration, and data recovery of the monitors can be found in the equipment operating manuals (Hydrolab Corporation, 1988 and 1991).

In 1991, water samples were analyzed for total and dissolved arsenic and mercury. Water samples for analysis of concentrations of nitrogen, phosphorus, organic carbon, and major dissolved constituents were taken at three separate times throughout the 1992 sampling season, using USGS sampling and preservation techniques (Ward and Harr, 1990) and analyzed at the NWQL. Samples were analyzed in the field for total ammonia in 1991 and 1992 using an ion-selective electrode (method described by Fishman and Friedman, 1985).

Water was sampled in 1991 and 1992 to determine concentrations of pesticides and related compounds using the solid-phase extraction method (Sandstrom and others, 1992). Only a few selected samples were chosen for analysis in 1991. Each week in 1992, three samples were randomly selected and up to three more samples were also analyzed if there was high bioassay mortality. Water was filtered through pre-ashed $0.7-\mu \mathrm{m}$, glass fiber filters, then pumped through cartridges packed with $500 \mathrm{mg}$ of silica with an octadecylsilane (C-18) phase covalently bonded to the silica surface. Most compounds of interest bond with the silica particles. The cartridges were eluted at the NWQL and samples analyzed using a gas chromatographic/mass spectrophotometric method (Steven D. Zaugg, U.S. Geological Survey, written commun., 1993).

A one-time fate study on the herbicide acrolein (Magnicide- $\mathrm{H}^{\circledR}$ ) was done in July 1992 along the 44 Drain and the J-7 and NCanals at the north end of Tule Lake Refuge (fig. 2). The purpose of this study was to determine if the herbicide persists in drainwater and whether it is transported to the refuge boundaries. The Endangered Species Consultation on use of acrolein in the Klamath Basin (U.S. Fish and Wildlife Service, 1989b) resulted in prohibiting the use of this herbicide on refuge land, but it is used extensively to clear vegetation from the drains and canals surrounding the refuge. Sites were selected downstream of the injection point of the herbicide (fig. 2) and sampled at regular intervals to determine the time and concentration of dispersement. Thirteen $40-\mathrm{mL}$ water samples were collected and preserved with hydrochloric acid to a $\mathrm{pH}$ of 4 . The samples were packed in ice and shipped to the NWQL and analyzed using the NWQL volatiles method. In this method, volatile organic compounds are purged from the sample matrix by bubbling helium through a $25-\mathrm{mL}$ aqueous 
sample. The analytes are trapped in a tube containing suitable sorbent materials and then thermally desorbed onto a megabore capillary column. The gas chromatograph is temperature-programmed to separate the analytes, which then are detected by the mass spectrometer. Target compound identifications are confirmed by analyzing standard reference materials under the same conditions as the samples, comparing retention times and mass spectra (Donna L. Rose, U.S. Geological Survey, written commun., 1993).

In 1992, deposition samplers (drift cards) were placed in and around leaseland waterway sampling sites (figs. 4 and 5). The drift cards were chemically clean, 7.0-cm-diameter cellulose filter paper. Water samples and drift cards were sent to the USFWS Patuxent Analytical Control Facility for analysis of methamidophos, EPN, acephate, aldicarb, azinphosmethyl, carbaryl, carbofuran, chlorpyrifos, coumaphos, and demeton. Methamidophos was the only compound detected in samples from leaseland waterway sampling sites. Methamidophos was detected at three sites on drift cards but not in sample water.

Daphnia magna and mallard duckling in situ bioassays also were done in these waterways using methods described below. These tests were done to determine if drift from aerial pesticide applications entered drainwater and was hazardous to nontarget aquatic organisms. Daphnia magna, mallard ducklings, and drift cards were placed in irrigation drains and canals adjacent to selected agricultural fields prior to aerial sprays. Water-quality measurements were taken at the time of deployment and retrieval of the in situ bioassays. This monitoring effort served to describe drainwater conditions and organism survival at sites adjacent to potato and grain fields.

\section{BIOTA}

Samples of the biota were collected for analysis of trace elements and pesticides. Blue-green algae, invertebrate composite samples (at least $10 \mathrm{~g}$ ), and fish composite samples (at least $20 \mathrm{~g}$ ) were collected and placed in clean containers. Bird eggs were hand collected, chilled prior to harvesting, and placed in clean glass jars. All samples were frozen prior to shipment.

Biological samples were analyzed for trace elements by one of the following laboratories under contract with the USFWS Patuxent Analytical Control Facility: Hazelton Laboratories America, Inc., Madison, Wisconsin; Environmental Trace Substances
Research Center, Columbia, Missouri; and Research Triangle Institute, Research Triangle Park, North Carolina. The analytical procedures were similar to those used in the reconnaissance study (Sorenson and Schwarzbach, 1991). The laboratories analyzed the samples for arsenic and selenium using hydridegeneration or graphite-furnace atomic absorption spectroscopy, and for mercury by cold vapor reduction. Other trace elements were analyzed by inductively coupled plasma atomic spectroscopy. Organochlorine pesticides and polychlorinated biphenyls were analyzed in invertebrate, fish, and avian-egg tissue using packed, capillary, or megabore column, electron-capture gas chromatography. Laboratory quality control was assured through the Patuxent Analytical Control Facility. The precision and accuracy of the laboratory analyses were confirmed with procedural blanks, duplicate analyses, test recoveries of spiked materials, and reference material analyses. Differences in significant figures in some tables result from use of different laboratories, analysis completed at different times, variation in tissue type and quantity, and sensitivity of instruments used.

Waterfowl carcasses were collected and brains extracted and analyzed for cholinesterase (ChE) activity using spectrophotometric methods developed by Hill and Fleming (1982).

Eggshell thickness was determined by taking measurements at three locations around the equator of each half shell using a micrometer with rounded contacts. The reported thickness is the average of the six measurements of the whole egg (U.S. Fish and Wildlife Service, 1990).

During 1992, water-column invertebrate communities were surveyed biweekly at primary sampling sites $1,2,3,5,7,9,11,12,13$, and 15 . Collection, handling, and subsampling procedures were based on methods described by Plafkin and others (1989) for rapid bioassessment of macroinvertebrates. Collections were made by duplicate draws of a student plankton net through the entire water column. The net was made of $80-\mu \mathrm{m}$ mesh equipped with a stainless steel Clarke-Bumpus plankton bucket with an 86$\mu \mathrm{m}$ mesh sieve. All organisms were preserved in 10percent ethanol in the field and then subsampled and identified in the laboratory. Subsampling entailed distributing the entire sample evenly over the surface of a gridded pan. Grids were randomly selected and all organisms within those grids removed until approximately 100 organisms were removed for identification. In cases in which a sample did not 
contain 100 organisms, all organisms were retained for identification. Invertebrates were identified to the family level using taxonomy recommended by Pennak (1978) and Merritt and Cummins (1984).

A benthic invertebrate survey was done in 1990 at sites in the Lost River, Tule Lake upper and lower (south) sumps, Lower Klamath Refuge, Clear Lake, J and N Canals, and the South Side Drain (figs. 1 and 2). In 1991, the inventory was limited to selected primary monitoring sites plus three sites in Tule Lake upper sump.

General methods for collection of benthic invertebrates were those described by Britton and Greeson (1987). Benthic invertebrates were collected using a petite Ponar grab sampler. A 533- $\mu \mathrm{m}$ mesh sieve was used to screen the samples. Samples were placed in wide-mouth containers with a 4-percent formalin solution containing Rose Bengal biological stain to aid in sorting the invertebrates from the other organic matter. Several days to several weeks later, samples were rinsed with tap water using a $250-\mu \mathrm{m}$ mesh sieve and then preserved in 70-percent ethanol. Three replicates (grabs) were taken at each site. Samples were sorted by hand using a binocular dissecting scope at $7 \times$ to $10 \times$ power.

Due to the large volume of benthic invertebrate samples and time constraints, identification of invertebrates to species level was not practical for this study. Larger samples were subsampled using techniques described by Britton and Greeson (1987). Taxa are reported to the family level where practical (Hirudinea, Gastropoda, and Insecta) using keys by Mason (1968), Merritt and Cummins (1984), and Pennak (1978).

Frog call surveys were done during the breeding season (May-July) in 1991 and 1992 at selected sites throughout Tule Lake and Lower Klamath Lake Refuges and at Indian Tom Lake (figs. 2 and 3). These surveys assessed the calling male-frog distribution and abundance. Methods described by Karns (1986) were used as the basis for the frog call surveys. Two-person teams would travel to the frog call survey sites on a weekly basis beginning at dusk. Each site was surveyed one night a week. The species calling and number of male frogs heard were recorded. Water-quality measurements were made at most survey sites where water was accessible.

Fish were surveyed from the G Canal, sites 11 and 12, and the Lost River in October 1991 during the Bureau of Reclamation's sucker salvage operation (when the irrigation system is drained for the winter months). Fish were surveyed again in June and September 1992 at the Lost River below Clear Lake Dam, Anderson Rose Dam, and selected primary sampling sites. These surveys were done to determine the biological condition of fish assemblages along the drainwater system. The Lost River site below Clear Lake Dam was selected to serve as a reference site because it is located above drainwater inputs. Surveys were based on methods for rapid bioassessment of fish communities (Plafkin and others, 1989). Collections were made with beach seines, dip nets, traps, and electroshock. Fish were sorted by species and size class, counted, and examined for external abnormalities.

Fish health surveys were done on fathead minnows (Pimephales promelas) collected in the 1991 and 1992 fish surveys to provide information on fish health. The fish health surveys consisted of an autopsy-based assessment on fathead minnows and use of an assessment index (Goede and Barton, 1990).

Bioassays were done weekly from June through August 1991 and 1992. Both static laboratory and in situ bioassays were run using standard test species relevant to the Klamath aquatic community. The static laboratory bioassays were done to determine if acutely hazardous chemical concentrations were present in drainwater. Static tests were done using Microtox $^{\circledR}$ (which uses bioluminescent marine bacteria, Photobacterium phosphoreum), green algae (Selenastrum capricornutum), duckweed (Lemna minor), water flea (Daphnia magna), amphipod (Hyalella azteca), midge larva (Chironomus tentans), fathead minnow (Pimephales promelas), and African clawed frog embryo (Xenopus laevis). Test organisms were exposed to water or sediment from selected primary sampling sites for 48 or 96 hours (depending upon species protocol). Static-test endpoints were percent luminescence (Microtox ${ }^{\circledR}$ ), percent growth (plants), or percent survival or percent malformations (animals). Bioassays were done using procedures described by Microbics Corporation (1988a,b; 1992), Nebeker and others (1984), Peltier and Weber (1985), Wang (1986), and Bantle and Sabourin (1990).

The in situ bioassays were used to determine if continuous field exposure to drainwater was hazardous to aquatic organisms. The species tested in situ were Daphnia magna, Hyalella azteca, Pimephales promelas, and mallard ducklings (Anas platyrynchos). Test organisms were placed in the drainwater at each sampling site weekly (mallards less frequently) for either 48 or 96 hours. The endpoint of the in situ 


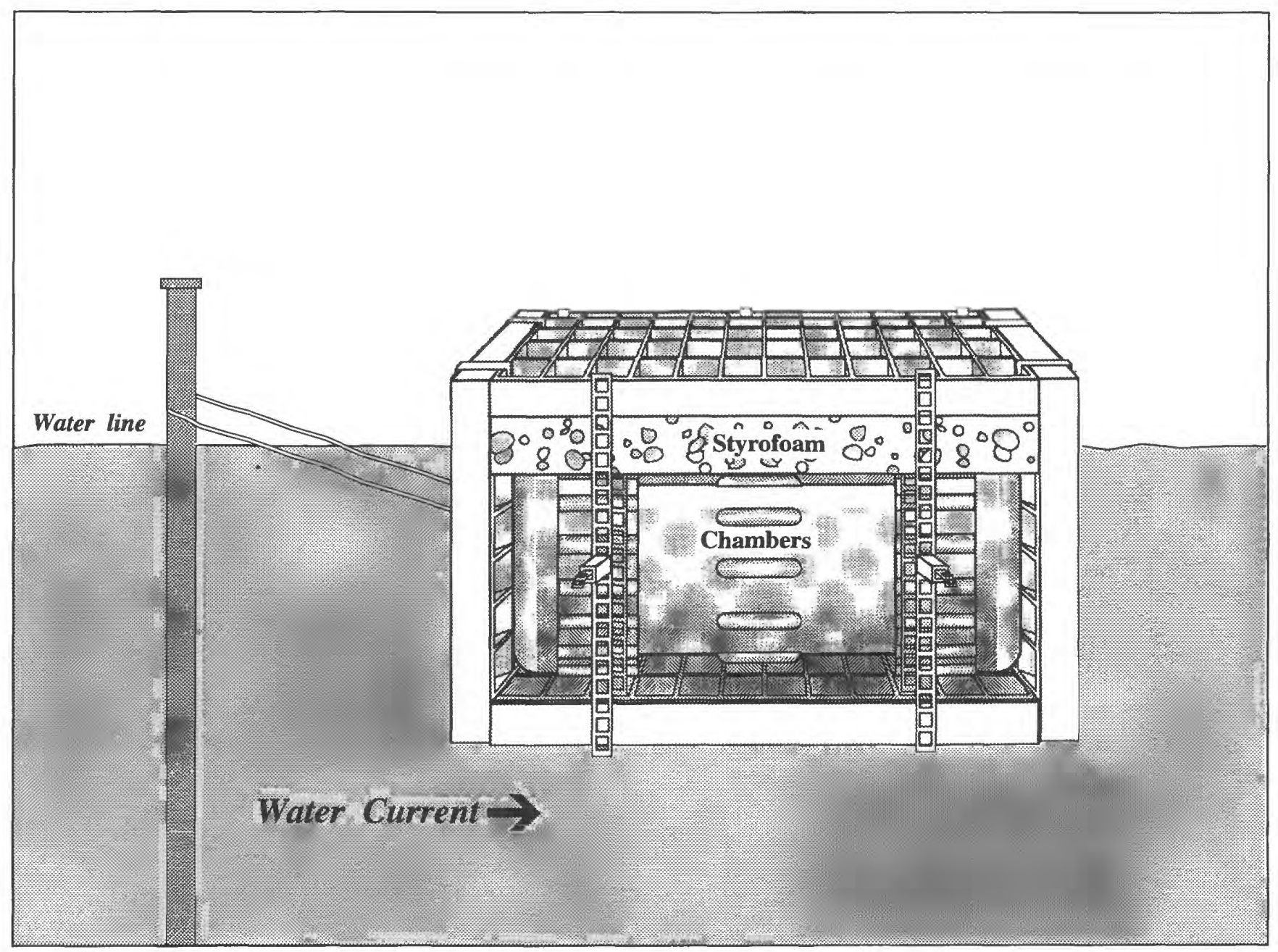

Figure 6. Diagram of in situ test chamber.

bioassays was percent survival. In situ test chambers, developed for this study to hold Daphnia magna, Hyalella azteca, and Pimephales promelas, were 5.7$\mathrm{cm}$-diameter polyvinyl-chloride compression couplers equipped with open screw-on endcaps. The endcap openings were covered with $500-\mu \mathrm{m}$ screening to contain the animals but permit waterflow through the chamber. The screens were glued into the endcap with nontoxic aquarium sealer to prevent gaps around the screen. Ten animals were placed in each test chamber and duplicate chambers were prepared for each species. Chambers were prepared the evening before deployment and were held overnight in plastic bags filled with culture water and placed in insulated coolers. After being transported to the field sites early the next morning, the test chambers were transferred under water to plastic mesh cages with a 2.6-mm layer of styrofoam on top to ensure that the chambers would remain suspended in the water column. Cages were tethered in place at each sam- pling site (fig. 6) (Jewel Bennett, U.S. Fish and Wildlife Service, written commun., 1993).

\section{COMPILATIONS OF PHYSICAL, CHEMICAL, AND BIOLOGICAL DATA}

Sites listed in table 1 with a USGS identification number correspond to numbers in WATSTORE (the USGS National WATer data STOrage and REtrieval system). Sites with no USGS identification number and those in table 2 are biological-tissue and biosurvey sampling sites. Bottom-sediment physical and chemical data are found in tables 3-5; surfacewater chemical data are in tables 6-14; biological tissue data are in tables 15-18; eggshell thickness data are in table 19; biological survey data are in tables 20-24; and bioassay data are in tables 25-36. Diel data from the continuous monitors are shown as graphs in figures 7 and 8. 

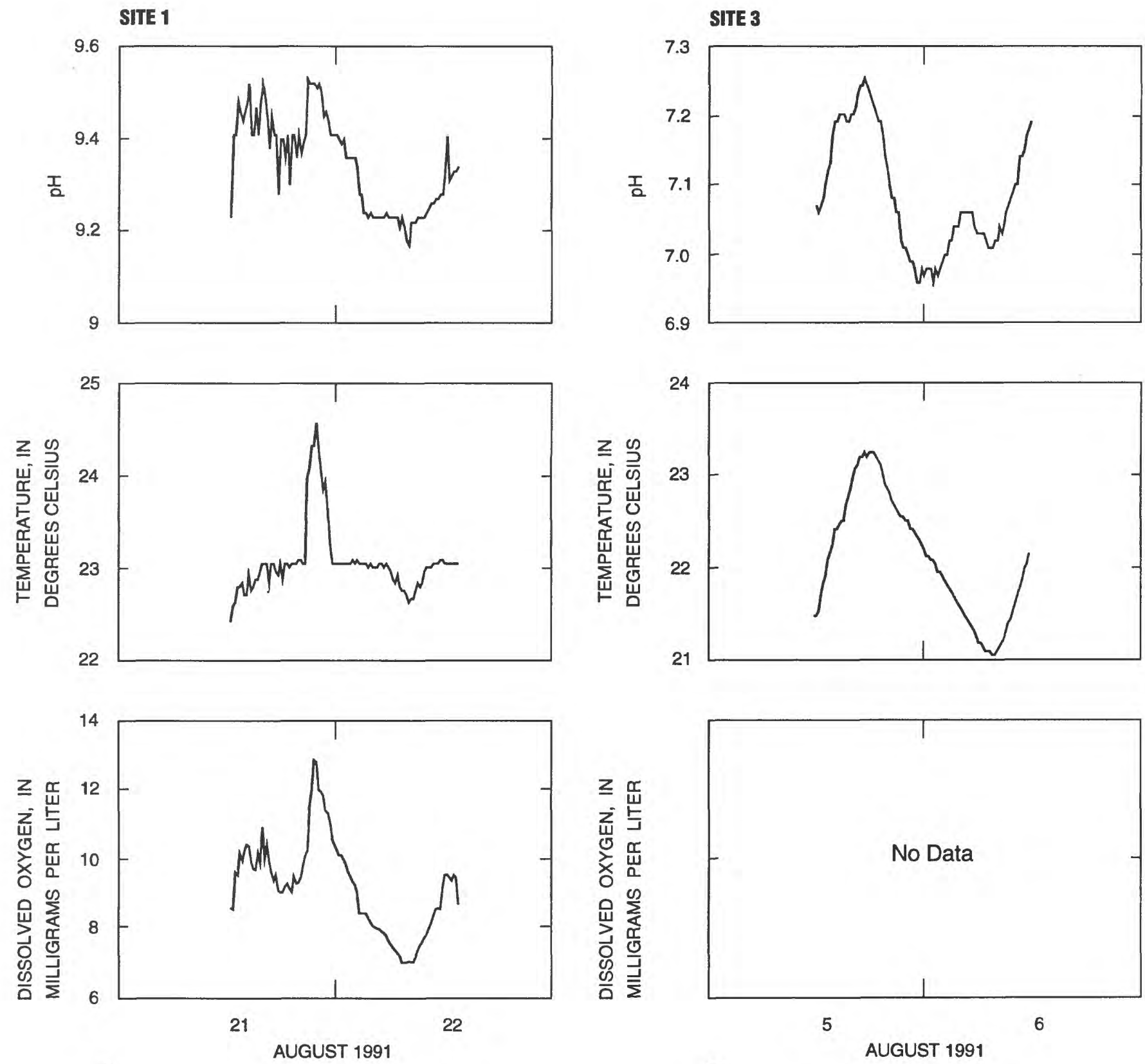

$A$

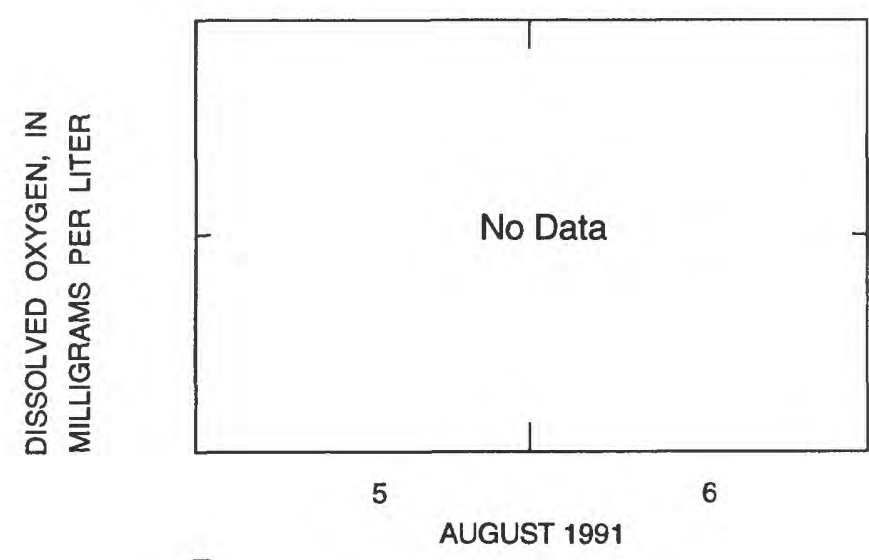

$\boldsymbol{B}$

Figure 7. Diel pH, temperature, and dissolved-oxygen data from selected sites in Klamath Basin, 1991. Scales vary by site for each property or constituent. Location of sites in figure 1. 

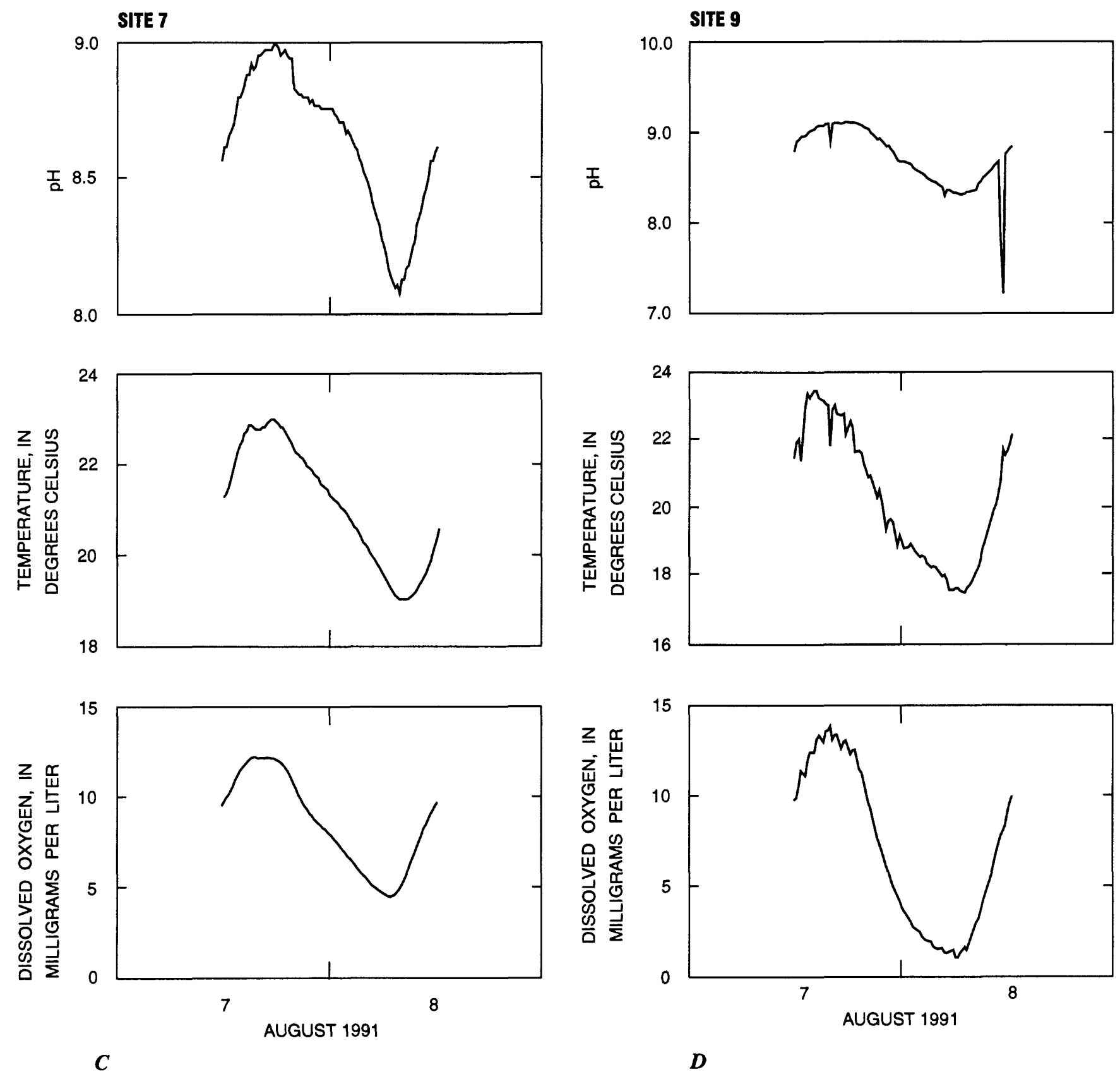

Figure 7. Continued. 

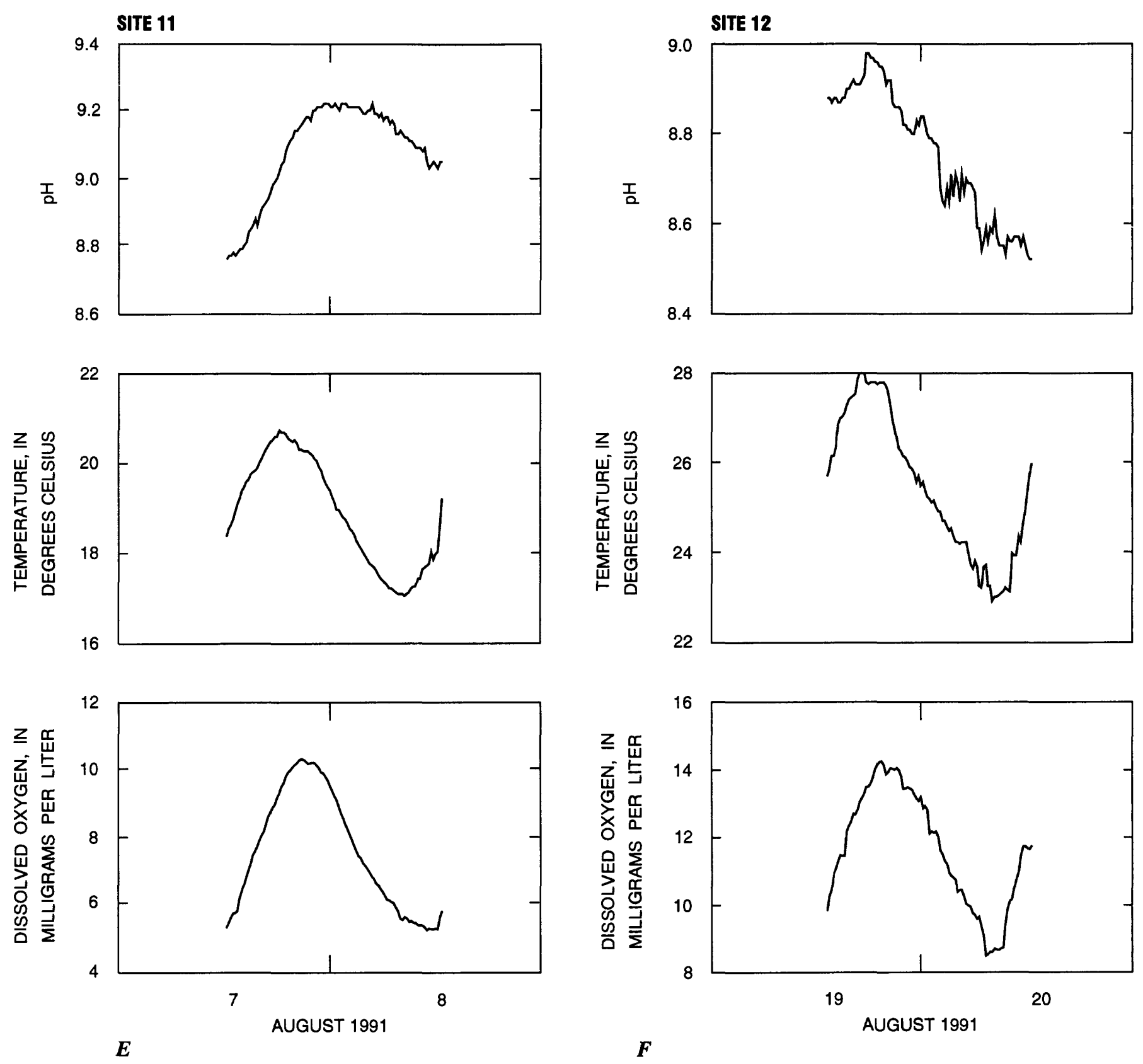

Figure 7. Continued. 

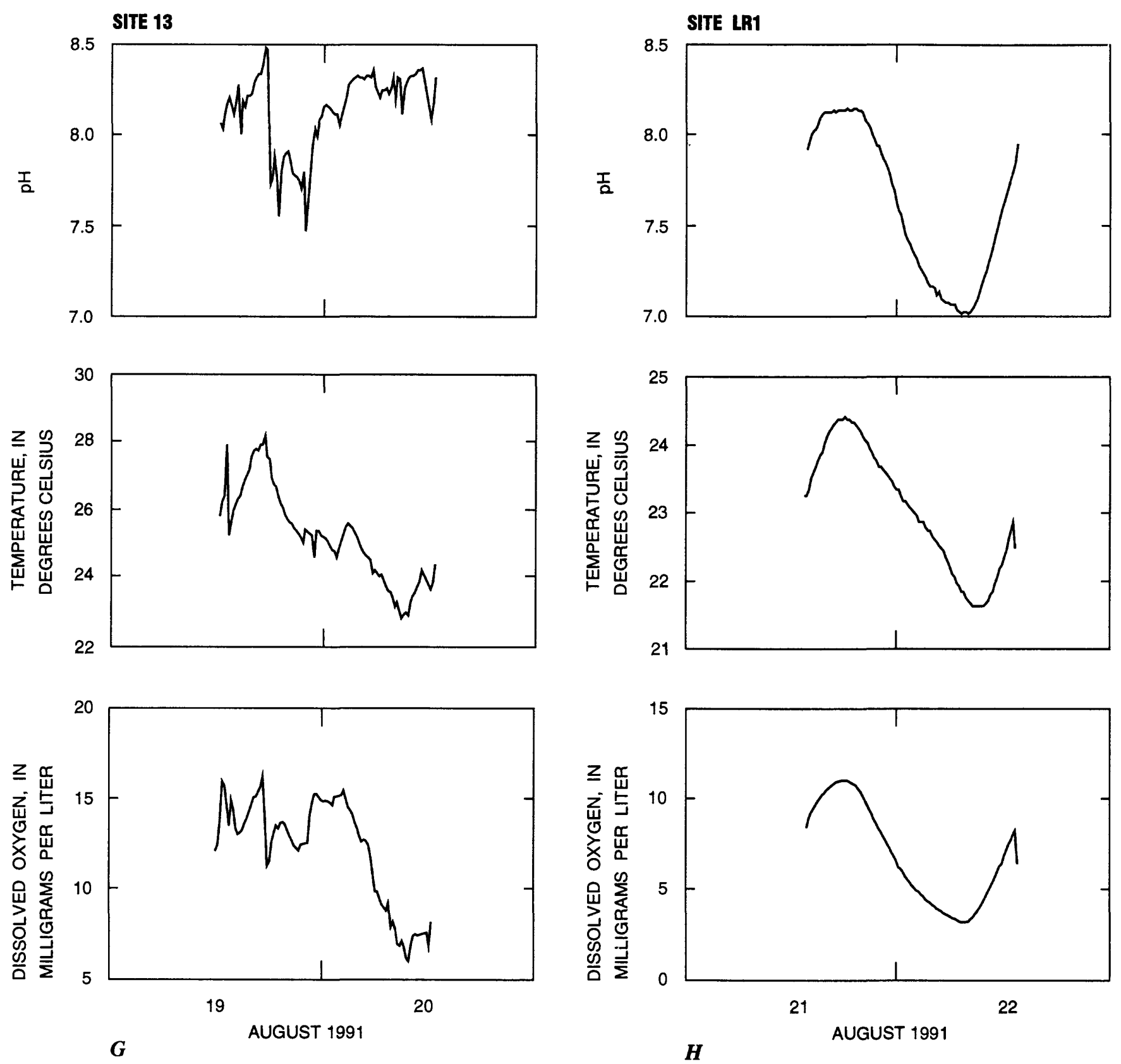

Figure 7. Continued. 

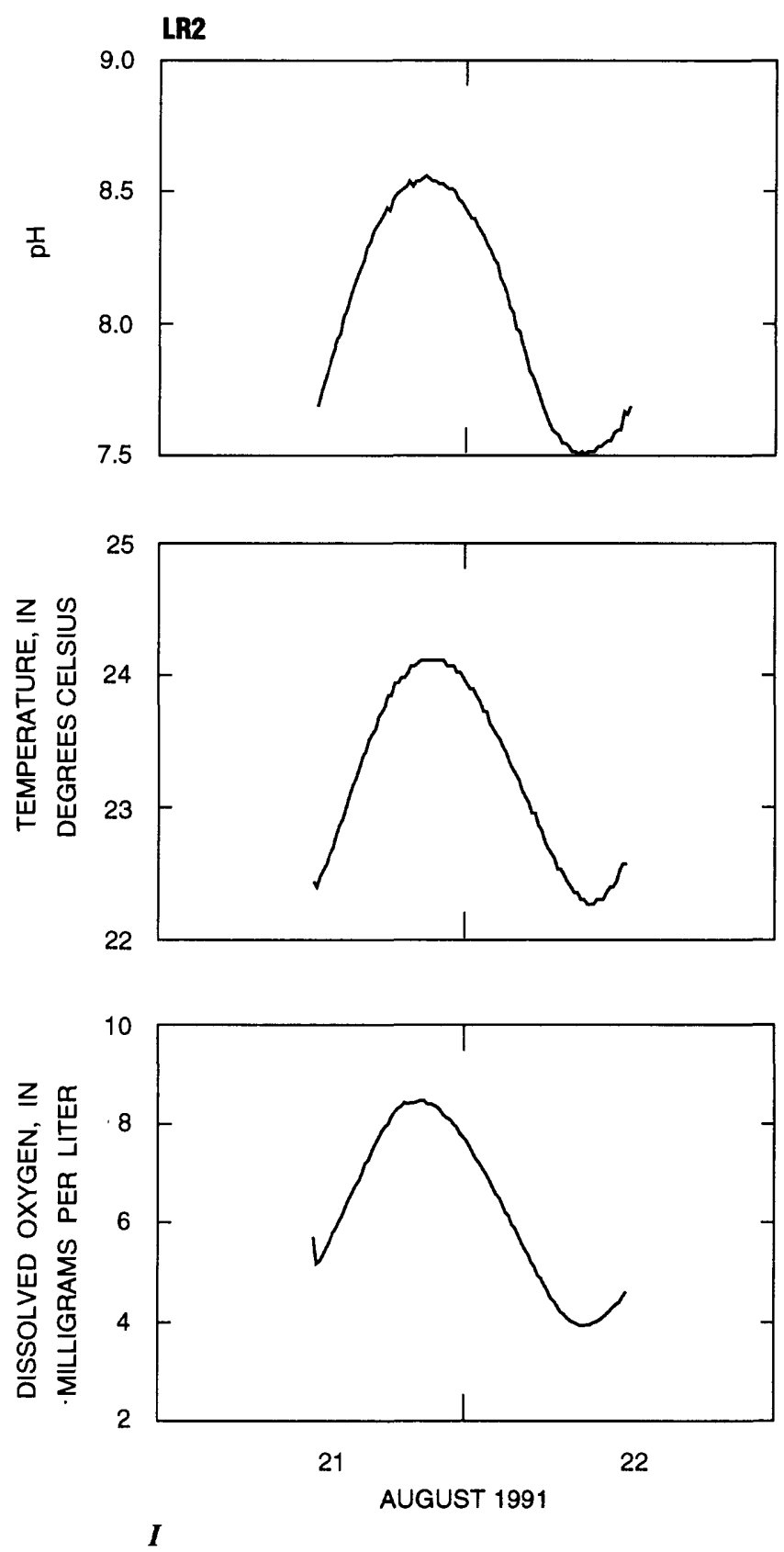

Figure 7. Continued. 

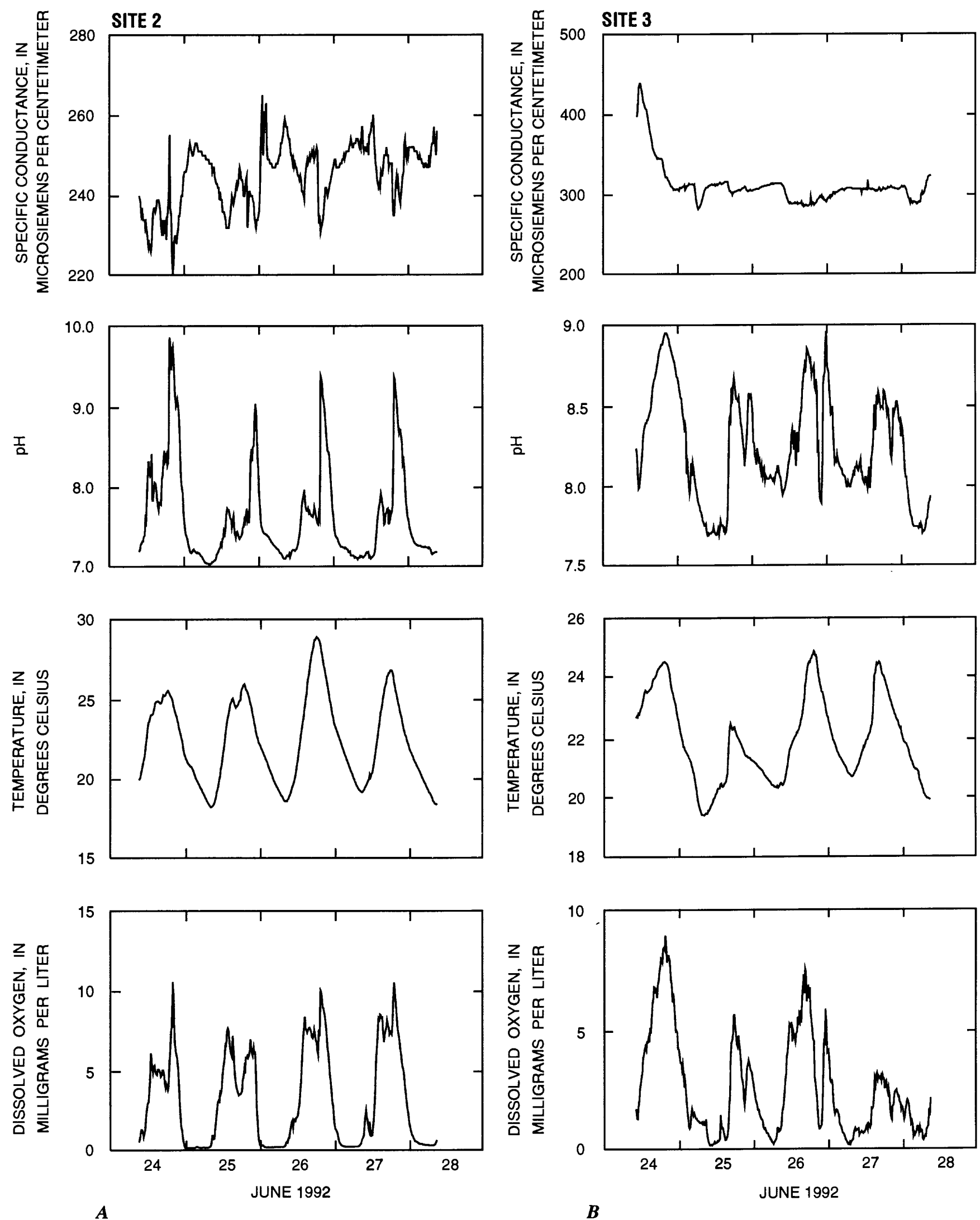

Figure 8. Diel specific conductance, $\mathrm{pH}$, temperature, and dissolved-oxygen data from selected sites in Klamath Basin, 1992. Scales vary by site for each property or constituent. Location of sites in figure 1. 

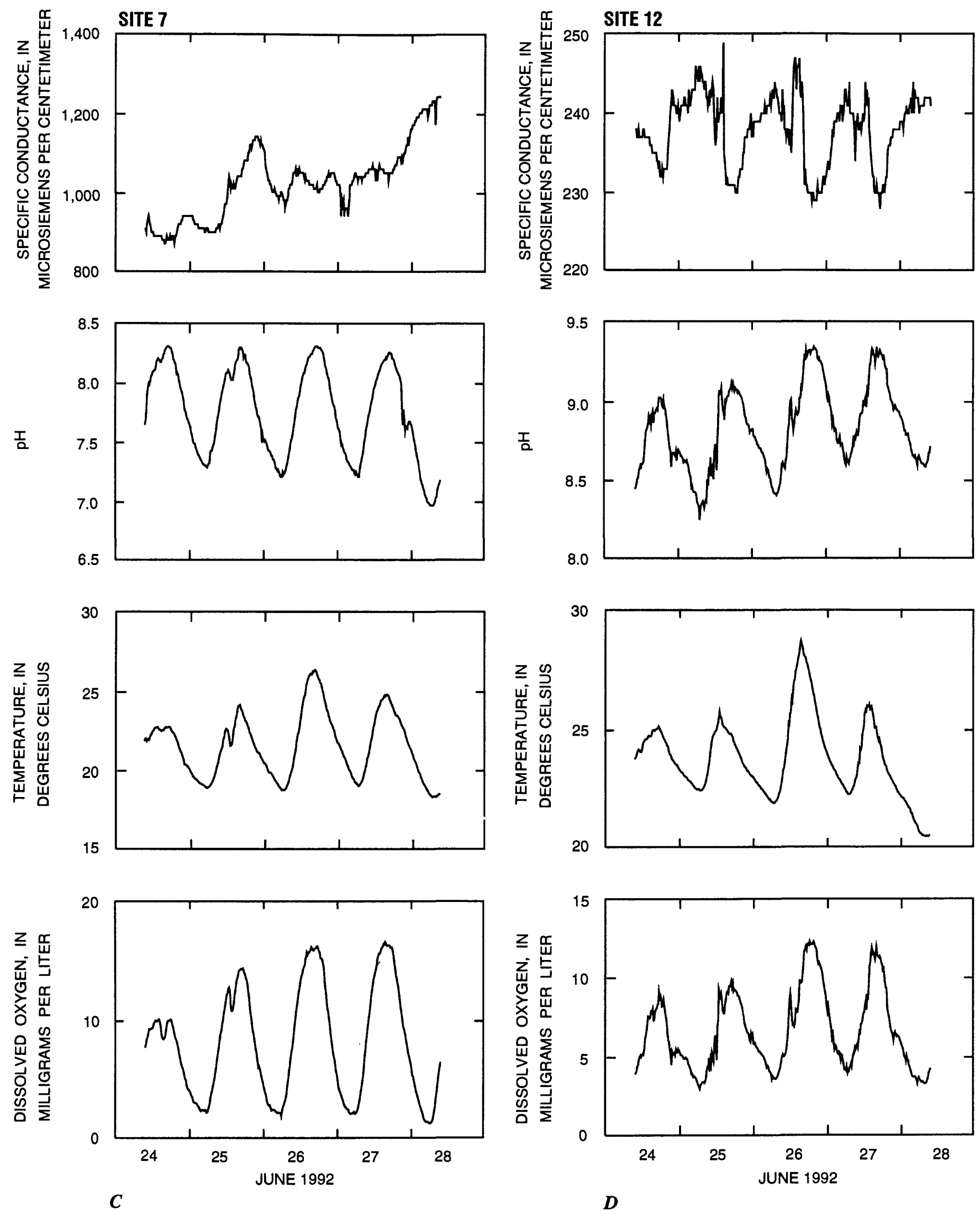

Figure 8. Continued. 

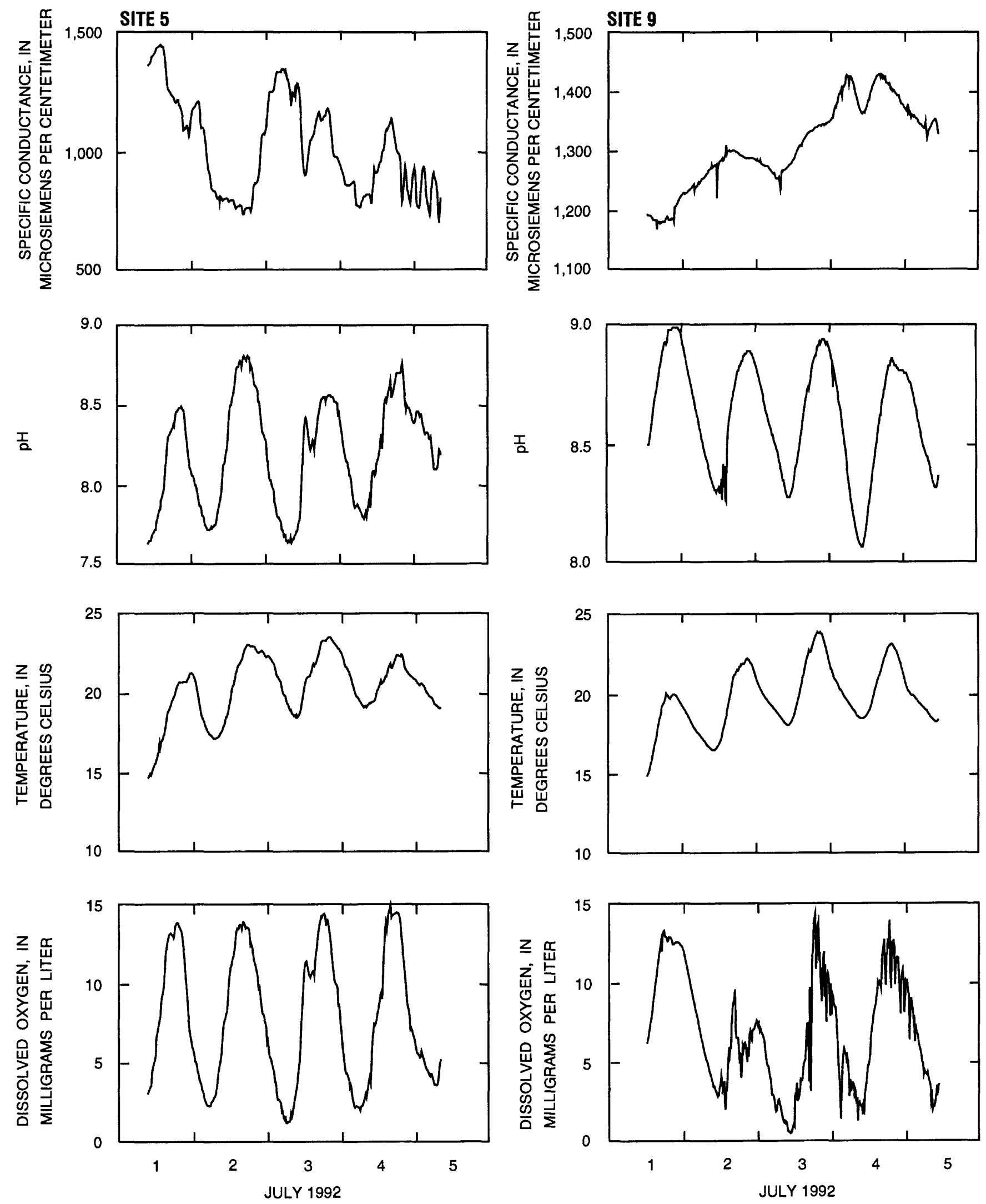

Figure 8. ${ }^{\boldsymbol{E}}$ Continued.

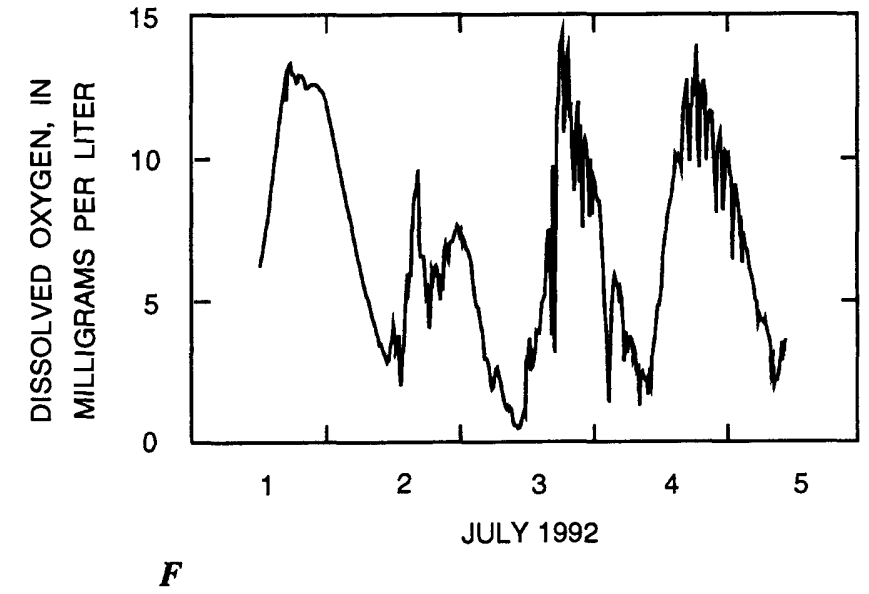



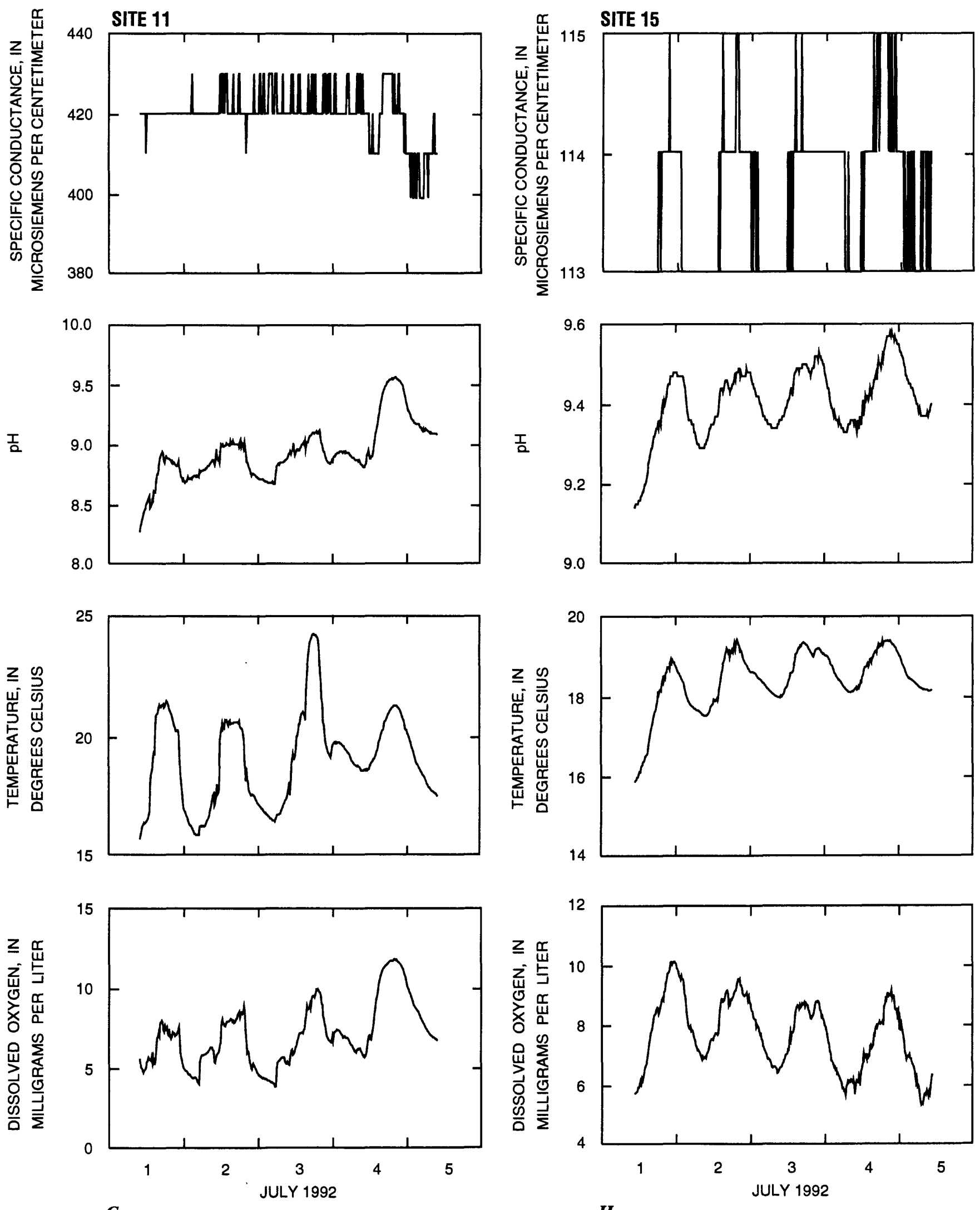

$\boldsymbol{G}$

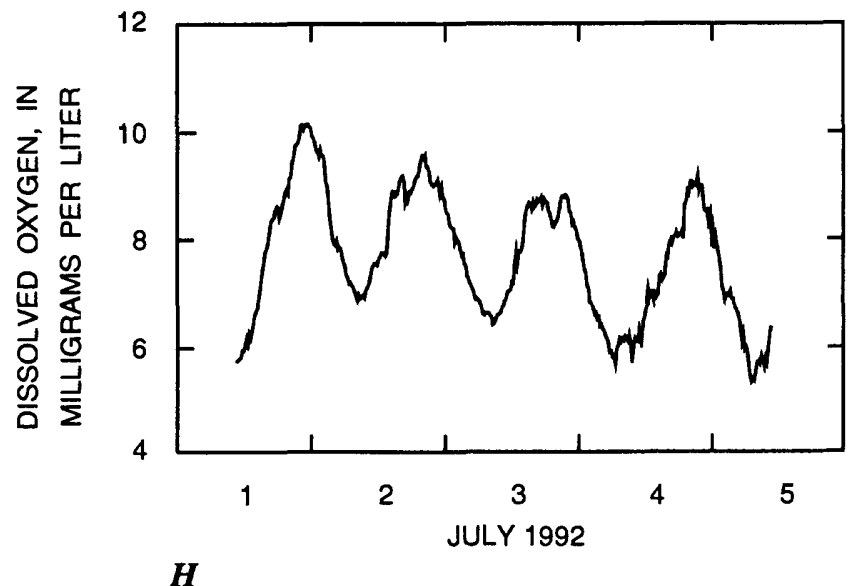

Figure 8. Continued. 

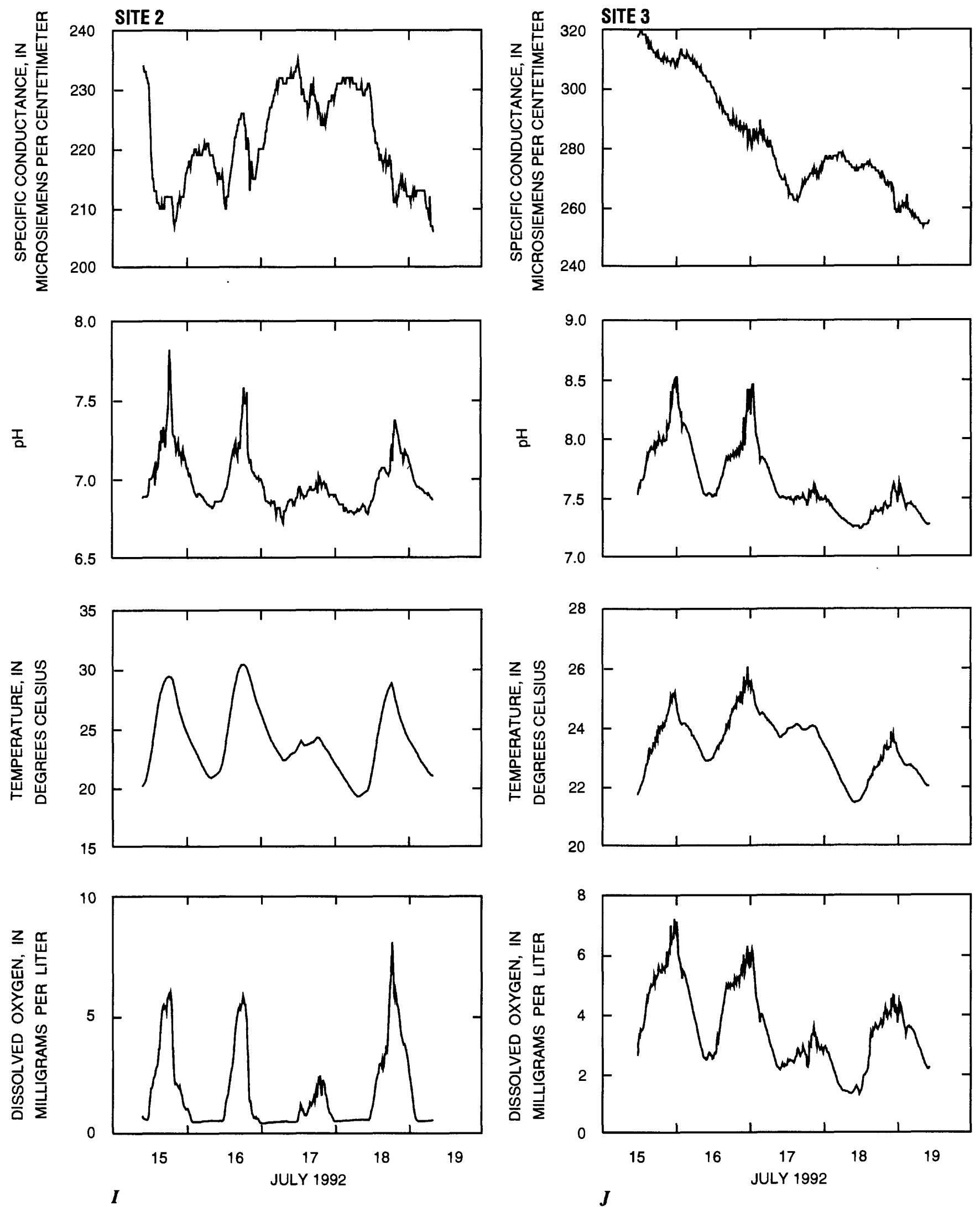

Figure 8. Continued. 

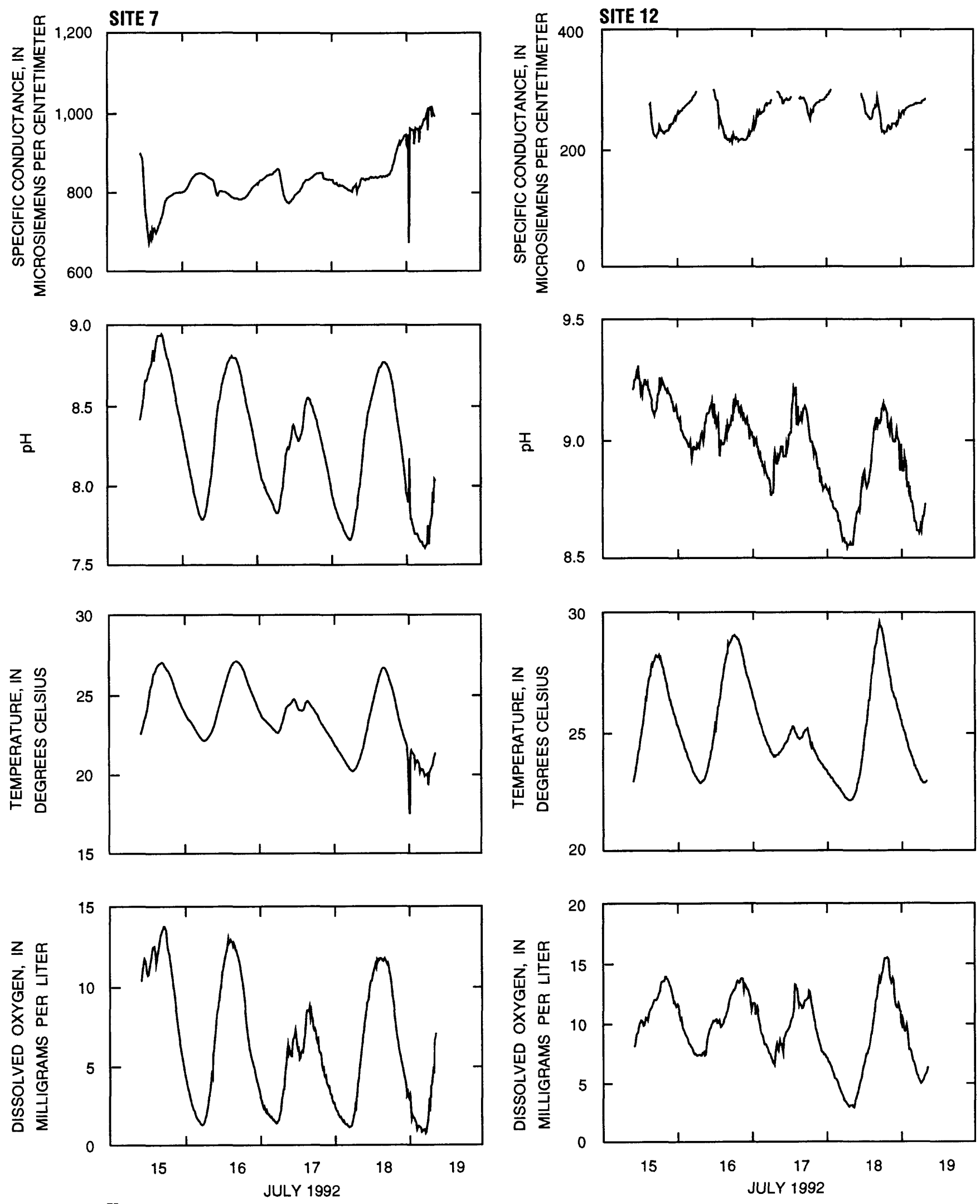

$K$

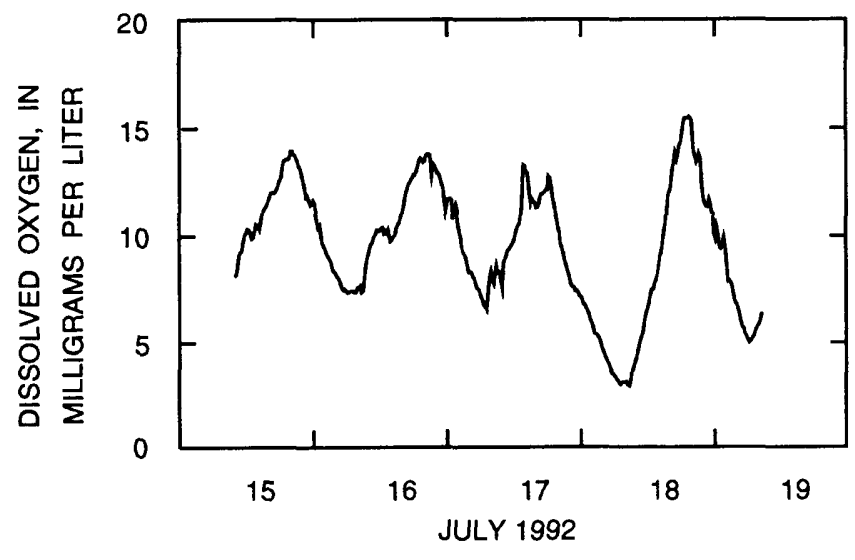

Figure 8. Continued. 

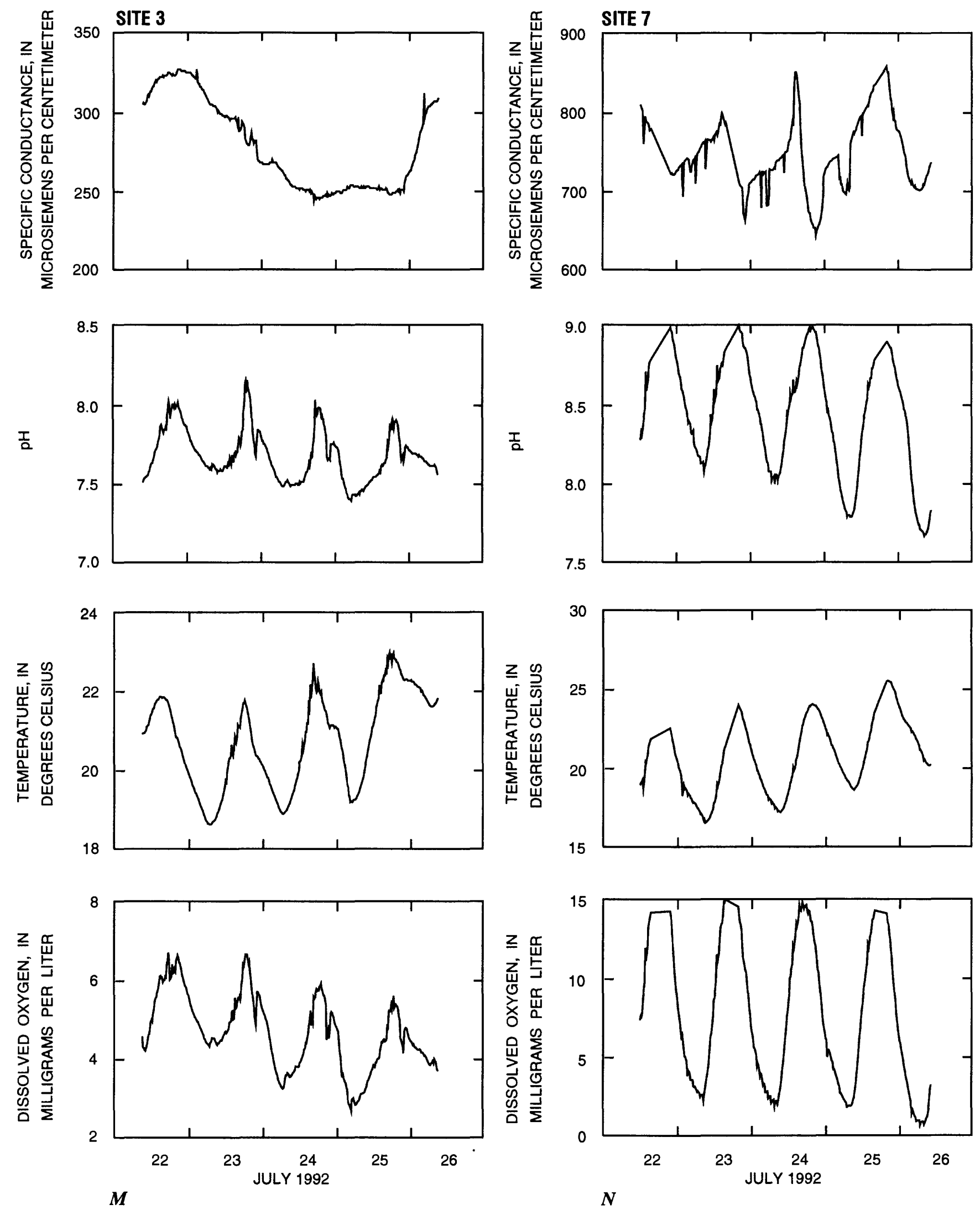

Figure 8. Continued. 

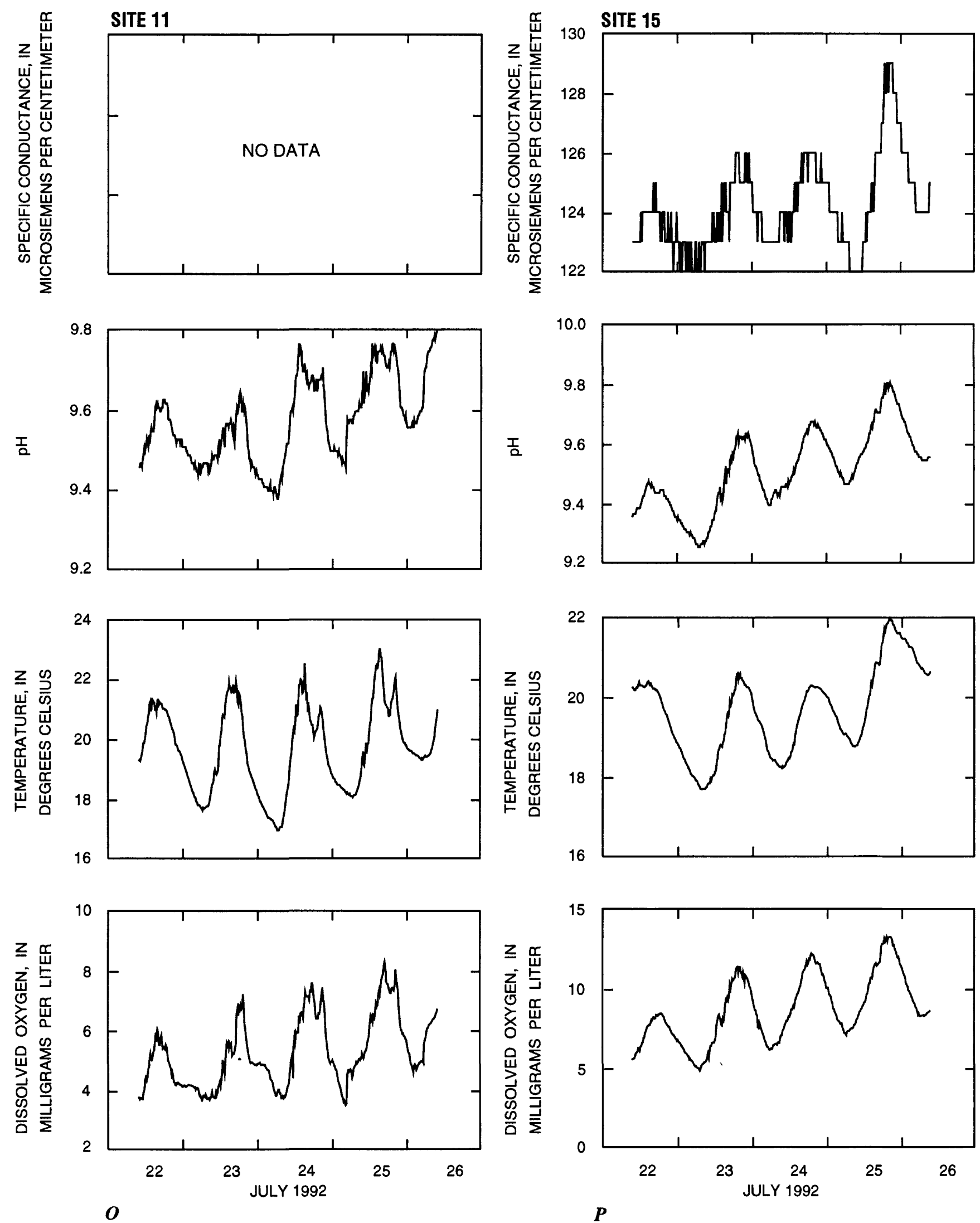

Figure 8. Continued. 

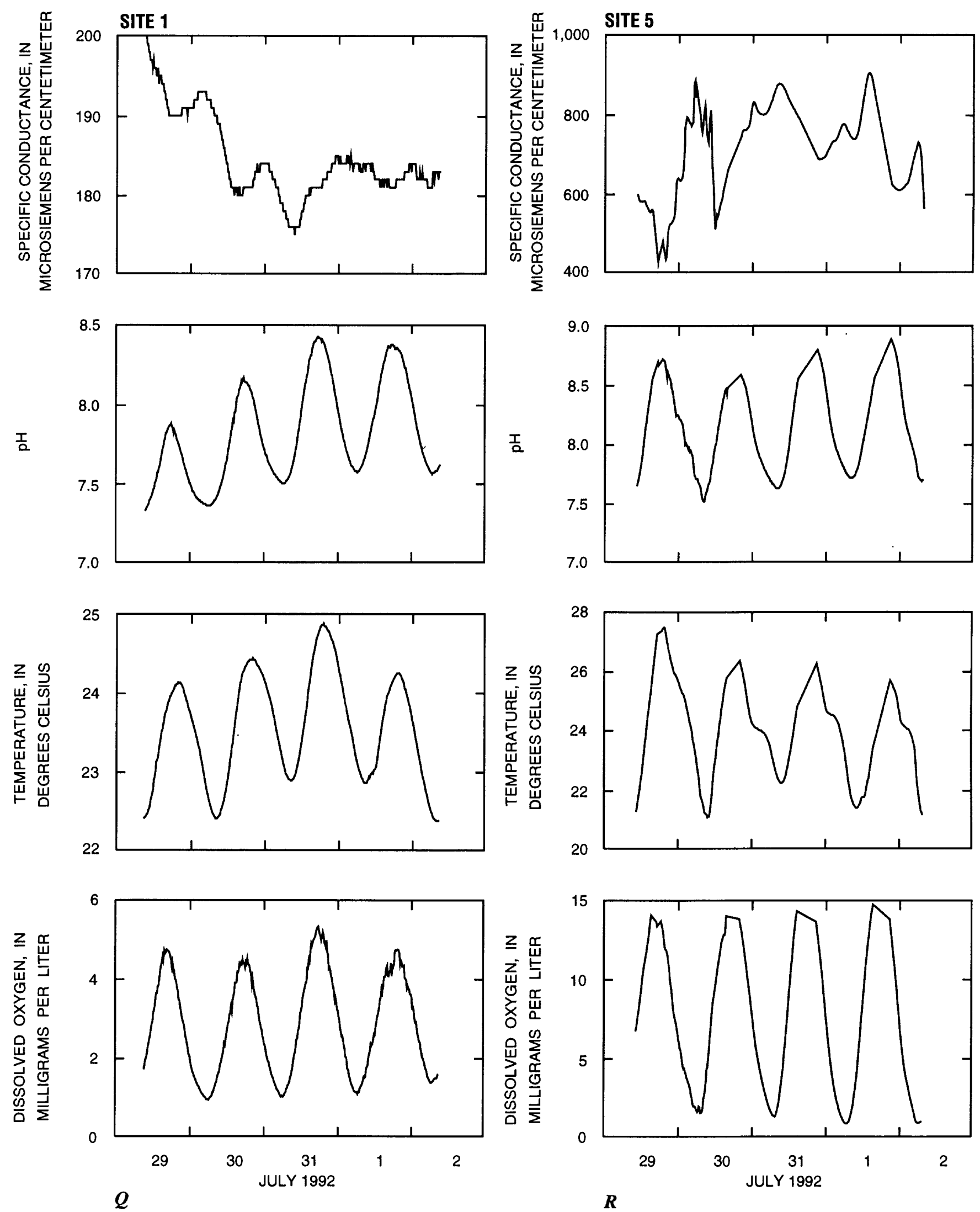

Figure 8. Continued. 

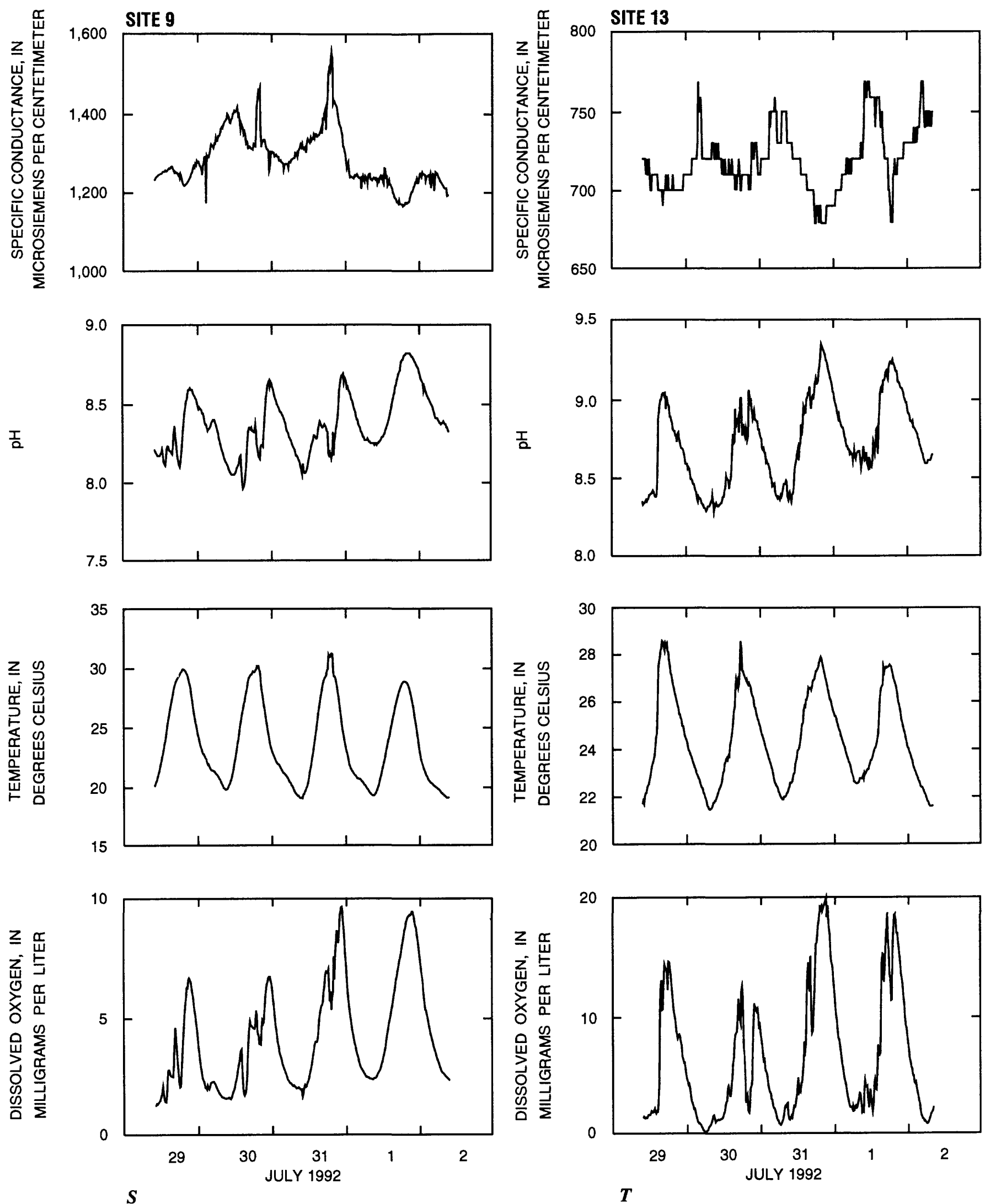

Figure 8. Continued. 

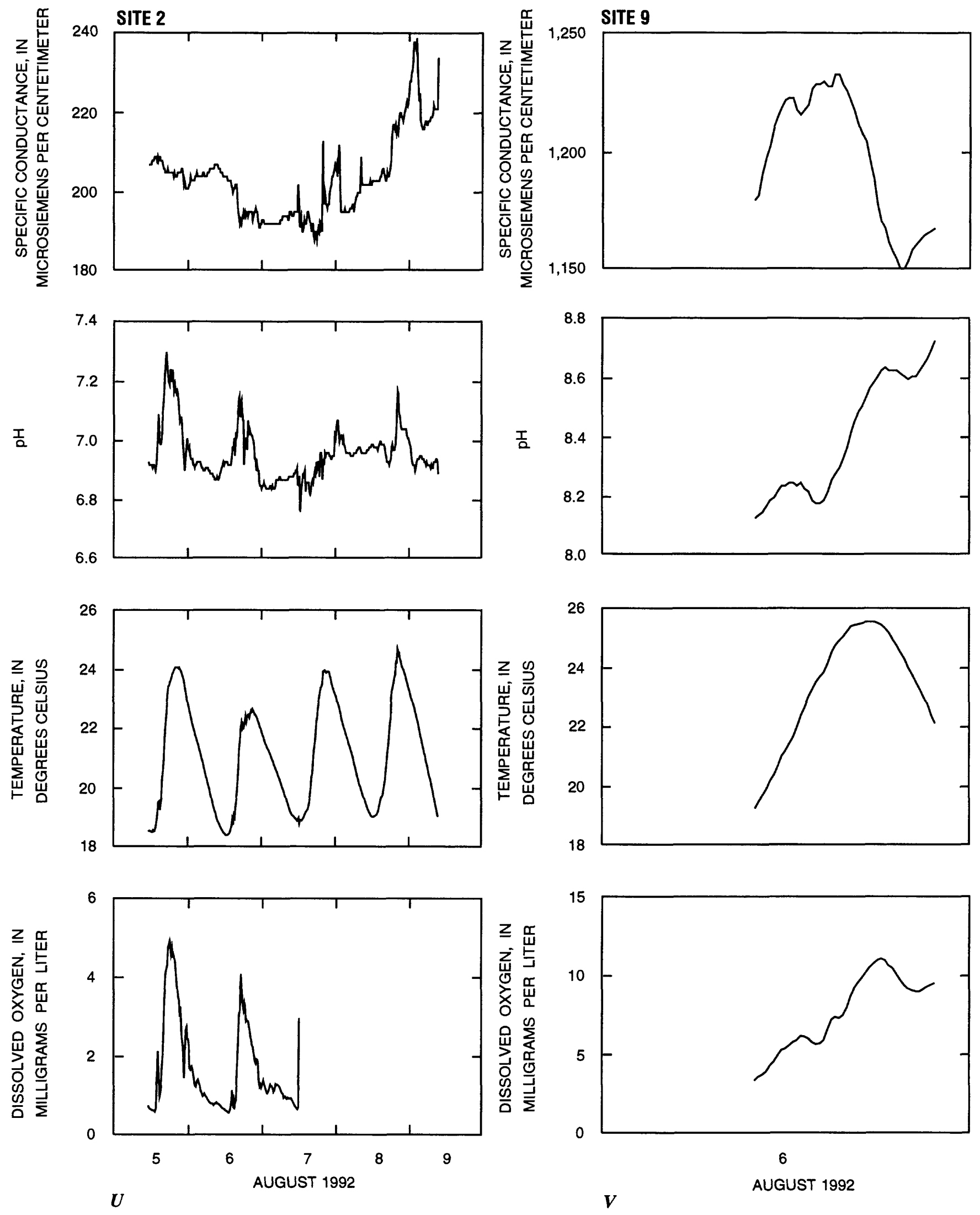

Figure 8. Continued. 

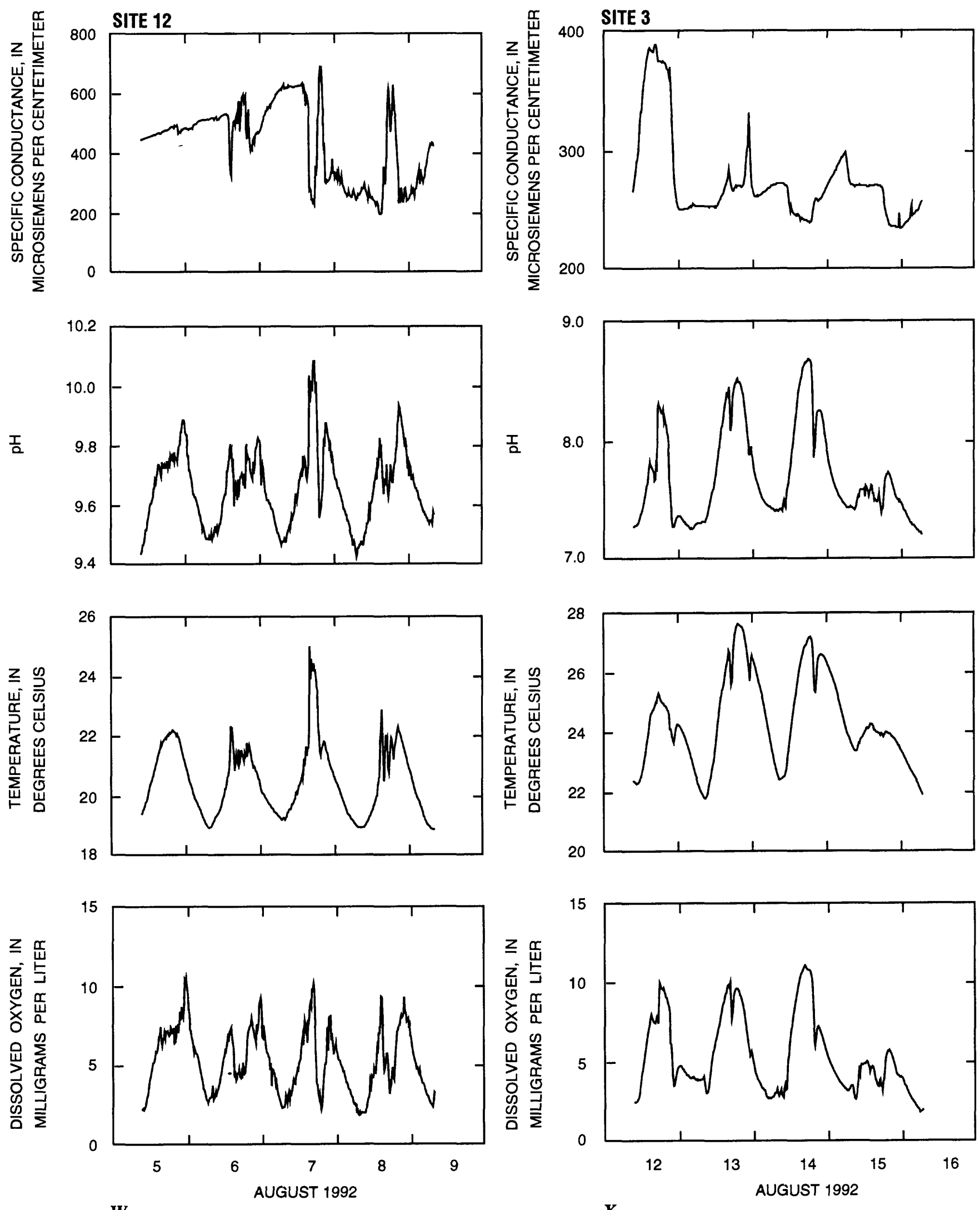

$\boldsymbol{W}$

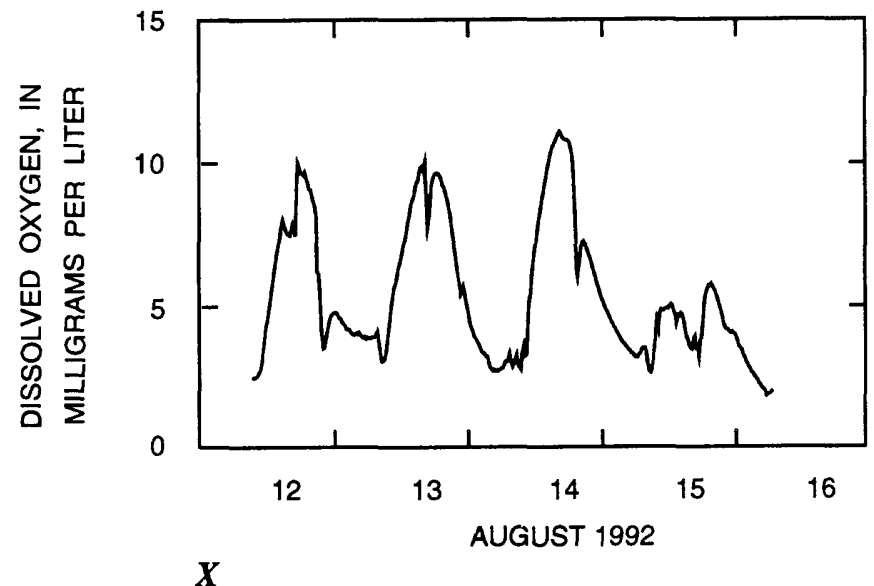

Figure 8. Continued. 

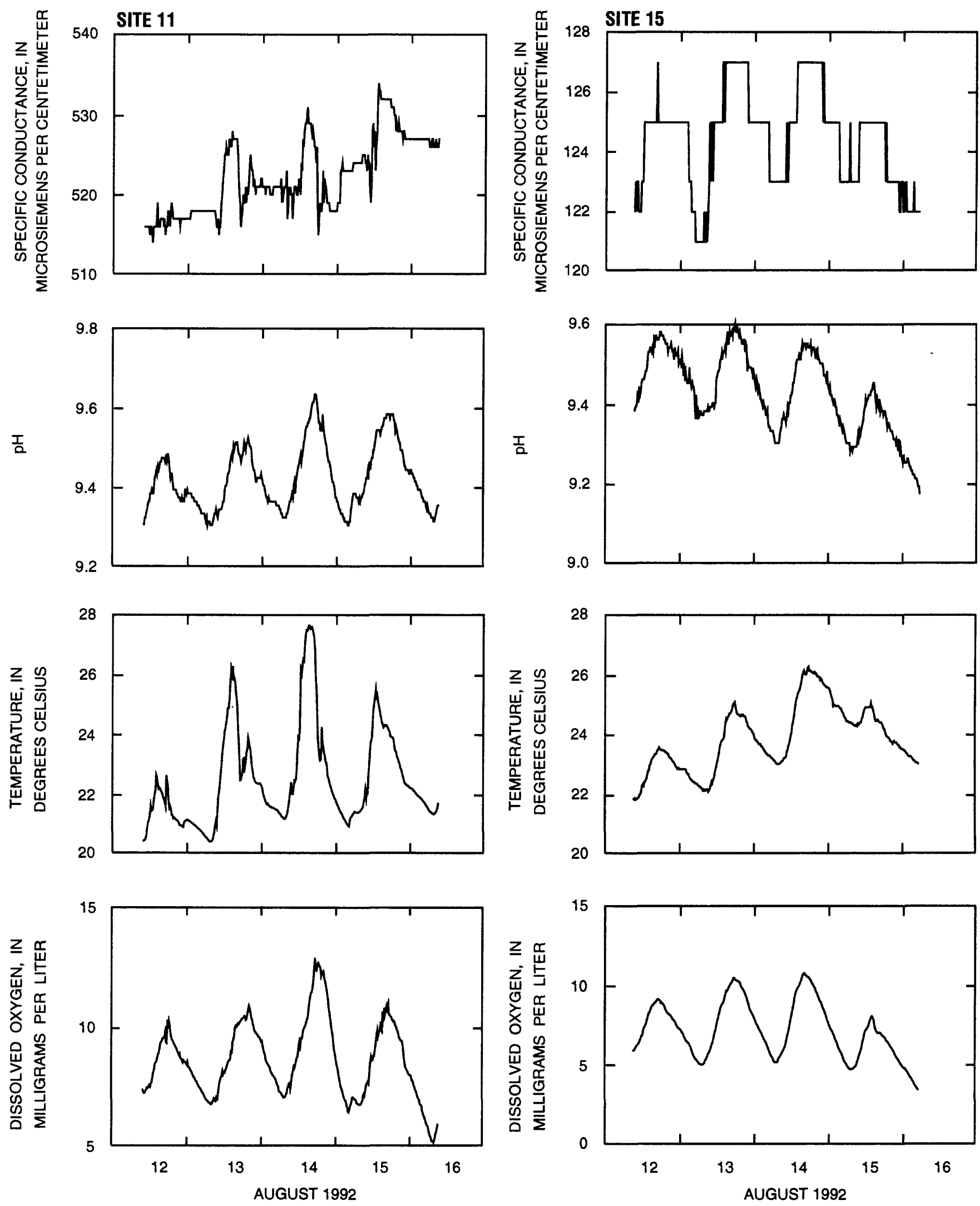

Figure 8. Continued.

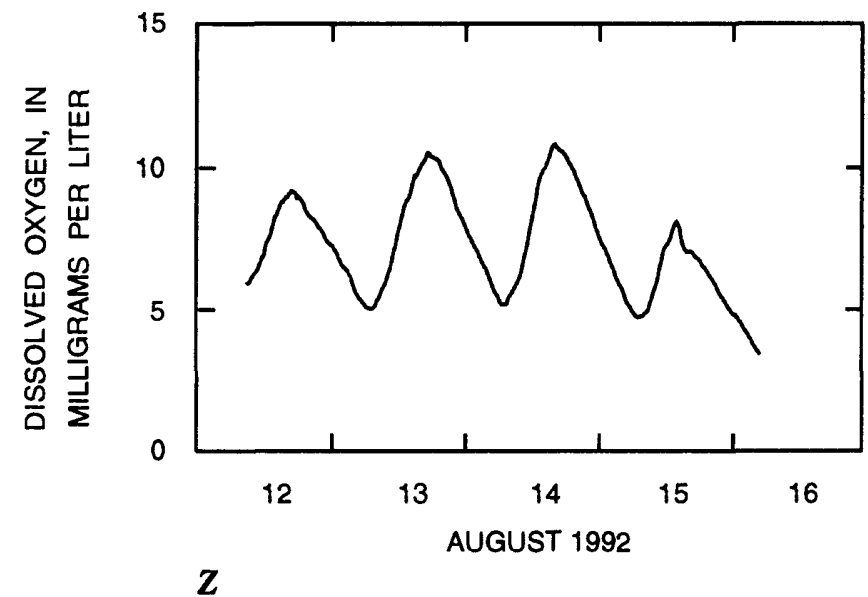



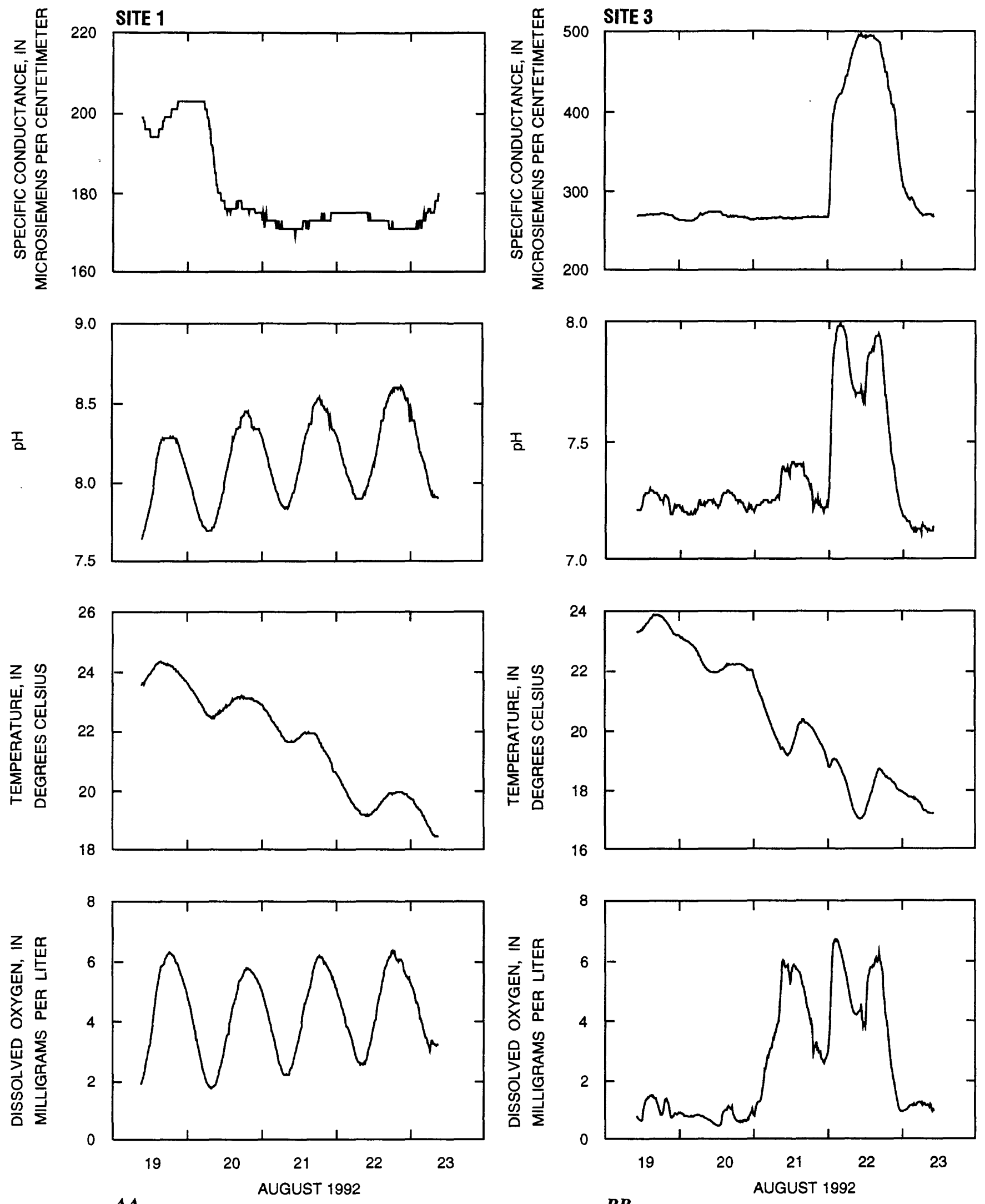

$\boldsymbol{A A}$

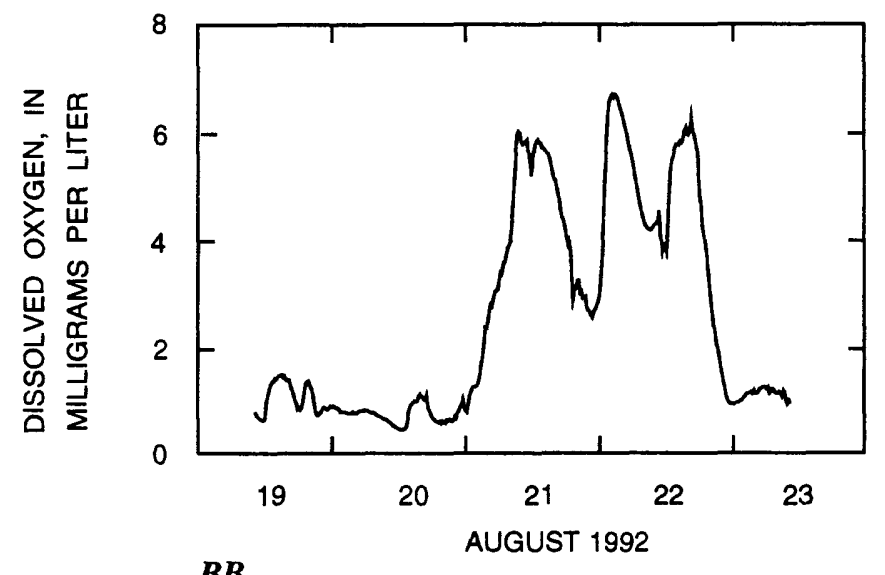

Figure 8. Continued. 

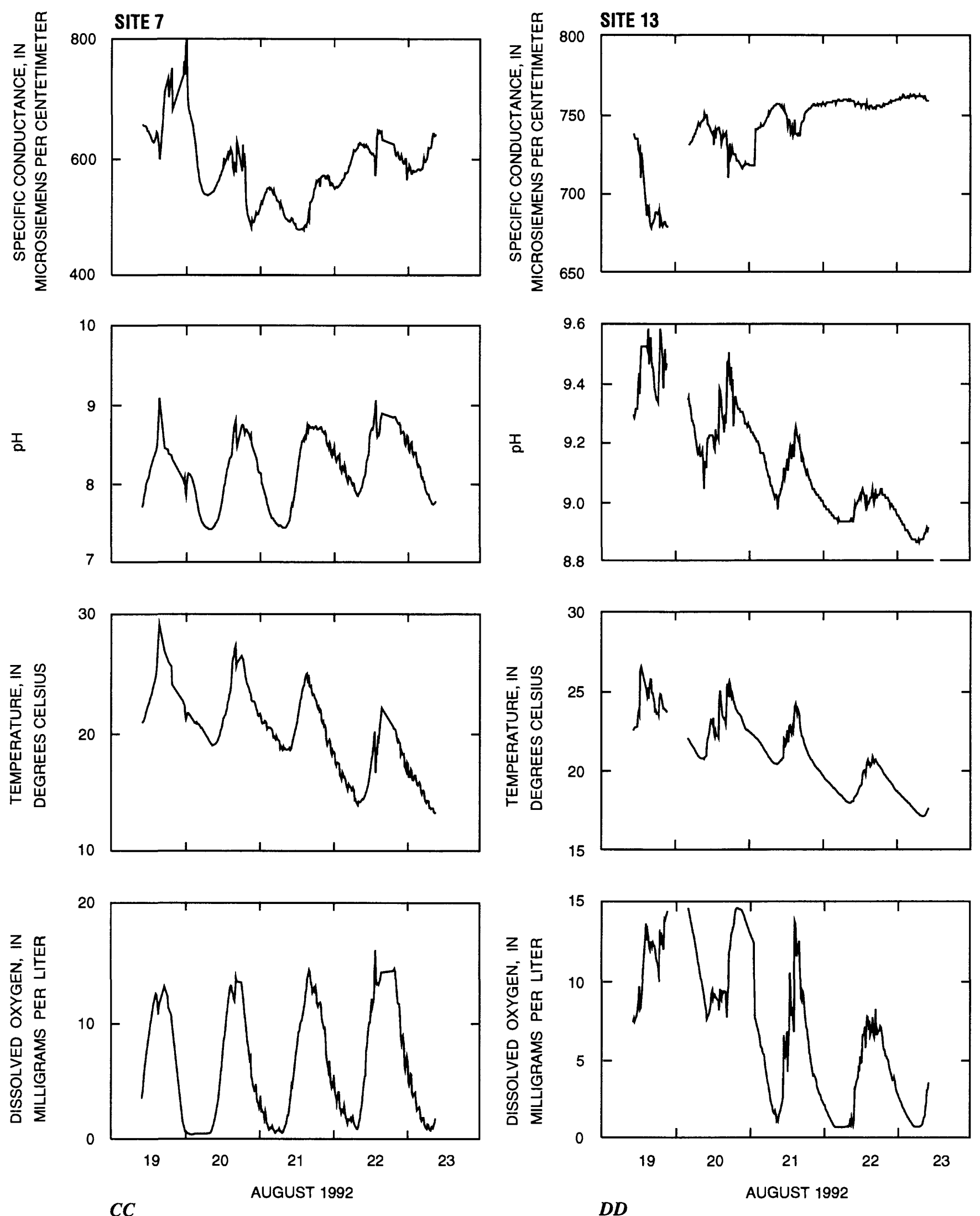

Figure 8. Continued.

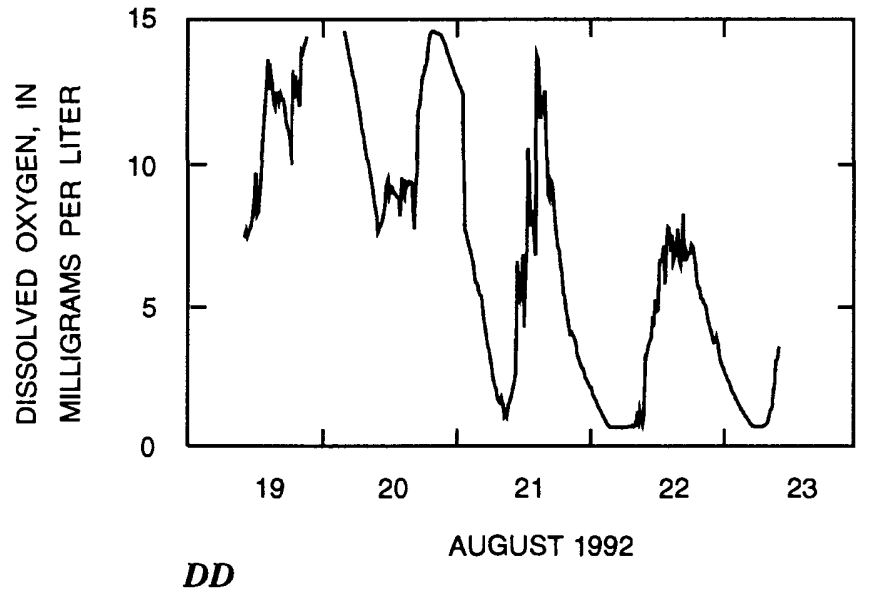



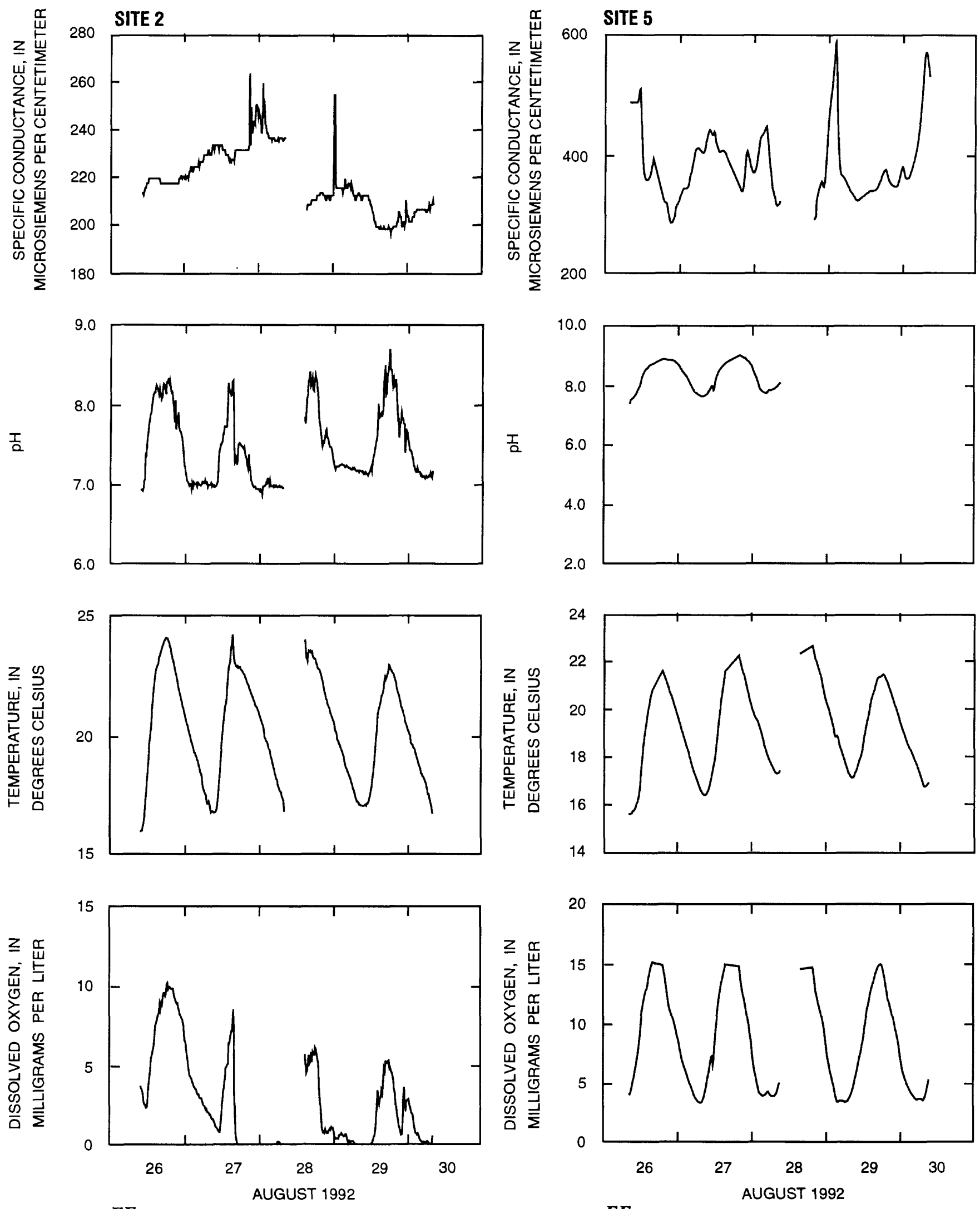

$E E$

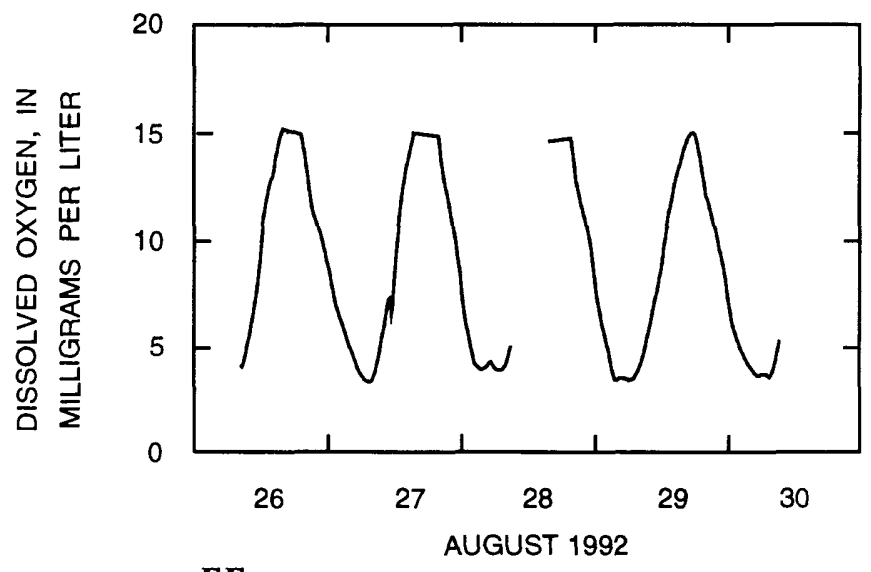

Figure 8. Continued. 

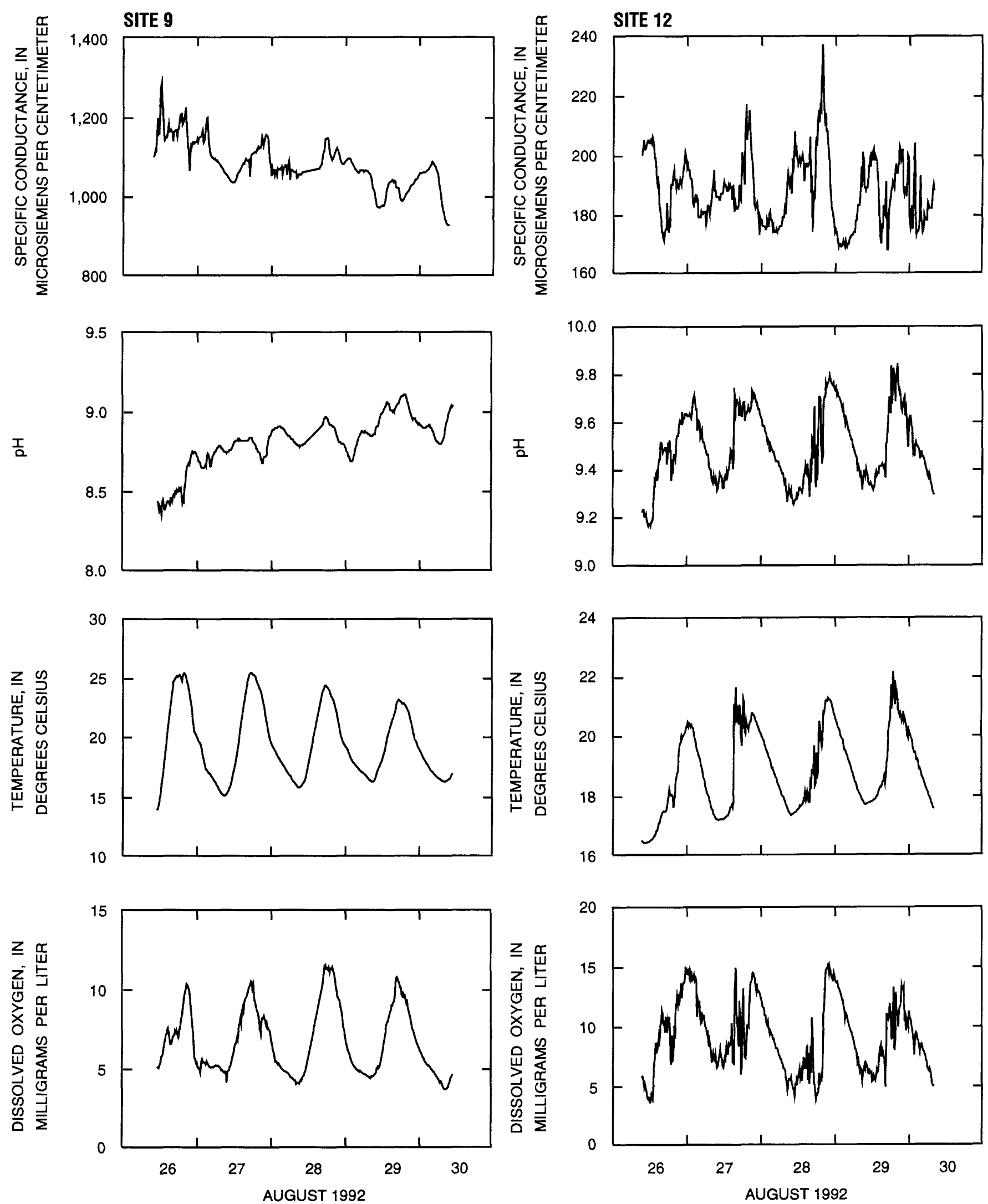

Figure 8. Continued.

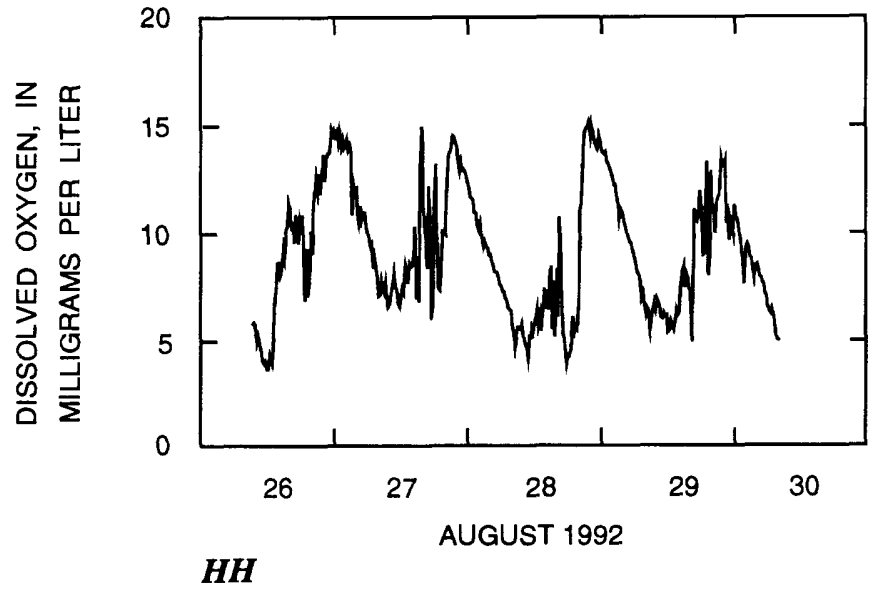




\section{References Cited}

Bantle, J.A., and Sabourin, T.D., 1990, Proposed new standard guide for conducting the frog embryo teratogenesis assay - Xenopus (FETAX): Proposed guideline for American Society of Testing and Materials, Committee E-47, Biological Effects and Environmental Fate.

Britton, L.J., and Greeson, P.E., eds., 1987, Methods for collection and analysis of aquatic biological and microbiological samples: U.S. Geological Survey Techniques of Water-Resources Investigations, Book 5, Chap. A4, 363 p.

Code of Federal Regulations, 1990, Protection of environment: Federal Register, 40, Part 136, Ch. 1, app. B, p. 537-539.

Fishman, M.J., and Friedman, L.C., eds., 1985, Methods for determination of inorganic substances in water and fluvial sediments: U.S. Geological Survey Techniques of Water-Resources Investigations, Book 5, Chap. A1, 709 p.

Friedman, L.C., and Erdman, D.E., 1982, Quality assurance practices for the chemical and biological analyses of water and fluvial sediments: U.S. Geological Survey Techniques of Water-Resources Investigations, Book 5, Chap. A6, $181 \mathrm{p}$.

Goede, R.W., and Barton, B.A., 1990, Organismic indices and an autopsy-based assessment as indicators of health and condition of fish: American Fisheries Society Symposium, v. 8, p. 145-166.

Hazelton Laboratories America, Inc., 1991a, Determination of organochlorine pesticides and polychlorinated biphenyls (PCBs) in soils and sediments, Method MPFWSS-MA: Madison, Wiscensin, Hazelton Laboratories America, Inc.

1991b, Determination of organochlorine pesticides and polychlorinated biphenyls (PCBs) in biological tissues, Method MP-FWST-MA: Madison, Wisconsin, Hazelton Laboratories America, Inc.

Hill, E.F., and Fleming, W.J., 1982, Anticholinesterase poisoning of birds--Field monitoring and diagnosis of acute poisoning: Environmental Toxicology and Chemistry, v. 1, p. 27-38.

Hydrolab Corporation, 1988, DataSonde 1 operating manual: Austin, Texas, Hydrolab Corporation, $34 \mathrm{p}$.

1991, DataSonde 3 multiparameter water quality datalogger-operating manual: Austin, Texas, Hydrolab Corporation, $83 \mathrm{p}$.

Karns, D.R., 1986, Field herpetology methods for the study of amphibians and reptiles in Minnesota: Minneapolis, Minnesota, University of Minnesota, Occasional Paper Number 18, $88 \mathrm{p}$.

Mason, W.T., 1968, An introduction to the identification of chironomid larvae: Cincinnati, Ohio, U.S. Department of Interior, Federal Water Pollution Control Administration, Division of Pollution Surveillance.

Merritt, R.W., and Cummins, K.W., 1984, An introduction to the aquatic insects of North America, 2d ed.: Dubuque, Iowa, Kendall Hunt.
Microbics Corporation, 1988a, A Microtox manual--How to run toxicity tests using the Microtox Model 500, Part No. 555880-R1: Carlsbad, California, Microbics Corporation.

1988b, A Microtox manual--How to reduce Microtox test data, Part No. 555881: Carlsbad, California, Microbics Corporation.

1992, A Microtox manual--A toxicity testing handbook, v. I-V, Part No. 55H550-1: Carlsbad, California, Microbics Corporation.

Nebeker, A.V., Cairns, M.A., Gakstatter, J.H., Malueg, K.M., Schuytema, G.S., and Krawczyk, D.F., 1984, Biological methods for determining toxicity of contaminated freshwater sediments to invertebrates: Environmental Toxicology and Chemistry, v. 3, p. 617-630.

Peltier, W.H., and Weber, C.I., 1985, Methods for measuring the acute toxicity of effluents to freshwater and marine organisms: Cincinnati, Ohio, U.S. Environmental Protection Agency Report EPA600/4-85/013, $216 \mathrm{p}$.

Pennak, R.W., 1978, Fresh-water invertebrates of the United States--Protozoa to mollusca, 3d: New York, Wiley and Sons, Inc., 628 p.

Plafkin, J.L., Barbour, M.T., Porter, K.D., Gross, S.K., and Hughes, R.M., 1989, Rapid bioassessment protocols for use in streams and rivers--Benthic macroinvertebrates and fish: U.S. Environmental Protection Agency Report EPA-444/4-89-001, 164 p.

Sandstrom, M.W., Wydoski, D.S., Schroeder, M.P., Zamboni, J.L., and Foreman, W.T., 1992, Methods of analysis by the U.S. Geological Survey National Water Quality Laboratory--Determination of organonitrogen herbicides in water by solid-phase extraction and capillary-column gas chromatography/mass spectrometry with selected-ion monitoring: U.S. Geological Survey Open-File Report 91-519, 26 p.

Sorenson, S.K., and Schwarzbach, S.E., 1991, Reconnaissance investigation of water quality, bottom sediment, and biota associated with irrigation drainage in the Klamath Basin, California and Oregon, 1988-89: U.S. Geological Survey Water-Resources Investigations Report 90-4203, 64 p.

U.S. Environmental Protection Agency, 1986, Test methods for evaluating solid waste--Physical/chemical methods: Environmental Protection Agency Publication No. SW846, Washington, D.C., Office of Solid Waste and Emergency Response.

1991, Statement of work for organic analysis, multimedia, and multi-concentration: Contract Laboratory Program, OLM1.2, January 1991.

U.S. Fish and Wildlife Service, 1986, Field operations manual for resource contaminant assessment.

1989a. Organophosphate/carbamate scanning method (0-25.00): Patuxent Analytical Control Facility, Analytical Chemistry Group, Standard Operating Procedures, April 28, 1989. 
U.S. Fish and Wildlife Service, 1989b, Formal endangered species consultation on the use of acrolein (Magnacide $\mathrm{H}^{\otimes}$ ) in canals and drainage ditches within the Klamath project service area, Klamath County, Oregon, and Siskiyou County, California: U.S. Fish and Wildlife Service, case number 1-1-89-F-43.

1990, Patuxent Analytical Control Facility reference manual: Laurel, Maryland, Patuxent Wildlife Research Center, $120 \mathrm{p}$.

Wang, W., 1986, Toxicity tests of aquatic pollutants by using common duckweed: Environmental Pollution (Series B), v. 11, p. 1-14.
Ward, J.R., and Harr, C.A., eds., 1990, Methods for collection and processing of surface-water and bedmaterial samples for physical and chemical analysis: U.S. Geological Survey Open-File Report 90-140, 71 p.

Wershaw, R.L., Fishman, M.J., Grabbe, R.R., and Lowe, L.E., eds., 1987, Methods for determination of organic substances in water and fluvial sediments: U.S. Geological Survey Techniques of Water-Resources Investigations, Book 5, Chap. A3, 80 p. 



\section{TABLES}


Table 1. Surface-water sampling sites and types of samples collected at each site, 1990-92

[See figures 1, 4, and 5 for site locations. Data from U.S. Geological Survey. Abbreviations: USGS, U.S. Geological Survey; NWR, National Wildlife Refuge. USGS identification No.: Unique number for each site based on the latitude and longitude of the site. First six digits are latitude, next seven digits are longitude, and final two digits are a sequence number to uniquely identify each site]

\begin{tabular}{|c|c|c|c|}
\hline $\begin{array}{l}\text { Site } \\
\text { No. }\end{array}$ & $\begin{array}{c}\text { USGS site } \\
\text { identification } \\
\text { No. } \\
\end{array}$ & Location & Types of samples collected \\
\hline \multicolumn{4}{|c|}{ Primary sampling sites } \\
\hline 1 & 420037121333001 & J Canal below Anderson Rose Dam & $\begin{array}{l}\text { Bioassay, biological tissue, invertebrates, } \\
\text { water }\end{array}$ \\
\hline 2 & 415635121301401 & Lost River at Tule Lake & Bioassay, invertebrates, water \\
\hline 3 & 415614121294201 & N Canal at pump $\mathbf{R}$ & $\begin{array}{l}\text { Bioassay, biological tissue, fish, } \\
\text { invertebrates, water }\end{array}$ \\
\hline 4 & 415615121294901 & Tule Lake at pump $\mathbf{R}$ & Bioassay, fish, water \\
\hline 5 & 415400121294201 & 101-C Drain at pump 11 & Bioassay, invertebrates, water \\
\hline 6 & 415400121294801 & Tule Lake at pump 11 & Bioassay, water \\
\hline 7 & 415217121294001 & 101-B Drain at pump 10 & Bioassay, fish, invertebrates, water \\
\hline 8 & 415217121294901 & Tule Lake at pump 10 & Bioassay, sediment, water \\
\hline 9 & 415223121323501 & 102 Drain at pump 9 & Bioassay, invertebrates, sediment, water \\
\hline 10 & 415227121323501 & Tule Lake at pump 9 & Bioassay, sediment, water \\
\hline 11 & 415526121335101 & Channel from upper sump Tule Lake to pump D & Bioassay, fish, invertebrates, water \\
\hline 12 & 415633121420601 & Klamath Straits drain at Lower Klamath NWR & $\begin{array}{l}\text { Bioassay, fish, invertebrates, sediment, } \\
\text { water }\end{array}$ \\
\hline 13 & 420232121474501 & Klamath Straits drain at pump E & $\begin{array}{l}\text { Bioassay, biological tissue, invertebrates, } \\
\text { sediment, water }\end{array}$ \\
\hline 15 & 421404121480101 & A Canal at Upper Klamath Lake & Bioassay, sediment, water \\
\hline
\end{tabular}

Additional sites at Tule Lake NWR

\begin{tabular}{llll} 
UTL2 & 415426121330401 & Upper sump & Invertebrates, sediment \\
UTL3 & 415335121330301 & Upper sump & Invertebrates, sediment, water \\
UTL4 & 415051121280901 & Upper sump & Invertebrates, sediment \\
UTL5 & 415519121314701 & Upper sump & Invertebrates, sediment, water \\
UTL6 & 415426121314601 & Upper sump & Invertebrates, sediment \\
UTL7 & 415334121314501 & Upper sump & Invertebrates, sediment, water \\
UTL8 & 415242121314501 & Upper sump & Invertebrates, sediment \\
UTL9 & 415427121300901 & Upper sump & Invertebrates, sediment \\
UTL10 & 415334121300801 & Upper sump & Invertebrates, sediment \\
UTL11 & 415242121300701 & Upper sump & Invertebrates, sediment \\
UTL13 & 415611121314601 & Upper sump & Invertebrates, sediment \\
LTL2 & 415054121291101 & Lower sump & Invertebrates, sediment \\
LTL4 & 415051121280901 & Lower sump & Invertebrates, sediment \\
LTL5 & 415051121265801 & Lower sump & Invertebrates, sediment \\
LTL6 & 415021121251501 & Lower sump & Invertebrates, sediment \\
SSD1 & 414949121255001 & South Side drain below lower sump & Bioassay, invertebrates, sediment \\
JCN1 & 415257121235101 & J Canal above lower sump & Invertebrates, sediment \\
NC1 & 415609121291301 & N Canal above Tule Lake & Invertebrates, sediment \\
B & & Southwest corner of lower sump & Sediment \\
\hline
\end{tabular}

Additional sites at Lower Klamath NWR

\begin{tabular}{llll}
\hline 4C & 415732121401101 & Unit 4 & Sediment, water \\
9A & 415603121384801 & Unit 9 & Sediment, water \\
13B & & Unit 13B & Sediment \\
LK1 & 415821121390701 & Unit 4A inflow & Water \\
LK2 & 415518121373401 & P Canal near unit 9C & Water
\end{tabular}


Table 1. Surface-water sampling sites and types of samples collected at each site, 1990-92--Continued

\begin{tabular}{|c|c|c|c|}
\hline $\begin{array}{l}\text { Site } \\
\text { No. }\end{array}$ & $\begin{array}{c}\text { USGS site } \\
\text { identification } \\
\text { No. }\end{array}$ & Location & Types of samples collected \\
\hline
\end{tabular}

\begin{tabular}{llll}
\hline & \multicolumn{3}{c}{ Additional sites at Lower Klamath NWR--Continued } \\
\hline LK3 & 415820121390301 & Pump E inflow to unit 4A & Water \\
LK4 & 415635121420801 & Unit 7B outflow & Sediment, water \\
LK5 & 415552121361001 & P Canal at P1 Canal & Water \\
P1 & 415907121370101 & P1 Canal at White Lake & Water \\
\hline
\end{tabular}

\begin{tabular}{|c|c|c|c|}
\hline \multicolumn{4}{|c|}{ Additional sites on Lost River } \\
\hline LR1 & & Lost River at California/Oregon State line & Sediment \\
\hline LR3 & & Lost River below Anderson Rose Dam & Fish, sediment \\
\hline LR4 & & Lost River below Anderson Rose Dam & Sediment \\
\hline LR5 & 420122121355701 & Lost River at Merrill, Oregon & Sediment, water \\
\hline \multicolumn{4}{|c|}{ Additional sites on Clear Lake } \\
\hline $\mathrm{Cl1}$ & 415348121101501 & Clear Lake & Invertebrates, sediment \\
\hline $\mathrm{Cl} 2$ & 415501121045501 & Clear Lake & Invertebrates, sediment \\
\hline
\end{tabular}

LL6

LL7

LL7A

LL8

LL10 415101121303701

LL13

LL14

LL16 415016121302901

LL17

LL18

LL25

LL26

LL27

LL28

LL29

LL30

LL31

LL32

LL33

LL34

LL35

LL41

LL46

LL56

LL57

LL70

415550121291301

415512121291201
102-C bypass drain at field 69 , lower sump fields

102-C-1 bypass drain at field 68 , lower sump fields

102-C-1 bypass drain at field 69 , lower sump fields

102-C-2 drain at field 68, lower sump fields

102-C-2 drain near SE corner of field 70, lower sump fields

102-C-1 drain at field 37, lower sump fields

102-C-1 drain at field 32, lower sump fields

$R$ canal near SE corner of field 35, lower sump fields

$\mathrm{R}-1$ canal at field 35 , lower sump fields

102-C-1-b drain at field 36, lower sump fields

Q-3-b canal at field 47 , lower sump fields

415010121325701 Q-3 canal near SW corner of field 47, lower sump fields

$102-\mathrm{H}$ drain at field 47 , lower sump fields

$102-\mathrm{H}$ drain at field 48 , lower sump fields

$102-\mathrm{H}$ drain at field 49 , lower sump fields

$102-\mathrm{H}$ drain at field 50 , lower sump fields

415028121330301 Q-3-a canal near SW corner of field 46, lower sump fields

Q-3 canal near SW corner of field 46, lower sump fields

102-G drain at field 39 , lower sump fields

415059121312201102 drain near SW corner of field 29 , lower sump fields

102-C-1-b drain at field 30, lower sump fields

102 drain at field 15 , lower sump fields

101 drain near SW corner of field 1, League of Nations fields

N-1 canal at field 4, League of Nations fields

N-1 canal at field 3, League of Nations fields

N-4 canal at field 19, League of Nations fields
Bioassay, water

Bioassay, water

Bioassay, water

Bioassay, water

Bioassay, water

Bioassay, water

Bioassay, water

Bioassay, water

Bioassay, water

Bioassay, water

Bioassay, water

Bioassay, water

Bioassay, water

Bioassay, water

Bioassay, water

Bioassay, water

Bioassay, water

Bioassay, water

Bioassay, water

Bioassay, water

Bioassay, water

Bioassay, water

Bioassay, water

Bioassay, water

Bioassay, water

Bioassay, water 
Table 1. Surface-water sampling sites and types of samples collected at each site, 1990-92--Continued

\begin{tabular}{lcc}
\hline $\begin{array}{l}\text { Site } \\
\text { No. }\end{array}$ & USGS site & Lentification \\
No. & Location & Types of samples collected \\
\hline & & Sites on Tule Lake NWR leaselands--Continued \\
\hline
\end{tabular}

Sites on Tule Lake NWR leaselands--Continued

LL71

LL77

LL78

LL79

LL87

LL88

LL89

LL90

LL91

LL92

LL93

LL146

LL148

LL172

LL173

LL174

LL175

LL176

LL201

LL205

LL206

LL214

LL215

LL218

LL220

LL221

LL222

LL223

LL224

LL225

LL226

LL227

LL230

LL232

LL233

LL234

LL235

LL237

LL238

LL248

LL251

LL255

LL258

LL259

LL260

LL261

LL262
415401121280001

415309121280001

415135121255401

415142121264601

415152121250101

415338121250502 .

415126121305001

415056121330801

415028121330303

415102121304802

415126121305002

415553121280201

415507121272601

415451121272601

415500121264902

415500121264901

415400121291101

415401121283801

415309121280002

415253121280001

415342121291101

415327121272501

415200121272401

415200121272402

415142121272401

415028121330304

415102121304801

415313121250403

415313121250404 .

415313121250401

415313121250402

415314121252802
101-C-4 drain at field 26, League of Nations fields

101-C-2 drain at field 19, League of Nations fields

101-C-2 drain at field 20, League of Nations fields

101-C drain near SE corner of field 20, League of Nations fields

$\mathrm{N}-12-\mathrm{c}$ canal at field 54, Frog Pond fields

$\mathrm{N}-12-\mathrm{c}$ canal at field 53, Frog Pond fields

N-12-c canal at field 52, Frog Pond fields

N-12-c canal near SE corner of field 51, Frog Pond fields

N-12-c canal at field 50, Frog Pond fields

N-12-c canal at field 49, Frog Pond fields

$\mathrm{N}-12$ canal at field 49, Frog Pond fields

N-15-b canal near NE corner of field 84, Frog Pond fields

N-15-b canal at field 84, Frog Pond fields

101-B-5 drain near NE corner of field 72, Frog Pond fields

101-B-5-a drain near SE corner of field 95, Frog Pond fields

101-B-5-a drain at field 95, Frog Pond fields

101-B-5-d drain at field 91, Frog Pond fields

$\mathrm{N}$ canal near NE corner of field 91, Frog Pond fields

$\mathrm{R}$ canal near NW corner of field 12 , lower sump fields

$\mathrm{Q}$ canal near SW corner of field 22, lower sump fields

102-F drain near NW corner of field 46, lower sump fields

$\mathrm{R}$ canal near SW corner of field 31, lower sump fields

R canal near SW corner of field 12, lower sump fields

N-4 canal near SE corner of field 15, League of Nations fields

N-6 canal near SE corner of field 22, League of Nations fields

N-6 canal near SE corner of field 23, League of Nations fields

101-C-6 drain near NW corner of field 27, League of Nations fields

101-C-6 drain near SW corner of field 27, League of Nations fields

101-C drain near SW corner of field 7, League of Nations fields

101-C drain near SE corner of field 14, League of Nations fields

101-B-2 drain near NW corner of field 51, Frog Pond fields

101-B-2 drain near NW corner of field 52, at Frog Pond fields

N-12 canal near NW corner of field 36, Frog Pond fields

N-12-b canal near NE corner of field 58, Frog Pond fields

N-12-b canal near NE corner of field 63, Frog Pond fields

N-12-b canal near NE corner of field 63, Frog Pond fields

N-12-b canal near SE corner of field 64, Frog Pond fields

101-B-5-b drain at field 93, Frog Pond fields

101-B-5-b drain at field 93, Frog Pond fields

102-F drain near NE corner of field 46, lower sump fields

$R$ canal near SE corner of field 31, lower sump fields

101-B-5-c drain near NE corner of field 92, Frog Pond fields

N-13 canal near NW corner of field 92, Frog Pond fields

N-13 canal near NW corner of field 92, Frog Pond fields

$\mathrm{N}$ canal near NE corner of field 92, Frog Pond fields

101-B-5-d drain at field 85, Frog Pond fields

N-13 canal near NE corner of field 86, Frog Pond fields
Bioassay, water

Bioassay, water

Bioassay, water

Bioassay, water

Bioassay, water

Bioassay, water

Bioassay, water

Bioassay, water

Bioassay, water

Bioassay, water

Bioassay, water

Bioassay, water

Bioassay, water

Bioassay, water

Bioassay, water

Bioassay, water

Bioassay, water

Bioassay, water

Bioassay, water

Bioassay, water

Bioassay, water

Bioassay, water

Bioassay, water

Bioassay, water

Bioassay, water

Bioassay, water

Bioassay, water

Bioassay, water

Bioassay, water

Bioassay, water

Bioassay, water

Bioassay, water

Bioassay, water

Bioassay, water

Bioassay, water

Bioassay, water

Bioassay, water

Bioassay, water

Bioassay, water

Bioassay, water

Bioassay, water

Bioassay, water

Bioassay, water

Bioassay, water

Bioassay, water

Bioassay, water

Bioassay, water 
Table 2. Principal biological tissue sampling locations, 1990-92

[Sample locations shown in figures 1, 2, and 3. NWR, National Wildlife Refuge]

\begin{tabular}{|c|c|c|}
\hline Sample Type & Sample No. & Location \\
\hline \multicolumn{3}{|c|}{ Aquatic invertebrates } \\
\hline Backswimmer & LK6BS & Lower Klamath NWR, near site 12 \\
\hline Chironomid larvae & $\begin{array}{l}\text { TLCH07A } \\
\text { TLCH08A }\end{array}$ & $\begin{array}{l}\text { Upper sump, Tule Lake, near site UTL7 } \\
\text { Upper sump, Tule Lake, near site UTL8 }\end{array}$ \\
\hline Leeches & TLL07A & Upper sump, Tule Lake, near site UTL7 \\
\hline Waterboatman & LK6WB & Lower Klamath NWR, near site 12 \\
\hline \multicolumn{3}{|r|}{ Fish } \\
\hline Fathead minnow & $\begin{array}{l}12 \mathrm{FH} 01 \mathrm{~J} \\
12 \mathrm{FH} 02 \mathrm{~J} \\
12 \mathrm{FH} 03 \mathrm{~J}\end{array}$ & $\begin{array}{l}\text { Klamath Straits drain near site } 12 \text {, Lower Klamath NWR } \\
\text { Klamath Straits drain near site } 12 \text {, Lower Klamath NWR } \\
\text { Klamath Straits drain near site } 12 \text {, Lower Klamath NWR }\end{array}$ \\
\hline Tui chub & $\begin{array}{l}\text { CLTC01A } \\
\text { CLTC01J } \\
\text { CLTC02A } \\
\text { CLTC02J } \\
\text { CLTC03A } \\
\text { CLTC03J } \\
\text { 2TC01A } \\
\text { 2TC01J } \\
\text { 2TC02A } \\
\text { 2TC02J } \\
\text { 2TC03A } \\
\text { 2TC03J } \\
\text { 3TC01J } \\
\text { 3TC02J } \\
\text { 3TC03J } \\
\text { 7TC01J } \\
\text { 7TC02J } \\
\text { 7TC03J } \\
11 T C 01 J \\
11 T C 02 J \\
11 T C 03 J\end{array}$ & $\begin{array}{l}\text { Lost River below Clear Lake Dam } \\
\text { Lost River below Clear Lake Dam } \\
\text { Lost River below Clear Lake Dam } \\
\text { Lost River below Clear Lake Dam } \\
\text { Lost River below Clear Lake Dam } \\
\text { Lost River below Clear Lake Dam } \\
\text { Lost River below Anderson Rose Dam } \\
\text { Lost River below Anderson Rose Dam } \\
\text { Lost River below Anderson Rose Dam } \\
\text { Lost River below Anderson Rose Dam } \\
\text { Lost River below Anderson Rose Dam } \\
\text { Lost River below Anderson Rose Dam } \\
\text { N Canal near site } 3 \\
\text { N Canal near site } 3 \\
\text { N Canal near site } 3 \\
\text { 101-B drain near site } 7 \\
\text { 101-B drain near site } 7 \\
\text { 101-B drain near site } 7 \\
\text { Channel to pump D near site } 11 \\
\text { Channel to pump D near site } 11 \\
\text { Channel to pump D near site } 11\end{array}$ \\
\hline
\end{tabular}

\section{Birds}

American avocet egg $\quad 4 \mathrm{BA} 1$

4BA2

$4 \mathrm{BA} 3$

$4 \mathrm{BA} 4$

4BA5

4BA6

4BA7

4BA8

4BA9

4BA10

4BA11

4BA12
Lower Klamath NWR, unit 4B

Lower Klamath NWR, unit 4B

Lower Klamath NWR, unit 4B

Lower Klamath NWR, unit 4B

Lower Klamath NWR, unit 4B

Lower Klamath NWR, unit 4B

Lower Klamath NWR, unit 4B

Lower Klamath NWR, unit 4B

Lower Klamath NWR, unit 4B

Lower Klamath NWR, unit 4B

Lower Klamath NWR, unit 4B

Lower Klamath NWR, unit 4B 
Table 2. Principal biological tissue sampling locations, 1990-92--Continued

\begin{tabular}{|c|c|c|}
\hline Sample Type & Sample No. & Location \\
\hline \multicolumn{3}{|c|}{ Birds--Continued } \\
\hline American coot egg & $\begin{array}{l}7 \mathrm{AC} 1 \\
7 \mathrm{AC} 2 \\
7 \mathrm{AC} 3 \\
7 \mathrm{AC} 4 \\
7 \mathrm{AC} 5 \\
\text { LK12CC1 } \\
\text { LK12CC2 } \\
\text { LK12CC3 } \\
\text { LK12CC4 } \\
\text { LK12CC5 }\end{array}$ & $\begin{array}{l}\text { Lower Klamath NWR, unit 7A } \\
\text { Lower Klamath NWR, unit 7A } \\
\text { Lower Klamath NWR, unit 7A } \\
\text { Lower Klamath NWR, unit 7A } \\
\text { Lower Klamath NWR, unit 7A } \\
\text { Lower Klamath NWR, unit 12C } \\
\text { Lower Klamath NWR, unit 12C } \\
\text { Lower Klamath NWR, unit 12C } \\
\text { Lower Klamath NWR, unit 12C } \\
\text { Lower Klamath NWR, unit 12C }\end{array}$ \\
\hline Egret egg & E1 & Upper sump, Tule Lake \\
\hline Mallard egg & $\begin{array}{l}\text { M441 } \\
\text { M447 } \\
\text { M459A } \\
\text { M459B } \\
\text { M499A } \\
\text { M499B } \\
\text { M516A } \\
\text { M516B } \\
\text { M563 } \\
\text { M574 } \\
92 M 2\end{array}$ & $\begin{array}{l}\text { Lower Klamath NWR, unit 8A } \\
\text { Lower Klamath NWR, unit 12A } \\
\text { Lower Klamath NWR, unit 7B } \\
\text { Lower Klamath NWR, unit 7B } \\
\text { Lower Klamath NWR, unit 6B } \\
\text { Lower Klamath NWR, unit 6B } \\
\text { Lower Klamath NWR, unit 6B } \\
\text { Lower Klamath NWR, unit 6B } \\
\text { Lower Klamath NWR, unit 6B } \\
\text { Lower Klamath NWR, unit 6B } \\
\text { Lower Klamath NWR, unit 13A }\end{array}$ \\
\hline
\end{tabular}


Table 2. Principal biological tissue sampling locations, 1990-92--Continued

\begin{tabular}{|c|c|c|}
\hline Sample Type & Sample No. & Location \\
\hline \multicolumn{3}{|c|}{ Birds--Continued } \\
\hline Western grebe egg--Continued & $\begin{array}{l}\text { 92TLWG6A } \\
\text { 92TLWG7A } \\
\text { 92TLWG8 }\end{array}$ & $\begin{array}{l}\text { Upper sump, Tule Lake } \\
\text { Upper sump, Tule Lake } \\
\text { Upper sump, Tule Lake }\end{array}$ \\
\hline White-faced ibis egg & $\begin{array}{l}\text { I1 } \\
\text { I2 } \\
\text { I3 } \\
\text { I4 } \\
\text { I5 } \\
\text { LK12AI1 } \\
\text { LK12AI2 } \\
\text { LK12AI3 } \\
\text { LK12AI4 } \\
\text { LK12AI5 } \\
\text { LK12AI7 } \\
\text { LK12AI8 } \\
\text { LK12AI9 } \\
\text { LK12AI10 } \\
\text { LK12AI11 } \\
\text { LK12AI12 } \\
\text { 92LKI1 } \\
\text { 92LKI2 } \\
\text { 92LKI3 } \\
\text { 92LKI4 } \\
\text { 92LKI5 } \\
\text { 92LKI6 } \\
\text { 92LKI7 } \\
\text { 92LKI8 } \\
\text { 92LKI9 } \\
\text { 92LKI10 }\end{array}$ & $\begin{array}{l}\text { Lower Klamath NWR, unit 12A } \\
\text { Lower Klamath NWR, unit 12A } \\
\text { Lower Klamath NWR, unit 12A } \\
\text { Lower Klamath NWR, unit 12A } \\
\text { Lower Klamath NWR, unit 12A } \\
\text { Lower Klamath NWR, unit 12A } \\
\text { Lower Klamath NWR, unit 12A } \\
\text { Lower Klamath NWR, unit 12A } \\
\text { Lower Klamath NWR, unit 12A } \\
\text { Lower Klamath NWR, unit 12A } \\
\text { Lower Klamath NWR, unit 12A } \\
\text { Lower Klamath NWR, unit 12A } \\
\text { Lower Klamath NWR, unit 12A } \\
\text { Lower Klamath NWR, unit 12A } \\
\text { Lower Klamath NWR, unit 12A } \\
\text { Lower Klamath NWR, unit 12A } \\
\text { Lower Klamath NWR, unit 13B } \\
\text { Lower Klamath NWR, unit 13B } \\
\text { Lower Klamath NWR, unit 12B } \\
\text { Lower Klamath NWR, unit 13B } \\
\text { Lower Klamath NWR, unit 13B } \\
\text { Lower Klamath NWR, unit 13B } \\
\text { Lower Klamath NWR, unit 13B } \\
\text { Lower Klamath NWR, unit 13B } \\
\text { Lower Klamath NWR, unit 13B } \\
\text { Lower Klamath NWR, unit 13B }\end{array}$ \\
\hline White-faced ibis nestling & I6Y & Lower Klamath NWR, unit 12A \\
\hline
\end{tabular}


Table 3. Concentrations of carbon and organochlorine compounds in bottom sediment, 1990

[See figure 1 for site locations. Carbon in grams per kilogram. Organochlorine compounds in micrograms per kilogram, wet weight. PCN, Polychlorinated naphthalene. PCB, Polychlorinated biphenyls. Detections are in bold print. <, actual value is less than value shown]

\begin{tabular}{|c|c|c|c|c|c|c|c|c|c|}
\hline Site & Date & $\begin{array}{c}\text { Carbon, } \\
\text { inor- } \\
\text { ganic }\end{array}$ & $\begin{array}{l}\text { Carbon, } \\
\text { inor- } \\
\text { ganic } \\
\text { plus } \\
\text { organic }\end{array}$ & $\mathrm{PCN}$ & PCB & Aldrin & $\begin{array}{l}\text { Chlor- } \\
\text { dane }\end{array}$ & DDD & $\mathrm{DDE}$ \\
\hline UTL2 & Aug. 22 & 19 & 100 & $<1.0$ & $<1.0$ & $<0.1$ & 1.0 & 0.9 & 0.8 \\
\hline UTL3 & Aug. 22 & 16 & 100 & $<1.0$ & $<1.0$ & $<.1$ & 1.0 & .7 & .7 \\
\hline UTL4 & Aug. 22 & 27 & 110 & $<1.0$ & $<1.0$ & $<.1$ & 1.0 & .6 & .9 \\
\hline UTL5 & Aug. 22 & 8 & 97 & $<1.0$ & $<1.0$ & $<.1$ & 1.0 & .6 & 1.0 \\
\hline UTL6 & Aug. 22 & 19 & 100 & $<1.0$ & $<1.0$ & $<.1$ & 1.0 & .7 & .9 \\
\hline UTL7 & Aug. 22 & 12 & 65 & $<1.0$ & $<1.0$ & $<.1$ & 1.0 & .5 & .5 \\
\hline UTL8 & Aug. 22 & 23 & 110 & $<1.0$ & $<1.0$ & $<.1$ & 1.0 & 1.1 & 1.2 \\
\hline UTL9 & Aug. 22 & 11 & 120 & $<1.0$ & $<1.0$ & $<.1$ & $<1.0$ & .2 & .3 \\
\hline UTL10 & Aug. 22 & 15 & 120 & $<1.0$ & $<1.0$ & $<.1$ & 1.0 & 1.2 & 1.3 \\
\hline UTL11 & Aug. 22 & 25 & 95 & $<1.0$ & $<1.0$ & $<.1$ & 1.0 & 1.2 & 1.8 \\
\hline UTL13 & Aug. 22 & 11 & 110 & $<1.0$ & $<1.0$ & $<.1$ & 1.0 & .8 & 1.5 \\
\hline LTL2 & $\begin{array}{l}\text { Aug. } 23 \\
\text { Aug. } 23\end{array}$ & $\overline{23}$ & 100 & $\begin{array}{l}<1.0 \\
<1.0\end{array}$ & $\begin{array}{l}<1.0 \\
<1.0\end{array}$ & $\begin{array}{l}<.1 \\
<.1\end{array}$ & $\begin{array}{l}1.0 \\
1.0\end{array}$ & $\begin{array}{l}.4 \\
.6\end{array}$ & $\begin{array}{l}.6 \\
.9\end{array}$ \\
\hline LTL4 & Aug. 23 & 19 & 95 & $<1.0$ & $<1.0$ & $<.1$ & 1.0 & .6 & .8 \\
\hline LTL5 & Aug. 23 & 20 & 100 & $<1.0$ & $<1.0$ & $<.1$ & $<1.0$ & 1.1 & 1.4 \\
\hline LTL6 & Aug. 23 & 15 & 73 & $<1.0$ & $<1.0$ & $<.1$ & $<1.0$ & 1.0 & .9 \\
\hline LR0 & Aug. 21 & 1 & 42 & $<1.0$ & $<1.0$ & $<.1$ & 4.0 & 1.6 & 2.7 \\
\hline LR5 & Aug. 28 & $<1$ & 25 & $<1.0$ & $<1.0$ & $<.1$ & 3.0 & 1.5 & 1.6 \\
\hline SSD1 & Aug. 27 & 8 & 58 & $<1.0$ & $<1.0$ & $<.1$ & 6.0 & 2.4 & 2.1 \\
\hline JCN1 & Aug. 27 & $<1$ & 37 & $<1.0$ & $<1.0$ & $<.1$ & 4.0 & 1.7 & 2.1 \\
\hline 1 & Aug. 23 & $<1$ & 14 & $<1.0$ & $<1.0$ & $<.1$ & 2.0 & .5 & .9 \\
\hline 12 & Aug. 24 & 14 & 100 & $<1.0$ & $<1.0$ & $<.1$ & 1.0 & .2 & .6 \\
\hline $\mathrm{NCl}$ & Aug. 27 & $<1$ & 62 & $<1.0$ & $<1.0$ & $<.1$ & 6.0 & 4.4 & 4.5 \\
\hline $4 \mathrm{C}$ & Aug. 24 & 25 & 140 & $<1.0$ & $<1.0$ & $<.1$ & 1.0 & .2 & .4 \\
\hline $9 \mathrm{~A}$ & Aug. 24 & 18 & 160 & $<1.0$ & $<1.0$ & $<.1$ & 1.0 & .3 & 1.0 \\
\hline CL1 & Aug. 21 & $<1$ & 7 & $<1.0$ & $<1.0$ & $<.1$ & $<1.0$ & .3 & .4 \\
\hline
\end{tabular}


Table 3. Concentrations of carbon and organichlorine compounds in bottom sediment, 1990--Continued

\begin{tabular}{|c|c|c|c|c|c|c|c|c|c|c|}
\hline Site & DDT & Dieldrin & $\begin{array}{l}\text { Endo- } \\
\text { sulfan }\end{array}$ & Endrin & $\begin{array}{l}\text { Hepta- } \\
\text { chlor }\end{array}$ & $\begin{array}{l}\text { Hepta- } \\
\text { chlor } \\
\text { epoxide }\end{array}$ & Lindane & $\begin{array}{l}\text { Meth- } \\
\text { oxy- } \\
\text { chlor }\end{array}$ & Mirex & $\begin{array}{l}\text { Toxa- } \\
\text { phene }\end{array}$ \\
\hline UTL2 & $<0.1$ & $<0.1$ & $<0.1$ & $<0.1$ & $<0.1$ & $<0.1$ & $<0.1$ & $<0.1$ & $<0.1$ & $<10.0$ \\
\hline UTL3 & $<.1$ & $<.1$ & $<.1$ & $<.1$ & $<.1$ & $<.1$ & $<.1$ & $<.1$ & $<.1$ & $<10.0$ \\
\hline UTL4 & $<.1$ & $<.1$ & $<.1$ & $<.1$ & $<.1$ & $<.1$ & $<.1$ & $<.1$ & $<.1$ & $<10.0$ \\
\hline UTL5 & $<.1$ & $<.1$ & $<.1$ & $<.1$ & $<.1$ & $<.1$ & $<.1$ & $<.1$ & $<.1$ & $<10.0$ \\
\hline UTL6 & $<.1$ & $<.1$ & $<.1$ & $<.1$ & $<.1$ & $<.1$ & $<.1$ & $<.1$ & $<.1$ & $<10.0$ \\
\hline UTL7 & $<.1$ & $<.1$ & $<.1$ & $<.1$ & $<.1$ & $<.1$ & $<.1$ & $<.1$ & $<.1$ & $<10.0$ \\
\hline UTL8 & $<.1$ & $<.1$ & $<.1$ & $<.1$ & $<.1$ & $<.1$ & $<.1$ & $<.1$ & $<.1$ & $<10.0$ \\
\hline UTL9 & $<.1$ & $<.1$ & $<.1$ & $<.1$ & $<.1$ & $<.1$ & $<.1$ & $<.1$ & $<1$ & $<10.0$ \\
\hline UTL10 & $<.1$ & $<.1$ & $<.1$ & $<.1$ & $<.1$ & $<.1$ & $<.1$ & $<.1$ & $<.1$ & $<10.0$ \\
\hline UTL11 & $<.1$ & $<.1$ & $<.1$ & $<.1$ & $<.1$ & $<.1$ & $<.1$ & $<.1$ & $<.1$ & $<10.0$ \\
\hline UTL13 & $<.1$ & $<.1$ & $<.1$ & $<.1$ & $<.1$ & $<.1$ & $<.1$ & $<.1$ & $<.1$ & $<10.0$ \\
\hline LTL2 & $\begin{array}{l}<.1 \\
<.1\end{array}$ & $\begin{array}{l}<.1 \\
<.1\end{array}$ & $\begin{array}{l}<.1 \\
<.1\end{array}$ & $\begin{array}{l}<.1 \\
<.1\end{array}$ & $\begin{array}{l}<.1 \\
<.1\end{array}$ & $\begin{array}{l}<.1 \\
<.1\end{array}$ & $\begin{array}{l}<.1 \\
<.1\end{array}$ & $\begin{array}{l}<.1 \\
<.1\end{array}$ & $\begin{array}{l}<.1 \\
<.1\end{array}$ & $\begin{array}{l}<10.0 \\
<10.0\end{array}$ \\
\hline LTL4 & $<.1$ & $<.1$ & $<.1$ & $<.1$ & $<.1$ & $<.1$ & $<.1$ & $<.1$ & $<.1$ & $<10.0$ \\
\hline LTL5 & $<.1$ & $<.1$ & $<.1$ & $<.1$ & $<.1$ & $<.1$ & $<.1$ & $<.1$ & $<.1$ & $<10.0$ \\
\hline LTL6 & $<.1$ & $<.1$ & $<.1$ & $<.1$ & $<.1$ & $<.1$ & $<.1$ & $<.1$ & $<.1$ & $<10.0$ \\
\hline LRO & .5 & .1 & $<.1$ & $<.1$ & $<.1$ & $<.1$ & $<.1$ & $<10$ & $<.1$ & $<10.0$ \\
\hline LR5 & .2 & $<.1$ & $<.1$ & $<.1$ & $<.1$ & $<.1$ & $<.1$ & $<.1$ & $<.1$ & $<10.0$ \\
\hline SSD1 & $<.1$ & $<.1$ & $<.1$ & $<.1$ & $<.1$ & $<.1$ & $<.1$ & $<.1$ & $<.1$ & $<10.0$ \\
\hline JCN1 & .2 & .1 & $<.1$ & $<.1$ & $<.1$ & $<.1$ & $<.1$ & $<.1$ & $<.1$ & $<10.0$ \\
\hline 1 & .3 & $<.1$ & $<.1$ & $<.1$ & $<.1$ & $<.1$ & $<.1$ & $<.1$ & $<.1$ & $<10.0$ \\
\hline 12 & $<.1$ & $<.1$ & $<.1$ & $<.1$ & $<.1$ & $<.1$ & $<.1$ & $<.1$ & $<.1$ & $<10.0$ \\
\hline $\mathrm{NC1}$ & .2 & .1 & $<.1$ & $<.1$ & $<.1$ & $<.1$ & $<.1$ & $<.1$ & $<.1$ & $<10.0$ \\
\hline $4 C$ & $<.1$ & $<.1$ & $<.1$ & $<.1$ & $<.1$ & $<.1$ & $<.1$ & $<.1$ & $<.1$ & $<10.0$ \\
\hline $9 \mathrm{~A}$ & $<.1$ & $<.1$ & $<.1$ & $<.1$ & $<.1$ & $<.1$ & $<.1$ & $<.1$ & $<.1$ & $<10.0$ \\
\hline CL1 & $<.1$ & $<.1$ & $<.1$ & $<.1$ & $<.1$ & $<.1$ & $<.1$ & $<1.0$ & $<.1$ & $<10.0$ \\
\hline
\end{tabular}


Table 4. Particle-size distribution in bottom sediment, 1990

[See figure 1 for site locations. --, all sediment is finer than indicated particle size]

\begin{tabular}{|c|c|c|c|c|c|c|c|c|c|c|c|c|c|}
\hline \multirow{2}{*}{ Site } & \multirow{2}{*}{ Date } & \multicolumn{12}{|c|}{$\begin{array}{l}\text { Percent finer than particle size, } \\
\text { in millimeters, indicated }\end{array}$} \\
\hline & & 16.0 & 8.00 & 4.00 & 2.00 & 1.00 & 0.500 & 0.250 & 0.125 & 0.062 & 0.031 & 0.016 & 0.004 \\
\hline UTL2 & Aug. 22 & -- & $\cdots$ & - & 100 & 90 & 63 & 45 & 38 & 35 & 34 & 31 & 23 \\
\hline UTL3 & Aug. 22 & -- & -- & -- & -- & 100 & 99 & 94 & 82 & 67 & 67 & 63 & 48 \\
\hline UTL4 & Aug. 22 & -- & -- & -. & -- & -- & -. & 100 & 92 & 84 & 83 & 80 & 65 \\
\hline UTL5 & Aug. 22 & -- & -- & 100 & 98 & 86 & 66 & 52 & 42 & 37 & 36 & 34 & 25 \\
\hline UTL7 & Aug. 22 & -- & -- & -- & -- & 100 & 83 & 68 & 58 & 53 & 53 & 49 & 36 \\
\hline UTL8 & Aug. 22 & -- & -- & -- & -- & 100 & 97 & 91 & 86 & 81 & 79 & 74 & 53 \\
\hline UTL9 & Aug. 22 & -- & -- & -- & -- & -- & -- & 100 & 99 & 95 & 94 & 86 & 57 \\
\hline UTL13 & Aug. 22 & -- & -- & -- & -- & -- & -- & 100 & 99 & 97 & 97 & 94 & 68 \\
\hline LTL2 & Aug. 23 & -- & -- & -- & -- & -- & 100 & 98 & 95 & 93 & 91 & 88 & 71 \\
\hline LTL4 & Aug. 23 & -- & -- & -- & 100 & 94 & 76 & 64 & 57 & 54 & 53 & 50 & 38 \\
\hline LTL5 & Aug. 23 & -- & -- & -- & -- & 100 & 99 & 95 & 91 & 88 & 88 & 82 & 57 \\
\hline LR0 & Aug. 21 & 100 & 72 & 72 & 64 & 59 & 56 & 52 & 46 & 28 & 25 & 22 & 13 \\
\hline LR5 & Aug. 28 & -- & -- & 100 & 99 & 97 & 89 & 68 & 46 & 38 & 33 & 27 & 18 \\
\hline SSD1 & Aug. 27 & -- & -- & 100 & 97 & 93 & 89 & 84 & 79 & 74 & 73 & 71 & 50 \\
\hline JCN1 & Aug. 27 & 100 & 96 & 95 & 93 & 91 & 85 & 55 & 46 & 43 & 40 & 35 & 27 \\
\hline 1 & Aug. 23 & -- & -- & -- & 100 & 94 & 88 & 62 & 25 & 14 & 13 & 12 & 8 \\
\hline $\mathrm{NCl}$ & Aug. 27 & -- & -- & 100 & 99 & 98 & 92 & 78 & 70 & 68 & 66 & 63 & 44 \\
\hline $4 C$ & Aug. 24 & -- & -- & -- & -. & 100 & 99 & 96 & 96 & 90 & 67 & 54 & 30 \\
\hline CL1 & Aug. 21 & 100 & 95 & 85 & 62 & 50 & 46 & 40 & 31 & 18 & 16 & 14 & 11 \\
\hline
\end{tabular}

Table 5. Moisture content, arsenic, mercury, and chlorophenoxy acid, organochlorine, organophosphate, and carbamate. pesticides in bottom sediment, 1992

[See figure 1 for site locations. Values (except moisture) in micrograms per gram $(\mu \mathrm{g} / \mathrm{g})$, wet weight; <, actual value is less than value shown; --, not analyzed]

\begin{tabular}{|c|c|c|c|c|c|c|c|c|c|c|}
\hline Site & Date & $\begin{array}{l}\text { Moisture } \\
\text { content } \\
\text { (percent) }\end{array}$ & Arsenic & Mercury & Dicamba & $\begin{array}{l}\text { Dichloro- } \\
\text { prop }\end{array}$ & $2,4-\mathrm{D}$ & $2,4,5-\mathrm{T}$ & 2,4-DB & PCP \\
\hline LR3 & July 27 & 61.2 & 1.36 & 0.03 & $<0.01$ & $<0.01$ & $<0.01$ & $<0.01$ & $<0.01$ & $<0.01$ \\
\hline LR4 & July 27 & 45.7 & 1.53 & .05 & -- & -- & -- & -- & -- & -- \\
\hline 8 & July 27 & 72.3 & .89 & .01 & -- & -- & -- & -- & -. & -- \\
\hline B & July 28 & 72.0 & $\ldots$ & -- & $<.01$ & $<.01$ & $<.01$ & $<.01$ & $<.01$ & $<.01$ \\
\hline 9 & June 1 & 72.6 & -- & -- & $<.01$ & $<.01$ & $<.01$ & $<.01$ & $<.01$ & $<.01$ \\
\hline 9 & June 16 & 71.8 & -- & -- & $<.01$ & $<.01$ & $<.01$ & $<.01$ & $<.01$ & $<.01$ \\
\hline 10 & June 16 & 77.4 & -. & -- & $<.01$ & $<.01$ & $<.01$ & $<.01$ & $<.01$ & $<.01$ \\
\hline 10 & July 28 & 83.0 & -- & .. & $<.01$ & $<.01$ & $<.01$ & $<.01$ & $<.01$ & $<.01$ \\
\hline 12 & June 28 & 78.8 & -- & -. & $<.01$ & $<.01$ & $<.01$ & $<.01$ & $<.01$ & $<.01$ \\
\hline 13 & June 15 & 38.8 & 2.65 & .06 & $<.01$ & $<.01$ & $<.01$ & $<.0 \mathrm{i}$ & $<.01$ & $<.01$ \\
\hline 15 & June 28 & 57.7 & 1.07 & .09 & -- & -- & -- & -- & -- & -- \\
\hline $4 \mathrm{C}$ & June 28 & 86.8 & 3.18 & .01 & $<.01$ & $<.01$ & $<.01$ & $<.01$ & $<.01$ & $<.01$ \\
\hline $9 \mathrm{~A}$ & May 31 & 70.6 & 4.25 & .04 & $<.01$ & $<.01$ & $<.01$ & $<.01$ & $<.01$ & $<.01$ \\
\hline $9 \mathrm{~A}$ & June 28 & 83.6 & 3.12 & .02 & $<.01$ & $<.01$ & $<.01$ & $<.01$ & $<.01$ & $<.01$ \\
\hline $9 \mathrm{~A}$ & July 27 & 85.0 & 5.95 & .03 & $<.01$ & $<.01$ & $<.01$ & $<.01$ & $<.01$ & $<.01$ \\
\hline $13 B$ & May 31 & 91.6 & 2.13 & $<.01$ & $<.01$ & $<.01$ & $<.01$ & $<.01$ & $<.01$ & $<.01$ \\
\hline $13 B$ & June 28 & 94.2 & 1.23 & .03 & $<.01$ & $<.01$ & $<.01$ & $<.01$ & $<.01$ & $<.01$ \\
\hline
\end{tabular}

Samples analyzed for listed chemicals but none detected:
10 July 28
Gamma BHC, gamma chlordane, heptachlor epoxide, mirex, o,p-DDD, o,p-DDE, o,p-DDT, oxychlor- dane,p,p-DDD, p,p-DDE, p,p-DDT, toxaphene, trans-nonachlor, HCB, total PCBs, alpha BHC, alpha chlordane, beta BHC, dieldrin, endrin. Detection limit $=0.01 \mu \mathrm{g} / \mathrm{g}$ wet weight.
$\begin{array}{ll}9 & \text { June } 16 \\ \text { LR2 } & \text { July } 27\end{array}$
EPN, acephate, azinphos-methyl, chlorpyrifos, coumaphos, demeton, diazinon, dichlorvos, dichrotophos, dimethoate, disulfoton, ethoprop, famphur, fensulfothion, fenthion, malathion, methami- dophos, methyl parathion, mevinphos, monocrotophos, parathion, phorate, terbufos, trichlorfon. Detection limit $=0.5 \mu \mathrm{g} / \mathrm{g}$ wet weight. 
Table 6. Field measurements of $\mathrm{pH}$ and dissolved oxygen in surface water from sediment-collection sites, 1990

[See figure 1 for site locations. $\mathrm{mg} / \mathrm{L}$, milligram per liter; $>$, actual value is greater than value shown; --, no data]

\begin{tabular}{|c|c|c|c|c|c|c|c|c|c|}
\hline Site & Date & Time & $\begin{array}{c}\mathrm{pH}, \\
\text { water, } \\
\text { whole, } \\
\text { field } \\
\text { (standard } \\
\text { units) }\end{array}$ & $\begin{array}{l}\text { Oxygen, } \\
\text { dis- } \\
\text { solved } \\
(\mathrm{mg} / \mathrm{L})\end{array}$ & Site & Date & Time & $\begin{array}{c}\mathrm{pH} \\
\text { water, } \\
\text { whole, } \\
\text { field } \\
\text { (standard } \\
\text { units) }\end{array}$ & $\begin{array}{c}\text { Oxygen } \\
\text { dis- } \\
\text { solved } \\
(\mathrm{mg} / \mathrm{L})\end{array}$ \\
\hline UTL2 & $\begin{array}{l}\text { July } 12 \\
\text { Aug. } 22\end{array}$ & $\begin{array}{l}1100 \\
0915\end{array}$ & $\begin{array}{r}9.8 \\
10.0\end{array}$ & $\begin{array}{r}11.6 \\
9.4\end{array}$ & LTL2 & Aug. 13 & 1630 & -- & 14.4 \\
\hline UTL3 & July 12 & 1150 & $\begin{array}{r}9.5 \\
100\end{array}$ & 8.8 & LTL4 & $\begin{array}{l}\text { Aug. } 13 \\
\text { Aug. } 23\end{array}$ & $\begin{array}{l}1530 \\
1100\end{array}$ & $\begin{array}{c}9.6 \\
10.1\end{array}$ & $\begin{array}{l}13.4 \\
12.9\end{array}$ \\
\hline UTL4 & Aug. 22 & 0945 & 10.0 & 10.1 & LTL5 & $\begin{array}{l}\text { Aug. } 13 \\
\text { Aug. } 23\end{array}$ & $\begin{array}{l}1430 \\
1030\end{array}$ & $\begin{array}{c}9.6 \\
10.2\end{array}$ & $\begin{array}{l}14.8 \\
13.8\end{array}$ \\
\hline & Aug. 22 & 1015 & 9.8 & 7.1 & LTL6 & Aug. 13 & 1330 & 8.9 & 8.8 \\
\hline UTL5 & $\begin{array}{l}\text { July } 19 \\
\text { Aug. } 22\end{array}$ & $\begin{array}{l}1615 \\
1230\end{array}$ & $\begin{array}{l}9.4 \\
9.1\end{array}$ & $\begin{array}{l}10.0 \\
11.2\end{array}$ & LR0 & Aug. 14 & 1200 & 7.3 & 11.4 \\
\hline UTL6 & $\begin{array}{l}\text { July } 19 \\
\text { Aug. } 22\end{array}$ & $\begin{array}{l}1530 \\
1155\end{array}$ & $\begin{array}{r}9.1 \\
10.3\end{array}$ & $\begin{array}{r}8.9 \\
15.6\end{array}$ & LR3 & Aug. 28 & 0730 & 7.6 & 3.9 \\
\hline UTL7 & $\begin{array}{l}\text { July } 19 \\
\text { Aug. } 22 \\
\text { Sept. } 18 \\
\text { Sept. } 18\end{array}$ & $\begin{array}{l}1440 \\
1130 \\
0945 \\
1600\end{array}$ & $\begin{array}{r}8.8 \\
10.2 \\
9.9 \\
--\end{array}$ & $\begin{array}{r}7.4 \\
16.8 \\
12.2 \\
17.8\end{array}$ & SSD1 & $\begin{array}{l}\text { Aug. } 27 \\
\text { Aug. } 27\end{array}$ & $\begin{array}{l}1445 \\
1715\end{array}$ & $\begin{array}{l}8.4 \\
8.9\end{array}$ & $\begin{array}{l}12.2 \\
11.9\end{array}$ \\
\hline UTL8 & $\begin{array}{l}\text { July } 12 \\
\text { Aug. } 22 \\
\text { Aug. } 28 \\
\text { Sept. } 19 \\
\text { Sept. } 19\end{array}$ & $\begin{array}{l}1320 \\
1050 \\
1015 \\
0915 \\
1345\end{array}$ & $\begin{array}{l}9.9 \\
9.8 \\
9.7 \\
9.5 \\
--\end{array}$ & $\begin{array}{r}11.9 \\
13.2 \\
12.9 \\
8.3 \\
17.0\end{array}$ & $\begin{array}{l}12 \\
\mathrm{NCl}\end{array}$ & $\begin{array}{l}\text { Aug. } 24 \\
\text { Aug. } 27\end{array}$ & $\begin{array}{l}1200 \\
1115\end{array}$ & $\begin{array}{l}9.3 \\
7.8\end{array}$ & $\begin{array}{l}2.9 \\
5.9\end{array}$ \\
\hline UTL9 & $\begin{array}{l}\text { July } 19 \\
\text { Aug. } 22\end{array}$ & $\begin{array}{l}1715 \\
1340\end{array}$ & $\begin{array}{l}8.8 \\
8.2\end{array}$ & $\begin{array}{l}17.4 \\
12.2\end{array}$ & $\begin{array}{l}4 \mathrm{C} \\
9 \mathrm{~A}\end{array}$ & Aug. 24 & 0945 & 10.0 & 10.3 \\
\hline UTL10 & $\begin{array}{l}\text { Aug. } 14 \\
\text { Aug. } 22\end{array}$ & $\begin{array}{l}1130 \\
1415\end{array}$ & $\begin{array}{l}9.1 \\
9.3\end{array}$ & $\begin{array}{r}9.6 \\
13.8\end{array}$ & CL1 & Aug. 21 & 1100 & 8.6 & 8.5 \\
\hline UTL11 & $\begin{array}{l}\text { Aug. } 14 \\
\text { Aug. } 22\end{array}$ & $\begin{array}{l}1215 \\
1450\end{array}$ & $\begin{array}{l}9.5 \\
9.7\end{array}$ & $\begin{array}{r}18.2 \\
>20.0\end{array}$ & CL2 & Aug. 21 & 1155 & 8.6 & 7.8 \\
\hline UTL13 & $\begin{array}{l}\text { Aug. } 14 \\
\text { Aug. } 22\end{array}$ & $\begin{array}{l}1300 \\
1305\end{array}$ & $\begin{array}{l}8.4 \\
8.6\end{array}$ & $\begin{array}{r}8.6 \\
11.0\end{array}$ & & & & & \\
\hline
\end{tabular}


Table 7. Field and laboratory measurements of selected properties and constituents in surface water from primary and additional sampling sites, 1991-92

[See figures 1,4 , and 5 for site locations. $\mu \mathrm{S} / \mathrm{cm}$, microsiemen per centimeter at $25^{\circ} \mathrm{C} ;{ }^{\circ} \mathrm{C}$, degree Celsius; $\mathrm{mg} / \mathrm{L}$, milligram per liter; $\mathrm{mm}$ of $\mathrm{Hg}$, millimeter of mercury; $\mathrm{mg} / \mathrm{L}$ as $\mathrm{N}$, data obtained from National Water Quality Laboratory; $\mathrm{mg} / \mathrm{L}$ as $\mathrm{NH}_{3}$, data obtained from ion selective electrode; <, actual value is less than value shown; --, no data]

\begin{tabular}{|c|c|c|c|c|c|c|c|c|c|c|}
\hline Site & Date & Time & $\begin{array}{c}\text { Specific } \\
\text { conductance } \\
(\mu \mathrm{S} / \mathrm{cm})\end{array}$ & $\begin{array}{c}\mathrm{pH} \\
\text { (standard } \\
\text { units) }\end{array}$ & $\begin{array}{c}\text { Temper- } \\
\text { ature } \\
\left({ }^{\circ} \mathrm{C}\right)\end{array}$ & $\begin{array}{c}\text { Oxygen, } \\
\text { dissolved } \\
\text { (mg/L) }\end{array}$ & $\begin{array}{l}\text { Oxygen, } \\
\text { dissolved } \\
\text { (percent } \\
\text { saturation) }\end{array}$ & $\begin{array}{l}\text { Barometric } \\
\text { pressure } \\
(\mathrm{mm} \text { of } \mathrm{Hg})\end{array}$ & $\begin{array}{c}\text { Nitrogen, } \\
\text { ammonia } \\
\text { (mg/L } \\
\text { as N) } \\
\text { (lab) }\end{array}$ & $\begin{array}{c}\text { Nitrogen, } \\
\text { ammonia } \\
\text { (mg/L } \\
\left.\text { as } \mathrm{NH}_{3}\right) \\
\text { (field) }\end{array}$ \\
\hline \multirow[t]{46}{*}{1} & $6-05-91$ & 0950 & 242 & 8.4 & 15.0 & 6.4 & 74 & 660 & -- & -- \\
\hline & 6-07-91 & 1135 & -- & 8.6 & 17.0 & 7.8 & -- & 660 & -- & -- \\
\hline & 6-09-91 & 0940 & 219 & 8.0 & 18.0 & 6.2 & 76 & 660 & -- & -- \\
\hline & $6-12-91$ & 1040 & 210 & 7.9 & 19.0 & 4.3 & 55 & 650 & -- & -- \\
\hline & $6-14-91$ & 0850 & 180 & 8.1 & 17.0 & 5.9 & 71 & 660 & -- & -- \\
\hline & $6-16-91$ & 0735 & 197 & 7.7 & 17.0 & 8.1 & 97 & 660 & -- & -- \\
\hline & 6-19-91 & 0950 & 187 & 7.9 & 18.0 & 4.8 & 60 & 650 & -- & -- \\
\hline & $6-20-91$ & 1045 & -- & 7.8 & 16.0 & 5.7 & -. & 650 & -- & -- \\
\hline & $6-21-91$ & 0900 & 220 & 7.2 & 16.5 & 6.2 & 75 & 650 & -- & -- \\
\hline & $6-23-91$ & 1128 & 240 & 8.0 & 17.5 & 5.8 & 70 & 660 & -- & -- \\
\hline & $6-26-91$ & 0930 & 224 & 7.9 & 17.0 & 6.2 & 74 & 660 & -. & -- \\
\hline & $6-28-91$ & 1000 & 231 & 8.2 & 17.0 & 6.1 & 74 & 650 & -- & -- \\
\hline & 6-30-91 & 0920 & 275 & 7.7 & 16.5 & 5.7 & 69 & 650 & -- & -- \\
\hline & 7-03-91 & 0910 & 315 & 7.9 & 22.0 & 3.5 & 46 & 660 & -- & -- \\
\hline & $7-05-91$ & 0925 & 220 & 7.5 & 23.0 & 3.8 & 51 & 660 & -- & -- \\
\hline & $7-07-91$ & 1010 & 208 & 8.8 & 23.0 & 3.3 & 45 & 660 & -- & -- \\
\hline & $7-10-91$ & 0947 & 180 & 8.7 & 20.5 & 3.2 & 41 & 660 & -- & -- \\
\hline & $7-12-91$ & 0929 & 187 & 8.5 & 22.0 & 2.5 & 34 & 650 & -- & -- \\
\hline & 7-14-91 & 1115 & 190 & 8.5 & 22.0 & 2.7 & 36 & 660 & -- & -- \\
\hline & $7-17-91$ & 0954 & 224 & 8.0 & 19.5 & 2.3 & 28 & 660 & -- & 0.37 \\
\hline & 7-19-91 & 0915 & 263 & 7.0 & 20.5 & 2.1 & 27 & 660 & -- & -- \\
\hline & $7-21-91$ & 0830 & 219 & 7.9 & 20.5 & 2.3 & 30 & 650 & -- & -- \\
\hline & $7-23-91$ & 1345 & 228 & 8.1 & 22.5 & 2.8 & 38 & 660 & -- & -- \\
\hline & $7-24-91$ & 1005 & 234 & 7.6 & 22.0 & 1.9 & 25 & 660 & -- & .31 \\
\hline & $7-26-91$ & 0830 & 250 & 7.7 & 21.5 & 1.7 & 22 & 660 & -- & -- \\
\hline & $7-28-91$ & 0820 & 192 & 7.7 & 22.0 & -- & -- & 660 & -- & -- \\
\hline & $7-31-91$ & 0900 & 255 & 7.3 & 22.0 & 1.4 & 19 & 660 & -- & .36 \\
\hline & $8-02-91$ & 0755 & 230 & 6.7 & 20.5 & 2.2 & 28 & 660 & -- & -- \\
\hline & 8-04-91 & 0800 & 239 & 6.8 & 22.0 & 2.5 & 34 & 650 & -- & -- \\
\hline & 8-07-91 & 0950 & 268 & 7.4 & 20.0 & 2.9 & 38 & 650 & -- & .20 \\
\hline & 8-09-91 & 1034 & 246 & 7.3 & 20.0 & 3.1 & 40 & 650 & -- & -- \\
\hline & $8-11-91$ & 0800 & 233 & 6.8 & 19.5 & 4.2 & 53 & 660 & -- & -- \\
\hline & $8-12-91$ & 1540 & 260 & 7.5 & 21.5 & 4.9 & 64 & 660 & -- & -- \\
\hline & 8-14-91 & 0900 & 226 & 7.2 & 20.0 & 3.3 & 42 & 660 & -- & .13 \\
\hline & $8-16-91$ & 0805 & 230 & 7.5 & 20.0 & 3.6 & 46 & 660 & -- & -- \\
\hline & 8-18-91 & 0755 & 234 & 7.5 & 22.0 & 4.3 & 57 & 660 & -- & -- \\
\hline & $8-21-91$ & 0715 & 238 & 7.8 & 20.5 & 3.9 & 50 & 660 & -- & $<.1$ \\
\hline & $8-21-91$ & 1240 & 224 & 7.9 & 22.5 & 4.3 & 58 & 660 & -- & -- \\
\hline & $8-21-91$ & 1420 & 205 & 8.1 & 23.0 & 5.1 & 69 & 660 & -- & -- \\
\hline & $8-21-91$ & 1515 & 225 & 8.4 & 23.0 & 6.0 & 81 & 660 & -- & -- \\
\hline & $8-21-91$ & 1600 & 204 & 8.5 & 23.5 & 6.2 & 85 & 660 & -- & -- \\
\hline & $8-21-91$ & 1720 & 204 & 8.5 & 24.0 & 6.2 & 86 & 660 & -- & -- \\
\hline & $8-21-91$ & 2030 & 167 & 8.5 & 24.0 & 8.2 & 113 & 660 & -- & -- \\
\hline & $8-21-91$ & 2200 & 178 & 8.8 & 23.0 & 8.1 & 110 & 660 & -- & -- \\
\hline & $8-21-91$ & 2400 & 199 & 8.7 & 23.0 & 7.4 & 100 & 660 & -- & -- \\
\hline & $8-22-91$ & 0158 & 173 & 8.4 & 22.5 & 6.2 & 83 & 660 & -- & -- \\
\hline
\end{tabular}


Table 7. Field and laboratory measurements of selected properties and constituents in surface water from primary and additional sampling sites, 1991-92--Continued

\begin{tabular}{|c|c|c|c|c|c|c|c|c|c|c|}
\hline Site & Date & Time & $\begin{array}{c}\text { Specific } \\
\text { conductance } \\
(\mu \mathrm{S} / \mathrm{cm})\end{array}$ & $\begin{array}{c}\mathrm{pH} \\
\text { (standard } \\
\text { units) }\end{array}$ & $\begin{array}{l}\text { Temper- } \\
\text { ature } \\
\left({ }^{\circ} \mathrm{C}\right)\end{array}$ & $\begin{array}{l}\text { Oxygen, } \\
\text { dissolved } \\
(\mathrm{mg} / \mathrm{L})\end{array}$ & $\begin{array}{l}\text { Oxygen, } \\
\text { dissolved } \\
\text { (percent } \\
\text { saturation) }\end{array}$ & $\begin{array}{l}\text { Barometric } \\
\text { pressure } \\
(\mathrm{mm} \text { of } \mathrm{Hg})\end{array}$ & $\begin{array}{c}\text { Nitrogen, } \\
\text { ammonia } \\
\text { (mg/L } \\
\text { as N) } \\
\text { (lab) }\end{array}$ & $\begin{array}{c}\text { Nitrogen, } \\
\text { ammonia } \\
(\mathrm{mg} / \mathrm{L} \\
\left.\text { as } \mathrm{NH}_{3}\right) \\
\text { (field) }\end{array}$ \\
\hline \multirow{47}{*}{ 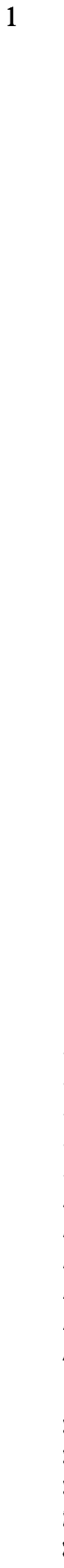 } & $8-22-91$ & 0615 & 198 & 7.7 & 22.5 & 3.8 & 51 & 660 & -- & -- \\
\hline & $8-22-91$ & 0825 & 203 & 7.7 & 22.0 & 3.2 & 42 & 660 & -- & -- \\
\hline & $8-22-91$ & 0940 & 208 & 7.7 & 21.5 & 3.2 & 42 & 660 & -- & -- \\
\hline & $8-22-91$ & 1201 & 237 & 7.8 & 22.0 & 3.9 & 52 & 660 & -- & -- \\
\hline & $8-22-91$ & 1345 & 174 & 8.3 & 22.5 & 5.4 & 72 & 660 & -- & -- \\
\hline & 8-23-91 & 1445 & 226 & 8.2 & 27.0 & 4.8 & 70 & 660 & -- & -- \\
\hline & $8-25-91$ & 0745 & 290 & 7.0 & 20.0 & 3.8 & 48 & 660 & -- & -- \\
\hline & $8-28-91$ & 0928 & 224 & 8.1 & 18.5 & 3.8 & 47 & 660 & -- & $<0.1$ \\
\hline & 8-30-91 & 1015 & 237 & 7.7 & 18.5 & 4.5 & 56 & 660 & -- & -- \\
\hline & 9-01-91 & 0714 & 228 & 7.3 & 19.0 & 4.4 & 55 & 660 & -- & -- \\
\hline & $6-03-92$ & 0930 & 195 & 8.0 & 21.0 & 4.8 & 63 & 655 & 0.10 & .22 \\
\hline & $6-05-92$ & 0730 & 174 & 8.7 & 20.0 & 4.8 & 61 & 660 & -- & -- \\
\hline & $6-07-92$ & 0719 & 169 & 8.5 & 20.0 & 5.0 & 64 & 655 & -- & -- \\
\hline & $6-10-92$ & 0700 & 168 & 8.5 & 19.5 & 4.7 & 60 & 650 & .10 & $<.1$ \\
\hline & $6-12-92$ & 0728 & 153 & 8.6 & 16.0 & 4.8 & 57 & 655 & -- & -- \\
\hline & $6-14-92$ & 0848 & 174 & 9.4 & 15.0 & .7 & 8 & 657 & -- & -- \\
\hline & $6-15-92$ & 1350 & 190 & 9.3 & 14.5 & 8.0 & 91 & 656 & -- & -- \\
\hline & $6-17-92$ & 0715 & 207 & 8.7 & 16.0 & 6.2 & 73 & 655 & .11 & $<.1$ \\
\hline & $6-19-92$ & 1215 & 220 & 8.7 & 19.0 & 4.5 & 56 & 659 & -- & -- \\
\hline & $6-21-92$ & 0855 & 225 & 8.7 & 20.0 & 3.6 & 46 & 658 & -- & -- \\
\hline & $6-24-92$ & 0915 & 183 & 8.2 & 21.0 & .9 & 12 & 656 & .51 & .64 \\
\hline & $6-26-92$ & 0754 & 202 & 7.4 & 20.0 & .8 & 10 & 657 & -- & -- \\
\hline & $6-28-92$ & 0845 & 204 & 7.4 & 21.0 & -- & -- & 652 & -- & -- \\
\hline & 7-01-92 & 0715 & 228 & 7.1 & 16.0 & 2.7 & 32 & 650 & .63 & .66 \\
\hline & $7-03-92$ & 0830 & 244 & 7.5 & 19.5 & 1.7 & 22 & 655 & -- & -- \\
\hline & $7-05-92$ & 0840 & 308 & -- & 20.0 & -- & -- & 654 & -- & -- \\
\hline & $7-08-92$ & 0707 & 219 & 8.0 & 20.0 & 4.4 & 56 & 660 & .20 & -- \\
\hline & 7-10-92 & 0805 & 239 & 7.7 & 21.0 & 3.7 & 48 & 660 & -- & -- \\
\hline & $7-10-92$ & 1725 & 233 & 8.0 & 23.0 & 6.2 & 84 & 657 & -- & -- \\
\hline & 7-12-92 & 0750 & 200 & 7.4 & 19.0 & 2.7 & 34 & 659 & -- & -- \\
\hline & $7-15-92$ & 0700 & 177 & 8.1 & 22.0 & 2.4 & 32 & 655 & .21 & -- \\
\hline & $7-17-92$ & 1015 & 153 & 8.1 & 23.0 & 2.0 & 27 & 660 & -- & -- \\
\hline & $7-19-92$ & 0743 & 206 & 7.4 & 22.5 & 1.0 & 13 & 660 & -- & -- \\
\hline & 7-22-92 & 0650 & 166 & 6.8 & 21.0 & 1.3 & 17 & 659 & .41 & -- \\
\hline & $7-24-92$ & 1055 & 190 & 6.8 & 20.0 & 2.4 & 31 & 660 & -- & -- \\
\hline & $7-24-92$ & 1300 & 190 & 6.9 & 20.0 & 2.5 & 32 & 660 & -- & -- \\
\hline & $7-26-92$ & 0814 & 199 & 7.0 & 21.5 & 1.4 & 18 & 662 & -- & -- \\
\hline & $7-27-92$ & 1205 & 220 & 7.2 & 22.0 & 2.1 & 28 & 660 & -- & -- \\
\hline & $7-28-92$ & 1550 & 210 & 7.3 & 24.0 & 4.2 & 58 & 662 & .41 & -- \\
\hline & $7-28-92$ & 1555 & -- & -- & -- & -- & -- & -- & .41 & -- \\
\hline & $7-29-92$ & 0640 & 196 & 6.9 & 23.0 & .7 & 9 & 660 & .46 & -- \\
\hline & $7-31-92$ & 0955 & 181 & 6.9 & 23.0 & 1.5 & 20 & 655 & -- & -- \\
\hline & $8-02-92$ & 1130 & 182 & 7.5 & 22.0 & 3.2 & 42 & 665 & -- & -- \\
\hline & $8-05-92$ & 0650 & -- & 7.4 & 23.0 & 1.9 & 26 & 659 & .18 & -- \\
\hline & $8-07-92$ & 0820 & 195 & 7.6 & 21.5 & 2.7 & 36 & 659 & -- & -- \\
\hline & $8-09-92$ & 0930 & 188 & 7.1 & 20.5 & 3.7 & 47 & 665 & -- & -- \\
\hline & $8-12-92$ & 0657 & 229 & 7.4 & 23.0 & 2.2 & 30 & 660 & .23 & -- \\
\hline
\end{tabular}


Table 7. Field and laboratory measurements of selected properties and constituents in surface water from primary and additional sampling sites, 1991-92--Continued

\begin{tabular}{|c|c|c|c|c|c|c|c|c|c|c|}
\hline Site & Date & Time & $\begin{array}{c}\text { Specific } \\
\text { conductance } \\
(\mu \mathrm{S} / \mathrm{cm})\end{array}$ & $\begin{array}{c}\mathrm{pH} \\
\text { (standard } \\
\text { units) }\end{array}$ & $\begin{array}{l}\text { Temper- } \\
\text { ature } \\
\left({ }^{\circ} \mathrm{C}\right)\end{array}$ & $\begin{array}{c}\text { Oxygen, } \\
\text { dissolved } \\
(\mathrm{mg} / \mathrm{L})\end{array}$ & $\begin{array}{l}\text { Oxygen, } \\
\text { dissolved } \\
\text { (percent } \\
\text { saturation) }\end{array}$ & $\begin{array}{l}\text { Barometric } \\
\text { pressure } \\
(\mathrm{mm} \text { of } \mathrm{Hg})\end{array}$ & $\begin{array}{c}\text { Nitrogen, } \\
\text { ammonia } \\
\text { (mg/L } \\
\text { as N) } \\
\text { (lab) }\end{array}$ & $\begin{array}{c}\text { Nitrogen, } \\
\text { ammonia } \\
(\mathrm{mg} / \mathrm{L} \\
\left.\text { as } \mathrm{NH}_{3}\right) \\
\text { (field) }\end{array}$ \\
\hline \multirow[t]{10}{*}{1} & 8-14-92 & 0923 & 220 & 7.4 & 23.0 & 2.4 & 32 & 660 & -- & -- \\
\hline & 8-16-92 & 0730 & 181 & 7.2 & 23.0 & 4.0 & 54 & 659 & -- & -- \\
\hline & 8-19-92 & 0650 & 221 & 6.7 & 24.0 & 3.1 & 43 & 655 & 0.21 & -- \\
\hline & $8-21-92$ & 1000 & 186 & 7.7 & 21.0 & 5.1 & 66 & 660 & -- & -- \\
\hline & $8-21-92$ & 1535 & 210 & 8.2 & 22.0 & 4.9 & 65 & 660 & -- & -- \\
\hline & 8-23-92 & 0725 & 165 & 7.8 & 18.5 & 3.4 & 42 & 660 & -- & -- \\
\hline & $8-24-92$ & 1445 & 231 & 7.2 & 18.0 & 5.0 & 61 & 660 & .18 & -- \\
\hline & $8-26-92$ & 0655 & 235 & 7.5 & 18.0 & 3.9 & 47 & 664 & .19 & -- \\
\hline & 8-28-92 & 0830 & 194 & 7.6 & 18.5 & 3.4 & 42 & 660 & -- & -- \\
\hline & 8-30-92 & 0945 & 247 & 7.3 & 18.0 & 2.7 & 33 & 660 & -- & -- \\
\hline \multirow[t]{38}{*}{2} & 6-05-91 & 0810 & 232 & 9.3 & 11.0 & 6.6 & 70 & 650 & -- & -- \\
\hline & $6-07-91$ & 1055 & -- & 8.8 & 16.5 & 8.7 & -- & 660 & -- & -- \\
\hline & 6-09-91 & 1105 & 216 & 8.7 & 20.0 & 8.4 & 107 & 660 & -- & -- \\
\hline & 6-12-91 & 0854 & 221 & 8.4 & 15.0 & 6.3 & 73 & 650 & -- & -- \\
\hline & 6-13-91 & 1115 & 222 & 8.7 & 12.0 & 9.4 & 102 & 653 & -- & -- \\
\hline & $6-14-91$ & 0815 & 237 & 7.6 & 12.5 & 5.8 & 63 & 660 & -- & -- \\
\hline & $6-16-91$ & 1009 & 191 & 7.9 & 14.5 & 6.3 & 72 & 660 & -- & -- \\
\hline & 6-19-91 & 0730 & 209 & 7.6 & 12.0 & 3.8 & 41 & 650 & -- & -- \\
\hline & $6-21-91$ & 0915 & 209 & 7.3 & 14.0 & 6.4 & 73 & 650 & -- & -- \\
\hline & 6-23-91 & 0919 & 216 & 7.5 & 15.0 & 5.5 & 64 & 650 & -- & -- \\
\hline & 6-26-91 & 0735 & 207 & 6.6 & 14.0 & 2.3 & 26 & 655 & -- & -- \\
\hline & $6-28-91$ & 1115 & 216 & 8.1 & 19.0 & 5.8 & 74 & 650 & -- & -- \\
\hline & $6-30-91$ & 0855 & 218 & 7.7 & 15.0 & 1.5 & 17 & 662 & -- & -- \\
\hline & 7-03-91 & 0733 & 263 & 6.3 & 20.0 & .5 & 6 & 650 & -- & -- \\
\hline & 7-05-91 & 0845 & 276 & 7.5 & 22.0 & 1.8 & 24 & 660 & -- & -- \\
\hline & 7-07-91 & 1110 & 279 & 8.4 & 22.5 & 6.2 & 83 & 660 & -- & -- \\
\hline & $7-10-91$ & 0755 & 249 & 6.8 & 17.0 & 1.5 & 18 & 660 & -- & -- \\
\hline & $7-12-91$ & 0922 & 226 & 7.6 & 20.0 & 3.8 & 49 & 650 & -- & -- \\
\hline & $7-14-91$ & 1115 & 255 & 9.6 & 23.0 & 12.6 & 173 & 650 & -- & -- \\
\hline & $7-17-91$ & 0730 & 243 & 6.0 & 16.0 & .6 & 7 & 660 & -- & $<0.10$ \\
\hline & 7-19-91 & 1035 & 251 & 8.2 & 18.5 & 1.6 & 20 & 660 & -- & -- \\
\hline & $7-21-91$ & 0820 & 220 & 6.8 & 18.0 & .7 & 9 & 650 & -- & -- \\
\hline & $7-24-91$ & 0735 & 233 & 6.6 & 18.5 & .5 & 6 & 655 & -- & $<.10$ \\
\hline & $7-26-91$ & 0900 & 208 & 7.3 & 18.0 & .4 & 5 & 660 & -- & -- \\
\hline & $7-28-91$ & 0810 & 230 & 6.6 & 18.0 & .3 & 4 & 660 & -- & -- \\
\hline & $7-30-91$ & 0955 & 210 & 6.9 & 19.0 & .5 & 6 & 661 & -- & -- \\
\hline & $7-31-91$ & 0720 & 234 & 6.9 & 19.5 & .3 & 4 & 660 & -- & $<.10$ \\
\hline & 8-02-91 & 0800 & 223 & 6.9 & 18.0 & .4 & 5 & 660 & -- & -- \\
\hline & 8-04-91 & 0945 & 184 & 7.1 & 20.0 & 1.8 & 23 & 650 & -- & -- \\
\hline & 8-05-91 & 1340 & 228 & 7.7 & 26.0 & 4.0 & 57 & 660 & -- & -- \\
\hline & 8-05-91 & 1600 & 228 & 8.5 & 25.5 & 6.7 & 95 & 660 & -- & -- \\
\hline & 8-05-91 & 1820 & 225 & 8.1 & 26.5 & 6.5 & 94 & 660 & -- & -- \\
\hline & 8-05-91 & 2000 & 205 & 8.5 & 25.0 & 9.2 & 129 & 660 & -- & -- \\
\hline & 8-05-91 & 2200 & 211 & 8.5 & 22.5 & 7.2 & 97 & 660 & -- & -- \\
\hline & 8-06-91 & 0004 & 214 & 7.6 & 21.5 & 4.0 & 53 & 660 & -- & -- \\
\hline & 8-06-91 & 0203 & 209 & 7.0 & 20.0 & 1.4 & 18 & 660 & -- & -- \\
\hline & 8-06-91 & 0400 & 205 & 7.0 & 19.0 & .4 & 5 & 660 & -- & -- \\
\hline & $8-06-91$ & 0545 & 216 & 6.4 & 18.5 & .1 & 1 & 660 & -- & -- \\
\hline
\end{tabular}


Table 7. Field and laboratory measurements of selected properties and constituents in surface water from primary and additional sampling sites, 1991-92--Continued

\begin{tabular}{|c|c|c|c|c|c|c|c|c|c|c|}
\hline Site & Date & Time & $\begin{array}{c}\text { Specific } \\
\text { conductance } \\
(\mu \mathrm{S} / \mathrm{cm})\end{array}$ & $\begin{array}{c}\mathrm{pH} \\
\text { (standard } \\
\text { units) }\end{array}$ & $\begin{array}{c}\text { Temper- } \\
\text { ature } \\
\left({ }^{\circ} \mathrm{C}\right)\end{array}$ & $\begin{array}{l}\text { Oxygen, } \\
\text { dissolved } \\
(\mathrm{mg} / \mathrm{L})\end{array}$ & $\begin{array}{l}\text { Oxygen, } \\
\text { dissolved } \\
\text { (percent } \\
\text { saturation) }\end{array}$ & $\begin{array}{l}\text { Barometric } \\
\text { pressure } \\
(\mathrm{mm} \text { of } \mathrm{Hg})\end{array}$ & $\begin{array}{c}\text { Nitrogen, } \\
\text { ammonia } \\
\text { (mg/L } \\
\text { as N) } \\
\text { (lab) }\end{array}$ & $\begin{array}{c}\text { Nitrogen, } \\
\text { ammonia } \\
\text { (mg/L } \\
\left.\text { as } \mathrm{NH}_{3}\right) \\
\text { (field) }\end{array}$ \\
\hline \multirow[t]{48}{*}{2} & $8-06-91$ & 0745 & 219 & 6.8 & 18.0 & .5 & 6 & 660 & -- & -- \\
\hline & $8-06-91$ & 1020 & 221 & 6.8 & 18.5 & 1.2 & 15 & 660 & -- & -- \\
\hline & $8-06-91$ & 1145 & 222 & 6.9 & 20.5 & 4.6 & 59 & 660 & -- & -- \\
\hline & 8-07-91 & 0724 & 204 & 6.2 & 16.5 & .6 & 7 & 650 & - & $<0.10$ \\
\hline & 8-09-91 & 1130 & 221 & 7.6 & 18.5 & 1.7 & 21 & 650 & -- & -- \\
\hline & $8-11-91$ & 1020 & 236 & 7.3 & 18.0 & 1.6 & 20 & 650 & -- & -- \\
\hline & $8-13-91$ & 1030 & 231 & 7.0 & 17.5 & 1.1 & 13 & 660 & -- & -- \\
\hline & $8-14-91$ & 0725 & 213 & 6.6 & 17.5 & .4 & 5 & 658 & -- & $<.10$ \\
\hline & $8-16-91$ & 0820 & 217 & 6.9 & 18.0 & 0.3 & 4 & 660 & -- & -- \\
\hline & $8-18-91$ & 0800 & 224 & 6.7 & 18.0 & .3 & 4 & 660 & -- & -- \\
\hline & $8-21-91$ & 0720 & 223 & 7.2 & 19.0 & .2 & 2 & 660 & -- & $<.10$ \\
\hline & $8-23-91$ & 1510 & 223 & 8.8 & 26.0 & 9.2 & 132 & 660 & -- & -- \\
\hline & $8-23-91$ & 1723 & 230 & 9.0 & 26.0 & 8.3 & 119 & 660 & -- & -- \\
\hline & $8-25-91$ & 0750 & 207 & 7.3 & 17.0 & .4 & 5 & 660 & -- & -. \\
\hline & $8-28-91$ & 0750 & 216 & 7.2 & 17.0 & .3 & 4 & 660 & -- & .17 \\
\hline & $8-30-91$ & 0840 & 273 & 7.2 & 16.0 & .7 & 8 & 663 & - & - \\
\hline & 9-01-91 & 0737 & 240 & 7.5 & 15.5 & .4 & 5 & 660 & -- & -- \\
\hline & 6-03-92 & 0655 & 250 & 7.3 & 15.5 & -- & -- & 655 & 0.33 & .34 \\
\hline & $6-05-92$ & 0914 & 226 & 7.7 & 14.0 & 4.2 & 47 & 660 & -- & -- \\
\hline & $6-07-92$ & 0735 & 226 & 7.9 & 13.0 & 1.0 & 11 & 651 & -- & -- \\
\hline & $6-10-92$ & 0700 & 228 & 6.9 & 13.5 & 1.2 & 14 & 650 & .08 & $<.12$ \\
\hline & $6-12-92$ & 0935 & 229 & 8.2 & 12.0 & 5.2 & 57 & 650 & -- & -- \\
\hline & $6-14-92$ & 0900 & 224 & 7.5 & 11.0 & 4.5 & 48 & 655 & -- & -- \\
\hline & $6-15-92$ & 1300 & 219 & 8.9 & 16.0 & 8.2 & 97 & 657 & -- & -- \\
\hline & $6-17-92$ & 0707 & 203 & 7.1 & 12.0 & 1.4 & 15 & 655 & .07 & $<.1$ \\
\hline & $6-21-92$ & 0802 & 243 & 6.5 & 15.5 & .6 & 7 & 660 & -- & -- \\
\hline & $6-24-92$ & 0710 & 244 & 7.1 & 19.0 & .4 & 5 & 655 & .70 & .88 \\
\hline & $6-26-92$ & 0755 & 244 & 7.1 & 19.0 & .6 & 7 & 660 & -- & -- \\
\hline & $6-26-92$ & 1600 & 240 & 8.5 & 28.0 & 10.5 & 157 & 655 & -- & -- \\
\hline & 6-28-92 & 0800 & 265 & 7.0 & 18.0 & .5 & 6 & 655 & -- & -- \\
\hline & $7-01-92$ & 0741 & 217 & 6.7 & 15.0 & .7 & 8 & 660 & .91 & .86 \\
\hline & 7-03-92 & 0755 & 238 & 6.9 & 18.0 & 1.1 & 14 & 656 & -- & -- \\
\hline & $7-05-92$ & 0810 & 237 & 7.0 & 17.5 & .9 & 11 & 650 & -- & -. \\
\hline & 7-08-92 & 0722 & 220 & 6.6 & 16.0 & .4 & 5 & 660 & .18 & -- \\
\hline & $7-10-92$ & 0805 & 228 & 6.7 & 18.5 & .2 & 2 & 660 & -- & -. \\
\hline & 7-12-92 & 0805 & 238 & 6.4 & 17.5 & .2 & 2 & 655 & -- & -- \\
\hline & $7-15-92$ & 0720 & 294 & 6.5 & 19.5 & .1 & 1 & 662 & .36 & -. \\
\hline & $7-17-92$ & 0815 & 245 & 7.0 & 21.5 & 1.0 & 13 & 665 & -- & -- \\
\hline & $7-17-92$ & 1643 & 244 & 6.6 & 24.0 & .4 & 6 & 660 & -- & -- \\
\hline & $7-19-92$ & 0755 & 201 & 6.7 & 20.5 & 1.1 & 14 & 664 & -- & -- \\
\hline & 7-22-92 & 0705 & 236 & 6.4 & 19.0 & .2 & 3 & 655 & .37 & -- \\
\hline & $7-24-92$ & 0800 & 210 & 6.6 & 17.0 & .7 & 8 & 663 & -- & -- \\
\hline & 7-24-92 & 1200 & 209 & 6.4 & 19.0 & .4 & 5 & 660 & -- & -- \\
\hline & $7-26-92$ & 0800 & 211 & 6.6 & 20.0 & 2.0 & 25 & 665 & -- & -- \\
\hline & $7-27-92$ & 1330 & 226 & 6.6 & 26.0 & 2.6 & 37 & 660 & -- & -- \\
\hline & $7-28-92$ & 1520 & 230 & 6.9 & 26.0 & 4.0 & 57 & 662 & .28 & -- \\
\hline & 7-29-92 & 0703 & 228 & 7.0 & 21.0 & .5 & 7 & 655 & .47 & -- \\
\hline & $7-31-92$ & 0605 & 210 & 6.6 & 23.0 & .7 & 9 & 661 & -- & -- \\
\hline
\end{tabular}


Table 7. Field and laboratory measurements of selected properties and constituents in surface water from primary and additional sampling sites, 1991-92--Continued

\begin{tabular}{|c|c|c|c|c|c|c|c|c|c|c|}
\hline Site & Date & Time & $\begin{array}{c}\text { Specific } \\
\text { conductance } \\
(\mu \mathrm{S} / \mathrm{cm})\end{array}$ & $\begin{array}{c}\mathrm{pH} \\
\text { (standard } \\
\text { units) }\end{array}$ & $\begin{array}{l}\text { Temper- } \\
\text { ature } \\
\left({ }^{\circ} \mathrm{C}\right)\end{array}$ & $\begin{array}{l}\text { Oxygen, } \\
\text { dissolved } \\
(\mathrm{mg} / \mathrm{L})\end{array}$ & $\begin{array}{l}\text { Oxygen, } \\
\text { dissolved } \\
\text { (percent } \\
\text { saturation) }\end{array}$ & $\begin{array}{l}\text { Barometric } \\
\text { pressure } \\
(\mathrm{mm} \text { of } \mathrm{Hg})\end{array}$ & $\begin{array}{c}\text { Nitrogen, } \\
\text { ammonia } \\
\text { (mg/L } \\
\text { as N) } \\
\text { (lab) }\end{array}$ & $\begin{array}{c}\text { Nitrogen, } \\
\text { ammonia } \\
(\mathrm{mg} / \mathrm{L} \\
\left.\text { as } \mathrm{NH}_{3}\right) \\
\text { (field) }\end{array}$ \\
\hline \multirow[t]{17}{*}{2} & 8-02-92 & 0545 & 232 & 6.8 & 21.5 & 0.5 & 7 & 660 & -- & -- \\
\hline & 8-05-92 & 0835 & 203 & 6.3 & 19.0 & .4 & 5 & 660 & 0.40 & -- \\
\hline & $8-07-92$ & 0935 & 206 & 6.5 & 19.0 & 1.0 & 12 & 660 & -- & -- \\
\hline & 8-07-92 & 1738 & 191 & 7.2 & 25.0 & 5.4 & 76 & 660 & -- & -- \\
\hline & 8-09-92 & 0709 & 205 & 6.9 & 19.0 & .7 & 9 & 662 & -- & -- \\
\hline & $8-12-92$ & 0700 & 255 & 6.6 & 23.0 & .9 & 12 & 655 & .46 & -- \\
\hline & $8-14-92$ & 0755 & 238 & 6.8 & 22.5 & .5 & 7 & 665 & -- & -- \\
\hline & $8-16-92$ & 0653 & 262 & 6.1 & 21.0 & 1.2 & 16 & 660 & -- & -- \\
\hline & $8-19-92$ & 0700 & 231 & 6.5 & 22.5 & .8 & 11 & 660 & 1.40 & -- \\
\hline & $8-19-92$ & 0705 & -- & -- & -- & -- & -- & -- & 1.40 & -- \\
\hline & $8-21-92$ & 0808 & 238 & 6.7 & 19.0 & 1.7 & 21 & 656 & -- & -- \\
\hline & $8-23-92$ & 0950 & 208 & 7.0 & 14.0 & 1.8 & 20 & 660 & -- & -- \\
\hline & $8-24-92$ & 1250 & 206 & 6.9 & 18.0 & 4.6 & 56 & 660 & .18 & -- \\
\hline & $8-26-92$ & 0708 & 225 & 6.8 & 15.0 & 2.5 & 28 & 665 & .37 & -- \\
\hline & $8-28-92$ & 0820 & 252 & 6.6 & 16.5 & 1.7 & 20 & 665 & -- & -- \\
\hline & $8-30-92$ & 0805 & 214 & 6.5 & 15.0 & 1.7 & 20 & 660 & -- & -- \\
\hline & 9-08-92 & 1055 & 216 & 7.5 & 16.5 & 7.1 & 84 & 661 & .15 & -- \\
\hline \multirow[t]{30}{*}{3} & $6-05-91$ & 0832 & 367 & 9.2 & 15.0 & 8.4 & 98 & 650 & -- & -- \\
\hline & 6-07-91 & 1255 & 343 & 9.2 & 18.0 & 11.8 & 145 & 660 & -- & -- \\
\hline & 6-09-91 & 0800 & 314 & 9.0 & 20.0 & 11.6 & 148 & 660 & -- & -- \\
\hline & $6-12-91$ & 0917 & 321 & 8.9 & 18.5 & 6.8 & 86 & 650 & -- & -- \\
\hline & 6-13-91 & 1125 & 307 & 8.9 & 17.0 & 8.2 & 99 & 653 & -- & -- \\
\hline & $6-14-91$ & 0855 & 239 & 9.1 & 13.0 & 5.8 & 64 & 660 & -- & -- \\
\hline & 6-19-91 & 0800 & 212 & 9.1 & 16.5 & 6.7 & 81 & 650 & -- & -- \\
\hline & 6-19-91 & 0951 & 245 & 9.0 & 17.0 & 8.9 & 107 & 660 & -- & -- \\
\hline & $6-21-91$ & 1009 & 207 & 8.5 & -- & 8.1 & -- & 650 & -- & -- \\
\hline & 6-23-91 & 0944 & 214 & 8.3 & 16.0 & 7.3 & 87 & 650 & -- & -- \\
\hline & $6-26-91$ & 0800 & 328 & 8.3 & 18.0 & 8.3 & 102 & 655 & -- & -- \\
\hline & $6-28-91$ & 1100 & 317 & 8.7 & 18.0 & 8.3 & 103 & 650 & -- & -- \\
\hline & 6-30-91 & 0925 & 334 & 8.9 & 17.0 & 8.0 & 96 & 662 & -- & -- \\
\hline & 7-03-91 & 0805 & 368 & 8.7 & 24.0 & 6.0 & 83 & 660 & -- & -- \\
\hline & 7-05-91 & 0815 & 380 & 8.5 & 24.0 & 5.1 & 70 & 660 & -- & -. \\
\hline & 7-07-91 & 1000 & 369 & 8.3 & 25.0 & 5.3 & 75 & 660 & -- & -- \\
\hline & $7-10-91$ & 0816 & 329 & 8.5 & 20.0 & 3.3 & 42 & 660 & -- & -- \\
\hline & $7-12-91$ & 0930 & 290 & 7.9 & 22.0 & 3.3 & 45 & 650 & -- & -- \\
\hline & 7-14-91 & 1035 & 650 & 8.5 & 24.0 & 4.6 & 65 & 650 & -- & -- \\
\hline & 7-17-91 & 0750 & 333 & 6.6 & 18.5 & 1.8 & 22 & 660 & -- & 0.37 \\
\hline & $7-19-91$ & 1020 & 334 & 8.5 & 22.0 & 6.9 & 92 & 660 & -- & -- \\
\hline & $7-21-91$ & 0845 & 290 & 7.8 & 21.0 & 4.1 & 54 & 650 & -- & -- \\
\hline & $7-24-91$ & 0805 & 345 & 7.6 & 21.5 & 2.7 & 36 & 655 & -- & .30 \\
\hline & $7-26-91$ & 0635 & 335 & 7.4 & 20.0 & 1.6 & 20 & 660 & -- & -- \\
\hline & 7-28-91 & 0830 & 244 & 7.0 & 21.0 & 2.0 & 26 & 660 & -- & -- \\
\hline & 7-30-91 & 1050 & 317 & 7.9 & 23.5 & 4.7 & 64 & 661 & -- & -- \\
\hline & $7-31-91$ & 0745 & 276 & 7.4 & 22.0 & 2.3 & 31 & 660 & -- & .16 \\
\hline & 8-02-91 & 0820 & -- & 7.2 & 18.0 & 1.9 & -- & 660 & -- & -- \\
\hline & $8-04-91$ & 0930 & 258 & 7.9 & 21.0 & 4.1 & 54 & 650 & -- & -- \\
\hline & 8-05-91 & 1220 & 322 & 7.4 & 21.0 & 4.7 & 61 & 660 & -- & -- \\
\hline
\end{tabular}


Table 7. Field and laboratory measurements of selected properties and constituents in surface water from primary and additional sampling sites, 1991-92--Continued

\begin{tabular}{|c|c|c|c|c|c|c|c|c|c|c|}
\hline Site & Date & Time & $\begin{array}{c}\text { Specific } \\
\text { conductance } \\
(\mu \mathrm{S} / \mathrm{cm})\end{array}$ & $\begin{array}{c}\mathrm{pH} \\
\text { (standard } \\
\text { units) }\end{array}$ & $\begin{array}{l}\text { Temper- } \\
\text { ature } \\
\left({ }^{\circ} \mathrm{C}\right)\end{array}$ & $\begin{array}{l}\text { Oxygen, } \\
\text { dissolved } \\
(\mathrm{mg} / \mathrm{L})\end{array}$ & $\begin{array}{l}\text { Oxygen, } \\
\text { dissolved } \\
\text { (percent } \\
\text { saturation) }\end{array}$ & $\begin{array}{l}\text { Barometric } \\
\text { pressure } \\
(\mathrm{mm} \text { of } \mathrm{Hg})\end{array}$ & $\begin{array}{c}\text { Nitrogen, } \\
\text { ammonia } \\
(\mathrm{mg} / \mathrm{L} \\
\text { as N) } \\
\text { (lab) }\end{array}$ & $\begin{array}{c}\text { Nitrogen, } \\
\text { ammonia } \\
(\mathrm{mg} / \mathrm{L} \\
\left.\text { as } \mathrm{NH}_{3}\right) \\
\text { (field) }\end{array}$ \\
\hline \multirow[t]{48}{*}{3} & $8-05-91$ & 1410 & 319 & 7.7 & 22.0 & 5.3 & 70 & 660 & -- & -- \\
\hline & $8-05-91$ & 1630 & 326 & 7.8 & 23.0 & 5.9 & 80 & 660 & -- & -- \\
\hline & $8-05-91$ & 1820 & 327 & 7.8 & 26.5 & 5.7 & 82 & 660 & -- & -- \\
\hline & $8-05-91$ & 2011 & 306 & 7.6 & 22.5 & 4.9 & 66 & 660 & -- & -- \\
\hline & $8-05-91$ & 2220 & 307 & 7.4 & 22.5 & 4.2 & 56 & 660 & -- & -- \\
\hline & $8-06-91$ & 0020 & 303 & 7.6 & 22.0 & 3.8 & 50 & 660 & -- & -- \\
\hline & $8-06-91$ & 0212 & 306 & 7.5 & 22.0 & 4.0 & 53 & 660 & -- & -- \\
\hline & $8-06-91$ & 0408 & 304 & 7.6 & 22.0 & 4.0 & 53 & 660 & -- & -- \\
\hline & $8-06-91$ & 0600 & 312 & 7.2 & 21.0 & 3.9 & 51 & 660 & -- & -- \\
\hline & $8-06-91$ & 0755 & 309 & 7.3 & 21.0 & 3.7 & 48 & 660 & -- & -- \\
\hline & $8-06-91$ & 1038 & 310 & 7.6 & 21.5 & 5.2 & 68 & 660 & -- & -- \\
\hline & $8-06-91$ & 1200 & 314 & 7.7 & 22.0 & 5.8 & 77 & 660 & -- & -- \\
\hline & $8-07-91$ & 0740 & 392 & 7.0 & 19.5 & 4.2 & 53 & 660 & -- & $<0.1$ \\
\hline & $8-09-91$ & 1110 & 327 & 8.2 & 20.5 & 5.2 & 68 & 650 & -- & -- \\
\hline & $8-11-91$ & 1005 & 314 & 8.0 & 20.0 & 5.8 & 75 & 650 & -- & -- \\
\hline & $8-13-91$ & 1125 & 336 & 8.4 & 21.5 & 7.6 & 100 & 660 & -- & -- \\
\hline & $8-14-91$ & 0740 & 325 & 7.1 & 20.0 & 3.5 & 45 & 660 & -- & $<.1$ \\
\hline & $8-16-91$ & 0845 & 327 & 7.4 & 20.0 & 5.2 & 66 & 660 & -- & -- \\
\hline & $8-18-91$ & 0815 & 458 & 7.8 & 20.0 & 2.7 & 34 & 660 & -- & -- \\
\hline & $8-21-91$ & 0740 & 294 & 7.3 & 21.5 & 1.5 & 20 & 660 & -- & $<.1$ \\
\hline & $8-21-91$ & 1710 & 310 & 8.8 & 25.0 & 9.5 & 134 & 660 & -- & -- \\
\hline & $8-23-91$ & 1525 & 291 & 8.4 & 25.0 & 8.6 & 121 & 660 & -- & -- \\
\hline & $8-25-91$ & 0810 & 300 & 7.4 & 20.0 & 1.7 & 22 & 660 & -- & -- \\
\hline & $8-28-91$ & 0810 & 292 & 7.6 & 18.0 & 2.9 & 36 & 660 & -- & .11 \\
\hline & $8-30-91$ & 0900 & 293 & 7.5 & 17.5 & 3.2 & 39 & 660 & -- & -- \\
\hline & $9-01-91$ & 0750 & 272 & 7.8 & 17.5 & 2.0 & 24 & 660 & -- & -- \\
\hline & $6-03-92$ & 0745 & 252 & 8.6 & 21.0 & -- & -- & 655 & 0.11 & .26 \\
\hline & $6-05-92$ & 0948 & 247 & 8.9 & 21.0 & 7.9 & 103 & 660 & -- & -- \\
\hline & $6-07-92$ & 0812 & 238 & 9.2 & 19.5 & 7.6 & 97 & 651 & -- & -- \\
\hline & $6-10-92$ & 0740 & 238 & 8.3 & 14.0 & 3.1 & 35 & 650 & .23 & .38 \\
\hline & $6-12-92$ & 1005 & 241 & 9.3 & -- & -- & -- & -. & -- & -- \\
\hline & $6-14-92$ & 0929 & 246 & 9.0 & 14.0 & 7.4 & 84 & 656 & -- & -- \\
\hline & $6-16-92$ & 1330 & 257 & 9.3 & 17.0 & 11.8 & 141 & 660 & -- & -- \\
\hline & $6-17-92$ & 0749 & 259 & 8.8 & 16.0 & 5.8 & 69 & 655 & .19 & .30 \\
\hline & $6-19-92$ & 0904 & 255 & 8.7 & 19.5 & 5.3 & 67 & 660 & -- & -- \\
\hline & $6-21-92$ & 0832 & 295 & 8.8 & 21.0 & 6.5 & 84 & 660 & -- & -- \\
\hline & $6-24-92$ & 0753 & -- & 7.9 & 22.0 & 1.1 & -- & 656 & .72 & .96 \\
\hline & $6-26-92$ & 0842 & 313 & 7.9 & 20.5 & 2.6 & 34 & 660 & -- & -- \\
\hline & $6-26-92$ & 1630 & 267 & 8.8 & 28.0 & 12.5 & 187 & 655 & -- & -- \\
\hline & $6-28-92$ & 0834 & 350 & 7.8 & 18.5 & 4.6 & 57 & 655 & -- & -- \\
\hline & $7-01-92$ & 0820 & 329 & 7.6 & 16.5 & 5.8 & 70 & 650 & .76 & .79 \\
\hline & 7-03-92 & 0835 & 342 & 7.6 & 20.0 & -- & -- & -- & -- & -- \\
\hline & $7-05-92$ & 0830 & 360 & 7.3 & 20.0 & 5.6 & 72 & 655 & -- & -- \\
\hline & 7-08-92 & 0817 & 282 & 7.4 & 17.5 & 3.1 & 38 & 660 & - & -- \\
\hline & $7-10-92$ & 0844 & 323 & 7.6 & 21.0 & 4.5 & 59 & 660 & -- & -- \\
\hline & $7-10-92$ & 1800 & 334 & 8.2 & 24.0 & 9.5 & 132 & 657 & - & -- \\
\hline & $7-12-92$ & 0845 & 294 & 7.2 & 20.0 & 4.3 & 55 & 660 & -. & -- \\
\hline & $7-15-92$ & 0825 & 341 & 6.9 & 21.5 & 2.2 & 29 & 662 & - & -- \\
\hline
\end{tabular}


Table 7. Field and laboratory measurements of selected properties and constituents in surface water from primary and additional sampling sites, 1991-92--Continued

\begin{tabular}{|c|c|c|c|c|c|c|c|c|c|c|}
\hline Site & Date & Time & $\begin{array}{c}\text { Specific } \\
\text { conductance } \\
(\mu \mathrm{S} / \mathrm{cm})\end{array}$ & $\begin{array}{c}\mathrm{pH} \\
\text { (standard } \\
\text { units) }\end{array}$ & $\begin{array}{l}\text { Temper- } \\
\text { ature } \\
\left({ }^{\circ} \mathrm{C}\right)\end{array}$ & $\begin{array}{c}\text { Oxygen, } \\
\text { dissolved } \\
(\mathrm{mg} / \mathrm{L})\end{array}$ & $\begin{array}{l}\text { Oxygen, } \\
\text { dissolved } \\
\text { (percent } \\
\text { saturation) }\end{array}$ & $\begin{array}{l}\text { Barometric } \\
\text { pressure } \\
(\mathrm{mm} \text { of } \mathrm{Hg})\end{array}$ & $\begin{array}{c}\text { Nitrogen, } \\
\text { ammonia } \\
\text { (mg/L } \\
\text { as N) } \\
\text { (lab) }\end{array}$ & $\begin{array}{c}\text { Nitrogen, } \\
\text { ammonia } \\
(\mathrm{mg} / \mathrm{L} \\
\left.\text { as } \mathrm{NH}_{3}\right) \\
\text { (field) }\end{array}$ \\
\hline \multirow[t]{28}{*}{3} & $7-17-92$ & 0848 & 276 & 7.3 & 23.5 & 4.8 & 65 & 665 & -- & -- \\
\hline & $7-17-92$ & 1702 & 276 & 7.3 & 24.0 & 2.4 & 32 & 660 & -- & -- \\
\hline & 7-19-92 & 0830 & 246 & 7.1 & 21.5 & 3.0 & 39 & 664 & -- & -- \\
\hline & $7-22-92$ & 0740 & 329 & 7.0 & 20.0 & 2.9 & 37 & 655 & -- & -- \\
\hline & 7-23-92 & 1000 & 287 & 6.8 & 18.5 & 4.2 & 52 & 664 & -- & -- \\
\hline & 7-24-92 & 0835 & 249 & 7.2 & 18.5 & 3.7 & 46 & 663 & -- & -- \\
\hline & $7-26-92$ & 0830 & 276 & 7.0 & 21.0 & 4.2 & 54 & 665 & -- & -- \\
\hline & $7-28-92$ & 1500 & 230 & 7.3 & -- & -- & -- & -- & -- & -- \\
\hline & 7-29-92 & 0745 & 251 & 6.7 & 22.0 & 1.6 & 21 & 655 & -- & -- \\
\hline & 7-30-92 & 0530 & 280 & 7.1 & 22.0 & 1.5 & 20 & 659 & 0.17 & -- \\
\hline & $7-31-92$ & 0640 & 272 & 7.0 & 22.0 & 1.8 & 24 & 663 & -- & -- \\
\hline & 8-02-92 & 0614 & 247 & 7.0 & 22.5 & 2.1 & 28 & 660 & -- & -- \\
\hline & $8-05-92$ & 0815 & 256 & 6.6 & 19.5 & .1 & 1 & 660 & .12 & -- \\
\hline & 8-07-92 & 0910 & 239 & 6.8 & 20.0 & 2.6 & 33 & 665 & -- & -- \\
\hline & 8-09-92 & 0740 & 267 & 7.2 & 20.0 & 2.0 & 25 & 662 & -- & -- \\
\hline & $8-12-92$ & 0733 & 225 & 7.0 & 23.0 & 2.0 & 27 & 655 & .38 & -- \\
\hline & $8-12-92$ & 0738 & -- & -- & -- & -- & -- & -- & .38 & -- \\
\hline & $8-14-92$ & 0835 & 267 & 6.9 & 22.5 & 5.2 & 69 & 665 & -- & -- \\
\hline & $8-14-92$ & 1635 & 252 & 8.3 & 31.0 & 6.6 & 103 & 660 & -- & -- \\
\hline & $8-16-92$ & 0714 & 274 & 6.1 & 21.5 & 1.7 & 22 & 660 & -- & -- \\
\hline & 8-19-92 & 0726 & 260 & 7.1 & 23.0 & .7 & 9 & 660 & .36 & -- \\
\hline & $8-21-92$ & 0815 & 240 & 6.9 & 19.0 & 4.1 & 52 & 655 & -- & -- \\
\hline & $8-21-92$ & 1606 & 272 & 7.3 & 20.0 & 3.3 & 42 & 660 & -- & -- \\
\hline & $8-23-92$ & 0930 & 270 & 7.0 & 17.0 & .8 & 10 & 660 & -- & -- \\
\hline & $8-24-92$ & 1335 & 268 & 6.5 & 18.0 & .4 & 5 & 660 & .16 & -- \\
\hline & $8-26-92$ & 0735 & 264 & 6.9 & 17.0 & 4.6 & 55 & 665 & .96 & -- \\
\hline & $8-30-92$ & 0835 & 248 & 7.3 & 19.5 & 3.8 & 48 & 660 & -- & -- \\
\hline & $9-08-92$ & 1150 & 276 & 7.3 & 16.0 & 6.6 & 77 & 661 & .07 & -- \\
\hline \multirow[t]{9}{*}{4} & $6-12-91$ & 0900 & 321 & 9.3 & 19.0 & 6.9 & 88 & 650 & -- & -- \\
\hline & 7-03-91 & 0740 & 367 & 8.8 & 23.0 & 6.0 & 81 & 660 & -- & -- \\
\hline & $7-24-91$ & 0800 & 350 & 6.2 & 21.0 & 2.4 & 32 & 650 & -- & -- \\
\hline & $8-19-91$ & 1540 & 303 & 8.2 & 23.5 & 7.8 & 107 & 660 & -- & -- \\
\hline & $7-20-92$ & 1320 & 312 & 7.3 & 24.0 & 2.5 & 35 & 659 & .25 & -- \\
\hline & $7-28-92$ & 1430 & 225 & 7.1 & -- & -- & -- & -- & -- & -- \\
\hline & $7-31-92$ & 1105 & 282 & 7.5 & 23.0 & 3.6 & 49 & 660 & .17 & -- \\
\hline & $8-20-92$ & 0900 & 274 & 6.6 & 22.0 & 1.2 & 16 & 660 & .26 & -- \\
\hline & $9-09-92$ & 0940 & 253 & 7.9 & 17.0 & 7.7 & 92 & 661 & .05 & -- \\
\hline \multirow[t]{5}{*}{5} & 6-05-91 & 0900 & 539 & 9.0 & 15.0 & 9.0 & 103 & 660 & -- & -- \\
\hline & $6-07-91$ & 1215 & 755 & 9.2 & 23.0 & 12.8 & 174 & 660 & -- & -- \\
\hline & $6-09-91$ & 1140 & 503 & 9.1 & 23.0 & 9.3 & 126 & 660 & -- & -- \\
\hline & $6-12-91$ & 0945 & 986 & 8.9 & 19.0 & 11.9 & 151 & 650 & -- & -- \\
\hline & $6-13-91$ & 1225 & 452 & 8.9 & 18.0 & 10.4 & 129 & 653 & -- & -- \\
\hline
\end{tabular}


Table 7. Field and laboratory measurements of selected properties and constituents in surface water from primary and additional sampling sites, 1991-92--Continued

\begin{tabular}{|c|c|c|c|c|c|c|c|c|c|c|}
\hline Site & Date & Time & $\begin{array}{c}\text { Specific } \\
\text { conductance } \\
(\mu \mathrm{S} / \mathrm{cm})\end{array}$ & $\begin{array}{c}\mathrm{pH} \\
\text { (standard } \\
\text { units) }\end{array}$ & $\begin{array}{l}\text { Temper- } \\
\text { ature } \\
\left({ }^{\circ} \mathrm{C}\right)\end{array}$ & $\begin{array}{l}\text { Oxygen, } \\
\text { dissolved } \\
\text { (mg/L) }\end{array}$ & $\begin{array}{l}\text { Oxygen, } \\
\text { dissolved } \\
\text { (percent } \\
\text { saturation) }\end{array}$ & $\begin{array}{l}\text { Barometric } \\
\text { pressure } \\
(\mathrm{mm} \text { of } \mathrm{Hg})\end{array}$ & $\begin{array}{c}\text { Nitrogen, } \\
\text { ammonia } \\
\text { (mg/L } \\
\text { as N) } \\
\text { (lab) }\end{array}$ & $\begin{array}{c}\text { Nitrogen, } \\
\text { ammonia } \\
(\mathrm{mg} / \mathrm{L} \\
\left.\text { as } \mathrm{NH}_{3}\right) \\
\text { (field) }\end{array}$ \\
\hline \multirow[t]{49}{*}{5} & $6-14-91$ & 0925 & 442 & 9.0 & 15.0 & 7.6 & 87 & 660 & -- & -- \\
\hline & $6-16-91$ & 0924 & 454 & 8.8 & 15.5 & 7.1 & 82 & 660 & -- & -- \\
\hline & $6-19-91$ & 0830 & 435 & 8.8 & 16.5 & 4.7 & 57 & 650 & -- & -- \\
\hline & $6-21-91$ & 1230 & 466 & 8.9 & -- & 11.0 & -- & 650 & -- & -- \\
\hline & 6-23-91 & 0959 & 526 & 8.4 & 17.5 & 6.3 & 78 & 650 & -- & -- \\
\hline & $6-26-91$ & 0828 & 758 & 7.8 & 15.5 & 5.5 & 64 & 655 & -- & -- \\
\hline & $6-28-91$ & 1020 & 736 & 8.2 & 17.0 & 7.9 & 96 & 650 & -- & -- \\
\hline & $6-30-91$ & 0800 & 811 & 8.4 & 17.0 & 7.5 & 90 & 662 & -- & -- \\
\hline & $7-03-91$ & 0825 & 632 & 8.3 & 22.5 & 4.5 & 60 & 660 & -- & -- \\
\hline & $7-05-91$ & 0935 & 684 & 8.1 & 24.0 & 6.0 & 83 & 660 & -- & -- \\
\hline & 7-07-91 & 1030 & 574 & 8.1 & 23.0 & 6.8 & 92 & 660 & -- & -- \\
\hline & $7-10-91$ & 0844 & 952 & 8.3 & 18.5 & 6.1 & 76 & 660 & -- & -- \\
\hline & $7-12-91$ & 1005 & 515 & 8.5 & 25.0 & 7.0 & 100 & 650 & -- & -- \\
\hline & 7-14-91 & 1015 & 763 & 8.7 & 23.0 & 8.4 & 116 & 650 & -- & -- \\
\hline & 7-17-91 & 0810 & 843 & 7.7 & 18.0 & 4.2 & 52 & 660 & -- & 0.20 \\
\hline & 7-19-91 & 0955 & 528 & 8.4 & 21.5 & 7.6 & 100 & 660 & -- & -- \\
\hline & $7-21-91$ & 0910 & 755 & 7.9 & 21.0 & 6.3 & 83 & 650 & -- & -- \\
\hline & 7-24-91 & 0830 & 386 & 7.7 & 21.0 & 5.0 & 66 & 655 & -- & $<.1$ \\
\hline & $7-26-91$ & 0715 & 528 & 8.2 & 20.0 & 3.1 & 40 & 660 & -- & -- \\
\hline & 7-28-91 & 0853 & 552 & 7.9 & 21.0 & 5.2 & 68 & 660 & -- & -- \\
\hline & $7-30-91$ & 1135 & 365 & 8.2 & 23.0 & 6.7 & 91 & 661 & -- & -- \\
\hline & $7-31-91$ & 0805 & 496 & 7.6 & 21.5 & 3.9 & 51 & 660 & -- & .19 \\
\hline & 8-02-91 & 0845 & 424 & 7.8 & 21.0 & 4.4 & 57 & 660 & -- & -- \\
\hline & 8-04-91 & 0905 & 420 & 7.7 & 20.0 & 4.2 & 54 & 650 & -- & -- \\
\hline & 8-05-91 & 1300 & 490 & 8.6 & 23.5 & 10.4 & 142 & 660 & -- & -. \\
\hline & 8-05-91 & 1420 & 445 & 8.4 & 24.0 & 11.0 & 152 & 660 & -- & -- \\
\hline & 8-05-91 & 1655 & 489 & 8.5 & 25.0 & 10.2 & 144 & 660 & -. & -- \\
\hline & 8-05-91 & 1800 & 538 & 8.5 & 24.0 & 10.1 & 139 & 660 & -- & -- \\
\hline & 8-05-91 & 2028 & 490 & 7.9 & 23.0 & 5.8 & 79 & 660 & -- & -- \\
\hline & 8-05-91 & 2240 & 458 & 7.6 & 22.0 & 4.0 & 53 & 660 & -- & -- \\
\hline & $8-06-91$ & 0040 & 460 & 7.7 & 22.0 & 3.7 & 49 & 660 & -- & -- \\
\hline & 8-06-91 & 0230 & 464 & 7.6 & 22.0 & 2.9 & 39 & 660 & -- & -- \\
\hline & 8-06-91 & 0420 & 482 & 7.5 & 21.0 & 2.6 & 34 & 660 & -- & -- \\
\hline & 8-06-91 & 0615 & 512 & 7.4 & 20.0 & 3.0 & 38 & 660 & -- & -- \\
\hline & 8-06-91 & 0810 & 515 & 7.3 & 20.0 & 4.6 & 59 & 660 & -- & -- \\
\hline & 8-06-91 & 1050 & 559 & 7.8 & 21.5 & 8.3 & 109 & 660 & -- & -- \\
\hline & 8-06-91 & 1230 & 575 & 8.0 & 23.0 & 10.1 & 137 & 660 & -- & -- \\
\hline & 8-07-91 & 0805 & 402 & 6.8 & 19.0 & 3.8 & 48 & 650 & -- & $<.1$ \\
\hline & 8-09-91 & 1045 & 348 & 8.1 & 20.5 & 7.8 & 102 & 650 & -- & -- \\
\hline & $8-11-91$ & 0945 & 440 & 7.9 & 19.0 & 6.4 & 81 & 650 & -- & -- \\
\hline & $8-12-91$ & 1020 & 502 & 7.8 & 20.5 & 8.2 & 105 & 662 & -- & -- \\
\hline & $8-14-91$ & 0755 & 423 & 7.3 & 19.5 & 3.7 & 47 & 660 & -- & $<.1$ \\
\hline & $8-16-91$ & 0915 & 461 & 7.9 & 20.0 & 6.8 & 87 & 660 & -- & -- \\
\hline & $8-18-91$ & 0835 & 478 & 7.5 & 20.0 & 3.7 & 47 & 660 & -- & -- \\
\hline & $8-21-91$ & 0750 & 381 & 7.5 & 20.0 & 4.1 & 52 & 660 & -- & $<.1$ \\
\hline & $8-21-91$ & 1648 & 520 & 8.2 & 25.5 & 11.2 & 159 & 660 & -- & -- \\
\hline & $8-23-91$ & 1540 & 292 & 8.3 & 25.0 & 4.2 & 59 & 660 & -- & -- \\
\hline & $8-25-91$ & 0825 & 544 & 7.5 & 18.0 & 3.2 & 39 & 660 & -- & -- \\
\hline & $8-28-91$ & 0825 & 357 & 7.7 & 17.0 & 5.6 & 67 & 660 & -- & .11 \\
\hline
\end{tabular}


Table 7. Field and laboratory measurements of selected properties and constituents in surface water from primary and additional sampling sites, 1991-92--Continued

\begin{tabular}{|c|c|c|c|c|c|c|c|c|c|c|}
\hline Site & Date & Time & $\begin{array}{c}\text { Specific } \\
\text { conductance } \\
(\mu \mathrm{S} / \mathrm{cm})\end{array}$ & $\begin{array}{c}\mathrm{pH} \\
\text { (standard } \\
\text { units) }\end{array}$ & $\begin{array}{c}\text { Temper- } \\
\text { ature } \\
\left({ }^{\circ} \mathrm{C}\right)\end{array}$ & $\begin{array}{l}\text { Oxygen, } \\
\text { dissolved } \\
(\mathrm{mg} / \mathrm{L})\end{array}$ & $\begin{array}{l}\text { Oxygen, } \\
\text { dissolved } \\
\text { (percent } \\
\text { saturation) }\end{array}$ & $\begin{array}{l}\text { Barometric } \\
\text { pressure } \\
(\mathrm{mm} \text { of } \mathrm{Hg})\end{array}$ & $\begin{array}{c}\text { Nitrogen, } \\
\text { ammonia } \\
\text { (mg/L } \\
\text { as N) } \\
\text { (lab) }\end{array}$ & $\begin{array}{c}\text { Nitrogen, } \\
\text { ammonia } \\
(\mathrm{mg} / \mathrm{L} \\
\left.\text { as } \mathrm{NH}_{3}\right) \\
\text { (field) }\end{array}$ \\
\hline \multirow[t]{47}{*}{5} & $8-30-91$ & 0925 & 392 & 7.7 & 17.0 & 5.3 & 64 & 660 & -- & -- \\
\hline & $9-01-91$ & 0803 & 420 & 8.0 & 17.0 & 4.4 & 53 & 660 & -- & -- \\
\hline & 6-03-92 & 0833 & 1,040 & 8.3 & 20.5 & -- & -- & 655 & 0.26 & 0.80 \\
\hline & $6-05-92$ & 1030 & 308 & 8.3 & 20.5 & 3.6 & 46 & 660 & -- & -- \\
\hline & 6-07-92 & 0855 & 409 & 9.0 & 18.0 & 5.0 & 62 & 651 & -- & -- \\
\hline & 6-10-92 & 0825 & 552 & 8.2 & 18.0 & 5.0 & 61 & 660 & .09 & $<.1$ \\
\hline & $6-12-92$ & 1040 & 643 & 8.9 & -- & -- & -- & -- & -- & -- \\
\hline & $6-14-92$ & 1000 & 617 & 8.5 & 14.0 & 6.8 & 77 & 655 & -- & -- \\
\hline & $6-16-92$ & 1220 & 520 & -- & 15.5 & 9.6 & 111 & 661 & -- & -- \\
\hline & $6-17-92$ & 0824 & 686 & 8.7 & 16.0 & 6.8 & 81 & 655 & .08 & $<.1$ \\
\hline & $6-19-92$ & 0930 & -- & 8.3 & 19.0 & 7.6 & -- & 660 & -- & -- \\
\hline & $6-21-92$ & 0904 & 566 & 7.8 & 21.0 & 7.2 & 94 & 660 & -- & -- \\
\hline & 6-24-92 & 0826 & 679 & 8.0 & 22.0 & 4.2 & 56 & 656 & .35 & .51 \\
\hline & $6-26-92$ & 0920 & 932 & 7.8 & 20.0 & 4.9 & 63 & 660 & -- & -- \\
\hline & $6-28-92$ & 0922 & 887 & 7.6 & 20.0 & 1.7 & 22 & 655 & -- & -- \\
\hline & $7-01-92$ & 0857 & 1,120 & 7.3 & 15.0 & 2.3 & 27 & 650 & .36 & .38 \\
\hline & 7-03-92 & 0905 & 1,130 & 7.5 & 19.0 & -- & -- & -- & -- & -- \\
\hline & 7-05-92 & 0850 & 786 & 7.7 & 19.0 & 2.3 & 29 & 655 & -- & -- \\
\hline & 7-08-92 & 0850 & .776 & 7.2 & 18.5 & 3.6 & 45 & 660 & .19 & -- \\
\hline & 7-10-92 & 0923 & 973 & 7.9 & 20.5 & 4.8 & 61 & 660 & -- & -- \\
\hline & 7-10-92 & 1823 & 992 & 8.4 & 24.0 & 16.0 & 222 & 657 & -- & -- \\
\hline & 7-12-92 & 0938 & 786 & 7.4 & 19.0 & 5.9 & 74 & 660 & -- & -- \\
\hline & $7-15-92$ & 0920 & 665 & 7.2 & 20.5 & 2.8 & 36 & 664 & .37 & -- \\
\hline & $7-17-92$ & 0920 & 629 & 7.6 & 22.5 & 4.7 & 63 & 665 & -- & -- \\
\hline & 7-19-92 & 0900 & 672 & 7.1 & 21.0 & 5.8 & 75 & 664 & -- & -- \\
\hline & $7-22-92$ & 0818 & 547 & 7.4 & 20.0 & 2.2 & 28 & 655 & .12 & -- \\
\hline & 7-23-92 & 1105 & 452 & 7.6 & 17.5 & 9.3 & 112 & 664 & -- & -- \\
\hline & 7-24-92 & 0905 & -- & 7.5 & 17.5 & .5 & -- & 664 & -- & -- \\
\hline & 7-26-92 & 0925 & 456 & 7.8 & 21.0 & 6.5 & 84 & 665 & -- & -- \\
\hline & $7-28-92$ & 1400 & 485 & 8.0 & 25.5 & 11.1 & 157 & 661 & -- & -- \\
\hline & 7-29-92 & 0820 & 710 & 7.3 & 20.0 & 3.5 & 45 & 655 & .10 & -- \\
\hline & 7-30-92 & 0635 & 785 & 7.4 & 22.0 & 1.7 & 23 & 660 & .24 & -- \\
\hline & 7-31-92 & 0715 & 822 & 7.3 & 22.5 & 1.5 & 20 & 662 & -- & -- \\
\hline & 8-02-92 & 0647 & 647 & 7.4 & 21.0 & 1.1 & 14 & 660 & -- & -- \\
\hline & 8-05-92 & 0750 & 749 & 7.0 & 19.0 & 1.3 & 16 & 660 & .19 & -- \\
\hline & 8-05-92 & 0755 & -- & -- & -- & -- & -- & -- & .19 & -- \\
\hline & 8-07-92 & 0830 & 965 & 6.9 & 19.0 & 3.2 & 40 & 665 & -- & -- \\
\hline & $8-07-92$ & 1756 & 495 & 8.7 & 24.0 & 15.4 & 211 & 664 & -- & -- \\
\hline & 8-09-92 & 0809 & 546 & 7.6 & 18.5 & 2.7 & 33 & 662 & -- & -- \\
\hline & $8-12-92$ & 0810 & 603 & 7.7 & 23.0 & 2.2 & 30 & 655 & .27 & -- \\
\hline & $8-14-92$ & 0920 & 771 & 7.2 & 21.5 & 4.7 & 61 & 665 & -- & -- \\
\hline & 8-14-92 & 1030 & -- & 7.8 & -- & -- & -- & -- & -- & -- \\
\hline & $8-16-92$ & 0737 & $\cdot 529$ & 6.7 & 21.0 & 2.9 & 38 & 660 & -- & -- \\
\hline & 8-19-92 & 0755 & 420 & 8.1 & 23.0 & 5.0 & 68 & 660 & .15 & -- \\
\hline & $8-21-92$ & 0840 & 562 & 7.0 & 19.0 & 4.6 & 58 & 660 & -- & -- \\
\hline & $8-21-92$ & 1325 & 523 & 7.9 & 21.0 & 10.7 & 140 & 656 & .06 & -- \\
\hline & $8-23-92$ & 0915 & 500 & 7.6 & 15.0 & 4.2 & 48 & 660 & -- & -- \\
\hline
\end{tabular}


Table 7. Field and laboratory measurements of selected properties and constituents in surface water from primary and additional sampling sites, 1991-92--Continued

\begin{tabular}{|c|c|c|c|c|c|c|c|c|c|c|}
\hline Site & Date & Time & $\begin{array}{c}\text { Specific } \\
\text { conductance } \\
(\mu \mathrm{S} / \mathrm{cm})\end{array}$ & $\begin{array}{c}\mathrm{pH} \\
\text { (standard } \\
\text { units) }\end{array}$ & $\begin{array}{c}\text { Temper- } \\
\text { ature } \\
\left({ }^{\circ} \mathrm{C}\right)\end{array}$ & $\begin{array}{l}\text { Oxygen, } \\
\text { dissolved } \\
(\mathrm{mg} / \mathrm{L})\end{array}$ & $\begin{array}{l}\text { Oxygen, } \\
\text { dissolved } \\
\text { (percent } \\
\text { saturation) }\end{array}$ & $\begin{array}{c}\text { Barometric } \\
\text { pressure } \\
(\mathrm{mm} \text { of } \mathrm{Hg})\end{array}$ & $\begin{array}{c}\text { Nitrogen, } \\
\text { ammonia } \\
\text { (mg/L } \\
\text { as N) } \\
\text { (lab) }\end{array}$ & $\begin{array}{l}\text { Nitrogen, } \\
\text { ammonia } \\
(\mathrm{mg} / \mathrm{L} \\
\left.\text { as } \mathrm{NH}_{3}\right) \\
\text { (field) }\end{array}$ \\
\hline \multirow[t]{4}{*}{5} & $8-26-92$ & 0805 & 514 & 7.4 & 16.0 & 4.4 & 51 & 665 & 0.12 & -- \\
\hline & $8-28-92$ & 0915 & 334 & 7.9 & 17.0 & 5.7 & 68 & 665 & -- & -- \\
\hline & $8-30-92$ & 0900 & 499 & 6.2 & 17.0 & 5.1 & 61 & 660 & -- & -- \\
\hline & $9-08-92$ & 1255 & 479 & 8.3 & 18.0 & 11.1 & 136 & 660 & .11 & -- \\
\hline \multirow[t]{9}{*}{6} & $6-12-91$ & 1000 & 341 & 9.1 & 18.0 & 4.8 & 60 & 650 & -- & -- \\
\hline & 7-03-91 & 0820 & 619 & 8.2 & 22.0 & 4.2 & 56 & 660 & -- & -- \\
\hline & $7-24-91$ & 0825 & 381 & 6.8 & 21.0 & 4.0 & 53 & 650 & -- & -- \\
\hline & 8-19-91 & 1625 & 411 & 8.7 & 28.0 & 14.2 & 211 & 660 & -- & -- \\
\hline & $7-20-92$ & 1250 & 590 & 8.3 & 25.0 & 5.0 & 70 & 659 & .05 & -- \\
\hline & $7-28-92$ & 1330 & 528 & 8.3 & 28.5 & 8.3 & 124 & 661 & -- & -- \\
\hline & $7-31-92$ & 1010 & 588 & 8.5 & 22.0 & 4.5 & 60 & 660 & .07 & -- \\
\hline & $8-20-92$ & 0835 & 496 & 7.6 & 20.5 & 1.8 & 23 & 660 & .32 & -- \\
\hline & 9-09-92 & 0930 & 396 & 8.3 & 16.0 & 6.7 & 79 & 660 & .03 & -- \\
\hline \multirow[t]{27}{*}{7} & $6-05-91$ & 0905 & 738 & 8.8 & 12.0 & 8.3 & 91 & 650 & -- & -- \\
\hline & 6-07-91 & 1155 & 902 & 9.0 & 17.5 & 18.1 & 220 & 660 & -- & -- \\
\hline & 6-09-91 & 1109 & 760 & 8.9 & 20.0 & 14.0 & 179 & 660 & -- & -- \\
\hline & $6-12-91$ & 0951 & 673 & 9.1 & 19.0 & 9.4 & 120 & 650 & -- & -- \\
\hline & $6-13-91$ & 1345 & 709 & 9.2 & 20.0 & 15.1 & 195 & 653 & -- & -- \\
\hline & $6-14-91$ & 1024 & 472 & 9.3 & 15.0 & 7.7 & 88 & 660 & -- & -- \\
\hline & 6-16-91 & 0850 & 462 & 9.2 & 16.5 & 6.1 & 72 & 660 & -- & -- \\
\hline & $6-19-91$ & 0840 & 718 & 8.5 & 16.0 & 2.8 & 33 & 650 & -- & -- \\
\hline & $6-21-91$ & 1145 & 660 & 8.9 & 20.5 & 9.0 & 118 & 650 & -- & -- \\
\hline & 6-23-91 & 1020 & 1,180 & 8.2 & 17.0 & 7.8 & 95 & 650 & -- & -- \\
\hline & $6-26-91$ & 0824 & 962 & 8.0 & 15.0 & 5.0 & 58 & 660 & -- & -- \\
\hline & $6-28-91$ & 0950 & 988 & 7.9 & 16.0 & 5.1 & 61 & 650 & -- & -- \\
\hline & $6-30-91$ & 1025 & 1,180 & 8.2 & 18.0 & 9.0 & 110 & 662 & -- & -- \\
\hline & 7-03-91 & 0820 & 1,500 & 7.7 & 20.0 & 2.7 & 35 & 660 & -- & -- \\
\hline & $7-05-91$ & 1015 & 1,250 & 8.1 & 23.0 & 8.7 & 118 & 660 & -- & -- \\
\hline & $7-07-91$ & 1000 & 1,090 & 7.9 & 22.0 & 8.9 & 118 & 660 & -- & -- \\
\hline & $7-10-91$ & 0856 & 995 & 8.1 & 19.0 & 6.2 & 78 & 660 & -- & -- \\
\hline & $7-12-91$ & 1043 & 716 & 8.6 & 22.5 & 7.7 & 105 & 650 & -. & -- \\
\hline & $7-14-91$ & 0945 & 826 & 8.2 & 22.0 & 6.3 & 85 & 650 & -- & -- \\
\hline & $7-17-91$ & 0822 & 653 & 7.3 & 18.0 & 2.8 & 34 & 660 & -- & 0.31 \\
\hline & 7-19-91 & 0935 & 696 & 8.1 & 21.0 & 5.8 & 76 & 660 & -- & -- \\
\hline & $7-21-91$ & 0935 & 795 & 8.2 & 21.0 & 10.6 & 138 & 660 & -- & -- \\
\hline & $7-24-91$ & 0843 & 573 & 8.2 & 21.0 & 5.8 & 76 & 660 & -- & .13 \\
\hline & $7-26-91$ & 0750 & 650 & 7.7 & 20.0 & 3.6 & 46 & 660 & -- & -- \\
\hline & $7-28-91$ & 0919 & 534 & 8.4 & 22.0 & 7.6 & 101 & 660 & -- & -- \\
\hline & $7-30-91$ & 1220 & 557 & 8.7 & 24.0 & 10.8 & 149 & 661 & -- & -- \\
\hline & $7-31-91$ & 0820 & 500 & 8.0 & 21.5 & 4.3 & 57 & 660 & -- & $<.1$ \\
\hline
\end{tabular}


Table 7. Field and laboratory measurements of selected properties and constituents in surface water from primary and additional sampling sites, 1991-92--Continued

\begin{tabular}{|c|c|c|c|c|c|c|c|c|c|c|}
\hline Site & Date & Time & $\begin{array}{c}\text { Specific } \\
\text { conductance } \\
(\mu \mathrm{S} / \mathrm{cm})\end{array}$ & $\begin{array}{c}\mathrm{pH} \\
\text { (standard } \\
\text { units) }\end{array}$ & $\begin{array}{l}\text { Temper- } \\
\text { ature } \\
\left({ }^{\circ} \mathrm{C}\right)\end{array}$ & $\begin{array}{l}\text { Oxygen, } \\
\text { dissolved } \\
(\mathrm{mg} / \mathrm{L})\end{array}$ & $\begin{array}{l}\text { Oxygen, } \\
\text { dissolved } \\
\text { (percent } \\
\text { saturation) }\end{array}$ & $\begin{array}{l}\text { Barometric } \\
\text { pressure } \\
(\mathrm{mm} \text { of } \mathrm{Hg})\end{array}$ & $\begin{array}{c}\text { Nitrogen, } \\
\text { ammonia } \\
\text { (mg/L } \\
\text { as N) } \\
\text { (lab) }\end{array}$ & $\begin{array}{c}\text { Nitrogen, } \\
\text { ammonia } \\
(\mathrm{mg} / \mathrm{L} \\
\left.\text { as } \mathrm{NH}_{3}\right) \\
\text { (field) } \\
\end{array}$ \\
\hline \multirow[t]{48}{*}{7} & $8-02-91$ & 0935 & 549 & 8.5 & 20.5 & 7.6 & 98 & 660 & -- & -- \\
\hline & $8-04-91$ & 0845 & 520 & 8.0 & 21.0 & 4.3 & 57 & 650 & -- & -- \\
\hline & $8-07-91$ & 0845 & 489 & 7.9 & 20.0 & 5.8 & 75 & 650 & -- & $<0.1$ \\
\hline & $8-07-91$ & 1200 & 502 & 7.6 & 22.0 & 10.3 & 137 & 660 & -- & -- \\
\hline & $8-07-91$ & 1401 & 490 & 8.2 & 23.0 & 12.0 & 163 & 660 & -- & -- \\
\hline & $8-07-91$ & 1602 & 478 & 8.4 & 23.0 & 12.4 & 168 & 660 & -- & -- \\
\hline & $8-07-91$ & 1805 & 509 & 8.2 & 23.0 & 12.1 & 164 & 660 & -- & -- \\
\hline & 8-07-91 & 2000 & 539 & 8.2 & 22.0 & 9.6 & 128 & 660 & -- & -- \\
\hline & $8-07-91$ & 2205 & 534 & 8.2 & 23.0 & 7.8 & 106 & 660 & -- & -- \\
\hline & $8-07-91$ & 2350 & 528 & 8.2 & 21.5 & 7.2 & 95 & 660 & -- & -- \\
\hline & $8-08-91$ & 0150 & 535 & 8.1 & 21.0 & 6.1 & 79 & 660 & -- & -- \\
\hline & 8-08-91 & 0330 & 538 & 7.9 & 20.5 & 4.9 & 63 & 660 & -- & -- \\
\hline & $8-08-91$ & 0555 & 495 & 7.5 & 19.5 & 4.1 & 52 & 660 & -- & -- \\
\hline & 8-08-91 & 0800 & 483 & 7.8 & 19.0 & 5.3 & 66 & 660 & -- & -- \\
\hline & 8-08-91 & 0952 & 473 & 8.0 & 19.5 & 7.9 & 100 & 660 & -- & -- \\
\hline & $8-08-91$ & 1205 & 462 & 8.4 & 21.0 & 11.1 & 145 & 660 & -- & -- \\
\hline & $8-09-91$ & 1025 & 488 & 8.2 & 20.0 & 8.2 & 106 & 650 & -- & -- \\
\hline & $8-11-91$ & 0920 & 650 & 8.5 & 19.0 & 7.0 & 89 & 650 & -- & -- \\
\hline & $8-12-91$ & 1100 & 585 & 8.7 & 21.0 & 9.8 & 127 & 662 & -- & -- \\
\hline & 8-14-91 & 0810 & 466 & 7.6 & 19.0 & 3.5 & 44 & 660 & -- & $<.1$ \\
\hline & 8-16-91 & 0950 & 507 & 8.2 & 20.0 & 7.0 & 89 & 660 & -- & -- \\
\hline & 8-18-91 & 0850 & 525 & 8.0 & 20.0 & 3.9 & 50 & 660 & -- & -- \\
\hline & $8-21-91$ & 0810 & 499 & 7.6 & 20.0 & 2.6 & 33 & 660 & -- & $<.1$ \\
\hline & $8-21-91$ & 1624 & 569 & 8.8 & 27.0 & 10.2 & 149 & 660 & -- & -- \\
\hline & $8-23-91$ & 1555 & 517 & 8.8 & 26.0 & 10.3 & 148 & 660 & -- & -- \\
\hline & $8-25-91$ & 0855 & 517 & 7.7 & 18.0 & 4.0 & 49 & 660 & -- & -- \\
\hline & $8-28-91$ & 0840 & 571 & 8.2 & 16.0 & 4.6 & 54 & 660 & -- & .12 \\
\hline & $8-30-91$ & 0945 & 416 & 7.7 & 16.5 & 3.6 & 43 & 660 & -- & -- \\
\hline & 9-01-91 & 0815 & 455 & 8.0 & 16.5 & 3.9 & 46 & 660 & -- & -- \\
\hline & 6-03-92 & 0915 & 1,130 & 8.1 & 19.0 & -- & -- & 655 & 0.28 & .56 \\
\hline & $6-05-92$ & 1100 & 843 & 8.6 & 20.5 & 13.0 & 168 & 660 & -- & -- \\
\hline & $6-07-92$ & 0930 & 1,070 & 8.6 & 18.0 & 7.0 & 87 & 651 & -- & -- \\
\hline & $6-10-92$ & 0920 & 760 & 8.2 & 17.0 & -- & -- & 650 & .29 & .41 \\
\hline & $6-12-92$ & 1100 & 655 & 8.7 & -- & -- & -- & -- & -- & -- \\
\hline & $6-14-92$ & 1030 & 641 & 8.7 & 14.5 & 9.7 & 111 & 655 & -- & -- \\
\hline & $6-16-92$ & 1100 & 578 & 9.2 & 16.0 & 11.2 & 132 & 660 & -- & -- \\
\hline & $6-17-92$ & 0900 & 677 & 8.6 & 16.0 & 6.2 & 73 & 655 & .06 & $<.1$ \\
\hline & $6-19-92$ & 1004 & -- & 8.7 & 19.5 & 10.2 & -- & 660 & -- & -- \\
\hline & $6-21-92$ & 0937 & 1,020 & 8.1 & 22.0 & 9.6 & 128 & 661 & -- & -- \\
\hline & $6-24-92$ & 0910 & 903 & 8.1 & 21.5 & 6.0 & 80 & 655 & .32 & .41 \\
\hline & $6-26-92$ & 1000 & 1,100 & 8.0 & 20.0 & 11.5 & 147 & 660 & -- & -- \\
\hline & $6-26-92$ & 1650 & 994 & 8.8 & 26.0 & 15.8 & 229 & 655 & -- & -- \\
\hline & $6-28-92$ & 0953 & 1,200 & 7.8 & 19.0 & 6.7 & 84 & 660 & -- & -- \\
\hline & $7-01-92$ & 0935 & 962 & 7.5 & 15.0 & 8.1 & 95 & 650 & .17 & .16 \\
\hline & $7-03-92$ & 0940 & $1 ; 260$ & 7.9 & 19.0 & -- & -- & -- & -- & -- \\
\hline & 7-05-92 & 0905 & 1,110 & 7.8 & 18.5 & 6.9 & 86 & 655 & -- & -- \\
\hline & 7-08-92 & 0924 & 1,050 & 7.8 & 18.5 & 8.9 & 110 & 660 & .05 & -- \\
\hline & $7-10-92$ & 1015 & 1,150 & 8.0 & 20.5 & 12.3 & 159 & 660 & -- & -- \\
\hline
\end{tabular}


Table 7. Field and laboratory measurements of selected properties and constituents in surface water from primary and additional sampling sites, 1991-92--Continued

\begin{tabular}{|c|c|c|c|c|c|c|c|c|c|c|}
\hline Site & Date & Time & $\begin{array}{c}\text { Specific } \\
\text { conductance } \\
(\mu \mathrm{S} / \mathrm{cm})\end{array}$ & $\begin{array}{c}\mathrm{pH} \\
\text { (standard } \\
\text { units) }\end{array}$ & $\begin{array}{c}\text { Temper- } \\
\text { ature } \\
\left({ }^{\circ} \mathrm{C}\right)\end{array}$ & $\begin{array}{l}\text { Oxygen, } \\
\text { dissolved } \\
(\mathrm{mg} / \mathrm{L})\end{array}$ & $\begin{array}{l}\text { Oxygen, } \\
\text { dissolved } \\
\text { (percent } \\
\text { saturation) }\end{array}$ & $\begin{array}{l}\text { Barometric } \\
\text { pressure } \\
(\mathrm{mm} \text { of } \mathrm{Hg})\end{array}$ & $\begin{array}{c}\text { Nitrogen, } \\
\text { ammonia } \\
\text { (mg/L } \\
\text { as N) } \\
\text { (lab) } \\
\end{array}$ & $\begin{array}{c}\text { Nitrogen, } \\
\text { ammonia } \\
(\mathrm{mg} / \mathrm{L} \\
\left.\text { as } \mathrm{NH}_{3}\right) \\
\text { (field) } \\
\end{array}$ \\
\hline \multirow[t]{31}{*}{7} & 7-12-92 & 1025 & 884 & 8.2 & 20.5 & 16.0 & 207 & 660 & -- & -- \\
\hline & $7-15-92$ & 1005 & 946 & 8.1 & 21.5 & 9.4 & 123 & 663 & 0.04 & -- \\
\hline & $7-17-92$ & 1002 & 750 & 8.2 & 23.5 & 7.3 & 98 & 665 & -- & - \\
\hline & $7-17-92$ & 1724 & 818 & 8.4 & 24.5 & 10.8 & 151 & 660 & -- & -- \\
\hline & 7-19-92 & 0935 & 1,000 & 7.8 & 22.0 & 9.6 & 127 & 664 & -- & -- \\
\hline & $7-22-92$ & 0853 & 786 & 8.0 & 19.0 & 8.0 & 101 & 655 & .08 & - \\
\hline & 7-23-92 & 1150 & 771 & 8.1 & 19.5 & 13.1 & 165 & 663 & -. & -- \\
\hline & $7-24-92$ & 0945 & 743 & 8.3 & 18.5 & 10.1 & 125 & 660 & -- & -- \\
\hline & $7-26-92$ & 0955 & 682 & 7.9 & 20.5 & 8.8 & 113 & 665 & -- & -- \\
\hline & $7-28-92$ & 1240 & 768 & 8.2 & 26.0 & 15.2 & 217 & 662 & -- & - \\
\hline & 7-29-92 & 0848 & 676 & 7.9 & 22.0 & 6.2 & 83 & 655 & .06 & -- \\
\hline & 7-29-92 & 0850 & -- & -- & -- & -- & -- & -- & .06 & -- \\
\hline & 7-30-92 & 0720 & 849 & 7.6 & 21.0 & 4.9 & 64 & 660 & .08 & -- \\
\hline & $7-31-92$ & 0755 & 697 & 7.6 & 20.5 & 1.7 & 22 & 663 & -- & -- \\
\hline & 8-02-92 & 0750 & 488 & 7.3 & 21.0 & 4.9 & 64 & 660 & -- & -- \\
\hline & 8-05-92 & 0710 & 671 & 7.7 & 19.5 & 1.5 & 19 & 660 & .07 & -- \\
\hline & 8-07-92 & 0807 & 697 & 7.2 & 18.5 & 4.8 & 59 & 667 & -- & -- \\
\hline & 8-09-92 & 0850 & 660 & 8.0 & 19.5 & 4.0 & 50 & 662 & -- & -- \\
\hline & $8-12-92$ & 0837 & 485 & 7.5 & 22.0 & 2.6 & 34 & 662 & .19 & -- \\
\hline & $8-14-92$ & 0945 & 658 & -- & 22.5 & 5.4 & 72 & 665 & -- & -- \\
\hline & $8-14-92$ & 1658 & 700 & 8.5 & 31.0 & 10.8 & 170 & 660 & -- & -- \\
\hline & 8-16-92 & 0808 & 685 & 7.0 & 20.5 & 4.1 & 53 & 660 & -- & -- \\
\hline & 8-19-92 & 0823 & 632 & 7.6 & 22.0 & 3.8 & 51 & 660 & .27 & -- \\
\hline & $8-21-92$ & 0900 & 412 & 7.4 & 18.5 & 5.4 & 67 & 655 & -- & -- \\
\hline & $8-21-92$ & 1050 & 474 & 7.8 & 21.0 & 9.7 & 127 & 655 & .10 & -- \\
\hline & $8-21-92$ & 1635 & 500 & 8.7 & 22.0 & 13.0 & 173 & 660 & -- & -- \\
\hline & $8-23-92$ & 0855 & 649 & 7.8 & 14.0 & 4.3 & 48 & 660 & -- & -- \\
\hline & 8-26-92 & 0836 & 647 & 7.6 & 14.5 & 3.5 & 40 & 665 & .67 & -- \\
\hline & $8-28-92$ & 0945 & 501 & 8.0 & 17.0 & 4.6 & 55 & 665 & -- & -- \\
\hline & $8-30-92$ & 0925 & 587 & 6.1 & 17.0 & 4.4 & 53 & 660 & -- & -- \\
\hline & 9-08-92 & 1355 & 460 & 7.9 & 19.0 & 11.2 & 140 & 660 & .37 & -- \\
\hline \multirow[t]{9}{*}{8} & $6-12-91$ & 1037 & 670 & 9.0 & 19.0 & 5.1 & 65 & 650 & - & -- \\
\hline & 7-03-91 & 0904 & 1,280 & 7.9 & 21.0 & 4.8 & 63 & 660 & - & -- \\
\hline & $7-24-91$ & 0840 & 554 & 7.4 & 21.0 & 3.3 & 44 & 650 & -- & -- \\
\hline & 8-19-91 & 1215 & 765 & 9.4 & 25.5 & 14.2 & 202 & 660 & -- & -- \\
\hline & 7-20-92 & 1220 & 923 & 7.7 & 24.0 & .2 & 3 & 659 & .95 & -- \\
\hline & $7-28-92$ & 1205 & 690 & 7.9 & 26.5 & 8.5 & 123 & 662 & -- & -- \\
\hline & $7-31-92$ & 0940 & 645 & 7.5 & 23.0 & 1.9 & 26 & 660 & .41 & -- \\
\hline & $8-20-92$ & 0815 & 643 & 7.9 & 21.5 & 1.3 & 17 & 660 & .25 & -- \\
\hline & 9-09-92 & 0825 & 660 & 8.6 & 17.0 & 4.8 & 58 & 660 & .15 & -- \\
\hline
\end{tabular}


Table 7. Field and laboratory measurements of selected properties and constituents in surface water from primary and additional sampling sites, 1991-92--Continued

\begin{tabular}{|c|c|c|c|c|c|c|c|c|c|c|}
\hline Site & Date & Time & $\begin{array}{c}\text { Specific } \\
\text { conductance } \\
(\mu \mathrm{S} / \mathrm{cm})\end{array}$ & $\begin{array}{c}\mathrm{pH} \\
\text { (standard } \\
\text { units) }\end{array}$ & $\begin{array}{l}\text { Temper- } \\
\text { ature } \\
\left({ }^{\circ} \mathrm{C}\right)\end{array}$ & $\begin{array}{l}\text { Oxygen, } \\
\text { dissolved } \\
(\mathrm{mg} / \mathrm{L})\end{array}$ & $\begin{array}{l}\text { Oxygen, } \\
\text { dissolved } \\
\text { (percent } \\
\text { saturation) }\end{array}$ & $\begin{array}{l}\text { Barometric } \\
\text { pressure } \\
(\mathrm{mm} \text { of } \mathrm{Hg})\end{array}$ & $\begin{array}{c}\text { Nitrogen, } \\
\text { ammonia } \\
\text { (mg/L } \\
\text { as N) } \\
\text { (lab) }\end{array}$ & $\begin{array}{c}\text { Nitrogen, } \\
\text { ammonia } \\
\left(\mathrm{mg}^{2} \mathrm{~L}\right. \\
\left.\text { as } \mathrm{NH}_{3}\right) \\
\text { (field) }\end{array}$ \\
\hline \multirow[t]{49}{*}{9} & 6-05-91 & 0830 & 970 & 8.8 & 13.0 & 8.0 & 89 & 650 & -- & -- \\
\hline & 6-07-91 & 1100 & 1,090 & 8.8 & 17.0 & 10.1 & 121 & 660 & -- & -- \\
\hline & 6-09-91 & 1038 & 1,010 & 8.8 & 18.0 & 10.5 & 129 & 660 & -- & -- \\
\hline & 6-12-91 & 0925 & 1,060 & 8.4 & 18.0 & 4.3 & 54 & 650 & -- & -- \\
\hline & $6-14-91$ & 1105 & 962 & 8.8 & 16.0 & 5.1 & 60 & 660 & -- & -- \\
\hline & 6-16-91 & 0850 & 998 & 8.3 & 15.0 & 5.2 & 60 & 660 & -- & -- \\
\hline & 6-19-91 & 0810 & 975 & 9.0 & 16.5 & 3.2 & 39 & 650 & -- & -- \\
\hline & 6-20-91 & 1000 & -- & 8.4 & 15.0 & 4.5 & -- & 650 & -- & -- \\
\hline & $6-21-91$ & 1100 & 1,040 & 8.5 & 17.0 & 4.5 & 55 & 650 & -- & -- \\
\hline & 6-23-91 & 1042 & 1,390 & 8.4 & 17.0 & 3.4 & 42 & 650 & -- & -- \\
\hline & 6-26-91 & 0800 & 1,180 & 8.2 & 15.0 & 3.4 & 39 & 660 & -- & -- \\
\hline & 6-28-91 & 0915 & 1,150 & 7.7 & 16.0 & 3.7 & 44 & 650 & -- & -- \\
\hline & $6-30-91$ & 1100 & 1,220 & 8.5 & 17.0 & 5.1 & 61 & 662 & -- & -- \\
\hline & 7-03-91 & 0755 & 1,120 & 8.2 & 22.5 & 1.8 & 24 & 660 & -- & -- \\
\hline & 7-05-91 & 1045 & 1,130 & 8.4 & 24.5 & 3.8 & 53 & 660 & -- & -- \\
\hline & 7-07-91 & 0930 & 1,040 & 8.0 & 23.0 & 3.4 & 46 & 660 & -- & -- \\
\hline & 7-10-91 & 0818 & 1,010 & 8.5 & 19.5 & 1.7 & 21 & 660 & -- & -- \\
\hline & 7-12-91 & 1111 & 1,170 & 8.6 & 23.0 & 4.4 & 61 & 650 & -- & -- \\
\hline & 7-14-91 & 0905 & 1,110 & 8.4 & 22.0 & 4.2 & 57 & 650 & -- & -- \\
\hline & 7-17-91 & 0755 & 1,110 & 8.2 & 17.0 & 3.0 & 36 & 660 & -- & 0.40 \\
\hline & $7-17-91$ & 0930 & 1,040 & 8.4 & 17.5 & 3.0 & 36 & 660 & -- & -- \\
\hline & 7-19-91 & 0850 & 1,050 & 8.1 & 21.5 & 5.0 & 66 & 660 & -- & -- \\
\hline & $7-21-91$ & 1005 & 1,110 & 8.5 & 21.5 & 6.6 & 88 & 650 & -- & -- \\
\hline & 7-24-91 & 0810 & 1,000 & 8.3 & 20.5 & 3.2 & 41 & 660 & -- & .24 \\
\hline & 7-26-91 & 0820 & 885 & 8.8 & 20.0 & 2.6 & 33 & 660 & -- & -- \\
\hline & $7-28-91$ & 0943 & 882 & 8.8 & 22.5 & 6.2 & 83 & 660 & -- & -- \\
\hline & $7-30-91$ & 1310 & 1,010 & 9.0 & 25.5 & 14.1 & 200 & 661 & -- & -- \\
\hline & $7-31-91$ & 0755 & 968 & 8.2 & 21.0 & 2.2 & 29 & 660 & -- & .18 \\
\hline & 8-02-91 & 0950 & 969 & 9.0 & 21.0 & 5.9 & 77 & 660 & -- & -- \\
\hline & 8-04-91 & 0800 & 849 & 8.5 & 21.0 & 2.1 & 28 & 650 & -- & -- \\
\hline & 8-07-91 & 0815 & 873 & 8.3 & 18.5 & 2.9 & 37 & 650 & -- & .37 \\
\hline & 8-07-91 & 1225 & 876 & 8.0 & 22.5 & 9.6 & 129 & 660 & -- & -- \\
\hline & 8-07-91 & 1420 & 878 & 8.5 & 24.0 & 11.4 & 158 & 660 & -- & -- \\
\hline & 8-07-91 & 1630 & 860 & 8.6 & 23.0 & 12.8 & 174 & 660 & -- & -- \\
\hline & 8-07-91 & 1820 & 874 & 8.4 & 23.0 & 11.6 & 157 & 660 & -- & -- \\
\hline & 8-07-91 & 2020 & 956 & 8.7 & -- & 8.8 & -- & 660 & -- & -- \\
\hline & 8-07-91 & 2230 & 959 & 8.3 & 19.0 & 5.2 & 65 & 660 & -- & -- \\
\hline & 8-08-91 & 0020 & 967 & 8.1 & 18.0 & 3.6 & 44 & 660 & -- & -- \\
\hline & 8-08-91 & 0215 & 965 & 8.0 & 19.0 & 2.8 & 35 & 660 & -- & -- \\
\hline & 8-08-91 & 0400 & 901 & 7.9 & 18.0 & 1.5 & 18 & 660 & -- & -- \\
\hline & 8-08-91 & 0620 & 950 & 7.8 & 17.5 & 1.3 & 16 & 660 & -- & -- \\
\hline & 8-08-91 & 0825 & 965 & 8.1 & 19.0 & 3.0 & 38 & 660 & -- & -- \\
\hline & 8-08-91 & 1007 & 972 & 8.6 & 25.0 & 9.9 & 140 & 660 & -- & -- \\
\hline & 8-08-91 & 1225 & 972 & 8.6 & 25.0 & 9.9 & 140 & 660 & -- & -- \\
\hline & 8-09-91 & 0940 & 950 & 8.2 & 21.0 & 5.5 & 73 & 650 & -- & -- \\
\hline & 8-11-91 & 0855 & 800 & 8.8 & 19.0 & 4.2 & 53 & 650 & -- & -- \\
\hline & $8-12-91$ & 1150 & 920 & 8.9 & 23.0 & 8.4 & 114 & 662 & -- & -- \\
\hline & $8-14-91$ & 0750 & 879 & 8.0 & 20.0 & 1.9 & 24 & 660 & -- & .45 \\
\hline & $8-16-91$ & 1015 & 1,120 & 8.4 & 21.5 & 5.6 & 74 & 660 & -- & -- \\
\hline
\end{tabular}


Table 7. Field and laboratory measurements of selected properties and constituents in surface water from primary and additional sampling sites, 1991-92--Continued

\begin{tabular}{|c|c|c|c|c|c|c|c|c|c|c|}
\hline Site & Date & Time & $\begin{array}{c}\text { Specific } \\
\text { conductance } \\
(\mu \mathrm{S} / \mathrm{cm})\end{array}$ & $\begin{array}{c}\mathrm{pH} \\
\text { (standard } \\
\text { units) }\end{array}$ & $\begin{array}{l}\text { Temper- } \\
\text { ature } \\
\left({ }^{\circ} \mathrm{C}\right)\end{array}$ & $\begin{array}{l}\text { Oxygen, } \\
\text { dissolved } \\
(\mathrm{mg} / \mathrm{L})\end{array}$ & $\begin{array}{l}\text { Oxygen, } \\
\text { dissolved } \\
\text { (percent } \\
\text { saturation) }\end{array}$ & $\begin{array}{l}\text { Barometric } \\
\text { pressure } \\
(\mathrm{mm} \text { of } \mathrm{Hg})\end{array}$ & $\begin{array}{c}\text { Nitrogen, } \\
\text { ammonia } \\
\text { (mg/L } \\
\text { as N) } \\
\text { (lab) }\end{array}$ & $\begin{array}{c}\text { Nitrogen, } \\
\text { ammonia } \\
(\mathrm{mg} / \mathrm{L} \\
\left.\text { as NH})_{3}\right) \\
\text { (field) }\end{array}$ \\
\hline \multirow[t]{47}{*}{9} & $8-18-91$ & 0915 & 1,030 & 8.5 & 21.0 & 3.6 & 47 & 660 & -- & -- \\
\hline & $8-21-91$ & 0740 & 966 & 8.0 & 19.5 & 1.7 & 22 & 660 & -- & 0.76 \\
\hline & $8-21-91$ & 1605 & 1,010 & 9.1 & 28.0 & 12.9 & 192 & 660 & -- & -- \\
\hline & $8-23-91$ & 1420 & 850 & 9.0 & 26.5 & 14.8 & 214 & 660 & -- & -- \\
\hline & $8-25-91$ & 0920 & 1,040 & 8.5 & 19.0 & 4.4 & 55 & 660 & -- & -- \\
\hline & $8-28-91$ & 0819 & 865 & 9.0 & 16.5 & 3.6 & 43 & 660 & -- & .31 \\
\hline & $8-30-91$ & 1015 & 946 & 8.9 & 19.0 & 7.9 & 99 & 660 & -- & -- \\
\hline & 9-01-91 & 0830 & 935 & 9.1 & 18.0 & 3.2 & 39 & 660 & -- & -- \\
\hline & 6-03-92 & 0725 & 1,210 & 8.2 & 17.5 & 3.5 & 43 & 655 & 0.55 & 1.12 \\
\hline & 6-05-92 & 1145 & 1,230 & 9.1 & 21.5 & 18.0 & 237 & 660 & -- & -- \\
\hline & 6-07-92 & 1005 & 1,040 & 9.1 & 18.5 & 10.6 & 133 & 651 & -- & -- \\
\hline & $6-10-92$ & 0755 & 1,090 & 8.6 & 17.0 & 4.8 & 59 & 650 & .38 & .52 \\
\hline & $6-12-92$ & 0820 & 1,010 & 8.8 & 12.0 & 4.5 & 49 & 655 & -- & -- \\
\hline & $6-14-92$ & 1110 & 1,080 & 8.9 & 13.0 & 7.9 & 88 & 655 & -- & -- \\
\hline & $6-16-92$ & 0730 & 1,000 & 8.8 & 12.5 & 4.2 & 46 & 660 & -- & -- \\
\hline & $6-17-92$ & 0810 & 1,190 & 8.4 & 14.0 & 5.1 & 58 & 655 & .33 & .45 \\
\hline & $6-19-92$ & 1035 & -- & 8.6 & 20.0 & 7.4 & -- & 660 & -- & -- \\
\hline & $6-21-92$ & 1011 & 1,130 & 8.6 & 22.0 & 6.9 & 92 & 660 & -- & -- \\
\hline & $6-24-92$ & 0715 & 1,100 & 8.4 & 20.5 & 1.7 & 22 & 656 & .44 & .52 \\
\hline & $6-26-92$ & 1045 & 1,090 & 8.7 & 22.0 & 8.4 & 112 & 660 & -- & -- \\
\hline & $6-28-92$ & 1031 & 1,150 & 8.5 & 20.0 & 4.8 & 62 & 655 & -- & -- \\
\hline & $7-01-92$ & 0915 & 1,220 & 8.2 & 16.0 & 5.8 & 69 & 650 & .24 & .22 \\
\hline & 7-03-92 & 1010 & 1,210 & 8.4 & 19.5 & -- & -- & -- & -- & -- \\
\hline & $7-05-92$ & 0950 & 1,240 & 8.2 & 18.0 & 7.5 & 93 & 655 & -- & -- \\
\hline & 7-08-92 & 0808 & 1,070 & 8.5 & 19.0 & 4.3 & 54 & 662 & .19 & -- \\
\hline & 7-10-92 & 1050 & 1,090 & 8.7 & 22.0 & 7.8 & 104 & 660 & -- & -- \\
\hline & 7-10-92 & 1850 & 1,110 & 8.9 & 25.0 & 14.9 & 211 & 657 & -- & -- \\
\hline & $7-12-92$ & 1112 & 1,110 & 8.4 & 21.5 & 9.5 & 125 & 660 & .- & -- \\
\hline & $7-15-92$ & 0755 & 1,180 & 8.1 & 21.0 & 1.3 & 17 & 655 & .34 & -- \\
\hline & $7-17-92$ & 1040 & 940 & 8.7 & 23.0 & 5.4 & 73 & 665 & -- & -- \\
\hline & 7-19-92 & 1005 & 1,070 & 8.2 & 22.5 & 4.2 & 56 & 664 & -- & -- \\
\hline & $7-22-92$ & 0840 & 871 & 8.3 & 17.0 & 3.5 & 42 & 660 & .43 & -- \\
\hline & $7-22-92$ & 0845 & -- & -- & -- & -- & -- & -- & .42 & -- \\
\hline & 7-23-92 & 1240 & 1,310 & 8.0 & 20.5 & 5.4 & 70 & 662 & -- & -- \\
\hline & 7-24-92 & 1020 & 1,320 & 8.1 & 19.0 & 3.1 & 39 & 660 & -- & -- \\
\hline & 7-26-92 & 1030 & 1,180 & 7.9 & 20.5 & 2.9 & 37 & 665 & -- & -- \\
\hline & $7-28-92$ & 0934 & 1,240 & 7.8 & 21.5 & .8 & 11 & 663 & -- & -- \\
\hline & $7-29-92$ & 0730 & 1,190 & 7.8 & 20.5 & .9 & 12 & 660 & 1.40 & -- \\
\hline & $7-30-92$ & 0830 & 1,330 & 8.0 & 20.5 & 1.6 & 21 & 660 & 1.40 & -- \\
\hline & $7-31-92$ & 0840 & 1,280 & 7.9 & 19.0 & 2.7 & 34 & 664 & -- & -- \\
\hline & $8-02-92$ & 0820 & 1,220 & 7.9 & 20.5 & 2.6 & 34 & 660 & -- & -- \\
\hline & 8-05-92 & 0810 & 1,160 & 8.1 & 19.0 & 2.8 & 35 & 659 & .58 & -- \\
\hline & $8-07-92$ & 0725 & 1,190 & 8.0 & 18.0 & 3.7 & 45 & 665 & -- & -- \\
\hline & 8-07-92 & 1822 & 1,290 & 8.6 & 26.0 & 15.3 & 219 & 663 & -- & -- \\
\hline & 8-09-92 & 0930 & 1,180 & 8.7 & 19.5 & 4.1 & 52 & 662 & -- & -- \\
\hline & $8-12-92$ & 0750 & 1,220 & 8.5 & 22.0 & 4.7 & 62 & 661 & .31 & -- \\
\hline & $8-14-92$ & 1015 & 1,160 & -- & 23.0 & 4.7 & 63 & 665 & -- & -- \\
\hline
\end{tabular}


Table 7. Field and laboratory measurements of selected properties and constituents in surface water from primary and additional sampling sites, 1991-92--Continued

\begin{tabular}{|c|c|c|c|c|c|c|c|c|c|c|}
\hline Site & Date & Time & $\begin{array}{c}\text { Specific } \\
\text { conductance } \\
(\mu \mathrm{S} / \mathrm{cm})\end{array}$ & $\begin{array}{c}\mathrm{pH} \\
\text { (standard } \\
\text { units) }\end{array}$ & $\begin{array}{c}\text { Temper- } \\
\text { ature } \\
\left({ }^{\circ} \mathrm{C}\right)\end{array}$ & $\begin{array}{l}\text { Oxygen, } \\
\text { dissolved } \\
(\mathrm{mg} / \mathrm{L})\end{array}$ & $\begin{array}{l}\text { Oxygen, } \\
\text { dissolved } \\
\text { (percent } \\
\text { saturation) }\end{array}$ & $\begin{array}{l}\text { Barometric } \\
\text { pressure } \\
(\mathrm{mm} \text { of } \mathrm{Hg})\end{array}$ & $\begin{array}{c}\text { Nitrogen, } \\
\text { ammonia } \\
\text { (mg/L } \\
\text { as N) } \\
\text { (lab) }\end{array}$ & $\begin{array}{c}\text { Nitrogen, } \\
\text { ammonia } \\
(\mathrm{mg} / \mathrm{L} \\
\left.\text { as } \mathrm{NH}_{3}\right) \\
\text { (field) }\end{array}$ \\
\hline \multirow[t]{10}{*}{9} & $8-14-92$ & 1130 & -- & 8.2 & -- & -- & -- & -- & -- & -- \\
\hline & $8-16-92$ & 0843 & 1,090 & 8.0 & 21.5 & 4.5 & 59 & 660 & -- & -- \\
\hline & $8-19-92$ & 0725 & 1,130 & 7.6 & 20.0 & 2.4 & 31 & 655 & 1.50 & -- \\
\hline & $8-21-92$ & 0930 & 817 & 8.7 & 19.0 & 6.1 & 77 & 655 & -- & -- \\
\hline & $8-21-92$ & 0955 & 926 & 8.9 & 20.0 & 7.3 & 93 & 660 & .33 & -- \\
\hline & $8-23-92$ & 0825 & 961 & 9.0 & 14.0 & 6.1 & 69 & 660 & -- & -- \\
\hline & $8-26-92$ & 0735 & 1,050 & 8.5 & 13.0 & 5.5 & 60 & 664 & .11 & -. \\
\hline & $8-28-92$ & 1010 & 1,060 & 8.7 & 17.0 & 6.6 & 79 & 665 & -- & -- \\
\hline & $8-30-92$ & 0950 & 918 & 8.1 & 17.5 & 6.4 & 78 & 660 & -- & -- \\
\hline & $9-08-92$ & 1445 & 962 & 9.0 & 23.0 & 12.4 & 168 & 660 & .46 & -- \\
\hline \multirow[t]{13}{*}{10} & $6-12-91$ & 1120 & 715 & 9.0 & 19.0 & 5.9 & 74 & 660 & -- & -- \\
\hline & 7-03-91 & 0940 & 1,160 & 8.4 & 23.5 & 3.8 & 52 & 660 & -- & -- \\
\hline & $7-24-91$ & 0910 & 1,110 & 6.7 & 21.0 & 3.3 & 44 & 650 & -- & -- \\
\hline & $8-18-91$ & 0831 & 658 & 9.1 & 20.0 & 4.1 & 52 & 660 & -- & -- \\
\hline & $8-19-91$ & 1300 & 905 & 9.4 & 27.5 & -- & -- & -- & -- & -- \\
\hline & 6-16-92 & 0930 & 1,010 & 8.9 & -- & -- & -- & 660 & -- & -- \\
\hline & $7-20-92$ & 1150 & 1,170 & 8.8 & 23.0 & 3.5 & 48 & 659 & .30 & -- \\
\hline & $7-28-92$ & 0845 & 1,080 & 8.4 & 24.0 & 1.5 & 21 & 663 & -- & -- \\
\hline & $7-31-92$ & 0700 & 1,230 & 8.3 & 22.0 & 2.3 & 31 & 660 & 1.10 & -- \\
\hline & $8-20-92$ & 0750 & 798 & 8.5 & 21.5 & 4.3 & 57 & 660 & .17 & -- \\
\hline & $8-20-92$ & 1520 & 1,110 & 8.6 & 26.0 & 20.0 & 286 & 660 & -- & -- \\
\hline & $8-21-92$ & 1610 & 999 & 8.5 & 23.0 & 19.0 & 260 & 655 & .53 & -- \\
\hline & $9-09-92$ & 0840 & 904 & 8.9 & 16.0 & 5.5 & 65 & 660 & .41 & -- \\
\hline \multirow[t]{18}{*}{11} & $6-05-91$ & 0805 & 816 & 8.6 & 12.0 & 7.6 & 83 & 650 & -- & -- \\
\hline & $6-07-91$ & 0955 & 788 & 8.6 & 15.0 & 7.6 & 87 & 660 & -- & -- \\
\hline & $6-09-91$ & 1035 & 812 & 8.9 & 18.0 & 7.2 & 88 & 660 & -- & -- \\
\hline & $6-12-91$ & 0854 & 767 & 8.8 & 16.0 & 6.5 & 77 & 650 & -- & -- \\
\hline & $6-13-91$ & 1515 & 794 & 8.9 & 18.0 & 9.2 & 114 & 653 & -- & -- \\
\hline & $6-14-91$ & 0815 & 745 & 8.9 & 13.0 & 7.9 & 87 & 660 & -- & -- \\
\hline & 6-16-91 & 0815 & 769 & 9.0 & 13.0 & 10.1 & 111 & 660 & -- & -- \\
\hline & 6-19-91 & 0729 & 810 & 9.7 & 15.0 & 6.6 & 77 & 650 & -- & -- \\
\hline & $6-21-91$ & 1000 & 827 & 9.1 & 16.0 & 8.4 & 100 & 650 & -- & -- \\
\hline & $6-23-91$ & 1105 & 915 & 9.4 & 18.0 & 13.8 & 169 & 660 & -- & -- \\
\hline & $6-26-91$ & 0735 & 779 & 9.2 & 14.0 & 7.3 & 82 & 660 & -- & -- \\
\hline & $6-27-91$ & 0950 & 780 & 9.2 & 15.5 & 6.6 & 78 & 653 & -- & -- \\
\hline & $6-28-91$ & 0930 & 880 & 9.3 & 16.0 & 7.6 & 91 & 650 & -- & -- \\
\hline & $6-30-91$ & 0945 & 765 & 8.6 & 16.0 & 7.4 & 88 & 650 & -- & -- \\
\hline & 7-03-91 & 0730 & 793 & 9.2 & 23.0 & 7.7 & 104 & 660 & -- & -- \\
\hline & 7-05-91 & 0845 & 729 & 8.5 & 22.0 & 3.5 & 47 & 660 & -- & -- \\
\hline & $7-07-91$ & 0930 & 754 & 8.9 & 27.0 & 2.8 & 41 & 660 & -- & -- \\
\hline & $7-10-91$ & 0748 & 726 & 8.9 & 18.0 & 1.2 & 15 & 660 & -- & -- \\
\hline
\end{tabular}


Table 7. Field and laboratory measurements of selected properties and constituents in surface water from primary and additional sampling sites, 1991-92--Continued

\begin{tabular}{|c|c|c|c|c|c|c|c|c|c|c|}
\hline Site & Date & Time & $\begin{array}{c}\text { Specific } \\
\text { conductance } \\
(\mu \mathrm{S} / \mathrm{cm})\end{array}$ & $\begin{array}{c}\mathrm{pH} \\
\text { (standard } \\
\text { units) }\end{array}$ & $\begin{array}{l}\text { Temper- } \\
\text { ature } \\
\left({ }^{\circ} \mathrm{C}\right)\end{array}$ & $\begin{array}{l}\text { Oxygen, } \\
\text { dissolved } \\
(\mathrm{mg} / \mathrm{L})\end{array}$ & $\begin{array}{l}\text { Oxygen, } \\
\text { dissolved } \\
\text { (percent } \\
\text { saturation) }\end{array}$ & $\begin{array}{l}\text { Barometric } \\
\text { pressure } \\
(\mathrm{mm} \text { of } \mathrm{Hg})\end{array}$ & $\begin{array}{c}\text { Nitrogen, } \\
\text { ammonia } \\
\text { (mg/L } \\
\text { as N) } \\
\text { (lab) }\end{array}$ & $\begin{array}{c}\text { Nitrogen, } \\
\text { ammonia } \\
(\mathrm{mg} / \mathrm{L} \\
\left.\text { as } \mathrm{NH}_{3}\right) \\
\text { (field) }\end{array}$ \\
\hline \multirow[t]{48}{*}{11} & $7-12-91$ & 0850 & 760 & 8.9 & 20.0 & 2.5 & 32 & 650 & -- & -- \\
\hline & $7-14-91$ & 1030 & 769 & 8.9 & 21.0 & 5.2 & 69 & 650 & -- & -- \\
\hline & $7-17-91$ & 0725 & 774 & 8.2 & 17.0 & 3.3 & 40 & 660 & -- & -- \\
\hline & 7-19-91 & 0847 & 680 & 9.2 & 19.0 & 4.0 & 50 & 660 & -- & -- \\
\hline & $7-21-91$ & 0918 & 619 & 9.0 & 19.5 & 4.6 & 59 & 650 & -. & -- \\
\hline & $7-23-91$ & 1250 & 647 & 9.5 & 23.0 & 6.6 & 89 & 660 & -- & -- \\
\hline & $7-24-91$ & 0740 & 599 & 8.8 & 20.5 & 4.5 & 58 & 660 & -- & 0.35 \\
\hline & $7-26-91$ & 0800 & 709 & 8.6 & 18.0 & 4.7 & 58 & 660 & -- & -- \\
\hline & $7-28-91$ & 0755 & 589 & 9.4 & 20.0 & 4.9 & 63 & 660 & -- & -- \\
\hline & $7-31-91$ & 0730 & 615 & 9.2 & 20.5 & 5.5 & 71 & 660 & -- & .23 \\
\hline & $8-02-91$ & 0825 & 691 & 9.3 & 19.0 & 4.6 & 58 & 660 & -- & -- \\
\hline & $8-04-91$ & 0815 & 578 & 9.1 & 21.0 & 4.7 & 62 & 660 & -- & -- \\
\hline & $8-07-91$ & 0735 & 731 & 9.2 & 17.5 & 4.6 & 57 & 660 & -- & .13 \\
\hline & $8-07-91$ & 1255 & 728 & 8.7 & 19.0 & 5.5 & 69 & 660 & -- & -- \\
\hline & $8-07-91$ & 1435 & 737 & 8.9 & 19.0 & 6.7 & 84 & 660 & -- & -- \\
\hline & $8-07-91$ & 1655 & 733 & 8.8 & 20.5 & 8.1 & 105 & 660 & -- & -- \\
\hline & $8-07-91$ & 1835 & 732 & 8.8 & 21.0 & 8.8 & 115 & 660 & -- & -- \\
\hline & $8-07-91$ & 2050 & 786 & 9.1 & 20.5 & 9.7 & 125 & 660 & -- & -- \\
\hline & $8-07-91$ & 2250 & 783 & 8.9 & 19.5 & 8.8 & 111 & 660 & -- & -- \\
\hline & $8-08-91$ & 0040 & 774 & 8.9 & 18.0 & 8.0 & 98 & 660 & -- & -- \\
\hline & $8-08-91$ & 0230 & 771 & 9.0 & 17.5 & 7.8 & 95 & 660 & -- & -- \\
\hline & $8-08-91$ & 0420 & 760 & 9.0 & 18.0 & 6.0 & 74 & 660 & -- & -- \\
\hline & $8-08-91$ & 0640 & 738 & 9.0 & 17.5 & 5.6 & 68 & 660 & -- & -- \\
\hline & 8-08-91 & 0850 & 726 & 9.2 & 17.5 & 5.4 & 66 & 660 & -- & -- \\
\hline & 8-08-91 & 1018 & 721 & 9.0 & 17.5 & 5.2 & 63 & 660 & -- & -- \\
\hline & 8-08-91 & 1250 & 759 & 9.2 & 19.5 & 6.9 & 87 & 660 & -- & -- \\
\hline & 8-09-91 & 1104 & 607 & 9.2 & 19.0 & 6.6 & 84 & 650 & -- & -- \\
\hline & $8-11-91$ & 0825 & 560 & 9.1 & 17.0 & 5.6 & 68 & 650 & -- & -- \\
\hline & $8-12-91$ & 1220 & 643 & 9.2 & 20.5 & 7.1 & 91 & 662 & -- & -- \\
\hline & $8-14-91$ & 0725 & 688 & 8.4 & 20.0 & 6.8 & 87 & 660 & -- & .21 \\
\hline & $8-16-91$ & 0840 & 720 & 9.3 & 20.0 & 5.8 & 74 & 660 & -. & -- \\
\hline & $8-21-91$ & 0730 & 595 & 9.1 & 20.5 & 3.3 & 43 & 660 & -. & .29 \\
\hline & $8-21-91$ & 1550 & 570 & 9.8 & 25.0 & 7.6 & 107 & 660 & -. & -- \\
\hline & 8-23-91 & 1356 & 622 & 9.3 & 23.0 & 7.1 & 98 & 650 & -- & -- \\
\hline & $8-25-91$ & 0830 & 585 & 9.1 & 18.0 & 4.2 & 51 & 660 & -- & -- \\
\hline & 8-28-91 & 0800 & 661 & 9.1 & 16.0 & 6.0 & 71 & 660 & -- & .22 \\
\hline & $8-30-91$ & 0955 & 560 & 9.2 & 17.5 & 4.5 & 55 & 660 & -- & -- \\
\hline & $9-01-91$ & 0847 & 463 & 8.9 & 17.0 & 4.4 & 53 & 660 & -- & -- \\
\hline & 6-03-92 & 0820 & 465 & 9.3 & 19.0 & 4.9 & 62 & 655 & 0.25 & .68 \\
\hline & 6-05-92 & 1214 & 438 & 9.8 & 22.0 & 6.5 & 86 & 660 & -- & -- \\
\hline & 6-07-92 & 1037 & 382 & 9.9 & 18.0 & 6.1 & 76 & 651 & -- & -. \\
\hline & $6-10-92$ & 0835 & 411 & 9.5 & 18.5 & 6.6 & 83 & 650 & .29 & .38 \\
\hline & $6-12-92$ & 0735 & 394 & 9.3 & 14.0 & 5.2 & 59 & 650 & -- & -- \\
\hline & $6-14-92$ & 1150 & 404 & 9.8 & 14.5 & 5.7 & 65 & 655 & -- & -- \\
\hline & $6-15-92$ & 1145 & 416 & 9.7 & 14.5 & 6.8 & 78 & 658 & -- & -- \\
\hline & $6-17-92$ & 0736 & 389 & 9.1 & 12.0 & 6.4 & 69 & 655 & .23 & .34 \\
\hline & $6-17-92$ & 1005 & 407 & 9.7 & 14.0 & 7.4 & 83 & 660 & -- & -- \\
\hline & 6-19-92 & 1110 & -- & 9.7 & 21.5 & 8.6 & -- & 660 & -- & -- \\
\hline
\end{tabular}


Table 7. Field and laboratory measurements of selected properties and constituents in surface water from primary and additional sampling sites, 1991-92--Continued

\begin{tabular}{|c|c|c|c|c|c|c|c|c|c|c|}
\hline Site & Date & Time & $\begin{array}{c}\text { Specific } \\
\text { conductance } \\
(\mu \mathrm{S} / \mathrm{cm})\end{array}$ & $\begin{array}{c}\mathrm{pH} \\
\text { (standard } \\
\text { units) }\end{array}$ & $\begin{array}{c}\text { Temper- } \\
\text { ature } \\
\left({ }^{\circ} \mathrm{C}\right)\end{array}$ & $\begin{array}{l}\text { Oxygen, } \\
\text { dissolved } \\
(\mathrm{mg} / \mathrm{L})\end{array}$ & $\begin{array}{l}\text { Oxygen, } \\
\text { dissolved } \\
\text { (percent } \\
\text { saturation) }\end{array}$ & $\begin{array}{l}\text { Barometric } \\
\text { pressure } \\
(\mathrm{mm} \text { of } \mathrm{Hg})\end{array}$ & $\begin{array}{c}\text { Nitrogen, } \\
\text { ammonia } \\
\text { (mg/L } \\
\text { as N) } \\
\text { (lab) }\end{array}$ & $\begin{array}{c}\text { Nitrogen, } \\
\text { ammonia } \\
\text { (mg/L } \\
\left.\text { as } \mathrm{NH}_{3}\right) \\
\text { (field) }\end{array}$ \\
\hline \multirow[t]{39}{*}{11} & $6-19-92$ & 1706 & 386 & 9.5 & 25.0 & 8.6 & 121 & 658 & -- & -- \\
\hline & $6-21-92$ & 1045 & $\cdot 374$ & 9.7 & 30.5 & 7.5 & 117 & 660 & -- & -- \\
\hline & $6-24-92$ & 0810 & 393 & 9.2 & 21.0 & 4.1 & 54 & 656 & 0.20 & 0.34 \\
\hline & $6-26-92$ & 1130 & 411 & 9.2 & 24.0 & 8.7 & 120 & 660 & -- & -- \\
\hline & $6-28-92$ & 1057 & 392 & 9.1 & 21.0 & 3.8 & 50 & 655 & -- & -- \\
\hline & 7-01-92 & 0945 & 444 & 9.0 & 16.5 & 4.8 & 58 & 650 & .33 & .37 \\
\hline & 7-03-92 & 1055 & 407 & -- & 20.0 & -- & -- & -- & -- & -- \\
\hline & 7-05-92 & 1000 & 396 & 9.1 & 17.0 & 5.2 & 63 & 655 & -- & -- \\
\hline & 7-08-92 & 0847 & 418 & 9.6 & 17.0 & 5.5 & 66 & 662 & .13 & -- \\
\hline & 7-10-92 & 1130 & -- & 9.0 & 22.0 & 7.3 & -- & 660 & -- & -- \\
\hline & 7-12-92 & 1200 & 402 & 8.8 & 23.5 & 4.6 & 63 & 660 & -- & -- \\
\hline & 7-15-92 & 0825 & 384 & 9.1 & 21.0 & 3.7 & 49 & 655 & .17 & -- \\
\hline & $7-17-92$ & 1110 & 338 & 9.2 & 22.0 & 5.9 & 79 & 655 & -- & -- \\
\hline & 7-19-92 & 1035 & 409 & 8.5 & 23.5 & 4.1 & 56 & 664 & -- & -- \\
\hline & $7-22-92$ & 0755 & 399 & 8.3 & 18.0 & 3.4 & 42 & 659 & .08 & -- \\
\hline & 7-23-92 & 1320 & 430 & 8.2 & 22.0 & 5.0 & 66 & 660 & -- & -- \\
\hline & 7-24-92 & 1100 & 428 & 8.8 & 19.5 & 5.1 & 64 & 663 & -- & -- \\
\hline & 7-26-92 & 1055 & 407 & 8.7 & 21.0 & 6.2 & 80 & 665 & -- & -- \\
\hline & $7-28-92$ & 0710 & 431 & 8.4 & 20.5 & 2.9 & 37 & 663 & -- & -- \\
\hline & 7-29-92 & 0815 & 462 & 8.5 & 21.0 & 4.4 & 57 & 660 & .09 & -- \\
\hline & 7-30-92 & 0945 & 453 & 9.1 & 22.0 & 5.4 & 72 & 660 & .07 & -- \\
\hline & $7-31-92$ & 0915 & 754 & 8.8 & 21.5 & 6.0 & 79 & 663 & -- & -- \\
\hline & 8-02-92 & 0925 & 449 & 8.9 & 22.5 & 4.3 & 58 & 660 & -- & -- \\
\hline & $8-05-92$ & 0740 & 488 & 9.5 & 19.0 & 4.9 & 61 & 659 & .04 & -- \\
\hline & 8-07-92 & 0655 & 512 & 9.4 & 19.0 & 5.0 & 62 & 665 & -- & -- \\
\hline & 8-07-92 & 1215 & 779 & 8.8 & 23.5 & 14.6 & 200 & 659 & -- & -- \\
\hline & 8-09-92 & 1010 & 512 & 9.3 & 20.0 & 6.7 & 85 & 662 & -- & -- \\
\hline & $8-12-92$ & 0840 & 508 & 8.8 & 20.5 & 6.9 & 89 & 661 & .05 & -- \\
\hline & 8-14-92 & 1040 & 515 & -- & 23.0 & 9.4 & 127 & 660 & -- & -- \\
\hline & 8-14-92 & 1725 & 538 & 9.4 & 29.0 & 14.8 & 224 & 660 & -- & -- \\
\hline & $8-16-92$ & 0911 & 549 & 8.5 & 22.0 & .4 & 5 & 660 & -- & -- \\
\hline & 8-19-92 & 0800 & 336 & 8.6 & 21.5 & 6.4 & 85 & 655 & .05 & -- \\
\hline & $8-21-92$ & 0840 & 530 & 9.1 & 19.0 & 7.5 & 95 & 655 & .06 & -- \\
\hline & $8-21-92$ & 1000 & 464 & 8.9 & 19.5 & 6.8 & 87 & 655 & -- & -- \\
\hline & 8-23-92 & 0755 & 554 & 9.4 & 15.0 & 6.3 & 72 & 660 & -- & -- \\
\hline & $8-26-92$ & 0800 & 566 & 9.4 & 16.0 & 10.2 & 119 & 664 & .03 & -- \\
\hline & $8-28-92$ & 1035 & 622 & 9.4 & 19.0 & 11.6 & 144 & 665 & -- & -- \\
\hline & $8-30-92$ & 1010 & 627 & 10.1 & 18.0 & 9.3 & 114 & 660 & -- & -- \\
\hline & $9-08-92$ & 1530 & 947 & 9.1 & 18.5 & 11.2 & 139 & 660 & .05 & -- \\
\hline \multirow[t]{8}{*}{12} & $6-05-91$ & 1040 & 947 & 8.5 & 17.0 & 7.5 & 90 & 660 & -- & -- \\
\hline & 6-07-91 & 1220 & -- & 8.6 & 21.0 & 8.9 & -- & 660 & -- & -- \\
\hline & $6-09-91$ & 1150 & 925 & 8.6 & 21.0 & 6.2 & 81 & 660 & -- & -- \\
\hline & $6-12-91$ & 1114 & 828 & 8.6 & 21.0 & 4.3 & 57 & 650 & -- & -- \\
\hline & $6-14-91$ & 0940 & 750 & 9.0 & 16.0 & 6.7 & 79 & 660 & -- & -- \\
\hline & $6-16-91$ & 1005 & 827 & 8.8 & 16.5 & 5.9 & 70 & 660 & -- & -- \\
\hline & 6-19-91 & 1030 & 847 & 9.2 & 18.5 & 6.3 & 79 & 650 & -- & -- \\
\hline & $6-20-91$ & 1127 & -- & 9.2 & 19.5 & 7.4 & -- & 650 & -- & -- \\
\hline
\end{tabular}


Table 7. Field and laboratory measurements of selected properties and constituents in surface water from primary and additional sampling sites, 1991-92--Continued

\begin{tabular}{|c|c|c|c|c|c|c|c|c|c|c|}
\hline Site & Date & Time & $\begin{array}{c}\text { Specific } \\
\text { conductance } \\
(\mu \mathrm{S} / \mathrm{cm})\end{array}$ & $\begin{array}{c}\mathrm{pH} \\
\text { (standard } \\
\text { units) }\end{array}$ & $\begin{array}{l}\text { Temper- } \\
\text { ature } \\
\left({ }^{\circ} \mathrm{C}\right)\end{array}$ & $\begin{array}{l}\text { Oxygen, } \\
\text { dissolved } \\
\text { (mg/L) }\end{array}$ & $\begin{array}{l}\text { Oxygen, } \\
\text { dissolved } \\
\text { (percent } \\
\text { saturation) }\end{array}$ & $\begin{array}{l}\text { Barometric } \\
\text { pressure } \\
(\mathrm{mm} \text { of } \mathrm{Hg})\end{array}$ & $\begin{array}{c}\text { Nitrogen, } \\
\text { ammonia } \\
(\mathrm{mg} / \mathrm{L} \\
\text { as N) } \\
\text { (lab) }\end{array}$ & $\begin{array}{c}\text { Nitrogen, } \\
\text { ammonia } \\
(\mathrm{mg} / \mathrm{L} \\
\left.\text { as } \mathrm{NH}_{3}\right) \\
\text { (field) }\end{array}$ \\
\hline \multirow[t]{48}{*}{12} & $6-21-91$ & 1055 & 920 & 8.7 & 18.5 & 4.4 & 55 & 650 & -- & -- \\
\hline & $6-23-91$ & 0940 & 1,770 & 8.6 & 18.0 & 5.6 & 69 & 660 & -- & -- \\
\hline & $6-26-91$ & 1004 & 1,850 & 8.5 & 18.0 & 8.6 & 106 & 660 & -- & -- \\
\hline & $6-28-91$ & 1045 & 2,020 & 8.5 & 17.0 & 6.2 & 76 & 650 & -- & -- \\
\hline & $6-30-91$ & 1000 & 2,200 & 8.5 & 17.0 & 6.8 & 83 & 650 & -- & -- \\
\hline & $7-03-91$ & 0935 & 2,440 & 8.9 & 25.0 & 5.3 & 75 & 660 & -- & -- \\
\hline & $7-05-91$ & 1020 & 2,320 & 8.6 & 25.0 & 6.2 & 88 & 660 & -- & -- \\
\hline & $7-07-91$ & 1050 & 2,430 & -- & 25.0 & 5.7 & 81 & 660 & -- & -- \\
\hline & $7-10-91$ & 1023 & 843 & 9.2 & 22.0 & 3.5 & 47 & 660 & -- & -- \\
\hline & $7-12-91$ & 1024 & 820 & 9.2 & 22.5 & 4.2 & 57 & 650 & -- & -- \\
\hline & $7-14-91$ & 0855 & 853 & 8.7 & 23.0 & 2.1 & 28 & 660 & -- & -- \\
\hline & $7-17-91$ & 1029 & 959 & 9.2 & 20.0 & 2.5 & 32 & 660 & -- & 0.58 \\
\hline & $7-19-91$ & 1006 & 920 & 9.1 & 22.0 & 5.5 & 73 & 660 & -- & -- \\
\hline & $7-21-91$ & 1000 & 845 & 9.2 & 22.0 & 6.9 & 93 & 650 & -- & -- \\
\hline & $7-23-91$ & 1020 & 1,010 & 9.2 & 23.0 & 5.6 & 76 & 660 & -- & -- \\
\hline & $7-24-91$ & 1035 & 1,080 & 9.4 & 23.5 & 6.3 & 86 & 660 & -- & $<.1$ \\
\hline & $7-26-91$ & 1020 & 877 & 8.7 & 23.0 & 6.4 & 87 & 660 & -- & -- \\
\hline & $7-28-91$ & 0905 & 779 & 9.3 & 23.0 & 6.4 & 87 & 660 & -- & -- \\
\hline & $7-31-91$ & 0930 & 1,060 & 9.3 & 23.5 & 8.1 & 111 & 660 & -- & .13 \\
\hline & $8-02-91$ & 0905 & 1,150 & 9.3 & 22.0 & 8.8 & 117 & 660 & -- & -- \\
\hline & 8-04-91 & 0855 & 1,090 & 9.4 & 22.5 & 7.2 & 98 & 650 & -- & -- \\
\hline & $8-07-91$ & 1026 & 1,590 & 9.2 & 20.0 & 8.7 & 113 & 650 & -- & $<.1$ \\
\hline & 8-09-91 & 1000 & 1,490 & 8.7 & 20.5 & 8.6 & 113 & 650 & -- & -- \\
\hline & 8-11-91 & 0910 & 1,300 & 9.0 & 20.0 & 6.7 & 86 & 660 & -- & -- \\
\hline & $8-12-91$ & 1340 & 1,290 & 9.0 & 24.0 & 10.6 & 147 & 660 & -- & -- \\
\hline & 8-14-91 & 0935 & 1,130 & 8.2 & 22.0 & 7.4 & 99 & 660 & -- & $<.1$ \\
\hline & $8-16-91$ & 0935 & 1,150 & 8.9 & 21.5 & 5.5 & 73 & 660 & -- & -- \\
\hline & 8-18-91 & 0915 & 1,260 & 8.9 & 22.0 & 6.7 & 89 & 660 & -- & -- \\
\hline & 8-19-91 & 1245 & 1,140 & 8.9 & 25.0 & 9.1 & 128 & 660 & -- & -- \\
\hline & 8-19-91 & 1425 & 1,160 & 8.9 & 27.0 & 8.9 & 130 & 660 & -- & -- \\
\hline & 8-19-91 & 1635 & 1,150 & 8.9 & 27.5 & 9.6 & 142 & 660 & -- & -- \\
\hline & 8-19-91 & 1800 & 1,150 & 8.9 & 27.5 & 12.4 & 183 & 660 & -- & -- \\
\hline & 8-19-91 & 2050 & 1,150 & 8.9 & 26.0 & 12.0 & 172 & 660 & -- & -- \\
\hline & 8-19-91 & 2230 & $1 ., 140$ & 9.0 & 25.5 & 11.1 & 158 & 660 & -- & -- \\
\hline & $8-20-91$ & 0040 & 1,150 & 9.0 & 25.0 & 10.2 & 144 & 660 & -- & -- \\
\hline & $8-20-91$ & 0230 & 1,160 & 9.0 & 24.0 & 9.1 & 126 & 660 & -- & -- \\
\hline & $8-20-91$ & 0425 & 1,180 & 9.0 & 24.0 & 8.2 & 113 & 660 & -- & -- \\
\hline & $8-20-91$ & 0640 & 1,140 & 8.9 & 23.0 & 7.4 & 100 & 660 & -- & -- \\
\hline & $8-20-91$ & 0920 & 1,140 & 8.9 & 22.5 & 7.6 & 102 & 660 & - & -- \\
\hline & $8-20-91$ & 1035 & 1,140 & 8.7 & 23.5 & 8.1 & 111 & 660 & -- & -- \\
\hline & $8-20-91$ & 1250 & 1,140 & 8.8 & 26.0 & 8.8 & 126 & 660 & -- & -- \\
\hline & $8-21-91$ & 0850 & 1,160 & 8.8 & 23.0 & 5.0 & 68 & 660 & -- & $<.1$ \\
\hline & $8-21-91$ & 1435 & 1,270 & 9.0 & 27.0 & 7.8 & 114 & 660 & -- & -- \\
\hline & $8-23-91$ & 1425 & 1,250 & 9.0 & 27.0 & 7.9 & 116 & 660 & -- & -- \\
\hline & $8-25-91$ & 0915 & 1,090 & 8.9 & 21.0 & 6.5 & 85 & 660 & -- & -- \\
\hline & $8-28-91$ & 0955 & 845 & 9.2 & 19.0 & 6.2 & 78 & 660 & -- & $<.1$ \\
\hline & $8-30-91$ & 0905 & 916 & 8.8 & 19.0 & 6.0 & 75 & 660 & -- & -- \\
\hline & 9-01-91 & 0922 & 818 & 9.3 & 19.0 & 6.6 & 83 & 660 & -- & -- \\
\hline
\end{tabular}


Table 7. Field and laboratory measurements of selected properties and constituents in surface water from primary and additional sampling sites, 1991-92--Continued

\begin{tabular}{|c|c|c|c|c|c|c|c|c|c|c|}
\hline Site & Date & Time & $\begin{array}{c}\text { Specific } \\
\text { conductance } \\
(\mu \mathrm{S} / \mathrm{cm})\end{array}$ & $\begin{array}{c}\mathrm{pH} \\
\text { (standard } \\
\text { units) }\end{array}$ & $\begin{array}{c}\text { Temper- } \\
\text { ature } \\
\left({ }^{\circ} \mathrm{C}\right)\end{array}$ & $\begin{array}{l}\text { Oxygen, } \\
\text { dissolved } \\
(\mathrm{mg} / \mathrm{L})\end{array}$ & $\begin{array}{l}\text { Oxygen, } \\
\text { dissolved } \\
\text { (percent } \\
\text { saturation) }\end{array}$ & $\begin{array}{l}\text { Barometric } \\
\text { pressure } \\
(\mathrm{mm} \text { of } \mathrm{Hg})\end{array}$ & $\begin{array}{c}\text { Nitrogen, } \\
\text { ammonia } \\
\text { (mg/L } \\
\text { as } \mathrm{N}) \\
\text { (lab) } \\
\end{array}$ & $\begin{array}{c}\text { Nitrogen, } \\
\text { ammonia } \\
(\mathrm{mg} / \mathrm{L} \\
\left.\text { as } \mathrm{NH}_{3}\right) \\
\text { (field) } \\
\end{array}$ \\
\hline \multirow[t]{48}{*}{12} & 6-03-92 & 0955 & 363 & 8.4 & 23.0 & 3.2 & 43 & 659 & 0.22 & 0.22 \\
\hline & $6-05-92$ & 0840 & 387 & 8.0 & 21.0 & 3.7 & 48 & 660 & -- & -- \\
\hline & $6-07-92$ & 0817 & 370 & 7.3 & 21.0 & 2.4 & 31 & 655 & -- & -- \\
\hline & $6-10-92$ & 0702 & 355 & 8.8 & 20.0 & 6.1 & 78 & 655 & .04 & $<.1$ \\
\hline & $6-12-92$ & 0855 & 317 & 8.2 & 14.0 & 6.2 & 70 & 655 & -- & -- \\
\hline & $6-14-92$ & 0955 & 308 & 8.1 & 15.0 & 4.2 & 49 & 655 & -- & -- \\
\hline & $6-15-92$ & 1040 & 312 & 8.2 & 16.0 & 4.0 & 47 & 657 & -- & -- \\
\hline & $6-17-92$ & 0800 & 276 & 8.5 & 17.0 & 6.2 & 74 & 659 & .36 & .42 \\
\hline & $6-17-92$ & 0805 & -- & -- & -- & -- & -- & -- & .36 & -- \\
\hline & $6-19-92$ & 1030 & 245 & 7.9 & 21.0 & 5.6 & 73 & 659 & -- & -- \\
\hline & $6-24-92$ & 0720 & 246 & 8.5 & 24.0 & 4.6 & 63 & 660 & .20 & .24 \\
\hline & $6-26-92$ & 0910 & 228 & 8.1 & 23.0 & 5.2 & 71 & 657 & -- & -- \\
\hline & $6-26-92$ & 1647 & 211 & 9.1 & 28.0 & 12.4 & 186 & 655 & -- & -- \\
\hline & $6-28-92$ & 1005 & 258 & 8.4 & 20.0 & -- & -- & 650 & -- & -- \\
\hline & $7-01-92$ & 0750 & 218 & 8.5 & 16.0 & 6.0 & 71 & 655 & .17 & .14 \\
\hline & 7-03-92 & 0915 & 201 & 8.8 & 21.5 & 4.8 & 64 & 655 & -- & -- \\
\hline & $7-05-92$ & 1005 & 262 & -- & 19.0 & 6.8 & 86 & 654 & -- & -- \\
\hline & 7-08-92 & 0737 & 228 & 8.9 & 20.5 & 8.5 & 109 & 665 & .04 & -- \\
\hline & $7-10-92$ & 0930 & 323 & 8.3 & 22.0 & 9.8 & 130 & 660 & -- & -- \\
\hline & $7-12-92$ & 0915 & 400 & 8.8 & 21.5 & 8.7 & 115 & 658 & -- & -- \\
\hline & $7-15-92$ & 0800 & 384 & 8.8 & 22.5 & 7.2 & 97 & 660 & .03 & - \\
\hline & $7-17-92$ & 0840 & 284 & 8.5 & 24.5 & 8.3 & 116 & 660 & -- & -- \\
\hline & $7-17-92$ & 1600 & 230 & 8.7 & 24.0 & 10.4 & 143 & 660 & -- & -- \\
\hline & $7-19-92$ & 0845 & 283 & 8.8 & 24.0 & 5.8 & 80 & 660 & -- & - \\
\hline & $7-22-92$ & 0738 & 315 & 8.7 & 20.5 & 6.4 & 83 & 655 & .03 & -- \\
\hline & 7-24-92 & 0800 & 322 & 8.9 & 20.0 & 13.0 & 166 & 660 & -- & -- \\
\hline & $7-24-92$ & 1035 & 268 & 9.2 & 23.0 & 16.0 & 217 & 660 & -- & -- \\
\hline & $7-26-92$ & 0930 & 250 & 9.1 & 20.5 & 7.0 & 90 & 662 & -- & - \\
\hline & $7-27-92$ & 1113 & 275 & 9.4 & 26.0 & 15.2 & 218 & 660 & -- & -- \\
\hline & $7-28-92$ & 1320 & 297 & 9.8 & 24.0 & 16.2 & 223 & 662 & .04 & -- \\
\hline & $7-29-92$ & 0730 & 199 & 9.0 & 19.0 & 5.1 & 64 & 660 & .03 & -- \\
\hline & $7-31-92$ & 0845 & 177 & 8.7 & 22.0 & 3.7 & 49 & 655 & -- & -- \\
\hline & $8-02-92$ & 0955 & 294 & 9.3 & 22.5 & 3.5 & 47 & 665 & -- & -- \\
\hline & $8-05-92$ & 0700 & 421 & 9.2 & 19.0 & 3.2 & 40 & 660 & .06 & -- \\
\hline & $8-07-92$ & 1045 & 229 & 9.5 & 22.5 & 11.8 & 158 & 659 & -- & -- \\
\hline & $8-07-92$ & 1658 & 232 & 10.1 & 25.0 & 14.5 & 203 & 663 & -- & -- \\
\hline & 8-09-92 & 0810 & 424 & 8.8 & 18.5 & 3.1 & 38 & 665 & -- & -- \\
\hline & $8-12-92$ & 0800 & 207 & 8.9 & 23.0 & .7 & 9 & 660 & .26 & -. \\
\hline & $8-14-92$ & 1120 & 200 & 9.5 & 25.0 & 9.0 & 127 & 660 & -- & -- \\
\hline & $8-16-92$ & 0835 & 183 & 8.8 & 21.0 & 5.0 & 65 & 660 & -- & -- \\
\hline & $8-19-92$ & 0825 & 171 & 8.5 & 22.0 & 1.5 & 20 & 655 & .15 & -- \\
\hline & $8-21-92$ & 0845 & 186 & 9.3 & 20.0 & 3.0 & 38 & 660 & -- & -- \\
\hline & $8-23-92$ & 0840 & 150 & 9.5 & 16.0 & 6.7 & 79 & 655 & -- & -- \\
\hline & $8-24-92$ & 1110 & 192 & 9.4 & 18.0 & 9.9 & 121 & 660 & .03 & -- \\
\hline & $8-26-92$ & 0705 & -- & 9.5 & 17.0 & 5.9 & 71 & 660 & .09 & -- \\
\hline & $8-28-92$ & 0915 & 194 & 9.7 & 17.5 & 8.7 & 105 & 660 & -- & -- \\
\hline & $8-30-92$ & 0835 & 196 & 9.4 & 17.0 & 5.8 & 70 & 660 & -- & -- \\
\hline & $9-08-92$ & 1730 & 183 & 10.0 & 20.0 & 17.0 & 217 & 660 & .05 & -- \\
\hline
\end{tabular}


Table 7. Field and laboratory measurements of selected properties and constituents in surface water from primary and additional sampling sites, 1991-92--Continued

\begin{tabular}{|c|c|c|c|c|c|c|c|c|c|c|}
\hline Site & Date & Time & $\begin{array}{c}\text { Specific } \\
\text { conductance } \\
(\mu \mathrm{S} / \mathrm{cm})\end{array}$ & $\begin{array}{c}\mathrm{pH} \\
\text { (standard } \\
\text { units) }\end{array}$ & $\begin{array}{l}\text { Temper- } \\
\text { ature } \\
\left({ }^{\circ} \mathrm{C}\right)\end{array}$ & $\begin{array}{l}\text { Oxygen, } \\
\text { dissolved } \\
\text { (mg/L) }\end{array}$ & $\begin{array}{l}\text { Oxygen, } \\
\text { dissolved } \\
\text { (percent } \\
\text { saturation) }\end{array}$ & $\begin{array}{l}\text { Barometric } \\
\text { pressure } \\
(\mathrm{mm} \text { of } \mathrm{Hg})\end{array}$ & $\begin{array}{c}\text { Nitrogen, } \\
\text { ammonia } \\
\text { (mg/L } \\
\text { as N) } \\
\text { (lab) }\end{array}$ & $\begin{array}{c}\text { Nitrogen, } \\
\text { ammonia } \\
(\mathrm{mg} / \mathrm{L} \\
\left.\text { as } \mathrm{NH}_{3}\right) \\
\text { (field) }\end{array}$ \\
\hline \multirow[t]{49}{*}{13} & $6-05-91$ & 1115 & 658 & 8.3 & 16.0 & 5.5 & 65 & 660 & -- & -- \\
\hline & $6-07-91$ & 1315 & -- & 8.3 & 19.5 & 4.2 & -- & 660 & -- & -- \\
\hline & 6-09-91 & 1230 & 492 & 8.0 & 22.0 & 3.5 & 46 & 660 & -. & -- \\
\hline & $6-12-91$ & 1154 & 455 & 7.8 & 21.0 & 3.0 & 40 & 650 & -- & -- \\
\hline & $6-14-91$ & 1015 & 574 & 8.9 & 15.5 & 4.4 & 51 & 660 & -- & -- \\
\hline & $6-16-91$ & 1040 & 429 & 7.8 & 16.5 & 5.9 & 70 & 660 & -- & -- \\
\hline & $6-19-91$ & 1113 & 391 & 8.1 & 19.0 & 4.3 & 55 & 650 & -- & -- \\
\hline & $6-20-91$ & 1225 & -- & 7.9 & 17.0 & 4.7 & -- & 650 & -- & -- \\
\hline & $6-21-91$ & 1145 & 351 & 7.8 & 18.0 & 4.9 & 61 & 650 & -- & -- \\
\hline & $6-23-91$ & 1045 & 608 & 8.2 & 18.0 & 4.3 & 53 & 660 & -- & -- \\
\hline & $6-26-91$ & 1042 & 965 & 8.3 & 18.0 & 4.6 & 56 & 660 & -- & -- \\
\hline & $6-28-91$ & 1120 & 1,280 & 8.4 & 17.0 & 4.1 & 50 & 650 & -- & -- \\
\hline & $6-30-91$ & 1050 & 1,190 & 8.1 & 18.0 & 4.8 & 60 & 650 & -- & -- \\
\hline & 7-03-91 & 1010 & 969 & 8.6 & 26.0 & 4.0 & 57 & 660 & -- & -- \\
\hline & 7-05-91 & 1110 & 683 & 8.4 & 26.5 & 1.8 & 26 & 660 & -- & -- \\
\hline & 7-07-91 & 1130 & 1,030 & -- & 26.0 & 2.7 & 39 & 660 & -- & -- \\
\hline & $7-10-91$ & 1053 & 714 & 8.4 & 22.0 & 5.3 & 70 & 660 & -- & -- \\
\hline & $7-12-91$ & 1114 & 390 & 8.3 & 23.0 & 3.5 & 48 & 650 & -- & -- \\
\hline & 7-14-91 & 0940 & 617 & 8.4 & 23.0 & 4.9 & 67 & 650 & -- & -- \\
\hline & $7-17-91$ & 1102 & 415 & 8.0 & 19.5 & 2.0 & 25 & 660 & -- & 0.43 \\
\hline & $7-21-91$ & 1031 & 436 & 7.7 & 22.0 & 3.0 & 40 & 650 & -- & -- \\
\hline & $7-21-91$ & 1045 & 471 & 7.7 & 22.0 & 3.0 & 40 & 660 & -- & -- \\
\hline & 7-23-91 & 1130 & 484 & 8.0 & 23.5 & 1.9 & 26 & 660 & -- & -- \\
\hline & $7-24-91$ & 1107 & 574 & 7.9 & 22.0 & 2.1 & 28 & 660 & -- & .96 \\
\hline & $7-26-91$ & 1055 & 376 & 7.7 & 22.0 & 1.6 & 21 & 660 & -- & -- \\
\hline & $7-28-91$ & 0845 & 521 & 8.5 & 21.0 & 5.3 & 69 & 660 & -- & -- \\
\hline & $7-31-91$ & 1000 & 602 & 9.0 & 23.5 & -- & 178 & 660 & -- & .10 \\
\hline & 8-02-91 & 0935 & 353 & 8.4 & 22.0 & 3.5 & 46 & 660 & -- & -- \\
\hline & 8-04-91 & 0920 & 458 & 8.3 & 23.5 & 3.4 & 47 & 650 & -- & -- \\
\hline & 8-07-91 & 1050 & 406 & 8.2 & 19.5 & 3.4 & 43 & 660 & -- & .38 \\
\hline & 8-09-91 & 0901 & 486 & 8.0 & 20.0 & 4.2 & 54 & 650 & -- & -- \\
\hline & $8-11-91$ & 1020 & 302 & 8.1 & 20.0 & 4.3 & 56 & 650 & -- & -- \\
\hline & $8-12-91$ & 1440 & 393 & 8.1 & 23.0 & 5.7 & 77 & 660 & -- & -- \\
\hline & $8-14-91$ & 1008 & 455 & 7.8 & 20.0 & 3.7 & 47 & 660 & -- & .28 \\
\hline & $8-16-91$ & 1010 & 382 & 7.6 & 21.0 & 2.6 & 34 & 660 & -- & -- \\
\hline & $8-18-91$ & 1000 & 323 & 7.6 & 22.0 & 3.0 & 40 & 660 & -- & -- \\
\hline & $8-19-91$ & 1205 & 441 & 8.8 & 25.0 & 11.0 & 155 & 660 & -- & -- \\
\hline & $8-19-91$ & 1400 & 500 & 8.9 & 26.0 & 11.3 & 162 & 660 & -- & -- \\
\hline & 8-19-91 & 1600 & 680 & 8.8 & 27.0 & 11.6 & 170 & 660 & -- & -- \\
\hline & 8-19-91 & 1745 & 493 & 8.6 & 27.0 & 11.5 & 168 & 660 & -- & -- \\
\hline & $8-19-91$ & 2010 & 502 & 8.3 & 25.5 & 9.7 & 138 & 660 & -- & -- \\
\hline & 8-19-91 & 2210 & 491 & 8.4 & 25.0 & 9.5 & 134 & 660 & -- & -- \\
\hline & $8-20-91$ & 0005 & 503 & 8.6 & 25.0 & 11.2 & 158 & 660 & -- & -- \\
\hline & $8-20-91$ & 0200 & 550 & 8.7 & 25.5 & 11.2 & 159 & 660 & -- & -- \\
\hline & $8-20-91$ & 0350 & 498 & 8.8 & 25.0 & 9.4 & 132 & 660 & -- & -- \\
\hline & $8-20-91$ & 0600 & 489 & 8.6 & 23.0 & 7.2 & 98 & 660 & -- & -- \\
\hline & $8-20-91$ & 0840 & 545 & 8.7 & 22.5 & 5.6 & 75 & 660 & -- & -- \\
\hline & $8-20-91$ & 1015 & 542 & 8.6 & 24.0 & 6.4 & 88 & 660 & -- & -- \\
\hline & $8-20-91$ & 1215 & 546 & 8.6 & 24.5 & 7.6 & 106 & 660 & -- & -- \\
\hline
\end{tabular}


Table 7. Field and laboratory measurements of selected properties and constituents in surface water from primary and additional sampling sites, 1991-92--Continued

\begin{tabular}{|c|c|c|c|c|c|c|c|c|c|c|}
\hline Site & Date & Time & $\begin{array}{c}\text { Specific } \\
\text { conductance } \\
(\mu \mathrm{S} / \mathrm{cm})\end{array}$ & $\begin{array}{l}\mathrm{pH} \\
\text { (standard } \\
\text { units) }\end{array}$ & $\begin{array}{c}\text { Temper- } \\
\text { ature } \\
\left({ }^{\circ} \mathrm{C}\right)\end{array}$ & $\begin{array}{l}\text { Oxygen, } \\
\text { dissolved } \\
(\mathrm{mg} / \mathrm{L})\end{array}$ & $\begin{array}{c}\text { Oxygen, } \\
\text { dissolved } \\
\text { (percent } \\
\text { saturation) }\end{array}$ & $\begin{array}{l}\text { Barometric } \\
\text { pressure } \\
(\mathrm{mm} \text { of } \mathrm{Hg})\end{array}$ & $\begin{array}{c}\text { Nitrogen, } \\
\text { ammonia } \\
\text { (mg/L } \\
\text { as N) } \\
\text { (lab) }\end{array}$ & $\begin{array}{c}\text { Nitrogen, } \\
\text { ammonia } \\
(\mathrm{mg} / \mathrm{L} \\
\left.\text { as } \mathrm{NH}_{3}\right) \\
\text { (field) }\end{array}$ \\
\hline \multirow[t]{47}{*}{13} & $8-21-91$ & 0917 & 522 & 8.2 & 22.0 & 3.5 & 47 & 660 & -- & 0.27 \\
\hline & $8-21-91$ & 1400 & 407 & 8.4 & 25.0 & 8.0 & 113 & 660 & -- & -- \\
\hline & $8-23-91$ & 1345 & 324 & 8.2 & 27.0 & 10.2 & 149 & 660 & -- & -- \\
\hline & $8-25-91$ & 0945 & 365 & 7.8 & 21.0 & 3.3 & 43 & 660 & -- & -- \\
\hline & 8-28-91 & 1020 & 302 & 8.6 & 18.5 & 3.0 & 37 & 660 & -- & .11 \\
\hline & 8-30-91 & 0830 & 530 & 7.7 & 18.0 & 3.6 & 44 & 660 & -- & -- \\
\hline & 9-01-91 & 0943 & 365 & 8.2 & 19.0 & 2.9 & 36 & 660 & -- & -- \\
\hline & $6-03-92$ & 0836 & 617 & 8.0 & 23.0 & 2.3 & 31 & 658 & 0.68 & .94 \\
\hline & $6-05-92$ & 0935 & 580 & 7.6 & 18.0 & 2.2 & 27 & 660 & -- & -- \\
\hline & 6-07-92 & 0905 & 441 & 7.0 & 19.5 & 2.0 & 25 & 655 & -- & -- \\
\hline & $6-10-92$ & 0758 & 524 & 7.5 & 17.0 & 1.6 & 19 & 655 & .58 & .68 \\
\hline & $6-10-92$ & 0800 & -- & -- & -- & -- & -- & -- & .57 & -- \\
\hline & $6-12-92$ & 1020 & 427 & 7.5 & 13.0 & 3.8 & 42 & 655 & -- & -- \\
\hline & 6-14-92 & 1110 & 485 & 7.4 & 15.0 & 5.2 & 60 & 656 & -- & -- \\
\hline & $6-15-92$ & 0845 & 429 & 7.7 & 13.5 & 4.1 & 46 & 650 & -- & -- \\
\hline & $6-17-92$ & 0950 & 312 & 8.9 & 18.0 & 4.5 & 55 & 659 & .16 & .45 \\
\hline & $6-19-92$ & 0840 & 451 & 7.4 & 21.0 & 4.5 & 59 & 659 & -- & -- \\
\hline & $6-24-92$ & 0830 & 630 & 8.3 & 22.0 & 4.4 & 58 & 660 & .05 & $<.1$ \\
\hline & $6-26-92$ & 1020 & 681 & 8.3 & 23.0 & 7.2 & 98 & 660 & -- & -- \\
\hline & $6-28-92$ & 1100 & 711 & 8.2 & 19.0 & -- & -- & 650 & -- & -- \\
\hline & $7-01-92$ & 0715 & 719 & 7.8 & 14.5 & 5.4 & 62 & 655 & 1.5 & 1.65 \\
\hline & 7-03-92 & 1015 & 695 & 8.6 & 22.5 & 8.2 & 111 & 655 & -- & -- \\
\hline & 7-05-92 & 1040 & 342 & -- & 20.0 & 3.4 & 44 & 654 & -- & -- \\
\hline & 7-08-92 & 0826 & 651 & 8.6 & 21.5 & 5.7 & 74 & 665 & .22 & -- \\
\hline & $7-10-92$ & 1005 & 659 & 8.1 & 23.0 & 4.4 & 60 & 660 & -- & -- \\
\hline & $7-12-92$ & 1005 & 835 & 8.1 & 22.0 & 4.4 & 59 & 659. & -- & -- \\
\hline & $7-15-92$ & 0855 & 665 & 8.3 & 22.0 & 3.7 & 49 & 660 & .49 & -- \\
\hline & $7-17-92$ & 0750 & 537 & 8.1 & 23.0 & 2.2 & 30 & 660 & -- & -- \\
\hline & 7-19-92 & 0935 & 431 & 7.8 & 23.0 & 3.0 & 41 & 660 & -- & -- \\
\hline & $7-22-92$ & 0820 & 564 & 7.3 & 20.0 & 1.6 & 21 & 655 & .44 & -- \\
\hline & 7-24-92 & 0900 & -- & 7.3 & 19.5 & 3.3 & -- & 660 & -- & -- \\
\hline & 7-24-92 & 0950 & 610 & 7.3 & 19.5 & 3.3 & 42 & 660 & -- & -- \\
\hline & $7-26-92$ & 1010 & 651 & 7.7 & 22.0 & 2.7 & 36 & 662 & -- & -- \\
\hline & $7-27-92$ & 0815 & 690 & 7.8 & 22.5 & 3.6 & 48 & 660 & -- & -- \\
\hline & $7-27-92$ & 1145 & 647 & 8.3 & 28.0 & 7.5 & 111 & 662 & .18 & -- \\
\hline & 7-29-92 & 0815 & 718 & 8.0 & 21.0 & 4.0 & 52 & 660 & .41 & -- \\
\hline & $7-31-92$ & 0752 & -- & 7.6 & 23.0 & 3.1 & -- & 655 & -- & -- \\
\hline & 8-02-92 & 0820 & 701 & 8.3 & 22.0 & 3.2 & 42 & 665 & -- & -- \\
\hline & 8-05-92 & 0755 & 770 & 8.3 & 19.0 & 4.0 & 50 & 660 & .04 & -- \\
\hline & 8-09-92 & 0720 & 679 & 8.5 & 17.5 & 7.4 & 89 & 665 & -- & -- \\
\hline & $8-12-92$ & 0840 & 789 & 8.6 & 24.0 & 8.6 & 119 & 660 & .04 & -- \\
\hline & 8-14-92 & 1046 & 708 & 9.0 & 23.0 & 10.8 & 146 & 660 & -- & -- \\
\hline & 8-16-92 & 0910 & 792 & 8.9 & 20.0 & 7.0 & 89 & 660 & -- & -- \\
\hline & $8-19-92$ & 0726 & 703 & 8.8 & 22.5 & 7.1 & 96 & 655 & .03 & -- \\
\hline & $8-21-92$ & 0804 & 686 & 8.6 & 19.0 & 4.3 & 54 & 660 & -- & -- \\
\hline & $8-21-92$ & 1730 & 672 & 9.1 & 20.0 & 8.3 & 106 & 660 & -- & -- \\
\hline & $8-23-92$ & 0900 & 503 & 9.1 & 17.5 & 3.3 & 40 & 660 & -- & -- \\
\hline
\end{tabular}


Table 7. Field and laboratory measurements of selected properties and constituents in surface water from primary and additional sampling sites, 1991-92--Continued

\begin{tabular}{|c|c|c|c|c|c|c|c|c|c|c|}
\hline Site & Date & Time & $\begin{array}{c}\text { Specific } \\
\text { conductance } \\
(\mu \mathrm{S} / \mathrm{cm})\end{array}$ & $\begin{array}{c}\mathrm{pH} \\
\text { (standard } \\
\text { units) }\end{array}$ & $\begin{array}{c}\text { Temper- } \\
\text { ature } \\
\left({ }^{\circ} \mathrm{C}\right)\end{array}$ & $\begin{array}{l}\text { Oxygen, } \\
\text { dissolved } \\
(\mathrm{mg} / \mathrm{L})\end{array}$ & $\begin{array}{l}\text { Oxygen, } \\
\text { dissolved } \\
\text { (percent } \\
\text { saturation) }\end{array}$ & $\begin{array}{l}\text { Barometric } \\
\text { pressure } \\
(\mathrm{mm} \text { of } \mathrm{Hg})\end{array}$ & $\begin{array}{c}\text { Nitrogen, } \\
\text { ammonia } \\
\text { (mg/L } \\
\text { as N) } \\
\text { (lab) }\end{array}$ & $\begin{array}{c}\text { Nitrogen, } \\
\text { ammonia } \\
(\mathrm{mg} / \mathrm{L} \\
\left.\text { as } \mathrm{NH}_{3}\right) \\
\text { (field) }\end{array}$ \\
\hline \multirow[t]{5}{*}{13} & $8-24-92$ & 0945 & 808 & 8.8 & 16.5 & 2.2 & 26 & 660 & 0.03 & -- \\
\hline & $8-26-92$ & 0755 & 699 & 8.9 & 14.0 & 2.2 & 25 & 660 & .03 & -- \\
\hline & $8-28-92$ & 0955 & 710 & 9.1 & 18.0 & 7.0 & 86 & 660 & -- & -- \\
\hline & 8-30-92 & 0750 & 716 & 9.2 & 17.0 & 7.0 & 84 & 660 & -- & -- \\
\hline & 9-08-92 & 1830 & 676 & 9.1 & 18.0 & 9.6 & 118 & 660 & .04 & -- \\
\hline \multirow[t]{42}{*}{15} & $6-03-92$ & 0640 & 116 & 9.7 & 19.5 & 8.6 & 109 & 656 & .06 & 0.13 \\
\hline & $6-05-92$ & 1205 & 137 & 9.8 & 19.5 & 10.4 & 132 & 660 & -- & -- \\
\hline & $6-07-92$ & 1048 & 121 & 9.8 & 19.0 & 9.0 & 113 & 655 & -- & -- \\
\hline & $6-10-92$ & 0944 & -- & 10.1 & 19.0 & 10.2 & -- & 655 & .05 & $<.1$ \\
\hline & $6-12-92$ & 1110 & 148 & 10.1 & 15.5 & 9.3 & 109 & 655 & -- & -- \\
\hline & 6-14-92 & 1200 & 144 & 10.2 & 14.5 & 10.3 & 117 & 660 & -- & -- \\
\hline & $6-15-92$ & 0700 & -- & 10.0 & 14.0 & 9.3 & -- & 654 & -- & -- \\
\hline & $6-17-92$ & 0640 & 134 & 10.5 & 15.5 & 9.2 & 107 & 657 & .05 & $<.1$ \\
\hline & $6-19-92$ & 0736 & 128 & 9.9 & 18.0 & 13.8 & 170 & 657 & -- & -- \\
\hline & $6-21-92$ & 0730 & 144 & 10.0 & 20.0 & 7.8 & 100 & 658 & -- & -- \\
\hline & $6-24-92$ & 1000 & 123 & 10.0 & 25.0 & 6.6 & 93 & 660 & .07 & $<.1$ \\
\hline & $6-26-92$ & 1109 & 117 & 9.7 & 22.0 & 7.0 & 94 & 655 & -- & -- \\
\hline & $6-28-92$ & 0730 & 111 & 9.5 & 20.5 & 4.4 & 57 & 652 & -- & -- \\
\hline & $7-01-92$ & 0625 & 114 & 9.0 & 16.0 & 4.6 & 54 & 654 & .22 & .24 \\
\hline & 7-03-92 & 1120 & 117 & 8.5 & 18.5 & 8.3 & 103 & 656 & -- & -- \\
\hline & 7-05-92 & 0725 & 128 & -- & 17.5 & 6.5 & 79 & 654 & -- & -- \\
\hline & 7-08-92 & 0640 & 117 & 9.2 & 19.0 & 6.6 & 82 & 660 & .05 & -- \\
\hline & 7-10-92 & 1051 & 121 & 9.3 & 21.0 & 6.2 & 81 & 660 & -- & -- \\
\hline & $7-12-92$ & 1055 & 122 & 9.1 & 19.5 & 6.9 & 87 & 657 & -- & -- \\
\hline & $7-15-92$ & 0630 & 123 & 8.8 & 22.0 & 5.8 & 77 & 660 & .05 & -- \\
\hline & $7-17-92$ & 0650 & 113 & 8.9 & 23.0 & 6.7 & 91 & 657 & -- & -- \\
\hline & 7-19-92 & 1020 & 102 & 9.5 & 23.0 & 7.6 & 103 & 660 & -- & -- \\
\hline & $7-22-92$ & 0620 & 125 & 8.6 & 20.0 & 4.7 & 60 & 655 & .05 & -- \\
\hline & $7-24-92$ & 0830 & 118 & 8.7 & 18.0 & 6.8 & 83 & 660 & -- & -- \\
\hline & $7-24-92$ & 1215 & -- & 8.7 & 20.0 & 9.2 & -- & 660 & -- & -- \\
\hline & $7-26-92$ & 1055 & 119 & 9.0 & 21.5 & 8.7 & 114 & 662 & -- & -- \\
\hline & $7-27-92$ & 0635 & 124 & 8.7 & 22.0 & 7.3 & 97 & 660 & -- & -- \\
\hline & $7-27-92$ & 1015 & 136 & 9.0 & 22.0 & 6.4 & 85 & 662 & .08 & -- \\
\hline & $7-29-92$ & 0625 & 119 & 9.3 & 22.0 & 5.1 & 68 & 660 & .05 & -- \\
\hline & $7-31-92$ & 0638 & 127 & 8.5 & 24.0 & 5.3 & 74 & 655 & -- & -- \\
\hline & 8-02-92 & 0720 & 140 & 9.0 & 22.5 & 4.1 & 55 & 660 & -- & -- \\
\hline & $8-05-92$ & 0840 & 125 & 9.2 & 20.5 & 4.3 & 55 & 660 & .05 & -- \\
\hline & $8-07-92$ & 1315 & 130 & 9.3 & 21.5 & 7.7 & 101 & 659 & -- & -- \\
\hline & 8-09-92 & 0622 & 114 & 8.7 & 19.0 & 5.1 & 63 & 665 & -- & -- \\
\hline & $8-12-92$ & 0625 & 122 & 9.1 & 21.0 & 6.3 & 82 & 660 & .04 & -- \\
\hline & $8-14-92$ & 0730 & 119 & 9.1 & 23.0 & 5.0 & 68 & 660 & -- & -- \\
\hline & $8-14-92$ & 1827 & 133 & 9.3 & 28.0 & 9.0 & 134 & 660 & -- & -- \\
\hline & $8-16-92$ & 0615 & 123 & 8.6 & 23.0 & 3.3 & 45 & 659 & -- & -- \\
\hline & 8-19-92 & 0635 & 120 & 8.6 & 24.0 & 5.7 & 79 & 655 & .05 & -- \\
\hline & $8-21-92$ & 0700 & 132 & 9.2 & 22.0 & 4.6 & 61 & 660 & -- & -- \\
\hline & $8-23-92$ & 0950 & 227 & 9.2 & 17.5 & 5.1 & 62 & 660 & -- & -- \\
\hline & 8-24-92 & 0800 & 135 & 8.3 & 16.0 & 6.2 & 73 & 660 & .01 & -- \\
\hline
\end{tabular}


Table 7. Field and laboratory measurements of selected properties and constituents in surface water from primary and additional sampling sites, 1991-92--Continued

\begin{tabular}{|c|c|c|c|c|c|c|c|c|c|c|}
\hline Site & Date & Time & $\begin{array}{c}\text { Specific } \\
\text { conductance } \\
(\mu \mathrm{S} / \mathrm{cm})\end{array}$ & $\begin{array}{c}\mathrm{pH} \\
\text { (standard } \\
\text { units) }\end{array}$ & $\begin{array}{l}\text { Temper- } \\
\text { ature } \\
\left({ }^{\circ} \mathrm{C}\right)\end{array}$ & $\begin{array}{l}\text { Oxygen, } \\
\text { dissolved } \\
(\mathrm{mg} / \mathrm{L})\end{array}$ & $\begin{array}{l}\text { Oxygen, } \\
\text { dissolved } \\
\text { (percent } \\
\text { saturation) }\end{array}$ & $\begin{array}{l}\text { Barometric } \\
\text { pressure } \\
(\mathrm{mm} \text { of } \mathrm{Hg})\end{array}$ & $\begin{array}{c}\text { Nitrogen, } \\
\text { ammonia } \\
\text { (mg/L } \\
\text { as } \mathrm{N}) \\
\text { (lab) }\end{array}$ & $\begin{array}{c}\text { Nitrogen, } \\
\text { ammonia } \\
(\mathrm{mg} / \mathrm{L} \\
\left.\text { as } \mathrm{NH}_{3}\right) \\
\text { (field) }\end{array}$ \\
\hline \multirow[t]{4}{*}{15} & $8-26-92$ & 0840 & 186 & 9.0 & 16.0 & 7.7 & 90 & 660 & 0.06 & -- \\
\hline & $8-28-92$ & 1035 & 128 & 8.9 & 18.5 & 6.9 & 85 & 660 & -- & -- \\
\hline & $8-30-92$ & 0650 & 128 & 9.0 & 18.0 & 5.5 & 67 & 660 & -- & -- \\
\hline & 9-09-92 & 1300 & 137 & 8.4 & 16.0 & 8.1 & 95 & 659 & .03 & -- \\
\hline \multirow[t]{2}{*}{ LL6 } & $7-10-92$ & 1730 & 1,510 & 8.5 & -- & -- & -- & -- & -- & -- \\
\hline & $7-11-92$ & 1653 & 1,540 & 7.7 & 21.0 & 5.4 & -- & -- & -- & -- \\
\hline \multirow[t]{2}{*}{ LL7 } & $7-10-92$ & 1700 & 1,560 & 8.1 & 24.5 & 11.8 & -- & -- & -- & -- \\
\hline & $7-11-92$ & 1625 & 1,460 & 7.5 & 21.0 & 16.0 & -- & -- & -- & -- \\
\hline \multirow[t]{2}{*}{ LL7A } & $7-06-92$ & 1605 & 1,490 & 8.9 & 21.0 & 10.8 & -- & -- & - & -- \\
\hline & $7-07-92$ & 1835 & 1,970 & 8.1 & 18.5 & 4.4 & -- & -- & -. & -- \\
\hline \multirow[t]{2}{*}{ LL8 } & $7-10-92$ & 1800 & 1,510 & 8.1 & 23.0 & 5.9 & -- & -- & -- & -- \\
\hline & $7-11-92$ & 1720 & 1,660 & 7.2 & 20.5 & 3.9 & -- & -- & -- & -- \\
\hline \multirow[t]{6}{*}{ LL13 } & $7-06-92$ & 1640 & 1,020 & 8.4 & 19.0 & 15.6 & -- & -- & -- & -- \\
\hline & $7-07-92$ & 2004 & 1,380 & 9.0 & 21.0 & 20.0 & -- & -- & -- & -- \\
\hline & 7-08-92 & 1825 & 1,450 & 8.5 & 22.0 & 20.0 & -- & -- & -- & -- \\
\hline & $7-09-92$ & 1855 & 1,460 & 8.8 & 23.5 & 20.0 & -- & -- & -- & -- \\
\hline & $7-10-92$ & 1830 & 1,290 & 8.7 & 22.0 & 20.0 & -- & -- & -- & -- \\
\hline & $7-11-92$ & 1825 & 1,420 & 8.2 & 21.0 & 20.0 & -- & -- & -- & -- \\
\hline \multirow[t]{2}{*}{ LL14 } & $7-06-92$ & 1715 & 1,460 & 7.9 & 20.0 & 10.8 & -- & -- & -- & -- \\
\hline & 7-07-92 & 1915 & 1,940 & 8.5 & 23.0 & 8.5 & -- & -- & -- & -- \\
\hline \multirow[t]{2}{*}{ LL16 } & $8-20-92$ & 1515 & 847 & 9.2 & 25.5 & 11.4 & 162 & 660 & -- & -- \\
\hline & $8-21-92$ & 1525 & 747 & 9.3 & 23.0 & 10.2 & 139 & 655 & .06 & -- \\
\hline \multirow[t]{4}{*}{ LL17 } & $7=08-92$ & 1940 & 720 & 9.5 & 24.0 & 10.8 & -- & -- & -- & -- \\
\hline & $7-09-92$ & 1815 & 711 & 9.6 & 27.5 & 13.8 & -- & -- & -- & -. \\
\hline & $7-10-92$ & 1900 & 741 & 9.5 & 24.0 & 11.8 & -- & -- & -- & -- \\
\hline & $7-11-92$ & 1853 & 746 & 9.1 & 24.0 & 11.3 & -- & -- & -- & -- \\
\hline LL18 & $7-08-92$ & 1915 & 983 & 9.0 & 22.0 & 10.0 & -- & -- & -- & -- \\
\hline LL25 & 7-04-92 & 2028 & 1,034 & 9.5 & 21.0 & 12.3 & -- & -- & -- & -- \\
\hline \multirow[t]{3}{*}{ LL26 } & 7-04-92 & 1947 & 1,040 & 9.5 & 21.5 & 12.1 & -- & -- & -- & -- \\
\hline & $8-20-92$ & 1445 & 1,140 & 8.8 & 25.5 & 13.0 & 185 & 660 & -- & -- \\
\hline & $8-21-92$ & 1500 & 972 & 8.8 & 23.5 & 10.8 & 149 & 655 & .06 & -- \\
\hline LL27 & 7-04-92 & 1738 & 1,580 & 8.1 & 21.0 & -- & -- & -- & -- & -- \\
\hline LL28 & $7-04-92$ & 1830 & 1,620 & 8.4 & 20.0 & 13.9 & -- & -- & -- & -- \\
\hline LL29 & 7-04-92 & 1853 & 1,670 & 8.4 & 20.0 & -- & -- & -- & -- & -- \\
\hline LL30 & 7-04-92 & 1915 & 1,770 & 8.5 & 19.5 & 12.4 & -- & -- & -- & -- \\
\hline
\end{tabular}


Table 7. Field and laboratory measurements of selected properties and constituents in surface water from primary and additional sampling sites, 1991-92--Continued

\begin{tabular}{|c|c|c|c|c|c|c|c|c|c|c|}
\hline Site & Date & Time & $\begin{array}{c}\text { Specific } \\
\text { conductance } \\
(\mu \mathrm{S} / \mathrm{cm})\end{array}$ & $\begin{array}{c}\mathrm{pH} \\
\text { (standard } \\
\text { units) }\end{array}$ & $\begin{array}{c}\text { Temper- } \\
\text { ature } \\
\left({ }^{\circ} \mathrm{C}\right)\end{array}$ & $\begin{array}{l}\text { Oxygen, } \\
\text { dissolved } \\
(\mathrm{mg} / \mathrm{L})\end{array}$ & $\begin{array}{l}\text { Oxygen, } \\
\text { dissolved } \\
\text { (percent } \\
\text { saturation) }\end{array}$ & $\begin{array}{l}\text { Barometric } \\
\text { pressure } \\
(\mathrm{mm} \text { of } \mathrm{Hg})\end{array}$ & $\begin{array}{c}\text { Nitrogen, } \\
\text { ammonia } \\
\text { (mg/L } \\
\text { as N) } \\
\text { (lab) } \\
\end{array}$ & $\begin{array}{c}\text { Nitrogen, } \\
\text { ammonia } \\
(\mathrm{mg} / \mathrm{L} \\
\left.\text { as } \mathrm{NH}_{3}\right) \\
\text { (field) } \\
\end{array}$ \\
\hline \multirow[t]{2}{*}{ LL31 } & $8-20-92$ & 1430 & 1,050 & 8.8 & 25.0 & 9.0 & 127 & 660 & -- & -- \\
\hline & $8-21-92$ & 1445 & 1,010 & 8.9 & 22.5 & 9.7 & 131 & 655 & 0.06 & -- \\
\hline \multirow[t]{2}{*}{ LL32 } & 8-20-92 & 1410 & 1,010 & 9.1 & 25.5 & 12.1 & 172 & 660 & -- & -- \\
\hline & $8-21-92$ & 1420 & 1,020 & 8.8 & 24.5 & 10.8 & 152 & 655 & .06 & -- \\
\hline \multirow[t]{2}{*}{ LL33 } & 7-08-92 & 1713 & 1,860 & 7.8 & 25.0 & 11.0 & -- & -- & -- & -- \\
\hline & $7-11-92$ & 1802 & 1,510 & 7.5 & 23.5 & 5.9 & -- & -- & -- & -- \\
\hline \multirow[t]{2}{*}{ LL34 } & $8-20-92$ & 1815 & 1,080 & 9.0 & 24.0 & 13.6 & 188 & 660 & -- & -- \\
\hline & $8-21-92$ & 1550 & 1,030 & 8.8 & 23.0 & 6.7 & 92 & 655 & .05 & -- \\
\hline LL35 & $7-08-92$ & 1629 & 1,450 & 8.7 & 22.0 & 20.0 & -- & -- & -- & -- \\
\hline LL41 & 7-05-92 & 2000 & 1,330 & 8.8 & 19.0 & -- & -- & -- & -- & -- \\
\hline \multirow[t]{2}{*}{ LL46 } & $8-19-92$ & 1445 & 1,630 & 7.9 & 29.0 & 16.5 & 250 & 661 & $\div-$ & -- \\
\hline & $8-20-92$ & 1517 & 1,650 & 7.9 & 26.0 & 6.0 & 87 & 657 & 16.0 & -- \\
\hline \multirow[t]{2}{*}{ LL56 } & 7-09-92 & 1700 & 563 & 9.2 & 25.5 & 17.8 & -- & -- & -- & -- \\
\hline & $\begin{array}{l}7-10-92 \\
7-11-92\end{array}$ & 1730 & 330 & 9.0 & 25.0 & 8.4 & -- & -- & -- & -- \\
\hline \multirow[t]{6}{*}{ LL57 } & $7-07-92$ & 1630 & 378 & 9.1 & 25.0 & 17.4 & -- & -- & -- & -- \\
\hline & $7-10-92$ & 1700 & 332 & 9.0 & 24.5 & 8.6 & -- & -- & -- & -- \\
\hline & $7-11-92$ & 1710 & 316 & 8.9 & 23.0 & 13.0 & -- & -- & -- & $\cdots$ \\
\hline & 8-19-92 & 1515 & 274 & 8.5 & 26.0 & 16.2 & 232 & 661 & -- & -- \\
\hline & $8-20-92$ & 1546 & 251 & 8.3 & 24.5 & 9.2 & 129 & 657 & .06 & -- \\
\hline & $8-20-92$ & 1551 & 251 & 8.3 & 24.5 & 9.2 & 129 & 657 & .06 & -- \\
\hline \multirow[t]{3}{*}{ LL70 } & 7-09-92 & 1855 & 374 & 8.8 & 26.0 & 5.9 & -- & -- & -- & -- \\
\hline & 7-10-92 & 1840 & 371 & 8.7 & 26.0 & 7.1 & -- & -- & -- & -- \\
\hline & $7-11-92$ & 1850 & 373 & 8.9 & 24.6 & 11.2 & -- & -- & -- & -- \\
\hline \multirow[t]{4}{*}{ LL71 } & 7-08-92 & 2005 & 1,960 & 6.4 & 19.5 & 7.4 & -- & -- & -- & -- \\
\hline & 7-09-92 & 1730 & 2,090 & 7.7 & -- & -- & -- & -- & -- & -- \\
\hline & $7-10-92$ & 1805 & 2,010 & 7.4 & 21.0 & 5.5 & -- & -- & -- & -- \\
\hline & $7-11-92$ & 1830 & 2,050 & 7.5 & 19.5 & 10.8 & -- & -- & -- & -- \\
\hline LL77 & 7-08-92 & 1830 & 1,940 & 7.5 & 23.5 & 2.2 & -- & -- & -- & -- \\
\hline LL78 & $7-08-92$ & 1905 & 1,890 & 7.5 & 25.0 & 2.7 & -- & -- & -- & -- \\
\hline \multirow[t]{2}{*}{ LL79 } & 8-19-92 & 1545 & 1,050 & 8.4 & 26.0 & 19.1 & 274 & 661 & -- & -- \\
\hline & $8-20-92$ & 1615 & 1,390 & 8.2 & 24.5 & 16.8 & 236 & 657 & .10 & -- \\
\hline \multirow[t]{2}{*}{ LL87 } & $7-10-92$ & 1930 & 418 & 8.3 & 25.0 & 9.8 & -- & -- & -- & -- \\
\hline & $7-11-92$ & 2030 & 930 & 8.6 & 22.0 & 7.8 & -- & -- & -- & -- \\
\hline
\end{tabular}


Table 7. Field and laboratory measurements of selected properties and constituents in surface water from primary and additional sampling sites, 1991-92--Continued

\begin{tabular}{|c|c|c|c|c|c|c|c|c|c|c|}
\hline Site & Date & Time & $\begin{array}{c}\text { Specific } \\
\text { conductance } \\
(\mu \mathrm{S} / \mathrm{cm})\end{array}$ & $\begin{array}{c}\mathrm{pH} \\
\text { (standard } \\
\text { units) }\end{array}$ & $\begin{array}{l}\text { Temper- } \\
\text { ature } \\
\left({ }^{\circ} \mathrm{C}\right)\end{array}$ & $\begin{array}{l}\text { Oxygen, } \\
\text { dissolved } \\
(\mathrm{mg} / \mathrm{L})\end{array}$ & $\begin{array}{l}\text { Oxygen, } \\
\text { dissolved } \\
\text { (percent } \\
\text { saturation) }\end{array}$ & $\begin{array}{c}\text { Barometric } \\
\text { pressure } \\
(\mathrm{mm} \text { of } \mathrm{Hg})\end{array}$ & $\begin{array}{c}\text { Nitrogen, } \\
\text { ammonia } \\
(\mathrm{mg} / \mathrm{L} \\
\text { as N) } \\
\text { (lab) }\end{array}$ & $\begin{array}{c}\text { Nitrogen, } \\
\text { ammonia } \\
(\mathrm{mg} / \mathrm{L} \\
\left.\text { as } \mathrm{NH}_{3}\right) \\
\text { (field) }\end{array}$ \\
\hline \multirow{3}{*}{ LL88 } & $7-03-92$ & 1830 & 307 & 7.6 & 23.0 & 7.9 & -- & -- & -- & -- \\
\hline & $7-10-92$ & 1950 & 415 & 8.8 & 24.5 & 6.8 & -- & -- & -- & -- \\
\hline & $7-11-92$ & 2016 & 795 & 8.4 & 22.0 & 7.0 & -- & -- & -- & -- \\
\hline \multirow{3}{*}{ LL89 } & $7-03-92$ & 1916 & 321 & 8.2 & -- & 6.7 & -- & -- & -- & -- \\
\hline & 7-10-92 & 2010 & 415 & 8.6 & 24.5 & 6.4 & -- & -- & -- & -- \\
\hline & $7-11-92$ & 1955 & 803 & 8.4 & 22.0 & 6.5 & -- & -- & -- & -- \\
\hline \multirow[t]{3}{*}{ LL90 } & $8-19-92$ & 1630 & 504 & 9.4 & 28.0 & 12.6 & 187 & 661 & -- & -- \\
\hline & $8-20-92$ & 1655 & 367 & 8.3 & 25.0 & 7.9 & 112 & 657 & 0.02 & -- \\
\hline & $8-22-92$ & 1455 & 398 & 8.6 & 22.0 & 12.0 & 160 & 659 & .06 & -- \\
\hline LL91 & 7-03-92 & 2020 & 311 & 7.9 & 23.0 & 6.0 & -- & -- & -- & -- \\
\hline LL92 & 7-03-92 & 2030 & 307 & 8.1 & 23.0 & 6.0 & -- & -- & -- & -- \\
\hline \multirow[t]{2}{*}{ LL93 } & 7-03-92 & 2100 & 300 & 8.9 & 25.0 & 9.3 & -- & -- & -- & -- \\
\hline & $8-20-92$ & 1635 & 318 & 8.1 & 22.0 & 7.2 & -- & -- & -- & -- \\
\hline \multirow[t]{2}{*}{ LL146 } & $8-19-92$ & 1720 & 283 & 8.1 & 27.0 & 12.2 & 178 & 661 & -- & -- \\
\hline & 8-20-92 & 1738 & 268 & 8.3 & 27.0 & 13.2 & 194 & 657 & .06 & -- \\
\hline \multirow[t]{4}{*}{ LL148 } & $7-04-92$ & 1700 & 274 & 9.0 & 25.0 & 14.4 & -- & -- & -- & -- \\
\hline & 7-05-92 & 1930 & 268 & 8.9 & 22.0 & 9.7 & -- & -- & -- & -- \\
\hline & 7-06-92 & 1825 & 265 & 9.6 & 25.0 & 14.9 & -- & -- & -- & -- \\
\hline & $7-08-92$ & 1850 & 416 & 8.8 & 26.0 & 12.8 & -- & -- & -- & -- \\
\hline \multirow[t]{2}{*}{ LL172 } & $8-19-92$ & 1700 & 320 & 8.4 & 27.0 & 12.0 & 175 & 660 & -- & -- \\
\hline & $8-20-92$ & 1718 & 336 & 8.4 & 27.0 & 12.2 & 179 & 657 & .06 & -- \\
\hline \multirow[t]{2}{*}{ LL173 } & $8-19-92$ & 1742 & 385 & 7.2 & 20.0 & 6.3 & 80 & 661 & -- & -- \\
\hline & $8-20-92$ & 1753 & 392 & 6.9 & 21.0 & 3.4 & 44 & 657 & .09 & -- \\
\hline \multirow[t]{3}{*}{ LL174 } & 7-06-92 & 1740 & 348 & 7.3 & 22.0 & 5.3 & -- & -- & -- & -- \\
\hline & $7-08-92$ & 1745 & 409 & 7.1 & 24.0 & 2.4 & -- & -- & -- & -- \\
\hline & 7-09-92 & 1725 & 381 & 7.5 & 25.0 & 2.6 & -- & -- & -- & -- \\
\hline LL175 & $7-06-92$ & 1705 & 548 & 7.2 & 19.0 & .4 & -- & -- & -- & -- \\
\hline \multirow[t]{2}{*}{ LL176 } & 8-19-92 & 1815 & 322 & 7.7 & 24.0 & 5.3 & 73 & 660 & -- & -- \\
\hline & $8-20-92$ & 1820 & 300 & 7.7 & 24.0 & 4.8 & 67 & 657 & .09 & -- \\
\hline \multirow[t]{2}{*}{ LL201 } & $7-31-92$ & 1640 & 773 & 9.2 & 28.0 & 12.4 & 184 & 662 & -- & -- \\
\hline & 8-01-92 & 1655 & 755 & 9.1 & 27.0 & 15.1 & 221 & 660 & .06 & -- \\
\hline \multirow[t]{4}{*}{ LL205 } & $8-10-92$ & 1817 & 1,050 & -- & 29.0 & 10.0 & 151 & 662 & -- & -- \\
\hline & $8-11-92$ & 1750 & 1,070 & 9.6 & 28.0 & 11.2 & 167 & 659 & .04 & -- \\
\hline & $8-12-92$ & 1711 & 955 & 9.1 & 26.0 & 9.1 & 131 & 659 & -- & -- \\
\hline & $8-14-92$ & 1715 & 888 & 9.4 & 29.0 & 9.2 & 139 & 660 & -- & -- \\
\hline
\end{tabular}


Table 7. Field and laboratory measurements of selected properties and constituents in surface water from primary and additional sampling sites, 1991-92--Continued

\begin{tabular}{|c|c|c|c|c|c|c|c|c|c|c|}
\hline Site & Date & Time & $\begin{array}{c}\text { Specific } \\
\text { conductance } \\
(\mu \mathrm{S} / \mathrm{cm})\end{array}$ & $\begin{array}{l}\mathrm{pH} \\
\text { (standard } \\
\text { units) }\end{array}$ & $\begin{array}{l}\text { Temper- } \\
\text { ature } \\
\left({ }^{\circ} \mathrm{C}\right)\end{array}$ & $\begin{array}{l}\text { Oxygen, } \\
\text { dissolved } \\
(\mathrm{mg} / \mathrm{L})\end{array}$ & $\begin{array}{l}\text { Oxygen, } \\
\text { dissolved } \\
\text { (percent } \\
\text { saturation) }\end{array}$ & $\begin{array}{l}\text { Barometric } \\
\text { pressure } \\
(\mathrm{mm} \text { of } \mathrm{Hg})\end{array}$ & $\begin{array}{l}\text { Nitrogen, } \\
\text { ammonia } \\
(\mathrm{mg} / \mathrm{L} \\
\text { as N) } \\
(1 \mathrm{ab})\end{array}$ & $\begin{array}{l}\text { Nitrogen, } \\
\text { ammonia } \\
(\mathrm{mg} / \mathrm{L} \\
\left.\text { as } \mathrm{NH}_{3}\right) \\
\text { (field) }\end{array}$ \\
\hline \multirow[t]{4}{*}{ LL206 } & $8-10-92$ & 1600 & 1,850 & 7.3 & 27.0 & 0.4 & 6 & 662 & -- & -- \\
\hline & $8-11-92$ & 1630 & 1,950 & 7.5 & 22.0 & .1 & 1 & 659 & 1.40 & -- \\
\hline & $8-12-92$ & 1608 & 1,740 & 7.4 & 23.0 & .6 & 8 & 659 & -- & -. \\
\hline & $8-14-92$ & 1615 & 1,750 & 7.1 & 25.0 & .7 & 10 & 660 & -- & -- \\
\hline \multirow[t]{2}{*}{ LL214 } & $7-31-92$ & 1800 & 816 & 9.2 & 27.0 & 11.8 & 172 & 662 & -- & -- \\
\hline & 8-01-92 & 1800 & 789 & 9.4 & 27.0 & 15.6 & 228 & 660 & -- & -- \\
\hline \multirow[t]{2}{*}{ LL215 } & $7-31-92$ & 1715 & 784 & 9.6 & 28.0 & 12.0 & 179 & 660 & -- & -- \\
\hline & 8-01-92 & 1730 & 790 & 9.4 & 27.0 & 13.6 & 199 & 660 & .03 & -- \\
\hline \multirow[t]{2}{*}{ LL218 } & 7-30-92 & 1655 & 293 & 8.9 & 31.0 & 10.5 & 165 & 660 & -- & -- \\
\hline & $7-31-92$ & 1830 & 271 & 8.5 & 25.0 & 9.6 & 135 & 660 & .03 & -- \\
\hline \multirow[t]{2}{*}{ LL220 } & 8-06-92 & 1800 & 268 & 8.0 & 25.0 & 9.8 & 137 & 665 & -- & -- \\
\hline & $8-07-92$ & 1713 & 256 & 8.8 & 27.0 & 11.8 & 170 & 667 & .02 & -- \\
\hline \multirow[t]{2}{*}{ LL221 } & $7-30-92$ & 1730 & 284 & 8.5 & 29.0 & 12.4 & 198 & 626 & -- & -- \\
\hline & $7-31-92$ & 1800 & 256 & 8.4 & 24.0 & 14.2 & 196 & 660 & .06 & -- \\
\hline \multirow[t]{4}{*}{ LL222 } & $8-06-92$ & 1735 & 609 & 7.8 & 25.0 & 5.7 & 80 & 665 & -- & -- \\
\hline & 8-07-92 & 1640 & 337 & 8.0 & 27.0 & 7.1 & 104 & 660 & .25 & -- \\
\hline & 8-08-92 & 1617 & 388 & 8.7 & 28.0 & 6.5 & 97 & 660 & .13 & -- \\
\hline & $8-08-92$ & 1620 & -- & -- & -- & -- & -- & -- & .13 & -- \\
\hline \multirow{3}{*}{ LL223 } & $8-06-92$ & 1705 & 892 & 7.5 & 20.0 & 3.4 & 43 & 665 & -- & -- \\
\hline & $8-07-92$ & 1545 & 351 & 7.5 & 25.0 & 4.0 & 57 & 657 & .25 & -- \\
\hline & $8-08-92$ & 1710 & 434 & 7.5 & 27.0 & 6.0 & 88 & 660 & .10 & -- \\
\hline \multirow[t]{2}{*}{ LL224 } & $8-09-92$ & 1725 & 314 & 8.8 & 25.0 & 12.2 & 172 & 660 & -- & -- \\
\hline & $8-10-92$ & 1700 & 396 & 8.7 & 27.0 & 14.6 & 213 & 662 & .09 & -- \\
\hline \multirow[t]{2}{*}{ LL225 } & $8-09-92$ & 1806 & 501 & 8.7 & 26.0 & 14.0 & 201 & 660 & -- & -- \\
\hline & $8-10-92$ & 1700 & 521 & -- & 27.0 & 14.4 & 210 & 662 & .05 & -- \\
\hline \multirow[t]{3}{*}{ LL226 } & $7-31-92$ & 2025 & 1,140 & 6.9 & 25.0 & .3 & 4 & 660 & - & -- \\
\hline & $8-01-92$ & 1930 & 1,060 & 7.1 & 21.0 & .7 & 9 & 665 & 1.70 & -- \\
\hline & $8-02-92$ & 1755 & 1,100 & 6.6 & 26.0 & .2 & 3 & 660 & 2.56 & -- \\
\hline \multirow[t]{4}{*}{ LL227 } & $7-31-92$ & 1955 & 932 & 7.8 & 25.0 & 5.3 & 75 & 660 & -- & -- \\
\hline & $8-01-92$ & 1825 & 1,610 & 7.5 & 21.0 & 1.5 & 19 & 665 & .29 & -- \\
\hline & $8-02-92$ & 1840 & 1,670 & 6.8 & 23.0 & 1.3 & 18 & 660 & .22 & -- \\
\hline & $8-07-92$ & 1335 & 1,670 & 6.8 & 23.0 & 1.3 & 18 & 660 & -- & -- \\
\hline \multirow[t]{2}{*}{ LL230 } & $7-30-92$ & 1830 & 753 & 7.4 & 24.0 & 3.7 & 51 & 660 & -- & -- \\
\hline & $7-31-92$ & 1905 & 566 & 8.1 & 23.0 & 1.6 & 22 & 660 & .27 & -- \\
\hline \multirow[t]{2}{*}{ LL232 } & $7-30-92$ & 1755 & 327 & 8.1 & 26.0 & 9.8 & 140 & 660 & -- & -- \\
\hline & $7-31-92$ & 1720 & 302 & 8.9 & 26.0 & 9.8 & 140 & 660 & .05 & -- \\
\hline
\end{tabular}


Table 7. Field and laboratory measurements of selected properties and constituents in surface water from primary and additional sampling sites, 1991-92--Continued

\begin{tabular}{|c|c|c|c|c|c|c|c|c|c|c|}
\hline Site & Date & Time & $\begin{array}{c}\text { Specific } \\
\text { conductance } \\
(\mu \mathrm{S} / \mathrm{cm})\end{array}$ & $\begin{array}{c}\mathrm{pH} \\
\text { (standard } \\
\text { units) }\end{array}$ & $\begin{array}{l}\text { Temper- } \\
\text { ature } \\
\left({ }^{\circ} \mathrm{C}\right)\end{array}$ & $\begin{array}{l}\text { Oxygen, } \\
\text { dissolved } \\
\text { (mg/L) }\end{array}$ & $\begin{array}{l}\text { Oxygen, } \\
\text { dissolved } \\
\text { (percent } \\
\text { saturation) }\end{array}$ & $\begin{array}{c}\text { Barometric } \\
\text { pressure } \\
(\mathrm{mm} \text { of } \mathrm{Hg})\end{array}$ & $\begin{array}{c}\text { Nitrogen, } \\
\text { ammonia } \\
\text { (mg/L } \\
\text { as N) } \\
\text { (lab) }\end{array}$ & $\begin{array}{c}\text { Nitrogen, } \\
\text { ammonia } \\
(\mathrm{mg} / \mathrm{L} \\
\left.\text { as } \mathrm{NH}_{3}\right) \\
\text { (field) }\end{array}$ \\
\hline \multirow[t]{5}{*}{ LL233 } & $8-10-92$ & 1959 & 390 & 8.0 & 23.0 & 7.5 & 102 & 660 & -- & -- \\
\hline & $8-11-92$ & 1715 & 472 & 7.8 & 23.0 & 4.9 & 66 & 659 & 0.12 & -- \\
\hline & $8-12-92$ & 2040 & 594 & 6.9 & 21.0 & .7 & 9 & 660 & -- & -- \\
\hline & $8-13-92$ & 1837 & 572 & 7.0 & 22.0 & 3.2 & 43 & 660 & -- & -- \\
\hline & $8-14-92$ & 1920 & 559 & 7.3 & 24.0 & 2.9 & 40 & 660 & -- & -- \\
\hline \multirow[t]{3}{*}{ LL234 } & $8-10-92$ & 1915 & 284 & 8.0 & 24.0 & 10.1 & 138 & 665 & -- & -- \\
\hline & $8-11-92$ & 1802 & 266 & 8.4 & 25.0 & 10.6 & 150 & 658 & .03 & -- \\
\hline & $8-12-92$ & 2030 & 284 & 8.4 & 25.0 & 9.1 & 128 & 660 & -- & -- \\
\hline \multirow[t]{5}{*}{ LL235 } & $8-10-92$ & 1932 & 300 & 8.1 & 23.0 & 9.3 & 125 & 665 & -- & -- \\
\hline & $8-11-92$ & 1825 & 271 & 8.2 & 25.0 & 10.8 & 152 & 660 & .03 & -- \\
\hline & $8-12-92$ & 2000 & 284 & 8.4 & 25.0 & 9.1 & 128 & 660 & -- & -- \\
\hline & $8-13-92$ & 1815 & 285 & 8.2 & 26.0 & 12.2 & 175 & 660 & -- & -- \\
\hline & $8-14-92$ & 1855 & 284 & 8.4 & 27.0 & 12.6 & 184 & 660 & -- & - \\
\hline LL237 & $7-24-92$ & 1705 & 318 & 8.0 & 23.5 & 10.4 & -- & -- & -- & -- \\
\hline LL238 & $7-24-92$ & 1645 & 486 & 7.0 & 22.5 & .6 & -- & -- & -- & -- \\
\hline \multirow[t]{4}{*}{ LL248 } & $8-10-92$ & 1625 & 2,240 & 7.2 & 25.0 & .8 & 11 & 662 & -- & -- \\
\hline & $8-11-92$ & 1710 & 1,940 & 7.4 & 23.0 & 1.4 & 19 & 659 & .70 & -- \\
\hline & $8-12-92$ & 1642 & 2,020 & 7.0 & 23.0 & .4 & 5 & 659 & -- & -- \\
\hline & $8-14-92$ & 1645 & 2,000 & 7.2 & 24.0 & .7 & 10 & 660 & -- & -- \\
\hline \multirow[t]{2}{*}{ LL251 } & $7-31-92$ & 1910 & 921 & 9.3 & 26.0 & 11.2 & 160 & 662 & -- & -- \\
\hline & $8-01-92$ & 1855 & 788 & 9.2 & 26.0 & 11.1 & 159 & 660 & .02 & -- \\
\hline \multirow[t]{4}{*}{ LL255 } & $8-10-82$ & 1640 & 588 & 6.5 & 23.0 & .8 & 11 & 665 & -- & -- \\
\hline & $8-11-92$ & 1950 & 543 & 6.7 & 20.0 & 1.3 & 17 & 660 & .85 & -- \\
\hline & $8-12-92$ & 1900 & 776 & 6.7 & 22.0 & .6 & 8 & 660 & -- & -- \\
\hline & $8-13-92$ & 1740 & 786 & 7.9 & 21.0 & .7 & 9 & 645 & -- & -- \\
\hline \multirow[t]{5}{*}{ LL259 } & $8-10-92$ & 1810 & 255 & -- & 29.0 & 18.2 & 273 & 665 & -- & -- \\
\hline & $8-11-92$ & 2032 & 242 & 9.2 & 26.0 & 13.2 & 189 & 660 & .05 & -- \\
\hline & $8-12-92$ & 1720 & 250 & 9.3 & 28.0 & 12.0 & 178 & 660 & -- & -- \\
\hline & $8-13-92$ & 1703 & 297 & 8.9 & 29.0 & 14.6 & 221 & 660 & - & -- \\
\hline & $8-14-92$ & 1753 & 328 & 7.8 & 26.5 & 14.6 & 221 & 660 & -- & -- \\
\hline \multirow[t]{4}{*}{ LL260 } & $8-10-92$ & 1620 & 319 & 7.9 & 24.0 & 6.7 & 91 & 668 & -- & -- \\
\hline & $8-11-92$ & 1928 & 264 & 7.3 & 24.0 & 3.7 & 51 & 660 & .14 & -- \\
\hline & $8-13-92$ & 1724 & 320 & 7.1 & 25.0 & 4.3 & 60 & 660 & -- & -- \\
\hline & $8-14-92$ & 1715 & 341 & 7.3 & 27.0 & 5.0 & 73 & 660 & -- & -- \\
\hline LL261 & $7-24-92$ & 1730 & 324 & 8.2 & 25.5 & 14.8 & -- & -- & -- & -- \\
\hline \multirow[t]{5}{*}{ LL262 } & $8-10-92$ & 1755 & 260 & 9.3 & 29.5 & 16.0 & 247 & 665 & -- & -- \\
\hline & $8-11-92$ & 2011 & 293 & 9.0 & 28.0 & 11.2 & 166 & 660 & .03 & -- \\
\hline & $8-12-92$ & 0810 & 274 & 9.3 & 28.0 & 12.4 & 184 & 660 & -- & -- \\
\hline & $8-13-92$ & 1640 & 265 & 9.2 & 31.0 & 14.9 & 234 & 660 & -- & -- \\
\hline & $8-14-92$ & 1811 & 341 & 7.9 & 26.0 & 9.2 & 132 & 660 & -- & -- \\
\hline
\end{tabular}


Table 7. Field and laboratory measurements of selected properties and constituents in surface water from primary and additional sampling sites, 1991-92--Continued

\begin{tabular}{|c|c|c|c|c|c|c|c|c|c|c|}
\hline Site & Date & Time & $\begin{array}{c}\text { Specific } \\
\text { conductance } \\
(\mu \mathrm{S} / \mathrm{cm})\end{array}$ & $\begin{array}{c}\mathrm{pH} \\
\text { (standard } \\
\text { units) }\end{array}$ & $\begin{array}{l}\text { Temper- } \\
\text { ature } \\
\left({ }^{\circ} \mathrm{C}\right)\end{array}$ & $\begin{array}{l}\text { Oxygen, } \\
\text { dissolved } \\
(\mathrm{mg} / \mathrm{L})\end{array}$ & $\begin{array}{l}\text { Oxygen, } \\
\text { dissolved } \\
\text { (percent } \\
\text { saturation) }\end{array}$ & $\begin{array}{l}\text { Barometric } \\
\text { pressure } \\
(\mathrm{mm} \text { of } \mathrm{Hg})\end{array}$ & $\begin{array}{c}\text { Nitrogen, } \\
\text { ammonia } \\
\text { (mg/L } \\
\text { as N) } \\
\text { (lab) }\end{array}$ & $\begin{array}{c}\text { Nitrogen, } \\
\text { ammonia } \\
\text { (mg/L } \\
\left.\text { as } \mathrm{NH}_{3}\right) \\
\text { (field) }\end{array}$ \\
\hline LR5 & $\begin{array}{l}8-21-91 \\
8-21-91 \\
8-21-91 \\
8-21-91 \\
8-21-91 \\
8-21-91 \\
8-22-91 \\
8-22-91 \\
8-22-91 \\
8-22-91 \\
8-22-91 \\
8-22-91 \\
8-22-91\end{array}$ & $\begin{array}{l}1305 \\
1450 \\
1620 \\
1750 \\
2100 \\
2220 \\
0015 \\
0215 \\
0355 \\
0650 \\
0840 \\
1005 \\
1300\end{array}$ & $\begin{array}{l}211 \\
197 \\
195 \\
198 \\
178 \\
182 \\
196 \\
174 \\
183 \\
224 \\
210 \\
221 \\
208\end{array}$ & $\begin{array}{l}8.6 \\
8.8 \\
9.4 \\
8.8 \\
8.6 \\
8.7 \\
8.2 \\
7.9 \\
7.8 \\
7.8 \\
7.7 \\
8.0 \\
8.4\end{array}$ & $\begin{array}{l}23.0 \\
24.0 \\
24.5 \\
24.5 \\
23.5 \\
23.0 \\
23.0 \\
22.0 \\
22.0 \\
21.5 \\
22.0 \\
22.0 \\
22.0\end{array}$ & $\begin{array}{l}7.3 \\
8.8 \\
8.9 \\
9.4 \\
8.2 \\
9.2 \\
5.8 \\
4.7 \\
4.2 \\
3.1 \\
3.7 \\
3.8 \\
7.2\end{array}$ & $\begin{array}{r}99 \\
121 \\
124 \\
131 \\
112 \\
124 \\
78 \\
62 \\
56 \\
41 \\
49 \\
50 \\
96\end{array}$ & $\begin{array}{l}660 \\
660 \\
660 \\
660 \\
660 \\
660 \\
660 \\
660 \\
660 \\
660 \\
660 \\
660 \\
660\end{array}$ & $\begin{array}{l}-- \\
-- \\
-- \\
-- \\
- \\
- \\
-- \\
- \\
- \\
- \\
- \\
-\end{array}$ & $\begin{array}{l}-- \\
-- \\
-- \\
-- \\
-- \\
-- \\
-- \\
-- \\
-- \\
- \\
- \\
-- \\
-\end{array}$ \\
\hline LR6 & $\begin{array}{l}8-21-91 \\
8-21-91 \\
8-21-91 \\
8-21-91 \\
8-21-91 \\
8-21-91 \\
8-22-91 \\
8-22-91 \\
8-22-91 \\
8-22-91 \\
8-22-91 \\
8-22-91 \\
8-22-91\end{array}$ & $\begin{array}{l}1350 \\
1530 \\
1645 \\
1820 \\
2130 \\
2240 \\
0030 \\
0245 \\
0410 \\
0715 \\
0900 \\
1035 \\
1340\end{array}$ & $\begin{array}{l}131 \\
136 \\
143 \\
149 \\
119 \\
135 \\
117 \\
127 \\
118 \\
126 \\
128 \\
128 \\
130\end{array}$ & $\begin{array}{r}9.2 \\
9.5 \\
9.9 \\
10.0 \\
9.5 \\
9.5 \\
9.1 \\
9.3 \\
9.3 \\
8.8 \\
9.2 \\
9.2 \\
9.2\end{array}$ & $\begin{array}{l}27.0 \\
26.0 \\
27.0 \\
27.0 \\
24.5 \\
23.0 \\
23.0 \\
22.5 \\
22.0 \\
22.5 \\
22.0 \\
23.0 \\
24.0\end{array}$ & $\begin{array}{r}13.5 \\
13.2 \\
16.5 \\
19.8 \\
13.2 \\
12.0 \\
9.4 \\
9.4 \\
7.5 \\
6.4 \\
7.1 \\
8.4 \\
9.1\end{array}$ & $\begin{array}{r}197 \\
189 \\
241 \\
289 \\
184 \\
162 \\
127 \\
126 \\
100 \\
86 \\
94 \\
114 \\
126\end{array}$ & $\begin{array}{l}660 \\
660 \\
660 \\
660 \\
660 \\
660 \\
660 \\
660 \\
660 \\
660 \\
660 \\
660 \\
660\end{array}$ & $\begin{array}{l}-- \\
-- \\
-- \\
- \\
-- \\
-- \\
- \\
- \\
- \\
- \\
- \\
- \\
- \\
--\end{array}$ & $\begin{array}{l}-- \\
-- \\
-- \\
-- \\
- \\
-- \\
- \\
-- \\
- \\
- \\
-- \\
-\end{array}$ \\
\hline UTL3 & $\begin{array}{l}7-31-92 \\
8-20-92 \\
9-09-92\end{array}$ & $\begin{array}{l}0845 \\
0705 \\
1030\end{array}$ & $\begin{array}{l}984 \\
921 \\
986\end{array}$ & $\begin{array}{l}9.4 \\
8.8 \\
9.4\end{array}$ & $\begin{array}{l}22.0 \\
20.5 \\
17.0\end{array}$ & $\begin{array}{r}5.2 \\
3.3 \\
10.4\end{array}$ & $\begin{array}{r}69 \\
43 \\
125\end{array}$ & $\begin{array}{l}660 \\
660 \\
661\end{array}$ & $\begin{array}{r}0.03 \\
.04 \\
.05\end{array}$ & $\begin{array}{l}-- \\
-- \\
--\end{array}$ \\
\hline UTL5 & $\begin{array}{l}7-31-92 \\
8-20-92 \\
9-09-92\end{array}$ & $\begin{array}{l}1155 \\
1000 \\
1000\end{array}$ & $\begin{array}{l}401 \\
346 \\
300\end{array}$ & $\begin{array}{l}9.1 \\
8.5 \\
8.4\end{array}$ & $\begin{array}{l}25.0 \\
22.0 \\
18.0\end{array}$ & $\begin{array}{l}7.5 \\
3.0 \\
4.0\end{array}$ & $\begin{array}{r}106 \\
40 \\
49\end{array}$ & $\begin{array}{l}660 \\
660 \\
661\end{array}$ & $\begin{array}{l}.03 \\
.06 \\
.07\end{array}$ & $\begin{array}{l}-- \\
-- \\
--\end{array}$ \\
\hline UTL7 & $\begin{array}{l}7-31-92 \\
8-20-92 \\
9-09-92 \\
9-09-92\end{array}$ & $\begin{array}{l}0910 \\
0735 \\
0750 \\
0900\end{array}$ & $\begin{array}{r}1050 \\
934 \\
836 \\
866\end{array}$ & $\begin{array}{l}9.0 \\
8.9 \\
9.5 \\
9.6\end{array}$ & $\begin{array}{l}23.0 \\
22.0 \\
16.0 \\
16.0\end{array}$ & $\begin{array}{r}6.5 \\
5.8 \\
10.8 \\
11.2\end{array}$ & $\begin{array}{r}88 \\
77 \\
127 \\
132\end{array}$ & $\begin{array}{l}660 \\
660 \\
660 \\
660\end{array}$ & $\begin{array}{l}.02 \\
.04 \\
.06 \\
.04\end{array}$ & $\begin{array}{l}-- \\
-- \\
-- \\
--\end{array}$ \\
\hline $9 A$ & $\begin{array}{l}8-19-91 \\
8-19-91 \\
8-19-91 \\
8-19-91 \\
8-19-91 \\
8-19-91 \\
8-20-91 \\
8-20-91 \\
8-20-91 \\
8-20-91 \\
8-20-91 \\
8-20-91 \\
8-20-91\end{array}$ & $\begin{array}{l}1310 \\
1435 \\
1555 \\
1810 \\
2110 \\
2255 \\
0100 \\
0245 \\
0440 \\
0700 \\
0935 \\
1050 \\
1315\end{array}$ & $\begin{array}{l}952 \\
953 \\
879 \\
958 \\
878 \\
878 \\
883 \\
903 \\
882 \\
875 \\
948 \\
948 \\
950\end{array}$ & $\begin{array}{l}8.6 \\
8.7 \\
8.6 \\
8.6 \\
8.7 \\
8.7 \\
8.7 \\
8.7 \\
8.6 \\
8.5 \\
8.4 \\
8.1 \\
8.5\end{array}$ & $\begin{array}{l}27.0 \\
28.0 \\
28.0 \\
27.5 \\
27.0 \\
26.0 \\
25.5 \\
25.0 \\
24.5 \\
24.0 \\
24.0 \\
24.5 \\
28.5\end{array}$ & $\begin{array}{r}1.5 \\
2.5 \\
2.6 \\
2.7 \\
3.2 \\
2.7 \\
2.1 \\
1.5 \\
1.0 \\
.6 \\
.7 \\
1.0 \\
2.0\end{array}$ & $\begin{array}{r}22 \\
37 \\
39 \\
40 \\
47 \\
39 \\
30 \\
21 \\
14 \\
8 \\
10 \\
14 \\
30\end{array}$ & $\begin{array}{l}660 \\
660 \\
660 \\
660 \\
660 \\
660 \\
660 \\
660 \\
660 \\
660 \\
660 \\
660 \\
660\end{array}$ & $\begin{array}{l}-- \\
-- \\
-- \\
-- \\
-- \\
-- \\
-- \\
-- \\
-- \\
- \\
- \\
-\end{array}$ & $\begin{array}{l}-- \\
-- \\
-- \\
-- \\
-- \\
-- \\
- \\
-- \\
- \\
-- \\
- \\
-\end{array}$ \\
\hline
\end{tabular}


Table 8. Water-quality data from frog call survey sites, 1991-92

[See figures 2 and 3 for site locations. ${ }^{\circ} \mathrm{C}$, degree Celsius; $\mathrm{mg} / \mathrm{L}$, milligram per liter; $\mu \mathrm{S} / \mathrm{cm}$, microsiemen per centimeter at $25^{\circ} \mathrm{C} ;--$, no data]

\begin{tabular}{|c|c|c|c|c|c|c|c|c|c|}
\hline Date & Time & $\mathrm{pH}$ & $\begin{array}{c}\text { Water } \\
\text { tempera- } \\
\text { ture } \\
\left({ }^{\circ} \mathrm{C}\right)\end{array}$ & $\begin{array}{c}\text { Oxygen, } \\
\text { dissolved } \\
\text { (mg/L) }\end{array}$ & Date & Time & $\mathrm{pH}$ & $\begin{array}{c}\text { Water } \\
\text { tempera- } \\
\text { ture } \\
\left({ }^{\circ} \mathrm{C}\right)\end{array}$ & $\begin{array}{c}\text { Oxygen, } \\
\text { dissolved } \\
(\mathrm{mg} / \mathrm{L})\end{array}$ \\
\hline \multicolumn{5}{|c|}{ Indian Tom Lake (1991) } & \multicolumn{5}{|c|}{ Site 29--Continued } \\
\hline \multicolumn{5}{|c|}{ Site T1 } & July 10 & 2215 & 9.0 & 23.0 & 0.2 \\
\hline May 22 & 2055 & 8.9 & 14.5 & 9.6 & \multicolumn{5}{|c|}{ Site 34} \\
\hline May 29 & 2030 & 9.1 & 14.0 & 7.2 & & & & & \\
\hline June 5 & 2330 & 8.9 & 17.0 & 8.4 & May 23 & 0145 & 8.7 & 16.5 & 6.5 \\
\hline June 12 & 2345 & 9.0 & 17.0 & 7.9 & June 5 & 2025 & 8.6 & 14.0 & 6.6 \\
\hline June 19 & 2330 & 9.1 & 15.0 & 8.4 & June 12 & 2325 & 8.5 & 15.0 & 6.5 \\
\hline June 26 & 2025 & 9.3 & 16.0 & 10.2 & June 19 & 2315 & 8.9 & 16.0 & 3.3 \\
\hline July 3 & 2350 & 9.0 & 21.0 & 9.3 & June 26 & 2125 & 9.0 & 15.0 & 6.8 \\
\hline July 10 & 2040 & 9.4 & 21.0 & 11.0 & July 10 & 2320 & 9.5 & 21.0 & 4.0 \\
\hline \multicolumn{5}{|c|}{ Lower Klamath Refuge (1991) } & \multicolumn{5}{|c|}{ Tule Lake (1991) } \\
\hline \multicolumn{5}{|c|}{ Site 2} & \multicolumn{5}{|c|}{ Site 1} \\
\hline May 22 & 2045 & 8.8 & 21.0 & 8.5 & May 21 & 2045 & 9.1 & 18 & 11.7 \\
\hline May 29 & 2300 & 8.7 & 22.0 & 9.0 & May 30 & 0015 & 9.5 & 15.0 & 11.0 \\
\hline June 5 & 2135 & -- & 19.0 & -- & June 5 & 2340 & 8.3 & 17.0 & 8.4 \\
\hline June 12 & 2050 & 8.0 & 23.0 & 5.4 & June 12 & 2315 & 9.0 & 22.0 & 6.7 \\
\hline June 19 & 2300 & 7.4 & 20.0 & 5.4 & June 19 & 2235 & 8.8 & 16.0 & 6.6 \\
\hline June 26 & 2230 & 8.4 & 20.0 & 7.2 & June 26 & 2155 & 8.4 & 18.0 & 8.2 \\
\hline July 3 & 2100 & 8.1 & 28.0 & 8.1 & July 3 & 2045 & 8.7 & 24.0 & 7.4 \\
\hline \multicolumn{5}{|c|}{ Site 22} & \multicolumn{5}{|c|}{ Site 8} \\
\hline May 22 & 2130 & 8.8 & 18.5 & 7.4 & May 21 & 2150 & 8.8 & 17.5 & 7.7 \\
\hline May 29 & 2105 & 9.1 & 15.5 & 7.4 & May 29 & 2035 & 8.6 & 17.5 & 6.5 \\
\hline June 5 & 2105 & 9.0 & 19.0 & 10.0 & June 4 & 0035 & 8.7 & 16.5 & 7.4 \\
\hline June 12 & 2100 & 9.6 & 15.0 & 11.4 & June 13 & 0010 & 8.5 & 22.0 & 4.5 \\
\hline June 19 & 2050 & 9.9 & 15.0 & 11.2 & June 19 & 2325 & 8.6 & 16.0 & 6.4 \\
\hline June 26 & 2215 & 10.1 & 17.0 & 13.4 & June 26 & 2245 & 8.6 & 17.0 & 6.3 \\
\hline July 3 & 2325 & 10.1 & 24.0 & 5.6 & July 3 & 2145 & 8.8 & 25.0 & 6.8 \\
\hline July 10 & 2240 & 10.0 & 23.0 & 10.2 & July 10 & 2105 & 9.2 & 22.0 & 12.2 \\
\hline \multicolumn{5}{|c|}{ Site 28} & \multicolumn{5}{|c|}{ Site 13} \\
\hline May 22 & 2330 & 7.9 & 17.0 & 7.0 & May 21 & 2240 & 9.1 & 16.5 & 11.1 \\
\hline May 29 & 2200 & 8.1 & 20.0 & 2.2 & May 29 & 2100 & 9.3 & 16.0 & 11.0 \\
\hline June 5 & 2240 & 7.9 & 21.0 & 1.4 & June 5 & 2045 & 8.6 & 20.0 & 9.0 \\
\hline June 12 & 2225 & 8.0 & 15.0 & .7 & June 13 & 0100 & 9.5 & 23.0 & 7.5 \\
\hline June 19 & 2150 & 8.2 & 16.0 & 1.5 & June 20 & 0005 & 10.0 & 17.0 & 14.2 \\
\hline June 27 & 0020 & 8.2 & 19.0 & .7 & June 26 & 2325 & 9.8 & 17.0 & 11.2 \\
\hline July 10 & 2200 & 8.5 & 25.0 & 1.0 & July 4 & 2230 & 10.3 & 25.0 & 12.8 \\
\hline \multicolumn{5}{|c|}{ Site 29} & July 10 & 2200 & 10.3 & 23.0 & 10.6 \\
\hline & & & & & \multicolumn{5}{|c|}{ Site 23} \\
\hline $\begin{array}{l}\text { May } 22 \\
\text { May } 29\end{array}$ & $\begin{array}{l}2345 \\
2215\end{array}$ & $\begin{array}{l}8.3 \\
9.0\end{array}$ & $\begin{array}{l}18.5 \\
17.0\end{array}$ & $\begin{array}{l}5.3 \\
8.5\end{array}$ & May 22 & 0010 & 9.1 & 135 & 10.2 \\
\hline June 5 & 2255 & 9.0 & 19.0 & 7.4 & May 29 & 2240 & 9.3 & 16.0 & 11.3 \\
\hline June 12 & 2240 & 8.1 & 15.0 & .2 & June 5 & 2210 & 8.8 & 19.0 & 8.2 \\
\hline June 19 & 2205 & 9.2 & 16.0 & 2.5 & June 12 & 2200 & 9.6 & 23.0 & 8.6 \\
\hline
\end{tabular}


Table 8. Water-quality data from frog call survey sites, 1991-92--Continued

\begin{tabular}{|c|c|c|c|c|c|}
\hline Date & Tim & & $\mathrm{pH}$ & $\begin{array}{l}\text { Water } \\
\text { tempera- } \\
\text { ture } \\
\left({ }^{\circ} \mathrm{C}\right)\end{array}$ & $\begin{array}{c}\text { Oxygen, } \\
\text { dissolved } \\
(\mathrm{mg} / \mathrm{L})\end{array}$ \\
\hline \multicolumn{6}{|c|}{ Site 23--Continued } \\
\hline June 19 & 2100 & & 10.4 & 16.0 & 16.2 \\
\hline June 27 & 0040 & & 10.0 & 18.0 & 12.2 \\
\hline July 3 & 2340 & & 9.9 & 22.0 & 15.6 \\
\hline July 10 & 2250 & & 9.5 & 20.0 & 8.7 \\
\hline \multicolumn{6}{|c|}{ Site 28} \\
\hline May 22 & 0055 & & 9.3 & 17.0 & 8.6 \\
\hline June 5 & 2250 & & 8.7 & 19.0 & 7.2 \\
\hline June 12 & 2235 & & 9.0 & 24.0 & 1.0 \\
\hline June 19 & 2150 & & 9.9 & 16.5 & 1.0 \\
\hline June 26 & 2100 & & 7.9 & 17.0 & .3 \\
\hline July 4 & 0020 & & 9.2 & 20.0 & .4 \\
\hline July 10 & 2335 & & 9.2 & 20.0 & .3 \\
\hline Date & Time & $\mathrm{pH}$ & $\begin{array}{c}\text { Water } \\
\text { tempera- } \\
\text { ture } \\
\left({ }^{\circ} \mathrm{C}\right)\end{array}$ & $\begin{array}{l}\text { Oxygen, } \\
\text { dissolved } \\
(\mathrm{mg} / \mathrm{L})\end{array}$ & $\begin{array}{l}\text { Specific } \\
\text { conduct- } \\
\text { ance } \\
(\mu \mathrm{S} / \mathrm{cm})\end{array}$ \\
\hline \multicolumn{6}{|c|}{ Indian Tom Lake (1992) } \\
\hline \multicolumn{6}{|c|}{ Site T1 } \\
\hline May 17 & 2025 & 8.4 & 15.0 & 7.7 & -- \\
\hline May 24 & 2330 & 8.9 & 16.0 & 6.7 & -- \\
\hline May 30 & 2330 & 9.3 & 13.0 & -- & 1,450 \\
\hline June 6 & 2045 & 9.2 & 16.0 & 9.6 & 1,450 \\
\hline June 14 & 0005 & 9.6 & 14.0 & 7.3 & 1,500 \\
\hline June 20 & 2015 & 8.9 & 26.0 & 7.8 & 1,360 \\
\hline June 27 & 2315 & 9.2 & 15.0 & 8.2 & 1,530 \\
\hline \multicolumn{6}{|c|}{ Lower Klamath Refuge (1992) } \\
\hline \multicolumn{6}{|c|}{ Site 2} \\
\hline May 17 & 2100 & 8.8 & 16.0 & 7.7 & -- \\
\hline May 24 & 2230 & 9.1 & 19.0 & 7.3 & 215 \\
\hline May 30 & 2140 & 9.9 & 22.0 & -- & 178 \\
\hline June 6 & 2115 & 9.8 & 22.5 & 12.2 & 172 \\
\hline June 13 & 2320 & 9.1 & 17.0 & 6.4 & 159 \\
\hline June 20 & 2150 & 8.5 & 23.0 & 6.8 & 175 \\
\hline June 27 & 2100 & 9.1 & 24.5 & 10.0 & 160 \\
\hline July 4 & 2215 & 8.9 & 26.0 & 15.2 & 154 \\
\hline
\end{tabular}

Lower Klamath Refuge (1992)

Site 2

\begin{tabular}{|c|c|c|c|c|c|}
\hline Date & Tim & & $\mathrm{pH}$ & $\begin{array}{l}\text { Water } \\
\text { tempera- } \\
\text { ture } \\
\left({ }^{\circ} \mathrm{C}\right)\end{array}$ & $\begin{array}{c}\text { Oxygen, } \\
\text { dissolved } \\
(\mathrm{mg} / \mathrm{L})\end{array}$ \\
\hline \multicolumn{6}{|c|}{ Site 23--Continued } \\
\hline June 19 & 2100 & & 10.4 & 16.0 & 16.2 \\
\hline June 27 & 0040 & & 10.0 & 18.0 & 12.2 \\
\hline July 3 & 2340 & & 9.9 & 22.0 & 15.6 \\
\hline July 10 & 2250 & & 9.5 & 20.0 & 8.7 \\
\hline \multicolumn{6}{|c|}{ Site 28} \\
\hline May 22 & 0055 & & 9.3 & 17.0 & 8.6 \\
\hline June 5 & 2250 & & 8.7 & 19.0 & 7.2 \\
\hline June 12 & 2235 & & 9.0 & 24.0 & 1.0 \\
\hline June 19 & 2150 & & 9.9 & 16.5 & 1.0 \\
\hline June 26 & 2100 & & 7.9 & 17.0 & .3 \\
\hline July 4 & 0020 & & 9.2 & 20.0 & .4 \\
\hline July 10 & 2335 & & 9.2 & 20.0 & .3 \\
\hline Date & Time & $\mathrm{pH}$ & $\begin{array}{c}\text { Water } \\
\text { tempera- } \\
\text { ture } \\
\left({ }^{\circ} \mathrm{C}\right)\end{array}$ & $\begin{array}{l}\text { Oxygen, } \\
\text { dissolved } \\
(\mathrm{mg} / \mathrm{L})\end{array}$ & $\begin{array}{l}\text { Specific } \\
\text { conduct- } \\
\text { ance } \\
(\mu \mathrm{S} / \mathrm{cm})\end{array}$ \\
\hline \multicolumn{6}{|c|}{ Indian Tom Lake (1992) } \\
\hline \multicolumn{6}{|c|}{ Site T1 } \\
\hline May 17 & 2025 & 8.4 & 15.0 & 7.7 & -- \\
\hline May 24 & 2330 & 8.9 & 16.0 & 6.7 & -- \\
\hline May 30 & 2330 & 9.3 & 13.0 & -- & 1,450 \\
\hline June 6 & 2045 & 9.2 & 16.0 & 9.6 & 1,450 \\
\hline June 14 & 0005 & 9.6 & 14.0 & 7.3 & 1,500 \\
\hline June 20 & 2015 & 8.9 & 26.0 & 7.8 & 1,360 \\
\hline June 27 & 2315 & 9.2 & 15.0 & 8.2 & 1,530 \\
\hline \multicolumn{6}{|c|}{ Lower Klamath Refuge (1992) } \\
\hline \multicolumn{6}{|c|}{ Site 2} \\
\hline May 17 & 2100 & 8.8 & 16.0 & 7.7 & -- \\
\hline May 24 & 2230 & 9.1 & 19.0 & 7.3 & 215 \\
\hline May 30 & 2140 & 9.9 & 22.0 & -- & 178 \\
\hline June 6 & 2115 & 9.8 & 22.5 & 12.2 & 172 \\
\hline June 13 & 2320 & 9.1 & 17.0 & 6.4 & 159 \\
\hline June 20 & 2150 & 8.5 & 23.0 & 6.8 & 175 \\
\hline June 27 & 2100 & 9.1 & 24.5 & 10.0 & 160 \\
\hline July 4 & 2215 & 8.9 & 26.0 & 15.2 & 154 \\
\hline
\end{tabular}

Site 15

\begin{tabular}{lllllr}
\hline May 17 & 2300 & 8.0 & 16.0 & 7.2 & -- \\
May 24 & 2130 & 8.2 & 20.0 & 4.5 & 730 \\
May 30 & 2045 & 8.8 & 23.0 & -- & 1,350 \\
June 6 & 2325 & 8.2 & 18.0 & 4.9 & 507 \\
June 13 & 2245 & 8.3 & 16.0 & 6.2 & 364
\end{tabular}

Site 13

\begin{tabular}{|c|c|c|c|c|c|}
\hline & & & $\left({ }^{\circ} \mathrm{C}\right)$ & & $(\mu \mathrm{S} / \mathrm{cm})$ \\
\hline \multicolumn{6}{|c|}{ Site 15--Continued } \\
\hline June 20 & 2125 & 8.0 & 23.0 & 3.0 & 330 \\
\hline June 27 & 2315 & 8.8 & 19.0 & 5.8 & 283 \\
\hline July 4 & 2130 & 8.0 & 23.0 & 5.5 & 250 \\
\hline \multicolumn{6}{|c|}{ Site 21} \\
\hline May 17 & 2055 & 8.9 & 16.0 & 9.5 & -. \\
\hline May 24 & 2315 & 9.2 & 19.0 & 1.0 & 660 \\
\hline May 30 & 2130 & 7.8 & 19.0 & 3.6 & -- \\
\hline June 6 & 2105 & 9.8 & 19.0 & 12.0 & 824 \\
\hline \multicolumn{6}{|c|}{ Site 29} \\
\hline May 17 & 2210 & 8.8 & 16.0 & 8.8 & -- \\
\hline May 24 & 2110 & 9.5 & 22.0 & -- & 1,307 \\
\hline May 30 & 2235 & 9.8 & 16.0 & 7.1 & -- \\
\hline June 6 & 2200 & 9.4 & 12.0 & 6.6 & 1,120 \\
\hline June 13 & 2125 & 8.9 & 21.0 & 5.1 & 1,390 \\
\hline June 20 & 2300 & 8.7 & 22.0 & .6 & 1,480 \\
\hline July 4 & 2210 & -- & 10.0 & 4.9 & 1,530 \\
\hline \multicolumn{6}{|c|}{ Tule Lake (1992) } \\
\hline \multicolumn{6}{|c|}{ Site 1} \\
\hline May 17 & 2045 & 9.2 & 23.0 & 11.9 & 230 \\
\hline May 25 & 0020 & 8.8 & 20.0 & 5.5 & 218 \\
\hline May 30 & 2345 & 8.9 & 20.0 & 6.2 & 290 \\
\hline June 6 & 2320 & 8.8 & 17.0 & 3.2 & 258 \\
\hline June 13 & 2305 & 7.6 & 15.0 & 5.8 & 232 \\
\hline June 20 & 2200 & 8.0 & 26.0 & 4.1 & -- \\
\hline June 27 & 2100 & 7.5 & 17.0 & -- & 250 \\
\hline July 5 & 0040 & 7.4 & 19.0 & 3.3 & 262 \\
\hline \multicolumn{6}{|c|}{ Site 8} \\
\hline May 17 & 2055 & 8.2 & 22.0 & 5.8 & 1,000 \\
\hline May 24 & 2035 & 8.1 & 25.0 & 3.8 & 1,080 \\
\hline June 1 & 0050 & 7.9 & 17.0 & 7.3 & 1,080 \\
\hline June 13 & 2305 & 8.8 & 13.0 & 8.5 & 993 \\
\hline June 20 & 2250 & 7.2 & 22.0 & 2.6 & -- \\
\hline June 27 & 2210 & 8.2 & 15.0 & -- & 725 \\
\hline July 4 & 2055 & 9.0 & 18.0 & 12.9 & -- \\
\hline \multicolumn{6}{|c|}{ Site 13} \\
\hline
\end{tabular}

\begin{tabular}{|c|c|c|c|c|c|}
\hline Date & Time & $\mathrm{pH}$ & $\begin{array}{l}\text { Water } \\
\text { tempera- } \\
\text { ture } \\
\end{array}$ & $\begin{array}{l}\text { Oxygen, } \\
\text { dissolved } \\
(\mathrm{mg} / \mathrm{L})\end{array}$ & $\begin{array}{l}\text { Specific } \\
\text { conduct- } \\
\text { ance } \\
(u S / \mathrm{cm})\end{array}$ \\
\hline
\end{tabular}

Site 15--Continued

Tule Lake (1992)

$\begin{array}{lrrrrr}\text { May 17 } & 2125 & 9.2 & 22.0 & 12.0 & 901 \\ \text { May 24 } & 2105 & 9.2 & 23.0 & 8.4 & 950 \\ \text { May 30 } & 2030 & 10.3 & 23.0 & 9.2 & 813 \\ \text { June 7 } & 0045 & 10.1 & 21.0 & 9.1 & 798 \\ \text { June 13 } & 2325 & 9.4 & 14.0 & 8.0 & 714\end{array}$


Table 8. Water-quality data from frog call survey sites, 1991-92--Continued

\begin{tabular}{|c|c|c|c|c|c|c|c|c|c|c|c|}
\hline Date & Time & $\mathrm{pH}$ & $\begin{array}{c}\text { Water } \\
\text { tempera- } \\
\text { ture } \\
\left({ }^{\circ} \mathrm{C}\right)\end{array}$ & $\begin{array}{c}\text { Oxygen, } \\
\text { dissolved } \\
(\mathrm{mg} / \mathrm{L})\end{array}$ & $\begin{array}{l}\text { Specific } \\
\text { conduct- } \\
\text { ance } \\
(\mu \mathrm{S} / \mathrm{cm})\end{array}$ & Date & Time & $\mathrm{pH}$ & $\begin{array}{c}\text { Water } \\
\text { tempera- } \\
\text { ture } \\
\left({ }^{\circ} \mathrm{C}\right)\end{array}$ & $\begin{array}{c}\text { Oxygen, } \\
\text { dissolved } \\
(\mathrm{mg} / \mathrm{L})\end{array}$ & $\begin{array}{c}\text { Specific } \\
\text { conduct- } \\
\text { ance } \\
(\mu \mathrm{S} / \mathrm{cm})\end{array}$ \\
\hline \multicolumn{6}{|c|}{ Site 13--Continued } & \multicolumn{6}{|c|}{ Site 23--Continued } \\
\hline June 20 & 2315 & 9.6 & 25.0 & 6.5 & -- & June 28 & 0015 & 8.4 & 15.0 & -- & 620 \\
\hline June 27 & 2240 & 9.4 & 15.0 &.- & 690 & July 4 & 2320 & 8.3 & 17.0 & 0.6 & 800 \\
\hline \multirow[t]{3}{*}{ July 4} & 2150 & 8.9 & 19.0 & 8.4 & 872 & \multirow{2}{*}{\multicolumn{6}{|c|}{ Site 28}} \\
\hline & \multirow{2}{*}{\multicolumn{5}{|c|}{ Site 23}} & & & & & & \\
\hline & & & & & & May 18 & 0025 & 9.1 & 17.0 & 7.1 & 426 \\
\hline May 17 & 2310 & 8.9 & 17.0 & 9.2 & 932 & May 24 & 2340 & 9.2 & 20.0 & 4.4 & 410 \\
\hline May 24 & 2240 & 9.1 & 21.0 & 3.5 & 915 & May 30 & 2315 & 9.1 & 20.0 & 6.7 & 325 \\
\hline May 30 & 2220 & 10.1 & 20.0 & 13.2 & 820 & June 6 & 2240 & 7.9 & 18.0 & 3.4 & 298 \\
\hline June 6 & 2140 & 10.3 & 21.0 & 9.1 & 779 & June 20 & 2100 & 8.0 & 24.0 & 4.1 & -- \\
\hline June 13 & 2045 & 9.4 & 14.0 & 8.0 & 714 & June 28 & 0115 & 8.0 & 14.5 & -- & 312 \\
\hline June 21 & 0100 & 9.5 & - & 8.9 & - & July 5 & 0105 & 7.3 & 17.0 & 2.5 & 383 \\
\hline
\end{tabular}

Table 9. Concentrations of arsenic and mercury in surface water from selected sites in Lower Klamath National Wildlife Refuge, February 28, 1991

[See figure 1 for site locations. Concentrations in micrograms per liter. <, actual value is less than value shown]

\begin{tabular}{lccccc}
\hline Site & Time & $\begin{array}{c}\text { Arsenic, } \\
\text { dis- } \\
\text { solved }\end{array}$ & $\begin{array}{c}\text { Arsenic, } \\
\text { total }\end{array}$ & $\begin{array}{c}\text { Mercury, } \\
\text { dis- } \\
\text { solved }\end{array}$ & $\begin{array}{c}\text { Mercury, } \\
\text { total } \\
\text { recov- } \\
\text { erable }\end{array}$ \\
\hline LK1 & 1440 & 22 & 27 & $<0.1$ & $<0.10$ \\
LK2 & 1000 & 19 & 20 & $<.1$ & $<.10$ \\
LK3 & 1405 & 24 & 28 & $<.1$ & $<.10$ \\
LK4 & 1615 & 66 & 78 & $<.1$ & $<.10$ \\
LK5 & 1120 & 9 & 9 & $<.1$ & $<.10$ \\
12 & 1530 & 20 & 22 & $<.1$ & $<.10$ \\
P1 & 1150 & 10 & 10 & $<.1$ & $<.10$ \\
\hline
\end{tabular}


Table 10. Concentrations of total and dissolved nitrogen, phosphorus, and organic carbon in surface water, 1991-92

[See figure 1 for site locations. Concentrations in milligrams per liter. <, actual value is less than value shown; >, actual value is greater than value shown; --, no data]

\begin{tabular}{|c|c|c|c|c|c|c|c|c|}
\hline Site & Date & Time & $\begin{array}{c}\text { Nitrogen, } \\
\text { nitrite, } \\
\text { total }\end{array}$ & $\begin{array}{l}\text { Nitrogen } \\
\text { nitrite, } \\
\text { dis- } \\
\text { solved }\end{array}$ & $\begin{array}{l}\text { Nitrogen, } \\
\mathrm{NO}_{2}+\mathrm{NO}_{3} \\
\quad \text { total }\end{array}$ & $\begin{array}{c}\text { Nitrogen, } \\
\mathrm{NO}_{2}+\mathrm{NO}_{3}, \\
\text { dis- } \\
\text { solved }\end{array}$ & $\begin{array}{l}\text { Nitrogen, } \\
\text { ammonia, } \\
\text { total }\end{array}$ & $\begin{array}{l}\text { Nitrogen, } \\
\text { ammonia, } \\
\text { dis- } \\
\text { solved }\end{array}$ \\
\hline \multirow[t]{11}{*}{1} & $8-21-91$ & 1420 & -- & 0.030 & -- & 0.190 & -- & 0.070 \\
\hline & $8-21-91$ & 1720 & .. & .030 & -- & .210 & --- & .080 \\
\hline & $8-21-91$ & 2200 & -. & .030 & -- & .230 & -- & .070 \\
\hline & $8-22-91$ & 0158 & -. & .030 & -. & .230 & -- & .120 \\
\hline & $8-22-91$ & 0615 & -. & .030 & -- & .220 & -- & .110 \\
\hline & $8-22-91$ & 0940 & -- & .030 & -- & .250 & -- & .100 \\
\hline & $7-24-92$ & 1300 & -- & -- & -- & -- & -. & -- \\
\hline & $7-28-92$ & 1550 & 0.090 & .090 & 0.270 & .320 & 0.410 & .430 \\
\hline & $7-28-92$ & 1555 & .090 & .090 & .270 & .320 & .410 & .420 \\
\hline & $8-24-92$ & 1445 & .050 & .040 & .290 & .260 & .180 & .190 \\
\hline & $8-24-92$ & 1450 & -- & -- & -- & -- & -. & -- \\
\hline \multirow[t]{5}{*}{2} & $8-05-91$ & 1340 & -- & .010 & -- & .084 & -- & .100 \\
\hline & $7-24-92$ & 1200 & -- & -- & -. & -. & -- & -- \\
\hline & $7-28-92$ & 1520 & .070 & .070 & .130 & .170 & .280 & .270 \\
\hline & $8-24-92$ & 1250 & .070 & .060 & .260 & .240 & .180 & .160 \\
\hline & $9-08-92$ & 1055 & .030 & .030 & .140 & .150 & .150 & .110 \\
\hline \multirow[t]{5}{*}{3} & $8-05-91$ & 1220 & $\ldots$ & .050 & -. & .220 & -. & .160 \\
\hline & $7-23-92$ & 1000 & -- & .- & .- & .. & -. & .- \\
\hline & $7-30-92$ & 0530 & .060 & .060 & .180 & .200 & .170 & .170 \\
\hline & $8-24-92$ & 1335 & .070 & .060 & .230 & .200 & .160 & .180 \\
\hline & $9-08-92$ & 1150 & .020 & .020 & .250 & .250 & .070 & .070 \\
\hline \multirow[t]{3}{*}{4} & $7-31-92$ & 1105 & .050 & .050 & .150 & .160 & .170 & .160 \\
\hline & $8-20-92$ & 0900 & .070 & .060 & .190 & .180 & .256 & .230 \\
\hline & $9-09-92$ & 0940 & .020 & .020 & .170 & .170 & .050 & .040 \\
\hline \multirow[t]{5}{*}{5} & $8-05-91$ & 1300 & -- & .060 & -- & 1.30 & -- & .070 \\
\hline & $7-23-92$ & 1105 & -- & -- & -- & -- & -- & -- \\
\hline & $7-30-92$ & 0635 & .160 & .160 & 3.30 & 3.40 & .240 & .250 \\
\hline & $8-21-92$ & 1325 & .080 & .080 & 2.20 & 2.20 & .060 & .060 \\
\hline & $9-08-92$ & 1255 & .040 & .040 & .250 & .250 & .110 & .100 \\
\hline \multirow[t]{3}{*}{6} & $7-31-92$ & 1010 & .010 & $<.010$ & $<.050$ & $<.050$ & .070 & .040 \\
\hline & $8-20-92$ & 0835 & .020 & .010 & $<.050$ & $<.050$ & .320 & .310 \\
\hline & $9-09-92$ & 0930 & .020 & .020 & .086 & .073 & .030 & .030 \\
\hline \multirow[t]{2}{*}{7} & $8-07-91$ & 1200 & -- & .030 & -- & .750 & -- & .060 \\
\hline & $8-07-91$ & 1602 & .- & .030 & -- & .580 & -. & .050 \\
\hline
\end{tabular}


Table 10. Concentrations of total and dissolved nitrogen, phosphorus, and organic carbon in surface water, 1991-92--Continued

\begin{tabular}{|c|c|c|c|c|c|c|c|c|c|}
\hline Site & Date & $\begin{array}{l}\text { Nitrogen, } \\
\text { ammonia, } \\
\text { plus } \\
\text { organic, } \\
\text { total }\end{array}$ & $\begin{array}{l}\text { Nitrogen, } \\
\text { ammonia, } \\
\text { plus } \\
\text { organic, } \\
\text { dissolved }\end{array}$ & $\begin{array}{l}\text { Phos- } \\
\text { phorus, } \\
\text { total }\end{array}$ & $\begin{array}{l}\text { Phos- } \\
\text { phorus, } \\
\text { dis- } \\
\text { solved }\end{array}$ & $\begin{array}{l}\text { Phos- } \\
\text { phorus, } \\
\text { ortho, } \\
\text { total }\end{array}$ & $\begin{array}{l}\text { Phos- } \\
\text { phorus, } \\
\text { ortho, } \\
\text { dis- } \\
\text { solved }\end{array}$ & $\begin{array}{l}\text { Carbon, } \\
\text { organic, } \\
\text { dissolved }\end{array}$ & $\begin{array}{c}\text { Carbon, } \\
\text { organic, } \\
\text { sus- } \\
\text { pended, } \\
\text { total }\end{array}$ \\
\hline \multirow[t]{11}{*}{1} & $8-21-91$ & .. & 1.1 & -- & 0.290 & -- & 0.230 & -- & -- \\
\hline & $8-21-91$ & -- & 1.0 & -- & .280 & -- & .230 & -- & -- \\
\hline & $8-21-91$ & -. & 1.3 & .- & .290 & -- & .230 & -- & -. \\
\hline & $8-22-91$ & -- & 1.4 & -- & .290 & -- & .230 & -- & -- \\
\hline & $8-22-91$ & -- & 1.3 & -- & .300 & -- & .230 & -- & -- \\
\hline & $8-22-91$ & $\therefore$ & 1.2 & -- & .300 & -- & .240 & -- & -.- \\
\hline & $7-24-92$ & -- & -- & -- & -- & -- & -- & 28 & 0.4 \\
\hline & $7-28-92$ & 4.9 & 2.1 & 0.970 & .290 & 0.240 & .220 & -- & -- \\
\hline & $7-28-92$ & 2.5 & 1.7 & .390 & .270 & .240 & .220 & -- & -- \\
\hline & $8-24-92$ & 2.1 & .90 & .330 & .170 & .180 & .170 & 11 & .1 \\
\hline & $8-24-92$ & -. & -- & -- & $\ldots$ & -- & -- & 10 & .1 \\
\hline \multirow[t]{5}{*}{2} & 8-05-91 & -. & 1.5 & -- & .330 & -- & .270 & -- & -- \\
\hline & $7-24-92$ & -. & -- & -- & -- & - &.- & 32 & $>4.5$ \\
\hline & $7-28-92$ & 2.2 & 1.7 & .540 & .310 & .330 & .250 & -. & .- \\
\hline & $8-24-92$ & 2.0 & 1.2 & .300 & .210 & .180 & .150 & 10 & .8 \\
\hline & $9-08-92$ & 2.1 & 1.2 & .250 & .150 & .160 & .150 & 10 & .4 \\
\hline \multirow[t]{5}{*}{3} & 8-05-91 & -- & 1.2 & -- & .330 & -- & .280 & -- & -- \\
\hline & $7-23-92$ & -- & .. & -. & -. & -. & -- & 38 & 3.4 \\
\hline & $7-30-92$ & 1.5 & 1.3 & .420 & .360 & .330 & .310 & .. & -- \\
\hline & $8-24-92$ & 2.0 & 1.3 & .450 & .320 & .360 & .260 & 11 & .4 \\
\hline & $9-08-92$ & 1.2 & 1.0 & .260 & .180 & .210 & .200 & 9.7 & .2 \\
\hline \multirow[t]{3}{*}{4} & $7-31-92$ & 1.6 & 1.4 & .410 & .390 & .280 & .300 & 10 & .1 \\
\hline & $8-20-92$ & 1.9 & 1.2 & .560 & .320 & .330 & .280 & 11 & .2 \\
\hline & $9-09-92$ & 1.4 & 1.0 & .260 & .200 & .210 & .180 & 9.7 & .1 \\
\hline \multirow[t]{5}{*}{5} & $8-05-91$ & -- & 1.5 & -- & .340 & -. & .290 & -- & -- \\
\hline & $7-23-92$ & -- & -- & -- & -- & -- & -- & 43 & .4 \\
\hline & $7-30-92$ & 2.4 & 2.4 & .400 & .370 & .310 & .300 & -- & .- \\
\hline & $8-21-92$ & 1.8 & 1.1 & .400 & .280 & .290 & .270 & 13 & .2 \\
\hline & $9-08-92$ & 1.8 & 1.3 & .370 & .300 & .290 & .280 & 14 & .1 \\
\hline \multirow[t]{3}{*}{6} & $7-31-92$ & 3.3 & 2.4 & .540 & .440 & .320 & .330 & 23 & .2 \\
\hline & $8-20-92$ & 3.0 & 2.9 & .800 & .620 & .550 & .560 & 19 & .2 \\
\hline & $9-09-92$ & 2.5 & 1.3 & .390 & .260 & .270 & .220 & 14 & .1 \\
\hline \multirow[t]{2}{*}{7} & $8-07-91$ & -- & 2.0 & -- & .370 & -- & .310 & -- & -. \\
\hline & $8-07-91$ & .- & 1.3 & -- & .350 & -- & .300 & -- & -- \\
\hline
\end{tabular}


Table 10. Concentrations of total and dissolved nitrogen, phosphorus, and organic carbon in surface water, 1991-92--Continued

\begin{tabular}{|c|c|c|c|c|c|c|c|c|}
\hline Site & Date & Time & $\begin{array}{l}\text { Nitrogen, } \\
\text { nitrite, } \\
\text { total }\end{array}$ & $\begin{array}{l}\text { Nitrogen } \\
\text { nitrite, } \\
\text { dis- } \\
\text { solved }\end{array}$ & $\begin{array}{l}\text { Nitrogen, } \\
\mathrm{NO}_{2}+\mathrm{NO}_{3} \\
\quad \text { total }\end{array}$ & $\begin{array}{c}\text { Nitrogen, } \\
\mathrm{NO}_{2}+\mathrm{NO}_{3} \\
\text { dis- } \\
\text { solved }\end{array}$ & $\begin{array}{l}\text { Nitrogen, } \\
\text { ammonia, } \\
\text { total }\end{array}$ & $\begin{array}{l}\text { Nitrogen, } \\
\text { ammonia, } \\
\text { dis- } \\
\text { solved }\end{array}$ \\
\hline \multirow[t]{9}{*}{7} & $8-07-91$ & 2000 & -- & 0.030 & -- & 0.650 & -- & 0.060 \\
\hline & $8-07-91$ & 2350 & -- & .030 & -- & .530 & -- & .070 \\
\hline & 8-08-91 & 0330 & -- & .030 & -- & .530 & -- & .070 \\
\hline & $8-08-91$ & 0800 & -- & .010 & -- & .560 & -- & .050 \\
\hline & $8-08-91$ & 1205 & -- & .020 & -- & .350 & -- & .060 \\
\hline & $7-23-92$ & 1150 & -- & -- & -- & -- & -- & -- \\
\hline & $7-30-92$ & 0720 & 0.110 & .100 & 0.380 & .390 & 0.080 & .070 \\
\hline & $8-21-92$ & 1050 & .040 & .030 & .063 & .050 & .100 & .090 \\
\hline & $9-08-92$ & 1355 & .080 & .070 & .680 & .700 & .370 & .300 \\
\hline \multirow[t]{3}{*}{8} & $7-31-92$ & 0940 & .050 & .040 & .097 & .075 & .410 & .400 \\
\hline & $8-20-92$ & 0815 & .030 & .020 & $<.050$ & $<.050$ & .250 & .200 \\
\hline & $9-09-92$ & 0825 & .020 & .020 & $<.050$ & $<.050$ & .150 & .160 \\
\hline \multirow[t]{11}{*}{9} & $8-07-91$ & 1225 & -- & .080 & -- & .270 & -- & .360 \\
\hline & 8-07-91 & 1630 & -- & .070 & -- & .320 & -- & .340 \\
\hline & 8-07-91 & 2020 & -- & .080 & -- & .430 & -- & .480 \\
\hline & 8-08-91 & 0020 & -- & .080 & -- & .370 & -- & .300 \\
\hline & 8-08-91 & 0400 & -- & .100 & -- & .350 & -- & .460 \\
\hline & 8-08-91 & 0825 & -- & .080 & -- & .270 & -- & .480 \\
\hline & $8-08-91$ & 1225 & -- & .080 & -- & .300 & -- & .320 \\
\hline & $7-23-92$ & 1240 & -- & -- & -- & -- & -- & -- \\
\hline & $7-30-92$ & 0830 & .040 & .040 & .170 & .190 & 1.40 & 1.40 \\
\hline & $8-21-92$ & 0955 & .010 & $<.010$ & $<.050$ & $<.050$ & .330 & .340 \\
\hline & 9-08-92 & 1445 & .010 & .020 & .290 & .270 & .460 & .490 \\
\hline \multirow[t]{3}{*}{10} & $7-31-92$ & 0700 & .160 & .140 & .410 & .420 & 1.10 & 1.10 \\
\hline & $8-20-92$ & 0750 & .020 & .020 & $<.050$ & $<.050$ & .170 & .160 \\
\hline & $9-09-92$ & 0840 & .020 & .030 & .360 & .390 & .410 & .380 \\
\hline \multirow[t]{11}{*}{11} & $8-07-91$ & 1255 & -- & .030 & -- & .070 & -- & .230 \\
\hline & 8-07-91 & 1655 & -- & .020 & -- & .050 & -- & .120 \\
\hline & $8-07-91$ & 2050 & -- & .020 & -- & .085 & -- & .170 \\
\hline & $8-08-91$ & 0040 & -- & .020 & -- & .074 & -- & .160 \\
\hline & $8-08-91$ & 0420 & -- & .020 & -- & .082 & -- & .190 \\
\hline & 8-08-91 & 0850 & -- & .020 & -- & .062 & -- & .210 \\
\hline & $8-08-91$ & 1250 & -- & .020 & -- & .071 & -- & .230 \\
\hline & $7-23-92$ & 1320 & -- & -. & -- & -- & -- & -- \\
\hline & $7-30-92$ & 0945 & .010 & .010 & $<.050$ & $<.050$ & .070 & .060 \\
\hline & $8-21-92$ & 0840 & $<.010$ & $<.010$ & $<.050$ & $<.050$ & .060 & .050 \\
\hline & 9-08-92, & 1530 & $<.010$ & $<.010$ & $<.050$ & $<.050$ & .050 & .040 \\
\hline
\end{tabular}


Table 10. Concentrations of total and dissolved nitrogen, phosphorus, and organic carbon in surface water, 1991-92--Continued

\begin{tabular}{|c|c|c|c|c|c|c|c|c|c|}
\hline Site & Date & $\begin{array}{l}\text { Nitrogen, } \\
\text { ammonia, } \\
\text { plus } \\
\text { organic, } \\
\text { total }\end{array}$ & $\begin{array}{l}\text { Nitrogen, } \\
\text { ammonia, } \\
\text { plus } \\
\text { organic, } \\
\text { dissolved }\end{array}$ & $\begin{array}{l}\text { Phos- } \\
\text { phorus, } \\
\text { total }\end{array}$ & $\begin{array}{c}\text { Phos- } \\
\text { phorus, } \\
\text { dis- } \\
\text { solved }\end{array}$ & $\begin{array}{l}\text { Phos- } \\
\text { phorus, } \\
\text { ortho, } \\
\text { total }\end{array}$ & $\begin{array}{l}\text { Phos- } \\
\text { phorus, } \\
\text { ortho, } \\
\text { dis- } \\
\text { solved }\end{array}$ & $\begin{array}{l}\text { Carbon, } \\
\text { organic, } \\
\text { dissolved }\end{array}$ & $\begin{array}{c}\text { Carbon, } \\
\text { organic, } \\
\text { sus- } \\
\text { pended, } \\
\text { total }\end{array}$ \\
\hline \multirow[t]{9}{*}{7} & $8-07-91$ & -- & 1.3 & -- & 0.380 & -- & 0.330 & -- & -- \\
\hline & $8-07-91$ & -- & 1.4 & -- & .380 & -- & .320 & -- & -- \\
\hline & $8-08-91$ & -- & 1.2 & -- & .380 & -- & .310 & -- & -- \\
\hline & $8-08-91$ & -- & .70 & -- & .360 & -- & .340 & -- & -- \\
\hline & $8-08-91$ & -- & 1.3 & -- & .370 & -- & .320 & -- & -- \\
\hline & $7-23-92$ & -- & -- & -- & -- & -- & -. & 80 & 0.5 \\
\hline & $7-30-92$ & 2.3 & 2.2 & 0.580 & .550 & 0.470 & .460 & -- & -- \\
\hline & $8-21-92$ & 2.0 & 1.0 & .510 & .330 & .380 & .290 & 14 & .1 \\
\hline & 9-08-92 & 2.5 & 1.6 & .470 & .340 & .360 & .310 & 15 & .2 \\
\hline \multirow[t]{3}{*}{8} & $7-31-92$ & 2.8 & 2.0 & .640 & .510 & .500 & .480 & 17 & .1 \\
\hline & $8-20-92$ & 3.5 & 1.7 & .630 & .320 & .330 & .250 & 21 & .1 \\
\hline & $9-09-92$ & 2.8 & 2.1 & .300 & .190 & .180 & .170 & 21 & -- \\
\hline \multirow[t]{11}{*}{9} & $8-07-91$ & -- & 2.7 & -- & .530 & -- & .480 & -- & -- \\
\hline & $8-07-91$ & -. & 2.7 & -- & .510 & -. & .450 & -- & -- \\
\hline & $8-07-91$ & -- & 2.7 & -- & .460 & -- & .410 & -- & -- \\
\hline & $8-08-91$ & -. & 2.4 & -- & .430 & -- & .370 & -- & -- \\
\hline & $8-08-91$ & -- & 2.7 & -- & .480 & -- & .430 & -- & -- \\
\hline & $8-08-91$ & -- & 3.1 & -- & .500 & -. & .450 & -- & -. \\
\hline & $8-08-91$ & -- & 2.8 & -- & .490 & -- & .410 & -- & -- \\
\hline & $7-23-92$ & -- & -- & -- & -- & -. & .- & 110 & 1.2 \\
\hline & $7-30-92$ & 5.9 & 5.1 & 1.10 & .410 & .420 & .350 & -- & -- \\
\hline & $8-21-92$ & 3.0 & 2.5 & .260 & .170 & .140 & .110 & 27 & .1 \\
\hline & 9-08-92 & 4.4 & 2.9 & .370 & .090 & .120 & .060 & 30 & .2 \\
\hline \multirow[t]{3}{*}{10} & $7-31-92$ & 5.0 & 4.5 & .410 & .310 & .260 & .240 & 32 & .2 \\
\hline & $8-20-92$ & 3.3 & 2.4 & .220 & .110 & .090 & .080 & 27 & .1 \\
\hline & $9-09-92$ & 5.2 & 2.8 & .430 & .120 & .160 & .100 & 29 & .2 \\
\hline \multirow[t]{11}{*}{11} & $8-07-91$ & -- & 2.2 & -. & .600 & -- & .550 & -- & -- \\
\hline & $8-07-91$ & $\therefore$ & 2.5 & -- & .530 & - & .470 & -- & -- \\
\hline & $8-07-91$ & -- & 2.6 & -- & .490 & -- & .430 & -- & -- \\
\hline & $8-08-91$ & -- & 2.4 & -- & .510 & .. & .430 & -- & -- \\
\hline & $8-08-91$ & -- & 2.5 & -- & .530 & -- & .460 & -- & -- \\
\hline & $8-08-91$ & -- & 2.5 & -- & .550 & -. & .500 & -- & -- \\
\hline & $8-08-91$ & -- & 2.3 & -- & .600 & -- & .520 & -- & -- \\
\hline & $7-23-92$ & -- & -- & -- & -- & -- & -- & 40 & .1 \\
\hline & $7-30-92$ & 1.8 & 1.7 & .330 & .270 & .220 & .200 & -- & -- \\
\hline & $8-21-92$ & 2.5 & 1.6 & .190 & .050 & .040 & .020 & 20 & -- \\
\hline & 9-08-92 & 4.4 & 2.3 & .190 & .020 & .020 & .010 & 30 & .2 \\
\hline
\end{tabular}


Table 10. Concentrations of total and dissolved nitrogen, phosphorus, and organic carbon in surface water, 1991-92-Continued

\begin{tabular}{|c|c|c|c|c|c|c|c|c|}
\hline Site & Date & Time & $\begin{array}{l}\text { Nitrogen, } \\
\text { nitrite, } \\
\text { total }\end{array}$ & $\begin{array}{l}\text { Nitrogen } \\
\text { nitrite, } \\
\text { dis- } \\
\text { solved }\end{array}$ & $\begin{array}{l}\text { Nitrogen, } \\
\mathrm{NO}_{2}+\mathrm{NO}_{3} \\
\quad \text { total }\end{array}$ & $\begin{array}{c}\text { Nitrogen, } \\
\mathrm{NO}_{2}+\mathrm{NO}_{3} \\
\text { dis- } \\
\text { solved }\end{array}$ & $\begin{array}{l}\text { Nitrogen, } \\
\text { ammonia, } \\
\text { total }\end{array}$ & $\begin{array}{l}\text { Nitrogen, } \\
\text { ammonia, } \\
\text { dis- } \\
\text { solved }\end{array}$ \\
\hline \multirow[t]{12}{*}{12} & $2-28-91$ & 1530 & -- & 0.020 & -- & 0.055 & -- & 0.260 \\
\hline & $8-19-91$ & 1245 & -- & $<.010$ & -. & .058 & -- & .050 \\
\hline & $8-19-91$ & 1635 & -- & $<.010$ & -- & $<.050$ & -- & .040 \\
\hline & $8-19-91$ & 2050 & -- & $<.010$ & -- & $<.050$ & -- & .050 \\
\hline & $8-20-91$ & 0040 & -- & $<.010$ & -- & $<.050$ & -- & .040 \\
\hline & $8-20-91$ & 0425 & -- & $<.010$ & -- & $<.050$ & -- & .040 \\
\hline & $8-20-91$ & 0920 & -- & $<.010$ & -- & $<.050$ & -- & .050 \\
\hline & $8-20-91$ & 1250 & -. & $<.010$ & -- & .073 & -- & .100 \\
\hline & $7-24-92$ & 1035 & -- & -- & -- & -- & -- & -- \\
\hline & $7-28-92$ & 1320 & $<0.010$ & $<.010$ & $<0.050$ & .078 & 0.040 & .040 \\
\hline & $8-24-92$ & 1110 & $<.010$ & $<.010$ & $<.050$ & $<.050$ & .030 & .030 \\
\hline & 9-08-92 & 1730 & .020 & $<.010$ & $<.050$ & $<.050$ & .050 & .030 \\
\hline \multirow[t]{11}{*}{13} & $8-19-91$ & 1205 & -- & .070 & -. & .230 & -- & .100 \\
\hline & $8-19-91$ & 1600 & -. & .130 & -- & .330 & -- & .170 \\
\hline & $8-19-91$ & 2010 & -- & .140 & -- & .260 & -- & .180 \\
\hline & $8-20-91$ & 0005 & -- & .090 & -- & .180 & -- & .120 \\
\hline & $8-20-91$ & 0350 & -- & .070 & -- & .170 & -- & .200 \\
\hline & $8-20-91$ & 0840 & -- & .080 & -- & .200 & -- & .280 \\
\hline & $8-20-91$ & 1215 & -- & .090 & -- & .240 & -- & .320 \\
\hline & 7-24-92 & 0950 & -- & -- & -- & -- & -- & -- \\
\hline & $7-27-92$ & 1145 & .020 & .020 & $<.050$ & .110 & .180 & .170 \\
\hline & $8-24-92$ & 0945 & $<.010$ & $<.010$ & $<.050$ & $<.050$ & .030 & .040 \\
\hline & $9-08-92$ & 1830 & $<.010$ & $<.010$ & $<.050$ & $<.050$ & .040 & .030 \\
\hline \multirow[t]{4}{*}{15} & $7-24-92$ & 0830 & -- & -- & -- & -- & -- & -- \\
\hline & $7-27-92$ & 1015 & .010 & .010 & $<.050$ & .091 & .080 & .080 \\
\hline & $8-24-92$ & 0800 & $<.010$ & $<.010$ & $<.050$ & $<.050$ & .010 & .010 \\
\hline & $9-09-92$ & 1300 & $<.010$ & $<.010$ & $<.050$ & $<.050$ & .030 & .020 \\
\hline \multirow[t]{3}{*}{ UTL3 } & $7-31-92$ & 0845 & $<.010$ & $<.010$ & $<.050$ & $<.050$ & .030 & .030 \\
\hline & $8-20-92$ & 0705 & $<.010$ & $<.010$ & $<.050$ & $<.050$ & .040 & .040 \\
\hline & $9-09-92$ & 1030 & $<.010$ & $<.010$ & $<.050$ & $<.050$ & .050 & .040 \\
\hline \multirow[t]{3}{*}{ UTL5 } & $7-31-92$ & 1155 & $<.010$ & $<.010$ & $<.050$ & $<.050$ & .030 & .030 \\
\hline & $8-20-92$ & 1000 & .020 & $<.010$ & $<.050$ & $<.050$ & .056 & .020 \\
\hline & 9-09-92 & 1000 & $<.010$ & $<.010$ & $<.050$ & $<.050$ & .070 & .040 \\
\hline \multirow[t]{4}{*}{ UTL7 } & $7-31-92$ & 0910 & $<.010$ & $<.010$ & $<.050$ & $<.050$ & .020 & .030 \\
\hline & $8-20-92$ & 0735 & .020 & $<.010$ & $<.050$ & $<.050$ & .040 & .056 \\
\hline & 9-09-92 & 0750 & $<.010$ & $<.010$ & $<.050$ & $<.050$ & .060 & .040 \\
\hline & 9-09-92 & 0900 & $<.010$ & $<.010$ & $<.050$ & $<.050$ & .040 & .020 \\
\hline
\end{tabular}


Table 10. Concentrations of total and dissolved nitrogen, phosphorus, and organic carbon in surface water, 1991-92--Continued

\begin{tabular}{|c|c|c|c|c|c|c|c|c|c|}
\hline Site & Date & $\begin{array}{l}\text { Nitrogen, } \\
\text { ammonia, } \\
\text { plus } \\
\text { organic, } \\
\text { total }\end{array}$ & $\begin{array}{l}\text { Nitrogen, } \\
\text { ammonia, } \\
\text { plus } \\
\text { organic, } \\
\text { dissolved }\end{array}$ & $\begin{array}{l}\text { Phos- } \\
\text { phorus, } \\
\text { total }\end{array}$ & $\begin{array}{l}\text { Phos- } \\
\text { phorus, } \\
\text { dis- } \\
\text { solved }\end{array}$ & $\begin{array}{l}\text { Phos- } \\
\text { phorus, } \\
\text { ortho, } \\
\text { total }\end{array}$ & $\begin{array}{l}\text { Phos- } \\
\text { phorus, } \\
\text { ortho, } \\
\text { dis- } \\
\text { solved }\end{array}$ & $\begin{array}{l}\text { Carbon, } \\
\text { organic, } \\
\text { dissolved }\end{array}$ & $\begin{array}{l}\text { Carbon, } \\
\text { organic, } \\
\text { sus- } \\
\text { pended, } \\
\text { total }\end{array}$ \\
\hline \multirow[t]{12}{*}{12} & $2-28-91$ & -- & 2.8 & -- & 0.290 & -- & 0.220 & -- & -- \\
\hline & $8-19-91$ & -. & 3.7 & -- & 1.10 & -- & 1.00 & -- & -- \\
\hline & $8-19-91$ & -- & 3.5 & -- & 1.10 & -- & 1.00 & -- & -- \\
\hline & 8-19-91 & -- & 2.7 & -- & 1.10 & -- & 1.00 & -- & -- \\
\hline & $8-20-91$ & -- & 2.1 & -- & 1.10 & -. & 1.00 & -- & -- \\
\hline & $8-20-91$ & -- & 3.1 & -- & 1.10 & -- & 1.00 & -- & -- \\
\hline & $8-20-91$ & -- & 2.3 & -- & 1.10 & -- & .990 & -. & -- \\
\hline & $8-20-91$ & -- & 2.6 & -- & 1.10 & -- & 1.00 & -- & -- \\
\hline & $7-24-92$ & -- & -- & -- & -- & -- & -- & 40 & 0.2 \\
\hline & $7-28-92$ & 5.2 & 2.4 & 0.460 & .100 & 0.100 & .040 & -- & -- \\
\hline & $8-24-92$ & 5.7 & 1.6 & .440 & .050 & .040 & $<.010$ & 16 & .1 \\
\hline & $9-08-92$ & 7.4 & 1.0 & .510 & .020 & .080 & $<.010$ & 17 & .2 \\
\hline \multirow[t]{11}{*}{13} & $8-19-91$ & -- & 1.9 & -- & .230 & -- & .180 & -- & -- \\
\hline & 8-19-91 & -- & 2.6 & -- & .260 & -- & .200 & -- & -- \\
\hline & $8-19-91$ & -- & 1.9 & -- & .230 & -- & .180 & -- & -- \\
\hline & $8-20-91$ & -- & 1.9 & -- & .210 & -- & .160 & -- & -- \\
\hline & $8-20-91$ & -- & 2.0 & -- & .230 & -- & .180 & -- & -- \\
\hline & $8-20-91$ & -- & 2.0 & -- & .270 & -- & .230 & -- & -- \\
\hline & $8-20-91$ & -- & 2.2 & -- & .300 & -- & .250 & -- & -- \\
\hline & $7-24-92$ & -- & -- & -- & -- & -- & -- & 90 & .2 \\
\hline & $7-27-92$ & 6.3 & 2.5 & .990 & .350 & .460 & .270 & -- & -- \\
\hline & $8-24-92$ & 6.4 & 2.4 & 1.50 & .730 & .640 & .560 & 28 & .2 \\
\hline & $9-08-92$ & 5.1 & 1.7 & .990 & .260 & .310 & .240 & 29 & .2 \\
\hline \multirow[t]{4}{*}{15} & $7-24-92$ & -- & -- & -- & -- & -- & -- & 38 & 2.5 \\
\hline & $7-27-92$ & 4.0 & 1.7 & .270 & .040 & .030 & $<.010$ & -- & -- \\
\hline & $8-24-92$ & 3.7 & .80 & .280 & .020 & .030 & $<.010$ & 10 & .4 \\
\hline & $9-09-92$ & 2.0 & .80 & .100 & $<.010$ & .030 & $<.010$ & 8.7 & .3 \\
\hline \multirow[t]{3}{*}{ UTL3 } & $7-31-92$ & 3.0 & 2.9 & .080 & .080 & .020 & .030 & 31 & .1 \\
\hline & $8-20-92$ & 2.9 & 2.6 & .060 & .070 & .020 & .020 & 29 & .1 \\
\hline & $9-09-92$ & 5.4 & 2.4 & .210 & .020 & .040 & $<.010$ & 32 & .2 \\
\hline \multirow[t]{3}{*}{ UTL5 } & $7-31-92$ & 2.1 & 1.6 & .630 & .540 & .440 & .430 & 14 & .1 \\
\hline & $8-20-92$ & 2.8 & 1.2 & .880 & .530 & .600 & .560 & 15 & .1 \\
\hline & $9-09-92$ & 2.4 & 1.2 & .440 & .320 & .300 & .270 & 13 & .2 \\
\hline \multirow[t]{4}{*}{ UTL7 } & $7-31-92$ & 2.9 & 2.6 & .170 & .120 & .080 & .070 & 28 & .1 \\
\hline & $8-20-92$ & 3.0 & 2.8 & .160 & .080 & .056 & .030 & 29 & .2 \\
\hline & $9-09-92$ & 5.9 & 2.4 & .310 & .030 & .050 & .010 & 29 & .2 \\
\hline & $9-09-92$ & 6.2 & 2.5 & .330 & .040 & .060 & $<.010$ & 31 & .3 \\
\hline
\end{tabular}


Table 10. Concentrations of total and dissolved nitrogen, phosphorus, and organic carbon in surface water, 1991-92--Continued

\begin{tabular}{|c|c|c|c|c|c|c|c|c|}
\hline Site & Date & Time & $\begin{array}{l}\text { Nitrogen, } \\
\text { nitrite, } \\
\text { total }\end{array}$ & $\begin{array}{l}\text { Nitrogen } \\
\text { nitrite, } \\
\text { dis- } \\
\text { solved }\end{array}$ & $\begin{array}{l}\text { Nitrogen, } \\
\mathrm{NO}_{2}+\mathrm{NO}_{3} \\
\quad \text { total }\end{array}$ & $\begin{array}{c}\text { Nitrogen, } \\
\mathrm{NO}_{2}+\mathrm{NO}_{3}, \\
\text { dis- } \\
\text { solved }\end{array}$ & $\begin{array}{l}\text { Nitrogen, } \\
\text { ammonia, } \\
\text { total }\end{array}$ & $\begin{array}{c}\text { Nitrogen, } \\
\text { ammonia, } \\
\text { dis- } \\
\text { solved }\end{array}$ \\
\hline LK1 & $2-28-92$ & 1440 & -- & 0.050 & -- & 0.076 & -- & 0.100 \\
\hline LK2 & $2-28-92$ & 1405 & -- & .080 & -- & .081 & -- & .020 \\
\hline \multirow[t]{6}{*}{ LR5 } & $8-21-91$ & 1450 & 0.030 & .280 & 0.070 & .280 & -. & .070 \\
\hline & $8-21-91$ & 1750 & .030 & .240 & .040 & .240 & -- & .040 \\
\hline & $8-21-91$ & 2220 & .030 & .230 & .040 & .230 & -- & .040 \\
\hline & $8-22-91$ & 0215 & .030 & .260 & .090 & .260 & -- & .090 \\
\hline & $8-22-91$ & 0650 & .040 & .270 & .120 & .270 & -- & .120 \\
\hline & $8-22-91$ & 1005 & .030 & .260 & .050 & .260 & -- & .050 \\
\hline \multirow[t]{6}{*}{ LR6 } & $8-21-91$ & 1530 & .010 & $<.050$ & .090 & $<.050$ & -- & .090 \\
\hline & $8-21-91$ & 1820 & $<.010$ & $<.050$ & .070 & $<.050$ & -- & .070 \\
\hline & $8-21-91$ & 2240 & .010 & .098 & .100 & .098 & -- & .100 \\
\hline & $8-22-91$ & 0245 & $<.010$ & .063 & .090 & .063 & -- & .090 \\
\hline & $8-22-91$ & 0715 & $<.010$ & $<.050$ & .100 & $<.050$ & -- & .100 \\
\hline & $8-22-91$ & 1035 & $<.010$ & $<.050$ & .080 & $<.050$ & -- & .080 \\
\hline \multirow[t]{7}{*}{$9 \mathrm{~A}$} & 8-19-91 & 1310 & .020 & .120 & .600 & .120 & -- & .600 \\
\hline & 8-19-91 & 1555 & .020 & .110 & .610 & .110 & -- & .610 \\
\hline & 8-19-91 & 2110 & .020 & $<.050$ & .570 & $<.050$ & -- & .570 \\
\hline & $8-20-91$ & 0100 & .020 & $<.050$ & .620 & $<.050$ & -- & .620 \\
\hline & $8-20-91$ & 0440 & .020 & $<.050$ & .640 & $<.050$ & -- & .640 \\
\hline & $8-20-91$ & 0935 & .010 & .056 & .640 & .056 & -- & .640 \\
\hline & $8-20-91$ & 1315 & .010 & .091 & .600 & .091 & -- & .600 \\
\hline
\end{tabular}


Table 10. Concentrations of total and dissolved nitrogen, phosphorus, and organic carbon in surface water, 1991-92--Continued

\begin{tabular}{|c|c|c|c|c|c|c|c|c|c|}
\hline Site & Date & $\begin{array}{l}\text { Nitrogen, } \\
\text { ammonia, } \\
\text { plus } \\
\text { organic, } \\
\text { total }\end{array}$ & $\begin{array}{l}\text { Nitrogen, } \\
\text { ammonia, } \\
\text { plus } \\
\text { organic, } \\
\text { dissolved }\end{array}$ & $\begin{array}{l}\text { Phos- } \\
\text { phorus, } \\
\text { total }\end{array}$ & $\begin{array}{l}\text { Phos- } \\
\text { phorus, } \\
\text { dis- } \\
\text { solved }\end{array}$ & $\begin{array}{l}\text { Phos- } \\
\text { phorus, } \\
\text { ortho, } \\
\text { total }\end{array}$ & $\begin{array}{l}\text { Phos- } \\
\text { phorus, } \\
\text { ortho, } \\
\text { dis- } \\
\text { solved }\end{array}$ & $\begin{array}{l}\text { Carbon, } \\
\text { organic, } \\
\text { dissolved }\end{array}$ & $\begin{array}{l}\text { Carbon, } \\
\text { organic, } \\
\text { sus- } \\
\text { pended, } \\
\text { total }\end{array}$ \\
\hline LK1 & $2-28-91$ & -- & 2.7 & -- & 0.120 & -- & 0.060 & -- & -- \\
\hline LK2 & $2-28-91$ & -- & 3.4 & -- & .110 & -- & .040 & -- & -- \\
\hline LR5 & $\begin{array}{l}8-21-91 \\
8-21-91 \\
8-21-91 \\
8-22-91 \\
8-22-91 \\
8-22-91\end{array}$ & $\begin{array}{l}- \\
-- \\
-- \\
-- \\
-- \\
--\end{array}$ & $\begin{array}{l}1.4 \\
1.2 \\
1.5 \\
1.5 \\
1.4 \\
1.1\end{array}$ & $\begin{array}{l}-- \\
-- \\
-- \\
-- \\
-- \\
--\end{array}$ & $\begin{array}{l}.270 \\
.280 \\
.270 \\
.290 \\
.300 \\
.290\end{array}$ & $\begin{array}{l}-- \\
-- \\
-- \\
-- \\
-- \\
--\end{array}$ & $\begin{array}{l}.210 \\
.190 \\
.210 \\
.230 \\
.240 \\
.220\end{array}$ & $\begin{array}{l}-- \\
-- \\
-- \\
-- \\
-- \\
--\end{array}$ & $\begin{array}{l}-- \\
-- \\
-- \\
-- \\
-- \\
--\end{array}$ \\
\hline LR6 & $\begin{array}{l}8-21-91 \\
8-21-91 \\
8-21-91 \\
8-22-91 \\
8-22-91 \\
8-22-91\end{array}$ & $\begin{array}{l}-- \\
-- \\
-- \\
-- \\
-- \\
--\end{array}$ & $\begin{array}{l}1.1 \\
1.3 \\
1.4 \\
1.2 \\
1.2 \\
1.1\end{array}$ & $\begin{array}{l}-- \\
-- \\
-- \\
-- \\
-- \\
--\end{array}$ & $\begin{array}{l}.160 \\
.160 \\
.160 \\
.170 \\
.170 \\
.160\end{array}$ & $\begin{array}{l}-- \\
-- \\
-- \\
-- \\
-- \\
--\end{array}$ & $\begin{array}{l}.100 \\
.100 \\
.120 \\
.110 \\
.120 \\
.100\end{array}$ & $\begin{array}{l}-- \\
-- \\
-- \\
- \\
- \\
--\end{array}$ & $\begin{array}{l}-- \\
-- \\
-- \\
-- \\
-- \\
--\end{array}$ \\
\hline $9 \mathrm{~A}$ & $\begin{array}{l}8-19-91 \\
8-19-91 \\
8-19-91 \\
8-20-91 \\
8-20-91 \\
8-20-91 \\
8-20-91\end{array}$ & $\begin{array}{l}-- \\
-- \\
-- \\
-- \\
-- \\
-- \\
--\end{array}$ & $\begin{array}{l}3.9 \\
3.3 \\
3.3 \\
3.2 \\
3.4 \\
2.9 \\
3.5\end{array}$ & $\begin{array}{l}-- \\
-- \\
-- \\
-- \\
-- \\
-- \\
--\end{array}$ & $\begin{array}{l}.860 \\
.830 \\
.850 \\
.920 \\
.850 \\
.910 \\
.840\end{array}$ & $\begin{array}{l}-- \\
-- \\
-- \\
-- \\
-- \\
-- \\
--\end{array}$ & $\begin{array}{l}.850 \\
.790 \\
.790 \\
.770 \\
.810 \\
.860 \\
.800\end{array}$ & $\begin{array}{l}-- \\
-- \\
-- \\
-- \\
-- \\
-- \\
--\end{array}$ & $\begin{array}{l}-- \\
-- \\
-- \\
-- \\
-- \\
-- \\
--\end{array}$ \\
\hline
\end{tabular}


Table 11. Concentrations of major dissolved constituents in surface water. 1992

[See figure 1 for site locations. $\mathrm{mg} / \mathrm{L}$, milligrams per liter; $\mu \mathrm{g} / \mathrm{L}$, micrograms per liter; $<$, actual value is less than value shown]

\begin{tabular}{|c|c|c|c|c|c|c|c|c|c|c|c|c|c|}
\hline Site & Date & Time & $\begin{array}{c}\text { Cal- } \\
\text { cium } \\
(\mathrm{mg} / \mathrm{L})\end{array}$ & $\begin{array}{c}\text { Magne- } \\
\text { sium } \\
(\mathrm{mg} / \mathrm{L})\end{array}$ & $\begin{array}{l}\text { Sodium } \\
(\mathrm{mg} / \mathrm{L})\end{array}$ & $\begin{array}{c}\text { Potas- } \\
\text { sium } \\
(\mathrm{mg} / \mathrm{L})\end{array}$ & $\begin{array}{c}\text { Sul- } \\
\text { fate } \\
(\mathrm{mg} / \mathrm{L}) \\
\end{array}$ & $\begin{array}{l}\text { Chlor- } \\
\text { ride } \\
(\mathrm{mg} / \mathrm{L})\end{array}$ & $\begin{array}{c}\text { Fluo- } \\
\text { ride } \\
(\mathrm{mg} / \mathrm{L})\end{array}$ & $\begin{array}{c}\text { Silica } \\
(\mathrm{mg} / \mathrm{L})\end{array}$ & $\begin{array}{c}\text { Boron } \\
(\mathrm{mg} / \mathrm{L})\end{array}$ & $\begin{array}{c}\text { Iron } \\
(\mu \mathrm{g} / \mathrm{L})\end{array}$ & $\begin{array}{c}\text { Manga- } \\
\text { nese } \\
(\mu \mathrm{g} / \mathrm{L})\end{array}$ \\
\hline \multirow[t]{3}{*}{1} & July 28 & 1550 & 12 & 7.0 & 18 & 3.1 & 9.2 & 6.1 & 0.20 & 50 & 0.09 & 65 & 36 \\
\hline & July 28 & 1555 & 12 & 7.2 & 20 & 3.0 & 8.9 & 5.9 & .20 & 50 & .08 & 65 & 37 \\
\hline & Aug. 24 & 1445 & 14 & 8.3 & 22 & 3.5 & 13 & 4.3 & .20 & $<.10$ & .09 & 28 & 17 \\
\hline \multirow[t]{3}{*}{2} & July 28 & 1520 & 14 & 9.0 & 24 & 3.5 & 11 & 4.9 & .20 & 32 & .09 & 56 & 33 \\
\hline & Aug. 24 & 1250 & 12 & 6.8 & 19 & 3.3 & 10 & 5.7 & .10 & 43 & .08 & 31 & 30 \\
\hline & Sept. 8 & 1055 & 14 & 7.7 & 21 & 3.5 & 12 & 6.2 & .20 & 38 & .09 & 26 & 42 \\
\hline \multirow[t]{3}{*}{3} & July 30 & 0530 & 17 & 9.8 & 25 & 3.2 & 22 & 5.7 & .20 & 39 & .08 & 62 & 45 \\
\hline & Aug. 24 & 1335 & 18 & 9.3 & 25 & 3.5 & 21 & 6.5 & .20 & 46 & .08 & 29 & 49 \\
\hline & Sept. 8 & 1150 & 16 & 9.4 & 25 & 4.0 & 18 & 8.8 & .20 & 47 & .09 & 24 & 10 \\
\hline \multirow[t]{3}{*}{4} & July 31 & 1105 & 18 & 9.9 & 25 & 3.9 & 27 & 7.9 & .20 & 40 & .91 & 120 & 46 \\
\hline & Aug. 20 & 0900 & 17 & 9.6 & 24 & 4.1 & 19 & 6.6 & .20 & 47 & .09 & 27 & 26 \\
\hline & Sept. 9 & 0940 & 16 & 9.4 & 24 & 4.0 & 18 & 7.2 & $<.10$ & 47 & .10 & 26 & 3 \\
\hline \multirow[t]{3}{*}{5} & July 30 & 0635 & 68 & 26 & 63 & 4.1 & 180 & 9.0 & .30 & 38 & .12 & 44 & 34 \\
\hline & Aug. 21 & 1325 & 37 & 16 & 42 & 4.8 & 83 & 8.5 & .30 & 56 & .11 & 22 & 21 \\
\hline & Sept. 8 & 1255 & 39 & 17 & 42 & 5.0 & 82 & 10 & .30 & 43 & .10 & 18 & 37 \\
\hline \multirow[t]{3}{*}{6} & July 31 & 1010 & 31 & 24 & 59 & 13 & 72 & 19 & .40 & 36 & .26 & 34 & 8 \\
\hline & Aug. 20 & 0835 & 37 & 21 & 46 & 9.3 & 59 & 9.1 & .30 & 37 & .18 & 15 & 73 \\
\hline & Sept. 9 & 0930 & 30 & 16 & 36 & 6.0 & 46 & 9.9 & .30 & 38 & .12 & 16 & 6 \\
\hline \multirow[t]{3}{*}{7} & July 30 & 0720 & 64 & 28 & 76 & 6.4 & 170 & 12 & .30 & 35 & .14 & 22 & 120 \\
\hline & Aug. 21 & 1050 & 33 & 17 & 41 & 6.9 & 66 & 9.9 & .30 & 41 & .11 & 16 & 40 \\
\hline & Sept. 8 & 1355 & 35 & 16 & 41 & 6.7 & 54 & 11 & .30 & 41 & .12 & 20 & 58 \\
\hline \multirow[t]{3}{*}{8} & July 31 & 0940 & 47 & 23 & 62 & 7.8 & 140 & 15 & .30 & 35 & .15 & 19 & 64 \\
\hline & Aug. 20 & 0815 & 41 & 25 & 74 & 11 & 130 & 16 & .40 & 47 & .21 & 6 & 26 \\
\hline & Sept. 9 & 0825 & 33 & 24 & 79 & 9.5 & 140 & 17 & .40 & 36 & .16 & 5 & 5 \\
\hline \multirow[t]{3}{*}{9} & July 30 & 0830 & 66 & 47 & 180 & 16 & 420 & 30 & .30 & 28 & .16 & 37 & 320 \\
\hline & Aug. 21 & 0955 & 34 & 26 & 130 & 11 & 270 & 21 & .30 & 32 & .14 & 15 & 51 \\
\hline & Sept. 8 & 1445 & 36 & 28 & 130 & 14 & 280 & 23 & .40 & 31 & .15 & 19 & 60 \\
\hline \multirow[t]{3}{*}{10} & July 31 & 0700 & 59 & 41 & 160 & 14 & 370 & 33 & .50 & 27 & .18 & 21 & 170 \\
\hline & Aug. 20 & 0750 & 33 & 28 & 120 & 12 & 250 & 23 & .40 & 32 & .08 & 6 & 15 \\
\hline & Sept. 9 & 0840 & 40 & 29 & 120 & 12 & 240 & 22 & .40 & 36 & .17 & 12 & 44 \\
\hline \multirow[t]{3}{*}{11} & July 30 & 0945 & 29 & 10 & 47 & 6.8 & 90 & 8.4 & .30 & 33 & .12 & 16 & 3 \\
\hline & Aug. 21 & 0840 & 26 & 10 & 69 & 7.8 & 130 & 13 & .30 & 43 & .12 & 5 & 3 \\
\hline & Sept. 8 & 1530 & 31 & 27 & 120 & 12 & 250 & 22 & .40 & 36 & .15 & $<3$ & 2 \\
\hline \multirow[t]{3}{*}{12} & July 28 & 1320 & 20 & 6.2 & 35 & 5.0 & 37 & 7.8 & .30 & 33 & .13 & 21 & 10 \\
\hline & Aug. 24 & 1110 & 14 & 5.5 & 22 & 4.3 & 9.6 & 6.1 & .20 & 49 & .11 & 19 & 4 \\
\hline & Sept. 8 & 1730 & 12 & 4.7 & 18 & 3.7 & 6.3 & 5.6 & .20 & 58 & .10 & 12 & 4 \\
\hline \multirow[t]{3}{*}{13} & July 27 & 1145 & 53 & 28 & 61 & 9.3 & 100 & 10 & .50 & 42 & .31 & 95 & 85 \\
\hline & Aug. 24 & 0945 & 49 & 29 & 86 & 2.7 & 110 & 17 & .50 & 44 & .36 & 16 & 59 \\
\hline & Sept. 8 & 1830 & 40 & 22 & 86 & 16 & 76 & 16 & .60 & 47 & .40 & 23 & 7 \\
\hline
\end{tabular}


Table 11. Concentrations of major dissolved constituents in surface water, 1992--Continued

\begin{tabular}{|c|c|c|c|c|c|c|c|c|c|c|c|c|c|}
\hline Site & Date & Time & $\begin{array}{l}\text { Cal- } \\
\text { cium } \\
(\mathrm{mg} / \mathrm{L})\end{array}$ & $\begin{array}{l}\text { Magne- } \\
\text { sium } \\
(\mathrm{mg} / \mathrm{L})\end{array}$ & $\begin{array}{l}\text { Sodium } \\
\text { (mg/L) }\end{array}$ & $\begin{array}{l}\text { Potas- } \\
\text { sium } \\
(\mathrm{mg} / \mathrm{L})\end{array}$ & $\begin{array}{l}\text { Sul- } \\
\text { fate } \\
(\mathrm{mg} / \mathrm{L})\end{array}$ & $\begin{array}{l}\text { Chlor- } \\
\text { ride } \\
(\mathrm{mg} / \mathrm{L})\end{array}$ & $\begin{array}{l}\text { Fluo- } \\
\text { ride } \\
(\mathrm{mg} / \mathrm{L})\end{array}$ & $\begin{array}{l}\text { Silica } \\
(\mathrm{mg} / \mathrm{L})\end{array}$ & $\begin{array}{l}\text { Boron } \\
(\mathrm{mg} / \mathrm{L})\end{array}$ & $\begin{array}{c}\text { Iron } \\
(\mu \mathrm{g} / \mathrm{L})\end{array}$ & $\begin{array}{c}\text { Manga- } \\
\text { nese } \\
(\mu \mathrm{g} / \mathrm{L})\end{array}$ \\
\hline \multirow[t]{3}{*}{15} & July 27 & 1015 & 8.1 & 3.8 & 12 & 2.1 & 2.9 & 4.3 & 0.10 & 50 & 0.07 & 130 & 14 \\
\hline & Aug. 24 & 0800 & 8.4 & 3.8 & 12 & 2.2 & 3.7 & 4.4 & .10 & 42 & .08 & 42 & 5 \\
\hline & Sept. 9 & 1300 & 9.3 & 4.6 & 13 & 2.3 & 4.6 & 4.5 & .20 & 57 & .08 & 44 & 2 \\
\hline \multirow[t]{3}{*}{ UTL3 } & July 31 & 0845 & 25 & 27 & 140 & 11 & 310 & 29 & .50 & 13 & .11 & 7 & 1 \\
\hline & Aug. 20 & 0705 & 25 & 28 & 140 & 13 & 260 & 22 & .40 & 29 & .04 & 4 & 1 \\
\hline & Sept. 9 & 1030 & 30 & 32 & 140 & 13 & 300 & 25 & .40 & 35 & .17 & 4 & 2 \\
\hline \multirow[t]{3}{*}{ UTL5 } & July 31 & 1155 & 30 & 18 & 31 & 8.5 & 27 & 10 & .30 & 29 & .16 & 20 & 21 \\
\hline & Aug. 20 & 1000 & 27 & 14 & 29 & 6.6 & 22 & 10 & .30 & 41 & .13 & 38 & 350 \\
\hline & Sept. 9 & 1000 & 22 & 11 & 26 & 4.7 & 20 & 8.1 & .30 & 22 & .10 & 29 & 9 \\
\hline \multirow[t]{4}{*}{ UTL7 } & July 31 & 0910 & 35 & 34 & 130 & 10 & 300 & 23 & .40 & 17 & .16 & 15 & 2 \\
\hline & Aug. 20 & 0735 & 33 & 29 & 120 & 12 & 230 & 17 & .30 & 29 & .15 & 32 & 3 \\
\hline & Sept. 9 & 0750 & 29 & 28 & 110 & 12 & 220 & 21 & .40 & 35 & .17 & 9 & 2 \\
\hline & Sept. 9 & 0900 & 28 & 28 & 120 & 12 & 240 & 21 & .40 & 36 & .17 & 17 & 4 \\
\hline
\end{tabular}


Table 12. Concentrations of pesticides and related compounds detected in surface water, 1991-92

[See figure 1 for site locations. Other elements analyzed for but not detected are shown at the end of this table. Concentrations in microgram per liter. Detections are shown in bold print. <, below method-detection limit]

\begin{tabular}{|c|c|c|c|c|c|c|c|c|c|}
\hline Site & Date & $\begin{array}{c}\text { Atra- } \\
\text { zine }\end{array}$ & $\begin{array}{l}\text { Benflu- } \\
\text { ralin }\end{array}$ & $\begin{array}{l}\text { Chlor- } \\
\text { pyrifos }\end{array}$ & $\begin{array}{l}\text { Cyana- } \\
\text { zine }\end{array}$ & $\begin{array}{l}\text { Disul- } \\
\text { foton }\end{array}$ & DDE & Eptam & $\begin{array}{l}\text { Etho- } \\
\text { prop }\end{array}$ \\
\hline \multirow[t]{5}{*}{1} & $6-10-92$ & $<0.002$ & $<0.005$ & $<0.002$ & $<0.010$ & $<0.010$ & $<0.002$ & 0.012 & $<0.005$ \\
\hline & $6-17-92$ & $<.002$ & $<.005$ & $<.004$ & $<.010$ & $<.050$ & $<.002$ & .011 & $<.005$ \\
\hline & $7-08-92$ & $<.002$ & $<.005$ & $<.010$ & $<.020$ & $<.050$ & $<.010$ & $<.002$ & $<.005$ \\
\hline & $7-29-92$ & $<.002$ & $<.005$ & $<.010$ & $<.020$ & $<.050$ & $<.010$ & $<.002$ & $<.005$ \\
\hline & $8-19-92$ & $<.002$ & $<.005$ & $<.010$ & $<.020$ & $<.050$ & $<.010$ & $<.002$ & $<.005$ \\
\hline \multirow[t]{7}{*}{2} & $7-10-91$ & $<.002$ & $<.005$ & $<.010$ & .021 & .050 & $<.010$ & $<.005$ & $<.005$ \\
\hline & $6-03-92$ & .010 & .005 & .018 & $<.010$ & $<.050$ & $<.002$ & .320 & $<.005$ \\
\hline & $6-24-92$ & $<.002$ & $<.005$ & $<.002$ & $<.010$ & $<.050$ & $<.002$ & .015 & $<.005$ \\
\hline & $7-08-92$ & $<.002$ & $<.005$ & $<.010$ & $<.020$ & $<.050$ & $<.002$ & $<.002$ & $<.005$ \\
\hline & $7-15-92$ & $<.002$ & $<.005$ & $<.010$ & $<.020$ & $<.050$ & $<.002$ & $<.002$ & $<.005$ \\
\hline & $7-22-92$ & $<.002$ & $<.005$ & $<.010$ & $<.020$ & $<.050$ & $<.002$ & $<.002$ & $<.005$ \\
\hline & $8-12-92$ & $<.002$ & $<.005$ & $<.010$ & $<.020$ & $<.050$ & $<.002$ & $<.002$ & $<.005$ \\
\hline \multirow[t]{7}{*}{3} & $7-10-91$ & $<.004$ & $<.010$ & $<.020$ & $<.020$ & $<.100$ & $<.004$ & $<.004$ & $<.005$ \\
\hline & $7-24-91$ & $<.004$ & $<.010$ & $<.020$ & $<.020$ & $<.100$ & $<.004$ & $<.004$ & $<.005$ \\
\hline & 8-14-91 & $<.002$ & $<.010$ & $<.020$ & $<.020$ & $<.100$ & $<.004$ & $<.004$ & $<.005$ \\
\hline & $6-24-92$ & $<.002$ & $<.005$ & $<.002$ & $<.010$ & $<.050$ & $<.002$ & .020 & $<.005$ \\
\hline & 7-08-92 & $<.002$ & $<.005$ & $<.010$ & $<.020$ & $<.050$ & $<.010$ & $<.002$ & $<.005$ \\
\hline & $7-22-92$ & $<.002$ & $<.005$ & $<.010$ & $<.020$ & $<.050$ & $<.010$ & $<.002$ & $<.005$ \\
\hline & $7-29-92$ & $<.002$ & $<.005$ & $<.010$ & $<.020$ & $<.050$ & $<.010$ & $<.002$ & $<.005$ \\
\hline \multirow[t]{11}{*}{5} & 7-03-91 & $<.004$ & $<.010$ & $<.020$ & $<.020$ & $<.050$ & $<.010$ & $<.002$ & $<.005$ \\
\hline & $7-10-91$ & $<.004$ & $<.010$ & $<.020$ & $<.020$ & $<.050$ & $<.010$ & $<.002$ & $<.005$ \\
\hline & $7-17-91$ & $<.004$ & $<.010$ & $<.020$ & $<.020$ & $<.050$ & $<.010$ & $<.002$ & $<.005$ \\
\hline & $7-24-91$ & $<.004$ & $<.010$ & $<.020$ & $<.020$ & $<.050$ & $<.010$ & $<.002$ & $<.005$ \\
\hline & $6-03-92$ & $<.002$ & $<.005$ & .004 & $<.010$ & $<.050$ & $<.002$ & .040 & .002 \\
\hline & $6-17-92$ & $<.002$ & $<.005$ & $<.002$ & $<.010$ & $<.050$ & $<.002$ & .015 & $<.005$ \\
\hline & $6-24-92$ & $<.002$ & $<.005$ & $<.002$ & $<.010$ & $<.050$ & $<.002$ & .013 & $<.005$ \\
\hline & $7-01-92$ & $<.002$ & $<.005$ & $<.002$ & $<.010$ & $<.050$ & $<.002$ & .016 & $<.005$ \\
\hline & $7-08-92$ & $<.055$ & $<.005$ & $<.002$ & $<.010$ & $<.100$ & $<.002$ & $<.010$ & $<.005$ \\
\hline & $7-29-92$ & $<.002$ & $<.005$ & $<.010$ & $<.020$ & $<.050$ & $<.002$ & $<.002$ & $<.005$ \\
\hline & $8-05-92$ & $<.002$ & $<.005$ & $<.010$ & $<.020$ & $<.050$ & $<.002$ & .006 & $<.005$ \\
\hline \multirow[t]{11}{*}{7} & $7-10-91$ & $<.002$ & $<.010$ & $<.010$ & $<.020$ & $<.050$ & $<.010$ & $<.005$ & $<.005$ \\
\hline & $7-17-91$ & $<.002$ & $<.010$ & $<.010$ & $<.020$ & .026 & $<.010$ & $<.005$ & $<.005$ \\
\hline & $7-24-91$ & $<.002$ & $<.010$ & $<.010$ & $<.020$ & $<.050$ & $<.010$ & $<.005$ & $<.005$ \\
\hline & $6-17-92$ & $<.002$ & .003 & .011 & $<.010$ & $<.050$ & .002 & .028 & $<.005$ \\
\hline & $6-24-92$ & $<.002$ & $<.005$ & $<.002$ & $<.010$ & $<.050$ & $<.002$ & .021 & .004 \\
\hline & $7-01-92$ & $<.002$ & $<.005$ & $<.010$ & $<.010$ & $<.010$ & $<.002$ & $<.001$ & $<.005$ \\
\hline & $7-08-92$ & $<.002$ & $<.005$ & $<.010$ & $<.020$ & $<.050$ & $<.010$ & .014 & $<.005$ \\
\hline & $7-15-92$ & $<.002$ & $<.005$ & $<.010$ & $<.020$ & $<.050$ & $<.010$ & .010 & $<.005$ \\
\hline & $7-29-92$ & $<.002$ & $<.005$ & $<.010$ & $<.020$ & $<.050$ & $<.010$ & $<.002$ & $<.005$ \\
\hline & $8-05-92$ & $<.002$ & $<.005$ & $<.010$ & $<.020$ & $<.050$ & $<.010$ & .006 & $<.005$ \\
\hline & 8-19-92 & .003 & $<.005$ & $<.010$ & $<.020$ & $<.050$ & $<.010$ & $<.002$ & $<.005$ \\
\hline
\end{tabular}


Table 12. Concentrations of pesticides and related compounds detected in surface water, 1991-92--Continued

\begin{tabular}{|c|c|c|c|c|c|c|c|c|c|}
\hline Site & Date & $\begin{array}{l}\text { Mala- } \\
\text { thion }\end{array}$ & $\begin{array}{l}\text { Methyl } \\
\text { parathion }\end{array}$ & $\begin{array}{l}\text { Metola- } \\
\text { chlor }\end{array}$ & $\begin{array}{l}\text { Metri- } \\
\text { buzin }\end{array}$ & $\begin{array}{l}\text { Prona- } \\
\text { mide }\end{array}$ & $\begin{array}{l}\text { Sima- } \\
\text { zine }\end{array}$ & $\begin{array}{l}\text { Terbu- } \\
\text { fos }\end{array}$ & $\begin{array}{l}\text { Triflu- } \\
\text { ralin }\end{array}$ \\
\hline \multirow[t]{5}{*}{1} & $6-10-92$ & $<0.010$ & $<0.005$ & $<0.005$ & $<0.010$ & $<0.010$ & $<0.002$ & $<0.010$ & $<0.005$ \\
\hline & $6-17-92$ & $<.010$ & $<.005$ & .002 & $<.010$ & .008 & .011 & $<.010$ & $<.005$ \\
\hline & $7-08-92$ & $<.005$ & $<.005$ & $<.005$ & .024 & $<.010$ & .008 & $<.050$ & $<.005$ \\
\hline & $7-29-92$ & $<.005$ & $<.005$ & $<.005$ & $<.005$ & $<.010$ & $<.002$ & $<.050$ & $<.005$ \\
\hline & $8-19-92$ & $<.005$ & $<.005$ & $<.005$ & $<.005$ & $<.010$ & $<.002$ & $<.050$ & $<.005$ \\
\hline \multirow[t]{7}{*}{2} & $7-10-91$ & $<.005$ & $<.005$ & .029 & .077 & $<.010$ & .005 & $<.010$ & $<.010$ \\
\hline & 6-03-92 & .010 & $<.005$ & .002 & $<.010$ & .010 & .006 & $<.010$ & .005 \\
\hline & $6-24-92$ & $<.010$ & $<.005$ & $<.002$ & .012 & $<.010$ & .004 & .037 & $<.005$ \\
\hline & $7-08-92$ & $<.005$ & $<.005$ & $<.005$ & .023 & $<.010$ & .004 & $<.050$ & $<.005$ \\
\hline & $7-15-92$ & $<.005$ & $<.005$ & $<.005$ & .035 & $<.010$ & .005 & $<.050$ & $<.005$ \\
\hline & $7-22-92$ & $<.005$ & $<.005$ & $<.005$ & $<.005$ & $<.010$ & $<.002$ & $<.050$ & $<.005$ \\
\hline & $8-12-92$ & $<.005$ & $<.005$ & $<.005$ & $<.005$ & $<.010$ & .009 & $<.050$ & $<.005$ \\
\hline \multirow[t]{7}{*}{3} & $7-10-91$ & $<.010$ & $<.005$ & .008 & .430 & $<.010$ & $<.005$ & $<.010$ & $<.010$ \\
\hline & $7-24-91$ & $<.010$ & $<.005$ & .005 & .039 & $<.010$ & .010 & $<.010$ & $<.010$ \\
\hline & $8-14-91$ & $<.010$ & $<.005$ & $<.005$ & $<.005$ & $<.010$ & $<.005$ & $<.010$ & $<.010$ \\
\hline & $6-24-92$ & $<.010$ & $<.005$ & $<.002$ & .043 & $<.010$ & .007 & $<.050$ & $<.005$ \\
\hline & $7-08-92$ & $<.005$ & $<.005$ & .042 & .056 & $<.010$ & .004 & $<.050$ & $<.005$ \\
\hline & $7-22-92$ & .013 & $<.005$ & $<.005$ & $<.005$ & $<.010$ & .007 & $<.050$ & $<.005$ \\
\hline & $7-29-92$ & $<.010$ & $<.005$ & $<.005$ & $<.005$ & $<.010$ & .005 & $<.050$ & $<.005$ \\
\hline \multirow[t]{11}{*}{5} & 7-03-91 & $<.005$ & $<.005$ & $<.005$ & $<.010$ & $<.010$ & $<.005$ & $<.050$ & $<.005$ \\
\hline & $7-10-91$ & $<.005$ & $<.005$ & $<.005$ & $<.010$ & $<.010$ & $<.005$ & $<.050$ & $<.005$ \\
\hline & $7-17-91$ & $<.005$ & $<.005$ & $<.005$ & $<.010$ & $<.010$ & $<.005$ & $<.050$ & $<.005$ \\
\hline & $7-24-91$ & $<.005$ & $<.005$ & $<.005$ & $<.010$ & $<.010$ & $<.005$ & $<.050$ & $<.005$ \\
\hline & 6-03-92 & $<.010$ & $<.005$ & .002 & .012 & $<.005$ & .005 & $<.010$ & $<.005$ \\
\hline & $6-17-92$ & $<.010$ & $<.005$ & .003 & .008 & $<.010$ & .005 & .024 & $<.005$ \\
\hline & $6-24-92$ & $<.010$ & $<.005$ & $<.002$ & .028 & $<.010$ & .005 & .029 & $<.005$ \\
\hline & $7-01-92$ & $<.010$ & $<.005$ & .060 & .045 & $<.010$ & .005 & .020 & $<.005$ \\
\hline & 7-08-92 & $<.010$ & $<.005$ & $<.005$ & $<.005$ & $<.010$ & $<.010$ & $<.050$ & $<.005$ \\
\hline & $7-29-92$ & $<.005$ & $<.005$ & $<.005$ & $<.005$ & $<.010$ & .008 & $<.050$ & $<.005$ \\
\hline & $8-05-92$ & $<.005$ & $<.005$ & $<.005$ & $<.005$ & $<.010$ & .003 & $<.050$ & $<.005$ \\
\hline \multirow[t]{11}{*}{7} & $7-10-91$ & $<.010$ & $<.005$ & $<.005$ & $<.010$ & $<.010$ & $<.010$ & $<.010$ & $<.010$ \\
\hline & $7-17-91$ & $<.010$ & $<.005$ & .006 & .190 & $<.010$ & .007 & $<.010$ & $<.010$ \\
\hline & $7-24-91$ & $<.010$ & $<.005$ & .005 & .053 & $<.010$ & $<.010$ & $<.010$ & $<.010$ \\
\hline & $6-17-92$ & $<.010$ & $<.005$ & .004 & .012 & $<.005$ & .004 & $<.050$ & .003 \\
\hline & $6-24-92$ & $<.010$ & $<.005$ & $<.002$ & .015 & $<.010$ & .004 & .024 & $<.005$ \\
\hline & $7-01-92$ & $<.010$ & $<.005$ & $<.002$ & $<.005$ & $<.010$ & $<.002$ & $<.050$ & $<.005$ \\
\hline & $7-08-92$ & $<.005$ & $<.005$ & .020 & .088 & $<.010$ & .003 & $<.050$ & $<.005$ \\
\hline & $7-15-92$ & $<.005$ & $<.005$ & .022 & .069 & $<.010$ & .005 & $<.050$ & $<.005$ \\
\hline & $7-29-92$ & $<.005$ & $<.005$ & $<.005$ & $<.005$ & $<.010$ & $<.002$ & $<.050$ & $<.005$ \\
\hline & $8-05-92$ & $<.005$ & .025 & $<.005$ & $<.005$ & $<.010$ & .006 & $<.050$ & $<.005$ \\
\hline & $8-19-92$ & $<.005$ & $<.005$ & $<.005$ & $<.005$ & $<.010$ & .006 & $<.050$ & $<.005$ \\
\hline
\end{tabular}


Table 12. Concentrations of pesticides and related compounds detected in surface water, 1991-92--Continued

\begin{tabular}{|c|c|c|c|c|c|c|c|c|c|}
\hline Site & Date & $\begin{array}{c}\text { Atra- } \\
\text { zine }\end{array}$ & $\begin{array}{l}\text { Benflu- } \\
\text { ralin }\end{array}$ & $\begin{array}{l}\text { Chlor- } \\
\text { pyrifos }\end{array}$ & $\begin{array}{c}\text { Cyana- } \\
\text { zine }\end{array}$ & $\begin{array}{l}\text { Disul- } \\
\text { foton }\end{array}$ & DDE & Eptam & $\begin{array}{l}\text { Etho- } \\
\text { prop }\end{array}$ \\
\hline \multirow[t]{9}{*}{9} & $7-10-91$ & $<0.002$ & $<0.010$ & $<0.010$ & $<0.010$ & $<0.050$ & $<0.010$ & $<0.005$ & $<0.005$ \\
\hline & $7-17-91$ & $<.002$ & $<.010$ & $<.010$ & $<.010$ & $<.050$ & $<.010$ & $<.005$ & $<.005$ \\
\hline & $7-24-91$ & $<.002$ & $<.010$ & $<.010$ & $<.010$ & $<.050$ & $<.010$ & $<.005$ & $<.005$ \\
\hline & $8-14-91$ & $<.002$ & $<.010$ & $<.010$ & $<.010$ & $<.050$ & $<.010$ & $<.005$ & $<.005$ \\
\hline & $6-10-92$ & $<.002$ & .003 & $<.002$ & $<.010$ & $<.050$ & $<.002$ & .027 & $<.005$ \\
\hline & $6-24-92$ & $<.002$ & $<.005$ & $<.002$ & $<.010$ & $<.050$ & $<.002$ & .008 & .004 \\
\hline & $7-08-92$ & $<.002$ & $<.005$ & $<.002$ & $<.010$ & $<.050$ & $<.002$ & .007 & $<.005$ \\
\hline & 7-29-92 & $<.002$ & $<.005$ & $<.010$ & $<.020$ & $<.050$ & $<.010$ & $<.002$ & $<.005$ \\
\hline & $8-26-92$ & $<.002$ & $<.005$ & $<.010$ & $<.020$ & $<.050$ & $<.010$ & $<.002$ & $<.005$ \\
\hline \multirow[t]{9}{*}{11} & $7-10-91$ & $<.002$ & $<.005$ & $<.010$ & $<.020$ & $<.050$ & $<.010$ & $<.005$ & .007 \\
\hline & $7-17-91$ & $<.002$ & $<.005$ & $<.010$ & $<.020$ & $<.050$ & $<.010$ & $<.005$ & $<.010$ \\
\hline & $7-24-91$ & $<.002$ & $<.005$ & $<.010$ & $<.020$ & $<.050$ & $<.010$ & $<.005$ & $<.010$ \\
\hline & $6-10-92$ & $<.002$ & $<.005$ & $<.002$ & $<.010$ & $<.050$ & $<.002$ & .042 & $<.005$ \\
\hline & $6-24-92$ & $<.002$ & $<.005$ & $<.002$ & $<.010$ & $<.050$ & $<.002$ & .041 & $<.005$ \\
\hline & $7-01-92$ & $<.002$ & $<.005$ & $<.002$ & $<.010$ & $<.050$ & $<.002$ & .023 & $<.005$ \\
\hline & $7-22-92$ & $<.002$ & $<.005$ & $<.002$ & $<.010$ & $<.050$ & $<.010$ & $<.002$ & $<.005$ \\
\hline & $7-29-92$ & $<.002$ & $<.005$ & $<.010$ & $<.020$ & $<.050$ & $<.010$ & $<.002$ & $<.005$ \\
\hline & $8-05-92$ & $<.002$ & $<.005$ & $<.010$ & $<.020$ & $<.050$ & $<.010$ & $<.002$ & $<.005$ \\
\hline \multirow[t]{3}{*}{12} & $6-03-92$ & $<.002$ & $<.005$ & $<.002$ & $<.010$ & $<.050$ & $<.002$ & $<.002$ & $<.005$ \\
\hline & $6-24-92$ & $<.002$ & .003 & $<.002$ & $<.010$ & $<.050$ & .001 & .006 & $<.005$ \\
\hline & $7-15-92$ & $<.002$ & $<.005$ & $<.002$ & $<.010$ & $<.050$ & $<.002$ & $<.002$ & $<.005$ \\
\hline \multirow[t]{7}{*}{13} & $6-03-92$ & $<.002$ & $<.005$ & $<.010$ & $<.020$ & $<.050$ & $<.010$ & .002 & $<.005$ \\
\hline & $6-17-92$ & $<.002$ & $<.005$ & $<.010$ & $<.020$ & $<.050$ & $<.010$ & $<.002$ & $<.005$ \\
\hline & $7-15-92$ & $<.002$ & $<.005$ & $<.010$ & $<.020$ & $<.050$ & $<.010$ & $<.002$ & $<.005$ \\
\hline & $7-29-92$ & $<.002$ & $<.005$ & $<.010$ & $<.020$ & $<.050$ & $<.010$ & $<.002$ & $<.005$ \\
\hline & $8-05-92$ & $<.002$ & $<.005$ & $<.010$ & $<.020$ & $<.050$ & $<.010$ & $<.002$ & $<.005$ \\
\hline & $8-12-92$ & $<.002$ & $<.005$ & $<.010$ & $<.020$ & $<.050$ & $<.010$ & $<.002$ & $<.005$ \\
\hline & $8-26-92$ & $<.002$ & $<.005$ & $<.010$ & $<.020$ & $<.050$ & $<.010$ & $<.002$ & $<.005$ \\
\hline \multirow[t]{7}{*}{15} & $6-10-92$ & $<.002$ & $<.005$ & $<.002$ & $<.010$ & $<.050$ & $<.002$ & .001 & $<.005$ \\
\hline & $6-17-92$ & $<.002$ & $<.005$ & $<.002$ & $<.010$ & $<.050$ & $<.002$ & $<.002$ & $<.005$ \\
\hline & $6-24-92$ & $<.002$ & $<.005$ & $<.002$ & $<.010$ & $<.050$ & $<.002$ & $<.002$ & $<.005$ \\
\hline & $7-01-92$ & $<.002$ & $<.005$ & $<.002$ & $<.010$ & $<.050$ & $<.010$ & $<.002$ & $<.005$ \\
\hline & $7-22-92$ & $<.002$ & $<.005$ & $<.010$ & $<.020$ & $<.050$ & $<.010$ & $<.002$ & $<.005$ \\
\hline & $8-12-92$ & $<.002$ & $<.005$ & $<.010$ & $<.020$ & $<.050$ & $<.010$ & $<.002$ & $<.005$ \\
\hline & $8-26-92$ & $<.002$ & $<.005$ & $<.010$ & $<.020$ & $<.050$ & $<.010$ & $<.002$ & $<.005$ \\
\hline
\end{tabular}

Other compounds analyzed for but not detected

\begin{tabular}{llll}
\hline Alachlor & Diethylanaline, 2,6- & Dieldrin & $\alpha-\mathrm{HCH}$ \\
Butylate & Dacthal & Dimethoate & $\gamma-\mathrm{HCH}$ (Lindane) \\
Carbaryl & Desethylatrazine & Ethalfluralin & Linuron \\
Carbofuron & Diazinon & Fonofos & \\
\hline
\end{tabular}


Table 12. Concentrations of pesticides and related compounds detected in surface water, 1991-92--Continued

\begin{tabular}{|c|c|c|c|c|c|c|c|c|c|}
\hline Site & Date & $\begin{array}{l}\text { Mala- } \\
\text { thion }\end{array}$ & $\begin{array}{c}\text { Methyl } \\
\text { parathion }\end{array}$ & $\begin{array}{l}\text { Metola- } \\
\text { chlor }\end{array}$ & $\begin{array}{l}\text { Metri- } \\
\text { buzin }\end{array}$ & $\begin{array}{l}\text { Prona- } \\
\text { mide }\end{array}$ & $\begin{array}{l}\text { Sima- } \\
\text { zine }\end{array}$ & $\begin{array}{l}\text { Terbu- } \\
\text { fos }\end{array}$ & $\begin{array}{l}\text { Triflu- } \\
\text { ralin }\end{array}$ \\
\hline \multirow[t]{9}{*}{9} & $7-10-91$ & $<0.010$ & $<0.005$ & 0.005 & 0.028 & $<0.010$ & 0.006 & $<0.010$ & $<0.010$ \\
\hline & $7-17-91$ & $<.010$ & $<.005$ & .002 & $<.010$ & $<.010$ & .004 & $<.010$ & $<.010$ \\
\hline & $7-24-91$ & $<.010$ & $<.005$ & .005 & .047 & $<.010$ & $<.005$ & $<.010$ & $<.010$ \\
\hline & 8-14-91 & $<.010$ & $<.005$ & .008 & $<.010$ & $<.010$ & $<.005$ & $<.010$ & $<.010$ \\
\hline & $6-10-92$ & $<.010$ & $<.005$ & $<.002$ & .010 & .006 & .005 & $<.050$ & .003 \\
\hline & 6-24-92 & $<.010$ & $<.005$ & $<.002$ & .015 & $<.010$ & .004 & .024 & $<.005$ \\
\hline & 7-08-92 & $<.010$ & $<.005$ & $<.005$ & $<.010$ & $<.010$ & .004 & $<.050$ & $<.005$ \\
\hline & 7-29-92 & $<.005$ & $<.005$ & $<.005$ & $<.010$ & $<.010$ & $<.002$ & $<.050$ & $<.005$ \\
\hline & $8-26-92$ & $<.005$ & $<.005$ & $<.005$ & $<.010$ & $<.010$ & .004 & $<.050$ & $<.005$ \\
\hline \multirow[t]{9}{*}{11} & 7-10-91 & $<.010$ & $<.010$ & .005 & $<.010$ & $<.010$ & .010 & $<.010$ & $<.010$ \\
\hline & $7-17-91$ & $<.010$ & $<.010$ & .005 & .013 & $<.010$ & .010 & $<.010$ & $<.010$ \\
\hline & $7-24-91$ & $<.010$ & $<.010$ & .005 & $<.010$ & $<.010$ & $<.005$ & $<.010$ & $<.010$ \\
\hline & $6-10-92$ & $<.010$ & $<.005$ & $<.002$ & $<.010$ & .009 & .010 & $<.005$ & $<.005$ \\
\hline & $6-24-92$ & $<.010$ & $<.005$ & $<.002$ & $<.010$ & $<.010$ & .005 & .039 & $<.005$ \\
\hline & 7-01-92 & $<.010$ & $<.005$ & $<.002$ & .008 & $<.010$ & .007 & .020 & $<.005$ \\
\hline & $7-22-92$ & $<.010$ & $<.005$ & $<.005$ & $<.010$ & $<.010$ & .005 & $<.005$ & $<.005$ \\
\hline & $7-29-92$ & $<.010$ & $<.005$ & .012 & $<.010$ & $<.010$ & .004 & $<.005$ & $<.005$ \\
\hline & 8-05-92 & $<.010$ & $<.005$ & $<.005$ & $<.010$ & $<.010$ & .003 & $<.005$ & $<.005$ \\
\hline \multirow[t]{3}{*}{12} & 6-03-92 & $<.010$ & $<.005$ & $<.002$ & $<.010$ & .011 & $<.005$ & $<.010$ & $<.005$ \\
\hline & $6-24-92$ & $<.010$ & $<.005$ & $<.002$ & $<.010$ & $<.010$ & $<.005$ & .027 & .003 \\
\hline & $7-15-92$ & $<.005$ & $<.005$ & $<.005$ & $<.005$ & $<.010$ & $<.005$ & $<.050$ & $<.005$ \\
\hline \multirow[t]{7}{*}{13} & 6-03-92 & $<.005$ & $<.005$ & $<.005$ & .003 & .007 & $<.010$ & $<.010$ & $<.005$ \\
\hline & $6-17-92$ & $<.005$ & $<.005$ & $<.005$ & $<.005$ & $<.010$ & $<.020$ & $<.010$ & $<.005$ \\
\hline & $7-15-92$ & $<.005$ & $<.005$ & $<.005$ & $<.005$ & $<.010$ & $<.020$ & $<.010$ & $<.005$ \\
\hline & 7-29-92 & & & & & $<.010$ & $<.020$ & $<.010$ & $<.005$ \\
\hline & 8-05-92 & $<.005$ & $<.005$ & $<.005$ & $<.005$ & $<.010$ & $<.020$ & $<.010$ & $<.005$ \\
\hline & $8-12-92$ & $<.005$ & $<.005$ & $<.005$ & $<.005$ & $<.010$ & $<.020$ & $<.010$ & $<.005$ \\
\hline & $8-26-92$ & $<.005$ & $<.005$ & $<.005$ & $<.005$ & $<.010$ & $<.020$ & $<.010$ & $<.005$ \\
\hline \multirow[t]{7}{*}{15} & $6-10-92$ & $<.010$ & $<.005$ & .001 & $<.010$ & .008 & $<.005$ & $<.010$ & $<.005$ \\
\hline & $6-17-92$ & $<.010$ & $<.005$ & $<.002$ & $<.010$ & $<.010$ & $<.005$ & $<.010$ & $<.005$ \\
\hline & 6-24-92 & $<.010$ & $<.005$ & $<.002$ & $<.010$ & $<.010$ & $<.005$ & $<.010$ & $<.005$ \\
\hline & $7-01-92$ & $<.010$ & $<.005$ & $<.002$ & $<.010$ & $<.010$ & $<.005$ & $<.010$ & $<.005$ \\
\hline & $7-22-92$ & $<.010$ & $<.050$ & $<.005$ & $<.010$ & $<.010$ & $<.005$ & $<.010$ & $<.005$ \\
\hline & $8-12-92$ & $<.005$ & $<.005$ & $<.005$ & $<.005$ & $<.010$ & $<.005$ & $<.010$ & $<.005$ \\
\hline & $8-26-92$ & $<.005$ & $<.005$ & $<.005$ & $<.005$ & $<.010$ & $<.050$ & $<.010$ & $<.005$ \\
\hline
\end{tabular}

Other compounds analyzed for but not detected

\begin{tabular}{llll}
\hline Methyl azimphos & Pebulate & Prometon & Tebuthiuron \\
Molinate & Pendimethalin & Propachlor & Terbacil \\
Napropamide & Permethrin & Propanil & Thiobencarb \\
Parathion & Phorate & Propargite & Triallate \\
\hline
\end{tabular}


Table 13. Concentrations of acrolein (Magnicide$H^{(B)}$ in sites downstream from an acrolein application in the J-7 Canal, July 1992

[See figure 2 for location of injection point. Postapplication water samples were collected from the 44 Drain and the $\mathrm{J}-7$ and $\mathrm{N}$ Canals. $\mathrm{km}$, kilometer; $\mathrm{mg} / \mathrm{L}$, milligram per liter; <, actual value is less than value shown]

\begin{tabular}{cccc}
\hline Date & Time & $\begin{array}{c}\text { Distance } \\
\text { downstream } \\
(\mathrm{km})\end{array}$ & $\begin{array}{c}\text { Acrolein } \\
(\mathrm{mg} / \mathrm{L})\end{array}$ \\
\hline July 13 & 0950 & 1.0 & $<0.02$ \\
& 1035 & 1.0 & $<.02$ \\
& 1120 & 1.0 & $<.02$ \\
& 1235 & .6 & 4.5 \\
& 1305 & 2.6 & $<.02$ \\
& 1405 & 3.2 & $<.02$ \\
& 1605 & Injection point & $<.02$ \\
July 14 & 1605 & 3.5 & $<.02$ \\
July 15 & 1620 & 1.0 & $<.02$ \\
& 1100 & 4.8 & $<.02$ \\
& 0830 & Injection point & $<.02$ \\
& 0903 & 3.5 & $<.02$ \\
& 0930 & 4.8 & .027 \\
\hline
\end{tabular}

Table 14. Concentrations of methamidophos recovered from deposition samplers placed in leaseland waterways at Tule Lake National Wildlife Refuge, 1992

[See figure 4 for site locations. The method detection limit for methamidophos was 2.0 total $\mu \mathrm{g}$. $\mu \mathrm{g}$, microgram; $\mu \mathrm{g} / \mathrm{m}^{2}$, microgram per square meter]

\begin{tabular}{lcccc}
\hline Site & $\begin{array}{c}\text { Sample } \\
\text { identification }\end{array}$ & Date & $\begin{array}{c}\text { Total } \\
\text { recovery } \\
(\mu \mathrm{g})\end{array}$ & $\begin{array}{c}\text { Concentration } \\
\left(\mu \mathrm{g} / \mathrm{m}^{2}\right)\end{array}$ \\
\hline LL224 & $224 \mathrm{~A} 0810$ & Aug. 10 & 4.0 & 1,039 \\
& $224 \mathrm{~B} 0810$ & Aug. 10 & 2.4 & 624 \\
LL226 & $224 \mathrm{C} 0810$ & Aug. 10 & 3.6 & 935 \\
& $226 \mathrm{A0802}$ & Aug. 2 & 4.4 & 1,143 \\
& $226 \mathrm{~B} 0802$ & Aug. 2 & 3.6 & 935 \\
LL227 & $226 \mathrm{C} 0802$ & Aug. 2 & 3.6 & 935 \\
& $227 \mathrm{A0802}$ & Aug. 2 & 4.4 & 1,143 \\
& $227 \mathrm{~B} 0802$ & Aug. 2 & 4.0 & 1,039 \\
& $227 \mathrm{C} 0802$ & Aug. 2 & 4.8 & 1,247 \\
\hline
\end{tabular}

Table 15. Concentrations of trace elements in blue-green algae (Aphanezomenon flos-aquae) samples, September 1991

[See figure 1 for site locations. Trace-element values in micrograms per gram, wet weight; <, actual value is less than value shown]

\begin{tabular}{|c|c|c|c|}
\hline & \multicolumn{3}{|c|}{ Site } \\
\hline & 1 & 3 & 13 \\
\hline Moisture content (percent) & 98.7 & 96.1 & 97.8 \\
\hline Aluminum $\ldots \ldots \ldots \ldots$ & 6.2 & 69.4 & 14.2 \\
\hline Arsenic $\ldots \ldots \ldots \ldots \ldots$ & .02 & .07 & .06 \\
\hline Barium & .12 & .40 & .28 \\
\hline Beryllium & $<.001$ & $<.001$ & $<.001$ \\
\hline Boron ... & .16 & .47 & .29 \\
\hline Cadmium & .007 & .012 & .022 \\
\hline Chromium & $<.007$ & .055 & .016 \\
\hline Copper . . . & .06 & .32 & .24 \\
\hline Iron $\ldots$ & 8.6 & 56.1 & 18.4 \\
\hline Lead. & $<.03$ & .04 & .04 \\
\hline Magnesium & 35.1 & 82.6 & 42.8 \\
\hline Manganese & 1.4 & 4.7 & 4.2 \\
\hline Mercury . . & $<.001$ & .002 & .002 \\
\hline Molybdenum & .04 & .08 & .04 \\
\hline Nickel .... & .04 & .26 & .14 \\
\hline Selenium & .004 & $<.008$ & .031 \\
\hline Strontium & .38 & .85 & .44 \\
\hline Vanadium & .04 & .43 & .08 \\
\hline Zinc $\ldots$. & .1 & .51 & 38.2 \\
\hline
\end{tabular}


Table 16. Moisture content and concentrations of arsenic, mercury, and selenium in biological tissue samples, 1990-92

[Sample locations described in table 2. Trace-element values in milligrams per kilogram, wet weight; <, actual value is less than value shown; --, no data; aquatic invertebrates, - 10-gram composites; fish, 20-gram composites]

\begin{tabular}{|c|c|c|c|c|c|c|}
\hline $\begin{array}{l}\text { Sample } \\
\text { type }\end{array}$ & $\begin{array}{l}\text { Sample } \\
\text { No. }\end{array}$ & Date & $\begin{array}{l}\text { Moisture content } \\
\quad \text { (percent) }\end{array}$ & Arsenic & Mercury & Selenium \\
\hline \multicolumn{7}{|c|}{ Aquatic invertebrates } \\
\hline Backswimmer & LK6BS & $6-23-92$ & 75.6 & 2.70 & 0.029 & 4.41 \\
\hline Waterboatman & LK6WB & $6-23-92$ & 76.0 & .72 & .048 & 2.88 \\
\hline \multicolumn{7}{|c|}{ Fish } \\
\hline \multirow[t]{3}{*}{ Fathead minnow } & 12FH01J & $6-23-92$ & 88.2 & -- & 0.038 & -- \\
\hline & $12 \mathrm{FH} 02 \mathrm{~J}$ & $6-23-92$ & 88.3 & -- & .027 & -. \\
\hline & $12 \mathrm{FH} 03 \mathrm{~J}$ & $6-23-92$ & 83.5 & -- & .039 & -- \\
\hline \multirow[t]{21}{*}{ Tui chub } & CLTC01A & $6-24-92$ & 73.8 & -- & .098 & -- \\
\hline & CLTC01J & $6-24-92$ & 75.3 & -- & .042 & -. \\
\hline & CLTC02A & $6-24-92$ & 75.0 & -- & .090 & -- \\
\hline & CLTC02J & $6-24-92$ & 73.4 & -- & .056 & -- \\
\hline & CLTC03A & $6-24-92$ & 74.7 & -- & .098 & -- \\
\hline & CLTC03J & $6-24-92$ & 75.3 & -- & .045 & -- \\
\hline & $2 \mathrm{TC} 01 \mathrm{~A}$ & $6-24-92$ & 74.5 & -- & .074 & -- \\
\hline & $2 \mathrm{TC} 01 \mathrm{~J}$ & $6-24-92$ & 76.7 & -- & .057 & -- \\
\hline & $2 \mathrm{TC} 02 \mathrm{~A}$ & $6-24-92$ & 74.8 & -- & .077 & -- \\
\hline & $2 \mathrm{TC} 02 \mathrm{~J}$ & $6-24-92$ & 75.8 & -- & .055 & -- \\
\hline & $2 \mathrm{TC} 03 \mathrm{~A}$ & $6-24-92$ & 75.8 & -- & .055 & -- \\
\hline & $2 \mathrm{TC} 03 \mathrm{~J}$ & $6-24-92$ & 74.8 & -- & .050 & -- \\
\hline & $3 \mathrm{TC} 02 \mathrm{~J}$ & $6-25-92$ & 88.6 & -- & .061 & -- \\
\hline & $3 \mathrm{TC} 03 \mathrm{~J}$ & $6-25-92$ & 84.1 & -- & .062 & -- \\
\hline & 7TC01J & $6-26-92$ & 84.4 & -- & .031 & -- \\
\hline & 7ТC02J & $6-26-92$ & 85.4 & -- & .032 & -- \\
\hline & 7TC03J & $6-26-92$ & 19.8 & -- & .029 & -- \\
\hline & $11 \mathrm{TC} 01 \mathrm{~J}$ & $6-25-92$ & 80.6 & -- & .017 & -- \\
\hline & $11 \mathrm{TC} 02 \mathrm{~J}$ & $6-25-92$ & 80.1 & -- & .027 & -- \\
\hline & $11 \mathrm{TC} 03 \mathrm{~J}$ & $6-25-92$ & 79.5 & -- & .026 & -- \\
\hline & \multicolumn{6}{|c|}{ Birds } \\
\hline \multirow[t]{11}{*}{ American avocet egg } & 4BA1 & $6-15-91$ & 73.7 & $<0.13$ & 0.028 & 0.33 \\
\hline & $4 \mathrm{BA} 2$ & $6-15-91$ & 73.0 & $<.13$ & .086 & .77 \\
\hline & $4 \mathrm{BA} 3$ & $6-15-91$ & 74.0 & $<.13$ & .030 & .57 \\
\hline & 4BA4 & $6-15-91$ & 74.0 & $<.13$ & .040 & .25 \\
\hline & $4 \mathrm{BA} 6$ & $6-15-91$ & 72.9 & $<.13$ & .066 & .25 \\
\hline & $4 \mathrm{BA} 7$ & $6-15-91$ & 74.6 & $<.13$ & .046 & .33 \\
\hline & 4BA8 & $6-15-91$ & 73.9 & $<.13$ & .060 & .33 \\
\hline & 4BA9 & $6-15-91$ & 73.6 & $<.13$ & .027 & .49 \\
\hline & 4BA10 & $6-15-91$ & 72.1 & $<.13$ & .071 & .33 \\
\hline & 4BA11 & $6-15-91$ & 75.4 & $<.13$ & .042 & .40 \\
\hline & 4BA12 & $6-15-91$ & 73.0 & $<.13$ & .054 & $<.17$ \\
\hline
\end{tabular}


Table 16. Moisture content and concentrations of arsenic, mercury, and selenium in biological tissue samples, 1990-92--Continued

\begin{tabular}{|c|c|c|c|c|c|c|}
\hline $\begin{array}{l}\text { Sample } \\
\text { type }\end{array}$ & $\begin{array}{c}\text { Sample } \\
\text { No. }\end{array}$ & Date & $\begin{array}{l}\text { Moisture content } \\
\quad \text { (percent) }\end{array}$ & Arsenic & Mercury & Selenium \\
\hline \multicolumn{7}{|c|}{ Birds--Continued } \\
\hline \multirow[t]{10}{*}{ American coot egg } & $7 \mathrm{AC} 1$ & $8-15-91$ & 73.9 & $<0.13$ & 0.050 & -- \\
\hline & $7 \mathrm{AC} 2$ & $8-15-91$ & 73.6 & $<.13$ & .055 & -- \\
\hline & $7 \mathrm{AC} 3$ & $8-15-91$ & 73.6 & $<.13$ & .063 & -- \\
\hline & $7 \mathrm{AC} 4$ & $8-15-91$ & 75.9 & $<.13$ & .123 & -- \\
\hline & $7 \mathrm{AC5}$ & $8-15-91$ & 76.1 & $<.13$ & .076 & -- \\
\hline & LK12CC1 & $6-29-91$ & 70.3 & $<.13$ & .188 & -- \\
\hline & LK12CC2 & $6-29-91$ & 74.7 & $<.13$ & .161 & -- \\
\hline & LK12CC3 & $6-29-91$ & 74.6 & $<.13$ & .142 & -- \\
\hline & LK12CC4 & $6-29-91$ & 75.6 & $<.13$ & .196 & -- \\
\hline & LK12CC5 & $6-29-91$ & 76.0 & $<.13$ & .113 & -- \\
\hline Egret egg & E1 & $7-02-90$ & 79.0 & -- & .084 & -- \\
\hline \multirow[t]{11}{*}{ Mallard egg } & M441 & $6-16-90$ & 61.9 & -- & .104 & -- \\
\hline & M447 & $5-15-90$ & 58.5 & -- & .244 & -- \\
\hline & M459A & $5-15-90$ & 56.5 & -- & .080 & -- \\
\hline & M459B & $5-15-90$ & 52.8 & -- & .147 & -- \\
\hline & M499A & $6-12-90$ & 63.0 & -- & .330 & -- \\
\hline & M499B & $6-12-90$ & 62.8 & -- & .050 & -- \\
\hline & M516A & $6-08-90$ & 59.5 & -- & .036 & -- \\
\hline & M516B & $6-08-90$ & 62.1 & -- & .033 & -- \\
\hline & M563 & $6-19-90$ & 57.7 & -- & .080 & -- \\
\hline & M574 & $6-19-90$ & 61.5 & -- & $<.025$ & -- \\
\hline & $92 \mathrm{M} 2$ & $7-01-92$ & 70.1 & -- & .149 & -- \\
\hline \multirow[t]{25}{*}{ Western grebe egg } & $3 \mathrm{AG} 1$ & $6-20-91$ & 77.6 & $<.13$ & .093 & -- \\
\hline & $3 \mathrm{AG} 2$ & $6-20-91$ & 76.7 & $<.13$ & .123 & -- \\
\hline & 3AG3 & $6-20-91$ & 76.7 & -- & .065 & -- \\
\hline & $3 \mathrm{AG} 4$ & $6-20-91$ & 78.1 & -- & .115 & -- \\
\hline & 3AG5 & $6-20-91$ & 75.8 & $<.13$ & .066 & -- \\
\hline & 3AG6 & $6-20-91$ & 77.2 & $<.13$ & .097 & -- \\
\hline & TLG1 & $6-29-91$ & 77.8 & $<.13$ & .077 & -- \\
\hline & TLG2 & $6-29-91$ & 76.7 & $<.13$ & .063 & -- \\
\hline & TLG3 & $6-29-91$ & 77.9 & $<.13$ & .072 & -- \\
\hline & TLG4 & $6-29-91$ & 78.2 & $<.13$ & .098 & -- \\
\hline & TLG5 & $6-29-91$ & 76.7 & $<.13$ & .139 & -- \\
\hline & TLG6 & $6-29-91$ & 78.2 & -- & .054 & -- \\
\hline & TLG7 & $6-29-91$ & 74.5 & -- & .084 & -- \\
\hline & TLG8 & $6-29-91$ & 78.2 & -- & .043 & -- \\
\hline & TLG9 & $6-29-91$ & 78.5 & -- & .063 & -- \\
\hline & TLG10 & $6-29-91$ & 78.6 & -- & .050 & -- \\
\hline & 92TLWG1 & $6-08-91$ & 80.4 & -- & .081 & -- \\
\hline & 92 $\mathrm{TLWG} 2 \mathrm{~A}$ & $6-08-91$ & 74.3 & -- & .053 & -- \\
\hline & 92TLWG2B & $6-08-91$ & 78.9 & -- & .053 & -- \\
\hline & 92TLWG3A & $6-08-91$ & 80.0 & -- & .078 & -- \\
\hline & 92TLWG3B & $6-08-91$ & 79.9 & -- & .075 & -- \\
\hline & 92TLWG5A & $6-08-91$ & 74.6 & -- & .079 & -- \\
\hline & 92TLWG6A & $6-08-91$ & 77.9 & -- & .072 & -- \\
\hline & 92TLWG7A & $6-08-91$ & 78.0 & -- & .089 & -- \\
\hline & 92TLWG8 & $6-08-91$ & 78.0 & -- & .108 & -- \\
\hline
\end{tabular}


Table 16. Moisture content and concentrations of arsenic, mercury, and selenium in biological tissue samples , 1990-92--Continued

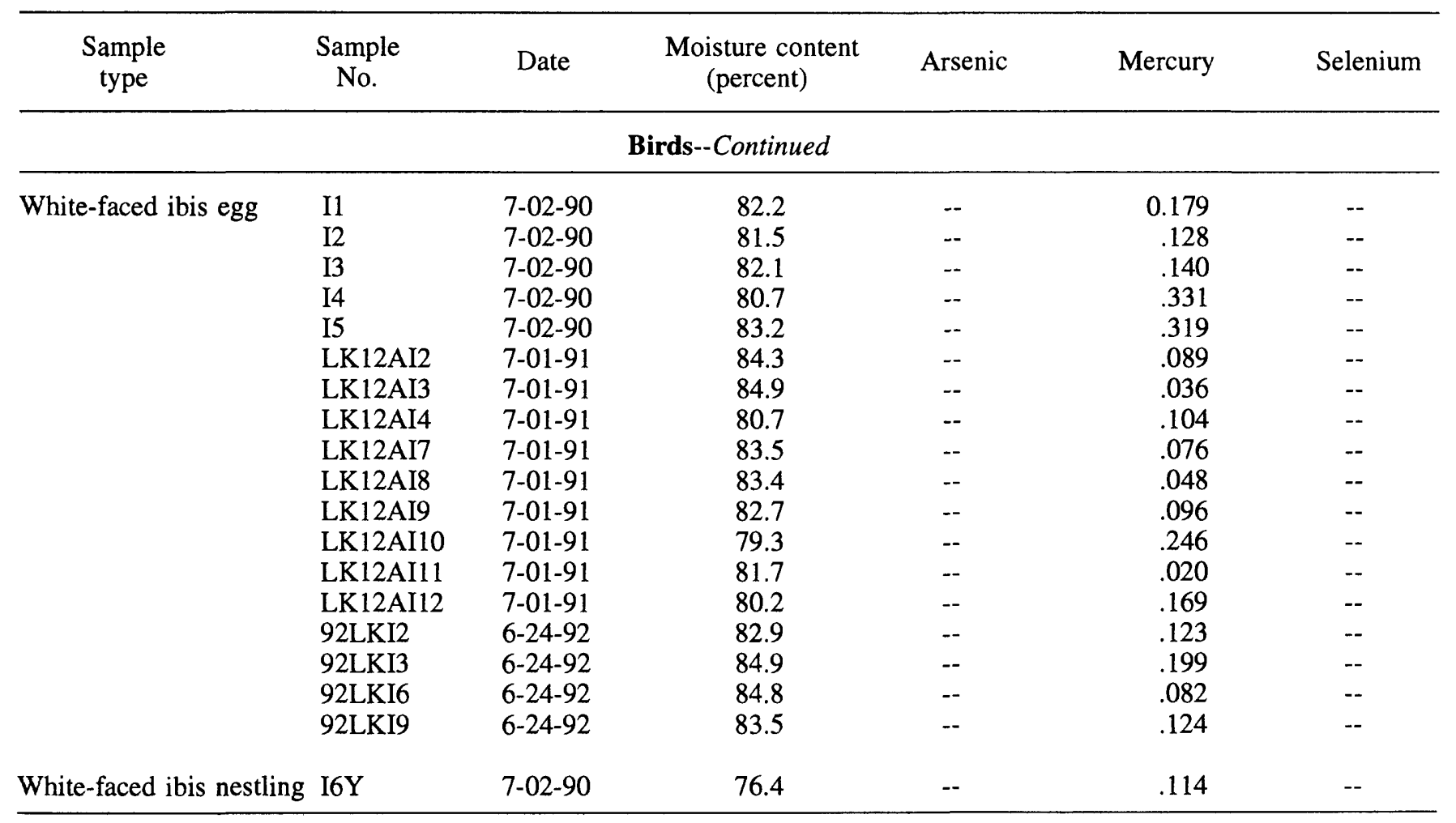


Table 17. Moisture content, lipid content, and concentrations of organochlorine compounds in biological tissue samples, 1990-92

[Sample locations described in table 2. Detections are shown in bold print. Concentrations in micrograms per gram, wet weight; <, actual value is less than value shown; --, no data; aquatic invertebrates, 10-gram composites; fish, 20-gram composites]

\begin{tabular}{|c|c|c|c|c|c|c|c|c|c|}
\hline Sample type & $\begin{array}{c}\text { Sample } \\
\text { No. }\end{array}$ & Date & $\begin{array}{l}\text { Moisture } \\
\text { content } \\
\text { (percent) }\end{array}$ & $\begin{array}{c}\text { Lipid } \\
\text { content } \\
\text { (percent) }\end{array}$ & $\begin{array}{l}\text { BHC } \\
\text { alpha }\end{array}$ & $\begin{array}{l}\text { BHC } \\
\text { beta }\end{array}$ & $\begin{array}{l}\text { BHC } \\
\text { delta }\end{array}$ & $\begin{array}{c}\text { BHC } \\
\text { gamma }\end{array}$ & $\begin{array}{l}\text { Alpha- } \\
\text { chlor- } \\
\text { dane }\end{array}$ \\
\hline Chironomid larvae & $\begin{array}{l}\text { TLCH07A } \\
\text { TLCH08A }\end{array}$ & $\begin{array}{l}8-91 \\
8-91\end{array}$ & $\begin{array}{l}91.1 \\
96.0\end{array}$ & $\begin{array}{r}1.2 \\
.5\end{array}$ & $\begin{array}{r}<0.01 \\
<.01\end{array}$ & $\begin{array}{r}<0.01 \\
<.01\end{array}$ & $\begin{array}{r}<0.01 \\
<.01\end{array}$ & $\begin{array}{r}<0.01 \\
<.01\end{array}$ & $\begin{array}{r}<0.01 \\
<.01\end{array}$ \\
\hline Leeches & TLL07A & $8-91$ & 89.0 & .9 & $<.01$ & $<.01$ & $<.01$ & $<.01$ & $<.01$ \\
\hline \multicolumn{10}{|c|}{ Fish } \\
\hline Fathead minnow & $12 \mathrm{FH} 01 \mathrm{~J}$ & $6-23-92$ & 87.2 & 2.4 & $<0.01$ & $<0.01$ & -- & $<0.01$ & $<0.01$ \\
\hline \multicolumn{10}{|c|}{ Birds } \\
\hline Western grebe egg & $\begin{array}{l}\text { 3AG3 } \\
\text { 3AG4 } \\
\text { 3AG7 } \\
\text { TLG6 } \\
\text { TLG7 } \\
\text { TLG8 } \\
\text { TLG9 } \\
\text { TLG10 } \\
\text { 92TLWG1 } \\
\text { 92TLWG2A } \\
\text { 92TLWG2B } \\
\text { 92TLWG3A } \\
\text { 92TLWG3B } \\
\text { 92TLWG5A } \\
\text { 92TLWG6A } \\
\text { 92TLWG7A } \\
\text { 92TLWG8 }\end{array}$ & $\begin{array}{l}6-20-91 \\
6-20-91 \\
6-20-91 \\
6-29-91 \\
6-29-91 \\
6-29-92 \\
6-29-91 \\
6-29-91 \\
7-08-92 \\
7-08-92 \\
7-08-92 \\
7-08-92 \\
7-08-92 \\
7-08-92 \\
7-08-92 \\
7-08-92 \\
7-08-92\end{array}$ & $\begin{array}{l}74.5 \\
75.8 \\
75.8 \\
76.2 \\
80.8 \\
77 \\
75.4 \\
76.0 \\
79.9 \\
74.3 \\
77.7 \\
80.8 \\
78.9 \\
75.9 \\
77.5 \\
79.1 \\
78.8\end{array}$ & $\begin{array}{r}10.1 \\
9.5 \\
9.1 \\
10.4 \\
11.1 \\
10.3 \\
10.2 \\
9.5 \\
10.4 \\
10.1 \\
10.9 \\
7.6 \\
7.8 \\
9.8 \\
8.5 \\
10.8 \\
9.1\end{array}$ & $\begin{array}{l}<.01 \\
<.01 \\
<.02 \\
<.01 \\
<.01 \\
<.01 \\
<.01 \\
<.01 \\
<.01 \\
<.01 \\
<.01 \\
<.01 \\
<.01 \\
<.01 \\
<.01 \\
<.01 \\
<.01\end{array}$ & $\begin{array}{l}<.01 \\
<.01 \\
<.02 \\
<.01 \\
<.01 \\
<.01 \\
<.01 \\
<.01 \\
<.01 \\
<.01 \\
<.01 \\
<.01 \\
<.01 \\
<.01 \\
<.01 \\
<.01 \\
<.01\end{array}$ & $\begin{array}{l}-- \\
-- \\
-- \\
-- \\
-- \\
-- \\
-- \\
-- \\
-- \\
-- \\
-- \\
-- \\
-- \\
-- \\
- \\
- \\
--\end{array}$ & $\begin{array}{l}<.01 \\
<.01 \\
<.02 \\
<.01 \\
<.01 \\
<.01 \\
<.01 \\
<.01 \\
<.01 \\
<.01 \\
<.01 \\
<.01 \\
<.01 \\
<.01 \\
<.01 \\
<.01 \\
<.01\end{array}$ & $\begin{array}{r}<.01 \\
<.01 \\
<.02 \\
<.01 \\
<.01 \\
.01 \\
<.01 \\
<.01 \\
<.01 \\
<.01 \\
<.01 \\
<.01 \\
<.01 \\
<.01 \\
<.01 \\
<.01 \\
<.01\end{array}$ \\
\hline
\end{tabular}


Table 17. Moisture content, lipid content, and concentrations of organochlorine compounds in biological tissue samples, 1990-92--Continued

\begin{tabular}{|c|c|c|c|c|c|c|c|c|c|c|}
\hline Sample type & $\begin{array}{c}\text { Sample } \\
\text { No. }\end{array}$ & Date & $\begin{array}{c}\text { Gamma } \\
\text { chlor- } \\
\text { dane }\end{array}$ & $\begin{array}{l}\text { Oxychlor- } \\
\text { dane }\end{array}$ & $\begin{array}{c}o, p^{\prime} \\
\text { DDD }\end{array}$ & $\begin{array}{l}\text { p,p' } \\
\text { DDD }\end{array}$ & $\begin{array}{l}\text { p,p' } \\
\text { DDE }\end{array}$ & $\begin{array}{l}o, p^{\prime} \\
\text { DDE }\end{array}$ & $\begin{array}{l}\text { p,p' } \\
\text { DDT }\end{array}$ & $\begin{array}{l}o, p^{\prime} \\
\text { DDT }\end{array}$ \\
\hline Chironomid larvae & $\begin{array}{l}\text { TLCH07A } \\
\text { TLCH08A }\end{array}$ & $\begin{array}{l}8-91 \\
8-91\end{array}$ & $\begin{array}{r}<0.01 \\
<.01\end{array}$ & $\begin{array}{r}<0.01 \\
<.01\end{array}$ & $\begin{array}{r}<0.01 \\
<.01\end{array}$ & $\begin{array}{r}<0.01 \\
<.01\end{array}$ & $\begin{array}{r}<0.01 \\
<.01\end{array}$ & $\begin{array}{r}<0.01 \\
<.01\end{array}$ & $\begin{array}{r}<0.01 \\
<.01\end{array}$ & $\begin{array}{r}<0.01 \\
<.01\end{array}$ \\
\hline \multicolumn{11}{|c|}{ Fish } \\
\hline Fathead minnow & $12 \mathrm{FH} 01 \mathrm{~J}$ & $6-23-92$ & $<0.01$ & $<0.01$ & $<0.01$ & $<0.01$ & $<0.01$ & $<0.01$ & $<0.01$ & $<0.01$ \\
\hline Tui chub & $\begin{array}{l}\text { CLTC01A } \\
\text { CLTC03J } \\
2 T C 01 A \\
2 T C 01 J \\
2 T C 02 A \\
2 T C 03 A \\
3 T C 01 J \\
7 T C 01 J \\
7 T C 02 J \\
7 T C 03 J \\
11 T C 01 J \\
11 T C 02 J \\
11 T C 03 J\end{array}$ & $\begin{array}{l}6-24-92 \\
6-24-92 \\
6-24-92 \\
6-24-92 \\
6-24-92 \\
6-24-92 \\
6-25-92 \\
6-26-92 \\
6-26-92 \\
6-26-92 \\
6-25-92 \\
6-25-92 \\
6-25-92\end{array}$ & $\begin{array}{l}<.01 \\
<.01 \\
<.01 \\
<.01 \\
<.01 \\
<.01 \\
<.01 \\
<.01 \\
<.01 \\
<.01 \\
<.01 \\
<.01 \\
<.01\end{array}$ & $\begin{array}{l}<.01 \\
<.01 \\
<.01 \\
<.01 \\
<.01 \\
<.01 \\
<.01 \\
<.01 \\
<.01 \\
<.01 \\
<.01 \\
<.01 \\
<.01\end{array}$ & $\begin{array}{l}<.01 \\
<.01 \\
<.01 \\
<.01 \\
<.01 \\
<.01 \\
<.01 \\
<.01 \\
<.01 \\
<.01 \\
<.01 \\
<.01 \\
<.01\end{array}$ & $\begin{array}{l}<.01 \\
<.01 \\
<.01 \\
<.01 \\
<.01 \\
<.01 \\
<.01 \\
<.01 \\
<.01 \\
<.01 \\
<.01 \\
<.01 \\
<.01\end{array}$ & $\begin{array}{r}<.01 \\
<.01 \\
<.01 \\
<.01 \\
.01 \\
.01 \\
<.01 \\
<.01 \\
<.01 \\
<.01 \\
<.01 \\
<.01 \\
<.01\end{array}$ & $\begin{array}{l}<.01 \\
<.01 \\
<.01 \\
<.01 \\
<.01 \\
<.01 \\
<.01 \\
<.01 \\
<.01 \\
<.01 \\
<.01 \\
<.01 \\
<.01\end{array}$ & $\begin{array}{l}<.01 \\
<.01 \\
<.01 \\
<.01 \\
<.01 \\
<.01 \\
<.01 \\
<.01 \\
<.01 \\
<.01 \\
<.01 \\
<.01 \\
<.01\end{array}$ & $\begin{array}{l}<.01 \\
<.01 \\
<.01 \\
<.01 \\
<.01 \\
<.01 \\
<.01 \\
<.01 \\
<.01 \\
<.01 \\
<.01 \\
<.01 \\
<.01\end{array}$ \\
\hline \multicolumn{11}{|c|}{ Birds } \\
\hline Western grebe egg & $\begin{array}{l}\text { 3AG3 } \\
\text { 3AG4 } \\
\text { 3AG7 } \\
\text { TLG6 } \\
\text { TLG7 } \\
\text { TLG8 } \\
\text { TLG9 } \\
\text { TLG10 } \\
\text { 92TLWG1 } \\
\text { 92TLWG2A } \\
\text { 92TLWG2B } \\
\text { 92TLWG3A } \\
\text { 92TLWG3B } \\
\text { 92TLWG5A } \\
\text { 92TLWG6A } \\
\text { 92TLWG7A } \\
\text { 92TLWG8 }\end{array}$ & $\begin{array}{l}6-20-91 \\
6-20-91 \\
6-20-91 \\
6-29-91 \\
6-29-91 \\
6-29-92 \\
6-29-91 \\
6-29-91 \\
7-08-92 \\
7-08-92 \\
7-08-92 \\
7-08-92 \\
7-08-92 \\
7-08-92 \\
7-08-92 \\
7-08-92 \\
7-08-92\end{array}$ & $\begin{array}{l}<.01 \\
<.01 \\
<.02 \\
<.01 \\
<.01 \\
<.01 \\
<.01 \\
<.01 \\
<.01 \\
<.01 \\
<.01 \\
<.01 \\
<.01 \\
<.01 \\
<.01 \\
<.01 \\
<.01\end{array}$ & $\begin{array}{l}<.01 \\
<.01 \\
<.02 \\
<.01 \\
<.01 \\
<.01 \\
<.01 \\
<.01 \\
<.01 \\
<.01 \\
<.01 \\
<.01 \\
<.01 \\
<.01 \\
<.01 \\
<.01 \\
.01\end{array}$ & $\begin{array}{l}<.01 \\
<.01 \\
<.02 \\
<.01 \\
<.01 \\
<.01 \\
<.01 \\
<.01 \\
<.01 \\
<.01 \\
<.01 \\
<.01 \\
<.01 \\
<.01 \\
<.01 \\
<.01 \\
<.01\end{array}$ & $\begin{array}{l}.38 \\
.03 \\
.09 \\
.22 \\
.36 \\
.02 \\
.05 \\
.18 \\
.06 \\
.13 \\
.15 \\
.38 \\
.36 \\
.25 \\
.21 \\
.94 \\
.96\end{array}$ & $\begin{array}{l}.93 \\
.35 \\
.70 \\
.56 \\
.91 \\
.38 \\
.52 \\
.47 \\
.35 \\
.42 \\
.46 \\
.91 \\
.87 \\
.7 \\
.3 .0 \\
2.6 \\
4.6\end{array}$ & $\begin{array}{l}<.15 \\
<.01 \\
<.01 \\
<.01 \\
<.01 \\
<.01 \\
<.025 \\
<.01 \\
<.01 \\
<.01 \\
<.01 \\
<.01 \\
<.01 \\
<.01 \\
<.01 \\
<.01 \\
<.01\end{array}$ & $\begin{array}{r}<.01 \\
<.01 \\
<.01 \\
<.01 \\
<.01 \\
<.01 \\
<.01 \\
<.01 \\
<.01 \\
.03 \\
.02 \\
.02 \\
<.01 \\
<.01 \\
.04 \\
.02 \\
.04\end{array}$ & $\begin{array}{r}<.01 \\
<.01 \\
<.01 \\
<.01 \\
<.01 \\
<.01 \\
<.01 \\
<.01 \\
<.01 \\
<.01 \\
<.01 \\
<.01 \\
<.01 \\
.01 \\
<.01 \\
<.01 \\
<.01\end{array}$ \\
\hline
\end{tabular}


Table 17. Moisture content, lipid content, and concentrations of organochlorine compounds in biological tissue samples, 1990-92--Continued

\begin{tabular}{|c|c|c|c|c|c|c|c|c|c|c|c|}
\hline Sample type & $\begin{array}{c}\text { Sample } \\
\text { No. }\end{array}$ & Date & Dieldrin & Endrin & HCB & $\begin{array}{c}\text { Heptachlor } \\
\text { epoxide }\end{array}$ & Mirex & $\begin{array}{c}\text { cis-Non } \\
\text { achlor }\end{array}$ & $\begin{array}{l}t \text {-Non- } \\
\text { achlor }\end{array}$ & $\begin{array}{l}\text { PCBs, } \\
\text { total }\end{array}$ & $\begin{array}{l}\text { Toxa- } \\
\text { phene }\end{array}$ \\
\hline Chironomid larvae & $\begin{array}{l}\text { TLCH07A } \\
\text { TLCH08A }\end{array}$ & $\begin{array}{l}8-91 \\
8-91\end{array}$ & $\begin{array}{r}<0.01 \\
<.01\end{array}$ & $\begin{array}{r}<0.01 \\
<.01\end{array}$ & $\begin{array}{r}<0.01 \\
<.01\end{array}$ & $\begin{array}{r}<0.01 \\
<.01\end{array}$ & $\begin{array}{r}<0.01 \\
<.01\end{array}$ & $\begin{array}{r}<0.01 \\
<.01\end{array}$ & $\begin{array}{r}<0.01 \\
<.01\end{array}$ & $\begin{array}{r}<0.05 \\
<.05\end{array}$ & $\begin{array}{r}<0.05 \\
<.05\end{array}$ \\
\hline \multicolumn{12}{|c|}{ Fish } \\
\hline Fathead minnow & $12 \mathrm{FH} 01 \mathrm{~J}$ & $6-23-92$ & $<0.01$ & $<0.01$ & $<0.01$ & $<0.01$ & $<0.01$ & -- & $<0.01$ & $<0.1$ & $<0.1$ \\
\hline Tui chub & $\begin{array}{l}\text { CLTC01A } \\
\text { CLTC03J } \\
\text { 2TC01A } \\
\text { 2TC01J } \\
\text { 2TC02A } \\
\text { 2TC03A } \\
3 T C 01 J \\
7 T C 01 J \\
7 T C 02 J \\
7 T C 03 J \\
11 T C 01 J \\
11 T C 02 J \\
11 T C 03 J\end{array}$ & $\begin{array}{l}6-24-92 \\
6-24-92 \\
6-24-92 \\
6-24-92 \\
6-24-92 \\
6-24-92 \\
6-25-92 \\
6-26-92 \\
6-26-92 \\
6-26-92 \\
6-25-92 \\
6-25-92 \\
6-25-92\end{array}$ & $\begin{array}{l}<.01 \\
<.01 \\
<.01 \\
<.01 \\
<.01 \\
<.01 \\
<.01 \\
<.01 \\
<.01 \\
<.01 \\
<.01 \\
<.01 \\
<.01\end{array}$ & $\begin{array}{l}<.01 \\
<.01 \\
<.01 \\
<.01 \\
<.01 \\
<.01 \\
<.01 \\
<.01 \\
<.01 \\
<.01 \\
<.01 \\
<.01 \\
<.01\end{array}$ & $\begin{array}{l}<.01 \\
<.01 \\
<.01 \\
<.01 \\
<.01 \\
<.01 \\
<.01 \\
<.01 \\
<.01 \\
<.01 \\
<.01 \\
<.01 \\
<.01\end{array}$ & $\begin{array}{l}<.01 \\
<.01 \\
<.01 \\
<.01 \\
<.01 \\
<.01 \\
<.01 \\
<.01 \\
<.01 \\
<.01 \\
<.01 \\
<.01 \\
<.01\end{array}$ & $\begin{array}{l}<.01 \\
<.01 \\
<.01 \\
<.01 \\
<.01 \\
<.01 \\
<.01 \\
<.01 \\
<.01 \\
<.01 \\
<.01 \\
<.01 \\
<.01\end{array}$ & $\begin{array}{l}-- \\
-- \\
-- \\
-- \\
-- \\
-- \\
-- \\
-- \\
-- \\
-- \\
-- \\
-- \\
--\end{array}$ & $\begin{array}{l}<.01 \\
<.01 \\
<.01 \\
<.01 \\
<.01 \\
<.01 \\
<.01 \\
<.01 \\
<.01 \\
<.01 \\
<.01 \\
<.01 \\
<.01\end{array}$ & $\begin{array}{l}<.1 \\
<.1 \\
<.1 \\
<.1 \\
<.1 \\
<.1 \\
<.1 \\
<.1 \\
<.1 \\
<.1 \\
<.1 \\
<.1 \\
<.1\end{array}$ & $\begin{array}{l}<.1 \\
<.1 \\
<.1 \\
<.1 \\
<.1 \\
<.1 \\
<.1 \\
<.1 \\
<.1 \\
<.1 \\
<.1 \\
<.1 \\
<.1\end{array}$ \\
\hline
\end{tabular}

\section{Birds}

\begin{tabular}{|c|c|c|c|c|c|c|c|c|c|c|c|}
\hline \multirow[t]{4}{*}{ Eared grebe egg } & 92TLEG4A & $7-08-92$ & $<0.01$ & $<0.01$ & $<0.01$ & $<0.01$ & $<0.01$ & -- & $<0.01$ & $<0.1$ & $<0.1$ \\
\hline & 92TLEG4B & $7-08-92$ & $<.01$ & $<.01$ & $<.01$ & $<.01$ & $<.01$ & -- & $<.01$ & $<.1$ & $<.1$ \\
\hline & 92TLEG9A & $7-08-92$ & $<.01$ & $<.01$ & $<.01$ & $<.01$ & $<.01$ & -- & $<.01$ & $<.1$ & $<.1$ \\
\hline & 92TLEG9B & $7-08-92$ & $<.01$ & $<.01$ & $<.01$ & $<.01$ & $<.01$ & -- & $<.01$ & $<.1$ & $<.1$ \\
\hline Mallard egg & $92 \mathrm{M} 2$ & $7-01-92$ & $<.01$ & $<.01$ & $<.01$ & $<.01$ & $<.01$ & -- & $<.01$ & $<.1$ & $<.1$ \\
\hline \multirow[t]{17}{*}{ Western grebe egg } & $3 \mathrm{AG} 3$ & $6-20-91$ & .02 & $<.01$ & $<.01$ & $<.01$ & $<.01$ & -- & .03 & .82 & $<.1$ \\
\hline & $3 \mathrm{AG} 4$ & $6-20-91$ & $<.01$ & $<.01$ & $<.01$ & $<.01$ & $<.01$ & -- & .01 & .49 & $<.1$ \\
\hline & $3 \mathrm{AG} 7$ & $6-20-91$ & $<.01$ & $<.01$ & $<.01$ & $<.01$ & $<.01$ & -- & $<.01$ & .23 & $<.1$ \\
\hline & TLG6 & $6-29-91$ & .03 & $<.01$ & .01 & $<.01$ & $<.01$ & -- & .05 & 1.37 & $<.1$ \\
\hline & TLG7 & $6-29-91$ & .01 & $<.01$ & $<.01$ & $<.01$ & $<.01$ & -- & .02 & .4 & $<.1$ \\
\hline & TLG8 & $6-29-92$ & $<.01$ & $<.01$ & $<.01$ & $<.01$ & $<.01$ & -- & $<.01$ & .38 & $<.1$ \\
\hline & TLG9 & $6-29-91$ & $<.01$ & $<.01$ & $<.01$ & $<.01$ & $<.01$ & -- & .02 & .99 & $<.1$ \\
\hline & TLG10 & $6-29-91$ & $<.01$ & $<.01$ & .01 & $<.01$ & $<.01$ & -- & .01 & .27 & $<.1$ \\
\hline & 92TLWG1 & $7-08-92$ & $<.01$ & $<.01$ & $<.01$ & $<.01$ & $<.01$ & -- & .01 & .38 & $<.1$ \\
\hline & 92TLWG2A & $7-08-92$ & $<.01$ & $<.01$ & .01 & $<.01$ & $<.01$ & -- & .02 & .2 & $<.1$ \\
\hline & 92TLWG2B & $7-08-92$ & .01 & $<.01$ & .01 & $<.01$ & $<.01$ & -- & .02 & .22 & $<.1$ \\
\hline & 92TLWG3A & $7-08-92$ & $<.01$ & $<.01$ & .01 & $<.01$ & $<.01$ & -- & .03 & 1.4 & $<.1$ \\
\hline & 92TLWG3B & $7-08-92$ & $<.01$ & $<.01$ & $<.01$ & $<.01$ & $<.01$ & -- & .03 & 1.5 & $<.1$ \\
\hline & 92TLWG5A & $7-08-92$ & $<.01$ & $<.01$ & $<.01$ & $<.01$ & $<.01$ & -- & .07 & 3.8 & $<.1$ \\
\hline & 92TLWG6A & $7-08-92$ & $<.01$ & $<.01$ & $<.01$ & $<.01$ & $<.01$ & -- & .05 & .89 & $<.1$ \\
\hline & 92TLWG7A & $7-08-92$ & $<.01$ & $<.01$ & .01 & $<.01$ & $<.01$ & -- & .09 & .59 & $<.1$ \\
\hline & 92TLWG8 & $7-08-92$ & $<.01$ & $<.01$ & .04 & .01 & $<.01$ & $-\cdot$ & .10 & 3.1 & $<.1$ \\
\hline
\end{tabular}


Table 17. Moisture content, lipid content, and concentrations of organochlorine compounds in biological tissue samples, 1990-92--Continued




Table 17. Moisture content, lipid content, and concentrations of organochlorine compounds in biological tissue samples, 1990-92--Continued

\begin{tabular}{|c|c|c|c|c|c|c|c|c|c|c|}
\hline Sample type & $\begin{array}{c}\text { Sample } \\
\text { No. }\end{array}$ & Date & $\begin{array}{c}\text { Gamma } \\
\text { chlor- } \\
\text { dane }\end{array}$ & $\begin{array}{l}\text { Oxychlor- } \\
\text { dane }\end{array}$ & $\begin{array}{c}o, p^{\prime} \\
\text { DDD }\end{array}$ & $\begin{array}{c}\mathrm{p}, \mathrm{p}^{\prime} \\
\text { DDD }\end{array}$ & $\begin{array}{c}\text { p,p' } \\
\text { DDE }\end{array}$ & $\begin{array}{c}o, p^{\prime} \\
\text { DDE }\end{array}$ & $\begin{array}{l}\mathrm{p}, \mathrm{p}^{\prime} \\
\mathrm{DDT}\end{array}$ & $\begin{array}{c}o, p^{\prime} \\
\text { DDT }\end{array}$ \\
\hline \multirow{16}{*}{ White-faced ibis egg } & I1 & $7-20-90$ & $<0.01$ & 0.04 & $<0.01$ & $<0.01$ & 1.3 & $<0.01$ & $<0.01$ & $<0.01$ \\
\hline & I2 & $7-20-90$ & $<.01$ & .01 & $<.01$ & $<.01$ & 1.4 & $<.01$ & $<.01$ & $<.01$ \\
\hline & I5 & $7-20-90$ & $<.01$ & .02 & $<.01$ & .05 & 3.4 & $<.01$ & $<.01$ & $<.01$ \\
\hline & LK12AI1 & $7-01-91$ & $<.01$ & .07 & $<.01$ & .08 & 9.08 & $<.01$ & .27 & $<.01$ \\
\hline & LK12AI2 & $7-01-91$ & $<.01$ & .03 & $<.01$ & .26 & 13.36 & $<.01$ & $<.01$ & $<.01$ \\
\hline & LK12AI5 & $7-01-91$ & $<.01$ & .04 & $<.01$ & .29 & 14.14 & $<.01$ & $<.01$ & $<.01$ \\
\hline & LK12AI10 & $7-01-91$ & $<.01$ & $<.01$ & $<.01$ & .02 & .68 & $<.01$ & $<.01$ & $<.01$ \\
\hline & LK12AI11 & $7-01-91$ & $<.01$ & $<.01$ & $<.01$ & .22 & 19.71 & $<.01$ & $<.01$ & $<.01$ \\
\hline & 92LKI3 & $6-24-92$ & $<.01$ & $<.01$ & $<.01$ & $<.01$ & 2.5 & $<.01$ & 1.0 & $<.01$ \\
\hline & 92LKI4 & $6-24-92$ & $<.01$ & $<.01$ & $<.01$ & $<.01$ & .29 & $<.01$ & $<.01$ & $<.01$ \\
\hline & 92LKI5 & $6-24-92$ & $<.01$ & $<.01$ & $<.01$ & $<.01$ & .85 & $<.01$ & $<.01$ & $<.01$ \\
\hline & 92LKI6 & $6-24-92$ & $<.01$ & $<.01$ & $<.01$ & $<.01$ & 9.7 & $<.01$ & .13 & $<.01$ \\
\hline & 92LKI7 & $6-24-92$ & $<.01$ & .03 & $<.01$ & $<.01$ & .6 & $<.01$ & $<.01$ & $<.01$ \\
\hline & 92LKI8 & $6-24-92$ & $<.01$ & $<.01$ & $<.01$ & $<.01$ & 11.0 & $<.01$ & .22 & $<.01$ \\
\hline & 92LKI9 & $6-24-92$ & $<.01$ & .02 & $<.01$ & $<.01$ & .34 & $<.01$ & .05 & $<.01$ \\
\hline & 92LKI10 & $6-24-92$ & $<.01$ & $<.01$ & $<.01$ & $<.01$ & .32 & $<.01$ & .02 & $<.01$ \\
\hline White-faced ibis nestling & $16 \mathrm{Y}$ & $7-02-90$ & $<.01$ & $<.01$ & $<.01$ & .01 & .16 & $<.01$ & .01 & $<.01$ \\
\hline
\end{tabular}


Table 17. Moisture content, lipid content, and concentrations of organochlorine compounds in biological tissue samples, 1990-92--Continued

\begin{tabular}{|c|c|c|c|c|c|c|c|c|c|c|c|}
\hline Sample type & $\begin{array}{l}\text { Sample } \\
\text { No. }\end{array}$ & Date & Dieldrin & Endrin & HCB & $\begin{array}{l}\text { Heptachlor } \\
\text { epoxide }\end{array}$ & Mirex & $\begin{array}{l}\text { cis-Non } \\
\text { achlor }\end{array}$ & $\begin{array}{l}t \text {-Non- } \\
\text { achlor }\end{array}$ & $\begin{array}{c}\text { PCBs, } \\
\text { total }\end{array}$ & $\begin{array}{l}\text { Toxa- } \\
\text { phene }\end{array}$ \\
\hline \multicolumn{12}{|c|}{ Birds--Continued } \\
\hline \multirow[t]{21}{*}{ White-faced ibis egg } & I1 & $7-20-90$ & 0.02 & $<0.01$ & 0.57 & 0.03 & $<0.01$ & $<0.01$ & 0.03 & 0.34 & $<0.1$ \\
\hline & I2 & $7-20-90$ & $<.01$ & $<.01$ & .01 & .01 & $<.01$ & $<.01$ & .01 & .31 & $<.1$ \\
\hline & I3 & $7-20-90$ & .02 & $<.01$ & .21 & .01 & $<.01$ & $<.01$ & .02 & $<.1$ & $<.1$ \\
\hline & I4 & $7-20-90$ & .03 & $<.01$ & $<.01$ & .01 & $<.01$ & $<.01$ & .01 & $<.1$ & $<.1$ \\
\hline & I5 & $7-20-90$ & .03 & $<.01$ & .12 & .02 & $<.01$ & $<.01$ & .03 & .84 & $<1$ \\
\hline & LK12AI1 & $7-01-91$ & .20 & $<.01$ & .02 & .09 & $<.01$ & -- & .17 & $<.1$ & $<.1$ \\
\hline & LK12AI2 & $7-01-91$ & .17 & .05 & .45 & .05 & $<.01$ & $\cdots$ & .08 & $<.1$ & $<.1$ \\
\hline & LK12AI5 & $7-01-91$ & .11 & .11 & 8.02 & .08 & $<.01$ & -- & .10 & .13 & $<.1$ \\
\hline & LK12AI10 & $7-01-91$ & $<.01$ & $<.01$ & $<.01$ & .01 & $<.01$ & -- & .02 & $<.1$ & $<.1$ \\
\hline & LK12AI11 & $7-01-91$ & .22 & $<.01$ & .06 & $<.01$ & $<.01$ & -- & .04 & $<.1$ & $<.1$ \\
\hline & LK12AI12 & $7-01-91$ & $<.01$ & $<.01$ & .01 & $<.01$ & $<.01$ & -- & $<.01$ & $<.1$ & $<.1$ \\
\hline & 92LKI1 & $6-24-92$ & $<.01$ & $<.01$ & .02 & $<.01$ & $<.01$ & -- & $<.01$ & $<.1$ & $<.1$ \\
\hline & $92 \mathrm{LKI} 2$ & $6-24-92$ & .2 & .02 & .12 & .01 & $<.01$ & -- & .02 & $<.1$ & $<.1$ \\
\hline & $92 \mathrm{LKI} 3$ & $6-24-92$ & .07 & $<.01$ & .19 & .12 & $<.01$ & -- & .03 & $<.1$ & $<.1$ \\
\hline & 92LKI4 & $6-24-92$ & $<.01$ & $<.01$ & $<.01$ & $<.01$ & $<.01$ & -- & $<.01$ & $<.1$ & $<.1$ \\
\hline & 92LKI5 & $6-24-92$ & $<.01$ & $<.01$ & $<.01$ & $<.01$ & $<.01$ & -- & $<.01$ & $<.1$ & $<.1$ \\
\hline & 92LKI6 & $6-24-92$ & .04 & $<.01$ & .24 & $<.01$ & $<.01$ & -- & .02 & $<.1$ & $<.1$ \\
\hline & 92LKI7 & $6-24-92$ & .01 & $<.01$ & .03 & .01 & $<.01$ & -- & .13 & 1.5 & $<.1$ \\
\hline & 92LKI8 & $6-24-92$ & .12 & .06 & .1 & .03 & $<.01$ & -- & .03 & $<.1$ & $<.1$ \\
\hline & 92LKI9 & $6-24-92$ & .03 & $<.01$ & .02 & .04 & $<.01$ & -. & .02 & $<.1$ & $<.1$ \\
\hline & 92LKI10 & $6-24-92$ & $<.01$ & $<.01$ & $<.01$ & $<.01$ & $<.01$ & $-\cdot$ & $<.01$ & $<.1$ & $<.1$ \\
\hline White-faced ibis nestling & $16 \mathrm{Y}$ & $7-02-90$ & $<.01$ & $<.01$ & $<.01$ & $<.01$ & $<.01$ & $<.01$ & $<.01$ & $<.1$ & $<.1$ \\
\hline
\end{tabular}


Table 18. Cholinesterase (ChE) activity of waterfowl brain tissue collected, 1991-92

[Sample number refers to Fish and Wildlife Service identification number; no specific site locations given for these samples. ChE activity is expressed as micromoles of substrate hydrolyzed per minute per gram of tissue. LK is Lower Klamath National Wildlife Refuge. TL is Tule Lake National Wildlife Refuge]

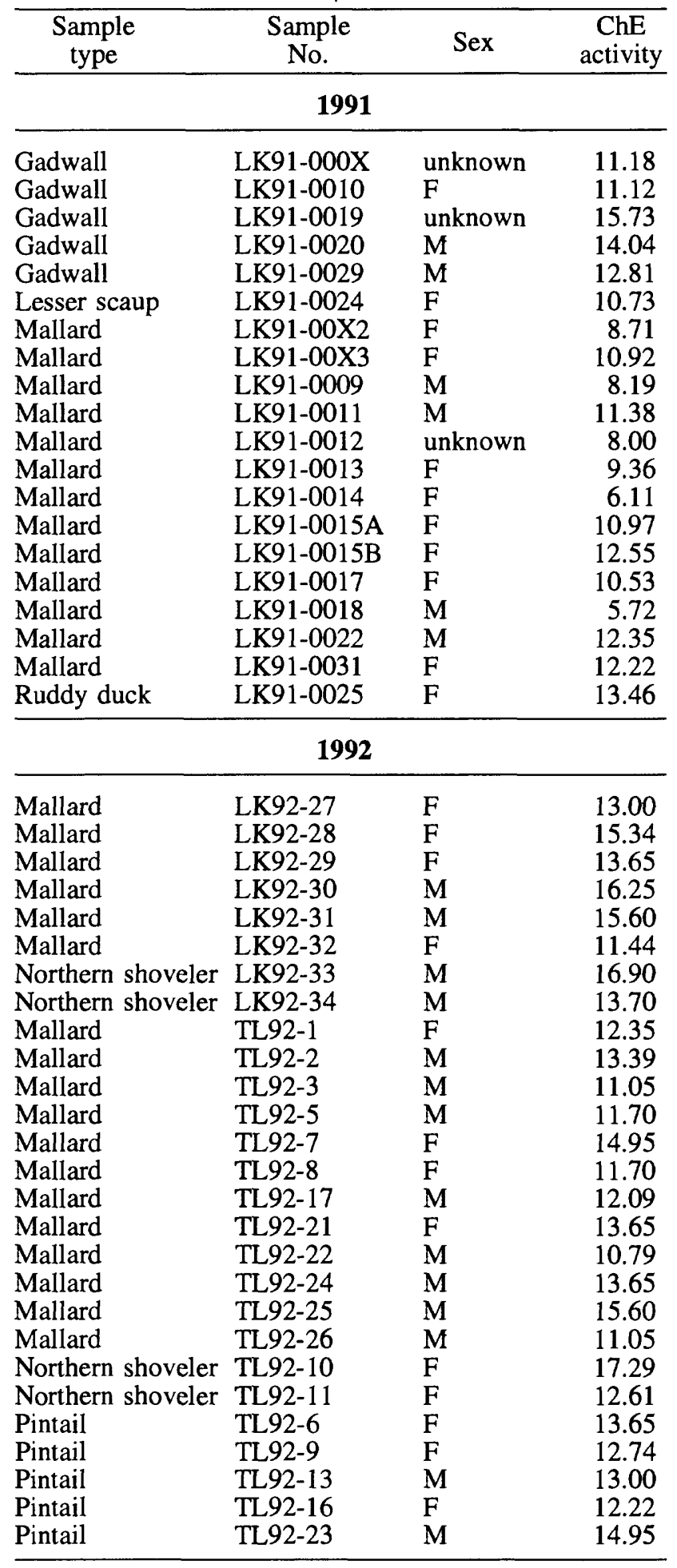


Table 19. Eggshell thickness of avian eggs collected, 1990-92

[Sample locations described in table 2. Values for eggshell thickness in millimeters, accurate to $0.01 \mathrm{~mm}$, third fractional digit estimated]

\begin{tabular}{|c|c|c|c|c|c|c|c|}
\hline $\begin{array}{l}\text { Sample } \\
\text { type }\end{array}$ & $\begin{array}{l}\text { Sample } \\
\text { No. }\end{array}$ & Date & $\begin{array}{l}\text { Eggshell } \\
\text { thickness }\end{array}$ & $\begin{array}{l}\text { Sample } \\
\text { type }\end{array}$ & $\begin{array}{c}\text { Sample } \\
\text { no. }\end{array}$ & Date & $\begin{array}{l}\text { Eggshell } \\
\text { thickness }\end{array}$ \\
\hline \multirow[t]{9}{*}{ American avocet } & 4BA1 & $6-15-91$ & 0.240 & \multirow{19}{*}{$\begin{array}{l}\text { Western grebe-- } \\
\text { Continued }\end{array}$} & TLG2 & 6-29-91 & .496 \\
\hline & $4 \mathrm{BA} 2$ & $6-15-91$ & .240 & & TLG3 & $6-29-91$ & .498 \\
\hline & $4 \mathrm{BA} 3$ & $6-15-91$ & .251 & & TLG4 & 6-29-91 & .513 \\
\hline & 4BA4 & $6-15-91$ & .235 & & TLG5 & 6-29-91 & .463 \\
\hline & $4 \mathrm{BA} 6$ & $6-15-91$ & .266 & & TLG6 & $6-29-91$ & .416 \\
\hline & $4 \mathrm{BA} 7$ & $6-15-91$ & .239 & & TLG7 & $6-29-91$ & .435 \\
\hline & $4 \mathrm{BA} 8$ & $6-15-91$ & .258 & & TLG8 & $6-29-91$ & .415 \\
\hline & $4 \mathrm{BA} 9$ & $6-15-91$ & .254 & & TLG9 & 6-29-91 & .482 \\
\hline & & & & & TLG10 & $6-29-91$ & .448 \\
\hline \multirow[t]{11}{*}{ American coot } & $7 \mathrm{AC} 1$ & $8-15-91$ & .330 & & 92TLWG1 & 6-08-92 & .354 \\
\hline & $7 \mathrm{AC} 2$ & $8-15-91$ & .341 & & 92TLWG2A & $6-08-92$ & .402 \\
\hline & $7 \mathrm{AC} 3$ & $8-15-91$ & .344 & & 92TLWG2B & 6-08-92 & .396 \\
\hline & 7AC4 & $8-15-91$ & .302 & & 92TLWG3A & 6-08-92 & .389 \\
\hline & $7 \mathrm{AC5}$ & $8-15-91$ & .313 & & 92TLWG3B & $6-08-92$ & .406 \\
\hline & LK12CC1 & $6-29-91$ & .325 & & 92TLWG5A & $6-08-92$ & .411 \\
\hline & LK12CC2 & $6-29-91$ & .336 & & 92TLWG6A & $6-08-92$ & .425 \\
\hline & LK12CC3 & $6-29-91$ & .331 & & 92TLWG7A & 6-08-92 & .454 \\
\hline & LK 12 CC4 & $6-29-91$ & .341 & & 92TLWG8 & $6-08-92$ & .411 \\
\hline & LK12CC5 & $6-29-91$ & .284 & & & & \\
\hline & & & & \multirow[t]{25}{*}{ White-faced ibis } & I1 & $7-02-90$ & .316 \\
\hline \multirow[t]{5}{*}{ Eared grebe } & 92TLEG4A & $7-08-92$ & .304 & & 12 & $7-02-90$ & .320 \\
\hline & 92TLEG4B & $7-08-92$ & .302 & & I3 & 7-02-90 & .343 \\
\hline & 92TLEG9A & $7-08-92$ & .288 & & I4 & $7-02-90$ & .295 \\
\hline & 92TLEG9B & $7-08-92$ & .290 & & I5 & $7-02-90$ & .315 \\
\hline & & & & & LK12AI2 & $7-01-91$ & .284 \\
\hline \multirow[t]{11}{*}{ Mallard } & M441 & $5-16-90$ & .359 & & LK12AI3 & $7-01-91$ & .282 \\
\hline & M447 & $5-15-90$ & .346 & & LK12AI4 & $7-01-91$ & .279 \\
\hline & M459A & $5-15-90$ & .316 & & LK12AI5 & 7-01-91 & .291 \\
\hline & M459B & $5-15-90$ & .317 & & LK12AI7 & $7-01-91$ & .331 \\
\hline & M499A & $6-12-90$ & .330 & & LK12AI8 & 7-01-91 & .304 \\
\hline & M499B & $6-12-90$ & .318 & & LK12AI9 & $7-01-91$ & .297 \\
\hline & M516A & 6-08-90 & .326 & & LK12AI10 & $7-01-91$ & .316 \\
\hline & M516B & $6-08-90$ & .342 & & LK12AI11 & 7-01-91 & .247 \\
\hline & M563 & $6-19-90$ & .374 & & LK12AI12 & $7-01-91$ & .332 \\
\hline & M574 & $6-19-90$ & .363 & & 92LKI1 & $6-24-92$ & .310 \\
\hline & & & & & 92LKI2 & $6-24-92$ & .334 \\
\hline \multirow[t]{8}{*}{ Western grebe } & $3 \mathrm{AG} 1$ & $6-20-91$ & .443 & & 92LKI3 & $6-24-92$ & .308 \\
\hline & $3 \mathrm{AG} 2$ & $6-20-91$ & .390 & & 92LKI4 & $6-24-92$ & .319 \\
\hline & $3 \mathrm{AG} 3$ & $6-20-91$ & .407 & & 92LKI5 & $6-24-92$ & .301 \\
\hline & $3 \mathrm{AG} 4$ & $6-20-91$ & .420 & & 92LKI6 & $6-24-92$ & .297 \\
\hline & $3 \mathrm{AG} 5$ & $6-20-91$ & .409 & & 92LKI7 & $6-24-92$ & .323 \\
\hline & $3 \mathrm{AG} 6$ & $6-20-91$ & .399 & & 92LKI8 & $6-24-92$ & .270 \\
\hline & $3 \mathrm{AG} 7$ & $6-20-91$ & .499 & & $92 \mathrm{LKI} 9$ & $6-24-92$ & .313 \\
\hline & TLG1 & $6-29-91$ & .466 & & $92 \mathrm{LKI} 10$ & $6-24-92$ & .309 \\
\hline
\end{tabular}


Table 20. Benthic invertebrate surveys, 1990-91

[See figure 1 for site locations.]

Following are notes regarding taxonomic entries.

Coelenterata: few specimens were found. All belong to the genus Hydra.

Oligochaeta: minimum of three species were found, representing at least two families, Lumbriculidae and Naididae.

Hirudinea: at least two species of Glossiphoniidae were found including a widespread and easily recognizable species, Helobdella stagnalis (L.). Erpobdellidae may be represented by more than one species.

Gastropoda: only one species of Valvatidae, Valvata sp., probably only one species of Hydrobiidae, one to three species of Lymnaeidae, more than one species of Physidae, and six or more species of Planorbidae are represented in the samples.

Pelecypoda: at lease two species were found.

Ephemeroptera: only one species of Caenidae, Caenis sp., was found. All Baetidae able to be keyed to genus (specimens with gills and/or head and caudal appendages intact) were identified as Callibaetis $s p$. There may be more than one genus (most likely Baetis) and more than one species of Callibaetis, however. Only a single species of Ephemeridae was present, Hexagenia sp., and it appears to be confined to Clear Lake and a short stretch of Lost River just below the Clear Lake Dam.

Trichoptera: only one genus, Oecetis sp., was found. One, possibly two, species occur within the study area.

Coleoptera: a single species of Haliplidae was found, Haliplus sp., represented mainly by larvae. Few specimens, but several species, of Dytiscidae were found. Most sample sites were too deep for most aquatic coleopteran species.

Chironomidae: many specimens from the 1990 samples were identified to genus. The dominate genus within the study area is Chironomus. Other common genera within the study area are Cryptochironomus, Cryptotendipes, Glyptotendipes, Einfeldia, Microtendipes, Cricotopus, and Psectrocladius. Only one to a very few specimens of Paramerina, Rheotanytarsus, Cladopelma, Dicrotendipes, Goeldichironomus, Paralanterborniella, Polypedilum, and Corynoneura were found.

Cladocera, Corixidae, and Ephydridae (pupae): also found in samples, but not included because they occur primarily in the water column or at the water surface, rather than on or within the substrate, and entered the sampling apparatus as it traveled through the water column to the substrate. This is also true of adult Coleoptera to some extent. Organisms occurring on vegetation anchored to the substrate, such as gastropods and baetids, are included and are considered part of the benthic community because they do not surface for air and often occur very near to or on the substrate. Floating and emergent vegetation was avoided while sampling. 
Table 20. Benthic invertebrate surveys, 1990-91--Continued

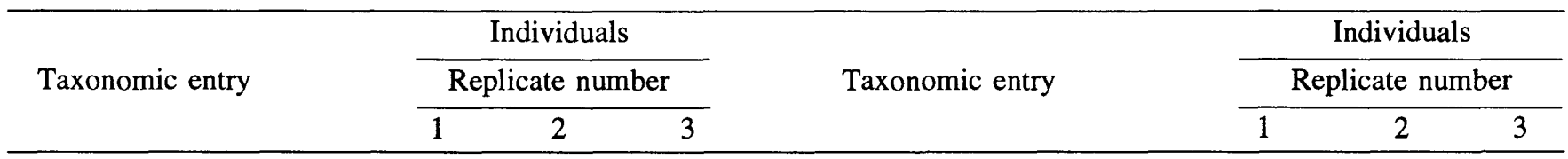

\section{Site 1}

Sampling date: June 20, 1991
Site 1--Continued

Sampling date: August 12, 1991

$\begin{array}{lrrr}\text { Coelenterata } & 0 & 1 & 0 \\ \text { Platyhelminthes } & & & \\ \quad \text { Tubellaria } & 2 & 17 & 1 \\ \text { Nematoda } & 29 & 18 & 13 \\ \text { Annelida } & & & \\ \quad \text { Oligochaeta } & 59 & 52,700 & 970 \\ \quad \text { Hirudinea } & & & \\ \quad \text { Erpobdellidae } & 0 & 31 & 9 \\ \quad \text { Glossiphoniidae } & 2 & 12 & 103 \\ \text { Mollusca } & & & \\ \text { Gastropoda } & & & \\ \quad \text { Valvatidae } & 0 & 2 & 1 \\ \quad \text { Hydrobiidae } & 30 & 73 & 535 \\ \quad \text { Planorbidae } & 0 & 2 & 2 \\ \quad \text { Pelecypoda } & 21 & 346 & 381 \\ \text { Arthropoda } & & & \\ \text { Crustacea } & & & \\ \quad \text { Conchostraca } & 0 & 1 & 0 \\ \quad \text { Copepoda } & 0 & 0 & 5 \\ \quad \text { Amphipoda } & 16 & 25 & 0 \\ \quad \text { Isopoda } & 0 & 14 & 0 \\ \text { Chelicerata } & & & \\ \quad \text { Hydrachnellae } & 0 & 1 & 0 \\ \text { Insecta } & & & \\ \quad \text { Diptera } & & & \\ \quad \text { Chironomidae } & 47 & 0 & 2\end{array}$

Sampling date: July 23, 1991

$\begin{array}{lrrr}\text { Nematoda } & 1 & 3 & 1 \\ \begin{array}{c}\text { Annelida } \\ \text { Oligochaeta }\end{array} & 4 & 9 & 7 \\ \quad \text { Hirudinea } & & & \\ \quad \text { Erpobdellidae } & 0 & 1 & 0 \\ \begin{array}{c}\text { Mollusca } \\ \text { Gastropoda }\end{array} & & & \\ \quad \text { Hydrobiidae } & 107 & 44 & 17 \\ \quad \text { Planorbidae } & 2 & 0 & 0 \\ \quad \begin{array}{c}\text { Pelecypoda } \\ \text { Arthropoda }\end{array} & 1 & 0 & 1 \\ \quad \text { Crustacea } & & & \\ \quad \text { Amphipoda } & 1 & 1 & 1 \\ \text { Insecta } & & & \\ \quad \text { Diptera } & & & \\ \quad \text { Chironomidae } & 5 & 1 & 4\end{array}$

\begin{tabular}{|c|c|c|c|}
\hline Coelenterata & 0 & 0 & 4 \\
\hline \multicolumn{4}{|l|}{ Platyhelminthes } \\
\hline Tubellaria & 0 & 0 & 4 \\
\hline Nematoda & 8 & 0 & 4 \\
\hline \multicolumn{4}{|l|}{ Annelida } \\
\hline Oligochaeta & 0 & 8 & 8 \\
\hline \multicolumn{4}{|l|}{ Hirudinea } \\
\hline Erpobdellidae & 4 & 0 & 0 \\
\hline \multicolumn{4}{|l|}{ Mollusca } \\
\hline \multicolumn{4}{|l|}{ Gastropoda } \\
\hline Valvatidae & 0 & 4 & 4 \\
\hline Hydrobiidae & 232 & 112 & 252 \\
\hline Planorbidae & 0 & 0 & 12 \\
\hline Pelecypoda & 12 & 4 & 0 \\
\hline \multicolumn{4}{|l|}{ Arthropoda } \\
\hline \multicolumn{4}{|l|}{ Crustacea } \\
\hline Amphipoda & 4 & 0 & 12 \\
\hline \multicolumn{4}{|l|}{ Insecta } \\
\hline \multicolumn{4}{|l|}{ Diptera } \\
\hline Simuliidae & 0 & 0 & 4 \\
\hline Chironomidae & 28 & 12 & 20 \\
\hline
\end{tabular}

Site 2

Sampling date: June 13, 1991

\begin{tabular}{|c|c|c|c|}
\hline Nematoda & 4 & 0 & 0 \\
\hline \multicolumn{4}{|l|}{ Annelida } \\
\hline Oligochaeta & 1,376 & 464 & 816 \\
\hline \multicolumn{4}{|l|}{ Hirudinea } \\
\hline Erpobdellidae & 4 & 0 & 4 \\
\hline Glossiphonidae & 0 & 8 & 20 \\
\hline \multicolumn{4}{|l|}{ Mollusca } \\
\hline \multicolumn{4}{|l|}{ Gastropoda } \\
\hline Valvatidae & 0 & 8 & 16 \\
\hline \multicolumn{4}{|l|}{ Arthropoda } \\
\hline \multicolumn{4}{|l|}{ Crustacea } \\
\hline Copepoda & 0 & 0 & 4 \\
\hline Amphipoda & 4 & 16 & 8 \\
\hline \multicolumn{4}{|l|}{ Chelicerata } \\
\hline Hydrachnellae & 4 & 0 & 0 \\
\hline \multicolumn{4}{|l|}{ Insecta } \\
\hline Diptera & & & \\
\hline Chironomidae & 12 & 4 & 0 \\
\hline
\end{tabular}


Table 20. Benthic invertebrate surveys, 1990-91--Continued

\begin{tabular}{|c|c|c|c|c|c|c|c|}
\hline \multirow{3}{*}{ Taxonomic entry } & \multicolumn{3}{|c|}{ Individuals } & \multirow{3}{*}{ Taxonomic entry } & \multicolumn{3}{|c|}{ Individuals } \\
\hline & \multicolumn{3}{|c|}{ Replicate number } & & \multicolumn{3}{|c|}{ Replicate number } \\
\hline & 1 & 2 & 3 & & 1 & 2 & 3 \\
\hline \multicolumn{4}{|c|}{$\begin{array}{l}\text { Site 2--Continued } \\
\text { Sampling date: July 30, } 1991\end{array}$} & \multicolumn{4}{|c|}{$\begin{array}{l}\text { Site 2--Continued } \\
\text { Sampling date: August 13, 1991--Continued }\end{array}$} \\
\hline $\begin{array}{l}\text { Nematoda } \\
\text { Annelida }\end{array}$ & 56 & 104 & 32 & $\begin{array}{l}\text { Arthropoda--Continued } \\
\text { Chelicerata }\end{array}$ & & & \\
\hline $\begin{array}{l}\text { Oligochaeta } \\
\text { Hirudinea }\end{array}$ & 484 & 876 & 200 & $\begin{array}{l}\text { Hydrachnellae } \\
\text { Insecta }\end{array}$ & 5 & 3 & 8 \\
\hline Erpobdellidae & 0 & 0 & 4 & Ephemeroptera & & & \\
\hline Glossiphoniidae & 32 & 68 & 60 & Caenidae & 2 & 1 & 0 \\
\hline Mollusca & & & & Baetidae & 7 & 13 & 13 \\
\hline Gastropoda & & & & Odonata & & & \\
\hline Valvatidae & 4 & 24 & 60 & Aeshnidae & 0 & 0 & 1 \\
\hline Planorbidae & 0 & 4 & 0 & Libellulidae & 1 & 0 & 0 \\
\hline Pelecypoda & 0 & 0 & 16 & Coenagrionidae & 2 & 3 & 5 \\
\hline Arthropoda & & & & Coleoptera & & & \\
\hline Crustacea & & & & Haliplidae & 5 & 4 & 0 \\
\hline Conchostraca & 0 & 4 & 4 & Dytiscidae & 0 & 0 & 1 \\
\hline Copepoda & 36 & 4 & 56 & Diptera & & & \\
\hline Amphipoda & 0 & 0 & 12 & Ceratopogonidae & 0 & 5 & 1 \\
\hline Chelicerata & & & & Chironomidae & 79 & 58 & 45 \\
\hline
\end{tabular}

Site 3

Insecta

$\begin{array}{lrrr}\begin{array}{l}\text { Coleoptera } \\ \text { Haliplidae }\end{array} & 16 & 16 & 0 \\ \begin{array}{l}\text { Diptera } \\ \text { Chironomidae }\end{array} & 52 & 84 & 152\end{array}$

Sampling date: August 13, 1991

\begin{tabular}{|c|c|c|c|}
\hline Coelenterata & 0 & 4 & \\
\hline \multicolumn{4}{|l|}{ Platyhelminthes } \\
\hline Tubellaria & 1 & 11 & \\
\hline Nematoda & 117 & 97 & \\
\hline \multicolumn{4}{|l|}{ Annelida } \\
\hline Oligochaeta & 592 & 1,523 & 5 \\
\hline \multicolumn{4}{|l|}{ Hirudinea } \\
\hline Erpobdellidae & 1 & 0 & \\
\hline Glossiphoniidae & 61 & 107 & \\
\hline \multicolumn{4}{|l|}{ Mollusca } \\
\hline \multicolumn{4}{|l|}{ Gastropoda } \\
\hline Valvatidae & 29 & 30 & \\
\hline Physidae & 2 & 3 & \\
\hline Planorbidae & 8 & 7 & \\
\hline Pelecypoda & 6 & 6 & \\
\hline \multicolumn{4}{|l|}{ Arthropoda } \\
\hline \multicolumn{4}{|l|}{ Crustacea } \\
\hline Conchostraca & 0 & 10 & \\
\hline Copepoda & 137 & 183 & \\
\hline Amphipoda & 5 & 18 & \\
\hline
\end{tabular}

$\begin{array}{lrrr}\text { Coelenterata } & 1 & 1 & 0 \\ \begin{array}{l}\text { Platyhelminthes } \\ \text { Tubellaria }\end{array} & 1 & 0 & 0 \\ \begin{array}{l}\text { Nematoda } \\ \text { Annelida }\end{array} & 33 & 17 & 33 \\ \quad \text { Oligochaeta } & 358 & 262 & 263 \\ \quad \text { Hirudinea } & & & \\ \quad \text { Erpobdellidae } & 0 & 3 & 2 \\ \quad \text { Glossiphoniidae } & 7 & 4 & 2\end{array}$

Mollusca

Gastropoda $\begin{array}{llll}\text { Hydrobiidae } & 0 & 1 & 2\end{array}$ $\begin{array}{llll}\text { Planorbidae } & 1 & 0 & 0\end{array}$

$\begin{array}{llll}\text { Pelecypoda } & 1 & 3 & 2\end{array}$

Arthropoda

Crustacea $\begin{array}{llll}\text { Conchostraca } & 16 & 1 & 2\end{array}$ $\begin{array}{llll}\text { Copepoda } & 5 & 1 & 0\end{array}$ $\begin{array}{llll}\text { Amphipoda } & 4 & 3 & 2\end{array}$

Chelicerata $\begin{array}{llll}\text { Hydrachnellae } & 2 & 0 & 1\end{array}$

Insecta Ephemeroptera $\begin{array}{llll}\text { Caenidae } & 4 & 0 & 3\end{array}$

Odonata Coenagrionidae 
Table 20. Benthic invertebrate surveys, 1990-91--Continued

\begin{tabular}{|c|c|c|c|c|c|c|c|}
\hline \multirow{3}{*}{ Taxonomic entry } & \multicolumn{3}{|c|}{ Individuals } & \multirow{3}{*}{ Taxonomic entry } & \multicolumn{3}{|c|}{ Individuals } \\
\hline & \multicolumn{3}{|c|}{ Replicate number } & & \multicolumn{3}{|c|}{ Replicate number } \\
\hline & 1 & 2 & 3 & & 1 & 2 & 3 \\
\hline \multicolumn{4}{|c|}{$\begin{array}{l}\text { Site 3--Continued } \\
\text { Sampling date: June 13, 1991--Continued }\end{array}$} & \multicolumn{4}{|c|}{$\begin{array}{l}\text { Site 3--Continued } \\
\text { ampling date: August 13, 1991--Continued }\end{array}$} \\
\hline $\begin{array}{l}\text { Arthropoda--Continued } \\
\text { Insecta--Continued }\end{array}$ & & & & $\begin{array}{l}\text { Mollusca } \\
\text { Gastropoda }\end{array}$ & & & \\
\hline Diptera & & & & Valvatidae & 0 & 1 & 0 \\
\hline \multirow{3}{*}{ Chironomidae } & 146 & 27 & 69 & Hydrobiidae & 12 & 12 & 3 \\
\hline & & & & Lymnaeidae & 2 & 0 & 0 \\
\hline & \multirow{2}{*}{\multicolumn{3}{|c|}{ July 30, 1991}} & Physidae & 1 & 0 & 1 \\
\hline Sampling date: & & & & Planorbidae & 4 & 5 & 6 \\
\hline \multicolumn{4}{|l|}{ Platyhelminthes } & Pelecypoda & 3 & 6 & 3 \\
\hline Tubellaria & 72 & 278 & 84 & $\begin{array}{l}\text { Arthropoda } \\
\text { Crustacea }\end{array}$ & & & \\
\hline Nematoda & 328 & 20 & 14 & $\begin{array}{l}\text { Crustacea } \\
\text { Conchostraca }\end{array}$ & 0 & 0 & 2 \\
\hline Annelida & & & & Copepoda & 28 & 20 & 14 \\
\hline Oligochaeta & 1,828 & 270 & 134 & Amphipoda & 1 & 14 & 12 \\
\hline Hirudinea & & & & Chelicerata & & & \\
\hline Erpobdellidae & 8 & 6 & 0 & Hydrachnellae & 1 & 6 & 2 \\
\hline $\begin{array}{l}\text { Glossiphoniidae } \\
\text { Mollusca }\end{array}$ & 28 & 16 & 10 & Insecta & & & \\
\hline $\begin{array}{l}\text { Mollusca } \\
\text { Gastropoda }\end{array}$ & & & & Diptera & & & \\
\hline Valvatidae & 2 & 10 & 0 & Chironomidae & 0 & 3 & 2 \\
\hline Hydrobiidae & 14 & 10 & 13 & \\
\hline Lymnaeidae & 2 & 0 & 0 & \multirow{2}{*}{\multicolumn{4}{|c|}{ Sampling date: August 19, 1991}} \\
\hline Physidae & 4 & 14 & 1 & & & & \\
\hline Planorbidae & 18 & 18 & 7 & Coelenterata & 12 & 4 & 28 \\
\hline Pelecypoda & 6 & 8 & 2 & Platyhelminthes & & & \\
\hline Arthropoda & & & & Tubellaria & 192 & 1,132 & 36 \\
\hline Crustacea & & & & Nematoda & 0 & 0 & 92 \\
\hline Conchostraca & 2 & 4 & 0 & Annelida & & & \\
\hline Copepoda & 14 & 42 & 0 & Oligochaeta & 628 & 1,476 & 804 \\
\hline Amphipoda & 4 & 6 & 0 & Hirudinea & & & \\
\hline $\begin{array}{l}\text { Chelicerata } \\
\text { Hydrachnellae }\end{array}$ & & & & Erpobdellidae & 0 & 8 & 0 \\
\hline Hydrachnellae & 8 & 8 & 3 & Glossiphoniidae & 16 & 84 & 36 \\
\hline $\begin{array}{l}\text { Insecta } \\
\text { Odonata }\end{array}$ & & & & Mollusca & & & \\
\hline $\begin{array}{l}\text { Odonata } \\
\text { Coenagrionidae }\end{array}$ & 0 & 2 & & Gastropoda & & & \\
\hline Coleoptera & & & 0 & Valvatidae & 8 & 92 & 0 \\
\hline Haliplidae & 0 & 4 & 0 & Hydrobiidae & 24 & 164 & 0 \\
\hline Diptera & & & 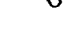 & Lymnaeidae & 4 & 0 & 0 \\
\hline Chironomidae & 0 & 14 & 1 & Physidae & 0 & 4 & 0 \\
\hline & 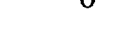 & 14 & 1 & Planorbidae & 8 & 8 & 0 \\
\hline & & & & Pelecypoda & 144 & 132 & 0 \\
\hline Sampling date: & August 1 & 991 & & Arthropoda & & & \\
\hline & & & & Crustacea & & & \\
\hline Coelenterata & 0 & 8 & 50 & Conchostraca & 4 & 0 & 0 \\
\hline Platyhelminthes & & & & Ostracoda & 0 & 4 & 0 \\
\hline Tubellaria & 15 & 172 & 175 & Copepoda & 4 & 0 & 12 \\
\hline Nematoda & 63 & 31 & 28 & Amphipoda & 156 & 152 & 384 \\
\hline Annelida & & & & Chelicerata & & & \\
\hline Oligochaeta & 198 & 32 & 162 & Hydrachnellae & 4 & 4 & 4 \\
\hline Hirudinea & & & & $\begin{array}{l}\text { Insecta } \\
\text { Fnhemerontera }\end{array}$ & & & \\
\hline Erpobdellidae & 8 & 0 & 1 & Caenidae & 0 & 0 & 12 \\
\hline Glossiphoniidae & 18 & 3 & 4 & Baetidae & 0 & 0 & 16 \\
\hline
\end{tabular}


Table 20. Benthic invertebrate surveys, 1990-91--Continued

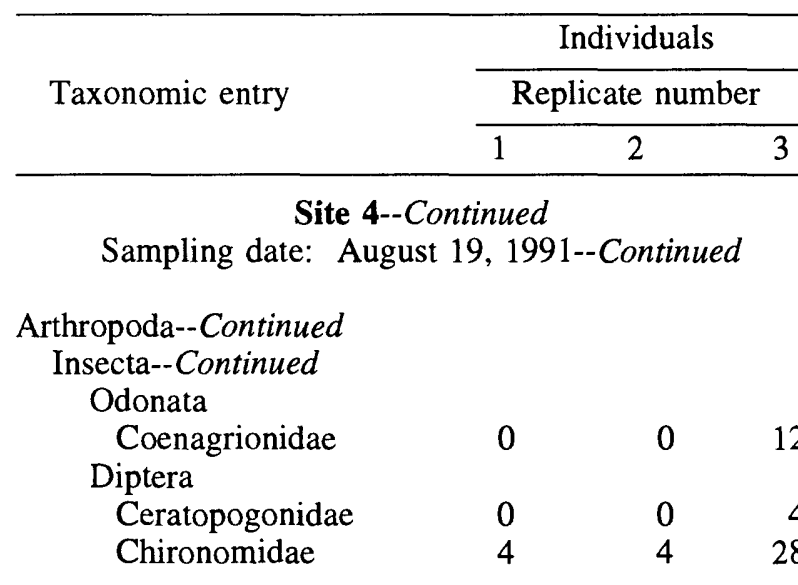

Site 5

Sampling date: June 13, 1991

Sampling date: August 12, 1991

Annelida

$\begin{array}{lccc}\quad \text { Oligochaeta } & 48 & 128 & 4 \\ \text { Hirudinea } & & & \\ \quad \text { Erpobdellidae } & 0 & 8 & 0 \\ \quad \text { Glossiphoniidae } & 4 & 0 & 4 \\ \begin{array}{l}\text { Arthropoda } \\ \quad \text { Insecta } \\ \quad \text { Diptera }\end{array} & & & \\ \quad \text { Chironomidae } & 0 & 4 & 0\end{array}$

Sampling date: July 30, 1991

$\begin{array}{lrrr}\text { Nematoda } & 12 & 24 & 24 \\ \begin{array}{l}\text { Annelida } \\ \text { Oligochaeta }\end{array} & 548 & 720 & 1,220 \\ \quad \text { Hirudinea } & & & \\ \quad \text { Erpobdellidae } & 4 & 0 & 36 \\ \quad \text { Glossiphoniidae } & 8 & 0 & 16\end{array}$

Mollusca

Gastropoda

$\begin{array}{llll}\text { Valvatidae } & 0 & 0 & 4\end{array}$

Planorbidae $\quad 0 \quad 1004$

Pelecypoda

$4 \quad 0 \quad 0$

Arthropoda

Crustacea

Conchostraca

Copepoda

Amphipoda

$\begin{array}{lll}4 & 4 & 20\end{array}$

$8 \quad 0 \quad 12$

Chelicerata

Hydrachnellae

Insecta

$4 \quad 0 \quad 12$

Ephemeroptera

$\begin{array}{llll}\begin{array}{c}\text { Baetidae } \\ \text { Odonata } \\ \text { Coenagrionidae }\end{array} & 0 & 0 & 8 \\ & 0 & 0 & 4\end{array}$

Coelenterata

Platyhelminthes

Tubellaria

Nematoda

Annelida

Oligochaeta

Hirudinea

Erpobdellidae

Glossiphoniidae

Mollusca

Gastropoda

Site 5--Continued

Individuals

\begin{tabular}{|c|c|c|}
\hline \multicolumn{3}{|c|}{ Replicate number } \\
\hline 1 & 2 & 3 \\
\hline
\end{tabular}

3

Sampling date: July 30, 1991--Continued

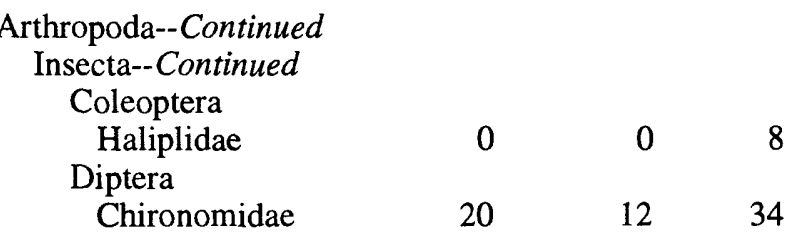

$\begin{array}{lllr}\text { Valvatidae } & 4 & 2 & 76 \\ \text { Lymnaeidae } & 4 & 0 & 24 \\ \text { Physidae } & 0 & 0 & 4 \\ \text { Planorbidae } & 4 & 0 & 48 \\ \text { Pelecypoda } & 0 & 2 & 0\end{array}$

Arthropoda

Crustacea

$\begin{array}{lrrr}\text { Conchostraca } & 12 & 0 & 20\end{array}$

$\begin{array}{llll}\text { Copepoda } & 0 & 0 & 104\end{array}$

$\begin{array}{llll}\text { Amphipoda } & 4 & 22 & 224\end{array}$

Insecta

Ephemeroptera

$\begin{array}{llll}\text { Caenidae } & 0 & 0 & 8\end{array}$

$\begin{array}{llll}\text { Baetidae } & 0 & 4 & 16\end{array}$

Coleoptera

Haliplidae $\quad \begin{array}{llll}0 & 0 & 4\end{array}$

$\begin{array}{llll}\text { Dytiscidae } & 0 & 0 & 8\end{array}$

Diptera

$\begin{array}{llll}\text { Ceratopogonidae } & 0 & 2 & 28\end{array}$

$\begin{array}{llll}\text { Chironomidae } & 0 & 6 & 8\end{array}$

Site 6

Sampling date: August 19, 1991

Coelenterata

Nematoda

$\begin{array}{lll}0 & 20 & 12\end{array}$

Annelida

Oligochaeta

$\begin{array}{lll}52 & 212 & 152\end{array}$

$2,044 \quad 1,324 \quad 280$ 
Table 20. Benthic invertebrate surveys, 1990-91--Continued

\begin{tabular}{|c|c|c|c|c|c|c|c|}
\hline \multirow{3}{*}{ Taxonomic entry } & \multicolumn{3}{|c|}{ Individuals } & \multirow{3}{*}{ Taxonomic entry } & \multicolumn{3}{|c|}{ Individuals } \\
\hline & \multicolumn{3}{|c|}{ Replicate number } & & \multicolumn{3}{|c|}{ Replicate number } \\
\hline & 1 & 2 & 3 & & 1 & 2 & 3 \\
\hline \multicolumn{4}{|c|}{$\begin{array}{l}\text { Site 6--Continued } \\
\text { Sampling date: August 19, 1991--Continued }\end{array}$} & \multicolumn{4}{|c|}{$\begin{array}{l}\text { Site 7--Continued } \\
\text { Sampling date: June 13, 1991--Continued }\end{array}$} \\
\hline $\begin{array}{l}\text { Annelida--Continued } \\
\text { Hirudinea }\end{array}$ & & & & $\begin{array}{l}\text { Arthropoda--Continued } \\
\text { Crustacea--Continued }\end{array}$ & & & \\
\hline Erpobdellidae & 4 & 4 & 8 & Amphipoda & 4 & 5 & 11 \\
\hline Glossiphoniidae & 40 & 88 & 116 & Chelicerata & & & \\
\hline Mollusca & & & & Hydrachnellae & 0 & 0 & 2 \\
\hline Gastropoda & & & & Insecta & & & \\
\hline Valvatidae & 4 & 24 & 100 & Ephemeroptera & & & \\
\hline Lymnaeidae & 0 & 0 & 32 & Caenidae & 4 & 6 & 0 \\
\hline Physidae & 0 & 0 & 20 & Baetidae & 0 & 0 & 1 \\
\hline Planorbidae & 0 & 4 & 20 & Coleoptera & & & \\
\hline Pelecypoda & 16 & 0 & 4 & Hydrophilidae & 0 & 1 & 0 \\
\hline Arthropoda & & & & Diptera & & & \\
\hline Crustacea & & & & Chironomidae & 187 & 286 & 6 \\
\hline Conchostraca & 0 & 16 & 12 & & & & \\
\hline Ostracoda & 4 & 4 & 0 & & & & \\
\hline Copepoda & 32 & 8 & 16 & \multicolumn{4}{|c|}{ Sampling date: July 30, 1991} \\
\hline Amphipoda & 16 & 16 & 36 & \multirow{2}{*}{\multicolumn{4}{|c|}{ Platyhelminthes }} \\
\hline Insecta & & & & & & & \\
\hline Ephemeroptera & & & & Tubellaria & 1 & 0 & 0 \\
\hline Caenidae & 0 & 0 & 4 & Nematoda & 28 & 90 & 74 \\
\hline Baetidae & 4 & 20 & 112 & Annelida & & & \\
\hline Odonata & & & & Oligochaeta & 84 & 1,498 & 195 \\
\hline Coenagrionidae & 4 & 0 & 8 & Hirudinea & & & \\
\hline Coleoptera & & & & Erpobdellidae & 0 & 4 & 0 \\
\hline Haliplidae & 0 & 8 & 0 & Glossiphoniidae & 75 & 26 & 13 \\
\hline Diptera & & & & Mollusca & & & \\
\hline Ceratopogonidae & 0 & 4 & 0 & Gastropoda & & & \\
\hline \multirow{3}{*}{ Chironomidae } & 12 & 8 & 76 & Valvatidae & 34 & 6 & 0 \\
\hline & & & & Lymnaeidae & 1 & 0 & 5 \\
\hline & & & & Physidae & 1 & 0 & 2 \\
\hline \multirow{2}{*}{\multicolumn{4}{|c|}{$\begin{array}{l}\text { Site } 7 \\
\text { Sampling date: June 13, } 1991\end{array}$}} & Pelecypoda & 2 & 0 & 1 \\
\hline & & & & Arthropoda & & & \\
\hline & & & & Crustacea & & & \\
\hline Nematoda & 41 & 42 & 88 & Conchostraca & 0 & 0 & 6 \\
\hline Annelida & & & & Copepoda & 19 & 0 & 38 \\
\hline Oligochaeta & 64 & 671 & 2,471 & Amphipoda & 48 & 0 & 6 \\
\hline Hirudinea & & & & Chelicerata & & & \\
\hline Erpobdellidae & 1 & 1 & 18 & Hydrachnellae & 2 & 2 & 0 \\
\hline Glossiphoniidae & 20 & 4 & 34 & Insecta & & & \\
\hline Mollusca & & & & Ephemeroptera & & & \\
\hline Gastropoda & & & & Caenidae & 0 & 0 & 3 \\
\hline Valvatidae & 7 & 4 & 2 & Baetidae & 1 & 0 & 0 \\
\hline Lymnaeidae & 1 & 1 & 2 & Coleoptera & & & \\
\hline Planorbidae & 1 & 1 & 0 & Haliplidae & 4 & 0 & 0 \\
\hline Pelecypoda & 0 & 2 & 2 & Dytiscidae & 0 & 0 . & 1 \\
\hline Anthropoda & & & & Hydrophilidae & 0 & 0 & 1 \\
\hline Crustacea & & & & Diptera & & & \\
\hline Conchostraca & 6 & 8 & 2 & Chironomidae & 9 & 4 & 488 \\
\hline Copepoda & 10 & 1 & 1 & & & & \\
\hline
\end{tabular}


Table 20. Benthic invertebrate surveys, 1990-91--Continued

\begin{tabular}{|c|c|c|c|c|c|c|c|}
\hline \multirow{3}{*}{ Taxonomic entry } & \multirow{2}{*}{\multicolumn{3}{|c|}{$\frac{\text { Individuals }}{\text { Replicate number }}$}} & \multirow{3}{*}{ Taxonomic entry } & \multirow{2}{*}{\multicolumn{3}{|c|}{$\begin{array}{c}\text { Individuals } \\
\text { Replicate number }\end{array}$}} \\
\hline & & & & & & & \\
\hline & 1 & 2 & 3 & & 1 & 2 & 3 \\
\hline \multicolumn{4}{|c|}{$\begin{array}{l}\text { Site 7--Continued } \\
\text { Sampling date: August 12, } 1991\end{array}$} & \multicolumn{4}{|c|}{$\begin{array}{l}\text { Site 9--Continued } \\
\text { Sampling date: June 20, 1991--Continued }\end{array}$} \\
\hline Nematoda & 127 & 60 & 5 & Arthropoda--Continued & & & \\
\hline $\begin{array}{l}\text { Annelida } \\
\text { Oligochaeta }\end{array}$ & 1,765 & 167 & 381 & $\begin{array}{l}\text { Insecta--Continued } \\
\text { Diptera }\end{array}$ & & & \\
\hline Hirudinea & & & & Chironomidae & 1,209 & 897 & 1,180 \\
\hline $\begin{array}{l}\text { Erpobdellidae } \\
\text { Glossiphoniidae }\end{array}$ & $\begin{array}{l}47 \\
42\end{array}$ & $\begin{array}{r}22 \\
124\end{array}$ & $\begin{array}{l}13 \\
55\end{array}$ & & & & \\
\hline $\begin{array}{l}\text { Mollusca } \\
\text { Gastropoda }\end{array}$ & & & & Sampling & July 30,1 & & \\
\hline Valvatidae & 1 & 18 & 5 & Nematoda & 4 & 3 & 3 \\
\hline Lymnaeidae & 1 & 5 & 0 & Annelida & & & \\
\hline Physidae & 1 & 8 & 2 & Oligochaeta & 230 & 210 & 280 \\
\hline Planorbidae & 0 & 0 & 2 & Hirudinea & & & \\
\hline Pelecypoda & 7 & 1 & 0 & Erpobdellidae & 0 & 1 & 3 \\
\hline Arthropoda & & & & Glossiphoniidae & 2 & 22 & 3 \\
\hline Crustacea & & & & Arthropoda & & & \\
\hline Conchostraca & 2 & 2 & 0 & Crustacea & & & \\
\hline Copepoda & 3 & 13 & 0 & Conchostraca & 2 & 0 & 0 \\
\hline Amphipoda & 0 & 12 & 10 & Copepoda & 2 & 1 & 0 \\
\hline Chelicerata & & & & Amphipoda & 24 & 7 & 16 \\
\hline Hydrachnellae & 0 & 2 & 1 & Insecta & & & \\
\hline Insecta & & & & Coleoptera & & & \\
\hline Ephemeroptera & & & & Dytiscidae & 0 & 1 & 0 \\
\hline Caenidae & 0 & 3 & 0 & Diptera & & & \\
\hline Baetidae & 4 & 39 & 48 & Chironomidae & 100 & 50 & 9 \\
\hline
\end{tabular}

$\begin{array}{llll}\text { Coenagrionidae } & 0 & 3 & 0\end{array}$

Coleoptera

Haliplidae $\quad \begin{array}{llll}0 & 3 & 1\end{array}$

$\begin{array}{llll}\text { Dytiscidae } & 0 & 4 & 1\end{array}$

Diptera

Chironomidae

$\begin{array}{lll}0 & 7 & 0\end{array}$

Site 9

Sampling date: June 20, 1991

\begin{tabular}{|c|c|c|c|}
\hline \multirow{2}{*}{\multicolumn{4}{|c|}{$\begin{array}{l}\text { Nematoda } \\
\text { Annelida }\end{array}$}} \\
\hline & & & \\
\hline Oligochaeta & 75 & 144 & 30 \\
\hline \multicolumn{4}{|l|}{ Hirudinea } \\
\hline Glossiphoniidae & 1 & 13 & 4 \\
\hline \multicolumn{4}{|l|}{ Arthropoda } \\
\hline \multicolumn{4}{|l|}{ Crustacea } \\
\hline Conchostraca & 0 & 2 & 2 \\
\hline Copepoda & 0 & 10 & 1 \\
\hline \multicolumn{4}{|l|}{ Chelicerata } \\
\hline Hydrachnellae & 1 & 2 & 1 \\
\hline \multicolumn{4}{|l|}{ Insecta } \\
\hline \multicolumn{4}{|l|}{ Ephemeroptera } \\
\hline Caenidae & 1 & 0 & 0 \\
\hline Baetidae & 0 & 1 & 0 \\
\hline
\end{tabular}

Mollusca

Nematoda

Sampling date: August 12, 1991

Annelida

Oligochaeta

Hirudinea

Erpobdellidae

Glossiphoniidae

Gastropoda

Valvatidae

Arthropoda

$$
\text { Physidae }
$$

$\begin{array}{rrr}2 & 2 & 1 \\ 168 & 181 & 89 \\ 2 & 2 & 1 \\ 178 & 209 & 89\end{array}$

Crustacea Copepoda Amphipoda

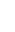

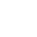

3


Table 20. Benthic invertebrate surveys, 1990-91--Continued

\begin{tabular}{|c|c|c|c|}
\hline \multirow{3}{*}{ Taxonomic entry } & \multicolumn{3}{|c|}{ Individuals } \\
\hline & \multicolumn{3}{|c|}{ Replicate number } \\
\hline & 1 & 2 & 3 \\
\hline \multicolumn{4}{|c|}{ Site 10} \\
\hline Coelenterata & 24 & 8 & 16 \\
\hline \multicolumn{4}{|l|}{ Platyhelminthes } \\
\hline Tubellaria & 8 & 12 & 0 \\
\hline Nematoda & 4 & 0 & 42 \\
\hline \multicolumn{4}{|l|}{ Annelida } \\
\hline Oligochaeta & 498 & 8 & 528 \\
\hline \multicolumn{4}{|l|}{ Hirudinea } \\
\hline Erpobdellidae & 14 & 0 & 12 \\
\hline Glossiphoniidae & 94 & 162 & 84 \\
\hline \multicolumn{4}{|l|}{ Mollusca } \\
\hline \multicolumn{4}{|l|}{ Gastropoda } \\
\hline Valvatidae & 22 & 72 & 56 \\
\hline Lymnaeidae & 4 & 6 & 0 \\
\hline Physidae & 2 & 6 & 6 \\
\hline Planorbidae & 10 & 16 & 14 \\
\hline \multicolumn{4}{|l|}{ Pelecypoda } \\
\hline \multicolumn{4}{|l|}{ Arthropoda } \\
\hline \multicolumn{4}{|l|}{ Crustacea } \\
\hline Conchostraca & 14 & 0 & 4 \\
\hline Ostracoda & 0 & 0 & 2 \\
\hline Copepoda & 70 & 46 & 28 \\
\hline Amphipoda & 58 & 130 & 26 \\
\hline \multicolumn{4}{|l|}{ Chelicerata } \\
\hline Hydrachnellae & 104 & 0 & 68 \\
\hline \multicolumn{4}{|l|}{ Insecta } \\
\hline \multicolumn{4}{|l|}{ Ephemeroptera } \\
\hline Caenidae & 4 & 10 & 0 \\
\hline Baetidae & 12 & 18 & 8 \\
\hline \multicolumn{4}{|l|}{ Odonata } \\
\hline Coenagrionidae & 10 & 6 & 8 \\
\hline \multicolumn{4}{|l|}{ Coleoptera } \\
\hline Dytiscidae & 0 & 2 & 0 \\
\hline \multicolumn{4}{|l|}{ Diptera } \\
\hline Ceratopogonidae & 4 & 0 & 0 \\
\hline Chironomidae & 10 & 2 & 4 \\
\hline
\end{tabular}

Site 11

Sampling date: June 13, 1991

$\begin{array}{lrrr}\text { Coelenterata } & 0 & 12 & 0 \\ \begin{array}{l}\text { Nematoda } \\ \text { Annelida }\end{array} & 8 & 0 & 0 \\ \quad \begin{array}{l}\text { Oligochaeta } \\ \text { Hirudinea }\end{array} & 776 & 840 & 558 \\ \quad \text { Glossiphoniidae } & 72 & 28 & 68\end{array}$

Taxonomic entry

Individuals

\begin{tabular}{ccc}
\hline \multicolumn{3}{c}{ Replicate number } \\
\hline 1 & 2 & 3
\end{tabular}

Site 11--Continued

Sampling date: June 13, 1991--Continued

Mollusca

Gastropoda

$\begin{array}{llll}\text { Planorbidae } & 4 & 4 & 0\end{array}$

Arthropoda

Crustacea

$\begin{array}{lrrr}\text { Conchostraca } & 8 & 0 & 0\end{array}$

$\begin{array}{llll}\text { Copepoda } & 148 & 108 & 58\end{array}$

Amphipoda $\quad 0 \quad 006$

Chelicerata

$\begin{array}{llll}\text { Hydrachnellae } & 4 & 4 & 2\end{array}$

Insecta

Ephemeroptera

$\begin{array}{llll}\text { Caenidae } & 16 & 12 & 12\end{array}$

Odonata

$\begin{array}{llll}\text { Coenagrionidae } & 0 & 0 & 2\end{array}$

Diptera

$\begin{array}{llll}\text { Chironomidae } & 48 & 96 & 14\end{array}$

Sampling date: July 23, 1991

Nematoda

Annelida

$\begin{array}{llll}\text { Oligochaeta } & 928 & 1,800 & 162\end{array}$

Hirudinea

$\begin{array}{lrrr}\text { Erpobdellidae } & 16 & 6 & 0\end{array}$

Mollusca

$\begin{array}{llll}\text { Glossiphoniidae } & 144 & 88 & 14\end{array}$

Gastropoda

$\begin{array}{llll}\text { Lymnaeidae } & 16 & 0 & 0\end{array}$

Arthropoda

Planorbidae

$\begin{array}{lll}0 & 0 & 4\end{array}$

Crustacea

$\begin{array}{llll}\text { Conchostraca } & 0 & 8 & 8\end{array}$

$\begin{array}{llll}\text { Copepoda } & 24 & 50 & 28\end{array}$

$\begin{array}{llll}\text { Amphipoda } & 28 & 4 & 0\end{array}$

Chelicerata

$\begin{array}{llll}\text { Hydrachnellae } & 16 & 4 & 4\end{array}$

Insecta

Ephemeroptera

Caenidae

Baetidae

Odonata

$\begin{array}{llll}\text { Coenagrionidae } & 4 & 0 & 0\end{array}$

Coleoptera

$\begin{array}{llll}\text { Haliplidae } & 20 & 24 & 2\end{array}$

Diptera

Chironomidae

$\begin{array}{lll}4 & 12 & 2\end{array}$

$0 \quad 8 \quad 6$

$56 \quad 36 \quad 12$ 
Table 20. Benthic invertebrate surveys, 1990-91--Continued

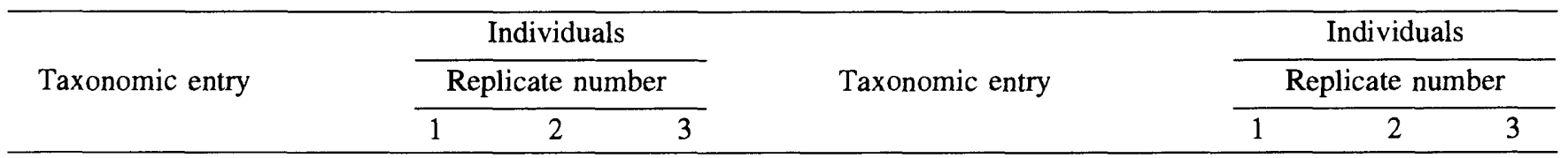

Site 11--Continued

Sampling date: August 12, 1991

Annelida

\begin{tabular}{|c|c|c|c|}
\hline Oligochaeta & 1,102 & 4,920 & 2 \\
\hline \multicolumn{4}{|l|}{ Hirudinea } \\
\hline Erpobdellidae & 10 & 24 & \\
\hline Glossiphoniidae & 76 & 168 & 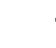 \\
\hline \multicolumn{4}{|l|}{ ollusca } \\
\hline \multicolumn{4}{|l|}{ Gastropoda } \\
\hline Lymnaeidae & 4 & 36 & 1 \\
\hline Physidae & 2 & 24 & 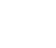 \\
\hline Planorbidae & 4 & 66 & \\
\hline \multicolumn{4}{|l|}{ thropoda } \\
\hline \multicolumn{4}{|l|}{ Crustacea } \\
\hline Conchostraca & 0 & 8 & \\
\hline Copepoda & 24 & 58 & \\
\hline Amphipoda & 0 & 88 & \\
\hline \multicolumn{3}{|l|}{ Chelicerata } & \\
\hline Hydrachnellae & 8 & 62 & s \\
\hline \multicolumn{4}{|l|}{ Insecta } \\
\hline \multicolumn{4}{|l|}{ Ephemeroptera } \\
\hline Caenidae & 8 & 10 & \\
\hline Baetidae & 2 & 24 & \\
\hline \multicolumn{3}{|l|}{ Odonata } & \\
\hline Coenagrionidae & 0 & 10 & ( \\
\hline \multicolumn{3}{|l|}{ Coleoptera } & \\
\hline Haliplidae & 8 & 30 & 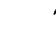 \\
\hline \multicolumn{4}{|l|}{ Diptera } \\
\hline Chironomidae & 20 & 54 & \\
\hline
\end{tabular}

Site 12

Sampling date: August 24, 1990

$\begin{array}{lllr}\text { Nematomorpha } & 0 & 0 & 1 \\ \begin{array}{l}\text { Nematoda } \\ \text { Annelida } \\ \quad \text { Oligochaeta }\end{array} & 2 & 1 & 1 \\ \quad \text { Hirudinea } & 5 & 0 & 28 \\ \quad \begin{array}{l}\text { Erpobdellidae } \\ \text { Glossiphoniidae }\end{array} & 0 & 0 & 1 \\ \text { Mollusca } & 0 & 2 & 4\end{array}$

Gastropoda

Planorbidae

Arthropoda

Crustacea

Conchostraca

Copepoda

Amphipoda

$5 \quad 0 \quad 3$

Chelicerata

Hydrachnellae
Site 12--Continued

Sampling date: August 24, 1990--Continued

$\begin{array}{llll}\begin{array}{c}\text { Arthropoda--Continued } \\ \text { Insecta } \\ \text { Ephemeroptera }\end{array} & & & \\ \begin{array}{c}\text { Caenidae } \\ \text { Coleoptera }\end{array} & 1 & 0 & 0 \\ \quad \begin{array}{l}\text { Haliplidae } \\ \text { Dytiscidae }\end{array} & 1 & 1 & 0 \\ \begin{array}{c}\text { Diptera } \\ \text { Chironomidae }\end{array} & 1 & 0 & 0 \\ & 56 & 13 & 42\end{array}$

Sampling date: June 20, 1991

\begin{tabular}{|c|c|c|c|}
\hline Coelenterata & 0 & 2 & 0 \\
\hline Nematoda & 252 & 0 & 6 \\
\hline \multicolumn{4}{|l|}{ Annelida } \\
\hline Oligochaeta & 1,136 & 138 & 79 \\
\hline \multicolumn{4}{|l|}{ Hirudinea } \\
\hline Erpobdellidae & 8 & 0 & 0 \\
\hline Glossiphoniidae & 0 & 4 & 0 \\
\hline \multicolumn{4}{|l|}{ Mollusca } \\
\hline \multicolumn{4}{|l|}{ Gastropoda } \\
\hline Planorbidae & 0 & 2 & 0 \\
\hline \multicolumn{4}{|l|}{ Arthropoda } \\
\hline \multicolumn{4}{|l|}{ Crustacea } \\
\hline Conchostraca & 20 & 24 & 2 \\
\hline Ostracoda & 516 & 8 & 0 \\
\hline Copepoda & 1,156 & 234 & 1,260 \\
\hline Amphipoda & 12 & 6 & 0 \\
\hline \multicolumn{4}{|l|}{ Chelicerata } \\
\hline Hydrachnellae & 4 & 2 & 0 \\
\hline \multicolumn{4}{|l|}{ Insecta } \\
\hline \multicolumn{4}{|l|}{ Ephemeroptera } \\
\hline Baetidae & 0 & 2 & 0 \\
\hline \multicolumn{4}{|l|}{ Coleoptera } \\
\hline Dytiscidae & 0 & 2 & 0 \\
\hline \multicolumn{4}{|l|}{ Diptera } \\
\hline Chironomidae & 408 & 170 & 482 \\
\hline
\end{tabular}

Sampling date: July 23, 1991

$\begin{array}{lrrr}\text { Nematoda } & 52 & 26 & 2 \\ \text { Annelida } & & & \\ \quad \text { Oligochaeta } & 934 & 132 & 8 \\ \quad \begin{array}{l}\text { Hirudinea } \\ \quad \text { Glossiphoniidae }\end{array} & 49 & 2 & 2 \\ \begin{array}{l}\text { Mollusca } \\ \text { Gastropoda } \\ \quad \text { Planorbidae }\end{array} & 1 & 2 & 0\end{array}$


Table 20. Benthic invertebrate surveys, 1990-91--Continued

\begin{tabular}{|c|c|c|c|}
\hline \multirow{3}{*}{ Taxonomic entry } & \multirow{2}{*}{\multicolumn{3}{|c|}{$\frac{\text { Individuals }}{\text { Replicate number }}$}} \\
\hline & & & \\
\hline & 1 & 2 & 3 \\
\hline \multicolumn{4}{|c|}{$\begin{array}{l}\text { Site 12--Continued } \\
\text { Sampling date: July 23, 1991--Continued }\end{array}$} \\
\hline \multicolumn{4}{|l|}{ Arthropoda } \\
\hline \multicolumn{4}{|l|}{ Crustacea } \\
\hline Conchostraca & 5 & 0 & 20 \\
\hline Copepoda & 216 & 40 & 10 \\
\hline Amphipoda & 27 & 0 & 11 \\
\hline \multicolumn{4}{|l|}{ Chelicerata } \\
\hline Hydrachnellae & 1 & 0 & C \\
\hline \multicolumn{4}{|l|}{ Insecta } \\
\hline \multicolumn{4}{|l|}{ Ephemeroptera } \\
\hline Baetidae & 8 & 0 & c \\
\hline \multicolumn{4}{|l|}{ Odonata } \\
\hline Coenagrionidae & 19 & 0 & ( \\
\hline \multicolumn{4}{|l|}{ Coleoptera } \\
\hline Haliplidae & 1 & 2 & 0 \\
\hline Dytiscidae & 11 & 0 & \\
\hline \multicolumn{4}{|l|}{ Diptera } \\
\hline Chironomidae & 356 & 612 & $r$ \\
\hline
\end{tabular}

Sampling date: August 12, 1991

\begin{tabular}{|c|c|c|c|}
\hline Coelenterata & 0 & 0 & 6 \\
\hline Nematoda & 40 & 24 & 6 \\
\hline \multicolumn{4}{|l|}{ Annelida } \\
\hline Oligochaeta & 260 & 324 & 9 \\
\hline \multicolumn{4}{|l|}{ Hirudinea } \\
\hline Erpobdellidae & 0 & 4 & 0 \\
\hline Glossiphoniidae & 188 & 220 & 162 \\
\hline \multicolumn{4}{|l|}{ Mollusca } \\
\hline \multicolumn{4}{|l|}{ Gastropoda } \\
\hline Physidae & 0 & 4 & 0 \\
\hline Planorbidae & 4 & 0 & 12 \\
\hline \multicolumn{4}{|l|}{ Arthropoda } \\
\hline \multicolumn{4}{|l|}{ Crustacea } \\
\hline Conchostraca & 60 & 20 & 90 \\
\hline Ostracoda & 0 & 0 & 90 \\
\hline Copepoda & 48 & 28 & 576 \\
\hline Amphipoda & 148 & 96 & 321 \\
\hline \multicolumn{4}{|l|}{ Chelicerata } \\
\hline Hydrachnellae & 4 & 4 & 6 \\
\hline \multicolumn{4}{|l|}{ Insecta } \\
\hline \multicolumn{4}{|l|}{ Ephemeroptera } \\
\hline Caenidae & 0 & 0 & 3 \\
\hline Baetidae & 28 & 60 & 129 \\
\hline \multicolumn{4}{|l|}{ Odonata } \\
\hline Coenagrionidae & 8 & 4 & 24 \\
\hline \multicolumn{4}{|l|}{ Coleoptera } \\
\hline Haliplidae & 4 & 8 & 0 \\
\hline Dytiscidae & 0 & 0 & 12 \\
\hline \multicolumn{4}{|l|}{ Diptera } \\
\hline Chironomidae & 424 & 320 & 102 \\
\hline
\end{tabular}

\begin{tabular}{|c|c|c|c|}
\hline Coelenterata & 0 & 10 & 50 \\
\hline \multicolumn{4}{|l|}{ Platyhelminthes } \\
\hline Tubellaria & 0 & 4 & 48 \\
\hline Nematoda & 4 & 6 & 5 \\
\hline \multicolumn{4}{|l|}{ Annelida } \\
\hline Oligochaeta & 2,104 & 1,407 & 1,065 \\
\hline \multicolumn{4}{|l|}{ Hirudinea } \\
\hline Erpobdellidae & 15 & 3 & 5 \\
\hline Glossiphoniidae & 37 & 14 & 72 \\
\hline \multicolumn{4}{|l|}{ Mollusca } \\
\hline \multicolumn{4}{|l|}{ Gastropoda } \\
\hline Lymnaeidae & 0 & 0 & 2 \\
\hline Physidae & 0 & 1 & 0 \\
\hline Planorbidae & 0 & 2 & 41 \\
\hline Pelecypoda & 37 & 48 & 8 \\
\hline \multicolumn{4}{|l|}{ Arthropoda } \\
\hline \multicolumn{4}{|l|}{ Crustacea } \\
\hline Conchostraca & 5 & 1 & 2 \\
\hline Copepoda & 2 & 0 & 0 \\
\hline Amphipoda & 4 & 2 & 29 \\
\hline Isopoda & 0 & 0 & 1 \\
\hline \multicolumn{4}{|l|}{ Insecta } \\
\hline \multicolumn{4}{|l|}{ Ephemeroptera } \\
\hline Caenidae & 0 & 0 & 4 \\
\hline \multicolumn{4}{|l|}{ Odonata } \\
\hline Coenagrionidae & 0 & 0 & 3 \\
\hline \multicolumn{4}{|l|}{ Diptera } \\
\hline Chironomidae & 14 & 0 & 0 \\
\hline
\end{tabular}

Site 13

Sampling date: June 20, 1991

Taxonomic entry

Individuals

Replicate number

123


Table 20. Benthic invertebrate surveys, 1990-91--Continued

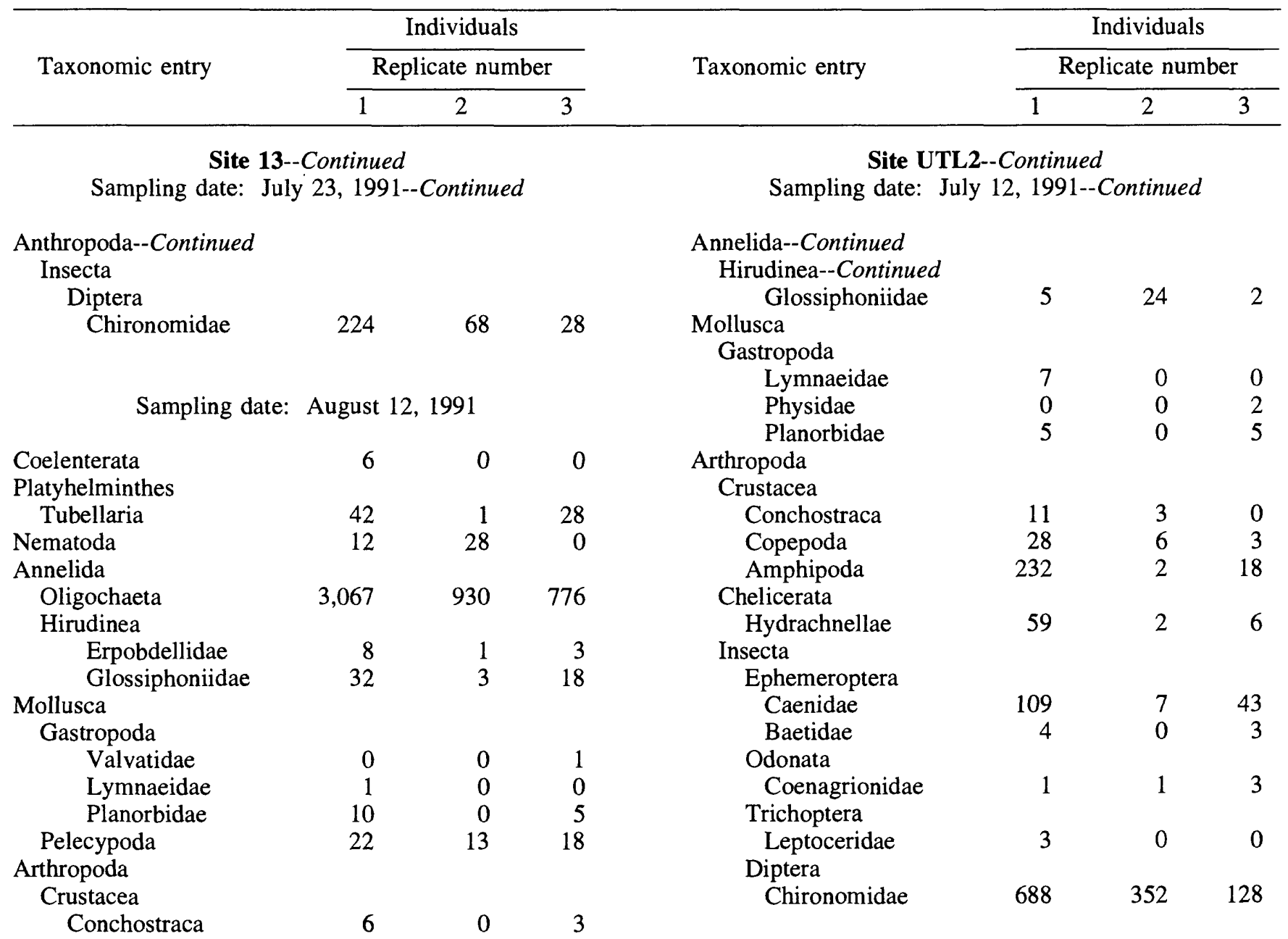

$\begin{array}{llll}\text { Copepoda } & 32 & 0 & 5 \\ \text { Amphipoda } & 27 & 0 & 1\end{array}$

$\begin{array}{llll}\text { Isopoda } & 7 & 0 & 2\end{array}$

Insecta

$\begin{array}{lccc}\begin{array}{l}\text { Ephemeroptera } \\ \text { Baetidae }\end{array} & 4 & 0 & 0 \\ \begin{array}{l}\text { Odonata } \\ \text { Coenagrionidae }\end{array} & 2 & 0 & 0 \\ \begin{array}{l}\text { Coleoptera } \\ \quad \text { Haliplidae }\end{array} & 0 & 1 & 0 \\ \begin{array}{l}\text { Dytiscidae } \\ \text { Diptera }\end{array} & 2 & 0 & 1 \\ \quad \text { Chironomidae } & 31 & 33 & 4\end{array}$

Site UTL2

Sampling date: July 12, 1990

Coelenterata

Nematoda

Annelida

Oligochaeta

Hirudinea

Erpobdellidae

$\begin{array}{rrr}9 & 0 & 1 \\ 4 & 29 & 0\end{array}$

519

385

0
1

4

0
Nematoda

Nematomorpha

Annelida

Oligochaeta

Hirudinea

Erpobdellidae

Glossiphoniidae

Arthropoda

Crustacea

Conchostraca

Copepoda

Chelicerata

Hydrachnellae

Insecta

Ephemeroptera

Caenidae

Baetidae

Diptera

Tipulidae

Chironomidae
Site UTL3

Sampling date: July 12, 1990

0 
Table 20. Benthic invertebrate surveys, 1990-91--Continued

\begin{tabular}{|c|c|c|}
\hline \multirow[t]{2}{*}{ Taxonomic entry } & \multicolumn{2}{|c|}{$\frac{\text { Individu }}{\text { Replicate } \mathrm{n} u}$} \\
\hline & 1 & 2 \\
\hline \multicolumn{3}{|c|}{$\begin{array}{l}\text { Site UTL4 } \\
\text { Sampling date: July } 12,1990\end{array}$} \\
\hline Nematoda & 1 & 2 \\
\hline $\begin{array}{l}\text { Annelida } \\
\text { Oligochaeta } \\
\text { Hirudinea }\end{array}$ & 2 & 1 \\
\hline $\begin{array}{l}\text { Glossiphoniidae } \\
\text { Arthropoda }\end{array}$ & 0 & 2 \\
\hline $\begin{array}{l}\text { Crustacea } \\
\text { Conchostraca }\end{array}$ & 1 & 3 \\
\hline $\begin{array}{l}\text { Copepoda } \\
\text { Chelicerata }\end{array}$ & 0 & 17 \\
\hline $\begin{array}{l}\text { Hydrachnellae } \\
\text { Insecta } \\
\text { Dintera }\end{array}$ & 0 & 3 \\
\hline Tipulidae & 0 & 1 \\
\hline Chironomidae & 42 & 31 \\
\hline
\end{tabular}

Taxonomic entry

Individuals

Replicate number

\begin{tabular}{lll}
\hline 1 & 2 & 3
\end{tabular}

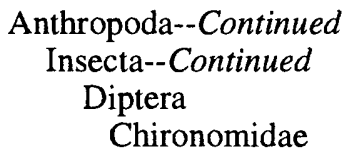

Site UTL5--Continued

Sampling date: July 19, 1990--Continued

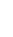

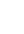

Site UTL5

Sampling date: July 19, 1990

3

17

3

$\begin{array}{lrrr}\text { Nematoda } & 1 & 5 & 3 \\ \text { Annelida } & & & \\ \quad \text { Oligochaeta } & 115 & 167 & 95 \\ \quad \text { Hirudinea } & & & \\ \quad \text { Erpobdellidae } & 2 & 3 & 1 \\ \quad \text { Glossiphoniidae } & 4 & 14 & 8 \\ \text { Mollusca } & & & \\ \quad \text { Gastropoda } & & & \\ \quad \text { Valvatidae } & 0 & 3 & 0 \\ \quad \text { Lymnaeidae } & 28 & 5 & 0 \\ \quad \text { Physidae } & 0 & 1 & 0 \\ \text { Arthropoda } & & & \\ \text { Crustacea } & & & \\ \quad \text { Conchostraca } & 1 & 2 & 0 \\ \quad \text { Copepoda } & 2 & 1 & 3 \\ \quad \text { Amphipoda } & 5 & 81 & 3 \\ \text { Chelicerata } & & & \\ \quad \text { Hydrachnellae } & 1 & 4 & 1 \\ \text { Insecta } & & & \\ \text { Ephemeroptera } & & & 1 \\ \quad \text { Caenidae } & 0 & 15 & 1 \\ \quad \text { Baetidae } & 1 & 45 & 5 \\ \quad \text { Trichoptera } \\ \quad \text { Leptoceridae } & 5 & 7 & 11\end{array}$

Site UTL6

Sampling date: July 19, 1990

\begin{tabular}{|c|c|c|c|}
\hline Coelenterata & 0 & 0 & 2 \\
\hline \multicolumn{4}{|l|}{ Annelida } \\
\hline Oligochaeta & 92 & 137 & 97 \\
\hline \multicolumn{4}{|l|}{ Hirudinea } \\
\hline Glossiphoniidae & 18 & 30 & 50 \\
\hline \multicolumn{4}{|l|}{ Mollusca } \\
\hline \multicolumn{4}{|l|}{ Gastropoda } \\
\hline Planorbidae & 1 & 0 & 0 \\
\hline \multicolumn{4}{|l|}{ Arthropoda } \\
\hline \multicolumn{4}{|l|}{ Crustacea } \\
\hline Conchostraca & 3 & 2 & 2 \\
\hline Copepoda & 1 & 0 & 1 \\
\hline Amphipoda & 18 & 4 & 3 \\
\hline \multicolumn{4}{|l|}{ Chelicerata } \\
\hline Hydrachnellae & 10 & 3 & 2 \\
\hline \multicolumn{4}{|l|}{ Insecta } \\
\hline \multicolumn{4}{|l|}{ Ephemeroptera } \\
\hline Caenidae & 1 & 2 & 0 \\
\hline Baetidae & 7 & 5 & 0 \\
\hline \multicolumn{4}{|l|}{ Odonata } \\
\hline Coenagrionidae & 3 & 0 & 0 \\
\hline \multicolumn{4}{|l|}{ Trichoptera } \\
\hline Leptoceridae & 2 & 2 & 2 \\
\hline \multicolumn{4}{|l|}{ Diptera } \\
\hline Chironomidae & 52 & 12 & 25 \\
\hline
\end{tabular}

Sampling date: August 19, 1991

$\begin{array}{llrr}\text { Nematoda } & 2 & 0 & 4 \\ \text { Annelida } & & & \\ \quad \text { Oligochaeta } & 6 & 18 & 672 \\ \text { Hirudinea } & & & \\ \quad \text { Erpobdellidae } & 0 & 0 & 4 \\ \quad \text { Glossiphoniidae } & 2 & 10 & 32\end{array}$


Table 20. Benthic invertebrate surveys, 1990-91--Continued

\begin{tabular}{|c|c|c|c|}
\hline \multirow{3}{*}{ Taxonomic entry } & Individuals & \multirow{3}{*}{ Taxonomic entry } & Individuals \\
\hline & Replicate number & & Replicate number \\
\hline & 2 & & 1 \\
\hline
\end{tabular}

Site UTL6--Continued

Sampling date: August 19, 1991--Continued

\section{Site UTL8}

Sampling date: July 12,1990
Mollusca

Gastropoda

Physidae

Arthropoda

Crustacea

Conchostraca

Copepoda

Amphipoda

Chelicerata

Hydrachnellae

Insecta

Ephemeroptera

Baetidae

Diptera

Chironomidae $\begin{array}{lll}0 & 0 & 4\end{array}$

$\begin{array}{lll}1 & 4 & 24\end{array}$

$3 \quad 4 \quad 8$

$\begin{array}{lll}0 & 2 & 0\end{array}$

$\begin{array}{lll}2 & 10 & 8\end{array}$

$15 \quad 0 \quad 4$

$\begin{array}{lll}16 & 2 & 32\end{array}$

Site UTL7

Sampling date: July 19, 1990

Annelida

Oligochaeta

Hirudinea

Erpobdellidae

Glossiphoniidae

Mollusca

Gastropoda

Lymnaeidae

Arthropoda

Crustacea

Conchostraca

Copepoda

Amphipoda

Chelicerata

Hydrachnellae

Insecta

Ephemeroptera

Caenidae

Baetidae

Trichoptera

Leptoceridae

Diptera

Tipulidae

Chironomidae
262

$92 \quad 98$

0

60

20

$59 \quad 64$

0

$0 \quad 2$

$17 \quad 6 \quad 0$

$\begin{array}{lll}5 & 2 & 2\end{array}$

0

2

12

1$$
0
$$

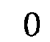

$\begin{array}{rrr}3 & 2 & 6 \\ 0 & 0 & 4 \\ 1 & 1 & 3 \\ 3 & 3 & 2 \\ 104 & 49 & 48\end{array}$

Coelenterata

Nematoda

Annelida

Oligochaeta

Hirudinea

$\begin{array}{llll}\text { Erpobdellidae } & 0 & 0 & 2\end{array}$

Mollusca

Glossiphoniidae

Gastropoda

Lymnaeidae

Physidae

Planorbidae

Arthropoda

Crustacea

Conchostraca

Copepoda

Amphipoda

Chelicerata

Hydrachnellae

Insecta

Ephemeroptera

Caenidae

Baetidae

Diptera

Chironomidae

Sampling date: August 19, 1991

Nematoda

Annelida

Oligochaeta

Hirudinea

Erpobdellidae

Glossiphoniidae

Mollusca

Gastropoda

$\begin{array}{lll}\text { Planorbidae } & 20 & 8\end{array}$

Arthropoda

Crustacea

Conchostraca

Ostracoda

Copepoda

Amphipoda

Chelicerata

Hydrachnellae $\begin{array}{lll}0 & 0 & 3\end{array}$

$307 \quad 305$

322

$\begin{array}{lll}117 & 70 & 48\end{array}$

$\begin{array}{lll}0 & 2 & 4\end{array}$

$\begin{array}{lll}0 & 3 & 0\end{array}$

$\begin{array}{lll}4 & 3 & 5\end{array}$

$\begin{array}{lll}3 & 4 & 6\end{array}$

$\begin{array}{lll}40 & 48 & 9\end{array}$

$19 \quad 11 \quad 5$

$181 \quad 130 \quad 193$

$\begin{array}{lll}0 & 0 & 2\end{array}$

$25-0 \quad 5$ 
Table 20. Benthic invertebrate surveys, 1990-91--Continued

\begin{tabular}{|c|c|c|c|c|c|c|c|}
\hline \multirow{3}{*}{ Taxonomic entry } & \multicolumn{3}{|c|}{ Individuals } & \multirow{3}{*}{ Taxonomic entry } & \multicolumn{3}{|c|}{ Individuals } \\
\hline & \multicolumn{3}{|c|}{ Replicate number } & & \multicolumn{3}{|c|}{ Replicate number } \\
\hline & 1 & 2 & 3 & & 1 & 2 & 3 \\
\hline \multirow{2}{*}{\multicolumn{4}{|c|}{$\begin{array}{l}\text { Site UTL8--Continued } \\
\text { Sampling date: August 19,1991--Continued }\end{array}$}} & \multirow{2}{*}{\multicolumn{4}{|c|}{$\begin{array}{l}\text { Site UTL10--Continued } \\
\text { Sampling date: August 14, 1990--Continued }\end{array}$}} \\
\hline & & & & & & & \\
\hline \multirow{2}{*}{\multicolumn{4}{|c|}{$\begin{array}{l}\text { Arthropoda--Continued } \\
\text { Insecta }\end{array}$}} & \multicolumn{4}{|l|}{ Annelida--Continued } \\
\hline & & & & \multicolumn{4}{|l|}{ Hirudinea } \\
\hline Ephemeroptera & & & & Glossiphoniidae & 18 & 5 & 2 \\
\hline Caenidae & 16 & 0 & & \multicolumn{4}{|l|}{ Mollusca } \\
\hline Baetidae & 2 & 1 & & \multicolumn{4}{|l|}{ Gastropoda } \\
\hline Diptera & & & & Valvatidae & 7 & 3 & 1 \\
\hline \multirow[t]{3}{*}{ Chironomidae } & \multirow[t]{3}{*}{254} & \multirow{3}{*}{\multicolumn{2}{|c|}{51}} & Lymnaeidae & 1 & 18 & 1 \\
\hline & & & & Physidae & 1 & 3 & 0 \\
\hline & & & & Planorbidae & 2 & 5 & 2 \\
\hline \multirow{3}{*}{\multicolumn{4}{|c|}{$\begin{array}{l}\text { Site UTL9 } \\
\text { Sampling date: July 19, } 1990\end{array}$}} & Pelecypoda & 17 & 4 & 1 \\
\hline & & & & \multirow{2}{*}{\multicolumn{4}{|c|}{$\begin{array}{l}\text { Arthropoda } \\
\text { Crustacea }\end{array}$}} \\
\hline & & & & & & & \\
\hline Nematoda & 5 & 6 & 34 & Conchostraca & 9 & 12 & 6 \\
\hline Annelida & & & & Copepoda & 7 & 1 & 5 \\
\hline Oligochaeta & 65 & 94 & 130 & Amphipoda & 2 & 0 & 0 \\
\hline Hirudinea & & & & Chelicerata & & & \\
\hline Erpobdellidae & 16 & 19 & 15 & Hydrachnellae & 1 & 0 & 3 \\
\hline Glossiphoniidae & 8 & 11 & 10 & Insecta & & & \\
\hline Mollusca & & & & Ephemeroptera & & & \\
\hline Gastropoda & & & & Caenidae & 0 & 0 & 1 \\
\hline Valvatidae & 13 & 2 & 6 & Baetidae & 1 & 5 & 2 \\
\hline Lymnaeidae & 8 & 3 & 6 & Diptera & & & \\
\hline Physidae & 1 & 3 & 1 & Chironomidae & 2 & 12 & 4 \\
\hline Planorbidae & 11 & 1 & 4 & & & & \\
\hline Arthropoda & & & & & & & \\
\hline Crustacea & & & & & 11 & & \\
\hline Conchostraca & 3 & 0 & 3 & Sampling d & gust & & \\
\hline Copepoda & 6 & 6 & 63 & & & & \\
\hline Amphipoda & 4 & 7 & 19 & Nematoda & 1 & 0 & 0 \\
\hline Chelicerata & & & & Annelida & & & \\
\hline Hydrachnellae & 1 & 2 & 3 & Oligochaeta & 29 & 80 & 3 \\
\hline Insecta & & & & Hirudinea & & & \\
\hline Ephemeroptera & & & & Erpobdellidae & 3 & 10 & 0 \\
\hline Caenidae & 2 & 2 & 9 & Glossiphoniidae & 9 & 21 & 18 \\
\hline Baetidae & 1 & 1 & 3 & Mollusca & & & \\
\hline Odonata & & & & Gastropoda & & & \\
\hline Coenagrionidae & 0 & 0 & 1 & Valvatidae & 5 & 4 & 0 \\
\hline Trichoptera & & & & Lymnaeidae & 10 & 1 & 2 \\
\hline Leptoceridae & 1 & 0 & 0 & Planorbidae & 0 & 2 & 1 \\
\hline Diptera & & & & Arthropoda & & & \\
\hline Chironomidae & 7 & 4 & 47 & Crustacea & & & \\
\hline & & & & Conchostraca & 1 & 6 & 0 \\
\hline & & & & Copepoda & 7 & 3 & 0 \\
\hline & L10 & & & Amphipoda & 11 & 9 & 1 \\
\hline Sampling $c$ & ugust & 990 & & Chelicerata & & & \\
\hline & & & & Hydrachnellae & 10 & 6 & 1 \\
\hline Nematoda & 5 & 1 & 0 & Insecta & & & \\
\hline Annelida & & & & Ephemeroptera & & & \\
\hline Oligochaeta & 62 & 34 & 20 & Caenidae & 2 & 6 & 0 \\
\hline Erpobdellidae & 2 & 0 & 1 & Baetidae & 8 & 6 & 2 \\
\hline
\end{tabular}


Table 20. Benthic invertebrate surveys, 1990-91--Continued

\begin{tabular}{|c|c|c|c|c|c|c|c|}
\hline \multirow{3}{*}{ Taxonomic entry } & \multirow{2}{*}{\multicolumn{3}{|c|}{$\frac{\text { Individuals }}{\text { Replicate number }}$}} & \multirow{3}{*}{ Taxonomic entry } & \multicolumn{3}{|c|}{ Individuals } \\
\hline & & & & & \multicolumn{3}{|c|}{ Replicate number } \\
\hline & 1 & 2 & 3 & & 1 & 2 & 3 \\
\hline \multicolumn{4}{|c|}{ Site UTL11--Continued } & \multicolumn{4}{|c|}{ Site UTL13--Continued } \\
\hline \multicolumn{4}{|c|}{ Sampling date: August 14, 1990--Continued } & \multicolumn{4}{|c|}{ Sampling date: August 19, 1991--Continued } \\
\hline Arthropoda--Continued & & & & Annelida--Continued & & & \\
\hline Insecta--Continued & & & & Hirudinea--Continued & & & \\
\hline Trichoptera & & & & Glossiphoniidae & 44 & 148 & 64 \\
\hline Leptoceridae & 1 & 1 & 4 & Mollusca & & & \\
\hline Diptera & & & & Gastropoda & & & \\
\hline Tipulidae & 0 & 0 & 2 & Valvatidae & 24 & 56 & 8 \\
\hline \multirow{3}{*}{ Chironomidae } & 2 & 43 & 8 & Lymnaeidae & 0 & 0 & 48 \\
\hline & & & & Planorbidae & 4 & 20 & 8 \\
\hline & & & & Arthropoda & & & \\
\hline \multirow{3}{*}{\multicolumn{4}{|c|}{$\begin{array}{l}\text { Site UTL13 } \\
\text { Sampling date: August 14, } 1990\end{array}$}} & Crustacea & & & \\
\hline & & & & Conchostraca & 8 & 0 & 4 \\
\hline & & & & Ostracoda & 4 & 0 & 0 \\
\hline Nematoda & 2 & 4 & 3 & Copepoda & 20 & 4 & 124 \\
\hline Annelida & & & & Amphipoda & 20 & 16 & 12 \\
\hline Oligochaeta & 96 & 54 & 133 & Chelicerata & & & \\
\hline Hirudinea & & & & Hydrachnellae & 4 & 4 & 0 \\
\hline Erpobdellidae & 13 & 6 & 4 & Insecta & & & \\
\hline Glossiphoniidae & 40 & 23 & 9 & Ephemeroptera & & & \\
\hline Mollusca & & & & Caenidae & 4 & 4 & 0 \\
\hline Gastropoda & & & & Baetidae & 24 & 48 & 24 \\
\hline Valvatidae & 60 & 21 & 37 & Trichoptera & & & \\
\hline Lymnaeidae & 453 & 36 & 18 & Leptoceridae & 0 & 4 & 0 \\
\hline Physidae & 1 & 2 & 1 & Diptera & & & \\
\hline Planorbidae & 121 & 35 & 34 & Chironomidae & 8 & 68 & 12 \\
\hline
\end{tabular}

Arthropoda

Crustacea

$\begin{array}{llll}\text { Conchostraca } & 2 & 6 & 0\end{array}$

$\begin{array}{llll}\text { Copepoda } & 9 & 11 & 2\end{array}$

$\begin{array}{lrrr}\text { Amphipoda } & 15 & 3 & 39\end{array}$

Chelicerata

$\begin{array}{llll}\text { Hydrachnellae } & 6 & 4 & 2\end{array}$

Insecta

Ephemeroptera

$\begin{array}{llll}\text { Caenidae } & 1 & 3 & 0\end{array}$

$\begin{array}{llll}\text { Baetidae } & 79 & 71 & 16\end{array}$

Odonata

$\begin{array}{llll}\text { Coenagrionidae } & 3 & 0 & 2\end{array}$

Diptera

$\begin{array}{llll}\text { Chironomidae } & 121 & 40 & 295\end{array}$

Sampling date: August 19, 1991

$\begin{array}{lrrr}\text { Coelenterata } & 16 & 44 & 8 \\ \text { Nematoda } & 8 & 8 & 12 \\ \begin{array}{l}\text { Annelida } \\ \quad \text { Oligochaeta }\end{array} & 176 & 372 & 632 \\ \begin{array}{l}\text { Hirudinea } \\ \quad \text { Erpobdellidae }\end{array} & 4 & 0 & 8\end{array}$

Site LTL2

Sampling date: August 13, 1990

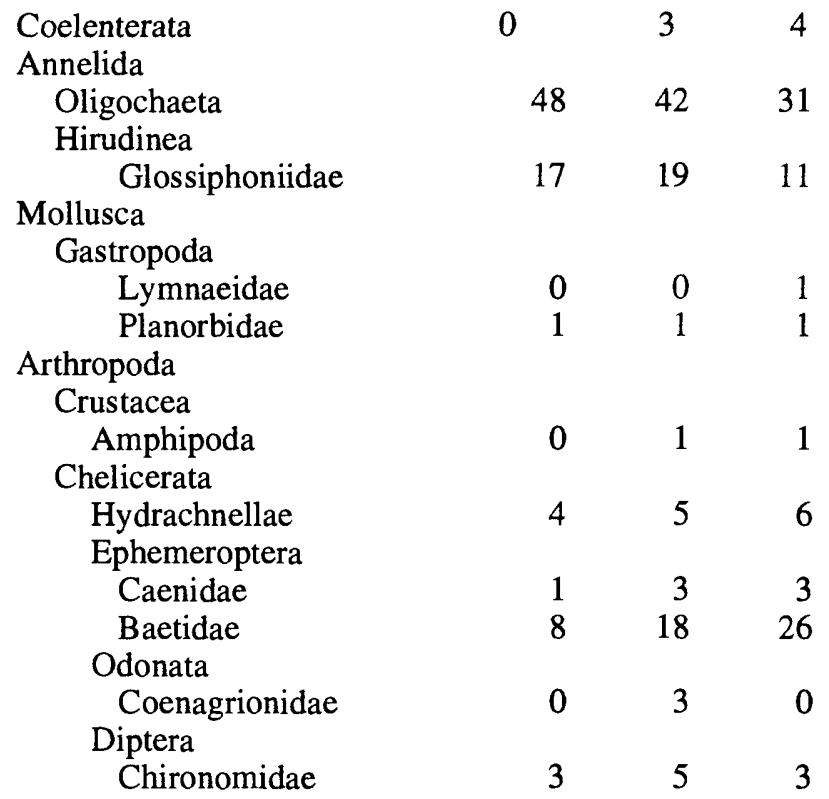


Table 20. Benthic invertebrate surveys, 1990-91--Continued

\begin{tabular}{|c|c|c|c|c|c|c|c|}
\hline \multirow{3}{*}{ Taxonomic entry } & \multirow{2}{*}{\multicolumn{3}{|c|}{$\begin{array}{c}\text { Individuals } \\
\text { Replicate number }\end{array}$}} & \multirow{3}{*}{ Taxonomic entry } & \multirow{2}{*}{\multicolumn{3}{|c|}{$\begin{array}{c}\text { Individuals } \\
\text { Replicate number }\end{array}$}} \\
\hline & & & & & & & \\
\hline & 1 & 2 & 3 & & 1 & 2 & 3 \\
\hline \multicolumn{4}{|c|}{$\begin{array}{l}\text { Site LTL4 } \\
\text { Sampling date: August } 13,1990\end{array}$} & \multicolumn{4}{|c|}{$\begin{array}{l}\text { Site LTL5--Continued } \\
\text { Sampling date: August 13, 1990--Continued }\end{array}$} \\
\hline Coelenterata & 0 & 1 & 0 & \multicolumn{4}{|l|}{ Arthropoda--Continued } \\
\hline Nematoda & 4 & 0 & 1 & \multicolumn{4}{|l|}{ Chelicerata } \\
\hline Annelida & & & & Hydrachnellae & 3 & 0 & 6 \\
\hline Oligochaeta & 0 & 6 & 25 & \multicolumn{4}{|l|}{ Insecta } \\
\hline Hirudinea & & & & \multicolumn{4}{|l|}{ Ephemeroptera } \\
\hline Erpobdellidae & 0 & 1 & 0 & Caenidae & 1 & 1 & 0 \\
\hline Glossiphoniidae & 3 & 10 & 10 & Baetidae & 21 & 0 & 5 \\
\hline Mollusca & & & & \multicolumn{4}{|l|}{ Odonata } \\
\hline Gastropoda & & & & Coenagrionidae & 3 & 1 & 0 \\
\hline Physidae & 0 & 3 & 1 & \multicolumn{4}{|l|}{ Diptera } \\
\hline Planorbidae & 0 & 14 & 39 & Stratiomyidae & 1 & 0 & 0 \\
\hline Arthropoda & & & & \multirow[t]{2}{*}{ Chironomidae } & \multirow[t]{2}{*}{6} & \multicolumn{2}{|c|}{1} \\
\hline \multicolumn{6}{|l|}{ Crustacea } & & \\
\hline Conchostraca & 0 & 1 & 0 & \multirow{2}{*}{\multicolumn{4}{|c|}{ Site LTL6 }} \\
\hline Copepoda & 4 & 14 & 9 & & & & \\
\hline Amphipoda & 0 & 3 & 8 & \multicolumn{4}{|c|}{ Sampling date: August 13, 1990} \\
\hline \multicolumn{8}{|l|}{ Chelicerata } \\
\hline Hydrachnellae & 0 & 3 & 4 & Coelenterata & 0 & 0 & 11 \\
\hline Insecta & & & & Nematoda & 0 & 1 & 0 \\
\hline Ephemeroptera & & & & Annelida & & & \\
\hline Caenidae & 0 & 28 & 42 & Oligochaeta & 1 & 1 & 510 \\
\hline Baetidae & 0 & 65 & 69 & Hirudinea & & & \\
\hline Odonata & & & & Erpobdellidae & 1 & 2 & 4 \\
\hline Libellulidae & 0 & 0 & 1 & Glossiphoniidae & 0 & 3 & 5 \\
\hline Coenagrionidae & 0 & 4 & 18 & Mollusca & & & \\
\hline Trichoptera & & & & Gastropoda & & & \\
\hline Leptoceridae & 0 & 1 & 0 & Lymnaeidae & 0 & 0 & 10 \\
\hline Diptera & & & & Planorbidae & 0 & 0 & 4 \\
\hline Chironomidae & 1 & 8 & 24 & Arthropoda & & & \\
\hline & & & & Crustacea & & & \\
\hline & & & & Conchostraca & 1 & 5 & 0 \\
\hline & & & & Copepoda & 5 & 4 & 1 \\
\hline Sampling da & gust 1 & 990 & & Amphipoda & 0 & 5 & 13 \\
\hline & & & & Chelicerata & & & \\
\hline Coelenterata & 5 & 0 & 0 & Hydrachnellae & 1 & 1 & 6 \\
\hline Annelida & & & & Insecta & & & \\
\hline Oligochaeta & 122 & 99 & 54 & Ephemeroptera & & & \\
\hline Hirudinea & & & & Caenidae & 1 & 2 & 3 \\
\hline Erpobdellidae & 1 & 1 & 1 & Baetidae & 0 & 1 & 10 \\
\hline Glossiphoniidae & 6 & 46 & 6 & Odonata & & & \\
\hline Mollusca & & & & Coenagrionidae & 0 & 0 & 2 \\
\hline Gastropoda & & & & Trichoptera & & & \\
\hline Lymnaeidae & 1 & 0 & 0 & Leptoceridae & 0 & 1 & 1 \\
\hline Planorbidae & 10 & 0 & 6 & Diptera & & & \\
\hline Arthropoda & & & & Chironomidae & 9 & 7 & 130 \\
\hline Crustacea & & & & & & & \\
\hline Copepoda & 3 & 0 & 1 & & & & \\
\hline Amphipoda & 3 & 1 & 0 & & & & \\
\hline
\end{tabular}


Table 20. Benthic invertebrate surveys, 1990-91--Continued

\begin{tabular}{|c|c|c|c|c|c|c|c|}
\hline \multirow{3}{*}{ Taxonomic entry } & \multicolumn{3}{|c|}{ Individuals } & \multirow{3}{*}{ Taxonomic entry } & \multicolumn{3}{|c|}{ Individuals } \\
\hline & \multicolumn{3}{|c|}{ Replicate number } & & \multicolumn{3}{|c|}{ Replicate number } \\
\hline & 1 & 2 & 3 & & 1 & 2 & 3 \\
\hline \multicolumn{4}{|c|}{$\begin{array}{c}\text { Site SSD1 } \\
\text { Sampling date: August 27, } 1990\end{array}$} & \multicolumn{4}{|c|}{$\begin{array}{l}\text { Site JCN1--Continued } \\
\text { Sampling date: August 27, 1990--Continued }\end{array}$} \\
\hline Coelenterata & 16 & 7 & 15 & Mollusca & & & \\
\hline Platyhelminthes & & & & Gastropoda & & & \\
\hline Tubellaria & 6 & 0 & 0 & Valvatidae & 33 & 27 & 187 \\
\hline Nematoda & 5 & 10 & 9 & Hydrobiidae & 1 & 0 & 0 \\
\hline Annelida & & & & Lymnaeidae & 0 & 1 & 36 \\
\hline Oligochaeta & 73 & 49 & 105 & Physidae & 0 & 0 & 2 \\
\hline Hirudinea & & & & Planorbidae & 2 & 7 & 28 \\
\hline Erpobdellidae & 2 & 2 & 6 & Pelecypoda & 29 & 38 & 280 \\
\hline Glossiphoniidae & 21 & 20 & 23 & Arthropoda & & & \\
\hline Mollusca & & & & Crustacea & & & \\
\hline Gastropoda & & & & Conchostraca & 5 & 8 & 0 \\
\hline Valvatidae & 159 & 155 & 159 & Ostracoda & 1 & 0 & 0 \\
\hline Hydrobiidae & 0 & 0 & 4 & Copepoda & 0 & 1 & 0 \\
\hline Lymnaeidae & 16 & 3 & 7 & Amphipoda & 14 & 10 & 195 \\
\hline Physidae & 44 & 19 & 18 & Chelicerata & & & \\
\hline Planorbidae & 98 & 26 & 91 & Hydrachnellae & 0 & 8 & 4 \\
\hline Pelecypoda & 35 & 14 & 5 & Insecta & & & \\
\hline Arthropoda & & & & Ephemeroptera & & & \\
\hline Crustacea & & & & Caenidae & 19 & 8 & 55 \\
\hline Conchostraca & 2 & 2 & 0 & Baetidae & 1 & 10 & 13 \\
\hline Copepoda & 22 & 9 & 3 & Odonata & & & \\
\hline Amphipoda & 108 & 48 & 53 & Coenagrionidae & 0 & 1 & 7 \\
\hline Chelicerata & & & & Coleoptera & & & \\
\hline Hydrachnellae & 0 & 4 & 0 & Haliplidae & 0 & 6 & 44 \\
\hline Insecta & & & & Trichoptera & & & \\
\hline Ephemeroptera & & & & Leptoceridae & 0 & 0 & 2 \\
\hline Caenidae & 6 & 22 & 27 & Diptera & & & \\
\hline Baetidae & 6 & 7 & 7 & Ceratopogonidae & 2 & 0 & 0 \\
\hline Odonata & & & & Chironomidae & 7 & 8 & 9 \\
\hline
\end{tabular}

Site NC1

Coleoptera

Haliplidae

Diptera

Chironomidae

$3 \quad 0 \quad 0$

$\begin{array}{lll}0 & 1 & 0\end{array}$

Sampling date: August 27, 1990

$\begin{array}{lrrr}\text { Coelenterata } & 2 & 2 & 86 \\ \begin{array}{l}\text { Platyhelminthes } \\ \text { Tubellaria }\end{array} & 2 & 32 & 23 \\ \begin{array}{l}\text { Nematoda } \\ \text { Annelida }\end{array} \quad \text { Oligochaeta } & 9 & 14 & 22 \\ \quad \text { Hirudinea } & 304 & 84 & 549 \\ \quad \text { Erpobdellidae } & 3 & 13 & 11 \\ \quad \text { Glossiphoniidae } & 9 & 20 & 19 \\ \text { Mollusca } & & & \\ \quad \text { Gastropoda } & & & \\ \quad \text { Valvatidae } & 42 & 32 & 83 \\ \quad \text { Hydrobiidae } & 77 & 8 & 41 \\ \quad \text { Physidae } & 0 & 0 & 4 \\ \quad \text { Planorbidae } & 4 & 3 & 3 \\ \text { Pelecypoda } & 16 & 0 & 5\end{array}$

$\begin{array}{lrrr}\text { Coelenterata } & 0 & 0 & 138 \\ \begin{array}{l}\text { Platyhelminthes } \\ \quad \text { Tubellaria }\end{array} & 1 & 0 & 2 \\ \begin{array}{l}\text { Nematoda } \\ \text { Annelida }\end{array} & 83 & 58 & 169 \\ \quad \begin{array}{l}\text { Oligochaeta } \\ \text { Hirudinea }\end{array} & 355 & 225 & 477 \\ \quad \text { Erpobdellidae } & & & \\ \quad \text { Glossiphoniidae } & 0 & 2 & 14 \\ & 58 & 4 & 338\end{array}$


Table 20. Benthic invertebrate surveys, 1990-91--Continued

\begin{tabular}{|c|c|c|c|c|c|c|c|}
\hline \multirow{3}{*}{ Taxonomic entry } & \multicolumn{3}{|c|}{ Individuals } & \multirow{3}{*}{ Taxonomic entry } & \multicolumn{3}{|c|}{ Individuals } \\
\hline & \multicolumn{3}{|c|}{ Replicate number } & & \multicolumn{3}{|c|}{ Replicate number } \\
\hline & 1 & 2 & 3 & & 1 & 2 & 3 \\
\hline \multicolumn{4}{|c|}{$\begin{array}{l}\text { Site NC1--Continued } \\
\text { Sampling date: August 27, 1990--Continued }\end{array}$} & \multirow{2}{*}{\multicolumn{4}{|c|}{$\begin{array}{l}\text { Site 9A } \\
\text { Sampling date: } \text { August } 24,1990\end{array}$}} \\
\hline Sampling date: & & Contir & & & & & \\
\hline \multicolumn{4}{|l|}{ Arthropoda } & \multicolumn{4}{|l|}{ Annelida } \\
\hline \multicolumn{4}{|l|}{ Crustacea } & Oligochaeta & 0 & 4 & 12 \\
\hline Conchostraca & 0 & 0 & 1 & \multicolumn{4}{|l|}{ Arthropoda } \\
\hline Copepoda & 0 & 3 & 4 & \multicolumn{4}{|l|}{ Crustacea } \\
\hline Amphipoda & 6 & 13 & 140 & \multirow{2}{*}{$\begin{array}{l}\text { Conchostraca } \\
\text { Copepoda }\end{array}$} & \multirow{2}{*}{$\begin{array}{l}8 \\
0\end{array}$} & 12 & 16 \\
\hline \multicolumn{4}{|l|}{ Chelicerata } & & & 4 & 16 \\
\hline Hydrachnellae & 5 & 1 & 4 & Amphipoda & 4 & 0 & 0 \\
\hline Insecta & & & & Insecta & & & \\
\hline Ephemeroptera & & & & Ephemeroptera & & & \\
\hline Caenidae & 5 & 4 & 6 & Baetidae & 12 & 4 & 16 \\
\hline Baetidae & 2 & 0 & 11 & Diptera & & & \\
\hline Odonata & & & & Chironomidae & 24 & 16 & 100 \\
\hline Coenagrionidae & 0 & 6 & 7 & & & & \\
\hline Coleoptera & & & & & & & \\
\hline Haliplidae & 1 & 1 & 0 & Site & & & \\
\hline Dytiscidae & 0 & 0 & 1 & Sampling date: & gust 14 , & & \\
\hline Diptera & & & & & & & \\
\hline Chironomidae & 13 & 7 & 52 & Coelenterata & 11 & 5 & 0 \\
\hline & & & & Platyhelminthes & & & \\
\hline & & & & Tubellaria & 0 & 0 & 1 \\
\hline & & & & Nematoda & 16 & 9 & 23 \\
\hline Sampling d & gust & 990 & & Annelida & & & \\
\hline & & & & Oligochaeta & 652 & 471 & 612 \\
\hline Annelida & & & & Hirudinea & & & \\
\hline Oligochaeta & 173 & 148 & 516 & Erpobdellidae & 64 & 44 & 39 \\
\hline Hirudinea & & & & Glossiphoniidae & 41 & 25 & 55 \\
\hline Glossiphoniidae & 0 & 4 & 0 & Mollusca & & & \\
\hline Mollusca & & & & Gastropoda & & & \\
\hline Gastropoda & & & & Valvatidae & 511 & 433 & 351 \\
\hline Lymnaeidae & 2 & 0 & 4 & Planorbidae & 283 & 202 & 70 \\
\hline Physidae & 1 & 0 & 0 & Pelecypoda & 197 & 256 & 223 \\
\hline Planorbidae & 59 & 16 & 72 & Arthropoda & & & \\
\hline Pelecypoda & 0 & 4 & 0 & Crustacea & & & \\
\hline Arthropoda & & & & Copepoda & 0 & 4 & 13 \\
\hline Crustacea & & & & Amphipoda & 3 & 6 & 4 \\
\hline Ostracoda & 136 & 176 & 156 & Chelicerata & & & \\
\hline Copepoda & 36 & 64 & 12 & Hydrachnellae & 7 & 7 & 4 \\
\hline Amphipoda & 165 & 176 & 64 & Insecta & & & \\
\hline Chelicerata & & & & Ephemeroptera & & & \\
\hline Hydrachnellae & 2 & 0 & 0 & Caenidae & 0 & 1 & 0 \\
\hline Insecta & & & & Baetidae & 6 & 26 & 10 \\
\hline Ephemeroptera & & & & Odonata & & & \\
\hline Caenidae & 0 & 0 & 4 & Coenagrionidae & 2 & 4 & 1 \\
\hline Baetidae & 25 & 36 & 20 & Trichoptera & & & \\
\hline Odonata & & & & Leptoceridae & 1 & 1 & 1 \\
\hline Coenagrionidae & 6 & 0 & 12 & Diptera & & & \\
\hline Trichoptera & & & & Ceratopogonidae & 0 & 1 & 2 \\
\hline Leptoceridae & 3 & 0 & 0 & Chironomidae & 4 & 12 & 4 \\
\hline Diptera & & & & & & & \\
\hline Chironomidae & 177 & 188 & 256 & & & & \\
\hline
\end{tabular}


Table 20. Benthic invertebrate surveys, 1990-91--Continued

\begin{tabular}{|c|c|c|c|c|c|c|c|}
\hline \multirow{3}{*}{ Taxonomic entry } & \multicolumn{3}{|c|}{ Individuals } & \multirow{3}{*}{ Taxonomic entry } & \multicolumn{3}{|c|}{ Individuals } \\
\hline & \multicolumn{3}{|c|}{ Replicate number } & & \multicolumn{3}{|c|}{ Replicate number } \\
\hline & 1 & 2 & 3 & & 1 & 2 & 3 \\
\hline \multirow{2}{*}{\multicolumn{4}{|c|}{$\begin{array}{c}\text { Site LR5 } \\
\text { Sampling date: August 28, } 1990\end{array}$}} & \multirow{2}{*}{\multicolumn{4}{|c|}{$\begin{array}{c}\text { Site CL1--Continued } \\
\text { Sampling date: August 21, 1990--Continued }\end{array}$}} \\
\hline & & & & & & & \\
\hline Coelenterata & 0 & 1 & 11 & Annelida--Continued & & & \\
\hline Nematoda & 99 & 74 & 107 & Hirudinea & & & \\
\hline Annelida & & & & Erpobdellidae & 0 & 1 & 0 \\
\hline Oligochaeta & 729 & 669 & 953 & Mollusca & & & \\
\hline Hirudinea & & & & Gastropoda & & & \\
\hline Erpobdellidae & 25 & 28 & 12 & Valvatidae & 1 & 2 & 0 \\
\hline Glossiphoniidae & 53 & 32 & 130 & Planorbidae & 0 & 0 & 2 \\
\hline Mollusca & & & & Arthropoda & & & \\
\hline Gastropoda & & & & Crustacea & & & \\
\hline Valvatidae & 6 & 25 & 71 & Copepoda & 1 & 4 & 13 \\
\hline Hydrobiidae & 23 & 1 & 2 & Amphipoda & 1 & 0 & 0 \\
\hline Lymnaeidae & 0 & 0 & 1 & Insecta & & & \\
\hline Physidae & 0 & 0 & 11 & Ephemeroptera & & & \\
\hline Planorbidae & 0 & 0 & 17 & Ephemeridae & 0 & 0 & 1 \\
\hline Pelecypoda & 41 & 51 & 237 & Diptera & & & \\
\hline \multirow{2}{*}{\multicolumn{4}{|c|}{$\begin{array}{l}\text { Arthropoda } \\
\text { Crustacea }\end{array}$}} & Chironomidae & 1 & 2 & 3 \\
\hline & & & & & & & \\
\hline Conchostraca & 0 & 1 & 1 & & & & \\
\hline Copepoda & 2 & 3 & 2 & \multirow{2}{*}{\multicolumn{4}{|c|}{$\begin{array}{c}\text { Site CL2 } \\
\text { Sampling date: August 21, } 1990\end{array}$}} \\
\hline Amphipoda & 1 & 2 & 12 & & & & \\
\hline \multicolumn{8}{|l|}{ Insecta } \\
\hline Ephemeroptera & & & & Nematoda & 19 & 9 & 22 \\
\hline Caenidae & 0 & 0 & 1 & Annelida & & & \\
\hline Baetidae & 2 & 0 & 4 & Oligochaeta & 13 & 9 & 10 \\
\hline Odonata & & & & Mollusca & & & \\
\hline Coenagrionidae & 0 & 0 & 57 & Gastropoda & & & \\
\hline Neuroptera & & & & Valvatidae & 1 & $\mathrm{c}$ & 0 \\
\hline Sialidae & 1 & 0 & 3 & Lymnaeidae & 0 & 1 & 0 \\
\hline Coleoptera & & & & Arthropoda & & & \\
\hline Haliplidae & 0 & 0 & 1 & Crustacea & & & \\
\hline Dytiscidae & 0 & 0 & 2 & Copepoda & 5 & $\mathrm{c}$ & 9 \\
\hline Diptera & & & & Insecta & & & \\
\hline \multirow[t]{3}{*}{ Chironomidae } & 31 & 7 & 134 & Ephemeroptera & & & \\
\hline & & & & Ephemeridae & 1 & c & 0 \\
\hline & & & & Trichoptera & & & \\
\hline & & & & Leptoceridae & 1 & $\mathrm{c}$ & 0 \\
\hline \multirow{2}{*}{\multicolumn{4}{|c|}{ Sampling date: August 21, 1990}} & Diptera & & & \\
\hline & & & & Chironomidae & 3 & 2 & 0 \\
\hline Nematoda & 0 & 0 & 2 & & & & \\
\hline Annelida & & & & & & & \\
\hline Oligochaeta & 2 & 1 & 4 & & & & \\
\hline
\end{tabular}


Table 21. Water-column invertebrate surveys, 1992

[See figure 1 for site locations. Number of organisms identified in duplicate collections (A and B) done at 2-week intervals at each site]

\begin{tabular}{|c|c|c|c|c|c|c|c|c|c|c|c|c|}
\hline \multirow{2}{*}{ Taxonomic entry } & \multicolumn{2}{|c|}{ June 15} & \multicolumn{2}{|c|}{ July 5} & \multicolumn{2}{|c|}{ July 17} & \multicolumn{2}{|c|}{ July 31} & \multicolumn{2}{|c|}{ August 14} & \multicolumn{2}{|c|}{ August 26} \\
\hline & $\overline{\mathrm{A}}$ & $\bar{B}$ & $\overline{\mathrm{A}}$ & $\bar{B}$ & $\overline{\mathrm{A}}$ & $\mathrm{B}$ & $\overline{\mathrm{A}}$ & $\bar{B}$ & $\overline{\mathrm{A}}$ & $\mathrm{B}$ & $\bar{A}$ & $\mathrm{~B}$ \\
\hline \multicolumn{13}{|c|}{ Site 1} \\
\hline \multicolumn{13}{|l|}{ Annelida } \\
\hline Oligochaeta & & & 1 & & & 4 & & & 2 & & & \\
\hline \multicolumn{13}{|l|}{ Mollusca } \\
\hline \multicolumn{13}{|l|}{ Gastropoda } \\
\hline Lymnaeidae & & & & & & & & & 1 & 1 & & \\
\hline Planorbidae & & & & & 1 & & & & & 1 & & \\
\hline \multirow{2}{*}{\multicolumn{13}{|c|}{$\begin{array}{l}\text { Arthropoda } \\
\text { Crustacea }\end{array}$}} \\
\hline & & & & & & & & & & & & \\
\hline Cladocera & 51 & & 32 & 14 & 6 & 5 & 2 & & 11 & 7 & 29 & 65 \\
\hline Copepoda & 1 & & & & 13 & 5 & 2 & & 1 & 14 & 70 & 14 \\
\hline Amphipoda & & & & & & & & & & & & \\
\hline Talitridae & & & & & & & & 3 & 3 & 5 & & 2 \\
\hline \multirow{2}{*}{\multicolumn{13}{|c|}{$\begin{array}{l}\text { Insecta } \\
\text { Ephemeroptera }\end{array}$}} \\
\hline Ephemeroptera & & & & & & & & & & & & \\
\hline Baetidae & & & & & & & & & & 22 & & \\
\hline Odonata & & & & & & & & & & & & \\
\hline Coenagrionidae & & & & & & & 1 & & & 2 & & 1 \\
\hline Hemiptera & & & & & & & & & & & & \\
\hline Gerridae & & & & & & & & & & 1 & & \\
\hline Notonectidae & & & & & & & & & & 3 & & \\
\hline Coleoptera & & & & & & & & & & & & \\
\hline Halpidae & & & & & 1 & & & & & 1 & & \\
\hline Diptera & & & & & & & & & & & & \\
\hline Chironomidae & & & 3 & & 5 & 7 & 34 & 17 & 8 & 49 & 1 & \\
\hline Total organisms & 52 & & 36 & 14 & 26 & 21 & 39 & 20 & 26 & 106 & 100 & 82 \\
\hline
\end{tabular}

Site 2

\begin{tabular}{|c|c|c|c|c|c|c|c|c|c|c|c|c|}
\hline \\
\hline Oligochaeta & 1 & & & 12 & 50 & & 11 & & & & & \\
\hline Hirudinea & & & & & & 95 & & & 3 & & & \\
\hline \multicolumn{13}{|l|}{ Mollusca } \\
\hline \multicolumn{13}{|l|}{ Gastropoda } \\
\hline Physidae & & & 1 & & & & & & & & & \\
\hline Lymnaeidae & 2 & 2 & 1 & & & & & 2 & 3 & & & \\
\hline Planorbidae & & 19 & 2 & 4 & & 1 & & 1 & 64 & 1 & 1 & 1 \\
\hline \multicolumn{13}{|l|}{ Arthropoda } \\
\hline \multicolumn{13}{|l|}{ Crustacea } \\
\hline Ostracoda & & & & & & & & & & & & 5 \\
\hline Cladocera & 56 & 30 & 56 & 65 & 21 & & 14 & 23 & 2 & 65 & 100 & 100 \\
\hline Copepoda & 2 & 12 & 16 & 7 & 9 & 4 & & 6 & & 13 & 4 & 7 \\
\hline \multicolumn{13}{|l|}{ Amphipoda } \\
\hline Talitridae & 3 & 16 & 4 & 3 & 3 & & & & 20 & 21 & & 1 \\
\hline Hydracarina & 3 & & 4 & & & & & & 6 & & & \\
\hline \multicolumn{13}{|l|}{ Insecta } \\
\hline \multicolumn{13}{|l|}{ Ephemeroptera } \\
\hline Baetidae & 1 & & & & & & & & & & & \\
\hline
\end{tabular}


Table 21. Water-column invertebrate surveys, 1992--Continued

\begin{tabular}{|c|c|c|c|c|c|c|c|c|c|c|c|c|}
\hline \multirow{2}{*}{ Taxonomic entry } & \multicolumn{2}{|c|}{ June 15} & \multicolumn{2}{|c|}{ July 5} & \multicolumn{2}{|c|}{ July 17} & \multicolumn{2}{|c|}{ July 31} & \multicolumn{2}{|c|}{ August 14} & \multicolumn{2}{|c|}{ August 26} \\
\hline & $\mathrm{A}$ & B & A & $\mathrm{B}$ & $\mathrm{A}$ & B & A & B & A & B & A & $\bar{B}$ \\
\hline \multicolumn{13}{|c|}{ Site 2--Continued } \\
\hline \multicolumn{13}{|l|}{$\begin{array}{l}\text { Arthropoda--Continued } \\
\text { Insecta--Continued } \\
\text { Odonata }\end{array}$} \\
\hline Aeshnidae & 3 & & 1 & & & & & & & & & \\
\hline Coenagrionidae & & 1 & 3 & 1 & & & 4 & & & 3 & 2 & 1 \\
\hline Hemiptera & & & & & & & & & & & & \\
\hline Corixidae & 17 & 8 & 9 & 2 & & & & & & & & \\
\hline Notonectidae & & 3 & & 3 & & & & & & & & \\
\hline Coleoptera & & & & & & & & & & & & \\
\hline Halpidae & 2 & & & & 1 & & & & 2 & & & \\
\hline Dytiscidae & 2 & 3 & 1 & & 1 & & & & & & & \\
\hline Hydrophilidae & & 1 & & & & & & & 1 & & & \\
\hline Diptera & & & & & & & & & & & & \\
\hline Chironomidae & 2 & 4 & 1 & & 3 & & & & & & & \\
\hline Total organisms & 94 & 99 & 99 & 97 & 88 & 100 & 29 & 32 & 101 & 103 & 107 & 115 \\
\hline
\end{tabular}

Site 3

Annelida

Oligochaeta

4

2

$9 \quad 10$

Mollusca

Gastropoda

Physidae

Lymnaeidae

Planorbidae

$\begin{array}{llll} & 2 & & \\ 2 & 1 & 2 & 1\end{array}$

2

Arthropoda

Crustacea

Ostracoda

Cladocera

Copepoda

Amphipoda

Talitridae

Hydracarina

Insecta

$\begin{array}{rrrrrrrrrrr}78 & 71 & 85 & 93 & 68 & & 28 & 85 & 82 & 20 & 49 \\ & 9 & 8 & & 4 & 96 & 12 & 5 & 4 & 10 & 12 \\ & 7 & & 2 & 6 & & 17 & 1 & 5 & 5 & 17 \\ 2 & & & 1 & 2 & 1 & 1 & & 2 & 1 & 2\end{array}$

Ephemeroptera

Baetidae

Odonata

Aeshnidae

Coenagrionidae

1

3

$4 \quad 1$

Hemiptera

Corixidae

Notonectidae

$\begin{array}{ll}1 & 1\end{array}$

11

Coleoptera

Halpidae

Dytiscidae

Diptera

Chironomidae

Total organisms

5

$\begin{array}{ll}3 & 1\end{array}$

8

1

$\begin{array}{llll}3 & 4 & 1 & 2\end{array}$

2

2

\begin{tabular}{|c|c|c|c|c|c|c|c|c|c|c|}
\hline 1 & & 2 & & 2 & 2 & & & & 2 & 1 \\
\hline 95 & 99 & 100 & 100 & 101 & 100 & 59 & 99 & 113 & 50 & 102 \\
\hline
\end{tabular}


Table 21. Water-column invertebrate surveys, 1992--Continued

\begin{tabular}{|c|c|c|c|c|c|c|c|c|c|c|c|c|}
\hline \multirow{2}{*}{ Taxonomic entry } & \multicolumn{2}{|c|}{ June 15} & \multicolumn{2}{|c|}{ July 5} & \multicolumn{2}{|c|}{ July 17} & \multicolumn{2}{|c|}{ July 31} & \multicolumn{2}{|c|}{ August 14} & \multicolumn{2}{|c|}{ August 26} \\
\hline & $\mathrm{A}$ & B & A & $\mathrm{B}$ & $\bar{A}$ & B & $\mathrm{A}$ & $\bar{B}$ & $\overline{\mathrm{A}}$ & $\bar{B}$ & $\overline{\mathrm{A}}$ & $\mathrm{B}$ \\
\hline \multicolumn{13}{|c|}{ Site 5} \\
\hline \multicolumn{13}{|l|}{ Annelida } \\
\hline Oligochaeta & & & & & 70 & 26 & 16 & & & & 6 & 15 \\
\hline Hirudinea & & & & & 1 & & 2 & & & & & \\
\hline \multicolumn{13}{|l|}{ Mollusca } \\
\hline \multicolumn{13}{|l|}{ Gastropoda } \\
\hline Physidae & & & & & 2 & & & & & & & \\
\hline Lymnaeidae & & & 2 & & 1 & & 2 & & & & & \\
\hline Planorbidae & 2 & & & 1 & 4 & 3 & 2 & 1 & & 1 & & 1 \\
\hline \multicolumn{13}{|l|}{ Arthropoda } \\
\hline \multicolumn{13}{|l|}{ Crustacea } \\
\hline Cladocera & 2 & 2 & 1 & & & & 1 & 5 & 1 & & 3 & \\
\hline Copepoda & & & & & & & 3 & 27 & & 2 & 8 & \\
\hline \multicolumn{13}{|l|}{ Amphipoda } \\
\hline Talitridae & & & & & 9 & & 39 & 5 & & 1 & 2 & 2 \\
\hline Hydracarina & 1 & & 1 & 8 & 3 & 1 & & & & 1 & 1 & \\
\hline \multirow{2}{*}{\multicolumn{13}{|c|}{$\begin{array}{l}\text { Insecta } \\
\text { Ephemeroptera }\end{array}$}} \\
\hline & & & & & & & & & & & & \\
\hline Baetidae & & & & & & & 11 & 1 & 1 & & 5 & 2 \\
\hline Caenidae & & & & & 1 & & & & & & & \\
\hline \multicolumn{13}{|l|}{ Odonata } \\
\hline Coenagrionidae & & & & & 1 & & 1 & & & & & 1 \\
\hline \multicolumn{13}{|l|}{ Hemiptera } \\
\hline Corixidae & & & 1 & 2 & & 1 & & & 1 & & 1 & \\
\hline \multicolumn{13}{|l|}{ Coleoptera } \\
\hline Halpidae & & & & & 4 & & & 1 & & & & \\
\hline Dytiscidae & & & & & & & 1 & & & & & \\
\hline \multicolumn{13}{|l|}{ Diptera } \\
\hline Chironomidae & 1 & 7 & 3 & 2 & 3 & & 2 & & & 2 & & 6 \\
\hline Total organisms & 6 & 9 & 8 & 13 & 99 & 31 & 80 & 40 & 3 & 7 & 26 & 27 \\
\hline
\end{tabular}

Site 7

Annelida

Oligochaeta

Hirudinea

1

Mollusca

Gastropoda

Physidae

Lymnaeidae

Planorbidae

Arthropoda

Crustacea

Cladocera

Copepoda

Amphipoda

Talitridae

Hydracarina

Insecta

Ephemeroptera

Baetidae

Caenidae

\begin{tabular}{|c|c|c|c|c|c|c|c|c|}
\hline 10 & $\begin{array}{l}3 \\
4\end{array}$ & 2 & 36 & $\begin{array}{l}9 \\
2\end{array}$ & $\begin{array}{r}53 \\
5\end{array}$ & $\begin{array}{l}18 \\
20\end{array}$ & $\begin{array}{r}4 \\
12\end{array}$ & $\begin{array}{r}1 \\
100\end{array}$ \\
\hline 2 & 5 & $\begin{array}{l}3 \\
2\end{array}$ & 8 & 7 & $\begin{array}{l}4 \\
1\end{array}$ & 8 & $\begin{array}{l}6 \\
1\end{array}$ & 1 \\
\hline
\end{tabular}

12

$\begin{array}{ll}31 & 3 \\ & 1\end{array}$

12

1 
Table 21. Water-column invertebrate surveys, 1992--Continued

\begin{tabular}{|c|c|c|c|c|c|c|c|c|c|c|c|c|}
\hline \multirow{2}{*}{ Taxonomic entry } & \multicolumn{2}{|c|}{ June 15} & \multicolumn{2}{|c|}{ July 5} & \multicolumn{2}{|c|}{ July 17} & \multicolumn{2}{|c|}{ July 31} & \multicolumn{2}{|c|}{ August 14} & \multicolumn{2}{|c|}{ August 26} \\
\hline & $\bar{A}$ & $\bar{B}$ & $\bar{A}$ & $\bar{B}$ & $\overline{\mathrm{A}}$ & $\bar{B}$ & $\bar{A}$ & $\bar{B}$ & $\overline{\mathrm{A}}$ & $\bar{B}$ & $\bar{A}$ & $\bar{B}$ \\
\hline \multicolumn{13}{|c|}{ Site 7--Continued } \\
\hline $\begin{array}{l}\text { Arthropoda--Continued } \\
\text { Insecta--Continued } \\
\text { Odonata }\end{array}$ & & & & & & & & & & & & \\
\hline $\begin{array}{l}\text { Coenagrionidae } \\
\text { Hemintera }\end{array}$ & & & & 1 & 2 & & & & & & & \\
\hline $\begin{array}{l}\text { Corixidae } \\
\text { Notonectidae }\end{array}$ & 10 & 9 & 19 & 13 & 29 & & 31 & $\begin{array}{l}2 \\
2\end{array}$ & & 10 & 1 & 5 \\
\hline $\begin{array}{l}\text { Coleoptera } \\
\text { Halpidae }\end{array}$ & & & 1 & & 1 & & & & & & 1 & \\
\hline Dytiscidae & & & 1 & 2 & 1 & & & 2 & & 1 & 1 & \\
\hline $\begin{array}{l}\text { Diptera } \\
\text { Chironomidae }\end{array}$ & 2 & & 3 & 1 & 1 & & 1 & & & 2 & 1 & 1 \\
\hline Total organisms & 26 & 20 & 67 & 30 & 100 & & 83 & 77 & 57 & 59 & 105 & 104 \\
\hline
\end{tabular}

Site 9

\begin{tabular}{|c|c|c|c|c|c|c|c|c|c|c|c|c|}
\hline Annelida & & & & & & & & & & & & \\
\hline $\begin{array}{l}\text { Oligochaeta } \\
\text { Hirudinea }\end{array}$ & & 38 & & 10 & 4 & & & & 1 & & & 2 \\
\hline Mollusca & & & & & & & & & 1 & & & \\
\hline Gastropoda & & & & & & & & & & & & \\
\hline Physidae & & & & & & & & 3 & 1 & 1 & & \\
\hline Lymnaeidae & & & & & & & & 11 & & 2 & & \\
\hline Planorbidae & & & 1 & & 1 & & & 1 & & & & \\
\hline Arthropoda & & & & & & & & & & & & \\
\hline Crustacea & & & & & & & & & & & & \\
\hline Cladocera & 99 & 10 & 1 & 4 & 32 & 10 & 6 & & & 4 & 1 & 1 \\
\hline Copepoda & & & & 3 & & 11 & 4 & & & & 2 & \\
\hline Amphipoda & & & & & & & & & & & & \\
\hline Talitridae & & & & & 26 & 5 & 21 & 55 & 1 & & 1 & 4 \\
\hline Hydracarina & & 1 & & & 2 & & 3 & & & & & 1 \\
\hline Insecta & & & & & & & & & & & & \\
\hline Ephemeroptera & & & & & & & & & & & & \\
\hline Baetidae & & & & & 9 & 3 & 9 & 1 & & & 1 & \\
\hline Caenidae & & & & & & 2 & & & & 2 & & \\
\hline Odonata & & & & & & & & & & & & \\
\hline Coenagrionidae & & & & & 12 & & 2 & 3 & & 1 & & \\
\hline Hemiptera & & & & & & & & & & & & \\
\hline Corixidae & 1 & & 1 & 6 & 10 & 3 & 3 & 2 & & & & 1 \\
\hline $\begin{array}{c}\text { Coleoptera } \\
\text { Halpidae }\end{array}$ & & & & & & & & & & & & \\
\hline $\begin{array}{l}\text { Halpıdae } \\
\text { Diptera }\end{array}$ & & & & 2 & 1 & 1 & & & & & & \\
\hline Chironomidae & & & & & 3 & 1 & 1 & 4 & 4 & & & 1 \\
\hline Total organisms & 100 & 49 & 3 & 25 & 100 & 36 & 49 & 8 & 7 & 10 & 5 & 10 \\
\hline
\end{tabular}

Site 11

Annelida

Oligochaeta 
Table 21. Water-column invertebrate surveys, 1992--Continued

\begin{tabular}{|c|c|c|c|c|c|c|c|c|c|c|c|c|}
\hline \multirow{2}{*}{ Taxonomic entry } & \multicolumn{2}{|c|}{ June 15} & \multicolumn{2}{|c|}{ July 5} & \multicolumn{2}{|c|}{ July 17} & \multicolumn{2}{|c|}{ July 31} & \multicolumn{2}{|c|}{ August 14} & \multicolumn{2}{|c|}{ August 26} \\
\hline & $\mathrm{A}$ & B & A & $\mathrm{B}$ & $\mathrm{A}$ & B & A & B & A & B & A & B \\
\hline \multicolumn{13}{|c|}{ Site 11--Continued } \\
\hline \multicolumn{13}{|l|}{ Mollusca } \\
\hline \multicolumn{13}{|l|}{ Gastropoda } \\
\hline Physidae & & & & 4 & & & 1 & 1 & 1 & & & \\
\hline Lymnaeidae & & & & & & 4 & & 6 & & & & \\
\hline Planorbidae & & & & & & & 7 & 27 & 1 & & & 1 \\
\hline \multicolumn{13}{|l|}{ Arthropoda } \\
\hline \multicolumn{13}{|l|}{ Crustacea } \\
\hline Cladocera & 3 & 2 & 8 & & 70 & 56 & 4 & 3 & 1 & 23 & 1 & 3 \\
\hline Copepoda & 24 & 26 & 13 & & & 1 & 4 & 2 & 1 & 7 & & 7 \\
\hline \multicolumn{13}{|l|}{ Amphipoda } \\
\hline Talitridae & & & & & & 3 & 1 & 2 & & 5 & & \\
\hline Hydracarina & 7 & 6 & & 10 & 3 & 2 & 4 & & & & & \\
\hline Insecta & & & & & & & & & & & & \\
\hline \multirow{2}{*}{\multicolumn{13}{|c|}{$\begin{array}{c}\text { Ephemeroptera } \\
\text { Baetidae }\end{array}$}} \\
\hline & & & 2 & 10 & 7 & 13 & 12 & 13 & 7 & 14 & & \\
\hline Caenidae & & & & & 1 & & & 1 & 6 & 1 & 1 & 5 \\
\hline \multicolumn{13}{|l|}{ Odonata } \\
\hline Coenagrionidae & & & & 11 & 1 & 5 & 3 & 4 & 1 & 1 & 2 & \\
\hline \multicolumn{13}{|l|}{ Hemiptera } \\
\hline Corixidae & 3 & 5 & 2 & 5 & & 1 & & & & 2 & & \\
\hline Notonectidae & & 1 & & 2 & & 1 & & 1 & & & & \\
\hline Saldidae & & & & & & & & & & 1 & & \\
\hline \multicolumn{13}{|l|}{ Coleoptera } \\
\hline Halpidae & & & & 1 & & 1 & & & & 1 & & \\
\hline Dytiscidae & & & & & & 5 & 1 & 1 & & & & \\
\hline \multicolumn{13}{|l|}{ Diptera } \\
\hline Chironomidae & 1 & 2 & 2 & 6 & 8 & 7 & 8 & 6 & 5 & 1 & 2 & 1 \\
\hline Total organisms & 38 & 42 & 27 & 49 & 90 & 99 & 45 & 67 & 24 & 56 & 6 & 17 \\
\hline
\end{tabular}

Site 12

Annelida

Hirudinea

Mollusca

Gastropoda

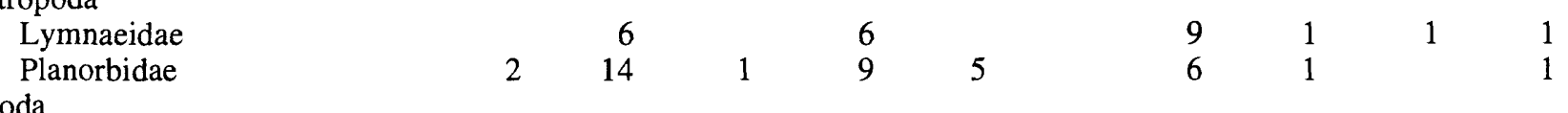

Arthropoda

Crustacea

Ostracoda

Cladocera

Copepoda

Amphipoda

$\begin{array}{rrrrrrrrrrr}80 & 90 & 8 & 16 & 26 & 60 & 8 & & 1 & & \\ 20 & 15 & 4 & & 31 & 1 & 34 & 95 & 64 & 57 & 90 \\ & & & & & 8 & & & 2 & 1 & 1\end{array}$

Insecta

Talitridae

Hydracarina

Ephemeroptera

Baetidae

Caenidae

Odonata

Coenagrionidae

$4 \quad 3$

$\begin{array}{llllll}5 & 20 & 3 & 4 & 4\end{array}$

1

$\begin{array}{llllll}3 & 2 & 9 & 4 & 5 & 6\end{array}$

1 
Table 21. Water-column invertebrate surveys, 1992--Continued

\begin{tabular}{|c|c|c|c|c|c|c|c|c|c|c|c|c|}
\hline \multirow{2}{*}{ Taxonomic entry } & \multicolumn{2}{|c|}{ June 15} & \multicolumn{2}{|c|}{ July 5} & \multicolumn{2}{|c|}{ July 17} & \multicolumn{2}{|c|}{ July 31} & \multicolumn{2}{|c|}{ August 14} & \multicolumn{2}{|c|}{ August 26} \\
\hline & $\mathrm{A}$ & $\mathrm{B}$ & A & $\bar{B}$ & $\mathrm{~A}$ & $\bar{B}$ & A & $\mathrm{B}$ & $\bar{A}$ & $\mathrm{~B}$ & $\mathrm{~A}$ & $\bar{B}$ \\
\hline \multicolumn{13}{|c|}{ Site 12--Continued } \\
\hline \multicolumn{13}{|l|}{$\begin{array}{l}\text { Arthropoda--Continued } \\
\text { Insecta--Continued } \\
\text { Hemiptera }\end{array}$} \\
\hline Corixidae & 1 & 1 & 11 & 6 & 4 & 4 & 1 & & 2 & 2 & 4 & 1 \\
\hline Notonectidae & & & 3 & 3 & & & & & 2 & 2 & & 1 \\
\hline Coleoptera & & & & & & & & & & & & \\
\hline Halpidae & & & & & & & 1 & & 1 & & & \\
\hline Diptera & & & & & & & & & & & & \\
\hline Chironomidae & & 2 & 2 & 3 & 1 & 6 & 8 & & 10 & 9 & 1 & 6 \\
\hline Total organisms & 101 & 108 & 34 & 65 & 86 & 106 & 65 & 99 & 105 & 22 & 65 & 103 \\
\hline
\end{tabular}

Site 13

Annelida

Oligochaeta

1

Mollusca

Gastropoda

Arthropoda

Lymnaeidae

Crustacea

Ostracoda

Cladocera

Copepoda

Hydracarina

Insecta

Hemiptera

Corixidae

Notonectidae

11

Coleoptera

Halpidae

Diptera

Chironomidae

Total organisms

$\begin{array}{rrrrr}15 & 26 & 2 & & \\ 7 & 10 & & 1 & 11 \\ & 1 & 1 & & 1\end{array}$

\begin{tabular}{|c|c|c|c|c|}
\hline & & 1 & 1 & \\
\hline $\begin{array}{r}85 \\
1\end{array}$ & 94 & $\begin{array}{r}90 \\
1\end{array}$ & 93 & $\begin{array}{r}93 \\
1\end{array}$ \\
\hline
\end{tabular}

1

$\begin{array}{llllll}1 & 4 & 5 & 10 & 4 \\ & 1 & & & 2\end{array}$

11

\begin{tabular}{|c|c|c|c|c|c|c|c|c|c|c|c|}
\hline \multirow{2}{*}{ Thironomidae } & \multicolumn{2}{|r|}{1} & \multicolumn{2}{|l|}{3} & 10 & \multicolumn{2}{|l|}{12} & \multicolumn{2}{|l|}{1} & \multicolumn{2}{|r|}{1} \\
\hline & 23 & 38 & 7 & 2 & 22 & 100 & 100 & 99 & 100 & 104 & 106 \\
\hline
\end{tabular}

Site 15

Annelida

Mollusca

Gastropoda

$\begin{array}{lr}\text { Physidae } & 1 \\ \text { Planorbidae } & 16\end{array}$

Arthropoda

Crustacea

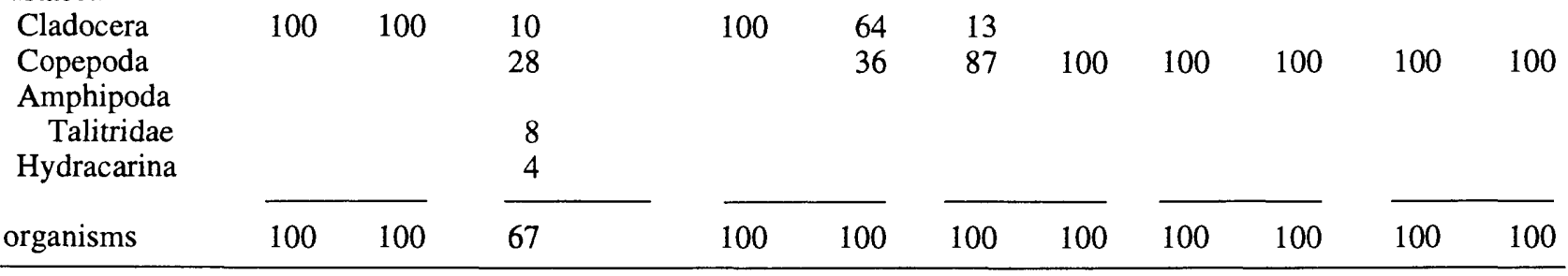


Table 22. Frog call surveys, 1991-92

[See figures 2 and 3 for site locations. Numbers separated by slash represent two different species heard; first number represents Pseudacris regilla, second number represents Rana catesbeiana; * denotes frog calls heard in canals adjacent to lake or impoundment units; --, site not surveyed; >, more than number shown]

\begin{tabular}{|c|c|c|c|c|c|c|c|c|c|c|c|c|c|c|c|c|}
\hline \multicolumn{17}{|c|}{ Indian Tom Lake } \\
\hline \multirow{2}{*}{ Site } & \multicolumn{2}{|c|}{ May 1991} & \multicolumn{3}{|c|}{ May 1992} & \multicolumn{4}{|c|}{ June 1991} & \multicolumn{4}{|c|}{ June 1992} & \multicolumn{2}{|c|}{ July 1991} & \multirow{2}{*}{$\frac{\text { July } 1992}{4}$} \\
\hline & 22 & 29 & 17 & 24 & 30 & 5 & 12 & 19 & 26 & 6 & 13 & 20 & 27 & 3 & 10 & \\
\hline $\mathrm{T} 1$ & $>5 / 0$ & $0 / 0$ & $0 / 0$ & $3 / 0$ & $3 / 0$ & $>5 / 0$ & $3 / 0$ & $4 / 0$ & $0 / 0$ & $2 / 0$ & $3 / 0$ & $0 / 0$ & $0 / 0$ & $4 / 0$ & $0 / 0$ & $0 / 0$ \\
\hline $\mathrm{T} 2$ & $0 / 0$ & $0 / 0$ & $0 / 0$ & $0 / 0$ & $0 / 0$ & $0 / 0$ & $2 / 0$ & $2 / 0$ & $0 / 0$ & $0 / 0$ & $0 / 0$ & $0 / 0$ & $0 / 0$ & $3 / 0$ & $0 / 0$ & $0 / 0$ \\
\hline
\end{tabular}

Lower Klamath National Wildlife Refuge

\begin{tabular}{|c|c|c|c|c|c|c|c|c|c|c|c|c|c|c|c|c|}
\hline \multirow{2}{*}{ Site } & \multicolumn{2}{|c|}{ May 1991} & \multicolumn{3}{|c|}{ May 1992} & \multicolumn{4}{|c|}{ June 1991} & \multicolumn{4}{|c|}{ June 1992} & \multicolumn{2}{|c|}{ July 1991} & \multirow{2}{*}{$\frac{\text { July } 1992}{4}$} \\
\hline & 22 & 29 & 17 & 24 & 30 & 5 & 12 & 19 & 26 & 6 & 13 & 20 & 27 & 3 & 10 & \\
\hline 1 & $0 / 0$ & $0 / 0$ & $0 / 0$ & $0 / 0$ & $0 / 0$ & $0 / 0$ & $0 / 0$ & $0 / 0$ & $0 / 0$ & $0 / 0$ & $0 / 0$ & $0 / 0$ & $0 / 0$ & $0 / 3 *$ & $0 / 3 *$ & $0 / 0$ \\
\hline 2 & $0 / 0$ & $0 / 0$ & $0 / 0$ & $0 / 0$ & $0 / 0$ & $0 / 0$ & $0 / 0$ & $0 / 0$ & $0 / 0$ & $0 / 0$ & $0 / 0$ & $0 / 0$ & $0 / 0$ & $0 / 0$ & $0 / 0$ & $0 / 4$ \\
\hline 3 & $1 / 0$ & $0 / 0$ & $0 / 0$ & $0 / 0$ & $0 / 0$ & $0 / 0$ & $0 / 0$ & $0 / 0$ & $0 / 0$ & $0 / 0$ & $0 / 0$ & $0 / 0$ & $0 / 0$ & $0 / 0$ & $0 / 0$ & $0 / 2$ \\
\hline 4 & $0 / 0$ & $0 / 0$ & $0 / 0$ & $0 / 0$ & $0 / 0$ & $0 / 0$ & $0 / 0$ & $0 / 0$ & $0 / 0$ & $0 / 0$ & $0 / 0$ & $0 / 0$ & $0 / 0$ & $0 / 0$ & $0 / 0$ & $0 / 0$ \\
\hline 5 & $0 / 0$ & $0 / 0$ & $0 / 0$ & $0 / 0$ & $0 / 0$ & $0 / 0$ & -- & $0 / 0$ & $0 / 0$ & $0 / 0$ & $0 / 0$ & $0 / 0$ & $0 / 0$ & $0 / 0$ & $0 / 0$ & -- \\
\hline 6 & $0 / 0$ & $0 / 0$ & $0 / 0$ & $0 / 0$ & $0 / 0$ & $0 / 0$ & $0 / 0$ & $0 / 0$ & $0 / 0$ & $0 / 0$ & $0 / 0$ & $0 / 0$ & $0 / 0$ & $0 / 0$ & $0 / 0$ & $0 / 0$ \\
\hline 7 & $0 / 0$ & $0 / 0$ & $0 / 0$ & $0 / 0$ & $0 / 0$ & $0 / 0$ & $0 / 0$ & $0 / 0$ & $0 / 0$ & $0 / 0$ & $0 / 0$ & $0 / 0$ & $0 / 0$ & $0 / 0$ & $0 / 0$ & $0 / 0$ \\
\hline 8 & $0 / 0$ & $0 / 0$ & $0 / 0$ & $0 / 0$ & $1 * / 0$ & $1 * / 0$ & $0 / 0$ & $0 / 0$ & $0 / 0$ & $1 / 0$ & $0 / 0$ & $0 / 0$ & $0 / 0$ & $0 / 0$ & $0 / 0$ & $0 / 0$ \\
\hline 9 & $0 / 0$ & $0 / 0$ & $0 / 0$ & $0 / 0$ & $0 / 0$ & $1 / 0$ & $0 / 0$ & $0 / 0$ & $0 / 0$ & $0 / 0$ & $0 / 0$ & $0 / 0$ & $0 / 0$ & $0 / 0$ & $0 / 0$ & $0 / 0$ \\
\hline 10 & $0 / 0$ & $0 / 0$ & $0 / 0$ & $0 / 0$ & $0 / 0$ & $0 / 0$ & $0 / 0$ & $0 / 0$ & $0 / 0$ & $0 / 0$ & $0 / 0$ & $0 / 0$ & $0 / 0$ & $0 / 0$ & $0 / 0$ & -- \\
\hline 11 & $0 / 0$ & $0 / 0$ & $0 / 0$ & $0 / 0$ & $0 / 0$ & $0 / 0$ & $0 / 0$ & $0 / 0$ & $0 / 0$ & $0 / 0$ & $0 / 0$ & $0 / 0$ & $0 / 0$ & $0 / 0$ & $0 / 0$ & -- \\
\hline 12 & $0 / 0$ & $0 / 0$ & $0 / 0$ & $0 / 0$ & $0 / 0$ & $0 / 0$ & $0 / 0$ & $0 / 0$ & $0 / 0$ & $0 / 0$ & $0 / 0$ & $0 / 0$ & $0 / 0$ & $0 / 0$ & $0 / 0$ & -- \\
\hline 13 & $0 / 0$ & $1 * / 0$ & -- & -- & -- & $0 / 0$ & $1 / 0$ & $0 / 0$ & -- & -- & -- & -- & -- & -- & -- & -- \\
\hline 14 & $0 / 0$ & $1 / 0$ & - & -- & -- & $0 / 0$ & $1 / 0$ & $0 / 0$ & $0 / 0$ & -- & -- & -- & -- & $1 / 0$ & $1 / 0$ & -- \\
\hline 15 & $5 / 0$ & $>5 / 0$ & $>5 / 0$ & $0 / 0$ & $0 / 0$ & $0 / 0$ & $3 / 0$ & $1 / 0$ & $2 / 0$ & $2 / 0$ & $3 / 0$ & $0 / 0$ & $2 / 0$ & $2 / 0$ & $0 / 0$ & $0 / 0$ \\
\hline 16 & $3,1 * / 0$ & $>5 / 0$ & $3 / 0$ & $2 / 0$ & $0 / 0$ & $0 / 0$ & $0 / 0$ & $0 / 0$ & $0 / 0$ & $2 / 0$ & $0 / 0$ & $0 / 0$ & $0 / 0$ & $0 / 0$ & $0 / 0$ & $0 / 0$ \\
\hline 17 & $2 / 0$ & $1 / 0$ & $0 / 0$ & $0 / 0$ & $0 / 0$ & $0 / 0$ & $0 / 0$ & $1 * / 0$ & $0 / 0$ & -- & -- & -- & -- & $0 / 0$ & $0 / 0$ & -- \\
\hline 18 & $1 * / 0$ & $1 / 0$ & $0 / 0$ & $0 / 0$ & $0 / 0$ & $0 / 0$ & $0 / 0$ & $0 / 0$ & $0 / 0$ & $0 / 0$ & $0 / 0$ & $0 / 0$ & $0 / 0$ & $1 / 0$ & $0 / 0$ & $0 / 0$ \\
\hline 19 & $1 / 0$ & $1 / 0$ & $0 / 0$ & $1 / 0$ & $0 / 0$ & $0 / 0$ & $0 / 0$ & $0 / 0$ & $0 / 0$ & $0 / 0$ & $0 / 0$ & $0 / 0$ & $0 / 0$ & $0 / 0$ & $0 / 0$ & $0 / 0$ \\
\hline 20 & $0 / 0$ & $0 / 0$ & $0 / 0$ & $1 / 0$ & $0 / 0$ & $0 / 0$ & $0 / 0$ & $0 / 0$ & $0 / 0$ & $0 / 0$ & $0 / 0$ & $0 / 0$ & $0 / 0$ & $0 / 0$ & $0 / 0$ & $0 / 0$ \\
\hline 21 & $1 / 0$ & $0 / 0$ & $0 / 0$ & $0 / 0$ & $0 / 0$ & $0 / 0$ & $0 / 0$ & $0 / 0$ & $0 / 0$ & $0 / 0$ & $1 / 0$ & $1 / 0$ & $0 / 0$ & $0 / 0$ & $0 / 0$ & $0 / 0$ \\
\hline 22 & $0 / 0$ & $1 / 0$ & $0 / 0$ & $0 / 0$ & $0 / 0$ & $0 / 0$ & $0 / 0$ & $0 / 0$ & $0 / 0$ & $0 / 0$ & $0 / 1$ & $0 / 0$ & $0 / 0$ & $0 / 0$ & $0 / 0$ & $0 / 0$ \\
\hline 23 & $2 / 0$ & $0 / 0$ & $0 / 0$ & $0 / 0$ & $0 / 0$ & $0 / 0$ & $0 / 0$ & $0 / 0$ & $0 / 0$ & $0 / 0$ & $0 / 0$ & $0 / 0$ & $0 / 0$ & $0 / 0$ & $0 / 0$ & $0 / 0$ \\
\hline 24 & $0 / 0$ & $0 / 0$ & $0 / 0$ & $0 / 0$ & $0 / 0$ & $0 / 0$ & $0 / 0$ & $0 / 0$ & $0 / 0$ & $0 / 0$ & $1 / 0$ & $2 / 0$ & $0 / 0$ & $0 / 0$ & $0 / 0$ & $0 / 0$ \\
\hline 25 & $0 / 0$ & $0 / 0$ & $0 / 0$ & $0 / 0$ & $0 / 0$ & $0 / 0$ & $0 / 0$ & $0 / 0$ & $0 / 1$ & $0 / 0$ & $0 / 0$ & $0 / 0$ & $0 / 0$ & $0 / 0$ & $0 / 0$ & $0 / 0$ \\
\hline 26 & $0 / 0$ & $0 / 0$ & -. & -- & -- & $0 / 0$ & $0 / 0$ & $0 / 0$ & $0 / 0$ & -- & -. & -- & -- & $0 / 0$ & $0 / 0$ & -- \\
\hline 27 & $5 / 0$ & $4 / 0$ & $3 / 0$ & -- & -- & $>5 / 0$ & $>5 / 0$ & $4 / 0$ & $5 / 0$ & -- & -- & -- & -- & $4 / 0$ & $1 / 0$ & -- \\
\hline 28 & $>5 / 0$ & $>5 / 0$ & $4 / 0$ & $2 / 0$ & $4 / 0$ & $>5 / 0$ & $>5 / 0$ & $4 / 0=$ & $>5 / 0$ & -- & -- & -- & -- & $5 / 0$ & $1 / 0$ & -- \\
\hline 29 & $2 / 0$ & $1 / 0$ & $4 / 0$ & $3 / 0$ & $>5 / 0$ & $2 / 0$ & $0 / 0$ & $0 / 0$ & $0 / 0$ & $1 / 0$ & $4 / 0$ & $2 / 0$ & $0 / 0$ & $3 / 0$ & $0 / 0$ & -- \\
\hline 30 & $3 / 0$ & $>5 / 0$ & $4 / 0$ & $2 / 0$ & $3 / 0$ & $0 / 0$ & $3 / 0$ & $2 / 0$ & $0 / 0$ & $2 / 0$ & $0 / 0$ & $1 / 0$ & $0 / 0$ & $3 / 0$ & $1 / 0$ & -- \\
\hline 31 & $0 / 0$ & $>5 / 0$ & $0 / 0$ & $0 / 0$ & $0 / 0$ & $0 / 0$ & $0 / 0$ & $0 / 0$ & $1 / 0$ & $0 / 0$ & $1 / 0$ & $0 / 0$ & $0 / 0$ & $1 / 0$ & $0 / 0$ & $0 / 0$ \\
\hline 32 & $0 / 0$ & $0 / 0$ & $0 / 0$ & $0 / 0$ & $0 / 0$ & $0 / 0$ & $0 / 0$ & $0 / 0$ & $0 / 0$ & $0 / 0$ & $0 / 0$ & $0 / 0$ & $0 / 0$ & $0 / 0$ & $0 / 0$ & $0 / 0$ \\
\hline 33 & $0 / 0$ & $>5 * / 0$ & $0 / 0$ & $>5 * / 0$ & $3 * / 0$ & $0 / 0$ & $0 / 0$ & $2 * / 0$ & $0 / 0$ & $0 / 0$ & $0 / 0$ & $0 / 0$ & $0 / 0$ & $0 / 0$ & $1 * / 0$ & $0 / 0$ \\
\hline 34 & $0 / 0$ & $0 / 0$ & $0 / 0$ & -- & -. & $\infty / 0$ & $0 / 0$ & $0 / 0$ & $4 * / 0$ & -- & -- & -- & -- & $0 / 0$ & $0 / 0$ & -- \\
\hline 35 & $0 / 0$ & $0 / 0$ & $0 / 0$ & -- & -- & $0 / 0$ & $0 / 0$ & $0 / 0$ & $0 / 0$ & -- & -- & -- & -- & $0 / 0$ & $0 / 0$ & -- \\
\hline 36 & $0 / 0$ & $0 / 0$ & $0 / 0$ & -- & -- & $0 / 0$ & $0 / 0$ & $0 / 0$ & $0 / 0$ & -- & -- & -- & -- & $0 / 0$ & $0 / 0$ & -- \\
\hline
\end{tabular}


Table 22. Frog call surveys, 1991-92--Continued

Tule Lake National Wildlife Refuge

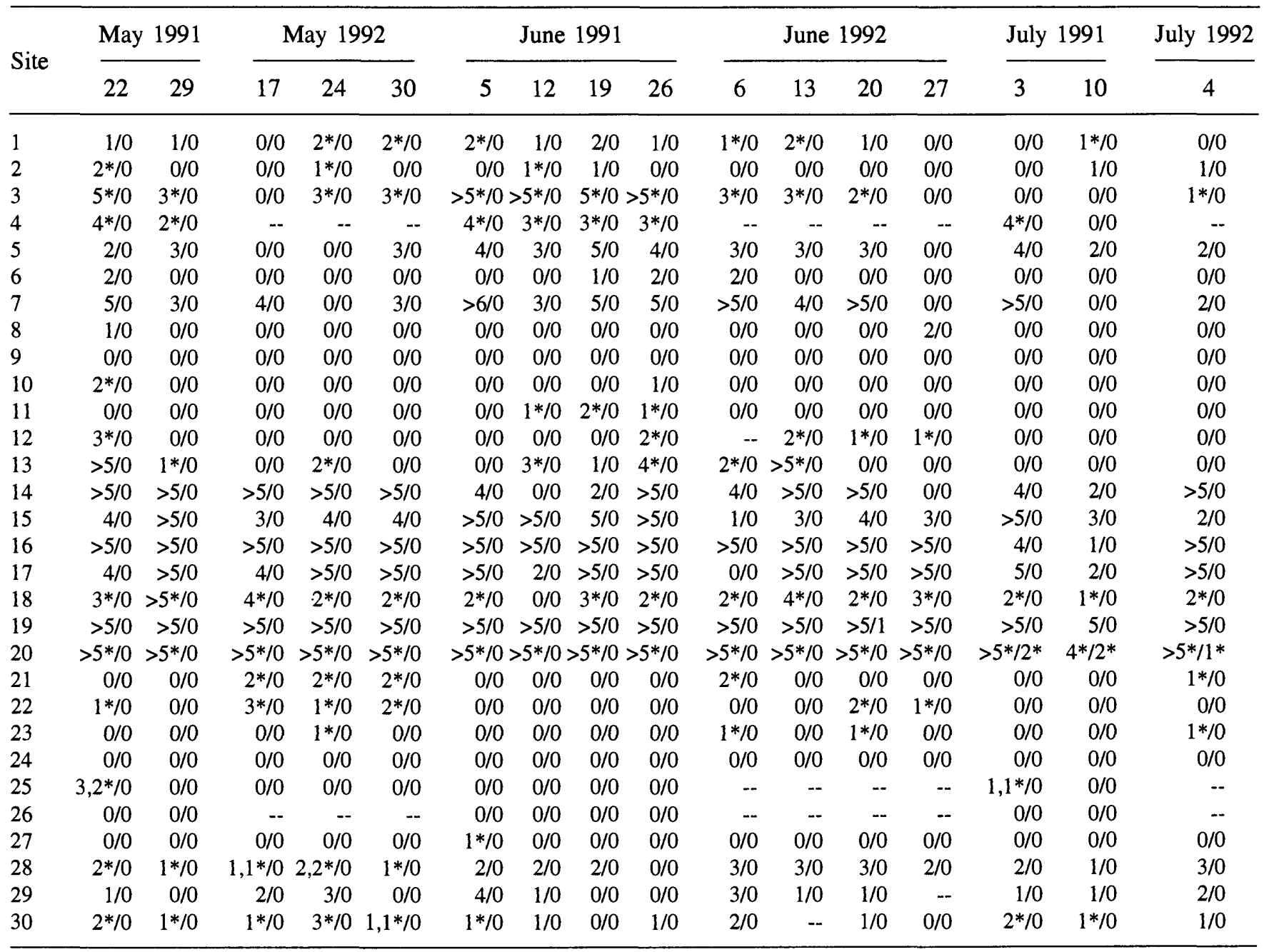


Table 23. Fish surveys, 1991-92

[See figure 1 for site locations. Site locations are approximations as collection covered a broad area. g, gram; --, no data]

\begin{tabular}{|c|c|c|c|c|c|c|}
\hline \multirow[b]{2}{*}{ Species } & \multirow[b]{2}{*}{$\begin{array}{c}\text { Total } \\
\text { caught }\end{array}$} & \multicolumn{2}{|c|}{ Adult } & \multicolumn{2}{|c|}{ Juvenile } & \multirow{2}{*}{$\begin{array}{c}\text { Total } \\
\text { external } \\
\text { anomolies }\end{array}$} \\
\hline & & $\begin{array}{l}\text { Number } \\
\text { surveyed }\end{array}$ & $\begin{array}{c}\text { Mean } \\
\text { weight }(\mathrm{g})\end{array}$ & $\begin{array}{l}\text { Number } \\
\text { surveyed }\end{array}$ & $\begin{array}{c}\text { Mean } \\
\text { weight (g) }\end{array}$ & \\
\hline \multicolumn{7}{|c|}{ Site LR1, October 20, 1991, electroshock } \\
\hline Tui chub & 63 & 8 & 13.5 & 55 & 1.9 & 8 \\
\hline Fathead minnow & 67 & 20 & 2.8 & 47 & 1.7 & 60 \\
\hline Total & 130 & 28 & & 102 & & 68 \\
\hline
\end{tabular}

Site 11, October 20, 1991, trap and dip net

\begin{tabular}{|c|c|c|c|c|c|c|}
\hline Tui chub & 37 & 13 & 5.6 & 24 & 1.9 & 10 \\
\hline Sacramento perch & 29 & 6 & -- & 23 & 1.0 & 2 \\
\hline Total & 66 & 19 & & 47 & & 12 \\
\hline
\end{tabular}

Site 12, October 19, 1991, seine

\begin{tabular}{|c|c|c|c|c|c|c|}
\hline Fathead minnows & 20 & 20 & 2.5 & -- & -- & 13 \\
\hline \multicolumn{7}{|c|}{ G Canal near Site LR6, October 22, 1991, seine and electroshock } \\
\hline Tui chub & 888 & 68 & 11.5 & 820 & 0.4 & 41 \\
\hline Large scale chub & 10 & 10 & 34.0 & 0 & -- & -- \\
\hline Sacramento perch & 1 & 1 & 17.0 & 0 & -- & -- \\
\hline Sculpin & 2 & 2 & 13.0 & 0 & -- & -- \\
\hline Shortnose sucker & 3 & 2 & -- & 1 & -- & -- \\
\hline Total & 904 & 83 & & 821 & & 41 \\
\hline
\end{tabular}

Lost River below Clear Lake Dam, June 24, 1992, seine

\begin{tabular}{|c|c|c|c|c|c|c|}
\hline Tui chub & 117 & 66 & 9.4 & 51 & 2.2 & 36 \\
\hline Sacramento perch & 5 & 0 & - & 5 & 3.9 & 0 \\
\hline Sculpin & 1 & 0 & -- & 1 & 7.6 & 0 \\
\hline Total & 123 & 66 & & 57 & & 36 \\
\hline
\end{tabular}

Site LR3, June 24, 1992, seine

\begin{tabular}{|c|c|c|c|c|c|c|}
\hline Tui chub & 252 & 100 & 19.6 & 152 & 6.7 & 104 \\
\hline Large scale chub & 20 & 15 & 18.3 & 5 & 8.9 & 10 \\
\hline Blue chub & 42 & 13 & 14.4 & 29 & 3.1 & 8 \\
\hline Fathead minnows & 94 & 94 & 2.4 & 0 & -- & 61 \\
\hline Sacramento perch & 2 & 2 & 18.1 & 0 & -- & 0 \\
\hline Total & 410 & 224 & & 186 & & 183 \\
\hline
\end{tabular}


Table 23. Fish surveys, 1991-92--Continued

\begin{tabular}{|c|c|c|c|c|c|c|}
\hline \multirow[b]{2}{*}{ Species } & \multirow[b]{2}{*}{$\begin{array}{l}\text { Total } \\
\text { caught }\end{array}$} & \multicolumn{2}{|c|}{ Adult } & \multicolumn{2}{|c|}{ Juvenile } & \multirow{2}{*}{$\begin{array}{c}\text { Total } \\
\text { external } \\
\text { anomolies }\end{array}$} \\
\hline & & $\begin{array}{l}\text { Number } \\
\text { surveyed }\end{array}$ & $\begin{array}{c}\text { Mean } \\
\text { weight (g) }\end{array}$ & $\begin{array}{l}\text { Number } \\
\text { surveyed }\end{array}$ & $\begin{array}{c}\text { Mean } \\
\text { weight }(\mathrm{g})\end{array}$ & \\
\hline
\end{tabular}

Site LR3, August 21, 1992, seine

\begin{tabular}{|c|c|c|c|c|c|c|}
\hline Tui chub & 297 & 297 & 15.0 & 0 & -- & 198 \\
\hline Blue chub & 14 & 14 & 9.2 & 0 & -- & 11 \\
\hline Fathead minnows & 748 & 748 & 1.9 & 0 & -- & $\begin{array}{r}28(100 \\
\text { examined })\end{array}$ \\
\hline Juvenile Cyprinidae & 459 & 0 & -- & 459 & 1.2 & 42 \\
\hline Sacramento perch & 4 & 0 & 0 & 4 & 2.4 & 0 \\
\hline Juvenile sucker & 1 & 0 & -- & 1 & -- & 0 \\
\hline Total & 1,523 & 1,059 & & 464 & & $\begin{array}{r}279 \text { (875 } \\
\text { examined) }\end{array}$ \\
\hline
\end{tabular}

Site 3, June 6, 1992, seine

\begin{tabular}{|c|c|c|c|c|c|c|}
\hline Tui chub & 481 & 1 & 5.4 & 480 & 0.2 & 13 \\
\hline Large scale chub & 12 & 9 & 4.1 & 3 & 2.5 & 2 \\
\hline Blue chub & 5 & 5 & 2.9 & 0 & -- & 2 \\
\hline Fathead minnow & 40 & 34 & 1.5 & 6 & 1.2 & $\begin{array}{r}19(34 \\
\text { examined) }\end{array}$ \\
\hline Sacramento perch & 1 & 1 & 7.0 & 0 & -- & 1 \\
\hline Yellow perch & 1 & 1 & 44.5 & 0 & -- & 0 \\
\hline Total & 540 & 51 & & 489 & & $\begin{array}{r}37(534 \\
\text { examined })\end{array}$ \\
\hline
\end{tabular}

Site 3, August 31, 1992, seine

\begin{tabular}{lrrrrrr}
\hline Blue chub & 1 & 0 & - & 1 & 1.0 & 0 \\
Fathead minnow & 526 & 4 & 1.1 & 522 & 0.3 & 69 \\
Sacramento perch & 2 & 0 & -- & 2 & 1.1 & 0 \\
$\quad$ Total . . . . . . . . . . . . . . . . . & $\overline{529}$ & $\overline{4}$ & & $\overline{525}$ & & $\overline{69}$ \\
\hline
\end{tabular}

Site 4, September 2, 1992, seine

\begin{tabular}{|c|c|c|c|c|c|c|}
\hline Tui chub & 1 & 1 & 3.0 & 0 & -- & 1 \\
\hline Fathead minnow & 92 & 39 & 0.8 & 53 & 0.4 & $\begin{array}{r}13(20 \\
\text { examined) }\end{array}$ \\
\hline Juvenile Cyprinidae & 245 & 0 & -- & 245 & 1.3 & 80 \\
\hline Sacramento perch & 1 & 0 & -- & 1 & -- & 0 \\
\hline Total & 339 & 40 & & 299 & & $\begin{array}{r}94(266 \\
\text { examined) }\end{array}$ \\
\hline
\end{tabular}

Site 7, June 25, 1992, seine

\begin{tabular}{|c|c|c|c|c|c|c|}
\hline Tui chub & 9 & 2 & 1.2 & 7 & 2.4 & 0 \\
\hline Fathead minnow & 20 & 20 & 1.7 & 0 & -- & 7 \\
\hline Juvenile Cyprinidae & 1,574 & 0 & -- & 1,574 & 0.5 & 72 \\
\hline Total & 1,603 & 22 & & 1,581 & & 79 \\
\hline
\end{tabular}


Table 23. Fish surveys, 1991-92--Continued

\begin{tabular}{|c|c|c|c|c|c|c|}
\hline \multirow[b]{2}{*}{ Species } & \multirow[b]{2}{*}{$\begin{array}{l}\text { Total } \\
\text { caught }\end{array}$} & \multicolumn{2}{|c|}{ Adult } & \multicolumn{2}{|c|}{ Juvenile } & \multirow{2}{*}{$\begin{array}{c}\text { Total } \\
\text { external } \\
\text { anomolies }\end{array}$} \\
\hline & & $\begin{array}{l}\text { Number } \\
\text { surveyed }\end{array}$ & $\begin{array}{c}\text { Mean } \\
\text { weight }(\mathrm{g})\end{array}$ & $\begin{array}{l}\text { Number } \\
\text { surveyed }\end{array}$ & $\begin{array}{c}\text { Mean } \\
\text { weight }(\mathrm{g})\end{array}$ & \\
\hline \multicolumn{7}{|c|}{ Site 7, September 2, 1992, seine } \\
\hline Tui chub & 10 & 4 & 18.1 & 6 & 1.3 & 3 \\
\hline Fathead minnow & 33 & 7 & 1.0 & 26 & 0.3 & 2 \\
\hline Sacramento perch & 12 & 1 & 2.0 & 11 & 0.5 & 0 \\
\hline Total & 55 & 12 & & 43 & & 5 \\
\hline \multicolumn{7}{|c|}{ Site 11, June 25, 1992, seine } \\
\hline Tui chub & 98 & 0 & -- & 98 & -- & 46 \\
\hline Large Scale chub & 30 & 0 & -- & 30 & 3.5 & 14 \\
\hline Blue chub & 57 & 0 & -- & 57 & 2.9 & 16 \\
\hline Fathead minnow & 71 & 71 & 1.2 & 0 & -- & 50 \\
\hline Juvenile Cyprinidae & 490 & 0 & -- & 490 & -- & -- \\
\hline Sacramento perch & 1 & 0 & -- & 1 & 5.5 & 0 \\
\hline Total & 747 & 71 & & 676 & & 126 \\
\hline \multicolumn{7}{|c|}{ Site 11, September 2, 1992, seine } \\
\hline Tui chub & 493 & 140 & 10.5 & 353 & 2.1 & 175 \\
\hline Sacramento perch & 101 & 0 & -- & 101 & 0.7 & 47 \\
\hline Fathead minnow & 13 & 13 & -- & -- & -- & 7 \\
\hline Total & 607 & 153 & & 454 & & 229 \\
\hline \multicolumn{7}{|c|}{ Site 12, June 23, 1992, seine } \\
\hline Fathead minnow & 807 & 66 & 2.9 & 745 & 0.2 & $\begin{array}{r}29(66 \\
\text { examined) }\end{array}$ \\
\hline \multicolumn{7}{|c|}{ Site 12 , August 31, 1992, seine } \\
\hline Tui chub & 3 & 3 & 19.7 & 0 & -- & 0 \\
\hline Fathead minnow & 122 & 92 & 1.4 & 30 & 0.6 & $\begin{array}{r}47(65 \\
\text { examined) }\end{array}$ \\
\hline Juvenile Cyprinidae & 1,758 & 0 & -- & 1,758 & 0.4 & 221 \\
\hline Sacramento perch & 3 & 3 & 3.6 & 0 & -- & 0 \\
\hline Total & 1,886 & 98 & & 1,788 & & $\begin{array}{l}268(1,829 \\
\text { examined })\end{array}$ \\
\hline
\end{tabular}


Table 24. Fish health surveys of native fathead minnows, 1991-92

[See figure 1 for site locations. Fish Health Index: Number, type, and severity of abnormalities is visually assessed for each fish. Extremities, gills, eyes, mesenteric fat, hind gut, liver, and kidney are assigned numbers representing degree of affected tissue and type of lesion or abnormality. For example, a fish with no abnormalities of the extremities would receive a 0 (zero) for that category, and a fish with severe, actively hemorrhaging bacterial lesions would receive a 3 or 4 (depending on type and extent of damage). The seven categories are added to produce an index total for each fish. The total range for overall health index is 0 (a perfectly healthy fish) to 25 (a severely affected fish)]

\begin{tabular}{|c|c|c|c|c|c|c|c|c|c|c|c|}
\hline $\begin{array}{l}\text { Fish } \\
\text { No. }\end{array}$ & $\begin{array}{c}\text { Index } \\
\text { total }\end{array}$ & $\begin{array}{l}\text { Fish } \\
\text { No. }\end{array}$ & $\begin{array}{c}\text { Index } \\
\text { total }\end{array}$ & $\begin{array}{l}\text { Fish } \\
\text { No. }\end{array}$ & $\begin{array}{c}\text { Index } \\
\text { total }\end{array}$ & $\begin{array}{l}\text { Fish } \\
\text { No. }\end{array}$ & $\begin{array}{c}\text { Index } \\
\text { total }\end{array}$ & $\begin{array}{l}\text { Fish } \\
\text { No. }\end{array}$ & $\begin{array}{r}\text { Index } \\
\text { total }\end{array}$ & $\begin{array}{l}\text { Fish } \\
\text { No. }\end{array}$ & $\begin{array}{c}\text { Index } \\
\text { total }\end{array}$ \\
\hline
\end{tabular}

\section{Site LR3}

October 20, 1991

$\begin{array}{rrrrr}1 & 5 & 6 & 7 & 11 \\ 2 & 2 & 7 & 8 & 12 \\ 3 & 6 & 8 & 8 & 13 \\ 4 & 4 & 9 & 2 & 14 \\ 5 & 5 & 10 & 2 & 15\end{array}$

$\begin{array}{ll}6 & 1 \\ 0 & 1 \\ 1 & 1 \\ 2 & 19 \\ 4 & 20\end{array}$

$\begin{array}{llllll}16 & 1 & 21 & 5 & 25 & 7 \\ 17 & 3 & 22 & 3 & 26 & 3 \\ 18 & 6 & 23 & 2 & 27 & 6 \\ 19 & 0 & 24 & 1 & 28 & 5 \\ 20 & 2 & & & \end{array}$

June 25, 1992

$\begin{array}{rlll}1 & 2 & 17 & 2 \\ 2 & 0 & 18 & 3 \\ 3 & 1 & 19 & 1 \\ 4 & 3 & 20 & 0 \\ 5 & 1 & 21 & 3 \\ 6 & 1 & 22 & 2 \\ 7 & 4 & 23 & 2 \\ 8 & 2 & 24 & 4 \\ 9 & 7 & 25 & 1 \\ 10 & 4 & 26 & 1 \\ 11 & 3 & 27 & 1 \\ 12 & 6 & 28 & 0 \\ 13 & 4 & 29 & 2 \\ 14 & 4 & 30 & 3 \\ 15 & 3 & 31 & 0 \\ 16 & 5 & 32 & 5\end{array}$

$\begin{array}{ll}33 & 0 \\ 34 & 3 \\ 35 & 1 \\ 36 & 1 \\ 37 & 1 \\ 38 & 0 \\ 39 & 1 \\ 40 & 1 \\ 41 & 3 \\ 42 & 2 \\ 43 & 4 \\ 44 & 3 \\ 45 & 2 \\ 46 & 1 \\ 47 & 3 \\ 48 & 4\end{array}$

$\begin{array}{ll}49 & 1 \\ 50 & 4 \\ 51 & 0 \\ 52 & 6 \\ 53 & 2 \\ 54 & 2 \\ 55 & 0 \\ 56 & 1 \\ 57 & 2 \\ 58 & 2 \\ 59 & 2 \\ 60 & 1 \\ 61 & 4 \\ 62 & 9 \\ 63 & 4 \\ 64 & 1\end{array}$

$\begin{array}{ll}65 & 2 \\ 66 & 3 \\ 67 & 4 \\ 68 & 4 \\ 69 & 4 \\ 70 & 5 \\ 71 & 1 \\ 72 & 6 \\ 73 & 4 \\ 74 & 5 \\ 75 & 5 \\ 76 & 2 \\ 77 & 1 \\ 78 & 5 \\ 79 & 5\end{array}$

$\begin{array}{ll}80 & 5 \\ 81 & 7 \\ 82 & 1 \\ 83 & 1 \\ 84 & 0 \\ 85 & 2 \\ 86 & 3 \\ 87 & 3 \\ 88 & 3 \\ 89 & 1 \\ 90 & 3 \\ 91 & 3 \\ 92 & 0 \\ 93 & 1 \\ 94 & 0\end{array}$

September 2, 1991

$\begin{array}{rlll}1 & 0 & 18 & 0 \\ 2 & 4 & 19 & 0 \\ 3 & 0 & 20 & 1 \\ 4 & 1 & 21 & 1 \\ 5 & 2 & 22 & 1 \\ 6 & 0 & 23 & 0 \\ 7 & 0 & 24 & 1 \\ 8 & 4 & 25 & 3 \\ 9 & 0 & 26 & 2 \\ 10 & 0 & 27 & 2 \\ 11 & 0 & 28 & 0 \\ 12 & 1 & 29 & 0 \\ 13 & 0 & 30 & 0 \\ 14 & 0 & 31 & 1 \\ 15 & 0 & 32 & 0 \\ 16 & 1 & 33 & 0 \\ 17 & 4 & 34 & 2\end{array}$

35
36
37
38
39
40
41
42
43
44
45
46
47
48
49
50
51

2
1
1
2
1
0
2
2
0
1
0
0
2
1
3
0
1

$\begin{array}{ll}52 & 0 \\ 53 & 1 \\ 54 & 0 \\ 55 & 0 \\ 56 & 0 \\ 57 & 6 \\ 58 & 1 \\ 59 & 3 \\ 60 & 1 \\ 61 & 3 \\ 62 & 0 \\ 63 & 1 \\ 64 & 4 \\ 65 & 3 \\ 66 & 2 \\ 67 & 1 \\ 68 & 0\end{array}$

69
70
71
72
73
74
75
76
77
78
79
80
81
82
83
84

$\begin{array}{rr}85 & 0 \\ 86 & \\ 87 & 1 \\ 88 & 0 \\ 89 & 0 \\ 90 & 1 \\ 91 & 0 \\ 92 & 0 \\ 93 & 0 \\ 94 & 1 \\ 95 & 1 \\ 96 & 2 \\ 97 & 0 \\ 98 & 1 \\ 99 & 1 \\ 100 & 0 \\ & \end{array}$


Table 24. Fish health surveys of native fathead minnows, 1991-92--Continued

\begin{tabular}{|c|c|c|c|c|c|c|c|c|c|c|c|}
\hline $\begin{array}{l}\text { Fish } \\
\text { No. }\end{array}$ & $\begin{array}{c}\text { Index } \\
\text { total }\end{array}$ & $\begin{array}{l}\text { Fish } \\
\text { No. }\end{array}$ & $\begin{array}{c}\text { Index } \\
\text { total }\end{array}$ & $\begin{array}{l}\text { Fish } \\
\text { No. }\end{array}$ & $\begin{array}{c}\text { Index } \\
\text { total }\end{array}$ & $\begin{array}{l}\text { Fish } \\
\text { No. }\end{array}$ & $\begin{array}{c}\text { Index } \\
\text { total }\end{array}$ & $\begin{array}{l}\text { Fish } \\
\text { No. }\end{array}$ & $\begin{array}{r}\text { Index } \\
\text { total }\end{array}$ & $\begin{array}{l}\text { Fish } \\
\text { No. }\end{array}$ & $\begin{array}{c}\text { Index } \\
\text { total }\end{array}$ \\
\hline \multicolumn{12}{|c|}{ Site 12} \\
\hline \multicolumn{12}{|c|}{ October 20,1991} \\
\hline $\begin{array}{l}1 \\
2 \\
3 \\
4\end{array}$ & $\begin{array}{l}1 \\
2 \\
2 \\
4\end{array}$ & $\begin{array}{l}5 \\
6 \\
7 \\
8\end{array}$ & $\begin{array}{l}2 \\
2 \\
3 \\
1\end{array}$ & $\begin{array}{r}9 \\
10 \\
11\end{array}$ & $\begin{array}{l}3 \\
5 \\
2\end{array}$ & $\begin{array}{l}12 \\
13 \\
14\end{array}$ & $\begin{array}{l}2 \\
2 \\
5\end{array}$ & $\begin{array}{l}15 \\
16 \\
17\end{array}$ & $\begin{array}{l}1 \\
5 \\
0\end{array}$ & $\begin{array}{l}18 \\
19 \\
20\end{array}$ & $\begin{array}{l}2 \\
4 \\
2\end{array}$ \\
\hline \multicolumn{12}{|c|}{ June 25,1992} \\
\hline $\begin{array}{r}1 \\
2 \\
3 \\
4 \\
5 \\
6 \\
7 \\
8 \\
9 \\
10 \\
11\end{array}$ & $\begin{array}{l}0 \\
0 \\
3 \\
3 \\
2 \\
7 \\
0 \\
1 \\
0 \\
0 \\
4\end{array}$ & $\begin{array}{l}12 \\
13 \\
14 \\
15 \\
16 \\
17 \\
18 \\
19 \\
20 \\
21 \\
22\end{array}$ & $\begin{array}{l}0 \\
1 \\
2 \\
0 \\
6 \\
2 \\
1 \\
7 \\
0 \\
0 \\
4\end{array}$ & $\begin{array}{l}23 \\
24 \\
25 \\
26 \\
27 \\
28 \\
29 \\
30 \\
31 \\
32 \\
33\end{array}$ & $\begin{array}{l}0 \\
1 \\
3 \\
7 \\
2 \\
2 \\
2 \\
0 \\
2 \\
1 \\
2\end{array}$ & $\begin{array}{l}34 \\
35 \\
36 \\
37 \\
38 \\
39 \\
40 \\
41 \\
42 \\
43 \\
44\end{array}$ & $\begin{array}{l}0 \\
1 \\
3 \\
0 \\
0 \\
1 \\
0 \\
0 \\
0 \\
0 \\
1\end{array}$ & $\begin{array}{l}45 \\
46 \\
47 \\
48 \\
49 \\
50 \\
51 \\
52 \\
53 \\
54 \\
55\end{array}$ & $\begin{array}{l}0 \\
1 \\
3 \\
3 \\
0 \\
1 \\
3 \\
2 \\
1 \\
1 \\
2\end{array}$ & $\begin{array}{l}56 \\
57 \\
58 \\
59 \\
60 \\
61 \\
62 \\
63 \\
64 \\
65 \\
66\end{array}$ & $\begin{array}{l}1 \\
0 \\
2 \\
1 \\
5 \\
6 \\
3 \\
1 \\
1 \\
1 \\
1\end{array}$ \\
\hline \multicolumn{12}{|c|}{ September 2, 1992} \\
\hline $\begin{array}{l}1 \\
2 \\
3 \\
4 \\
5 \\
6\end{array}$ & $\begin{array}{l}0 \\
1 \\
0 \\
2 \\
0 \\
1\end{array}$ & $\begin{array}{r}7 \\
8 \\
9 \\
10 \\
11 \\
12\end{array}$ & $\begin{array}{l}0 \\
2 \\
2 \\
2 \\
2 \\
1\end{array}$ & $\begin{array}{l}13 \\
14 \\
15 \\
16 \\
17 \\
18\end{array}$ & $\begin{array}{l}3 \\
3 \\
2 \\
0 \\
0 \\
3\end{array}$ & $\begin{array}{l}19 \\
20 \\
21 \\
22 \\
23 \\
24\end{array}$ & $\begin{array}{l}0 \\
3 \\
2 \\
0 \\
0 \\
1\end{array}$ & $\begin{array}{l}25 \\
26 \\
27 \\
28 \\
29 \\
30\end{array}$ & $\begin{array}{l}1 \\
0 \\
0 \\
2 \\
2 \\
2\end{array}$ & $\begin{array}{l}31 \\
32 \\
33 \\
34 \\
35\end{array}$ & $\begin{array}{l}2 \\
0 \\
2 \\
0 \\
0\end{array}$ \\
\hline
\end{tabular}

Composite of sites 3,7 , and 11

October 20, 1991

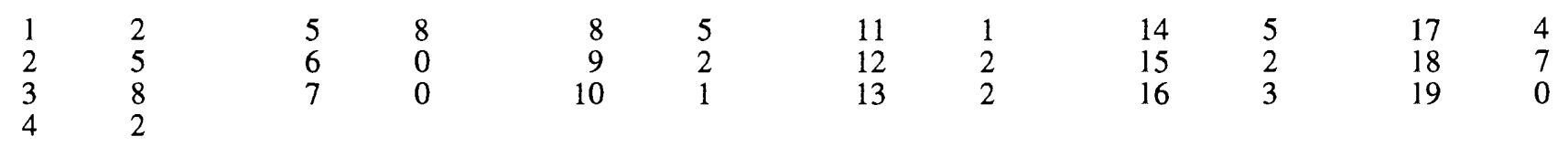

June 25, 1992

$\begin{array}{rrrrrrrrrrrr}1 & 6 & 18 & 3 & 35 & 1 & 52 & 0 & 69 & 2 & 86 & 4 \\ 2 & 2 & 19 & 2 & 36 & 5 & 53 & 0 & 70 & 0 & 87 & 0 \\ 3 & 5 & 20 & 1 & 37 & 1 & 54 & 2 & 71 & 0 & 88 & 0 \\ 4 & 3 & 21 & 1 & 38 & 1 & 55 & 0 & 72 & 2 & 89 & 3 \\ 5 & 1 & 22 & 1 & 39 & 1 & 56 & 0 & 73 & 2 & 90 & 5 \\ 6 & 0 & 23 & 0 & 40 & 0 & 57 & 1 & 74 & 6 & 91 & 0 \\ 7 & 1 & 24 & 2 & 41 & 1 & 58 & 3 & 75 & 1 & 92 & 3 \\ 8 & 2 & 25 & 4 & 42 & 2 & 59 & 1 & 76 & 3 & 93 & 1 \\ 9 & 4 & 26 & 0 & 43 & 0 & 60 & 1 & 77 & 0 & 94 & 0 \\ 10 & 4 & 27 & 5 & 44 & 2 & 61 & 1 & 78 & 0 & 95 & 4 \\ 11 & 1 & 28 & 1 & 45 & 1 & 62 & 1 & 79 & 8 & 96 & 4 \\ 12 & 5 & 29 & 2 & 46 & 1 & 63 & 1 & 70 & 1 & 97 & 2 \\ 13 & 2 & 30 & 1 & 47 & 1 & 64 & 3 & 71 & 0 & 98 & 1 \\ 14 & 7 & 31 & 4 & 48 & 3 & 65 & 1 & 82 & 2 & 99 & 2 \\ 15 & 1 & 32 & 0 & 49 & 1 & 66 & 1 & 83 & 3 & 100 & 4 \\ 16 & 4 & 33 & 4 & 50 & 1 & 67 & 1 & 84 & 1 & 101 & 5 \\ 17 & 4 & 34 & 3 & 51 & 0 & 68 & 1 & 85 & 2 & 102 & 3\end{array}$


Table 24. Fish health surveys of native fathead minnows, 1991-92--Continued

\begin{tabular}{|c|c|c|c|c|c|c|c|c|c|c|c|}
\hline $\begin{array}{l}\text { Fish } \\
\text { No. }\end{array}$ & $\begin{array}{c}\text { Index } \\
\text { total }\end{array}$ & $\begin{array}{l}\text { Fish } \\
\text { No. }\end{array}$ & $\begin{array}{c}\text { Index } \\
\text { total }\end{array}$ & $\begin{array}{l}\text { Fish } \\
\text { No. }\end{array}$ & $\begin{array}{c}\text { Index } \\
\text { total }\end{array}$ & $\begin{array}{l}\text { Fish } \\
\text { No. }\end{array}$ & $\begin{array}{c}\text { Index } \\
\text { total }\end{array}$ & $\begin{array}{l}\text { Fish } \\
\text { No. }\end{array}$ & $\begin{array}{r}\text { Index } \\
\text { total }\end{array}$ & $\begin{array}{l}\text { Fish } \\
\text { No. }\end{array}$ & $\begin{array}{c}\text { Index } \\
\text { total }\end{array}$ \\
\hline
\end{tabular}

Composite of sites 3, 7, and 11--Continued

June 25, 1992--Continued

\begin{tabular}{|c|c|c|c|c|c|c|c|c|c|c|}
\hline 103 & 0 & 107 & 0 & 111 & 3 & 115 & 0 & 119 & 0 & 123 \\
\hline 104 & 0 & 108 & 0 & 112 & 0 & 116 & 2 & 120 & 1 & 124 \\
\hline 105 & 2 & 109 & 1 & 113 & 1 & 117 & 0 & 121 & 0 & 125 \\
\hline 106 & 5 & 110 & 3 & 114 & 4 & 118 & 0 & 122 & 3 & \\
\hline
\end{tabular}

September 2, 1992

\begin{tabular}{rrrrrrrrrrrr}
1 & 2 & 7 & 2 & 13 & 1 & 19 & 1 & 25 & 2 & 30 & 1 \\
2 & 0 & 8 & 2 & 14 & 1 & 20 & 3 & 26 & 2 & 31 & 1 \\
3 & 1 & 9 & 1 & 15 & 1 & 21 & 0 & 27 & 1 & 32 & 1 \\
4 & 2 & 10 & 0 & 16 & 0 & 22 & 0 & 28 & 0 & 33 & 0 \\
5 & 3 & 11 & 2 & 17 & 0 & 23 & 3 & 29 & 1 & & \\
6 & 1 & 12 & 3 & 18 & 0 & 24 & 0 & & & \\
\hline
\end{tabular}


Table 25. Microtox ${ }^{\circledast}$ sediment (solid phase) bioassay, 1991

[See figure 1 for site locations. Value EC50 is percentage of sediment that causes 50-percent decrease in luminescence of Microtox ${ }^{\circledR}$ bacteria (Photobacterium phosphoreum); *, mean of three field replicates]

\begin{tabular}{|c|c|c|c|c|c|}
\hline Site & Date & $\begin{array}{l}\text { Value } \\
\text { EC50 }\end{array}$ & Site & Date & $\begin{array}{l}\text { Value } \\
\text { EC50 }\end{array}$ \\
\hline 1 & $\begin{array}{l}\text { July } 27 \\
\text { Aug. } 13\end{array}$ & $\begin{array}{l}1.5 \\
3.1\end{array}$ & 10 & $\begin{array}{l}\text { Aug. } 14 \\
\text { Aug. } 21\end{array}$ & $\begin{array}{c}.5 \\
1.2^{*}\end{array}$ \\
\hline 2 & Aug. 13 & 1.0 & 11 & $\begin{array}{l}\text { July } 26 \\
\text { Aug. } 14\end{array}$ & $\begin{array}{l}.3 \\
.6\end{array}$ \\
\hline 3 & Aug. 13 & 1.5 & 12 & July 26 & 6 \\
\hline 4 & $\begin{array}{l}\text { Aug. } 13 \\
\text { Aug. } 21\end{array}$ & $\begin{array}{l}1.0 \\
2.8^{*}\end{array}$ & & Aug. 14 & 1.1 \\
\hline 5 & Aug. 13 & 1.0 & 13 & $\begin{array}{l}\text { July } 27 \\
\text { Aug. } 14\end{array}$ & $\begin{array}{l}.5 \\
.7\end{array}$ \\
\hline 6 & $\begin{array}{l}\text { Aug. } 13 \\
\text { Aug. } 21\end{array}$ & $\begin{array}{l}1.3 \\
2.1^{*}\end{array}$ & CL2 & Aug. 13 & .9 \\
\hline 7 & Aug. 14 & .8 & UTL6 & Aug. 21 & $2.0^{*}$ \\
\hline 8 & Aug. 14 & .7 & UTL8 & Aug. 21 & $1.2 *$ \\
\hline 9 & Aug. 13 & .5 & & & \\
\hline
\end{tabular}

Table 26. Microtox ${ }^{\circledast}$ sediment porewater bioassay, 1991

[See figure 1 for site locations. Value EC50 is percentage of porewater that causes 50 -percent decrease in luminescence of Microtox ${ }^{\otimes}$ bacteria (Photobacterium phosphoreum); >, actual value is greater than value shown; *, mean of three field replicates]

\begin{tabular}{|c|c|c|c|c|c|}
\hline Site & Date & $\begin{array}{l}\text { Value } \\
\text { EC50 }\end{array}$ & Site & Date & $\begin{array}{l}\text { Value } \\
\text { EC50 }\end{array}$ \\
\hline 1 & $\begin{array}{l}\text { July } 9 \\
\text { July } 23 \\
\text { Aug. } 14\end{array}$ & $\begin{array}{c}>90 \\
15.6 \\
>100\end{array}$ & 10 & $\begin{array}{l}\text { Aug. } 14 \\
\text { Aug. } 20\end{array}$ & $\begin{array}{l}0.6 \\
1.1^{*}\end{array}$ \\
\hline 2 & $\begin{array}{l}\text { July } 9 \\
\text { Aug. } 14\end{array}$ & $\begin{array}{c}18.2 \\
>100\end{array}$ & 11 & $\begin{array}{r}\text { July } 23 \\
\text { Aug. } 14\end{array}$ & $\begin{array}{l}.2 \\
.1\end{array}$ \\
\hline 3 & $\begin{array}{l}\text { June } 19 \\
\text { Aug. } 14\end{array}$ & $\begin{array}{r}2.9 \\
65.4\end{array}$ & 12 & $\begin{array}{r}\text { July } 23 \\
\text { Aug. } 14\end{array}$ & $\begin{array}{l}.3 \\
.001\end{array}$ \\
\hline 4 & $\begin{array}{l}\text { Aug. } 14 \\
\text { Aug. } 20\end{array}$ & $\begin{array}{l}>100 \\
\quad 66.7 *\end{array}$ & 13 & $\begin{array}{r}\text { July } 23 \\
\text { Aug. } 14\end{array}$ & $\begin{aligned}> & 100 \\
& 24.0\end{aligned}$ \\
\hline 5 & $\begin{array}{l}\text { June } 19 \\
\text { Aug. } 14\end{array}$ & $\begin{array}{c}46.1 \\
>100\end{array}$ & CL2 & Aug. 14 & $>100$ \\
\hline 6 & $\begin{array}{l}\text { Aug. } 14 \\
\text { Aug. } 20\end{array}$ & $\begin{array}{l}>100 \\
9.6^{*}\end{array}$ & LR1 & June 19 & $>100$ \\
\hline 7 & $\begin{array}{l}\text { July } 9 \\
\text { Aug. } 14\end{array}$ & $\begin{array}{r}38.0 \\
7.0\end{array}$ & UTL6 & Aug. 20 & $13.1 *$ \\
\hline 8 & Aug. 14 & $>100$ & UTL8 & Aug. 20 & $5.4^{*}$ \\
\hline 9 & $\begin{array}{l}\text { July } 9 \\
\text { Aug. } 14\end{array}$ & $\begin{array}{l}>100 \\
33.6\end{array}$ & UTL13 & Aug. 20 & $10.7^{*}$ \\
\hline
\end{tabular}


Table 27. Microtox ${ }^{\circledR}$ sediment porewater bioassay, 1992

[See figure 1 for site locations. Value EC50 is percentage of porewater that causes 50-percent decrease in luminescence of Microtox ${ }^{\otimes}$ bacteria (Photobacterium phosphoreum). $>$, actual value is greater than value shown]

\begin{tabular}{|c|c|c|c|c|c|}
\hline Site & Date & $\begin{array}{l}\text { Value } \\
\text { EC50 }\end{array}$ & Site & Date & $\begin{array}{l}\text { Value } \\
\text { EC50 }\end{array}$ \\
\hline \multirow[t]{6}{*}{1} & June 18 & $>100$ & 10 & June 18 & 1.4 \\
\hline & July 1 & $>100$ & & July 29 & .4 \\
\hline & July 29 & $>100$ & & Aug. 13 & .4 \\
\hline & Aug. 13 & $>100$ & & & \\
\hline & Aug. 26 & $>100$ & 11 & June 18 & 36.5 \\
\hline & & & & July 1 & 30.1 \\
\hline \multirow[t]{6}{*}{2} & June 18 & $>100$ & & July 29 & 7.3 \\
\hline & July 1 & 36.4 & & Aug. 13 & $>100$ \\
\hline & July 29 & 47.4 & & Aug. 26 & 58.3 \\
\hline & Aug. 13 & $>100$ & & & \\
\hline & Aug. 26 & $>100$ & 12 & June 18 & $>100$ \\
\hline & & & & July 1 & 12.8 \\
\hline \multirow{6}{*}{3} & June 18 & $>100$ & & July 29 & 17.2 \\
\hline & July 1 & $>100$ & & Aug. 13 & $>100$ \\
\hline & July 29 & 24.3 & & Aug. 26 & 25.6 \\
\hline & Aug. 13 & $>100$ & & & \\
\hline & Aug. 26 & 41.1 & 13 & June 18 & $>100$ \\
\hline & & & & July 1 & $>100$ \\
\hline \multirow[t]{3}{*}{4} & June 18 & $>100$ & & July 29 & $>100$ \\
\hline & July 29 & 27.3 & & Aug. 13 & $>100$ \\
\hline & Aug. 13 & $>100$ & & Aug. 26 & $>100$ \\
\hline \multirow[t]{6}{*}{5} & June 18 & $>100$ & 15 & July 1 & $>100$ \\
\hline & July 1 & $>100$ & & July 29 & $>100$ \\
\hline & July 29 & $>100$ & & Aug. 13 & $>100$ \\
\hline & Aug. 13 & $>100$ & & Aug. 26 & $>100$ \\
\hline & Aug. 26 & $>100$ & & & \\
\hline & & & $4 C$ & July 1 & 2.5 \\
\hline \multirow[t]{4}{*}{6} & June 18 & $>100$ & & & \\
\hline & July 29 & 4.9 & $9 \mathrm{~A}$ & July 1 & 2.0 \\
\hline & Aug. 13 & $>100$ & & July 29 & .5 \\
\hline & & & & Aug. 26 & .3 \\
\hline \multirow[t]{6}{*}{7} & June 18 & $>100$ & & & \\
\hline & July 1 & $>100$ & $13 \mathrm{~B}$ & July 1 & .7 \\
\hline & July 29 & 4.8 & & July 29 & 23.3 \\
\hline & Aug. 13 & 11.6 & & Aug. 26 & $>100$ \\
\hline & Aug. 26 & 3.2 & & & \\
\hline & & & LR3 & July 1 & 34.0 \\
\hline \multirow[t]{4}{*}{8} & June 18 & 2.0 & & July 29 & 39.5 \\
\hline & July 29 & 2.4 & & Aug. 26 & 17.3 \\
\hline & Aug. 13 & .8 & & & \\
\hline & & & LR4 & July 1 & 100 \\
\hline \multirow[t]{5}{*}{9} & June 18 & 5.4 & & July 29 & 100 \\
\hline & July 1 & 4.3 & & Aug. 26 & 100 \\
\hline & July 29 & .8 & & & \\
\hline & Aug. 13 & 4.8 & & & \\
\hline & Aug. 26 & 2.5 & & & \\
\hline
\end{tabular}


Table 28. Aquatic plant, invertebrate, and vertebrate laboratory and in situ bioassays, 1991-92

[See figure 1 for site locations. Bioassays were done with Klamath Basin drainwater. --, no data]

\section{Green Algae (Selenastrum capricornutum) Laboratory Static Bioassay, 1991}

Results are the average response observed in duplicate test vessels expressed as a percent of control growth (number of algal cells)

\begin{tabular}{|c|c|c|c|c|c|c|c|c|c|}
\hline \multirow{2}{*}{ Date } & \multicolumn{9}{|c|}{ Site } \\
\hline & 1 & 2 & 3 & 5 & 7 & 9 & 11 & 12 & 13 \\
\hline July 10 & 4 & 4 & 2 & 5 & 5 & 3 & 2 & 4 & 5 \\
\hline July 17 & 30 & 10 & 10 & 20 & 20 & 30 & 30 & 50 & 20 \\
\hline July 24 & 10 & 20 & 10 & 10 & 10 & 20 & 10 & 20 & 10 \\
\hline July 31 & 20 & 20 & 0 & 10 & 30 & 30 & 100 & 30 & 10 \\
\hline Aug. 14 & 10 & 20 & 20 & 40 & 10 & 20 & 20 & 30 & 30 \\
\hline Aug. 21 & 20 & 10 & 10 & 20 & 20 & 20 & 0 & 0 & 10 \\
\hline
\end{tabular}

Daphnia (Daphnia magna) Laboratory Static Bioassay, 1991

Results are the average percent survival observed in duplicate test vessels

\begin{tabular}{|c|c|c|c|c|c|c|c|c|c|c|c|c|c|c|}
\hline \multirow{2}{*}{ Date } & \multicolumn{13}{|c|}{ Site } & \multirow[b]{2}{*}{ Control } \\
\hline & 1 & 2 & 3 & 4 & 5 & 6 & 7 & 8 & 9 & 10 & 11 & 12 & 13 & \\
\hline June 5 & 95 & 100 & 100 & -- & 85 & -- & 90 & -- & 90 & -- & 95 & 85 & 95 & 100 \\
\hline June 12 & 100 & 95 & 95 & 95 & 100 & 100 & 100 & 100 & 95 & 90 & 100 & 95 & 95 & 100 \\
\hline June 19 & 100 & 100 & 100 & -- & 100 & -- & 100 & -- & 100 & -- & 100 & 85 & 100 & 100 \\
\hline June 26 & 95 & 100 & 100 & -- & 100 & -- & 100 & -- & 95 & -- & 100 & 100 & 100 & 100 \\
\hline July 3 & 95 & 95 & 100 & 100 & 85 & 100 & 95 & 100 & 100 & 100 & 100 & 100 & 100 & 100 \\
\hline July 10 & 100 & 100 & 95 & -- & 100 & -- & 90 & -- & 100 & -- & 100 & 95 & 100 & 100 \\
\hline July 17 & 95 & 90 & 95 & -- & 100 & -- & 95 & -- & 100 & -- & 100 & 100 & 100 & 90 \\
\hline July 24 & 80 & 90 & 100 & 100 & 100 & 100 & 95 & 95 & 100 & 100 & 95 & 100 & 100 & 95 \\
\hline July 31 & 100 & 100 & 100 & -- & 100 & -- & 100 & -- & 100 & -- & 100 & 100 & 100 & 95 \\
\hline Aug. 7 & 100 & 100 & 100 & -- & 95 & -- & 75 & -- & 95 & -- & 100 & 100 & 100 & 100 \\
\hline Aug. 14 & 100 & 100 & 100 & -- & 95 & -- & 95 & --. & 95 & -- & 100 & 95 & 95 & 100 \\
\hline Aug. 21 & 100 & 95 & 100 & -- & 100 & -- & 100 & -- & 100 & -- & 95 & 100 & 100 & 100 \\
\hline Aug. 28 & 100 & 95 & 100 & -- & 95 & -- & 90 & -- & 95 & -- & 95 & 100 & 95 & 100 \\
\hline
\end{tabular}

Daphnia (Daphnia magna) In Situ Bioassay, 1991

Results are the average percent survival observed in duplicate test chambers

\begin{tabular}{lrrrrrrrrr}
\hline \multirow{2}{*}{ Date } & \multicolumn{7}{c}{ Site } & \multicolumn{7}{c}{ } \\
\cline { 2 - 10 } & 1 & 2 & 3 & 5 & 7 & 9 & 11 & 12 & 13 \\
\hline June 5 & 75 & 80 & 70 & 10 & 85 & 65 & 85 & 85 & 65 \\
June 12 & 30 & 70 & 85 & 0 & 65 & 35 & 75 & 70 & - \\
June 19 & 0 & 60 & 45 & 65 & 35 & 70 & 65 & 90 & 65 \\
June 26 & 80 & 50 & 55 & 45 & 70 & 55 & 55 & 65 & 85 \\
July 3 & 40 & 0 & 10 & 5 & 80 & 75 & 40 & 75 & 70 \\
July 10 & 65 & 55 & 60 & 35 & 80 & 95 & 70 & 80 & 100 \\
July 17 & 80 & 90 & 90 & 80 & 95 & 95 & 90 & 90 & 95 \\
July 24 & 25 & 30 & 50 & 5 & 25 & 30 & 45 & 75 & 15 \\
July 31 & 70 & 65 & 5 & 25 & 90 & 95 & 80 & 80 & 50
\end{tabular}


Table 28. Aquatic plant, invertebrate, and vertebrate laboratory and in situ bioassays, 1991-92--Continued

\begin{tabular}{lrrrrrrrrr}
\hline \multicolumn{10}{c}{ Daphnia (Daphnia magna) In Situ Bioassay, 1991--Continued } \\
\hline \multirow{2}{*}{ Date } & 1 & 2 & 3 & 5 & 7 & 9 & 11 & 12 & 13 \\
\hline & 0 & 80 & 35 & 0 & 0 & 5 & 50 & 0 & 10 \\
Aug. 7 & 90 & 65 & 0 & 40 & 60 & 65 & 85 & 85 & 85 \\
Aug. 14 & 30 & 85 & 30 & 30 & 65 & 10 & 50 & 45 & 90 \\
Aug. 21 & 45 & 100 & 10 & 55 & 80 & 60 & 70 & 100 & 80 \\
Aug. 28 & &
\end{tabular}

Hyalella (Hyalella azteca) Laboratory Static Bioassay, 1991

Results are the average percent survival observed in duplicate test vessels

\begin{tabular}{lrrrrrrrrrr}
\hline \multirow{2}{*}{ Date } & \multicolumn{10}{c}{ Site } \\
\cline { 2 - 11 } & 1 & 2 & 3 & 5 & 7 & 9 & 11 & 12 & 13 & Control \\
\hline June 12 & -- & 95 & 100 & 100 & 95 & 90 & 95 & -- & - & 95 \\
June 19 & -- & 50 & 45 & 60 & 0 & 0 & 90 & -- & - & 35 \\
June 26 & -- & 100 & 100 & 100 & 95 & 80 & 100 & 50 & - & 50 \\
July 3 & -- & 100 & 65 & 55 & 70 & 45 & 95 & 65 & -- & 95 \\
July 10 & 100 & 95 & 100 & 90 & 95 & 90 & 100 & 100 & 100 & 100 \\
July 17 & 90 & 90 & 95 & 80 & 80 & 80 & 75 & 90 & 95 & 80 \\
July 24 & 80 & 80 & 85 & 85 & 80 & 100 & 95 & 100 & 100 & 90 \\
July 31 & 90 & 100 & 100 & 85 & 65 & 90 & 95 & 95 & 80 & $10 C$ \\
Aug. 7 & -- & -- & 90 & 60 & 80 & -- & -- & -- & -- & -- \\
Aug. 14 & 100 & 100 & 100 & 100 & 100 & 100 & 100 & 90 & 100 & 100 \\
Aug. 21 & 100 & 95 & 90 & 95 & 100 & 95 & 85 & 100 & 95 & 95 \\
Aug. 28 & 85 & 95 & 100 & 95 & 90 & 95 & 90 & 90 & 95 & 100 \\
\hline
\end{tabular}

\section{Hyalella (Hyalella azteca) Laboratory In Situ Bioassay, 1991}

Results are the average percent survival observed in duplicate test chambers

\begin{tabular}{|c|c|c|c|c|c|c|c|c|c|}
\hline \multirow{2}{*}{ Date } & \multicolumn{9}{|c|}{ Site } \\
\hline & 1 & 2 & 3 & 5 & 7 & 9 & 11 & 12 & 13 \\
\hline June 19 & 5 & 20 & 0 & 5 & 5 & 0 & 5 & 15 & 5 \\
\hline June 26 & 75 & 95 & 100 & 80 & 65 & 55 & 90 & 85 & 90 \\
\hline July 3 & -- & -- & 65 & 35 & 45 & 35 & -- & -- & -- \\
\hline July 10 & 30 & 80 & 20 & 25 & 35 & 30 & 35 & 100 & 90 \\
\hline July 17 & 25 & 70 & 85 & 35 & 0 & 90 & 100 & 100 & 70 \\
\hline July 24 & 40 & 45 & 10 & 90 & 25 & 70 & 45 & 45 & 35 \\
\hline July 31 & 70 & 50 & 75 & 15 & 60 & 30 & 25 & 70 & 85 \\
\hline Aug. 7 & -- & -- & 0 & 0 & 0 & 10 & 25 & .. & -- \\
\hline Aug. 14 & 70 & 100 & 100 & 85 & 90 & 95 & 75 & 90 & 30 \\
\hline Aug. 21 & 75 & 30 & 35 & 50 & 45 & -- & 35 & 75 & 80 \\
\hline Aug. 28 & 100 & 80 & 95 & 85 & 100 & 95 & 100 & 85 & 95 \\
\hline
\end{tabular}


Table 28. Aquatic plant, invertebrate, and vertebrate laboratory and in situ bioassays, 1991-92--Continued

Fathead Minnow (Pimephales promelas) Laboratory Static Bioassay, 1991

Results are the average percent survival observed in duplicate test vessels

\begin{tabular}{lrrrrrrrrrr}
\hline \multirow{2}{*}{ Date } & \multicolumn{1}{c}{ Site } \\
\cline { 2 - 11 } & 1 & 2 & 3 & 5 & 7 & 9 & 11 & 12 & 13 & Control \\
\hline June 5 & -- & 100 & 95 & -- & 65 & -- & -- & -- & 60 & 100 \\
June 12 & 95 & 100 & 90 & 75 & 85 & 65 & 85 & 85 & 80 & 95 \\
June 19 & 100 & 100 & 95 & 80 & 100 & 85 & 90 & 100 & 80 & 100 \\
June 26 & 75 & 95 & 80 & 80 & -- & -- & 60 & - & -- & 95 \\
July 3 & 90 & 100 & 100 & 95 & 95 & 100 & 95 & 100 & 100 & 100 \\
July 10 & 100 & 100 & 80 & 90 & 90 & 100 & 80 & 100 & 55 & 100 \\
July 17 & 100 & 100 & 75 & 100 & 90 & 90 & 80 & 95 & 100 & 100 \\
July 24 & -- & 100 & 85 & 70 & 95 & 95 & 85 & 100 & 80 & 95 \\
July 31 & 100 & 100 & 100 & 100 & 95 & 100 & 100 & 100 & 100 & 100 \\
Aug. 7 & 90 & 100 & 90 & 85 & 90 & 90 & 100 & 95 & 70 & -- \\
Aug. 14 & 100 & 95 & 100 & 95 & 95 & 85 & 100 & 80 & 100 & 95 \\
Aug. 21 & 100 & 100 & 95 & 100 & 95 & 95 & 100 & 100 & 95 & 100 \\
Aug. 28 & 85 & 85 & 90 & 100 & 45 & 80 & 95 & 100 & 100 & 95 \\
\hline
\end{tabular}

Fathead Minnow (Pimephales promelas) In Situ Bioassays, 1991

Results are the average percent survival observed in duplicate test chambers

\begin{tabular}{lrrrrrrrrr}
\hline \multirow{2}{*}{ Date } & \multicolumn{1}{c}{ Site } \\
\cline { 2 - 9 } & 1 & 2 & 3 & 5 & 7 & 9 & 11 & 12 & 13 \\
\hline June 5 & 30 & 35 & 35 & 0 & 35 & 15 & 25 & 15 & 10 \\
June 12 & 25 & 55 & 30 & 50 & 20 & 25 & 20 & 65 & 0 \\
June 19 & 25 & 30 & 50 & 20 & 0 & 5 & 50 & 90 & 0 \\
June 26 & 35 & 0 & 10 & 5 & 40 & 0 & 40 & 50 & 35 \\
July 3 & 50 & 15 & 40 & 0 & 20 & 10 & 50 & 25 & 10 \\
July 10 & 60 & 40 & 25 & 0 & 60 & 25 & 20 & 45 & 5 \\
July 17 & 40 & 30 & 35 & 30 & 20 & 15 & 40 & 25 & 5 \\
July 24 & 10 & 10 & 10 & 5 & 20 & 0 & 25 & 15 & 0 \\
July 31 & 0 & 0 & 5 & 0 & 20 & 0 & 20 & 20 & 20 \\
Aug. 7 & 0 & 15 & 35 & 10 & 0 & 0 & 5 & 0 & 0 \\
Aug. 14 & 15 & 5 & 0 & 15 & 0 & 0 & 5 & 0 & 0 \\
Aug. 21 & 10 & 5 & 5 & 5 & 5 & 0 & 50 & 55 & 0 \\
Aug. 28 & 25 & 15 & 20 & 5 & 40 & 5 & 10 & 85 & 0 \\
\hline
\end{tabular}

African Clawed Frog (Xenopus laevis) Laboratory Static-Renewal Bioassay, 1991

Results are the average percent survival observed in duplicate test vessels

\begin{tabular}{lrrrrrrrrrr}
\hline \multirow{2}{*}{ Date } & \multicolumn{1}{c}{ Site } \\
\cline { 2 - 11 } & 1 & 2 & 3 & 5 & 7 & 9 & 11 & 12 & 13 & Control \\
\hline June 5 & 58 & 53 & 65 & 75 & 75 & 85 & 78 & 48 & 68 & 60 \\
June 19 & 100 & 93 & 98 & 98 & 98 & 88 & 93 & 100 & 95 & 100 \\
June 26 & 98 & 95 & 93 & 100 & 95 & 93 & 98 & 98 & 98 & 95 \\
July 10 & 98 & 85 & 93 & 95 & 100 & 95 & 98 & 95 & 98 & 98 \\
July 17 & 98 & 98 & 100 & 100 & 100 & 100 & 100 & 98 & 98 & 100 \\
Aug. 7 & -- & 90 & 100 & 98 & 93 & 95 & 95 & -- & -- & 100 \\
Aug. 14 & 98 & 100 & 88 & 90 & 98 & 88 & 98 & 95 & 88 & 100
\end{tabular}


Table 28. Aquatic plant, invertebrate, and vertebrate laboratory and in situ bioassays,

1991-92--Continued

\section{African Clawed Frog (Xenopus laevis) Laboratory Static-Renewal Bioassay, 1991}

Results are the average percent malformation observed in duplicate test vessels. ${ }^{*} \mathrm{n}=24$

\begin{tabular}{lrrrrrrrrrr}
\hline \multirow{2}{*}{ Date } & \multicolumn{10}{c}{ Site } \\
\cline { 2 - 11 } & 1 & 2 & 3 & 5 & 7 & 9 & 11 & 12 & 13 & Control \\
\hline June 5 & 33 & 53 & 35 & 75 & 75 & 70 & 20 & 48 & 53 & 48 \\
June 19 & 50 & 53 & 98 & 40 & 53 & 88 & 48 & 18 & 88 & 100 \\
June 26 & $8 *$ & 80 & 43 & 85 & 15 & 88 & 48 & 63 & 93 & 35 \\
July 10 & 63 & 40 & 88 & 85 & 18 & 60 & 75 & 45 & 10 & 5 \\
July 17 & 45 & 13 & 38 & 98 & 53 & 3 & 95 & 25 & 48 & 5 \\
Aug. 7 & -- & 30 & 10 & 10 & 3 & 18 & 5 & -- & -- & 23 \\
Aug. 14 & 35 & 13 & 13 & 40 & 40 & 13 & 18 & 18 & 8 & 0
\end{tabular}

African Clawed Frog (Xenopus laevis) Laboratory Static-Renewal Bioassay, 1991

Results are the average percent survival observed in duplicate test vessels (pilot larval/tadpole study)

\begin{tabular}{|c|c|c|c|c|c|c|c|c|c|c|}
\hline \multirow{2}{*}{ Date } & \multicolumn{9}{|c|}{ Site } & \multirow[b]{2}{*}{ Control } \\
\hline & 1 & 2 & 3 & 5 & 7 & 9 & 11 & 12 & 13 & \\
\hline \multicolumn{11}{|c|}{ 7-day-old larvae } \\
\hline July 17 & -- & -- & 100 & 100 & 100 & 100 & -- & -- & -- & 100 \\
\hline Aug. 21 & -- & -- & 100 & 100 & 100 & 100 & -- & -- & -. & 100 \\
\hline \multicolumn{11}{|c|}{ 14-day-old larvae } \\
\hline July 24 & -- & -- & 100 & 100 & 100 & 100 & -- & -- & -- & 100 \\
\hline
\end{tabular}

Mallard duckling (Anas platyrynchos) In Situ Bioassay, 1991

Results are the average percent survival observed in duplicate test cages

\begin{tabular}{lcccc}
\hline \multirow{2}{*}{ Date } & \multicolumn{3}{c}{ Site } & 7 \\
\cline { 2 - 5 } & 1 & 3 & 5 & 100 \\
June 5 & -- & 100 & 100 & 100 \\
June 19 & -- & 100 & 100 & 100 \\
July 3 & 100 & 100 & 100 & 100 \\
July 24 & 100 & 100 & 100 & 100 \\
Aug. 21 & 100 & 100 & 100 & \\
\hline
\end{tabular}

Duckweed (Lemna minor) Laboratory Static Bioassay, 1992

Results are the average response from duplicate test dishes expressed as a percent of control growth (increase in number of leaves)

\begin{tabular}{|c|c|c|c|c|c|c|c|c|c|c|}
\hline \multirow{2}{*}{ Date } & \multicolumn{9}{|c|}{ Site } & \multirow[b]{2}{*}{15} \\
\hline & 1 & 2 & 3 & 5 & 7 & 9 & 11 & 12 & 13 & \\
\hline June 3 & 74 & 68 & 82 & 69 & 74 & 84 & 80 & 83 & 46 & 82 \\
\hline June 10 & 58 & 108 & 103 & 92 & 116 & 97 & 100 & 103 & 104 & 63 \\
\hline June 17 & 63 & 60 & 51 & 87 & 54 & 84 & 63 & 58 & 60 & 40 \\
\hline June 24 & 20 & 46 & 63 & 80 & 49 & 61 & 41 & 37 & 37 & 34 \\
\hline July 1 & 69 & 64 & 56 & 93 & 87 & 91 & 56 & 53 & 53 & 60 \\
\hline July 8 & 58 & 58 & 72 & 56 & 67 & 48 & 52 & 53 & 67 & 53 \\
\hline July 15 & 51 & 49 & 57 & 46 & 63 & 57 & 63 & 63 & 46 & 57 \\
\hline July 22 & 49 & 50 & 51 & 46 & 43 & 51 & 48 & 49 & 49 & 43 \\
\hline July 29 & 92 & 82 & 68 & 76 & 64 & 78 & 66 & 58 & 68 & 50 \\
\hline Aug. 5 & 76 & 75 & 66 & 58 & 61 & 54 & 51 & 63 & 66 & 63 \\
\hline Aug. 12 & 74 & 71 & 71 & 65 & 65 & 70 & 59 & 73 & 62 & 47 \\
\hline Aug. 19 & 78 & 90 & 85 & 100 & 88 & 80 & 76 & 63 & 73 & 80 \\
\hline Aug. 26 & 67 & 76 & 73 & 79 & 80 & 76 & 66 & 70 & 70 & 72 \\
\hline
\end{tabular}


Table 28. Aquatic plant, invertebrate, and vertebrate laboratory and in situ bioassays, 1991-92--Continued

\section{Daphnia (Daphnia magna) Laboratory Static Bioassay, 1992}

Results are the average percent survival observed in duplicate test vessels

\begin{tabular}{|c|c|c|c|c|c|c|c|c|c|c|c|}
\hline \multirow{2}{*}{ Date } & \multicolumn{11}{|c|}{ Site } \\
\hline & 1 & 2 & 3 & 5 & 7 & 9 & 11 & 12 & 13 & 15 & Control \\
\hline June 3 & 100 & 100 & 95 & 100 & 100 & 100 & 100 & 100 & 100 & 100 & 100 \\
\hline June 10 & 100 & 100 & 100 & 100 & 100 & 100 & 100 & 75 & 100 & 100 & 100 \\
\hline June 17 & 100 & 100 & 90 & 100 & 100 & 100 & 100 & 100 & 100 & 100 & 95 \\
\hline June 24 & 100 & 100 & 100 & 100 & 100 & 100 & 100 & 100 & 100 & 100 & 100 \\
\hline July 1 & 95 & 100 & 100 & 100 & 100 & 100 & 100 & 100 & 100 & 100 & 100 \\
\hline July 8 & 85 & 100 & 100 & 100 & 100 & 100 & 90 & 100 & 100 & 100 & 90 \\
\hline July 15 & 100 & 100 & 100 & 100 & 100 & 100 & 100 & 100 & 100 & 100 & 100 \\
\hline July 22 & 100 & 100 & 85 & 100 & 100 & 100 & 90 & 100 & 100 & 100 & 100 \\
\hline July 29 & 100 & 100 & 100 & 100 & 100 & 100 & 95 & 95 & 100 & 95 & 100 \\
\hline Aug. 5 & 95 & 100 & 100 & 100 & 100 & 100 & 95 & 95 & 100 & 95 & -- \\
\hline Aug. 12 & 95 & 50 & 100 & 100 & 100 & 95 & 95 & 90 & 90 & 95 & 100 \\
\hline Aug. 19 & 80 & 95 & 100 & 85 & 80 & 80 & 90 & 95 & 100 & 100 & -- \\
\hline Aug. 26 & 95 & 100 & 95 & 95 & 100 & 100 & 95 & 80 & 95 & 75 & 75 \\
\hline
\end{tabular}

Daphnia (Daphnia magna) In Situ Bioassay, 1992

Results are the average percent survival observed in duplicate test chambers

\begin{tabular}{|c|c|c|c|c|c|c|c|c|c|c|c|}
\hline \multirow{2}{*}{ Date } & \multicolumn{11}{|c|}{ Site } \\
\hline & 1 & 2 & 3 & 5 & 7 & 9 & 11 & 12 & 13 & 15 & Control \\
\hline June 10 & 100 & 90 & 95 & 90 & 85 & 90 & 85 & 95 & 90 & 80 & -- \\
\hline June 17 & 65 & 35 & 100 & 85 & 60 & 40 & 55 & 100 & 100 & 30 & -- \\
\hline June 24 & 40 & 65 & 90 & 90 & 80 & 90 & 75 & 95 & 80 & 45 & 100 \\
\hline July 1 & 70 & 85 & 75 & 65 & 70 & 55 & 100 & 85 & 95 & 90 & 90 \\
\hline July 8 & 20 & 55 & 40 & 40 & 50 & 0 & 20 & 25 & 25 & 25 & 85 \\
\hline July 15 & 55 & 55 & 100 & 80 & 45 & 90 & 100 & 65 & 45 & 40 & 100 \\
\hline July 22 & 100 & 10 & 40 & 40 & 60 & 100 & 100 & 80 & 35 & 40 & 100 \\
\hline July 29 & 55 & 40 & 45 & 60 & 20 & 55 & 90 & 70 & 95 & 85 & 80 \\
\hline Aug. 5 & 30 & 0 & 60 & 80 & 80 & 100 & -- & 95 & 100 & 30 & 80 \\
\hline Aug. 12 & 85 & 0 & 50 & 40 & 95 & 30 & 90 & 85 & 95 & 75 & 95 \\
\hline Aug. 19 & 80 & 90 & 0 & 45 & 45 & 75 & 80 & 65 & 65 & 85 & 100 \\
\hline Aug. 26 & 70 & 90 & 85 & 80 & 85 & 95 & 65 & 85 & 75 & 100 & 95 \\
\hline
\end{tabular}


Table 28. Aquatic plant, invertebrate, and vertebrate laboratory and in situ bioassays, 1991-92--Continued

\section{Hyalella (Hyalella azteca) Laboratory Static Bioassay, 1992}

Results are the average percent survival observed in duplicate test vessels

\begin{tabular}{|c|c|c|c|c|c|c|c|c|c|c|c|}
\hline \multirow{2}{*}{ Date } & \multicolumn{11}{|c|}{ Site } \\
\hline & 1 & 2 & 3 & 5 & 7 & 9 & 11 & 12 & 13 & 15 & Control \\
\hline June 3 & 80 & 100 & 85 & 90 & 40 & 95 & 90 & 85 & 15 & 85 & 100 \\
\hline June 10 & 70 & 65 & 70 & 75 & 65 & 85 & 80 & 90 & 80 & 65 & 95 \\
\hline June 17 & 80 & 70 & 75 & 85 & 95 & 90 & 65 & 95 & 75 & 20 & 100 \\
\hline June 24 & 100 & 100 & 100 & 85 & 100 & 90 & 95 & 85 & 85 & 90 & 100 \\
\hline July 1 & 95 & 75 & 100 & 90 & 95 & 90 & 90 & 85 & 65 & 70 & 85 \\
\hline July 8 & 55 & 95 & 50 & 90 & 100 & 75 & 75 & 60 & 100 & 75 & 100 \\
\hline July 15 & 90 & 95 & 95 & 100 & 100 & 100 & 95 & 100 & 100 & 100 & -- \\
\hline July 22 & 90 & 95 & 100 & 100 & 100 & 95 & 100 & 100 & 100 & 90 & 100 \\
\hline July 29 & 100 & 90 & 85 & 90 & 100 & 65 & 85 & 95 & 95 & 90 & -- \\
\hline Aug. 5 & 100 & 95 & 90 & 100 & 90 & 100 & 100 & 90 & 80 & 80 & 90 \\
\hline Aug. 12 & 90 & 90 & 95 & 95 & 95 & 100 & 90 & 100 & 90 & 100 & 100 \\
\hline Aug. 19 & 100 & 90 & 100 & 85 & 95 & 90 & 100 & 85 & 90 & 100 & -- \\
\hline Aug. 26 & 95 & 85 & 90 & 100 & 80 & 60 & 65 & 85 & 85 & 85 & 95 \\
\hline
\end{tabular}

Hyalella (Hyalella azetca) In Situ Bioassay, 1992

Results are the average percent survival observed in duplicate test chambers

\begin{tabular}{lrrrrrrrrrrr}
\hline \multirow{2}{*}{ Date } & \multicolumn{10}{c}{} & \multicolumn{10}{c}{ Site } \\
\cline { 2 - 11 } & 1 & 2 & 3 & 5 & 7 & 9 & 11 & 12 & 13 & 15 & Control \\
\hline June 10 & 70 & -- & 100 & 70 & 100 & 100 & 90 & 95 & 80 & 0 & -- \\
June 17 & 95 & 35 & 85 & 80 & 100 & 95 & 65 & 95 & 90 & 5 & -- \\
June 24 & 85 & 85 & 100 & 100 & 100 & 100 & 90 & 100 & 100 & 60 & 100 \\
July 1 & 85 & 95 & 100 & 70 & 90 & 85 & 85 & 70 & 55 & 75 & 100 \\
July 8 & 100 & 75 & 75 & 60 & 65 & -- & 30 & 60 & 100 & 75 & 100 \\
July 15 & 55 & 65 & 85 & 60 & 85 & 60 & 90 & 90 & 45 & 40 & 100 \\
July 22 & 100 & 60 & 80 & 100 & 100 & 80 & 95 & 60 & 90 & 30 & 100 \\
July 29 & 75 & 65 & 45 & 80 & 65 & 65 & 65 & 80 & 80 & 40 & 100 \\
Aug. 5 & 65 & 90 & 95 & 90 & -- & 80 & -- & 75 & 60 & 50 & 100 \\
Aug. 12 & 80 & 0 & 60 & 70 & 50 & 80 & 85 & 50 & 65 & 50 & 100 \\
Aug. 19 & 60 & 95 & 40 & 60 & 85 & 90 & 85 & 70 & 85 & 55 & 100 \\
Aug. 26 & 90 & 100 & 85 & 95 & 80 & 100 & 90 & 85 & 100 & 100 & 100
\end{tabular}


Table 28. Aquatic plant, invertebrate, and vertebrate laboratory and in situ bioassays, 1991-92--Continued

Fathead Minnow (Pimephales promelas) Laboratory Static Bioassay, 1992

Results are the average percent survival observed in duplicate test vessels

\begin{tabular}{lrrrrrrrrrrr}
\hline \multirow{2}{*}{ Date } & \multicolumn{10}{c}{ 1 } & \multicolumn{10}{c}{ Site } \\
\cline { 2 - 11 } & 1 & 3 & 5 & 7 & 9 & 11 & 12 & 13 & 15 & Control \\
\hline June 3 & 100 & 100 & 100 & 100 & 100 & 100 & 100 & 100 & 100 & 100 & -- \\
June 10 & 90 & 100 & 100 & 100 & 100 & 95 & 90 & 100 & 95 & 100 & -- \\
June 17 & 100 & 100 & 90 & 80 & 70 & 100 & 85 & 95 & 90 & 100 & -- \\
June 24 & 100 & 100 & 100 & 95 & 90 & 90 & 95 & 95 & 100 & 95 & -- \\
July 1 & 100 & 95 & 95 & 95 & 75 & 100 & 100 & 100 & 85 & 95 & 100 \\
July 8 & 95 & 75 & 90 & 80 & 85 & 100 & 100 & 75 & 65 & 100 & 90 \\
July 15 & 100 & 100 & 95 & 100 & 70 & 100 & 100 & 100 & 95 & 100 & 95 \\
July 22 & 95 & 75 & 60 & 95 & 95 & 85 & 90 & 100 & 100 & 95 & 100 \\
July 29 & 100 & 100 & 100 & 100 & 100 & 95 & 95 & 95 & 85 & 100 & 95 \\
Aug. 5 & 100 & 90 & 100 & 50 & 95 & 80 & 95 & 90 & 35 & 80 & 100 \\
Aug. 12 & 95 & 100 & 85 & 85 & 80 & 100 & 100 & 70 & 40 & 100 & 100 \\
Aug. 19 & 100 & 100 & 100 & 100 & 95 & 100 & 100 & 80 & 85 & 100 & 100 \\
Aug. 26 & 80 & 100 & 75 & 95 & 85 & 95 & 90 & 100 & 70 & 85 & 90 \\
\hline
\end{tabular}

Fathead Minnow (Pimephales promelas) In Situ Bioassay, 1992

Results are the average percent survival observed in duplicate test chambers

\begin{tabular}{lrrrrrrrrrrr}
\hline Date & \multicolumn{10}{c}{ Site } \\
\cline { 2 - 13 } & 1 & 2 & 3 & 5 & 7 & 9 & 11 & 12 & 13 & 15 & Control \\
\hline June 17 & 25 & 0 & 45 & 50 & 80 & 35 & 60 & 60 & 45 & 5 & -- \\
June 24 & 0 & 0 & 50 & 65 & 40 & 40 & 40 & 60 & 40 & 65 & 90 \\
July 1 & 65 & 75 & 85 & 50 & 35 & 0 & 85 & 80 & 15 & 35 & 100 \\
July 8 & 60 & 5 & 60 & 25 & 50 & 10 & 30 & 65 & 55 & 65 & 100 \\
July 15 & 45 & 0 & 60 & 20 & 60 & 60 & 40 & 45 & 0 & 80 & 90 \\
July 22 & 25 & 0 & 90 & 30 & 60 & 0 & 55 & 35 & 45 & 80 & 70 \\
July 29 & 0 & 20 & 75 & 75 & 60 & 0 & 65 & 35 & 65 & 30 & 80 \\
Aug. 5 & 30 & 0 & 0 & 40 & 20 & 50 & -- & 30 & 0 & 0 & 75 \\
Aug. 12 & 60 & 0 & 25 & 40 & 45 & 50 & 70 & 50 & 0 & 70 & 75 \\
Aug. 19 & 65 & 40 & 0 & 20 & 30 & 10 & 55 & 0 & 0 & 90 & 90 \\
Aug. 26 & 0 & 35 & 20 & 30 & 35 & 20 & 60 & 5 & 10 & 50 & 70 \\
\hline
\end{tabular}

\section{African Clawed Frog (Xenopus laevis) Laboratory Static-Renewal Bioassay, 1992}

Results are the average percent survival observed in duplicate test vessels. Site results that are separated by / represent tests using $\mathrm{pH}$-nonadjusted and $\mathrm{pH}$-adjusted samples

\begin{tabular}{lrrrrrrrrrrr}
\hline \multirow{2}{*}{ Date } & \multicolumn{10}{c}{ Site } \\
\cline { 2 - 11 } & 1 & 2 & 3 & 5 & 7 & 9 & 11 & 12 & 13 & 15 & Control \\
\hline June 7 & -- & -- & $90 / 83$ & $85 / 70$ & $78 / 60$ & $88 / 98$ & $90 / 98$ & -- & -- & $90 / 88$ & 95 \\
June 24 & -- & -- & 73 & 60 & 75 & $60 / 60$ & $48 / 53$ & 73 & -- & $25 / 70$ & 90 \\
July 8 & 70 & 73 & 78 & 48 & 23 & $20 / 53$ & $60 / 83$ & $75 / 68$ & $65 / 75$ & $70 / 65$ & 95 \\
July 22 & 98 & 93 & $95 / 100$ & $95 / 100$ & 100 & 95 & 93 & $98 / 90$ & 98 & 95 & 100 \\
July 29 & -- & -- & 78 & 90 & 73 & 65 & 60 & -- & -- & - & 98 \\
Aug. 5 & -- & -- & 73 & 63 & 55 & 73 & 58 & -- & -- & -- & 93 \\
Aug. 12 & 83 & 78 & 88 & 70 & 55 & $65 / 83$ & $83 / 78$ & -- & -- & $83 / 60$ & 93 \\
Aug. 19 & -- & -- & 98 & 100 & 100 & $98 / 95$ & $95 / 98$ & -- & -- & -- & 100
\end{tabular}


Table 28. Aquatic plant, invertebrate, and vertebrate laboratory and in situ bioassays, 1991-92--Continued

\section{African Clawed Frog (Xenopus laevis) Laboratory Static-Renewal Bioassay, 1992}

Results are the average percent malformation observed in duplicate test dishes. Site results that are separated by / represent tests using $\mathrm{pH}$-nonadjusted and $\mathrm{pH}$-adjusted samples

\begin{tabular}{lrrrrrrrrrrr}
\hline \multirow{2}{*}{ Date } & \multicolumn{10}{c}{ Site } \\
\cline { 2 - 11 } & 1 & 2 & 3 & 5 & 7 & 9 & 11 & 12 & 13 & 15 & Control \\
\hline June 7 & -- & -- & $0 / 8$ & $5 / 10$ & $8 / 8$ & $5 / 5$ & $8 / 5$ & - & -- & $3 / 3$ & 0 \\
June 24 & -- & -- & 25 & 5 & 15 & $15 / 13$ & $18 / 28$ & 25 & - & $5 / 20$ & 0 \\
July 8 & 23 & 8 & 10 & 10 & 10 & $5 / 10$ & $20 / 25$ & $25 / 23$ & $33 / 43$ & $35 / 35$ & 5 \\
July 22 & 3 & 5 & $5 / 3$ & $3 / 5$ & 8 & 5 & 0 & $3 / 3$ & 3 & 5 & 0 \\
July 29 & -- & -- & 15 & 20 & 25 & 20 & 10 & -- & -- & -- & 5 \\
Aug. 5 & -- & -- & 15 & 10 & 5 & 15 & 28 & -- & -- & -- & 3 \\
Aug. 12 & - & 5 & 5 & 10 & 13 & $18 / 28$ & $33 / 40$ & -- & -- & $23 / 8$ & 8 \\
Aug. 19 & -- & -- & 5 & 0 & 8 & $0 / 0$ & $0 / 5$ & -- & -- & -- & -- \\
\hline
\end{tabular}

African Clawed Frog (Xenopus laevis) Laboratory Static-Renewal Bioassay, 1992

Results are the average percent survival of 7-day-old larvae observed in duplicate test dishes. Site results that are separated by / represent tests using $\mathrm{pH}$-nonadjusted and $\mathrm{pH}$-adjusted samples

\begin{tabular}{lrrrrrrrrrrr}
\hline \multirow{2}{*}{ Date } & \multicolumn{10}{c}{ Site } \\
\cline { 2 - 11 } & 1 & 2 & 3 & 5 & 7 & 9 & 11 & 12 & 13 & 15 & Control \\
\hline June 10 & -- & -- & $100 / 95$ & $100 / 95$ & $95 / 95$ & $95 / 100$ & $95 / 100$ & -- & -- & -- & 95 \\
June 17 & -- & -- & $95 / 90$ & $100 / 85$ & $100 / 100$ & $100 / 95$ & $100 / 100$ & -- & -- & -- & 100 \\
July 1 & -- & -- & 95 & 90 & 100 & 95 & 100 & -- & -- & -- & 100 \\
July 15 & -- & -- & 100 & 100 & 100 & 90 & -- & -- & -- & -- & 95 \\
July 22 & -- & -- & 95 & 90 & 95 & 100 & 100 & -- & -- & -- & 100 \\
Aug. 5 & -- & -- & 100 & 100 & 95 & 90 & $95 / 100$ & -- & -- & -- & 100 \\
Aug. 26 & -- & -- & 100 & 100 & 100 & $100 / 95$ & $90 / 100$ & -- & -- & -- & 100 \\
\hline
\end{tabular}


Table 29. Sodium chloride positive-control bioassays, 1991-92

[Results are the average percent organism survival observed in duplicate test vessels. Sodium chloride shown in parts per thousand (ppt)]

\begin{tabular}{|c|c|c|c|c|c|c|c|c|c|c|}
\hline \multicolumn{6}{|c|}{ Daphnia, 1991} & \multicolumn{5}{|c|}{ Daphnia, 1992} \\
\hline Date & 0 & 1.25 & 2.5 & 5.0 & 10.0 & Date & 0 & 2.5 & 5.0 & 10.0 \\
\hline June 5 & 100 & 100 & 100 & 70 & 0 & June 3 & 85 & 100 & 80 & 0 \\
\hline June 12 & 100 & 100 & 100 & 100 & 0 & June 10 & 100 & 100 & 80 & 0 \\
\hline June 19 & 100 & 100 & 100 & 90 & 0 & June 17 & 95 & 85 & 90 & 0 \\
\hline June 26 & 100 & 100 & 100 & 100 & 0 & June 24 & 100 & 100 & 70 & 0 \\
\hline July 3 & 100 & 100 & 95 & 95 & 0 & July 1 & 100 & 90 & 95 & 0 \\
\hline July 10 & 100 & 100 & 100 & 55 & 0 & July 8 & 90 & 100 & 80 & 0 \\
\hline July 17 & 90 & 100 & 85 & 100 & 0 & July 15 & 100 & 85 & 60 & 0 \\
\hline July 24 & 95 & 100 & 100 & 5 & 0 & July 22 & 100 & 100 & 90 & 0 \\
\hline July 31 & 95 & 100 & 100 & 0 & 0 & July 29 & 100 & 100 & 70 & 0 \\
\hline Aug. 7 & 100 & 100 & 100 & 35 & 0 & Aug. 5 & 0 & 95 & 90 & 0 \\
\hline Aug. 14 & 100 & 100 & 100 & 45 & 0 & Aug. 12 & 100 & 80 & 35 & 0 \\
\hline Aug. 21 & 100 & 100 & 100 & 100 & 0 & Aug. 19 & 0 & 65 & 35 & 0 \\
\hline Aug. 28 & 100 & 95 & 100 & 55 & 0 & Aug. 26 & 75 & 90 & 60 & 0 \\
\hline \multicolumn{6}{|c|}{ Hyalella, 1991} & \multicolumn{5}{|c|}{ Hyalella, 1992} \\
\hline Date & 0 & 1.25 & 2.5 & 5.0 & 10.0 & Date & 0 & 2.5 & 5.0 & 10.0 \\
\hline June 12 & 90 & -- & 10 & 10 & 0 & June 3 & 100 & 70 & 50 & 0 \\
\hline June 19 & 70 & 90 & 10 & 0 & 0 & June 10 & 95 & 100 & 85 & 50 \\
\hline June 26 & 50 & 100 & 70 & 0 & 0 & June 17 & 100 & 85 & 85 & 5 \\
\hline July 3 & 95 & 90 & 90 & 0 & 0 & June 24 & 100 & 90 & 85 & 15 \\
\hline July 10 & 100 & 00 & 100 & 0 & 0 & July 1 & 85 & 85 & 85 & 15 \\
\hline July 17 & 70 & 75 & 65 & 0 & 0 & July 8 & 100 & 100 & 90 & 70 \\
\hline July 24 & 90 & 95 & 80 & 0 & 0 & July 15 & 70 & 95 & 85 & 15 \\
\hline July 31 & 100 & 100 & 90 & 5 & 0 & July 22 & 100 & 95 & 100 & 30 \\
\hline Aug. 14 & 100 & 10 & 90 & 5 & 0 & July 29 & 70 & 85 & 85 & 0 \\
\hline Aug. 21 & 95 & 100 & 80 & 0 & 0 & Aug. 5 & 90 & 100 & 100 & 60 \\
\hline \multirow{3}{*}{ Aug. 28} & 100 & 100 & 80 & 0 & 0 & Aug. 12 & 100 & 95 & 100 & 60 \\
\hline & & & & & & Aug. 19 & 30 & 95 & 80 & 95 \\
\hline & & & & & & Aug. 26 & 95 & 70 & 85 & 5 \\
\hline \multicolumn{6}{|c|}{ Fathead minnow, 1991} & \multicolumn{5}{|c|}{ Fathead minnow, 1992} \\
\hline Date & 0 & 1.25 & 2.5 & 5.0 & 10.0 & Date & 0 & 2.5 & 5.0 & 10.0 \\
\hline June 5 & 100 & 100 & 100 & 60 & 0 & June 3 & 65 & 50 & 0 & 0 \\
\hline June 12 & 95 & 100 & 100 & 100 & 0 & June 10 & 55 & 45 & 0 & 0 \\
\hline June 19 & 100 & 100 & 100 & 100 & 0 & June 17 & 65 & 75 & 25 & 0 \\
\hline June 26 & 95 & 100 & 95 & 95 & 0 & June 24 & 60 & 65 & 0 & 0 \\
\hline July 3 & 100 & 100 & 100 & 100 & 0 & July 1 & 100 & 100 & 100 & 0 \\
\hline July 10 & 100 & 95 & 100 & 100 & 0 & July 8 & 85 & 100 & 100 & 0 \\
\hline July 17 & 100 & 100 & 100 & 95 & 0 & July 15 & 95 & 100 & 100 & 0 \\
\hline July 24 & 95 & 100 & 100 & 100 & 0 & July 22 & 100 & 100 & 100 & 0 \\
\hline July 31 & 100 & 100 & 100 & 95 & 0 & July 29 & 95 & 95 & 100 & 0 \\
\hline Aug. 7 & 0 & 20 & 30 & 20 & 0 & Aug. 5 & 100 & 100 & 65 & 0 \\
\hline Aug. 14 & 95 & 100 & 100 & 100 & 0 & Aug. 12 & 100 & 100 & 100 & 5 \\
\hline Aug. 21 & 100 & 100 & 95 & 90 & 0 & Aug. 19 & 100 & 100 & 100 & 10 \\
\hline Aug. 28 & 95 & 100 & 100 & 85 & 0 & Aug. 26 & 100 & 100 & 100 & 5 \\
\hline
\end{tabular}


Table 30. Frog embryo (Xenopus laevis) positivecontrol bioassays, 1991-92

[Tests conducted using the LD50 concentration of 6amino-nicotinamide. All malformed embryos were total body failures, and, consequently, were not predicted to survive. --, no data]

\begin{tabular}{ccc}
\hline Date & $\begin{array}{c}\text { Percent } \\
\text { mortality }\end{array}$ & $\begin{array}{c}\text { Percent } \\
\text { malformation } \\
\text { in survivors }\end{array}$ \\
\hline $6-05-91$ & 65 & 100 \\
$6-19-91$ & 85 & 100 \\
$6-29-91$ & 85 & 100 \\
$7-10-91$ & 68 & 100 \\
$7-17-91$ & 83 & 100 \\
$8-07-91$ & 88 & 100 \\
$8-14-91$ & 75 & 100 \\
$6-17-92$ & 88 & 100 \\
$6-24-92$ & 83 & 100 \\
$7-08-92$ & 100 & -- \\
$7-22-92$ & 63 & 100 \\
$7-30-92$ & 85 & 100 \\
$8-06-92$ & 100 & -- \\
$8-12-92$ & 100 & -- \\
$8-20-92$ & 58 & 100 \\
\hline
\end{tabular}


Table 31. Chironomus tentans sediment bioassay, 1991

[See figure 1 for site locations. ${ }^{\circ} \mathrm{C}$, degree Celsius; mg/L, milligram per liter; culture, the medium that test animals are raised in; control, clean potting soil; rep, replicate sample]

\begin{tabular}{|c|c|c|c|c|c|c|c|}
\hline $\begin{array}{c}\text { Sediment } \\
\text { collection } \\
\text { date } \\
\end{array}$ & $\begin{array}{l}\text { Test } \\
\text { start } \\
\text { date }\end{array}$ & $\begin{array}{c}\text { Percent } \\
\text { sample } \\
\text { sediment }\end{array}$ & $\begin{array}{l}\text { Percent } \\
\text { survival }\end{array}$ & $\begin{array}{c}\text { Sediment } \\
\text { collection } \\
\text { date }\end{array}$ & $\begin{array}{l}\text { Test } \\
\text { start } \\
\text { date }\end{array}$ & $\begin{array}{c}\text { Percent } \\
\text { sample } \\
\text { sediment }\end{array}$ & $\begin{array}{l}\text { Percent } \\
\text { survival }\end{array}$ \\
\hline \multicolumn{4}{|c|}{ Site 1} & \multicolumn{4}{|c|}{ Site 4--Continued } \\
\hline July 8 & July 9 & $\begin{array}{c}6.25 \\
12.5 \\
25 \\
50 \\
100\end{array}$ & $\begin{array}{r}90 \\
95 \\
90 \\
100 \\
100\end{array}$ & Aug. 19 & Aug. 28 & $\begin{array}{c}6.25 \\
12.5 \\
25 \\
50 \\
100\end{array}$ & $\begin{array}{l}85 \\
65 \\
95 \\
60 \\
75\end{array}$ \\
\hline \multirow[t]{2}{*}{ July 21} & \multirow[t]{2}{*}{ July 24} & \multirow[t]{2}{*}{$\begin{array}{c}6.25 \\
12.5 \\
25 \\
50 \\
100\end{array}$} & \multirow[t]{2}{*}{$\begin{array}{r}60 \\
100 \\
80 \\
90 \\
80\end{array}$} & Aug. 19 & $\begin{array}{l}\text { Aug. } 28 \\
\text { rep }\end{array}$ & $\begin{array}{c}6.25 \\
12.5 \\
25 \\
50 \\
100\end{array}$ & $\begin{array}{l}85 \\
80 \\
85 \\
95 \\
75\end{array}$ \\
\hline & & & & \multicolumn{4}{|c|}{ Site 5} \\
\hline \multirow[t]{2}{*}{ Aug. 12} & Aug. 14 & $\begin{array}{c}6.25 \\
12.5 \\
25 \\
50 \\
100\end{array}$ & $\begin{array}{l}80 \\
75 \\
85 \\
80 \\
75\end{array}$ & June 18 & June 21 & $\begin{array}{l}6.25 \\
12.5 \\
25 \\
50 \\
100\end{array}$ & $\begin{array}{l}55 \\
95 \\
90 \\
70 \\
90\end{array}$ \\
\hline & \multicolumn{2}{|c|}{ Site 2} & & June 18 & June 21 & 6.25 & 55 \\
\hline \multirow[t]{2}{*}{ July 8} & \multirow[t]{2}{*}{ July 9} & \multirow{2}{*}{$\begin{array}{l}6.25 \\
12.5 \\
25 \\
50 \\
100\end{array}$} & \multirow{2}{*}{$\begin{array}{r}90 \\
95 \\
100 \\
95 \\
95\end{array}$} & & & $\begin{array}{c}12.5 \\
25 \\
50 \\
100\end{array}$ & $\begin{array}{l}75 \\
95 \\
55 \\
90\end{array}$ \\
\hline & & & & \multicolumn{4}{|c|}{ Site 6} \\
\hline Aug. 12 & Aug. 14 & $\begin{array}{c}6.25 \\
12.5 \\
25 \\
50 \\
100\end{array}$ & $\begin{array}{r}85 \\
70 \\
100 \\
85 \\
70\end{array}$ & Aug. 12 & Aug. 14 & $\begin{array}{c}6.25 \\
12.5 \\
25 \\
50 \\
100\end{array}$ & $\begin{array}{l}85 \\
70 \\
85 \\
50 \\
65\end{array}$ \\
\hline \multicolumn{4}{|c|}{ Site 3} & \multirow[t]{2}{*}{ Aug. 19} & Aug. 27 & $\begin{array}{l}6.25 \\
12.5\end{array}$ & $\begin{array}{l}90 \\
85\end{array}$ \\
\hline \multirow[t]{2}{*}{ June 18} & \multirow[t]{2}{*}{ June 21} & $\begin{array}{l}6.25 \\
12.5 \\
25\end{array}$ & $\begin{array}{l}81 \\
75 \\
95\end{array}$ & & & $\begin{array}{r}25 \\
50 \\
100\end{array}$ & $\begin{array}{r}90 \\
95 \\
100\end{array}$ \\
\hline & & $\begin{array}{r}50 \\
100\end{array}$ & $\begin{array}{l}90 \\
80\end{array}$ & \multirow[t]{3}{*}{ Aug. 19} & \multirow[t]{3}{*}{$\begin{array}{l}\text { Aug. } 27 \\
\text { rep }\end{array}$} & $\begin{array}{l}6.25 \\
12.5\end{array}$ & $\begin{array}{l}90 \\
85\end{array}$ \\
\hline & & & & & & 50 & $\begin{array}{l}85 \\
75\end{array}$ \\
\hline \multirow{3}{*}{ Aug. 12} & \multirow{3}{*}{ Aug. 14} & 6.25 & 85 & & & 100 & 60 \\
\hline & & $\begin{array}{l}12.5 \\
25\end{array}$ & $\begin{array}{l}70 \\
90\end{array}$ & \multicolumn{4}{|c|}{ Site 7} \\
\hline & & $\begin{array}{r}50 \\
100\end{array}$ & $\begin{array}{l}70 \\
55\end{array}$ & July 8 & July 9 & $\begin{array}{l}6.25 \\
12.5\end{array}$ & $\begin{array}{r}90 \\
100\end{array}$ \\
\hline
\end{tabular}


Table 31. Chironomus tentans sediment bioassay, 1991-_Continued

\begin{tabular}{|c|c|c|c|c|c|c|c|}
\hline $\begin{array}{l}\text { Sediment } \\
\text { collection } \\
\text { date }\end{array}$ & $\begin{array}{l}\text { Test } \\
\text { start } \\
\text { date }\end{array}$ & $\begin{array}{c}\text { Percent } \\
\text { sample } \\
\text { sediment }\end{array}$ & $\begin{array}{l}\text { Percent } \\
\text { survival }\end{array}$ & $\begin{array}{l}\text { Sediment } \\
\text { collection } \\
\text { date }\end{array}$ & $\begin{array}{l}\text { Test } \\
\text { start } \\
\text { date }\end{array}$ & $\begin{array}{c}\text { Percent } \\
\text { sample } \\
\text { sediment }\end{array}$ & $\begin{array}{l}\text { Percent } \\
\text { survival }\end{array}$ \\
\hline \multicolumn{4}{|c|}{ Site 7--Continued } & \multicolumn{4}{|c|}{ Site 10--Continued } \\
\hline \multirow[t]{3}{*}{ July 8} & July 9 & $\begin{array}{l}25 \\
50\end{array}$ & $\begin{array}{r}85 \\
100\end{array}$ & Aug. 19 & Aug. 27 & 100 & 90 \\
\hline & & 100 & 100 & Aug. 19 & Aug. 28 & 6.25 & 85 \\
\hline & Aug 14 & & 60 & & rep & 12.5 & 85 \\
\hline \multirow{3}{*}{ Aug. 12} & Aug. 14 & $\begin{array}{l}0.25 \\
12.5\end{array}$ & $\begin{array}{l}00 \\
90\end{array}$ & & & $\begin{array}{l}25 \\
50\end{array}$ & 70 \\
\hline & & 25 & 70 & & & $\begin{array}{r}50 \\
100\end{array}$ & $\begin{array}{l}75 \\
55\end{array}$ \\
\hline & & 100 & 90 & \multicolumn{4}{|c|}{ Site 11} \\
\hline \multicolumn{4}{|c|}{ Site 8} & July 21 & July 24 & 6.25 & 100 \\
\hline \multirow[t]{5}{*}{ Aug. 12} & Aug. 14 & 6.25 & 55 & & & $\begin{array}{l}12.5 \\
25\end{array}$ & $\begin{array}{l}90 \\
90\end{array}$ \\
\hline & & 12.5 & 60 & & & 50 & 100 \\
\hline & & 25 & 65 & & & 100 & 90 \\
\hline & & 50 & 70 & & & & \\
\hline & & 100 & 75 & Aug. 12 & Aug. 14 & 6.25 & 80 \\
\hline \multirow[t]{4}{*}{ Aug. 19} & \multirow[t]{4}{*}{ Aug. 27} & \multirow{4}{*}{$\begin{array}{c}6.25 \\
12.5 \\
25 \\
50 \\
100\end{array}$} & \multirow{4}{*}{$\begin{array}{l}85 \\
80 \\
85 \\
85 \\
85\end{array}$} & & & $\begin{array}{l}12.5 \\
25\end{array}$ & $\begin{array}{l}80 \\
80\end{array}$ \\
\hline & & & & & & $\begin{array}{l}25 \\
50\end{array}$ & $\begin{array}{l}80 \\
65\end{array}$ \\
\hline & & & & & & 100 & 85 \\
\hline & & & & \multicolumn{4}{|c|}{ Site 12} \\
\hline \multirow[t]{5}{*}{ Aug. 19} & \multirow{5}{*}{$\begin{array}{l}\text { Aug. } 28 \\
\text { rep }\end{array}$} & 6.25 & 85 & & & & 90 \\
\hline & & 12.5 & 85 & July 21 & July 24 & $\begin{array}{l}6.25 \\
125\end{array}$ & $\begin{array}{l}90 \\
80\end{array}$ \\
\hline & & 25 & $\begin{array}{l}75 \\
75\end{array}$ & & & 25 & 70 \\
\hline & & $\begin{array}{r}50 \\
100\end{array}$ & $\begin{array}{l}75 \\
75\end{array}$ & & & 50 & 50 \\
\hline & & & & & & 100 & 40 \\
\hline \multicolumn{4}{|c|}{ Site 9} & \multirow{5}{*}{ Aug. 12} & \multirow{5}{*}{ Aug. 14} & & \\
\hline \multirow{5}{*}{ July 8} & \multirow{5}{*}{ July 9} & \multirow{5}{*}{$\begin{array}{c}6.25 \\
12.5 \\
25 \\
50 \\
100\end{array}$} & \multirow{5}{*}{$\begin{array}{r}95 \\
90 \\
95 \\
100 \\
100\end{array}$} & & & 12.5 & 70 \\
\hline & & & & & & 25 & 80 \\
\hline & & & & & & 50 & 75 \\
\hline & & & & & & 100 & 70 \\
\hline & & & & \multicolumn{4}{|c|}{ Site 13} \\
\hline \multicolumn{4}{|c|}{ Site 10} & \multirow{6}{*}{ July 21} & \multirow{6}{*}{ July 24} & & \\
\hline Aug. 12 & Aug. 14 & 6.25 & 60 & & & $\begin{array}{l}6.25 \\
12.5\end{array}$ & $\begin{array}{l}90 \\
90\end{array}$ \\
\hline & & 12.5 & 55 & & & 25 & 70 \\
\hline & & 25 & 65 & & & 50 & 100 \\
\hline & & 50 & 60 & & & 100 & 90 \\
\hline & & 100 & 50 & & & & \\
\hline & & & & Aug. 12 & Aug. 14 & 6.25 & 90 \\
\hline Aug. 19 & Aug. 27 & 6.25 & 85 & & & 12.5 & 85 \\
\hline & & 12.5 & 100 & & & 25 & 65 \\
\hline & & 25 & 95 & & & 50 & 80 \\
\hline & & 50 & 100 & & & 100 & 85 \\
\hline
\end{tabular}


Table 31. Chironomus tentans sediment bioassay, 1991--Continued

\begin{tabular}{|c|c|c|c|c|c|c|c|c|}
\hline $\begin{array}{c}\text { Sediment } \\
\text { collection } \\
\text { date }\end{array}$ & $\begin{array}{l}\text { Test } \\
\text { start } \\
\text { date }\end{array}$ & $\begin{array}{c}\text { Percent } \\
\text { sample } \\
\text { sediment }\end{array}$ & $\begin{array}{l}\text { Percent } \\
\text { survival }\end{array}$ & Date & $\begin{array}{c}\text { Tempera- } \\
\text { ture } \\
\left({ }^{\circ} \mathrm{C}\right) \\
\end{array}$ & $\begin{array}{c}\text { Alkalinity } \\
\text { as } \mathrm{CaCO} \\
(\mathrm{mg} / \mathrm{L})\end{array}$ & $\begin{array}{c}\mathrm{pH} \\
\text { (standard } \\
\text { units) }\end{array}$ & $\begin{array}{c}\text { Hardness } \\
\text { as } \mathrm{CaCO}{ }_{3} \\
(\mathrm{mg} / \mathrm{L})\end{array}$ \\
\hline \multicolumn{4}{|c|}{ Site UTL6 } & \multicolumn{5}{|c|}{ Water quality during $C$. tentans sediment bioassay } \\
\hline Aug. 19 & Aug. 27 & $\begin{array}{l}6.25 \\
12.5 \\
25 \\
50 \\
100\end{array}$ & $\begin{array}{l}75 \\
90 \\
85 \\
85 \\
75\end{array}$ & $\begin{array}{l}\text { June } 21 \\
\text { July } 9 \\
\text { July } 24 \\
\text { Aug. } 14 \\
\text { Aug. } 27 \\
\text { Aug. } 28\end{array}$ & $\begin{array}{l}21 \\
20 \\
22 \\
21 \\
22 \\
21\end{array}$ & $\begin{array}{l}147 \\
120 \\
143 \\
132 \\
163 \\
163\end{array}$ & $\begin{array}{l}7.4-8.1 \\
7.2-8.4 \\
7.4-8.0 \\
7.9-8.9 \\
7.5-8.1 \\
7.4-7.9\end{array}$ & $\begin{array}{l}240 \\
200 \\
260 \\
200 \\
280 \\
260\end{array}$ \\
\hline \multirow[t]{3}{*}{ Aug. 19} & $\begin{array}{l}\text { Aug. } 27 \\
\text { rep }\end{array}$ & $\begin{array}{l}6.25 \\
12.5\end{array}$ & $\begin{array}{l}90 \\
90\end{array}$ & \multicolumn{5}{|c|}{ Culture and control percent survival } \\
\hline & & & $\begin{array}{l}95 \\
85\end{array}$ & Date & & Culture & & Control \\
\hline & & 100 & 75 & June 18 & & 98 & & 100 \\
\hline \multicolumn{4}{|c|}{ Site UTL13 } & July 9 & & 100 & & 90 \\
\hline \multirow[t]{2}{*}{ Aug. 19} & Aug. 28 & $\begin{array}{l}6.25 \\
12.5 \\
25\end{array}$ & $\begin{array}{r}100 \\
90 \\
85\end{array}$ & $\begin{array}{l}\text { July } 24 \\
\text { Aug. } 12 \\
\text { Aug. } 19\end{array}$ & & $\begin{array}{r}100 \\
90 \\
95\end{array}$ & & $\begin{array}{l}90 \\
90 \\
90\end{array}$ \\
\hline & & $\begin{array}{r}50 \\
100\end{array}$ & $\begin{array}{l}80 \\
90\end{array}$ & \multicolumn{5}{|c|}{ Reference sediment } \\
\hline \multirow[t]{2}{*}{ Aug. 19} & $\begin{array}{l}\text { Aug. } 28 \\
\text { rep }\end{array}$ & $\begin{array}{l}6.25 \\
12.5\end{array}$ & $\begin{array}{l}60 \\
75\end{array}$ & Date & & $\begin{array}{c}\text { Percent } \\
\text { sample } \\
\text { sediment }\end{array}$ & & $\begin{array}{l}\text { Percent } \\
\text { survival }\end{array}$ \\
\hline & & $\begin{array}{r}25 \\
50 \\
100\end{array}$ & $\begin{array}{l}75 \\
65 \\
75\end{array}$ & July 9 & & $\begin{array}{l}\quad 6.25 \\
12.5 \\
25\end{array}$ & & $\begin{array}{l}95 \\
95 \\
90\end{array}$ \\
\hline \multicolumn{4}{|c|}{ Site CL1 } & & & $\begin{array}{r}50 \\
100\end{array}$ & & $\begin{array}{l}90 \\
75\end{array}$ \\
\hline Aug. 9 & Aug. 14 & $\begin{array}{l}6.25 \\
12.5 \\
25 \\
50 \\
100\end{array}$ & $\begin{array}{l}90 \\
95 \\
85 \\
85 \\
90\end{array}$ & July 24 & & $\begin{array}{l}6.25 \\
12.5 \\
25 \\
50 \\
100\end{array}$ & & $\begin{array}{l}60 \\
20 \\
20 \\
30 \\
20\end{array}$ \\
\hline
\end{tabular}


Table 32. Chironomus tentans sediment bioassay, 1992

[See figure 1 for site locations. Culture, the medium that test animals are raised in; control, clean potting soil]

\begin{tabular}{cccccc}
\hline $\begin{array}{l}\text { Sediment } \\
\begin{array}{c}\text { collection } \\
\text { date }\end{array}\end{array}$ & $\begin{array}{l}\text { Test } \\
\text { start } \\
\text { date }\end{array}$ & $\begin{array}{l}\text { Percent } \\
\text { survival }\end{array}$ & $\begin{array}{c}\text { Sediment } \\
\text { collection } \\
\text { date }\end{array}$ & $\begin{array}{l}\text { Test } \\
\text { start } \\
\text { date }\end{array}$ & $\begin{array}{c}\text { Percent } \\
\text { survival }\end{array}$ \\
\hline
\end{tabular}

\begin{tabular}{llr} 
& Site 1 & \\
\hline June 1 & June 2 & 85 \\
June 15 & June 16 & 65 \\
June 28 & June 30 & 75 \\
July 27 & July 28 & 90 \\
July 27 & July 28 & 90 \\
Aug. 10 & Aug. 11 & 75 \\
Aug. 25 & Aug. 26 & 100 \\
\hline
\end{tabular}

\section{Site 2}

\begin{tabular}{lll}
\hline May 31 & June 1 & 80 \\
May 31 & June 1 & 90 \\
June 15 & June 16 & 75 \\
June 29 & June 30 & 62 \\
July 27 & July 28 & 90 \\
Aug. 10 & Aug. 11 & 95 \\
Aug. 25 & Aug. 26 & 90 \\
\hline
\end{tabular}

\section{Site 3}

\begin{tabular}{llr}
\hline June 1 & June 2 & 75 \\
June 16 & June 17 & 90 \\
June 29 & June 30 & 70 \\
July 28 & July 29 & 100 \\
Aug. 11 & Aug. 12 & 90 \\
Aug. 25 & Aug. 26 & 100 \\
\hline
\end{tabular}

\section{Site 4}

\begin{tabular}{lll}
\hline June 16 & June 17 & 90 \\
July 28 & July 29 & 80 \\
Aug. 11 & Aug. 12 & 90 \\
\hline
\end{tabular}

\section{Site 5}

\begin{tabular}{llr}
\hline June 1 & June 2 & 65 \\
June 16 & June 17 & 95 \\
June 29 & June 30 & 70 \\
July 28 & July 29 & 95 \\
Aug. 11 & Aug. 12 & 50 \\
Aug. 25 & Aug. 26 & 100 \\
\hline
\end{tabular}

\section{Site 6}

June 16

July 28

Aug. 11
June 17

July 29

Aug. 12
85

100

95
Site 7

\begin{tabular}{lcc}
\hline June 1 & June 2 & 65 \\
June 16 & June 17 & 85 \\
June 29 & June 30 & 33 \\
June 29 & June 30 & 55 \\
July 28 & July 29 & 80 \\
Aug. 11 & Aug. 12 & 90 \\
Aug. 11 & Aug. 12 & 76 \\
Aug. 25 & Aug. 26 & 70 \\
\hline
\end{tabular}

\section{Site 8}

\begin{tabular}{lll}
\hline June 16 & June 17 & 80 \\
July 28 & July 29 & 90 \\
Aug. 11 & Aug. 12 & 85 \\
\hline
\end{tabular}

Site 9

\begin{tabular}{llr}
\hline June 1 & June 2 & 40 \\
June 16 & June 17 & 40 \\
June 29 & June 30 & 80 \\
July 28 & July 29 & 75 \\
Aug. 11 & Aug. 12 & 5 \\
Aug. 25 & Aug. 26 & 77 \\
\hline & Site 10 \\
\hline June 16 & June 17 & 70 \\
July 28 & July 29 & 50 \\
Aug. 11 & Aug. 12 & 50 \\
\hline
\end{tabular}

Site 11

\begin{tabular}{llr}
\hline June 1 & June 2 & 71 \\
June 15 & June 16 & 65 \\
June 15 & June 16 & 65 \\
June 29 & June 30 & 75 \\
July 28 & July 29 & 100 \\
Aug. 10 & Aug. 11 & 75 \\
Aug. 25 & Aug. 26 & 80 \\
Aug. 25 & Aug. 26 & 100 \\
\hline
\end{tabular}

Site 12

May 31

June 15

June 28

July 27

Aug. 10

Aug. 24

$\begin{array}{lr}\text { June } 1 & 65 \\ \text { June } 16 & 70 \\ \text { June } 30 & 40 \\ \text { July } 28 & 100 \\ \text { Aug. } 11 & 75 \\ \text { Aug. } 25 & 95\end{array}$


Table 32. Chironomus tentans sediment bioassay, 1992--Continued

\begin{tabular}{|c|c|c|c|c|c|}
\hline $\begin{array}{c}\text { Sediment } \\
\text { collection } \\
\text { date }\end{array}$ & $\begin{array}{l}\text { Test } \\
\text { start } \\
\text { date }\end{array}$ & $\begin{array}{l}\text { Percent } \\
\text { survival }\end{array}$ & $\begin{array}{c}\text { Sediment } \\
\text { collection } \\
\text { date }\end{array}$ & $\begin{array}{l}\text { Test } \\
\text { start } \\
\text { date }\end{array}$ & $\begin{array}{l}\text { Percent } \\
\text { survival }\end{array}$ \\
\hline \multicolumn{3}{|c|}{ Site 13} & \multicolumn{3}{|c|}{ Site 9A } \\
\hline May 31 & June 1 & 85 & May 31 & June 1 & 60 \\
\hline June 15 & June 16 & 86 & June 28 & June 30 & 55 \\
\hline June 15 & June 16 & 80 & July 27 & July 28 & 75 \\
\hline June 28 & June 30 & 80 & Aug. 24 & Aug. 25 & 50 \\
\hline $\begin{array}{l}\text { July } 27 \\
\text { Aug. } 10\end{array}$ & $\begin{array}{c}\text { July } 28 \\
\text { Aug. } 11\end{array}$ & $\begin{array}{l}90 \\
90\end{array}$ & \multicolumn{3}{|c|}{ Site 13B } \\
\hline Aug. 24 & Aug. 25 & 70 & \multirow{5}{*}{$\begin{array}{l}\text { May } 31 \\
\text { June } 28 \\
\text { June } 28 \\
\text { July } 27 \\
\text { Aug. } 11\end{array}$} & \multirow{4}{*}{$\begin{array}{l}\text { June } 1 \\
\text { June } 30 \\
\text { June } 30 \\
\text { July } 28 \\
\text { Aug. } 25\end{array}$} & \multirow{4}{*}{$\begin{array}{r}35 \\
70 \\
100 \\
90 \\
05\end{array}$} \\
\hline & Site 15 & & & & \\
\hline Mav 31 & Iune 1 & 81 & & & \\
\hline June 15 & June 16 & 70 & & & \\
\hline June 15 & June 16 & 65 & \multirow{2}{*}{\multicolumn{3}{|c|}{ Site B }} \\
\hline June 28 & June 30 & 45 & & & \\
\hline July 27 & July 28 & 90 & \multirow{4}{*}{\multicolumn{3}{|c|}{$\begin{array}{l}\text { June } 17 \\
\text { July } 29 \\
\text { Aug. } 12\end{array}$}} \\
\hline Aug. 11 & Aug. 12 & 90 & & & \\
\hline Aug. 24 & Aug. 25 & 95 & & & \\
\hline Aug. 25 & Aug. 26 & 65 & & & \\
\hline \multicolumn{3}{|c|}{ Site LR3 } & \multicolumn{3}{|c|}{ Culture and control percent survival } \\
\hline \multirow{3}{*}{$\begin{array}{l}\text { May } 31 \\
\text { June } 28 \\
\text { July } 27 \\
\text { Aug. } 24\end{array}$} & \multirow{3}{*}{$\begin{array}{l}\text { June } 1 \\
\text { June } 30 \\
\text { July } 28 \\
\text { Aug. } 25\end{array}$} & 85 & Date & Culture & Control \\
\hline & & $\begin{array}{l}65 \\
90\end{array}$ & \multirow{8}{*}{$\begin{array}{l}\text { June } 1 \\
\text { June } 2 \\
\text { June } 16 \\
\text { June } 17 \\
\text { June } 30 \\
\text { July 28 } \\
\text { July } 29 \\
\text { Aug. } 11 \\
\text { Aug. } 12 \\
\text { Aug. } 25 \\
\text { Aug. } 26\end{array}$} & \multirow{8}{*}{$\begin{array}{r}81 \\
97 \\
95 \\
92 \\
88 \\
94 \\
98 \\
100 \\
92 \\
100 \\
100\end{array}$} & \multirow{8}{*}{$\begin{array}{r}79 \\
80 \\
44 \\
72 \\
62 \\
93 \\
98 \\
90 \\
89 \\
100 \\
96\end{array}$} \\
\hline & & 85 & & & \\
\hline & Site LR4 & & & & \\
\hline Mav 31 & June 1 & 90 & & & \\
\hline June 28 & June 30 & 90 & & & \\
\hline July 27 & July 28 & 90 & & & \\
\hline \multirow[t]{2}{*}{ Aug. 24} & Aug. 25 & 95 & & & \\
\hline & Site $4 \mathrm{C}$ & & & & \\
\hline May 31 & June 1 & 95 & & & \\
\hline June 28 & June 30 & 70 & & & \\
\hline
\end{tabular}


Table 33. Mallard duckling bioassay, 1991

[See figure 1. AChE, brain acetylcholinesterase activity expressed as micromoles of substrate hydrolyzed per minute per gram of tissue. Weights are in grams. $\Delta \mathrm{T}$, change in body temperature of mallard duckling during cold stress test; ${ }^{\circ} \mathrm{C}$, degree Celsius; --, no data]

\begin{tabular}{cccccccccc}
\hline Site & $\begin{array}{c}\text { Bird } \\
\text { identi- } \\
\text { fication } \\
\text { No. }\end{array}$ & 0 & & Weight for indicated day of testing & & $\begin{array}{c}\text { Terminal } \\
\text { liver } \\
\text { weight, } \\
\text { day } 4 \text { of } \\
\text { testing }\end{array}$ & $\begin{array}{c}\text { Brain } \\
\text { weight, } \\
\text { day 4 of } \\
\text { testing }\end{array}$ & $\begin{array}{c}\text { AChE } \\
\text { activity }\end{array}$ & $\begin{array}{c}\Delta \mathrm{T} \\
\left({ }^{\circ} \mathrm{C}\right)\end{array}$ \\
\hline
\end{tabular}

Test period 1: June 2 to 5

\begin{tabular}{|c|c|c|c|c|c|c|c|c|c|c|}
\hline Control & 61 & 47.1 & 56.8 & 65.6 & 80.8 & 86.5 & 4.659 & 1.838 & 15.60 & -- \\
\hline Control & 62 & 53.1 & 59.5 & 69.8 & 84.3 & 93.4 & 4.314 & 1.879 & 11.37 & -- \\
\hline Control & 63 & 58.4 & 67.4 & 82.8 & 95.1 & 102.9 & 5.294 & 1.746 & 12.67 & -- \\
\hline Control & 64 & 58.7 & 71.7 & 87.4 & 102.1 & 112.5 & 7.053 & 1.862 & -- & -- \\
\hline Control & 65 & 59.5 & 66.0 & 80.6 & 95.1 & 101.8 & 5.166 & 1.828 & 14.30 & -- \\
\hline Control & 66 & 55.5 & 65.4 & 76.6 & 92.0 & 95.4 & 5.600 & 1.975 & 13.32 & -- \\
\hline Control & 67 & 52.4 & 62.3 & 75.5 & 88.6 & 99.8 & 5.015 & 2.133 & 13.97 & -- \\
\hline Control & 68 & 51.8 & 60.8 & 69.7 & 84.5 & 91.6 & 4.616 & 1.827 & 13.65 & -- \\
\hline Control & 69 & 47.8 & 55.5 & 64.6 & 79.8 & 84.5 & 4.238 & 1.756 & 11.70 & -- \\
\hline Control & 70 & 60.6 & 69.0 & 82.6 & 99.1 & 104.5 & 5.981 & 1.857 & 9.10 & -- \\
\hline Control & 71 & 47.2 & 51.5 & 61.6 & 68.3 & 77.4 & 4.142 & 1.803 & 15.27 & -- \\
\hline Control & 72 & 47.0 & 53.2 & 63.3 & 72.0 & 84.1 & 4.334 & 1.991 & 15.60 & -- \\
\hline Control & 73 & 54.2 & 63.0 & 78.0 & 84.9 & 96.3 & 5.305 & 1.994 & 13.65 & -- \\
\hline Control & 74 & 50.2 & 57.2 & 68.7 & 75.7 & 84.4 & 4.487 & 1.681 & 13.32 & -- \\
\hline Control & 75 & 49.7 & 59.4 & 72.5 & 84.2 & 93.1 & 4.922 & 1.736 & 15.27 & -- \\
\hline Control & 76 & 60.2 & 67.5 & 83.7 & 91.9 & 98.6 & 4.795 & 1.714 & 11.37 & -. \\
\hline Control & 77 & 57.2 & 64.8 & 78.8 & 86.9 & 96.0 & 5.653 & 1.922 & 14.62 & -- \\
\hline Control & 78 & 60.0 & 66.5 & 79.1 & 91.5 & 101.5 & 6.998 & 1.607 & 11.05 & -- \\
\hline Control & 79 & 53.6 & 62.6 & 76.0 & 81.4 & 95.0 & 4.982 & 1.874 & 15.92 & -- \\
\hline Control & 80 & 64.3 & 75.4 & 92.4 & 101.7 & 113.7 & 6.226 & 1.799 & 11.05 & -- \\
\hline 3 & 1 & 40.8 & 48.0 & 59.3 & 76.2 & 83.5 & 5.764 & 1.716 & 17.55 & -- \\
\hline 3 & 2 & 43.2 & 47.6 & 53.4 & 66.9 & 73.4 & 4.905 & 1.617 & 14.95 & -- \\
\hline 3 & 3 & 43.4 & 50.1 & 58.3 & 72.0 & 74.8 & 4.740 & 1.605 & 12.35 & -- \\
\hline 3 & 4 & 49.7 & 56.2 & 69.6 & 87.4 & 98.1 & 7.500 & 2.116 & 13.00 & -- \\
\hline 3 & 5 & 51.2 & 57.2 & 69.9 & 92.4 & 95.3 & 5.546 & 1.772 & 12.35 & -- \\
\hline 3 & 6 & 52.5 & 61.4 & 75.9 & 99.3 & 105.0 & 6.955 & 1.868 & 14.30 & -- \\
\hline 3 & 7 & 53.7 & 61.6 & 74.6 & 92.8 & 98.2 & 5.108 & 1.946 & 11.37 & -- \\
\hline 3 & 8 & 52.9 & 62.9 & 77.3 & 95.6 & 103.3 & 7.062 & 1.867 & 14.30 & -- \\
\hline 3 & 9 & 59.8 & 69.1 & 85.6 & 109.0 & 119.4 & 6.910 & 2.003 & 13.00 & -- \\
\hline 3 & 10 & 60.0 & 66.0 & 81.9 & 104.9 & 111.7 & 9.075 & 1.907 & 13.98 & -- \\
\hline 3 & 11 & 46.0 & 52.9 & 65.7 & 84.6 & 90.5 & 5.051 & 1.755 & 13.65 & -- \\
\hline 3 & 12 & 44.0 & 49.6 & 59.1 & 78.1 & 82.9 & 5.253 & 1.670 & 11.37 & -- \\
\hline 3 & 13 & 54.4 & 61.8 & 78.6 & 105.6 & 110.3 & 6.888 & 1.806 & 13.32 & -- \\
\hline 3 & 14 & 54.9 & 65.1 & 78.3 & 100.2 & 106.2 & 6.296 & 1.860 & 13.00 & -- \\
\hline 3 & 15 & 53.1 & 63.5 & 74.0 & 98.3 & 100.7 & 6.215 & 1.881 & 9.75 & -- \\
\hline 3 & 16 & 50.5 & 56.9 & 69.0 & 88.1 & 95.9 & 5.766 & 1.868 & 14.25 & -- \\
\hline 3 & 17 & 48.0 & 55.7 & 69.0 & 89.5 & 97.1 & 6.426 & 2.007 & 12.02 & -- \\
\hline 3 & 18 & 48.1 & 53.1 & 63.4 & 80.0 & 84.6 & 4.930 & 1.757 & 14.95 & -- \\
\hline 3 & 19 & 59.8 & 68.0 & 79.0 & 101.3 & 105.7 & 6.165 & 1.893 & 14.62 & -- \\
\hline 3 & 20 & 58.8 & 70.0 & 89.0 & 121.3 & 125.6 & 7.804 & 1.997 & 12.67 & -- \\
\hline 5 & 21 & 47.2 & 53.0 & 67.0 & 81.5 & 93.8 & 5.435 & 1.760 & 10.07 & -- \\
\hline 5 & 22 & 44.7 & 52.0 & 60.7 & 72.6 & 81.6 & 4.677 & 1.704 & 12.35 & -- \\
\hline 5 & 23 & 48.8 & 55.0 & 68.5 & 81.9 & 95.5 & 6.181 & 1.832 & 7.80 & -- \\
\hline 5 & 24 & 51.5 & 60.3 & 70.7 & 86.2 & 96.1 & 5.528 & 1.899 & 13.65 & -- \\
\hline
\end{tabular}


Table 33. Mallard duckling bioassay, 1991--Continued

\begin{tabular}{ccccccccc}
\hline & $\begin{array}{c}\text { Bird } \\
\text { identi- } \\
\text { fication } \\
\text { No. }\end{array}$ & 0 & Weight for indicated day of testing & & $\begin{array}{c}\text { Terminal } \\
\text { liver } \\
\text { weight, } \\
\text { day } 4 \text { of } \\
\text { testing }\end{array}$ & $\begin{array}{c}\text { Brain } \\
\text { weight, } \\
\text { day } 4 \text { of } \\
\text { testing }\end{array}$ & $\begin{array}{c}\mathrm{AChE} \\
\text { activity }\end{array}$ & $\begin{array}{c}\Delta \mathrm{T} \\
\left({ }^{\circ} \mathrm{C}\right)\end{array}$ \\
\hline
\end{tabular}

Test period 1: June 2 to 5--Continued

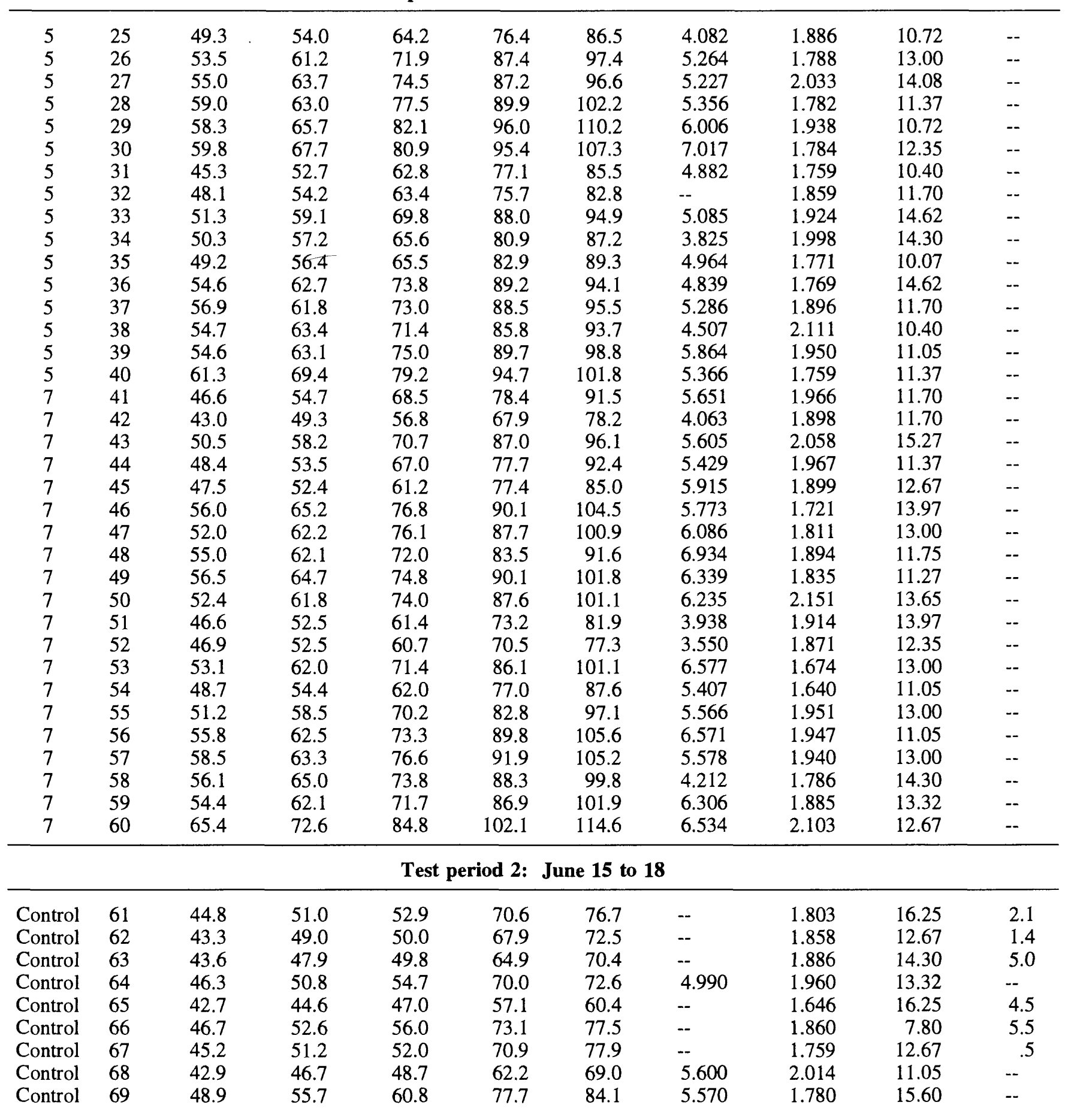


Table 33. Mallard duckling bioassay, 1991--Continued

\begin{tabular}{|c|c|c|c|c|c|c|c|c|c|c|}
\hline \multirow{2}{*}{ Site } & \multirow{2}{*}{$\begin{array}{l}\text { Bird } \\
\text { identi- } \\
\text { fication } \\
\text { No. }\end{array}$} & \multicolumn{5}{|c|}{ Weight for indicated day of testing } & \multirow{2}{*}{$\begin{array}{l}\text { Terminal } \\
\text { liver } \\
\text { weight, } \\
\text { day } 4 \text { of } \\
\text { testing }\end{array}$} & \multirow{2}{*}{$\begin{array}{c}\text { Brain } \\
\text { weight, } \\
\text { day } 4 \text { of } \\
\text { testing }\end{array}$} & \multirow{2}{*}{$\begin{array}{l}\mathrm{AChE} \\
\text { activity }\end{array}$} & \multirow{2}{*}{$\begin{array}{c}\Delta \mathrm{T} \\
\left({ }^{\circ} \mathrm{C}\right)\end{array}$} \\
\hline & & 0 & 1 & 2 & 3 & 4 & & & & \\
\hline
\end{tabular}

Test period 2: June 15 to 18--Continued

\begin{tabular}{|c|c|c|c|c|c|c|c|c|c|c|}
\hline Control & 70 & 45.5 & 51.2 & 55.0 & 68.0 & 74.0 & 4.748 & 1.962 & 14.95 & -- \\
\hline Control & 71 & 44.9 & 47.6 & 43.8 & 49.9 & 55.5 & -- & 1.571 & 10.07 & 6.0 \\
\hline Control & 72 & 44.0 & 54.2 & 57.0 & 73.3 & 80.8 & 6.159 & 1.922 & 12.67 & -- \\
\hline Control & 73 & 43.4 & 52.0 & 58.6 & 74.4 & 80.4 & -- & 1.792 & 14.95 & 4.2 \\
\hline Control & 74 & 46.8 & 51.4 & 56.8 & 70.0 & 76.1 & -- & 1.790 & 13.65 & -- \\
\hline Control & 75 & 43.1 & 48.9 & 45.8 & 62.0 & 63.6 & 3.683 & 1.622 & 12.02 & -- \\
\hline Control & 76 & 46.0 & 51.6 & 57.4 & 71.5 & 79.4 & 4.598 & 1.929 & 15.92 & -- \\
\hline Control & 77 & 45.0 & 53.5 & 58.5 & 74.2 & 77.7 & -- & 1.692 & 12.67 & 8.0 \\
\hline Control & 78 & 51.9 & 57.8 & 61.2 & 76.3 & 82.1 & 4.359 & 1.810 & 13.32 & -- \\
\hline Control & 79 & 49.8 & 54.5 & 60.0 & 77.1 & 81.5 & -- & 1.663 & 11.70 & 3.6 \\
\hline Control & 80 & 50.1 & 56.6 & 61.5 & 75.5 & 81.7 & -- & 1.814 & 13.65 & -- \\
\hline 3 & 1 & 47.2 & 50.0 & 61.5 & 69.7 & 81.1 & -- & 1.917 & 14.95 & .6 \\
\hline 3 & 2 & 43.9 & 48.6 & 58.8 & 67.5 & 83.1 & -- & 1.675 & 14.30 & .5 \\
\hline 3 & 3 & 46.3 & 51.1 & 61.0 & 74.1 & 88.4 & -. & 1.846 & 12.35 & 3.8 \\
\hline 3 & 4 & 43.0 & 48.9 & 59.2 & 66.6 & 75.5 & -- & 1.689 & 13.32 & 4.6 \\
\hline 3 & 5 & 45.9 & 51.4 & 63.7 & 77.7 & 96.4 & 7.182 & 1.787 & 13.00 & -- \\
\hline 3 & 6 & 45.6 & 52.1 & 63.0 & 73.7 & 85.6 & -- & 1.930 & 11.37 & 5.4 \\
\hline 3 & 7 & 45.5 & 50.9 & 57.5 & 66.8 & 77.6 & 6.253 & 1.856 & 15.27 & -- \\
\hline 3 & 8 & 50.5 & 55.8 & 67.0 & 80.8 & 94.0 & -- & 1.941 & 12.02 & 2.8 \\
\hline 3 & 9 & 50.0 & 52.0 & 63.4 & 73.7 & 85.9 & 5.477 & -- & -- & -- \\
\hline 3 & 10 & 55.1 & 58.1 & 69.1 & 83.0 & 91.8 & 6.259 & 1.963 & 13.97 & -- \\
\hline 3 & 11 & 43.6 & 48.1 & 57.2 & 70.0 & 82.9 & 5.183 & 1.940 & 10.40 & -- \\
\hline 3 & 12 & 46.9 & 53.1 & 59.9 & 70.9 & 82.3 & -. & 1.971 & 13.00 & 7.4 \\
\hline 3 & 13 & 46.6 & 51.7 & 60.6 & 71.3 & 84.6 & -- & 2.113 & 12.35 & 2.7 \\
\hline 3 & 14 & 44.8 & 49.4 & 58.6 & 69.6 & 83.0 & -- & 1.581 & 14.95 & .3 \\
\hline 3 & 15 & 49.9 & 54.0 & 58.4 & 71.1 & 83.1 & 5.078 & 2.149 & 15.27 & -- \\
\hline 3 & 16 & 56.7 & 59.1 & 71.2 & 80.6 & 98.6 & 7.400 & 1.932 & 10.40 & -- \\
\hline 3 & 17 & 51.8 & 58.0 & 69.9 & 78.7 & 97.8 & -- & 2.046 & 12.35 & 1.4 \\
\hline 3 & 18 & 48.7 & 53.2 & 63.6 & 72.2 & 86.4 & .. & 1.858 & 6.82 & 5.2 \\
\hline 3 & 19 & 49.5 & 56.5 & 65.5 & 76.1 & 92.1 & -- & -- & -- & -- \\
\hline 3 & 20 & 44.9 & 47.2 & 55.1 & 65.3 & 78.2 & 5.457 & 1.913 & 13.00 & -- \\
\hline 5 & 21 & 46.0 & 54.4 & 62.9 & 74.0 & 85.0 & 5.216 & -- & -- & -- \\
\hline 5 & 22 & 48.4 & 55.8 & 66.2 & 77.3 & 91.1 & 6.730 & 2.094 & 14.30 & -. \\
\hline 5 & 23 & 46.1 & 54.0 & 61.5 & 68.7 & 72.3 & 5.172 & 1.552 & 13.65 & -- \\
\hline 5 & 24 & 51.6 & 58.5 & 66.1 & 77.7 & 89.7 & 6.266 & 1.971 & 13.32 & -- \\
\hline 5 & 25 & 44.7 & 52.6 & 64.2 & 73.0 & 83.2 & 5.390 & 1.753 & 12.35 & -. \\
\hline 5 & 26 & 48.2 & 56.0 & 67.1 & 71.2 & 78.6 & 4.990 & -- & -- & -- \\
\hline 5 & 27 & 50.5 & 61.7 & 74.0 & 83.0 & 99.7 & 5.652 & -- & .- & -- \\
\hline 5 & 28 & 50.5 & 56.0 & 66.2 & 75.9 & 88.6 & 6.142 & 1.764 & 10.07 & -- \\
\hline 5 & 29 & 54.6 & 62.3 & 75.2 & 90.2 & 100.5 & 7.008 & -- & -- & -- \\
\hline 5 & 30 & 50.2 & 56.5 & 64.1 & 75.2 & 85.2 & 5.397 & -- & -- & -- \\
\hline 5 & 31 & 49.1 & 52.2 & 64.1 & 72.5 & 86.7 & 6.018 & 1.926 & 12.35 & -- \\
\hline 5 & 32 & 42.7 & 48.3 & 59.0 & 69.0 & 76.7 & 5.057 & -- & -- & -- \\
\hline 5 & 33 & 45.2 & 49.7 & 62.3 & 71.6 & 81.3 & 4.899 & 1.844 & 12.02 & -. \\
\hline 5 & 34 & 42.2 & 49.7 & 56.0 & 60.9 & 70.0 & 5.040 & 1.602 & 12.67 & -- \\
\hline 5 & 35 & 43.6 & 48.0 & 58.5 & 67.0 & 77.2 & 4.231 & 1.520 & 9.75 & -- \\
\hline 5 & 36 & 41.6 & 47.7 & 55.9 & 62.1 & 72.7 & 4.932 & -- & -- & -- \\
\hline 5 & 37 & 51.4 & 57.4 & 71.1 & 79.2 & 90.4 & 3.982 & -- & -- & -- \\
\hline
\end{tabular}


Table 33. Mallard duckling bioassay, 1991-Continued

\begin{tabular}{|c|c|c|c|c|c|c|c|c|c|c|}
\hline \multirow{2}{*}{ Site } & \multirow{2}{*}{$\begin{array}{c}\text { Bird } \\
\text { identi- } \\
\text { fication } \\
\text { No. }\end{array}$} & \multicolumn{5}{|c|}{ Weight for indicated day of testing } & \multirow{2}{*}{$\begin{array}{l}\text { Terminal } \\
\text { liver } \\
\text { weight, } \\
\text { day } 4 \text { of } \\
\text { testing }\end{array}$} & \multirow{2}{*}{$\begin{array}{l}\text { Brain } \\
\text { weight, } \\
\text { day } 4 \text { of } \\
\text { testing }\end{array}$} & \multirow{2}{*}{$\begin{array}{l}\mathrm{AChE} \\
\text { activity }\end{array}$} & \multirow{2}{*}{$\begin{array}{r}\Delta \mathrm{T} \\
\left({ }^{\circ} \mathrm{C}\right.\end{array}$} \\
\hline & & 0 & 1 & 2 & 3 & 4 & & & & \\
\hline
\end{tabular}

Test period 2: June 15 to 18--Continued

\begin{tabular}{|c|c|c|c|c|c|c|c|c|c|c|}
\hline 5 & 38 & 49.3 & 53.9 & 64.1 & 72.6 & 87.2 & 5.890 & -- & $\ldots$ & -- \\
\hline 5 & 39 & 51.8 & 56.8 & 69.6 & 83.5 & 93.6 & 5.815 & 1.967 & 12.35 & -- \\
\hline 5 & 40 & 53.4 & 59.9 & 79.0 & 91.3 & 103.5 & 6.425 & -- & - & -- \\
\hline 7 & 41 & 48.2 & 55.0 & 66.1 & 80.7 & 86.3 & 5.643 & 1.955 & 12.67 & -- \\
\hline 7 & 42 & 47.7 & 51.4 & 60.3 & 71.5 & 81.6 & 4.535 & -- & - & -- \\
\hline 7 & 43 & 45.0 & 51.0 & 59.9 & 71.2 & 83.4 & 5.375 & 2.063 & 12.02 & -- \\
\hline 7 & 44 & 48.1 & 52.7 & 64.1 & 72.5 & 84.8 & 5.165 & .- & - & -- \\
\hline 7 & 45 & 42.6 & 46.8 & 53.3 & 63.6 & 69.0 & 4.229 & -- & .- & -- \\
\hline 7 & 46 & 49.7 & 56.9 & 66.7 & 77.8 & 90.4 & 4.922 & -- & -- & -- \\
\hline 7 & 47 & 49.2 & 52.2 & 61.9 & 76.1 & 85.2 & 4.147 & 2.059 & 9.75 & -. \\
\hline 7 & 48 & 50.4 & 57.4 & 69.4 & 76.1 & 88.0 & 5.820 & -- & .. & -- \\
\hline 7 & 49 & 49.9 & 52.6 & 64.9 & 73.8 & 82.9 & 4.791 & 1.757 & 11.70 & -. \\
\hline 7 & 50 & 49.5 & 55.7 & 65.9 & 78.9 & 91.5 & 6.320 & 1.778 & 13.32 & -- \\
\hline 7 & 51 & 46.4 & 50.9 & 61.1 & 67.8 & 78.4 & 6.170 & -- & - & -- \\
\hline 7 & 52 & 47.6 & 51.1 & 60.4 & 74.0 & 82.3 & 5.090 & 1.565 & 13.00 & -- \\
\hline 7 & 53 & 45.0 & 53.5 & 62.8 & 72.4 & 82.7 & - & - & -- & -- \\
\hline 7 & 54 & 44.6 & 50.7 & 59.1 & 69.6 & 80.5 & 4.648 & 1.616 & 13.32 & -. \\
\hline 7 & 55 & 46.8 & 52.8 & 64.3 & 75.6 & 85.3 & 5.653 & 1.936 & 10.72 & -- \\
\hline 7 & 56 & 47.3 & 51.6 & 63.8 & 74.0 & 84.1 & 5.725 & -- & -- & -- \\
\hline 7 & 57 & 50.8 & 52.5 & 62.8 & 75.7 & 85.7 & 3.573 & 2.013 & 14.30 & -. \\
\hline 7 & 58 & 53.0 & 61.1 & 73.0 & 85.9 & 98.6 & 5.737 & .. & -- & -- \\
\hline 7 & 59 & 51.7 & 55.6 & 65.3 & 77.5 & 88.4 & 4.855 & 1.975 & 12.02 &.- \\
\hline 7 & 60 & 51.0 & 57.2 & 69.8 & 76.2 & 87.6 & 6.020 & -- & -- & -- \\
\hline
\end{tabular}

Test period 3: July 6 to 9

\begin{tabular}{|c|c|c|c|c|c|c|c|c|c|c|}
\hline Control & 61 & 56.6 & 71.9 & 81.3 & 99.5 & 108.2 & -- & 1.867 & 13.97 & 3.1 \\
\hline Control & 62 & 43.6 & 56.9 & 66.4 & 77.7 & 82.0 & 5.530 & 1.680 & 11.37 & -- \\
\hline Control & 63 & 40.0 & 52.3 & 58.0 & 74.0 & 75.1 & 4.440 & 1.856 & 10.07 & -. \\
\hline Control & 64 & 46.6 & 64.3 & 74.1 & 84.1 & 89.8 & 4.880 & 1.800 & 13.97 & -- \\
\hline Control & 65 & 50.2 & 63.7 & 73.2 & 86.1 & 90.8 & 5.490 & 2.088 & 14.95 & -- \\
\hline Control & 66 & 44.6 & 54.2 & 62.9 & 72.7 & 76.8 & 4.430 & 2.033 & 12.67 & -- \\
\hline Control & 67 & 46.3 & 55.8 & 61.9 & 70.3 & 84.8 & -- & 1.939 & 12.35 & 4.2 \\
\hline Control & 68 & 47.8 & 61.2 & 72.1 & 86.8 & 98.3 & -- & 1.960 & 15.27 & 3.9 \\
\hline Control & 69 & 51.8 & 65.5 & 73.0 & 86.5 & 92.1 & 6.890 & -- & -. & -- \\
\hline Control & 70 & 42.5 & 53.2 & 59.3 & 70.8 & 74.9 & 3.420 & -- & -- & -- \\
\hline Control & 71 & 44.9 & 57.3 & 68.9 & 87.8 & 86.7 & 6.049 & 1.918 & 12.67 & -- \\
\hline Control & 72 & 43.8 & 44.8 & 51.9 & 62.9 & 68.8 & $\ldots$ & 1.709 & 13.65 & 4.4 \\
\hline Control & 73 & 47.7 & 57.0 & 68.3 & 82.1 & 84.5 & 5.629 & -- & -- & -- \\
\hline Control & 74 & 41.4 & 49.0 & 57.6 & 70.5 & 77.4 & -- & -- & -. & 4.5 \\
\hline Control & 75 & 52.3 & 64.0 & 78.7 & 96.7 & 105.7 & -- & 2.130 & 12.02 & 5.2 \\
\hline Control & 76 & 46.3 & 58.1 & 69.3 & 87.0 & 85.7 & 5.912 & 1.928 & 13.32 & -- \\
\hline Control & 77 & 43.0 & 54.7 & 66.7 & 84.1 & 85.1 & 5.383 & -- & -- & -- \\
\hline Control & 78 & 45.2 & 52.4 & 65.1 & 77.1 & 80.0 & 4.445 & 1.658 & 14.30 & -- \\
\hline Control & 79 & 48.0 & 58.3 & 73.9 & 90.1 & 90.5 & 4.611 & 1.930 & 14.30 & -- \\
\hline Control & 80 & 50.5 & 61.3 & 74.6 & 92.0 & 95.3 & 4.932 & 1.868 & 16.90 & -- \\
\hline 3 & 1 & 42.2 & 46.6 & 55.4 & 75.6 & 84.4 & 4.786 & 1.949 & 13.65 & -- \\
\hline 3 & 2 & 55.6 & 59.9 & 74.5 & 93.2 & 102.5 & 6.872 & -- & -- & -- \\
\hline
\end{tabular}


Table 33. Mallard duckling bioassay, 1991--Continued

\begin{tabular}{|c|c|c|c|c|c|c|c|c|c|c|}
\hline \multirow{2}{*}{ Site } & \multirow{2}{*}{$\begin{array}{c}\text { Bird } \\
\text { identi- } \\
\text { fication } \\
\text { No. }\end{array}$} & \multicolumn{5}{|c|}{ Weight for indicated day of testing } & \multirow{2}{*}{$\begin{array}{l}\text { Terminal } \\
\text { liver } \\
\text { weight, } \\
\text { day } 4 \text { of } \\
\text { testing }\end{array}$} & \multirow{2}{*}{$\begin{array}{c}\text { Brain } \\
\text { weight, } \\
\text { day } 4 \text { of } \\
\text { testing }\end{array}$} & \multirow{2}{*}{$\begin{array}{l}\text { AChE } \\
\text { activity }\end{array}$} & \multirow{2}{*}{$\begin{array}{c}\Delta \mathrm{T} \\
\left({ }^{\circ} \mathrm{C}\right)\end{array}$} \\
\hline & & 0 & 1 & 2 & 3 & 4 & & & & \\
\hline
\end{tabular}

Test period 3: July 6 to 9--Continued

\begin{tabular}{|c|c|c|c|c|c|c|c|c|c|c|}
\hline 3 & 3 & 51.0 & 56.4 & 66.8 & 84.2 & 92.2 & 5.062 & 2.029 & 11.05 & -- \\
\hline 3 & 4 & 47.3 & 54.2 & 68.1 & 80.7 & 88.9 & -- & 1.794 & 11.70 & 4.4 \\
\hline 3 & 5 & 52.8 & 56.3 & 66.2 & 80.8 & 86.8 & 6.008 & -- & -- & -- \\
\hline 3 & 6 & 45.9 & 49.1 & 58.0 & 76.2 & 81.4 & 4.937 & 1.908 & 13.97 & -- \\
\hline 3 & 7 & 50.8 & 55.7 & 65.3 & 82.2 & 91.9 & -- & 1.961 & 12.35 & 3.7 \\
\hline 3 & 8 & 49.4 & 54.5 & 65.2 & 82.6 & 92.5 & -- & 1.962 & 13.00 & 1.3 \\
\hline 3 & 9 & 43.1 & 48.4 & 61.7 & 77.0 & 90.8 & 5.080 & 1.867 & 13.00 & -- \\
\hline 3 & 10 & 45.9 & 51.5 & 58.3 & 73.2 & 81.9 & 4.338 & 1.717 & 13.32 & -- \\
\hline 3 & 11 & 45.5 & 50.7 & 60.6 & 77.4 & 86.1 & -- & 1.865 & 12.35 & .5 \\
\hline 3 & 12 & 46.8 & 50.8 & 60.8 & 83.4 & 89.3 & 6.423 & 1.988 & 14.62 & -- \\
\hline 3 & 13 & 49.1 & 54.9 & 62.9 & 83.0 & 90.0 & 7.189 & 1.974 & 14.62 & -- \\
\hline 3 & 14 & 47.9 & 52.3 & 61.3 & 78.9 & 85.5 & 4.453 & -- & -- & -- \\
\hline 3 & 15 & 44.1 & 47.9 & 54.3 & 68.7 & 72.7 & 4.084 & -- & -- & -- \\
\hline 3 & 16 & 53.5 & 56.1 & 65.7 & 83.4 & 91.5 & -- & 1.894 & 12.35 & 3.5 \\
\hline 3 & 17 & 40.9 & 43.9 & 51.4 & 65.3 & 65.0 & 3.622 & 1.805 & 14.30 & -. \\
\hline 3 & 18 & 49.8 & 57.6 & 69.7 & 89.0 & 99.5 & -- & 2.222 & 14.62 & 3.5 \\
\hline 3 & 19 & 49.5 & 56.2 & 62.1 & 80.9 & 86.7 & 5.742 & 1.995 & 16.90 & -. \\
\hline 3 & 20 & 41.5 & 48.8 & 57.1 & 74.6 & 78.1 & 5.552 & 1.827 & 9.75 & -- \\
\hline 5 & 21 & 53.5 & 59.0 & 71.3 & 89.3 & 95.1 & -- & 1.972 & 16.90 & 3.8 \\
\hline 5 & 22 & 46.0 & 51.4 & 61.3 & 70.3 & 71.9 & 4.077 & 1.879 & 13.00 & -- \\
\hline 5 & 23 & 53.1 & 64.6 & 78.3 & 105.8 & 109.6 & 7.915 & 1.892 & 13.00 & -- \\
\hline 5 & 24 & 47.9 & 54.4 & 65.5 & 83.9 & 84.8 & 5.012 & 2.028 & 11.70 & -- \\
\hline 5 & 25 & 50.7 & 55.3 & 68.0 & 84.9 & 90.0 & -- & 1.468 & 11.05 & 2.9 \\
\hline 5 & 26 & 57.0 & 60.9 & 73.0 & 92.4 & 96.4 & 5.497 & -- & -- & -. \\
\hline 5 & 27 & 43.5 & 49.5 & 56.8 & 69.7 & 77.7 & -- & -- & -. & 4.5 \\
\hline 5 & 28 & 43.8 & 48.9 & 59.7 & 73.4 & 75.4 & 4.649 & 1.823 & 12.35 & -. \\
\hline 5 & 29 & 44.4 & 50.9 & 63.9 & 84.8 & 86.7 & 4.941 & -- & -- & -- \\
\hline 5 & 30 & 50.6 & 58.1 & 70.0 & 89.7 & 88.5 & 4.828 & 1.852 & 12.67 & -. \\
\hline 5 & 31 & 55.5 & 63.0 & 77.3 & 102.2 & 109.4 & -- & 2.138 & 11.70 & -- \\
\hline 5 & 32 & 48.9 & 56.6 & 65.6 & 87.8 & 96.6 & -- & 1.775 & 11.70 & 1.9 \\
\hline 5 & 33 & 51.0 & 56.0 & 69.3 & 88.2 & 94.2 & 5.784 & 1.940 & 13.32 & -- \\
\hline 5 & 34 & 41.4 & 46.5 & 56.1 & 75.3 & 78.8 & 5.090 & 1.721 & 13.00 & -- \\
\hline 5 & 35 & 42.3 & 47.8 & 56.2 & 74.5 & 83.4 & -- & 1.727 & 13.97 & 3.9 \\
\hline 5 & 36 & 50.1 & 53.3 & 59.7 & 73.8 & 77.5 & 4.144 & -- & -- & -- \\
\hline 5 & 37 & 44.4 & 49.0 & 62.1 & 80.9 & 84.8 & 5.302 & 1.974 & 13.00 & -- \\
\hline 5 & 38 & 49.1 & 56.8 & 67.5 & 89.1 & 92.1 & 5.249 & 2.012 & 12.35 & -- \\
\hline 5 & 39 & 45.1 & 52.0 & 62.9 & 83.5 & 85.6 & 6.044 & 1.921 & 13.00 & -- \\
\hline 5 & 40 & 44.0 & 49.7 & 60.8 & 74.2 & 82.1 & 4.935 & -- & -- & -- \\
\hline 7 & 41 & 56.2 & 63.9 & 78.6 & 93.1 & 101.0 & 6.828 & 1.965 & 12.67 & -- \\
\hline 7 & 42 & 52.0 & 60.5 & 67.9 & 82.5 & 88.5 & 5.733 & -. & -. & -- \\
\hline 7 & 43 & 49.1 & 55.3 & 67.6 & 79.2 & 87.8 & 4.959 & 1.882 & 12.02 & -- \\
\hline 7 & 44 & 44.3 & 52.2 & 65.6 & 77.6 & 90.0 & .. & 1.878 & 13.65 & 4.3 \\
\hline 7 & 45 & 49.8 & 56.3 & 68.9 & 82.3 & 89.3 & 5.912 & 1.717 & 13.97 & -- \\
\hline 7 & 46 & 44.9 & 51.6 & 63.2 & 77.1 & 86.1 & 4.891 & 1.872 & 12.02 & -- \\
\hline 7 & 47 & 43.3 & 45.5 & 55.1 & 76.8 & 90.2 & -- & -- & -- & 4.3 \\
\hline 7 & 48 & 47.7 & 54.2 & 67.6 & 82.1 & 86.4 & 4.678 & -- & -- & -. \\
\hline 7 & 49 & 49.1 & 54.3 & 68.7 & 79.7 & 92.9 & -- & 1.889 & 12.02 & 2.6 \\
\hline 7 & 50 & 47.6 & 54.9 & 67.7 & 87.2 & 95.9 & 5.504 & 1.878 & 14.62 & -.. \\
\hline 7 & 51 & 44.7 & 54.4 & 64.3 & 76.2 & 81.8 & -- & 1.825 & 14.95 & 5.9 \\
\hline
\end{tabular}


Table 33. Mallard duckling bioassay, 1991--Continued

\begin{tabular}{cccccccccc}
\hline Site & $\begin{array}{c}\text { Bird } \\
\text { identi- } \\
\text { fication } \\
\text { No. }\end{array}$ & 0 & & Weight for indicated day of testing & & $\begin{array}{c}\text { Terminal } \\
\text { liver } \\
\text { weight, } \\
\text { day } 4 \text { of } \\
\text { testing }\end{array}$ & $\begin{array}{c}\text { Brain } \\
\text { weight, } \\
\text { day } 4 \text { of } \\
\text { testing }\end{array}$ & $\begin{array}{c}\text { AChE } \\
\text { activity }\end{array}$ & $\begin{array}{c}\Delta \mathrm{T} \\
\left({ }^{\circ} \mathrm{C}\right)\end{array}$ \\
\hline
\end{tabular}

Test period 3: July 6 to 9--Continued

\begin{tabular}{|c|c|c|c|c|c|c|c|c|c|c|}
\hline 7 & 52 & 47.7 & 54.5 & 67.9 & 84.1 & 91.6 & -- & 2.039 & 13.32 & $\ldots$ \\
\hline 7 & 53 & 44.2 & 52.7 & 61.1 & 76.1 & 78.1 & 4.997 & 1.837 & 13.00 & $\ldots$ \\
\hline 7 & 54 & 48.1 & 54.4 & 63.0 & 78.3 & 82.1 & 4.281 & 1.814 & 10.40 & $\ldots$ \\
\hline 7 & 55 & 50.4 & 58.2 & 69.8 & 84.8 & 90.2 & 4.722 & 1.936 & 10.72 & $\ldots$ \\
\hline 7 & 56 & 50.8 & 57.9 & 68.7 & 82.9 & 91.6 & 5.518 & 2.041 & 13.97 & -. \\
\hline 7 & 57 & 48.0 & 53.8 & 65.5 & 78.1 & 87.6 & -- & 1.837 & 12.35 & 3.7 \\
\hline 7 & 58 & 42.6 & 48.2 & 60.2 & 73.2 & 77.8 & 4.420 & -- & -- & - \\
\hline 7 & 59 & 54.6 & 59.8 & 74.9 & 87.9 & 94.0 & 5.375 & 1.858 & 11.05 & $\ldots$ \\
\hline 7 & 60 & 48.3 & 51.2 & 58.7 & 70.5 & 76.5 & 4.051 & -- & -- & - \\
\hline 1 & 81 & 42.5 & 50.1 & 58.0 & 70.8 & 74.2 & 3.819 & -- & -- & .. \\
\hline 1 & 82 & 41.8 & 48.0 & 58.3 & 73.0 & 75.5 & 5.012 & 2.018 & 12.35 &.- \\
\hline 1 & 83 & 48.7 & 60.3 & 68.6 & 83.7 & 86.1 & 4.169 & 1.750 & 9.42 & -- \\
\hline 1 & 84 & 54.2 & 68.6 & 77.2 & 97.0 & 97.1 & 5.578 & -- & -- & - \\
\hline 1 & 85 & 51.2 & 59.2 & 66.9 & 80.3 & 82.6 & 4.549 & -- & -- & - \\
\hline 1 & 86 & 44.1 & 56.7 & 64.6 & 80.1 & 83.8 & 5.189 & -- & -. & -- \\
\hline 1 & 87 & 43.7 & 51.7 & 56.6 & 70.3 & 72.1 & 3.919 & 1.741 & 14.62 & - \\
\hline 1 & 88 & 54.0 & 63.1 & 73.5 & 90.6 & 98.6 & 5.653 & 1.874 & 11.05 & -- \\
\hline 1 & 89 & 46.3 & 59.3 & 65.9 & 76.8 & 84.1 & 4.110 & -- & -- & -- \\
\hline 1 & 90 & 51.1 & 58.7 & 67.2 & 78.7 & 82.8 & 4.726 & 1.910 & 16.57 & -. \\
\hline 1 & 91 & 48.0 & 57.1 & 64.9 & 80.5 & 85.0 & 4.050 & -- & -- & -- \\
\hline 1 & 92 & 53.8 & 70.2 & 73.0 & 94.3 & 93.3 & 5.200 & 1.855 & 12.67 & -. \\
\hline 1 & 93 & 46.9 & 56.3 & 61.7 & 76.2 & 79.1 & 3.800 & -- & -- & -- \\
\hline 1 & 94 & 51.1 & 59.0 & 69.2 & 86.6 & 90.1 & 4.724 & 1.837 & 12.02 & -- \\
\hline 1 & 95 & 38.9 & 46.1 & 51.5 & 63.1 & 65.2 & 3.407 & 1.770 & 14.95 & -. \\
\hline 1 & 96 & 46.2 & 52.6 & 58.2 & 71.7 & 75.1 & 3.631 & -- & -- & -- \\
\hline 1 & 97 & 38.9 & 45.3 & 50.1 & 62.7 & 65.1 & 3.914 & 1.818 & 13.32 & -- \\
\hline 1 & 98 & 44.5 & 51.8 & 58.9 & 73.6 & 76.7 & 4.115 & -- & -- & -- \\
\hline 1 & 99 & 48.3 & 60.6 & 68.9 & 89.3 & 89.0 & 4.490 & 1.927 & 11.05 & -- \\
\hline 1 & 100 & 50.8 & 59.6 & 67.4 & 83.2 & 88.7 & 4.279 & -- & -- & -- \\
\hline
\end{tabular}

Test period 4: July 25 to 29

\begin{tabular}{llllllllllr}
\hline Control & 61 & 32.7 & 43.1 & 52.2 & 60.0 & 66.3 & 3.499 & 1.660 & 15.27 & -- \\
Control & 62 & 31.4 & 42.5 & 51.1 & 57.5 & 68.4 & 4.453 & -- & - & - \\
Control & 63 & 35.8 & 46.8 & 58.6 & 74.6 & 85.1 & 5.784 & 1.839 & 9.75 & -- \\
Control & 64 & 33.5 & 44.3 & 50.8 & 66.7 & 74.5 & - & 1.728 & 13.00 & 5.0 \\
Control & 65 & 33.9 & 45.3 & 56.4 & 65.6 & 72.9 & 4.259 & 1.744 & 14.30 & -- \\
Control & 66 & 33.7 & 46.9 & 51.3 & 62.4 & 67.6 & 4.448 & 1.820 & 13.32 & -- \\
Control & 67 & 38.8 & 48.8 & 55.4 & 65.1 & 75.2 & 4.600 & -- & -- & -- \\
Control & 68 & 39.4 & 47.3 & 58.8 & 69.2 & 75.7 & - & 1.642 & 12.35 & 5.2 \\
Control & 69 & 35.5 & 44.3 & 53.3 & 61.6 & 68.1 & 4.272 & 1.800 & 12.35 & -- \\
Control & 70 & 36.2 & 47.2 & 56.6 & 68.9 & 81.6 & -- & 1.646 & 14.95 & 2.0 \\
Control & 71 & 33.8 & 47.0 & 58.7 & 73.5 & 80.8 & 4.235 & - & - & - \\
Control & 72 & 32.0 & 42.1 & 51.1 & 60.5 & 69.5 & 3.547 & 1.721 & 13.97 & -- \\
Control & 73 & 33.6 & 44.3 & 52.9 & 62.4 & 69.2 & 3.277 & 1.896 & 11.37 & - \\
Control & 74 & 33.7 & 44.6 & 56.6 & 66.1 & 74.5 & -- & 1.841 & 12.02 & 5.8 \\
Control & 75 & 31.6 & 42.8 & 54.2 & 63.3 & 67.6 & 3.247 & -- & - & - \\
Control & 76 & 32.8 & 41.4 & 51.0 & 61.1 & 68.7 & 3.737 & 1.784 & 13.97 & --
\end{tabular}


Table 33. Mallard duckling bioassay, 1991--Continued

\begin{tabular}{|c|c|c|c|c|c|c|c|c|c|c|}
\hline \multirow{2}{*}{ Site } & \multirow{2}{*}{$\begin{array}{c}\text { Bird } \\
\text { identi- } \\
\text { fication } \\
\text { No. }\end{array}$} & \multicolumn{5}{|c|}{ Weight for indicated day of testing } & \multirow{2}{*}{$\begin{array}{l}\text { Terminal } \\
\text { liver } \\
\text { weight, } \\
\text { day } 4 \text { of } \\
\text { testing }\end{array}$} & \multirow{2}{*}{$\begin{array}{c}\text { Brain } \\
\text { weight, } \\
\text { day } 4 \text { of } \\
\text { testing }\end{array}$} & \multirow{2}{*}{$\begin{array}{l}\text { AChE } \\
\text { activity }\end{array}$} & \multirow{2}{*}{$\begin{array}{r}\Delta \mathrm{T} \\
\left({ }^{\circ} \mathrm{C}\right)\end{array}$} \\
\hline & & 0 & 1 & 2 & 3 & 4 & & & & \\
\hline
\end{tabular}

Test period 4: July 25 to 29--Continued

\begin{tabular}{|c|c|c|c|c|c|c|c|c|c|c|}
\hline Control & 77 & 39.0 & 51.6 & 65.6 & 74.8 & 82.8 & -- & 1.772 & 12.35 & 3.1 \\
\hline Control & 78 & 36.5 & 46.1 & 56.1 & 66.2 & 77.0 & 4.194 & 1.915 & 13.65 & -- \\
\hline Control & 79 & 36.3 & 47.6 & 58.1 & 70.7 & 76.8 & -- & 1.709 & 11.05 & 3.1 \\
\hline Control & 80 & 39.6 & 54.5 & 66.9 & 74.9 & 87.2 & 4.143 & 1.886 & 13.32 & -- \\
\hline 3 & 1 & 35.2 & 43.6 & 52.3 & 62.5 & 68.9 & 3.271 & -- & -- & 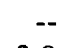 \\
\hline 3 & 2 & 33.3 & 41.4 & 47.3 & 57.4 & 63.8 & -- & 1.854 & 13.97 & 3.8 \\
\hline 3 & 3 & 34.1 & 41.5 & 47.1 & 56.4 & 67.0 & -- & 1.537 & 13.32 & 4.9 \\
\hline 3 & 4 & 33.9 & 41.0 & 48.3 & 57.3 & 63.8 & 3.224 & 1.649 & 13.97 & -- \\
\hline 3 & 5 & 31.3 & 37.4 & 44.6 & 55.8 & 62.3 & 3.104 & 1.799 & 10.40 & -- \\
\hline 3 & 6 & 35.4 & 38.1 & 46.1 & 56.7 & 61.6 & 3.321 & 1.586 & 14.30 & -- \\
\hline 3 & 7 & 35.8 & 43.7 & 48.7 & 55.3 & 63.3 & 3.703 & -- & -- & -- \\
\hline 3 & 8 & 36.6 & 43.5 & 48.6 & 55.0 & 64.1 & 3.727 & 1.786 & 14.62 & -- \\
\hline 3 & 9 & 36.9 & 43.8 & 47.6 & 58.7 & 62.2 & -- & .725 & 14.30 & 3.2 \\
\hline 3 & 10 & 32.2 & 39.4 & 45.0 & 56.1 & 62.6 & 3.347 & 1.612 & 14.62 & -- \\
\hline 3 & 11 & 34.4 & 41.8 & 45.3 & 53.6 & 61.7 & 3.721 & 1.862 & 14.95 & -- \\
\hline 3 & 12 & 31.5 & 37.5 & 40.7 & 47.9 & 55.3 & -- & 1.555 & 14.95 & 4.2 \\
\hline 3 & 13 & 33.0 & 42.8 & 50.0 & 63.4 & 71.5 & 3.916 & 1.672 & 15.60 & -- \\
\hline 3 & 14 & 33.6 & 45.0 & 54.7 & 65.8 & 74.0 & 3.785 & 1.918 & 14.62 & -- \\
\hline 3 & 15 & 34.7 & 40.7 & 45.9 & 56.5 & 64.5 & -- & 1.709 & 13.32 & 1.9 \\
\hline 3 & 16 & 34.1 & 41.3 & 47.2 & 55.3 & 61.2 & 3.073 & -- & -- & -- \\
\hline 3 & 17 & 40.1 & 49.4 & 54.9 & 63.1 & 70.4 & 4.243 & 1.787 & 14.62 & -- \\
\hline 3 & 18 & 35.7 & 40.8 & 45.4 & 50.0 & 57.4 & -- & 1.561 & -- & 5.0 \\
\hline 3 & 19 & 36.8 & 54.4 & 61.9 & 74.6 & 84.1 & 4.060 & 1.778 & 13.32 & -- \\
\hline 3 & 20 & 39.6 & 49.4 & 57.7 & 69.9 & 77.3 & 5.044 & -- & -- & -- \\
\hline 5 & 21 & 30.5 & 39.8 & 47.6 & 56.1 & 63.2 & -- & 1.787 & 13.32 & 3.6 \\
\hline 5 & 22 & 32.6 & 41.2 & 48.7 & 53.4 & 61.3 & 3.038 & 1.778 & 13.97 & -- \\
\hline 5 & 23 & 32.7 & 40.7 & 50.9 & 56.6 & 66.1 & 3.734 & 1.665 & 12.35 & -- \\
\hline 5 & 24 & 33.8 & 43.0 & 52.7 & 60.9 & 71.7 & -- & 1.792 & 13.32 & 5.3 \\
\hline 5 & 25 & 34.5 & 43.0 & 54.5 & 68.0 & 74.6 & 3.921 & 1.650 & 11.70 & -- \\
\hline 5 & 26 & 33.1 & 40.7 & 48.5 & 56.3 & 62.5 & 4.133 & 1.574 & 14.95 & -- \\
\hline 5 & 27 & 34.2 & 44.6 & 54.0 & 63.0 & 68.8 & 3.814 & 1.705 & 12.35 & -- \\
\hline 5 & 28 & 35.4 & 43.2 & 56.3 & 61.6 & 71.0 & - & 1.646 & 9.75 & 4.5 \\
\hline 5 & 29 & 39.5 & 47.6 & 60.0 & 71.3 & 80.0 & 4.107 & -- & -- & -- \\
\hline 5 & 30 & 36.2 & 45.6 & 48.7 & 59.7 & 63.1 & 3.431 & -- & -- & -- \\
\hline 5 & 31 & 34.3 & 46.6 & 54.2 & 60.3 & 62.5 & 3.488 & - & -- & -- \\
\hline 5 & 32 & 36.2 & 45.1 & 51.1 & 55.6 & 57.9 & -- & 1.950 & 13.32 & -- \\
\hline 5 & 33 & 33.1 & 42.3 & 51.4 & 62.0 & 62.3 & -- & 1.718 & 15.27 & 4.4 \\
\hline 5 & 34 & 34.0 & 41.2 & 49.1 & 54.1 & 56.2 & 2.158 & 1.684 & 14.62 & -- \\
\hline 5 & 35 & 32.6 & 39.6 & 46.3 & 53.8 & 55.6 & -- & 1.684 & 14.30 & 4.1 \\
\hline 5 & 36 & 38.5 & 48.1 & 58.6 & 65.1 & 68.0 & 3.360 & 1.760 & 14.62 & -- \\
\hline 5 & 37 & 35.4 & 43.3 & 53.3 & 62.5 & 62.8 & 3.268 & -- & -- & -- \\
\hline 5 & 38 & 40.6 & 50.6 & 58.3 & 64.6 & 68.9 & 3.204 & 1.784 & 14.30 & -- \\
\hline 5 & 39 & 36.4 & 46.4 & 52.5 & 60.7 & 63.3 & 2.643 & 1.827 & 15.27 & -- \\
\hline 5 & 40 & 40.0 & 47.6 & 57.2 & 65.0 & 65.2 & 3.023 & 1.782 & 13.65 & -- \\
\hline 7 & 41 & 34.0 & 42.7 & 50.6 & 64.0 & 70.1 & 4.085 & 1.865 & 14.62 & - \\
\hline 7 & 42 & 31.1 & 39.3 & 49.8 & 62.2 & 69.0 & 4.537 & 1.728 & 14.30 & -- \\
\hline 7 & 43 & 31.3 & 39.0 & 44.6 & 58.8 & 62.5 & -- & 1.594 & 14.95 & 5.2 \\
\hline 7 & 44 & 32.5 & 40.5 & 46.3 & 61.4 & 63.6 & 3.989 & 1.670 & 13.00 & -- \\
\hline 7 & 45 & 33.2 & 43.5 & 52.4 & 63.5 & 68.0 & -- & 1.605 & 14.30 & 4.3 \\
\hline
\end{tabular}


Table 33. Mallard duckling bioassay, 1991--Continued

\begin{tabular}{|c|c|c|c|c|c|c|c|c|c|c|}
\hline \multirow{2}{*}{ Site } & \multirow{2}{*}{$\begin{array}{l}\text { Bird } \\
\text { identi- } \\
\text { fication } \\
\text { No. }\end{array}$} & \multicolumn{5}{|c|}{ Weight for indicated day of testing } & \multirow{2}{*}{$\begin{array}{l}\text { Terminal } \\
\text { liver } \\
\text { weight, } \\
\text { day } 4 \text { of } \\
\text { testing }\end{array}$} & \multirow{2}{*}{$\begin{array}{l}\text { Brain } \\
\text { weight, } \\
\text { day } 4 \text { of } \\
\text { testing }\end{array}$} & \multirow{2}{*}{$\begin{array}{l}\mathrm{AChE} \\
\text { activity }\end{array}$} & \multirow{2}{*}{$\begin{array}{c}\Delta \mathrm{T} \\
\left({ }^{\circ} \mathrm{C}\right)\end{array}$} \\
\hline & & 0 & 1 & 2 & 3 & 4 & & & & \\
\hline
\end{tabular}

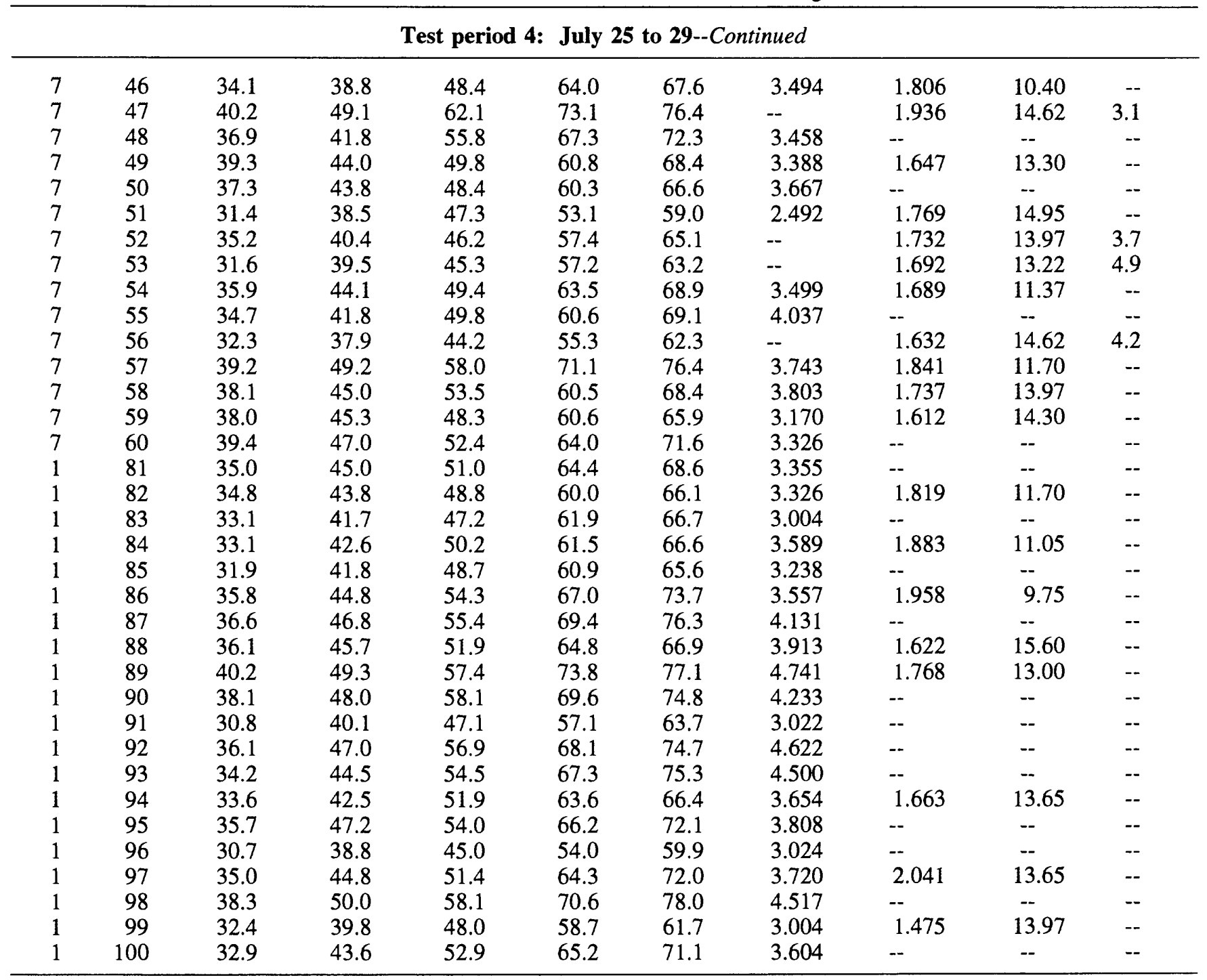

\section{Test period 5: August 22 to 26}

\begin{tabular}{llllllllllr}
\hline Control & 61 & 45.3 & 54.6 & 63.0 & 78.4 & 86.3 & - & 1.873 & 13.65 & 3.7 \\
Control & 62 & 47.9 & 58.5 & 66.6 & 72.7 & 83.1 & 5.200 & 1.729 & 15.27 & -- \\
Control & 63 & 51.0 & 62.7 & 68.8 & 89.3 & 95.0 & 6.700 & 1.813 & 13.97 & -- \\
Control & 64 & 45.1 & 56.2 & 64.4 & 80.3 & 86.2 & 5.500 & - & -- & -- \\
Control & 65 & 41.5 & 47.1 & 54.1 & 65.5 & 69.4 & 4.100 & 1.816 & 14.30 & - \\
Control & 66 & 44.6 & 56.3 & 63.9 & 81.3 & 91.9 & - & 1.784 & 13.97 & 3.6 \\
Control & 67 & 42.5 & 50.9 & 57.2 & 70.3 & 75.5 & 5.300 & 1.711 & 13.97 & - \\
Control & 68 & 45.2 & 52.3 & 60.8 & 73.0 & 81.5 & - & 1.892 & 13.32 & 1.8 \\
Control & 69 & 49.8 & 58.5 & 69.6 & 84.8 & 89.4 & 6.400 & 1.837 & 10.40 & -- \\
Control & 70 & 40.2 & 50.3 & 55.0 & 71.3 & 74.3 & 5.000 & -- & -- & --
\end{tabular}


Table 33. Mallard duckling bioassay, 1991--Continued

\begin{tabular}{|c|c|c|c|c|c|c|c|c|c|c|}
\hline \multirow{2}{*}{ Site } & \multirow{2}{*}{$\begin{array}{c}\text { Bird } \\
\text { identi- } \\
\text { fication } \\
\text { No. }\end{array}$} & \multicolumn{5}{|c|}{ Weight for indicated day of testing } & \multirow{2}{*}{$\begin{array}{l}\text { Terminal } \\
\text { liver } \\
\text { weight, } \\
\text { day } 4 \text { of } \\
\text { testing }\end{array}$} & \multirow{2}{*}{$\begin{array}{c}\text { Brain } \\
\text { weight, } \\
\text { day } 4 \text { of } \\
\text { testing }\end{array}$} & \multirow{2}{*}{$\begin{array}{l}\text { AChE } \\
\text { activity }\end{array}$} & \multirow{2}{*}{$\begin{array}{c}\Delta \mathrm{T} \\
\left({ }^{\circ} \mathrm{C}\right)\end{array}$} \\
\hline & & 0 & 1 & 2 & 3 & 4 & & & & \\
\hline
\end{tabular}

Test period 5: August 22 to 26--Continued

\begin{tabular}{|c|c|c|c|c|c|c|c|c|c|c|}
\hline Control & 71 & 40.3 & 44.8 & 55.2 & 68.3 & 77.5 & -- & 1.731 & 13.00 & 4.5 \\
\hline Control & 72 & 46.1 & 53.3 & 60.7 & 73.3 & 79.3 & 5.300 & 2.048 & 15.27 & -- \\
\hline Control & 73 & 46.5 & 56.4 & 62.2 & 78.3 & 83.0 & 5.900 & 1.852 & 14.95 & -- \\
\hline Control & 74 & 46.4 & 54.1 & 63.0 & 77.7 & 84.4 & 5.300 & -- & .- & .- \\
\hline Control & 75 & 41.8 & 50.6 & 58.3 & 75.4 & 80.5 & 5.500 & -- & -- & -- \\
\hline Control & 76 & 52.7 & 66.4 & 74.9 & 92.4 & 103.2 & 8.000 & 1.638 & 10.40 & -- \\
\hline Control & 77 & 41.7 & 47.2 & 57.0 & 68.0 & 76.9 & -- & 1.839 & 13.65 & 5.7 \\
\hline Control & 78 & 46.1 & 57.6 & 68.5 & 86.1 & 90.4 & 7.700 & 1.597 & 14.62 & -- \\
\hline Control & 79 & 49.0 & 57.7 & 70.2 & 85.8 & 96.4 & -- & 1.881 & 13.00 & 1.3 \\
\hline Control & 80 & 45.0 & 54.6 & 62.9 & 76.7 & 81.1 & 4.600 & 1.912 & 16.57 & -- \\
\hline 3 & 1 & 48.5 & 51.7 & 65.1 & 78.4 & 84.5 & 5.000 & 1.730 & 14.62 & -- \\
\hline 3 & 2 & 45.7 & 53.0 & 63.5 & 78.0 & 89.3 & -- & 1.818 & 13.97 & 3.4 \\
\hline 3 & 3 & 43.1 & 50.3 & 59.9 & 76.6 & 81.5 & 5.100 & 1.895 & 13.97 & -- \\
\hline 3 & 4 & 43.8 & 49.6 & 59.9 & 74.8 & 79.8 & 4.500 & 1.850 & 14.30 & -- \\
\hline 3 & 5 & 43.7 & 49.8 & 59.9 & 76.0 & 81.5 & 5.200 & -- & -- & - \\
\hline 3 & 6 & 45.6 & 49.6 & 58.4 & 76.0 & 79.7 & 4.900 & -- & -- & -- \\
\hline 3 & 7 & 46.3 & 52.5 & 66.0 & 83.9 & 92.2 & -- & 1.840 & 13.32 & 2.3 \\
\hline 3 & 8 & 48.7 & 56.5 & 66.9 & 81.6 & 86.7 & -- & 1.775 & 13.32 & 2.0 \\
\hline 3 & 9 & 40.9 & 44.6 & 54.6 & 55.0 & 56.9 & 4.300 & 1.807 & 15.60 & -- \\
\hline 3 & 10 & 41.1 & 46.6 & 56.7 & 70.3 & 74.4 & 4.500 & 1.913 & 12.02 & \\
\hline 3 & 11 & 40.8 & 47.3 & 55.5 & 69.9 & 75.9 & -- & 1.780 & 13.97 & 6.9 \\
\hline 3 & 12 & 48.3 & 55.5 & 65.7 & 81.4 & 85.1 & 4.600 & 1.808 & 12.35 & -- \\
\hline 3 & 13 & 45.8 & 54.5 & 63.4 & 74.8 & 75.1 & 4.800 & -- & -- & -- \\
\hline 3 & 14 & 43.0 & 49.3 & 59.3 & 74.0 & 78.0 & 4.700 & 1.850 & 13.97 & -- \\
\hline 3 & 15 & 41.6 & 48.2 & 57.2 & 72.1 & 74.4 & 4.400 & -- & -- & -- \\
\hline 3 & 16 & 49.7 & 56.8 & 66.4 & 82.7 & 89.0 & 4.800 & 1.906 & 12.67 & -- \\
\hline 3 & 17 & 49.0 & 59.5 & 69.3 & 85.8 & 88.7 & 5.300 & 1.952 & 13.32 & -- \\
\hline 3 & 18 & 46.5 & 55.5 & 65.3 & 81.1 & 83.8 & 5.200 & 1.970 & 13.65 & 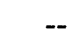 \\
\hline 3 & 19 & 47.5 & 56.3 & 69.3 & 86.8 & 93.3 & -- & 1.796 & 14.62 & 5.4 \\
\hline 3 & 20 & 44.8 & 52.1 & 60.3 & 73.2 & 81.7 & -- & 1.755 & 13.00 & 5.4 \\
\hline 5 & 21 & 46.5 & 53.6 & 63.3 & 85.6 & 86.9 & 5.800 & 1.877 & 13.65 & -- \\
\hline 5 & 22 & 41.2 & 46.6 & 54.6 & 69.8 & 73.3 & -- & 1.748 & 15.27 & 4.1 \\
\hline 5 & 23 & 48.7 & 55.5 & 62.5 & 82.0 & 86.2 & 4.900 & 1.769 & 13.65 & \\
\hline 5 & 24 & 48.3 & 53.7 & 64.0 & 79.4 & 87.4 & -- & 1.790 & 12.02 & 3.7 \\
\hline 5 & 25 & 47.0 & 54.2 & 66.6 & 83.9 & 88.2 & 5.900 & 1.668 & 12.35 & 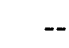 \\
\hline 5 & 26 & 44.2 & 50.1 & 58.6 & 73.2 & 77.9 & -- & 1.780 & 13.00 & 3.3 \\
\hline 5 & 27 & 43.5 & 49.3 & 58.3 & 74.4 & 74.7 & 4.000 & 1.765 & 11.70 & 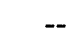 \\
\hline 5 & 28 & 44.8 & 49.9 & 58.9 & 72.9 & 77.0 & 4.800 & -- & -- & -- \\
\hline 5 & 29 & 48.3 & 53.4 & 67.2 & 86.9 & 89.4 & 5.700 & 1.809 & 13.32 & -- \\
\hline 5 & 30 & 47.1 & 55.3 & 66.4 & 84.4 & 89.8 & 5.400 & -- & -- & -- \\
\hline 5 & 31 & 46.4 & 51.3 & 60.4 & 73.6 & 80.9 & -- & 1.790 & 13.32 & 1.4 \\
\hline 5 & 32 & 46.9 & 51.8 & 60.5 & 75.7 & 79.4 & 3.800 & 1.777 & 15.60 & . \\
\hline 5 & 33 & 51.0 & 56.9 & 67.6 & 85.5 & 96.5 & -- & 1.790 & 12.35 & -- \\
\hline 5 & 34 & 40.0 & 46.3 & 55.6 & 69.4 & 72.5 & 4.000 & 1.790 & 14.73 & -- \\
\hline 5 & 35 & 48.5 & 56.2 & 65.7 & 81.6 & 90.1 & -- & 1.755 & 13.65 & 4.9 \\
\hline 5 & 36 & 40.0 & 66.7 & -- & -- & -- & -- & -- & -- & -- \\
\hline 5 & 37 & 45.3 & 52.0 & 62.4 & 77.1 & 82.1 & 5.200 & 1.820 & 13.65 & \\
\hline 5 & 38 & 45.3 & 53.5 & 61.5 & 80.6 & 82.1 & 5.900 & -- & -- & \\
\hline 5 & 39 & 39.8 & 45.7 & 55.7 & 70.5 & 72.0 & 3.500 & 1.633 & 14.30 & \\
\hline
\end{tabular}


Table 33. Mallard duckling bioassay, 1991--Continued

\begin{tabular}{|c|c|c|c|c|c|c|c|c|c|c|}
\hline \multirow{2}{*}{ Site } & \multirow{2}{*}{$\begin{array}{c}\text { Bird } \\
\text { identi- } \\
\text { fication } \\
\text { No. }\end{array}$} & \multicolumn{5}{|c|}{ Weight for indicated day of testing } & \multirow{2}{*}{$\begin{array}{c}\text { Terminal } \\
\text { liver } \\
\text { weight, } \\
\text { day } 4 \text { of } \\
\text { testing }\end{array}$} & \multirow{2}{*}{$\begin{array}{c}\text { Brain } \\
\text { weight, } \\
\text { day } 4 \text { of } \\
\text { testing }\end{array}$} & \multirow{2}{*}{$\begin{array}{l}\text { AChE } \\
\text { activity }\end{array}$} & \multirow{2}{*}{$\begin{array}{l}\Delta \mathrm{T} \\
\left({ }^{\circ} \mathrm{C}\right.\end{array}$} \\
\hline & & 0 & 1 & 2 & 3 & 4 & & & & \\
\hline
\end{tabular}

Test period 5: August 22 to 26--Continued

\begin{tabular}{|c|c|c|c|c|c|c|c|c|c|c|}
\hline 5 & 40 & 51.0 & 57.6 & 72.5 & 91.6 & 90.0 & 6.000 & 1.798 & 12.35 & -- \\
\hline 7 & 41 & 40.9 & 50.9 & 55.5 & 67.4 & 73.6 & 4.800 & 1.865 & 14.30 & -- \\
\hline 7 & 42 & 47.1 & 56.5 & 62.4 & 81.6 & 88.6 & -- & 1.899 & 12.67 & 2.0 \\
\hline 7 & 43 & 45.0 & 54.7 & 62.0 & 81.4 & 85.8 & 5.400 & 1.942 & 16.25 & -. \\
\hline 7 & 44 & 44.8 & 54.3 & 60.2 & 73.6 & 84.2 & -- & 1.861 & 14.95 & 4.2 \\
\hline 7 & 45 & 48.4 & 52.6 & 60.3 & 75.6 & 81.0 & -- & 1.878 & 15.27 & 2.2 \\
\hline 7 & 46 & 45.1 & 54.8 & 61.8 & 82.0 & 85.7 & 5.700 & 1.686 & 10.72 & -- \\
\hline 7 & 47 & 47.4 & 55.4 & 61.4 & 75.7 & 83.2 & 4.900 & -. & -- & -- \\
\hline 7 & 48 & 47.1 & 59.6 & 64.6 & 82.0 & 88.7 & 6.100 & 1.939 & 10.72 & -- \\
\hline 7 & 49 & 43.3 & 51.9 & 55.8 & 70.3 & 72.4 & 4.000 & 1.890 & 14.30 & -- \\
\hline 7 & 50 & 43.2 & 50.6 & 57.5 & 71.1 & 74.5 & 4.500 & -- & -- & -. \\
\hline 7 & 51 & 44.3 & 51.5 & 61.7 & 74.5 & 79.6 & -- & 1.477 & 14.95 & 5.6 \\
\hline 7 & 52 & 43.2 & 49.8 & 56.6 & 74.7 & 75.8 & 4.200 & -- & -- & -. \\
\hline 7 & 53 & 48.0 & 55.7 & 61.7 & 80.7 & 83.2 & 5.400 & 1.897 & 16.90 & -- \\
\hline 7 & 54 & 44.8 & 57.6 & 59.2 & 72.2 & 77.3 & 4.600 & -- & -- & -. \\
\hline 7 & 55 & 48.5 & 56.6 & 63.4 & 82.2 & 85.2 & 4.200 & 1.954 & 14.95 & -. \\
\hline 7 & 56 & 45.9 & 53.7 & 62.1 & 76.0 & 83.4 & -- & 1.721 & 13.00 & 2.6 \\
\hline 7 & 57 & 48.8 & 49.9 & 58.2 & 72.7 & 77.6 & 4.600 & 2.043 & 14.30 & -. \\
\hline 7 & 58 & 42.4 & 56.7 & 62.0 & 77.6 & 80.6 & 5.100 & 1.991 & 14.30 & -- \\
\hline 7 & 59 & 51.2 & 57.0 & 62.8 & 79.3 & 85.2 & -- & 1.801 & 11.37 & 1.9 \\
\hline 7 & 60 & 47.3 & 57.1 & 63.6 & 80.6 & 85.5 & 5.900 & 1.789 & 14.63 & -. \\
\hline 1 & 81 & 42.8 & 51.1 & 58.3 & 71.3 & 77.6 & 4.500 & 1.792 & 15.27 & -- \\
\hline 1 & 82 & 44.5 & 52.6 & 62.5 & 77.4 & 82.3 & 4.500 & 1.879 & 15.92 & -- \\
\hline 1 & 83 & 44.4 & 51.3 & 59.0 & 73.2 & 77.2 & 5.600 & -- & -- & -- \\
\hline 1 & 84 & 40.6 & 45.6 & 52.3 & 58.4 & 61.6 & 5.300 & 1.786 & 13.32 & -- \\
\hline 1 & 85 & 44.9 & 53.1 & 61.3 & 77.9 & 79.5 & 5.600 & 1.840 & 14.62 & -- \\
\hline 1 & 86 & 41.4 & 50.2 & 59.7 & 77.1 & 82.8 & 4.700 & -- & -- & -- \\
\hline 1 & 87 & 49.2 & 55.4 & 68.0 & 82.9 & 86.5 & 4.700 & -- & -- & -. \\
\hline 1 & 88 & 38.9 & 45.9 & 52.7 & 65.6 & 69.0 & 3.800 & -- & -- & -- \\
\hline 1 & 89 & 45.2 & 50.7 & 61.0 & 75.0 & 80.4 & 4.900 & 1.759 & 13.97 & -- \\
\hline 1 & 90 & 46.5 & 55.3 & 60.2 & 81.7 & 81.0 & 5.900 & -- & -- & -. \\
\hline 1 & 91 & 50.8 & 59.9 & 70.2 & 90.5 & 94.9 & 7.500 & -- & -- & -. \\
\hline 1 & 92 & 47.2 & 58.1 & 68.6 & 83.0 & 91.3 & 5.600 & -- & -- & -- \\
\hline 1 & 93 & 46.8 & 55.0 & 62.1 & 78.9 & 83.4 & 6.200 & 1.787 & 17.55 & -- \\
\hline 1 & 94 & 44.4 & 52.5 & 60.4 & 75.5 & 81.6 & 5.900 & -- & -- & -- \\
\hline 1 & 95 & 44.8 & 54.6 & 62.6 & 81.4 & 85.2 & 6.500 & 1.929 & 16.90 & -- \\
\hline 1 & 96 & 41.4 & 43.1 & 46.2 & 52.5 & 53.4 & 3.300 & -- & -- & -- \\
\hline 1 & 97 & 41.2 & 48.3 & 58.6 & 72.3 & 76.2 & 4.400 & 1.638 & 15.60 & -. \\
\hline 1 & 98 & 49.2 & 62.0 & 71.0 & 91.8 & 97.0 & 6.300 & -- & -- & -- \\
\hline 1 & 99 & 42.5 & 52.0 & 59.2 & 74.5 & 80.9 & 5.600 & 1.664 & 14.95 & -. \\
\hline 1 & 100 & 39.5 & 48.6 & 53.7 & 67.5 & 69.8 & 4.800 & 1.827 & 16.90 & -- \\
\hline
\end{tabular}


Table 34. In situ bioassay in sites downstream from an acrolein (Magnicide- $H^{\circledR}$ ) application in the J-7 Canal, July 13-14, 1992

[See figure 2 for location of injection point. Daphnia and minnow bioassay values in percent survival; a new set of animals was set out every 24 hours; $\mathrm{km}$, kilometers]

\begin{tabular}{|c|c|c|c|c|c|c|c|c|}
\hline \multirow{2}{*}{$\begin{array}{c}\text { Distance } \\
\text { downstream } \\
(\mathrm{km})\end{array}$} & \multicolumn{4}{|c|}{ Daphnia } & \multicolumn{4}{|c|}{ Minnows } \\
\hline & $\begin{array}{l}24 \text { hours } \\
\text { pre- } \\
\text { application }\end{array}$ & $\begin{array}{l}0 \text { hour } \\
\text { at } \\
\text { application }\end{array}$ & $\begin{array}{c}2 \text { hours } \\
\text { post- } \\
\text { application }\end{array}$ & $\begin{array}{l}24 \text { hours } \\
\text { post- } \\
\text { application }\end{array}$ & $\begin{array}{l}24 \text { hours } \\
\text { pre- } \\
\text { application }\end{array}$ & $\begin{array}{c}0 \text { hour } \\
\text { at } \\
\text { application }\end{array}$ & $\begin{array}{l}2 \text { hours } \\
\text { post- } \\
\text { application }\end{array}$ & $\begin{array}{l}24 \text { hours } \\
\text { post- } \\
\text { application }\end{array}$ \\
\hline 1.0 & 80 & 0 & 0 & 75 & 65 & 0 & 0 & 10 \\
\hline 3.2 & 90 & 80 & 95 & 65 & 60 & 70 & 10 & 10 \\
\hline 3.9 & 95 & 70 & 100 & 100 & 85 & 80 & 60 & 55 \\
\hline 4.8 & 95 & 95 & 95 & 100 & 85 & 85 & 90 & 25 \\
\hline
\end{tabular}


Table 35. In situ bioassay in leaseland waterways at Tule Lake National Wildlife Refuge, 1992

[See figures 4 and 5 for site locations. --, no data. Results of Daphnia (Daphnia magna) and mallard (Anas platyrynchos) in situ bioassays. Test results are the average percent survival observed in test chambers]

\begin{tabular}{|c|c|c|c|c|c|c|c|c|c|}
\hline \multicolumn{10}{|c|}{ Daphnia In Situ Bioassay } \\
\hline Site & $\begin{array}{l}\text { Water- } \\
\text { way }\end{array}$ & $\begin{array}{c}\text { Canal or } \\
\text { drain }\end{array}$ & $\begin{array}{l}\text { Test } \\
\text { date }\end{array}$ & $\begin{array}{l}\text { Daphnia } \\
\text { survival }\end{array}$ & Site & $\begin{array}{l}\text { Water- } \\
\text { way }\end{array}$ & $\begin{array}{c}\text { Canal or } \\
\text { drain }\end{array}$ & $\begin{array}{l}\text { Test } \\
\text { date }\end{array}$ & $\begin{array}{l}\text { Daphnia } \\
\text { survival }\end{array}$ \\
\hline \multirow[t]{3}{*}{ LL6 } & 102-C- & drain & July 10 & 25 & LL41 & 102 & drain & July 5 & 75 \\
\hline & Bypass & & & & LL46 & 101 & drain & July 8 & 10 \\
\hline & & & July 11 & 70 & & & & Aug. 2 & 85 \\
\hline \multirow[t]{2}{*}{ LL7 } & $102-\mathrm{C}-1$ & drain & July 10 & 5 & LL56 & $\mathrm{N}-1$ & canal & July 9 & 45 \\
\hline & & & July 11 & 55 & & & & July 10 & 20 \\
\hline \multirow[t]{2}{*}{ LL7A } & $102-\mathrm{C}-1$ & drain & July 6 & 85 & & & & July 11 & 40 \\
\hline & & & July 7 & 100 & LL57 & N-1 & canal & July 9 & 75 \\
\hline \multirow{2}{*}{ LL8 } & $102-C-2$ & dräin & July 10 & 40 & & & & July 10 & 25 \\
\hline & & & July 11 & 75 & & & & July 11 & 35 \\
\hline \multirow[t]{3}{*}{ LL10 } & $102-C-2$ & drain & July 6 & 90 & & & & Aug. 20 & 20 \\
\hline & & & July 7 & 75 & LL70 & $\mathrm{N}-4$ & canal & July 9 & 85 \\
\hline & & & Aug. 21 & 70 & & & & July 10 & 100 \\
\hline \multirow[t]{6}{*}{ LL13 } & 102-C-1 & drain & July 6 & 90 & & & & July 11 & 0 \\
\hline & & & July 7 & 65 & LL71 & $101-\mathrm{C}-4$ & drain & July 8 & 60 \\
\hline & & & July 8 & 100 & & & & July 9 & 0 \\
\hline & & & July 9 & 80 & & & & July 10 & 10 \\
\hline & & & July 10 & 80 & & & & July 11 & 0 \\
\hline & & & July 11 & 90 & LL77 & $101-\mathrm{C}-2$ & drain & July 8 & 10 \\
\hline \multirow[t]{2}{*}{ LL14 } & $102-\mathrm{C}-1$ & drain & July 6 & 65 & LL78 & $101-\mathrm{C}-2$ & drain & July 8 & 15 \\
\hline & & & July 7 & 95 & LL79 & $101-C$ & drain & July 8 & 30 \\
\hline \multirow{2}{*}{ LL16 } & R-1 & canal & July 8 & 75 & & & & Aug. 20 & 45 \\
\hline & & & Aug. 21 & 100 & LL87 & $N-12-c$ & canal & July 10 & 100 \\
\hline \multirow[t]{4}{*}{ LL17 } & R-1 & canal & July 8 & 15 & & & & July 11 & 35 \\
\hline & & & July 9 & 90 & LL88 & $\mathrm{N}-12-\mathrm{c}$ & canal & July 3 & 45 \\
\hline & & & July 10 & 55 & & & & July 10 & 50 \\
\hline & & & July 11 & 100 & & & & July 11 & 65 \\
\hline LL18 & $102-C-1-b$ & drain & July 8 & 55 & LL89 & $\mathrm{N}-12-\mathrm{c}$ & canal & July 3 & 90 \\
\hline LL25 & Q-3-b & canal & July 4 & 55 & & & & July 10 & 55 \\
\hline \multirow[t]{2}{*}{ LL26 } & Q-3 & canal & July 4 & 25 & & & & July 11 & 0 \\
\hline & & & Aug. 21 & 95 & LL90 & $\mathrm{N}-12-\mathrm{c}$ & canal & July 3 & 70 \\
\hline LL27 & $102-\mathrm{H}$ & drain & July 4 & 55 & & & & July 10 & 75 \\
\hline LL28 & $102-\mathrm{H}$ & drain & July 4 & 70 & & & & July 11 & 20 \\
\hline LL29 & $102-\mathrm{H}$ & drain & July 4 & 5 & & & & Aug. 22 & 100 \\
\hline LL30 & $102-\mathrm{H}$ & drain & July 4 & 55 & LL91 & $\mathrm{N}-12-\mathrm{c}$ & canal & July 3 & 85 \\
\hline \multirow[t]{2}{*}{ LL31 } & $\mathrm{Q}-3-\mathrm{a}$ & canal & July 3 & 90 & LL92 & $\mathrm{N}-12-\mathrm{c}$ & canal & July 3 & 80 \\
\hline & & & Aug. 21 & 100 & LL93 & $\mathrm{N}-12$ & canal & July 3 & 70 \\
\hline \multirow[t]{2}{*}{ LL32 } & Q-3 & canal & July 3 & 95 & & & & Aug. 20 & 0 \\
\hline & & & Aug. 21 & 100 & LL146 & N-15-b & canal & July 4 & 70 \\
\hline \multirow[t]{4}{*}{ LL33 } & $102-G$ & drain & July 8 & 75 & & & & July 5 & 45 \\
\hline & & & July 9 & 90 & & & & July 6 & 0 \\
\hline & & & July 10 & 85 & & & & July 8 & 0 \\
\hline & & & July 11 & 85 & & & & Aug. 20 & 100 \\
\hline \multirow[t]{3}{*}{ LL34 } & 102 & drain & July 5 & 60 & LL148 & N-15-b & canal & July 4 & 95 \\
\hline & & & July 8 & 65 & & & & July 5 & 60 \\
\hline & & & Aug. 21 & 95 & & & & July 6 & 60 \\
\hline LL35 & $102-\mathrm{C}-1$ & drain & July 8 & 60 & & & & July 8 & 55 \\
\hline
\end{tabular}


Table 35. In situ bioassay in leaseland waterways at Tule Lake National Wildlife Refuge, 1992--Continued

Daphnia In Situ Bioassay--Continued

\begin{tabular}{|c|c|c|c|c|c|c|c|c|c|}
\hline Site & $\begin{array}{l}\text { Water- } \\
\text { way }\end{array}$ & $\begin{array}{l}\text { Canal or } \\
\text { drain }\end{array}$ & $\begin{array}{l}\text { Test } \\
\text { date }\end{array}$ & $\begin{array}{l}\text { Daphnia } \\
\text { survival }\end{array}$ & Site & $\begin{array}{l}\text { Water- } \\
\text { way }\end{array}$ & $\begin{array}{c}\text { Canal or } \\
\text { drain }\end{array}$ & $\begin{array}{l}\text { Test } \\
\text { date }\end{array}$ & $\begin{array}{l}\text { Daphnia } \\
\text { survival }\end{array}$ \\
\hline \multirow[t]{3}{*}{ LL172 } & $101-B-5$ & drain & July 6 & 90 & LL233 & $101-B$ & drain & Aug. 11 & 0 \\
\hline & & & July 8 & 85 & & & & Aug. 12 & 0 \\
\hline & & & Aug. 20 & 0 & & & & Aug. 14 & 0 \\
\hline \multirow[t]{3}{*}{ LL173 } & 101-B-5-a & drain & July 6 & 65 & LL234 & $\mathrm{N}-12-\mathrm{b}$ & canal & Aug. 11 & 85 \\
\hline & & & July 8 & 20 & LL235 & N-12-b & canal & Aug. 11 & 90 \\
\hline & & & Aug. 20 & 100 & & & & Aug. 12 & 70 \\
\hline \multirow[t]{3}{*}{ LL174 } & $101-B-5-a$ & drain & July 6 & 55 & & & & Aug. 13 & 80 \\
\hline & & & July 8 & 10 & & & & Aug. 14 & 100 \\
\hline & & & July 9 & 35 & LL237 & 101-B-5-b & drain & July 24 & 20 \\
\hline LL175 & $101-B-5-d$ & drain & July 6 & 0 & LL238 & $101-B-5-b$ & drain & July 24 & 35 \\
\hline \multirow[t]{4}{*}{ LL176 } & & canal & July 6 & 95 & LL248 & $102-\mathrm{F}$ & drain & Aug. 11 & 70 \\
\hline & & & July 8 & 70 & & & & Aug. 12 & 0 \\
\hline & & & July 9 & 95 & & & & Aug. 13 & 0 \\
\hline & & & Aug. 20 & 0 & & & & Aug. 14 & 0 \\
\hline LL201 & $\mathbf{R}$ & canal & Aug. 1 & 55 & LL251 & $\mathbf{R}$ & canal & Aug. 1 & 90 \\
\hline \multirow{4}{*}{ LL205 } & $Q$ & canal & Aug. 11 & 70 & LL255 & $101-\mathrm{B}-5-\mathrm{c}$ & drain & Aug. 11 & 0 \\
\hline & & & Aug. 12 & 95 & & & & Aug. 12 & 0 \\
\hline & & & Aug. 13 & 90 & & & & Aug. 13 & 0 \\
\hline & & & Aug. 14 & 80 & LL259 & $\mathrm{N}-13$ & canal & Aug. 11 & 80 \\
\hline \multirow[t]{3}{*}{ LL206 } & $102-\mathrm{F}$ & drain & Aug. 11 & 5 & & & & Aug. 12 & 95 \\
\hline & & & Aug. 12 & 0 & & & & Aug. 13 & 85 \\
\hline & & & Aug. 13 & 5 & LL260 & $\mathbf{N}$ & canal & Aug. 11 & 90 \\
\hline LL214 & $\mathbf{R}$ & canal & Aug. 1 & 75 & & & & Aug. 14 & 80 \\
\hline LL215 & $\mathbf{R}$ & canal & Aug. 1 & 60 & LL261 & $101-B-5-d$ & drain & July 24 & 80 \\
\hline LL218 & $\mathrm{N}-4$ & canal & July 31 & 65 & LL262 & $\mathrm{N}-13$ & canal & Aug. 11 & 95 \\
\hline LL220 & $\mathrm{N}-6$ & canal & Aug. 7 & 100 & & & & Aug. 12 & 15 \\
\hline LL221 & N-6 & canal & July 31 & 60 & & & & & \\
\hline LL222 & $101-\mathrm{C}-6$ & drain & Aug. 7 & 35 & \multicolumn{5}{|c|}{ Mallard In Situ Bioassay } \\
\hline LL223 & $101-C-6$ & drain & $\begin{array}{l}\text { Aug. } 8 \\
\text { Aug. } 7 \\
\text { Aug. } 8\end{array}$ & $\begin{array}{r}40 \\
95 \\
100\end{array}$ & Site & $\begin{array}{l}\text { Water- } \\
\text { way }\end{array}$ & $\begin{array}{l}\text { Canal or } \\
\text { drain }\end{array}$ & $\begin{array}{l}\text { Test } \\
\text { date }\end{array}$ & $\begin{array}{l}\text { Mallard } \\
\text { survival }\end{array}$ \\
\hline LL224 & $101-\mathrm{C}-6$ & drain & Aug. 10 & 45 & LL201 & $\mathbf{R}$ & canal & Aug. 1 & 100 \\
\hline LL225 & $101-C$ & drain & Aug. 10 & 55 & LL215 & $\mathrm{R}$ & canal & Aug. 1 & 100 \\
\hline \multirow[t]{2}{*}{ LL226 } & $101-B-2$ & drain & Aug. 1 & 0 & LL226 & 101-B-2 & drain & Aug. 2 & 100 \\
\hline & & & Aug. 2 & 10 & LL227 & $101-\mathrm{B}-2$ & drain & Aug. 2 & 100 \\
\hline \multirow[t]{2}{*}{ LL227 } & $101-B-2$ & drain & Aug. 1 & 70 & Control & -- & -- & Aug. 2 & 100 \\
\hline & & & Aug. 2 & 100 & LL222 & $101-\mathrm{C}-6$ & drain & Aug. 8 & 100 \\
\hline LL230 & 101 & drain & July 31 & 5 & LL223 & $101-\mathrm{C}-6$ & drain & Aug. 8 & 100 \\
\hline \multirow[t]{2}{*}{ LL232 } & $\mathrm{N}-12-\mathrm{b}$ & canal & July 31 & 30 & LL224 & $101-\mathrm{C}$ & drain & Aug. 10 & 100 \\
\hline & & & & & Control & -- & -- & Aug. 10 & 100 \\
\hline
\end{tabular}


Table 36. Mallard duckling bioassay in leaseland waterways at Tule Lake National Wildlife Refuge, 1992

(See figures 4 and 5 for site locations. g, gram; --, no data. Cholinesterase activity expressed as micromoles of substrate hydrolyzed per minute per gram of tissue]

\begin{tabular}{|c|c|c|c|c|c|c|c|c|}
\hline \multirow{2}{*}{ Site } & \multirow{2}{*}{$\begin{array}{c}\text { Bird } \\
\text { identi- } \\
\text { fication }\end{array}$} & \multicolumn{2}{|c|}{ Weight change (g) } & \multirow{2}{*}{$\begin{array}{l}\text { Cholines- } \\
\text { terase } \\
\text { activity }\end{array}$} & \multirow{2}{*}{ Site } & \multirow{2}{*}{$\begin{array}{c}\text { Bird } \\
\text { identi- } \\
\text { fication }\end{array}$} & \multirow{2}{*}{$\begin{array}{c}\text { Weight } \\
\text { change (g) } \\
\text { 6-day-old }\end{array}$} & \multirow{2}{*}{$\begin{array}{c}\text { Cholines- } \\
\text { terase } \\
\text { activity }\end{array}$} \\
\hline & & 5-day-old & 6-day-old & & & & & \\
\hline \multicolumn{5}{|c|}{ August 1 and 2} & \multicolumn{4}{|c|}{ August 7 and 8} \\
\hline \multirow{10}{*}{ LL201 } & 21 & 3.30 & -- & 14.95 & \multirow{10}{*}{ LL222 } & 1 & 3.60 & 17.22 \\
\hline & 22 & 2.70 & -- & 17.55 & & 2 & 3.90 & 13.32 \\
\hline & 23 & 2.20 & -- & 15.92 & & 3 & 5.50 & 16.25 \\
\hline & 24 & 1.00 & -- & 12.35 & & 4 & 1.90 & 16.90 \\
\hline & 25 & .60 & -- & 14.62 & & 5 & 4.90 & 16.25 \\
\hline & 26 & -.10 & .- & 15.60 & & 6 & 5.60 & 14.95 \\
\hline & 27 & 5.80 & -- & 15.27 & & 7 & 4.20 & 17.55 \\
\hline & 28 & 1.60 & -- & 15.60 & & 8 & 3.40 & 16.90 \\
\hline & 29 & 4.60 & .- & 19.17 & & 9 & 4.30 & 17.22 \\
\hline & 30 & 2.50 & -- & 17.87 & & 10 & 3.90 & 15.60 \\
\hline \multirow[t]{9}{*}{ LL215 } & 11 & 4.30 & -- & 14.62 & \multirow[t]{10}{*}{ LL223 } & 11 & 5.70 & 13.32 \\
\hline & 12 & 3.00 & -- & 14.95 & & 12 & 6.10 & 16.25 \\
\hline & 13 & 5.40 & .- & 20.47 & & 13 & 6.50 & 15.92 \\
\hline & 14 & 5.90 & -- & 14.30 & & 14 & 2.70 & 13.97 \\
\hline & 15 & 3.40 & -. & 14.62 & & 15 & 6.60 & 17.87 \\
\hline & 16 & 4.10 & -- & 18.20 & & 16 & 3.20 & 15.60 \\
\hline & 17 & 3.50 & -- & 19.17 & & 17 & 5.30 & 14.30 \\
\hline & 18 & 3.80 & -- & 16.25 & & 18 & 5.80 & 16.90 \\
\hline & 19 & 3.20 & -- & 19.83 & & 19 & 2.80 & 13.65 \\
\hline LL226 & 1 & -- & 3.80 & 16.25 & & 20 & 2.80 & 18.20 \\
\hline & 2 & -. & 2.20 & 13.32 & Control & 21 & 7.40 & 19.82 \\
\hline & 3 & .. & 3.50 & 13.65 & & 22 & 4.20 & 16.25 \\
\hline & 4 & .. & 3.00 & 15.60 & & 23 & 7.30 & $\ldots$ \\
\hline & 5 & -- & 4.60 & 17.87 & & 24 & 5.10 & 18.52 \\
\hline & 6 & -- & 1.80 & 13.32 & & 25 & 6.40 & 14.95 \\
\hline & 7 & -- & 4.60 & 13.97 & & 26 & 8.10 & 15.92 \\
\hline & 8 & -- & 2.30 & 15.60 & & 27 & 7.60 & 13.32 \\
\hline & 9 & -- & .10 & 21.77 & & 28 & 8.10 & 14.62 \\
\hline & 10 & -- & 4.00 & 17.55 & & 29 & 6.30 & 16.25 \\
\hline LL227 & 51 & -- & 4.10 & 16.90 & & 30 & 4.10 & 17.22 \\
\hline & 52 & -- & $\begin{array}{r}6.80 \\
5.0\end{array}$ & 16.57 & & Bird & Weight & Cholines- \\
\hline & $\begin{array}{l}53 \\
54\end{array}$ & -- & $\begin{array}{l}5.20 \\
860\end{array}$ & 18.20 & Site & identi- & change $(\mathrm{g})$ & terase \\
\hline & $\begin{array}{l}54 \\
55\end{array}$ & $\begin{array}{l}-- \\
--\end{array}$ & $\begin{array}{l}8.60 \\
6.00\end{array}$ & $\begin{array}{l}17.55 \\
1690\end{array}$ & St & fication & 7-day-old & activity \\
\hline & 56 & -- & $\begin{array}{l}0.00 \\
7.90\end{array}$ & $\begin{array}{l}10.90 \\
17.87\end{array}$ & & & st 10 & \\
\hline & 57 & -- & 7.30 & 15.92 & $\overline{\text { LL224 }}$ & 31 & 9.4 & 12.35 \\
\hline & 58 & -- & -3.30 & 15.60 & & 32 & 8.4 & 16.58 \\
\hline & 59 & -- & 7.20 & 18.20 & & 33 & 8.1 & 18.52 \\
\hline & 60 & -- & 4.20 & 17.55 & & 34 & 6.5 & 13.97 \\
\hline LL251 & 41 & 2.00 & -- & 15.27 & & 35 & 4.9 & 15.92 \\
\hline & 42 & 1.80 & .- & 17.87 & & 36 & 7.3 & 17.55 \\
\hline & 43 & 3.50 & -- & 14.95 & & 37 & 3.5 & 16.90 \\
\hline & 44 & 4.10 & .- & 10.40 & & 38 & 8.5 & 13.98 \\
\hline & 45 & 2.10 & -- & 19.17 & & 39 & 11.1 & 11.70 \\
\hline & 46 & .60 & -- & 19.50 & & 40 & 4.7 & 16.25 \\
\hline & 47 & 2.90 & -- & 16.57 & Control & 41 & -- & - \\
\hline & 48 & $1.10^{\circ}$ & -- & 18.20 & & 42 & 1.7 & 16.57 \\
\hline & 49 & 6.70 & -- & 16.25 & & 43 & -- & - \\
\hline & 50 & 3.90 & -- & 15.27 & & 44 & -- & -- \\
\hline Control & 31 & -- & -- & 19.50 & & 45 & -- & -- \\
\hline & 32 & 2.70 & -- & 12.02 & & 46 & 2.1 & 15.27 \\
\hline & 33 & -- & -- & 21.45 & & 47 & -2.5 & 20.15 \\
\hline & 34 & -- & -- & 18.20 & & 48 & -.5 & 14.95 \\
\hline & 35 & 1.60 & -- & 13.65 & & 49 & $\ldots$ & $\ldots$ \\
\hline & 36 & 5.50 & .- & 17.55 & & 50 & 4.2 & 13.00 \\
\hline & 37 & 5.30 & -- & 16.90 & & & & \\
\hline & 38 &.- & -- & 19.50 & & & & \\
\hline & 39 & 4.70 & -- & 16.25 & & & & \\
\hline & 40 & -- & -- & 17.55 & & & & \\
\hline
\end{tabular}

